\title{
Fluorescent Dyes and Quenchers with Rigid Linkers
}

\author{
Dissertation \\ for the award of the degree \\ Doctor rerum naturalium \\ of the Georg-August-Universität Göttingen
}

within the doctoral program in Chemistry

of the Georg-August University School of Science (GAUSS)

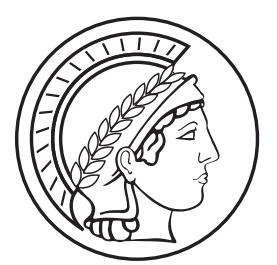

Department of NanoBiophotonics

Max Planck Institute for Biophysical Chemistry

Göttingen

Institute for Organic and Biomolecular Chemistry

Georg-August-Universität

Göttingen

\author{
submitted by \\ Dirk Norbert Hendrik Meineke \\ from Hamburg
}

Göttingen, 2017 



\section{Thesis committee}

Prof. Dr. Ulf Diederichsen, Institute of Organic and Biomolecular Chemistry, Georg-August-Universität Göttingen

Prof. Dr. Stefan W. Hell, Department of NanoBiophotonics, Max Planck Institute for Biophysical Chemistry

Dr. Vladimir N. Belov, Department of NanoBiophotonics, Max Planck Institute for Biophysical Chemistry

\section{Members of the examination board}

Reviewer: Prof. Dr. Ulf Diederichsen, Institute of Organic and Biomolecular Chemistry, Georg-August-Universität Göttingen

Second reviewer: Prof. Dr. Stefan W. Hell, Department of NanoBiophotonics, Max Planck Institute for Biophysical Chemistry

\section{Further members of the Examination board}

Prof. Dr. Marina Bennati, Max Planck Institute for Biophysical Chemistry and Institute of Organic and Biomolecular Chemistry, Georg-August-Universität Göttingen

Prof. Dr. Karl Helmut Grubmüller, Department of Theoretical and Computational Biophysics, Max Planck Institute for Biophysical Chemistry

Prof. Dr. Claudia Höbartner, Institute of Organic and Biomolecular Chemistry, Georg-August-Universität Göttingen

Prof. Dr. Konrad Koszinowski, Institute of Organic and Biomolecular Chemistry, Georg-August-Universität Göttingen

Date of oral examination: 02.03.2017 



\section{Affidavit}

I hereby declare that my doctoral thesis entitled "Fluorescent Dyes and Quenchers with Rigid Linkers" has been written independently and with no other sources and aids than quoted. I have indicated the parts which were performed by project collaborators.

Dirk N. H. Meineke

Göttingen, January 2017 

"An expert is a person who has found out by his own painful experience all the mistakes that one can make in a very narrow field." - Niels Bohr ${ }^{[1]}$ 



\section{Abstract}

Electronic energy transfer (EET) between chromophores is of fundamental importance for many natural processes and technical applications. However, common models fall short in fully describing the process, especially in bichromophoric compounds with a donor and acceptor connected by a linker, at very short distances in near perpendicular geometries. A fundamental problem is the role played by the linker, in particular its size, the angle between chromophores it imposes, and its ability to prevent rotations.

This thesis reports a novel strategy to prepare bichromophores containing adamantane or 2-(2-adamantylidene)adamantane as rigid spacers, providing access to bichromophores with fixed chromophore distance, and parallel or perpendicular relative orientation. Novel fluorophores were developed for the linkage to the spacers via spiroatoms, from which bichromophores with identical (blueblue) and different (blue-red) chromophores were synthesized, each in orthogonal and parallel geometry. These were fully characterized by absorption/fluorescence spectroscopy, time-resolved fluorescence anisotropy and antibunching measurements. Remarkably, efficient energy transfer has been found for all bichromophores, despite of the orthogonal arrangement. The observed EET efficiency was compared to the expected efficiency based on the Förster pointdipole approximation using density functional calculations. The results suggest, that Förster resonance energy transfer (FRET) is not sufficient to explain the EET efficiency of $100 \%$.

This novel strategy allows the synthesis of rigid bichromophores with a welldefined chromophore separation and relative orientation. 



\section{Contents}

I Introduction 1

1 Excitation energy transfer (EET) 3

1.1 Classification of EET processes . . . . . . . . . . . . 3

1.2 EET mechanisms . . . . . . . . . . . . . . . . 6

1.2.1 Förster resonance energy transfer . . . . . . . . 6

1.2.2 Dexter exchange mechanism . . . . . . . . . . 10

1.2.3 Through-bond excitation energy transfer . . . . . . . . 11

2 Bichromophoric compounds $\quad 15$

$2.1 \quad$ Molecular architecture of bichromophores . . . . . . . . . . . 15

2.2 Bichromophores with flexible linkers . . . . . . . . . . . . 15

2.3 Bichromophores with rigid and semi-rigid linkers . . . . . . . . 17

2.3.1 Bichromophores with helical linkers . . . . . . . . . . 17

2.3.2 Bichromophores with linear linkers . . . . . . . . . . . . . . 19

2.3.3 Bichromophores with scaffold linkers . . . . . . . . . . 23

2.4 Applications of multichromophoric compounds . . . . . . . . . . 29

2.4.1 Artificial photosynthesis . . . . . . . . . . . . . . . . 29

2.4.2 Bichromophores for DNA sequencing . . . . . . . . 30

2.4.3 Bichromophore in super-resolution microscopy . . . . . . . . 31

3 Experimental investigation of intramolecular EET 33

$3.1 \quad$ Steady-state fluorescence anisotropy measurements . . . . . . . . 33

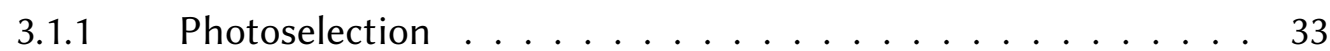

3.1.2 Fluorescence polarization ratio and anisotropy . . . . . . . 34

3.1.3 Influences on the fluorescence anisotropy . . . . . . . . . . 36

3.2 Time-resolved fluorescence anisotropy measurements . . . . . . . . 37

3.2.1 Anisotropy decays . . . . . . . . . . . . . . 37

3.2.2 Anisotropy decay due to excitation energy transfer . . . . . . . 38

3.3 Antibunching experiments . . . . . . . . . . . . . . . . 39

3.3.1 Hanbury Brown and Twiss (HBT) interferometer . . . . . . . . 39

3.3.2 Second-order correlation function . . . . . . . . . . . 41 
3.3.3 Photon antibunching in single molecule fluorescence spectroscopy . . . . . . . . . . . . . . . . . 42

3.3.4 Determination of emitters per molecule . . . . . . . . 43

3.3.5 Singlet-singlet annihilation . . . . . . . . . . . . . . 44

4 Aim and general strategy of this project 47

4.1 Aim of this project . . . . . . . . . . . . 47

4.2 General strategy . . . . . . . . . . . . . . . . . 47

II Results and Discussion $\quad 49$

5 Strategies to link fluorescent dyes to the adamantane scaffold 51

6 Spiroketal compounds $\quad 53$

6.1 Synthesis of bichromophore $36 \ldots \ldots$. . . . . . . . . . 53

6.2 Photophysical properties of model compounds 33a, 35a and bichromophore $36 \ldots \ldots$. . . . . . . . . . . . 53

6.3 DFT calculations for model compounds 33a, 35a and bichromophore $36 \ldots \ldots \ldots$. . . . . . . . . . . . 56

6.4 Estimation of energy transfer efficiency based on ideal Förster point-dipole approximation for bichromophore $36 \ldots$. . . . . . 58

7 Develelopment of spiroadamantylfluorene chromophores $\quad 61$

7.1 Spiroadamantylfluorene core build-up before chromophore modification . . . . . . . . . . . . . . . . 61

7.2 Synthesis of substituted spiroadamantylfluorene system from a precursor with already expanded aromatic system . . . . . . . 63

7.3 Photophysical properties of spiroadamantylfluorene $45 \ldots 66$

7.4 DFT calculations for model compound $45 \ldots \ldots 6$

7.5 Bathochromic modification of spiroadamantylfluorene 45 . . . . 67

8 Development of spiroadamantyl bithiophene based chro$\begin{array}{ll}\text { mophores } & \mathbf{7 1}\end{array}$

8.1 Photophysical properties of spiroadamantylbithiophene 77 . . . 73

8.2 Revised synthesis strategy of the red bithiophene based fluorophore 73

8.3 DFT calculations for model compound $77 \ldots \ldots 76$ 
9 Bichromophore $87 \quad 77$

9.1 Synthesis of bichromophore $87 \ldots \ldots \ldots 77$

9.2 Photophysical properties of bichromophore (87) . . . . . . . . . 78

9.3 DFT calculations for bichromophore $87 \ldots$. . . . . . . . . . . 81

9.4 Estimation of energy transfer efficiency based on ideal Förster point-dipole approximation for bichromophore $87 \ldots$. . . . . . 81

10 Advancement of the blue fluorophore $\quad 83$

10.1 Further development of the blue chromophore . . . . . . . . . 83

10.2 Development of model compound 105 and bichromophore 107 with symmetric blue fluorophores . . . . . . . . . . . . . 84

10.3 Photophysical properties of compounds $\mathbf{B}(\mathbf{1 0 5})$ and $\mathbf{B} \perp \mathbf{B}(\mathbf{1 0 7}) \ldots 88$

10.4 DFT calculations for model compound B (105) and bichromophore $\mathbf{B} \perp \mathbf{B}(\mathbf{1 0 7}) \ldots \ldots \ldots$. . . . . . . . . . 89

10.5 Estimation of energy transfer efficiency based on ideal Förster point-dipole approximation for bichromophore $\mathbf{B} \perp \mathbf{B}(\mathbf{1 0 7}) \ldots . .91$

11 Advancement of the red fluorophore 93

11.1 Disadvantages and suggested improvements of previous red chromophore ......................... 93

11.2 Synthesis of $\mathbf{R}(\mathbf{1 1 2}) \ldots \ldots \ldots$. . . . . . . . . . . . 93

11.3 Photophysical properties of compound R (112) . . . . . . . . . . . 94

11.4 DFT calculations for model compound $\mathbf{R}(\mathbf{1 1 2}) \ldots \ldots 5$

12 Bichromophore $B \perp R(114) \quad 97$

12.1 Synthesis of bichromopohore $\mathbf{B} \perp \mathbf{R}(\mathbf{1 1 4}) \ldots \ldots$. . . . . . . . 98

12.2 Photophysical properties of bichromophore $\mathbf{B} \perp \mathbf{R}(\mathbf{1 1 4}) \ldots$. . . . . 99

12.3 DFT calculations for bichromophore $\mathbf{B} \perp \mathbf{R}(\mathbf{1 1 4}) \ldots . . \ldots 100$

12.4 Estimation of energy transfer efficiency based on ideal Förster point-dipole approximation for bichromophore $\mathbf{B} \perp \mathbf{R}(\mathbf{1 1 4}) \ldots \ldots$

13 Bichromophores with parallel arranged fluorophores 103

13.1 Synthesis of compounds with parallel chromophores . . . . . . . 104

13.2 Photophysical properties of compounds with parallel chromophores 104

13.3 DFT calculations for bichromophores B $\| \mathbf{B}(\mathbf{1 1 6})$ and $\mathbf{B} \| \mathbf{R}(\mathbf{1 1 8})$. . 106 
13.4 Estimation of energy transfer efficiency based on ideal Förster point-dipole approximation for bichromophores B $\| \mathbf{B}(\mathbf{1 1 6})$ and

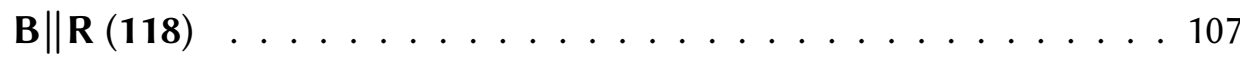

14 Time-resolved fluorescence anisotropy experiments to study intramolecular EET

$14.1 \quad$ EET in bichromophore $\mathbf{B} \perp \mathbf{B}(\mathbf{1 0 7}) \ldots \ldots$. . . . . . . . . 109

14.2 EET in bichromophores $\mathbf{B} \perp \mathbf{R}(\mathbf{1 1 4})$ and $\mathbf{B} \| \mathbf{R}(\mathbf{1 1 8}) \ldots 112$

14.3 Effective hydrodynamic volumes . . . . . . . . . . 120

15 Evidence for intramolecular EET by antibunching experiments 123

\section{Conclusion and Outlook}

\section{Experimental Part $\quad 131$}

16 General remarks 133

16.1 Solvents and reagents . . . . . . . . . . . . . . 133

16.2 Preparative methods . . . . . . . . . . . . . . 133

16.2.1 Thin layer chromatography (TLC) . . . . . . . . . . 133

16.2.2 Column chromatography . . . . . . . . . . . . 133

16.3 Instrumental analytics . . . . . . . . . . . . . . . . 134

16.3.1 Steady-state absorption and emission spectroscopy . . . . . 134

16.3.2 Nuclear magnetic resonance (NMR) . . . . . . . . . . . . 134

16.3.3 Mass spectrometry (MS) . . . . . . . . . . . . . . 135

16.3.4 High-performance liquid chromatography (HPLC) . . . . . . 135

17 Time-resolved fluorescence anisotropy measurements 137

18 Antibunching experiments 139

19 DFT and TD-DFT calculations 141

19.1 Ground state geometry optimization . . . . . . . . . . . . . 141

19.2 Excited state geometry optimization . . . . . . . . . . . . . 142

19.3 Electronic transitions . . . . . . . . . . . . . . . . . . . 142 
19.4 Transferring transition dipoles from excited to ground state geometries . . . . . . . . . . . . . . . . . . . . 143

19.5 Placement of the transition dipoles within the molecular framework 144

20 Estimation of FRET efficiency based on density functional calculations and model compound properties $\quad 145$

20.1 Calculation of orientation factor $\kappa^{2} \ldots \ldots$. . . . . . . . 145

20.2 Determination of spectral overlap integral $J_{\mathrm{DA}} \ldots \ldots$. . . . . . 145

20.3 Calculation of Förster radius $R_{0} \ldots \ldots \ldots \ldots$. . . . . . . 145

20.4 Calculation of FRET efficiency . . . . . . . . . . . . . 146

21 General procedures $\quad 147$

21.1 GP1: General procedure for formation of spiroketals . . . . . . . 147

21.2 GP2: General procedure for coupling of aryl bromide to adamantane scaffold . . . . . . . . . . . . . . . . . . . 147

21.3 GP3: General procedure for cyclization of tertiary alcohols . . . . 148

22 Compounds $\quad 149$

22.1 Compound 33a . . . . . . . . . . . . . . . . . . . . . . 149

22.2 Compound $33 \mathbf{b} \ldots \ldots \ldots \ldots$

22.3 Compound 35a . . . . . . . . . . . . . . . . 150

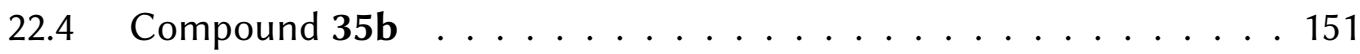

22.5 Compound $36 \ldots \ldots \ldots \ldots$

22.6 Compound $38 \ldots \ldots \ldots$

22.7 Compound 39b . . . . . . . . . . . . . . . . . . 154

22.8 Compound $40 \ldots \ldots \ldots \ldots$

22.9 Compound $42 \ldots \ldots \ldots \ldots$

22.10 Compound $43 \mathrm{~b} \ldots \ldots$. . . . . . . . . . . . . . . 157

22.11 Compound $44 \ldots \ldots \ldots \ldots$

22.12 Compound $45 \ldots \ldots \ldots$

22.13 4-[2'-Bromo-4'-(4-pyridyl)-4-biphenylyl]morpholine (48) . . . . 160

22.14 Compound $49 \ldots \ldots$. . . . . . . . . . . . . . . 161

22.15 4,4'-Dibromo-2-nitrobiphenyl (51) . . . . . . . . . . . . . 162

22.16 4'-Bromo-4-(4-pyridyl)-2-biphenylylamine (53) . . . . . . . . . 163

22.17 4-(4'-Bromo-2-nitro-4-biphenylyl)pyridine (54) . . . . . . . . . 164 
22.18 4'-Bromo-4-(4-pyridyl)-2 biphenylylamino-2,2-dimethylpropionate $(55) \ldots \ldots \ldots$. . . . . . . . . . . . 165

22.19 4-[2'-Nitro-4'-(4-pyridyl)-4-biphenylyl]morpholine (56) . . . . . . . 166

22.20 4'-Morpholino-4-(4-pyridyl)-2-biphenylylamine (58) . . . . . . . . 167

22.21 Compound $61 \ldots \ldots$. . . . . . . . . . . . . . . . 168

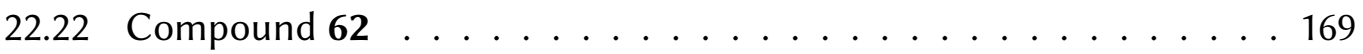

22.23 3-Bromo-2,2'-bithiophene $(64) \ldots \ldots$. . . . . . . . . . 170

$22.242,3$-Dibromothiophene $(68) \ldots \ldots \ldots$

22.25 Compound $70 \ldots \ldots$. . . . . . . . . . . . . . . 172

22.26 Compound $71 \ldots \ldots \ldots$. . . . . . . . . . . . . . . . . . . . . . . .

22.27 Compound $72 \ldots \ldots \ldots$. . . . . . . . . . . . . . . . . . . . . . . .

22.28 Compound $73 \ldots \ldots \ldots \ldots$

22.29 4-Methoxyphenylboronic acid (74) . . . . . . . . . . . . . 176

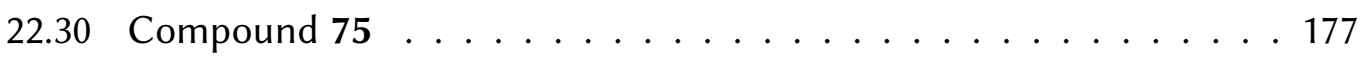

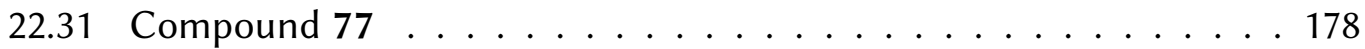

22.32 2,5-Dibromothiophene (79) . . . . . . . . . . . . . . . 179

22.33 2-Bromo-5-(4-methoxyphenyl)thiophene (80) . . . . . . . . 179

22.34 3-Bromo-5'-iodo-2,2'-bithienyl (81) . . . . . . . . . . . . . . . 180

22.35 3-Bromo-5'-( p-methoxyphenyl)-2,2'-bithienyl (82) $\ldots \ldots 181$

22.36 3-Bromo-5-(dimethoxymethyl)-5'-( -methoxyphenyl)-2,2'-bi-

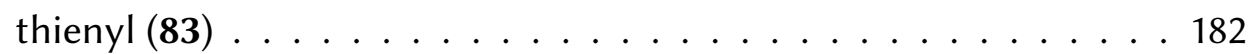

22.37 3-Bromo-5'-(p-methoxyphenyl)-2,2'-bithienyl-5-carbaldehyde (84) . 183

22.38 Compound 85 . . . . . . . . . . . . . . . . . . . . . . . . . . 184

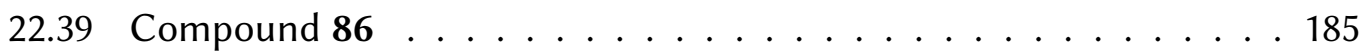

22.40 Compound $87 \ldots \ldots \ldots$. . . . . . . . . . . . . . 186

22.41 Compound $88 \ldots \ldots$. . . . . . . . . . . . . . . . . 187

22.42 Compound $89 \ldots \ldots$

22.43 2-[2',5'-Dibromo-4'-(2-thienyl)-4-biphenylyl]thiophene (90) . . . . 189

22.44 4,4'-Bis(2-thienyl)-2-biphenylylamine (91) . . . . . . . . . . . . 190

22.45 2-[2'-Bromo-4'-(2-thienyl)-4-biphenylyl]thiophene (92) . . . . . . . 191

22.46 1,2-Bis(tert-butoxycarbonyl)-1-phenylhydrazine (94) . . . . . . . 192

22.47 Di-tert-butyl-1-(3-bromophenyl)-2-phenylhydrazine-1,2-dicarboxylate (95) . . . . . . . . . . . . . . . . . . . . . . . . 194

22.48 3-lodobromobenzene $(97) \ldots \ldots$. . . . . . . . . . . . 195

22.49 2-Bromobiphenyl-4,4'-diamine (98) . . . . . . . . . . . . . 195 
22.50 5-Methyl-2-(tributylstannyl)thiophene (101) . . . . . . . . . 196

22.51 2-Bromo-4,4'-diiodobiphenyl (102) . . . . . . . . . . . . . . . 197

22.52 2-[2'-Bromo-4'-(5-methyl-2-thienyl)-4-biphenylyl]-5-methylthiophene (103) . . . . . . . . . . . . . . . . . . 198

22.53 Compound 104 . . . . . . . . . . . . . . . . . . . . 199

22.54 Compound 105 . . . . . . . . . . . . . . . . 200

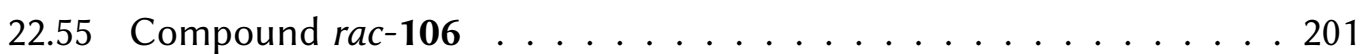

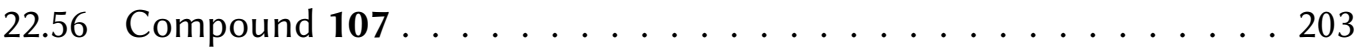

22.57 3-Bromo-5-iodo-5'-( $p$-methoxyphenyl)-2,2'-bithienyl (108) $\ldots . .204$

22.58 Compound 109 . . . . . . . . . . . . . . . . 205

22.59 3-Bromo-5'-(p-methoxyphenyl)-5-(4-pyridyl)-2,2'-bithienyl (110) . . 206

22.60 Compound $111 \ldots \ldots$. . . . . . . . . . . . . . . 207

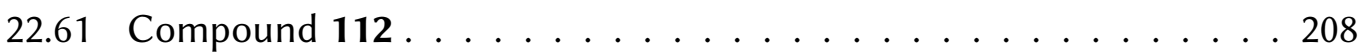

22.62 Compound 113 . . . . . . . . . . . . . . . . . . 209

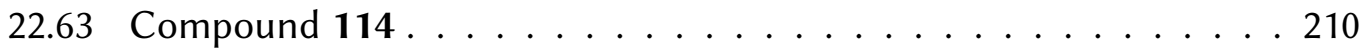

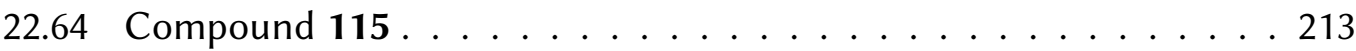

22.65 Compound 116 . . . . . . . . . . . . . . . . . 214

22.66 Compound 117 . . . . . . . . . . . . . . . . 215

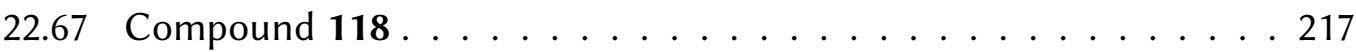

$\begin{array}{ll}\text { V Appendix } & 219\end{array}$

$\begin{array}{ll}\text { List of abbreviations } & 221\end{array}$

$\begin{array}{ll}\text { List of symbols } & 223\end{array}$

$\begin{array}{ll}\text { List of figures } & 225\end{array}$

List of schemes $\quad 229$

$\begin{array}{ll}\text { List of tables } & 231\end{array}$

$\begin{array}{ll}\text { References } & 233\end{array}$

$\begin{array}{lr}\text { Acknowledgments } & 245\end{array}$

$\begin{array}{lr}\text { Curriculum vitae } & 247\end{array}$ 



\section{Part I}

\section{Introduction}





\section{Excitation energy transfer (EET)}

Excitation energy transfer (EET) denotes a photophysical process where the excitation energy is transferred from an initially excited donor molecule $D^{*}$ to an acceptor molecule $\mathrm{A}^{\dagger}{ }^{\dagger}$ independent from the underlying transfer mechanism: ${ }^{[2]}$

$$
\mathrm{D}^{*}+\mathrm{A} \longrightarrow \mathrm{D}+\mathrm{A}^{*}
$$

In many cases EET is initiated by photoexcitation of the donor and it is the ubiquitous mechanism for energy transfer in multichromophoric systems. EET plays a key role in many natural ${ }^{[3]}$ and artificial processes. ${ }^{[4]}$ For example in natural photosynthesis, EET is of fundamental importance for harvesting and collecting of solar energy ${ }^{[3 a, 3 b, 3 c]}$ and plays a significant role in DNA damage due to absorption of ultraviolet radiation. ${ }^{[3 \mathrm{~d}, 3 \mathrm{e}]}$ But also for technical applications such as organic photovoltaics ${ }^{[4 \mathrm{~b}, 4 \mathrm{c}]}$ and organic light emitting diodes (OLEDs) ${ }^{[4 \mathrm{a}, 4 \mathrm{~d}]}$ EET represents a crucial step.

Although these examples show the immense importance of EET, the current models do not completely explain energy transfer at especially short distances and at perpendicular arrangement of transition dipole moments. ${ }^{[5]}$ In order to gain a better understanding of EET, it is reasonable to design molecules including both, the energy donor (D) and acceptor (A), which are connected by a linker (L). Such bichromophores of type A-L-D represent ideal model compounds for studying EET because of the constrained interchromophoric distance and in some cases even fixed relative chromophore orientation. The linker has a direct and significant impact on the EET in bichromophores. Besides defining the geometrical arrangement of chromophores and deformability of the assembly, the linker may also mediate the EET process. In the case of a mediated process, which is only possible because of the linker (e.g. superexchange interactions), the linker will be termed bridge in the following.

\subsection{Classification of EET processes}

EET can occur between different and two identical chromophores. The process is called heterotransfer, if the donor and acceptor molecules are not alike and homotransfer in the case of two identical molecules. ${ }^{[6,7]}$

\footnotetext{
${ }^{\dagger}$ Asterisk $\left({ }^{*}\right)$ marks excited molecules.
} 
Another distinction can be made between radiative processes, when the donor emits a photon which is absorbed by the acceptor, and non-radiative EET processes (scheme 1). ${ }^{[6]}$

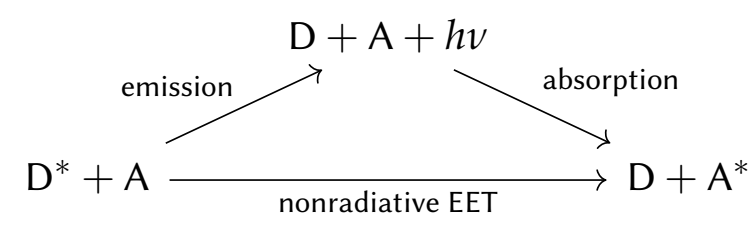

Scheme 1: Radiative and nonradiative energy transfer processes.

In bichromophores of type D-L-A, the transfer may be sequential or coherent (Scheme 2). In a sequential transfer at least one intermediate state, e.g. an excited bridge, is involved. Whereas in a coherent transfer the excitation of the acceptor and the relaxation of the donor occur simultaneously and no intermediate states are populated. ${ }^{[8]}$

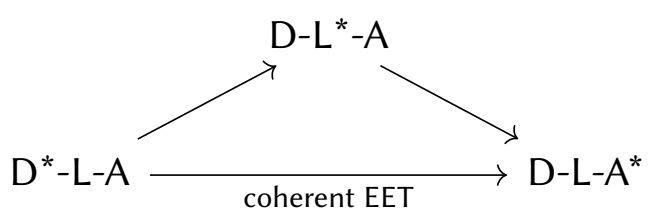

Scheme 2: Sequential and coherent energy transfer processes in donor-linker-acceptor (D-L-A) assemblies.

A sequential mechanism requires energies of the electronically excited bridge comparable to the energies of the excited donor and acceptor. ${ }^{[8]}$ For the bichromophores with non-conjugated linkers, the energies of the chromophores and the linker are usually very disparately. Thus, sequential mechanisms with an intermediate state comprising an excited bridge are unlikely.

According to Wigner's spin conversation rule in any allowed EET process, the overall spin angular momentum of the system should not change. ${ }^{[9]}$ Accordingly, an allowed EET transfer can be classified according to the maintained spin multiplicity: A distinction is made between singlet excitation energy transfer (SEET) and triplet excitation energy transfer (TEET) as illustrated in scheme 3. ${ }^{[8,10]}$ 


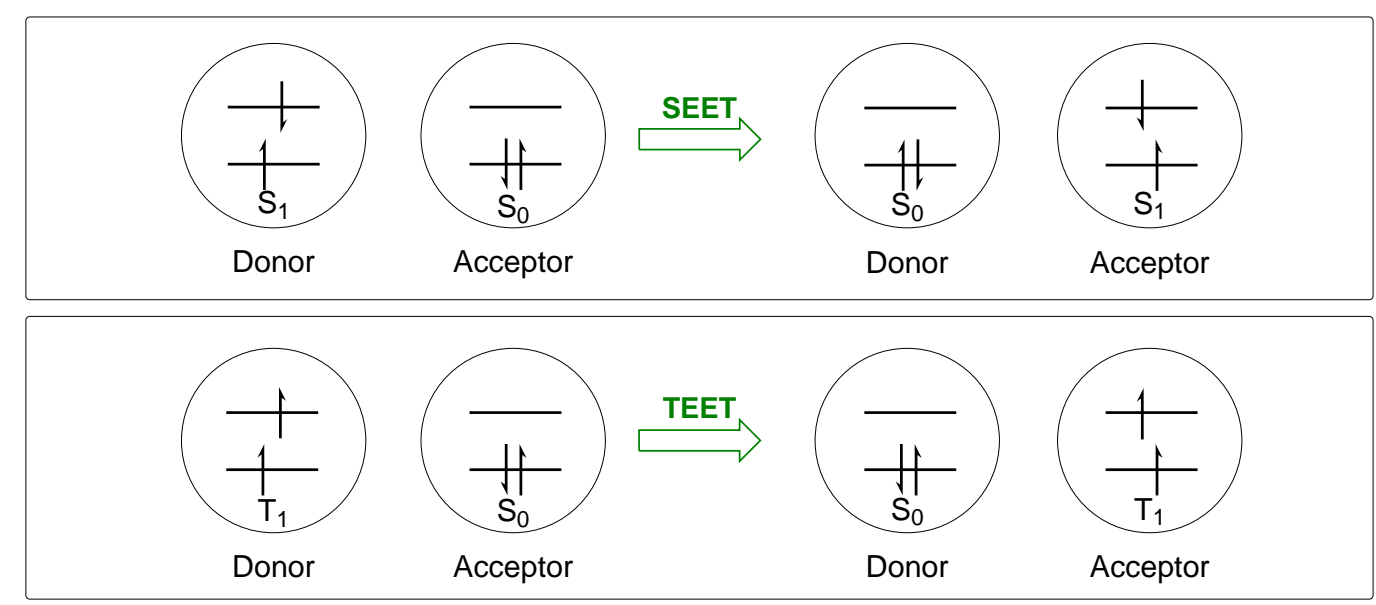

Scheme 3: Singlet excitation energy transfer (SEET) and triplet excitation energy transfer (TEET).

Scheme 4 gives an overview of the commonly used models to explain EET processes between donor and acceptor chromophores. The EET process between chromophores not connected by a linker has to occur through-space. These interactions can be attributed to Coulombic interactions, which are often approximated as Förster resonance energy transfer (FRET) or to classical Dexter exchange. For the latter orbital overlap (direct contact) of the chromophores is needed, as will be described later herein. In bichromophores, the two chromophores are connected by a linker and besides through-space interactions also through-bond interactions may occur. ${ }^{[11]}$ These interactions are often considered as long-range Dexter exchange interactions mediated by the bridging unit and often termed superexchange interactions. ${ }^{[12]}$ While SEET can be based on both (electrostatic Coulomb interactions and electron exchange interactions), TEET is exclusively possible by electron exchange interactions. ${ }^{[4 f]}$ 


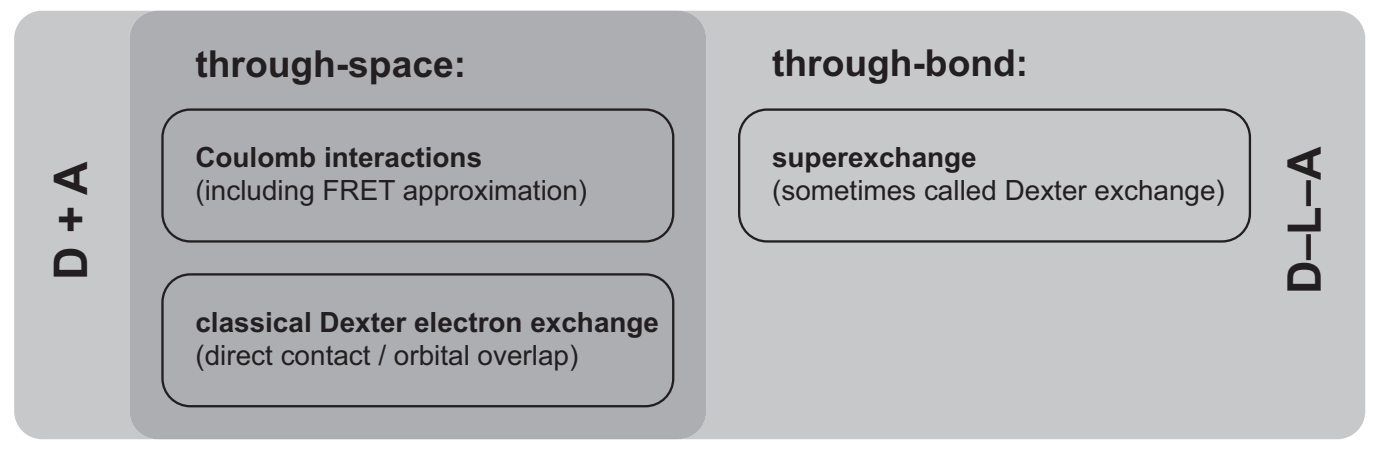

Scheme 4: Interactions enabling EET between independent $(D+A)$ and linked $(D-L-A)$ chromophores.

To which extent Coulombic or electron exchange interactions contribute to the EET process depends on several factors but most importantly on the distance. While FRET typically ranges up to interchromophoric distances of about $100 \AA$, Dexter exchange requires that the electron density distributions (i.e. orbitals) of the two chromophores overlap. ${ }^{[8]}$ Because the electron density decreases exponentially with distance from the nuclei, the overlap decreases correspondingly and Dexter EET is rarely observed to occur at distances over $15 \AA .{ }^{[13]}$ In the case of through-bond EET, the superexchange interactions can be effective over much larger distances $(>20 \AA)$, as will be described in section 1.2.3 in more detail.

\subsection{EET mechanisms}

The commonly used models to describe EET are the classical Förster ${ }^{[14-17]}$ and Dexter ${ }^{[18]}$ models, which will be described hereinafter. Also bridge mediated EET in bichromophores will be outlined afterwards. ${ }^{[8,12,19-24]}$

\subsubsection{Förster resonance energy transfer}

Förster (or fluorescence) energy transfer (FRET) is a non-radiative, throughspace energy transfer mechanism that describes the energy transfer from a donor fluorophore to an acceptor chromophore as dipole-dipole interaction between the donor excited state and acceptor ground state (scheme 5). ${ }^{[25]}$ According to the Franck-Condon principle, the donor fluorophore is in it's excited state geometry whereas the acceptor is in it's ground state geometry during the so called vertical transition. 


\section{SEET}

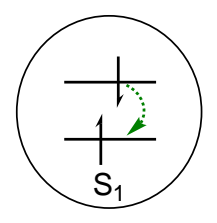

Donor

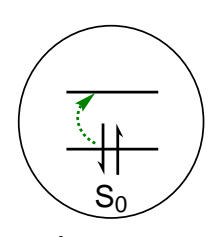

Acceptor

\section{FRET}

$\longrightarrow$

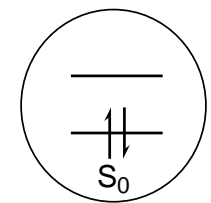

Donor

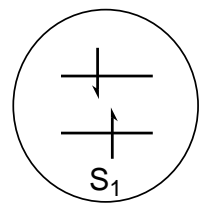

Acceptor

Scheme 5: Singlet excitation energy transfer (SEET) via Förster resonance energy transfer (FRET).

\section{Ideal dipole approximation}

The widely applied Förster approximation by the german physical chemist Theodor Förster ${ }^{[14-17]}$ involves two levels of approximation. The first level is, that the electronic coupling, $V$, can be completely represented by Coulomb in-

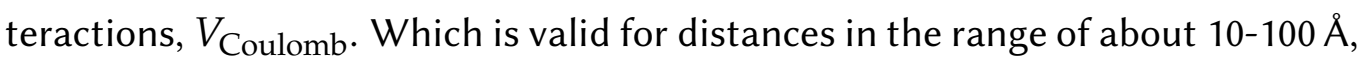
where the donor-acceptor distance is large enough to prevent orbital-overlap based interactions but not too large, where radiative mechanisms begin to dominate. ${ }^{[5 b]}$ To explain the second level of approximation, the Coulomb potential between two particles is represented as a multipole expansion, where the interaction is described by a series of multipole-multipole interactions: ${ }^{[26]}$

$$
\begin{aligned}
V_{\text {Coulomb }}\left(\vec{R}_{\mathrm{DA}}\right)= & \frac{1}{4 \pi \varepsilon_{0}}[\overbrace{\frac{\overbrace{\mathrm{D} q \mathrm{~A}}}{\left|\vec{R}_{\mathrm{DA}}\right|}}^{\begin{array}{c}
\text { charge-charge } \\
\text { interactions }
\end{array}}+\overbrace{\frac{q_{\mathrm{D}}\left(\vec{\mu}_{\mathrm{A}} \cdot \vec{R}_{\mathrm{DA}}\right)}{\left|\vec{R}_{\mathrm{DA}}\right|^{3}}-\frac{q_{\mathrm{A}}\left(\vec{\mu}_{\mathrm{D}} \cdot \vec{R}_{\mathrm{DA}}\right)}{\left|\vec{R}_{\mathrm{DA}}\right|^{3}}}^{\text {charge-dipole interactions }} \\
& +\underbrace{\left.\frac{\left|R_{\mathrm{DA}}\right|^{2}\left(\vec{\mu}_{\mathrm{D}} \cdot \vec{\mu}_{\mathrm{A}}\right)-3\left(\vec{\mu}_{\mathrm{D}} \cdot \vec{R}_{\mathrm{DA}}\right)\left(\vec{\mu}_{\mathrm{A}} \cdot \vec{R}_{\mathrm{DA}}\right)}{\left|\vec{R}_{\mathrm{DA}}\right|^{5}}+\ldots\right]}_{\text {dipole-dipole interactions }}
\end{aligned}
$$

with $\varepsilon_{0}$, vacuum permittivity, $q_{\mathrm{D}}$ and $q_{\mathrm{A}}$, electronic charges, $\vec{\mu}_{\mathrm{D}}$ and $\vec{\mu}_{\mathrm{A}}$, transition dipoles of donor and acceptor, respectively. $\vec{R}_{\mathrm{DA}}$ is the interconnecting vector between the donor and acceptor multipole expansion centers. $\left|\vec{R}_{\mathrm{DA}}\right|$ gives the distance between donor and acceptor multipole centers. The second level of the Förster approximation is assuming that only the dipole-dipole term is significant. Importantly, this holds only true for distances (much) larger than the dimensions of the chromophores. ${ }^{[5 b]}$ 


\section{FRET rate}

For a donor-acceptor pair separated by the distance $\left|\vec{R}_{\mathrm{DA}}\right|$, the rate of the FRET process $k_{\text {FRET }}$ is given by ${ }^{[6]}$

$$
k_{\mathrm{FRET}}=\underbrace{\frac{9 \ln (10)}{128 \pi^{5} n^{4} N_{\mathrm{A}}}}_{\text {solvent dependendconstant }} \cdot \underbrace{\frac{J_{\mathrm{DA}} \Phi_{\mathrm{D}}}{\tau_{\mathrm{D}}}}_{\begin{array}{c}
\text { photophysical } \\
\text { properties }
\end{array}} \cdot \underbrace{\frac{\kappa^{2}}{\left|\vec{R}_{\mathrm{DA}}\right|^{6}}}_{\begin{array}{c}
\text { geometrical } \\
\text { parameters }
\end{array}} .
$$

The expression can be divided in three parts: The first part gives a solvent depended constant which includes Avogadro's number $N_{\mathrm{A}}$ and the refractive index $n$ of the medium in which the donor and the acceptor are dissolved, to account for the screening effect of the medium on the dipole-dipole interaction. ${ }^{\text {[27] }}$ Furthermore, the rate depends on photophysical properties, such as the fluorescence quantum yield of the donor in absence of the acceptor $\Phi_{\mathrm{D}}$, the fluorescence lifetime of the donor in absence of the acceptor $\tau_{D}$ and the overlap integral $J_{\mathrm{DA}}$ of the donor emission and acceptor absorption. The important geometry factors include the center-to-center distance between the donor and acceptor $\left|\vec{R}_{\mathrm{DA}}\right|$ and the orientation factor $\kappa^{2}$, which depends on the relative alignment of the transition dipole moments as explained below.

\section{Overlap integral}

The overlap integral $J_{\mathrm{DA}}$ between the normalized donor emission and acceptor absorption in units of $\mathrm{M}^{-1} \mathrm{~cm}^{-1} \mathrm{~nm}^{4}$ is defined as

$$
J_{\mathrm{DA}}=\int_{0}^{\infty} \varepsilon_{\mathrm{A}}(\lambda) \lambda^{4} f_{\mathrm{D}}(\lambda) \mathrm{d} \lambda
$$

with $\varepsilon_{\mathrm{A}}(\lambda)$ the molar extinction of the acceptor and $f_{\mathrm{D}}(\lambda)$ the donor emission after normalizing the emission spectrum to an area of 1 . The overlap integral assures energy conservation and is related to the Franck-Condon weighted density of states. ${ }^{[8]}$ 


\section{Orientation factor}

The orientation factor $\kappa^{2}$ is defined by the relative orientation of the transition dipole moments of the donor $\left(\vec{\mu}_{\mathrm{D}}\right)$ and acceptor $\left(\vec{\mu}_{\mathrm{A}}\right)$ and is given by

$$
\begin{aligned}
\kappa^{2} & =\left[\cos \left(\theta_{\mathrm{DA}}\right)-3 \cos \left(\theta_{\mathrm{D}}\right) \cos \left(\theta_{\mathrm{A}}\right)\right]^{2} \\
& =\left[\vec{\mu}_{\mathrm{D}} \cdot \vec{\mu}_{\mathrm{A}}-3\left(\vec{R}_{\mathrm{DA}} \cdot \vec{\mu}_{\mathrm{D}}\right)\left(\vec{R}_{\mathrm{DA}} \cdot \vec{\mu}_{\mathrm{A}}\right)\right]^{2} .
\end{aligned}
$$

As shown in figure $1, \theta_{\mathrm{DA}}$ describes the angle between the two transition dipole moments of the energy donor $\vec{\mu}_{\mathrm{D}}$ and acceptor $\vec{\mu}_{\mathrm{A}}$. Whereas $\theta_{\mathrm{D}}$ and $\theta_{\mathrm{A}}$ are the angles between the two transition dipole moments and the interconnecting vector $\vec{R}_{\mathrm{DA}}$.

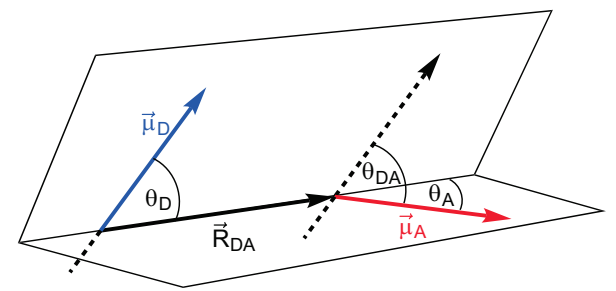

Figure 1: Relative orientation of energy donor and acceptor transition dipole moments $\vec{\mu}_{\mathrm{D}}$ and $\vec{\mu}_{\mathrm{A}}$ shown as red and blue vectors.

The orientation factor can have values between 0 and 4 .

\section{FRET efficiency}

The FRET efficiency can be represented as

$$
E_{\mathrm{FRET}}=\frac{k_{\mathrm{FRET}}}{k_{\mathrm{FRET}}+k_{\mathrm{D}}+\sum_{i} k_{\mathrm{D} i}}=\frac{1}{1+\frac{k_{\mathrm{D}}+\sum_{i} k_{\mathrm{D} i}}{k_{\mathrm{FRET}}}} .
$$

where $k_{\mathrm{D}}$ is the rate of the radiative deactivation and $\sum_{i} k_{\mathrm{D} i}$ is the rate of the nonradiative deactivations of excited state (other than FRET).

\section{Förster Radius}

The Förster radius (or critical distance) $R_{0}$ is defined as the distance between the energy donor and acceptor of a FRET pair at which the FRET efficiency $E_{\text {FRET }}$ is $50 \%$. According to equation 5 , in that case the spontaneous decay of the excited 
donor and FRET are equally probable $\left(k_{\mathrm{FRET}}=k_{\mathrm{D}}+\sum_{i} k_{\mathrm{D} i}\right)$. The Förster radius can be calculated by ${ }^{[6]}$

$$
R_{0}^{6}=\frac{9 \ln (10) \kappa^{2} \Phi_{\mathrm{D}} J_{\mathrm{DA}}}{128 \pi^{5} N_{\mathrm{A}} n^{4}} .
$$

The FRET efficiency can be calculated from the interchromophoric distance $\left|\vec{R}_{\mathrm{DA}}\right|$ and the Förster radius $R_{0}$ according to

$$
E_{\mathrm{FRET}}=\frac{1}{1+\left(\left|\vec{R}_{\mathrm{DA}}\right| / R_{0}\right)^{6}}
$$

\subsubsection{Dexter exchange mechanism}

The Dexter mechanism is often considered as a simultaneous exchange of two electrons between the donor in its electronically excited state and the acceptor in its ground state (scheme 6).

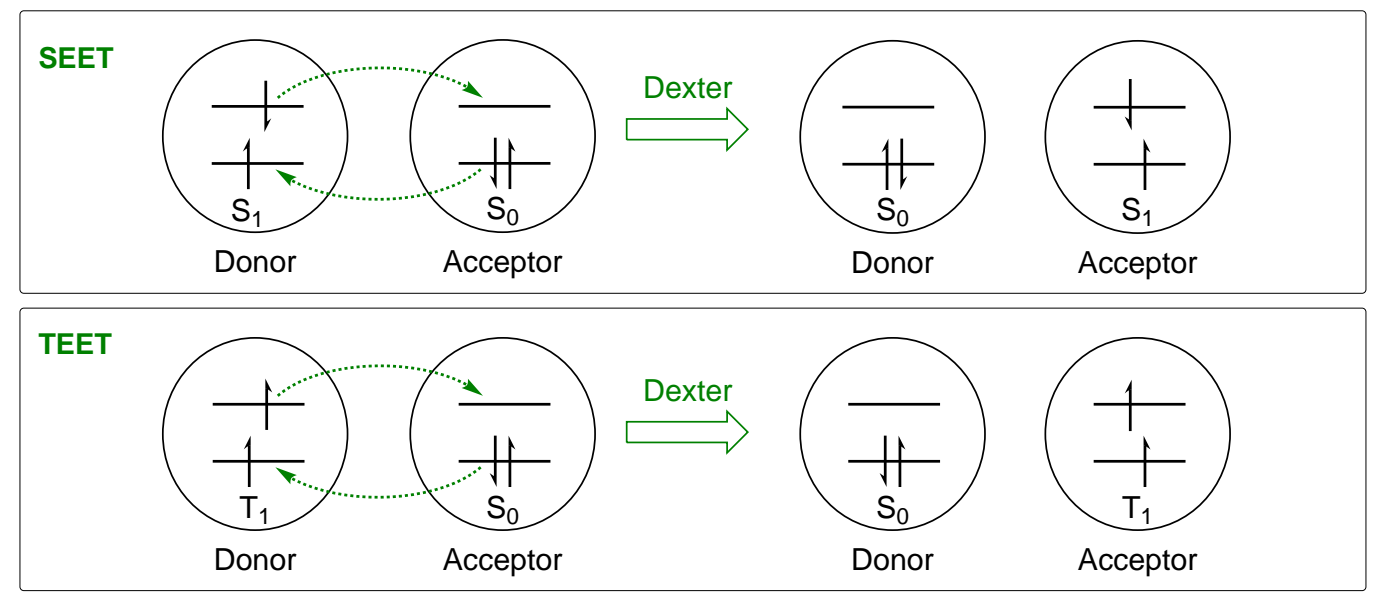

Scheme 6: Singlet and triplet excitation energy transfer (SEET, TEET) via Dexter electron exchange mechanism.

The Dexter exchange interaction is based on the orbital overlap between the donor and the acceptor which means physical contact between the chromphores electron densities. In contrast to the inverse sixth power dependence on the distance for the FRET mechanism, an exponential dependence is to be expected for the exchange mechanism. ${ }^{[6]}$ Dexter derived the following expression for the EET rate between donor and acceptor separated by $r_{\mathrm{DA}}$, with the edge-to-edge 
distance between chromophores in this case:

$$
k_{\text {Dexter }}=\frac{2 \pi}{h} K J_{\mathrm{DA}}^{\prime} \exp \left(\frac{-2 r_{\mathrm{DA}}}{L}\right)
$$

Where $L$ is the sum of van der Waals radii, $J_{\mathrm{DA}}^{\prime}$ is the normalized spectral overlap integral $J_{\mathrm{DA}}^{\prime}=\int_{0}^{\infty} f_{\mathrm{D}}(\lambda) \varepsilon_{\mathrm{A}}(\lambda) \mathrm{d} \lambda$ and $K$ is a constant. ${ }^{[6]}$ FRET cannot explain TEET because that would violate the Wigner spin conservation law. Consequently, the Dexter exchange mechanism plays a crucial role to depict the TEET process.

In bichromophores of the type D-B-A, the bridge (B) may extend the range of the electron exchange due to interactions with bridging orbitals. This through-bond EET, will be described in more detail in the next section.

\subsubsection{Through-bond excitation energy transfer}

Many deviations from the idealized Förster and Dexter models have been reported for EET between bridged chromophores. ${ }^{\left[{ }^{8,19-23]}\right.}$ The deviations are attributed to the mediating influence of the bridge on the EET and therefor often termed through-bond EET.
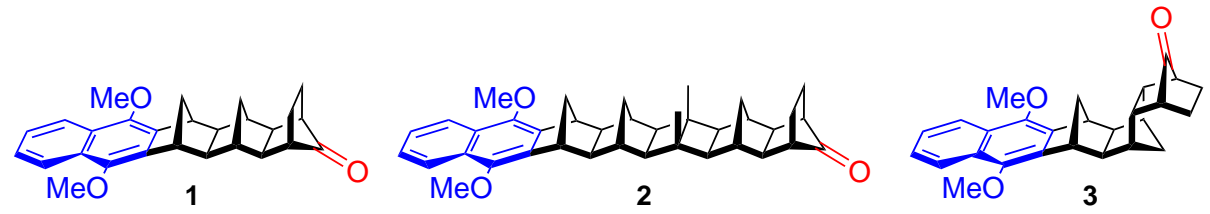

Figure 2: Bichromophores for studying through-bond SEET from 1,4-dimethoxynaphthalene (donor) via a rigid polynorbornyl system of varying length and geometry to a cyclic ketone (acceptor). ${ }^{[22,28]}$

In 1988 Verhoeven et al. reported fast singlet EET between 1,4-dimethoxynaphthalene as the energy donor and a cyclic ketone chromophores as acceptor which were connected by a rigid polynorbornyl systems of varying length (e.g. compounds 1 and 2 , see figure 2). The EET rates $k_{\text {SEET }}$ decreased exponentially with the number of $\sigma$-bonds between donor and acceptor. But the distance dependence was too weak for Dexter exchange interaction and too strong for FRET. ${ }^{[28]}$ Furthermore, it was shown later that the efficiency strongly depends on the conformation of the rigid bridge. The 


\section{\begin{tabular}{l|l} 
Chapter 1 & EXCITATION ENERGY TRANSFER (EET)
\end{tabular}}

energy transfer was more efficient in an all-s-trans than in a gauche or s-cis conformation (e.g. compounds $\mathbf{1}$ and $\mathbf{3}$, see Figure 2). ${ }^{[8,22]}$

These findings underline the important role of the linker in a bichromophoric compound and demonstrate the limitations of the idealized Förster and Dexter models.

In order to explain the through-bond singlet EET, a superexchange mechanism was assumed, which was originally developed by McConnel in order to explain the electron transfer (ET) in anionic radicals mediated by a molecular bridge. ${ }^{[29]}$ The link between Dexter electron exchange and electron transfer may be seen in formal description of electron exchange as two-electron exchange event. Due to the mediation of the bridge, the electronic exchange coupling decreases much slower with increasing donor-acceptor distance than expected from the decrease in orbital overlap.

Empirically was found, that the electron transfer rate follows the expression ${ }^{[8]}$

$$
k_{\mathrm{ET}}=k_{0} e^{-\beta r_{\mathrm{DA}}}
$$

where $k_{0}$ is the maximum rate constant at van der Waals contact, $r_{\mathrm{DA}}$ is the edge-to-edge distance between the donor and acceptor and $\beta$ is the so called attenuation factor which quantifies the mediating ability of a molecular framework.

Verhoeven et al. plotted $k_{\text {SEET }}$ versus the number of $\sigma$-bonds between the donor and the acceptor and found a slope of -1.45 per bond. Assuming a carbon-carbon bond length of $1.54 \AA$ this corresponds to a attenuation factor of $\beta=0.94 \AA^{-1}$. ${ }^{[28]}$ $\beta$ depends on the nature of the bridge, e.g. the bridge's rigidity, and on the energy gap between bridge electronic energies and the energy levels of the excited donor and acceptor. ${ }^{[30]}$

McConnel suggested that low lying "virtual" orbitals localized on the bridge could mediate electronic coupling between an electron donor and acceptor (scheme 7). This coherent process, where no intermediate states (with excited bridge) are involved, can be seen as a tunneling process. ${ }^{[31]}$ 


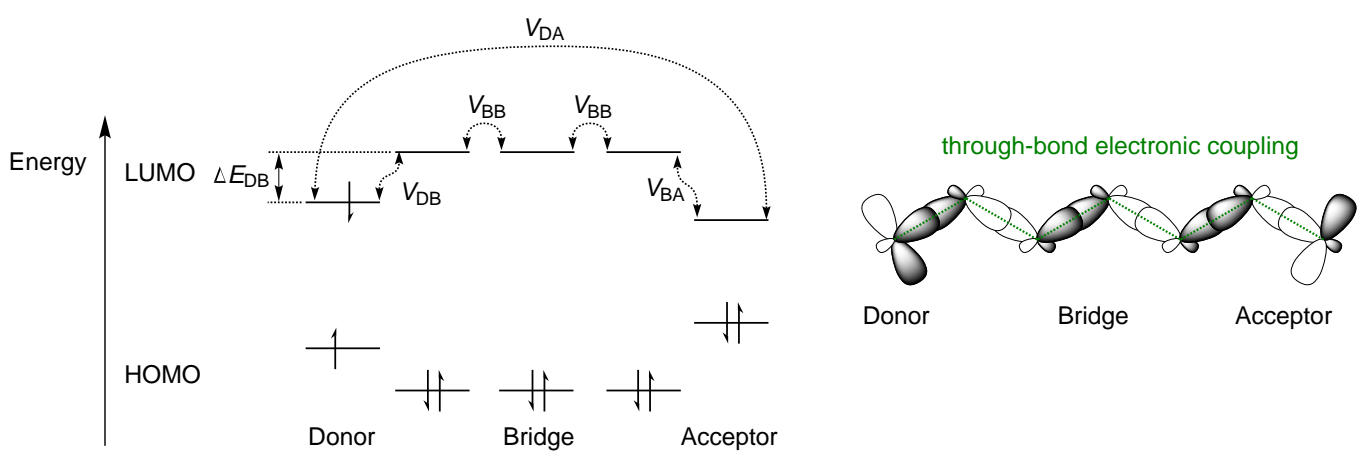

Scheme 7: McConnell superexchange model for electron tunneling through molecular structures. ${ }^{[31-34]}$

The total electronic coupling between a donor and acceptor $V_{\mathrm{DA}}$ mediated by a chain of $n$ identical units, is given by: ${ }^{[31]}$

$$
V_{\mathrm{DA}}=\frac{V_{\mathrm{DB}} V_{\mathrm{BA}}}{\Delta E_{\mathrm{DB}}}\left(\frac{V_{\mathrm{BB}}}{\Delta E_{\mathrm{DB}}}\right)^{n-1}
$$

The magnitude of the coupling is determined by the coupling of the donor and acceptor to the bridge ( $V_{\mathrm{DB}}$ and $V_{\mathrm{AB}}$ ), the nearest neighbor interactions, $V_{\mathrm{BB}}$, and the energy between the donor and bridge localized states $\Delta E_{\mathrm{DB}} \cdot{ }^{[32]}$

If the edge-to-edge distance between the donor and acceptor $r_{\mathrm{DA}}$ is equal to $n \cdot r_{\mathrm{B}}$, where $r_{\mathrm{B}}$ is the length of one repeating bridge unit, and the electronic coupling $V_{\mathrm{BB}}$ is much smaller than $\Delta E_{\mathrm{DB}}$, than the attenuation factor $\beta$ is given by: ${ }^{[8]}$

$$
\beta=\frac{2}{r_{\mathrm{B}}}\left|\frac{\Delta E_{\mathrm{DB}}}{V_{\mathrm{BB}}}\right|
$$

The influence of the energy gap between the donor and the bridge on $\beta$ was experimentally demonstrated by Albinsson et al. by using rod-like linkers with different lengths and energy levels (see section 2.3.2). ${ }^{[8,20]}$

Because the different EET mechanisms may be operative in parallel, the contributions of the individual processes needs to be considered in order to describe EET in bichromophores. ${ }^{[11,35,36]}$ A variety of molecular bichromophore architectures was developed for the study of EET mechanisms as will be presented in the following chapter. 



\section{Bichromophoric compounds}

A bichromophoric compound may be defined as a molecule which is built from two chromophores connected by a linker. The terms linker, bridge and spacer are often used synonymously. However, bridge is often used if the linker mediates electronic interactions between the chromophores, whereas spacer is commonly used if the linker does not mediate any electronic interaction.

Bichromophores have become a major tool for studying the mechanisms of ET and EET between a donor and an acceptor chromophore over the past decades. ${ }^{[36]}$ The limited conformational freedom, as well as the possibility to explore the effect of linker length (donor-acceptor separation) and the choice of appropriate chromophores allow systematically investigation of EET mechanisms. ${ }^{[12]}$ The well-defined separation, and in some cases also orientation of chromophores, facilitates the development of models which explain the experimental data.

\subsection{Molecular architecture of bichromophores}

The properties of the linker determine the flexibility of the whole bichromophore. The nature of linkers between chromophores is represented by a broad variety of chemical structures. ${ }^{[32,37]}$ In the following, the selected bichromophores are classified according to linker properties (molecular rigidity and geometry).

The first group includes bichromophores with a flexible linker. A flexible linker can adopt many possible conformations which may result in various distances between chromophores and orientations. The second group comprises bichromophores with a rigid and semi-rigid linker, which to some extent fix the distance between the chromophores. These include helical structures, linear and scaffold linkers. However, in most cases, the rotation around single bonds excludes any fixed orientations of chromophores. Examples of bichromophores with fixed distances between them and also fixed orientation are very rare.

\subsection{Bichromophores with flexible linkers}

The very first EET study in a bichromophoric molecule was done by Schnepp and Levy ${ }^{[38]}$ on naphthalene and anthracene connected by a polymethylene chain (4) 


\section{\begin{tabular}{l|l} 
Chapter 2 & BICHROMOPHORIC COMPOUNDS
\end{tabular}}

as shown in figure $3 .^{[30]}$

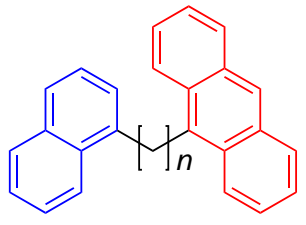

$4(n=1-9)$

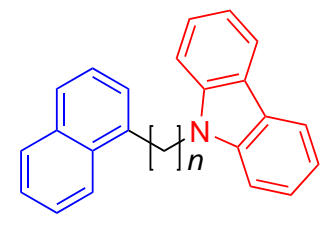

$5(n=4-12)$

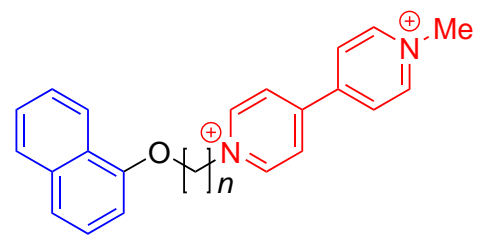

$6(n=3,6,8,10)$

Figure 3: Examples of bichromophoric compounds with flexible polymethylene linkers. ${ }^{[39,40]}$

Among many flexible linkers utilized for the design of bichromophores, ${ }^{\text {[39-53] }}$ polymethylene is certainly the simplest one. It's length can easily be modified by varying the number of methylene groups. Compounds of the type D- $\left(\mathrm{CH}_{2}\right)_{\mathrm{n}}-\mathrm{A}$ have been frequently used to study ET and EET processes between various chromophores. ${ }^{[39,40,47-53]}$ For example, bichromophore 5 was used to study intramolecular TEET, ${ }^{[39]}$ and bichromophore $\mathbf{6}$ - to investigate photoinduced $\mathrm{ET}^{[40]}$ (figure 3).

Another family of linkers are oligoamides which can be flexible, but also rather rigid, e.g. when the linker forms $\alpha$-helices, as it will be exemplified in section 2.3.1. Figure 4 shows bichromophore 7 which can be used as $\mathrm{pH}$ probe for intracellular measurements. ${ }^{[43]}$ While at neutral $\mathrm{pH}$ mainly fluorescence of the dansyl fluorophore is oberved, under acidic conditions mainly emission of the anthracene moiety is observed. ${ }^{[43]}$

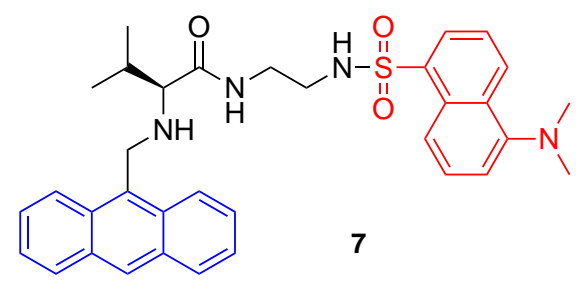

Figure 4: Bichromophore with a flexible peptide linker used as a $\mathrm{pH}$ probe for intracellular measurements. ${ }^{[43]}$

Although bichromophores with flexible linkers such as polymethylene or oligoamide chains are easier to prepare than the bichromophores with rigid linkers, the study on the distance dependence of intramolecular ET or EET processes 
in bichromophores with flexible linkers is usually complicated due to many possible conformations of the molecules. ${ }^{[40]}$ Accordingly, many studies deal with the design of bichromophores connected with rigid linkers.

\subsection{Bichromophores with rigid and semi-rigid linkers}

\subsubsection{Bichromophores with helical linkers}

Many helical structures have been used as linkers in bichromophoric compounds. ${ }^{[54-61]}$ Most of them are based on peptides forming $\alpha$-helices, which (besides $\beta$-sheets) are the most common elements of the secondary-structure of proteins. These studies typically aim at scaling down the natural ET processes to understand their basic mechanisms. Instead of using bichromophores, immobilized peptides decorated only with a donor chromophore have been studied recently. They were immobilized on a surface, and the electron-acceptor part was replaced by an electrode. ${ }^{[56-58]}$ However, in this short review the focus lies on bichromophores.

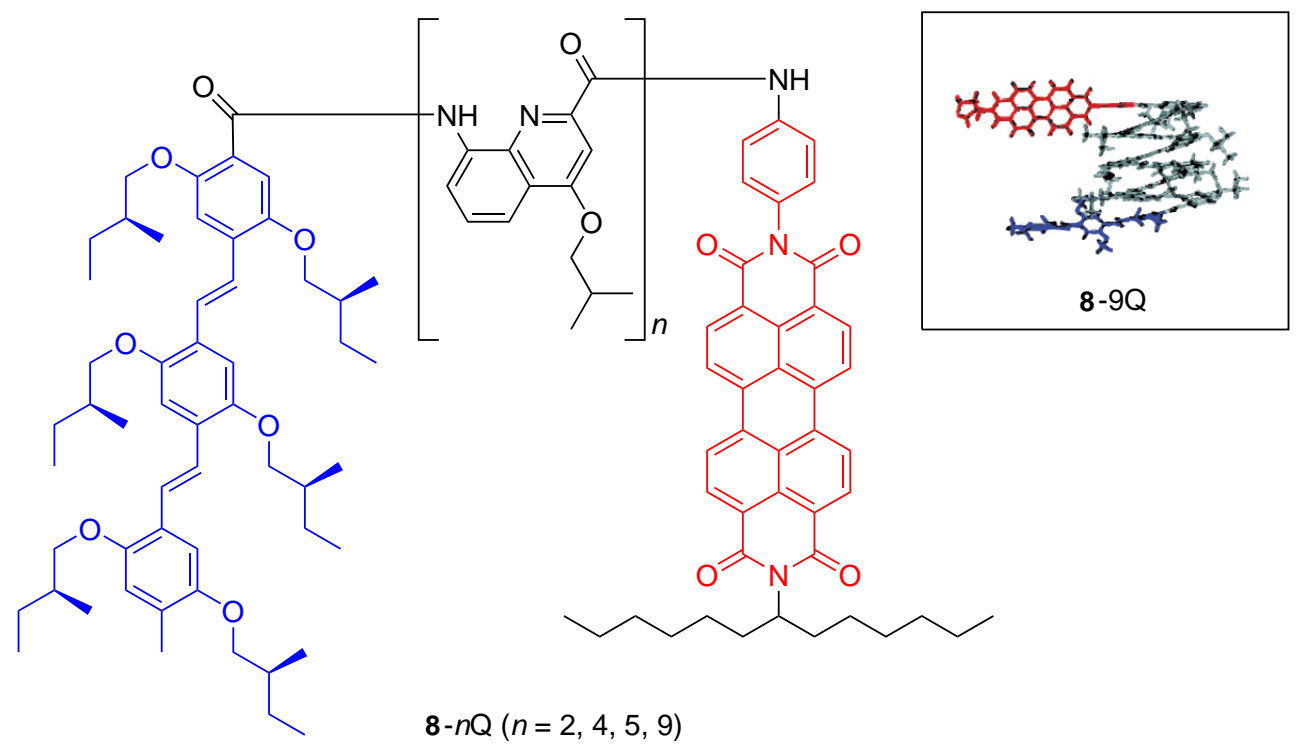

Figure 5: Bichromophore 8- $n \mathrm{Q}(n=2,4,5,9)$ with a quinoline based oligoamide linker forming rather rigid helices. The inset shows an energy minimized conformation of 8-9Q.

Wolffs et al. designed quinoline-derived foldamers 8- $n \mathrm{Q}$ with increasing oligomeric length depending on the number of quinoline units $n=2,4,5,9$ (fig- 


\section{\begin{tabular}{l|l} 
Chapter 2 & BICHROMOPHORIC COMPOUNDS
\end{tabular}}

ure 5). ${ }^{[55]}$ They investigated electron transfer (ET) from oligo( $p$-phenylene vinylene) chromophore to a perylene bisimide. The linker formed rather rigid helical foldamers in chloroform and toluene (figure 5, inset). They found evidence for two mechanisms of ET: a photoinduced charge transfer via through-space and through-bridge interactions.

Schuler et al. designed bichromophores 9- $n \mathrm{P}$ with Alexa488 and Alexa594 dyes connected by a polyproline linker of varying length (figure 6). ${ }^{[59-61]}$ They determined efficiencies for the EET from Alexa488 to Alexa594 and compared the results with the expected values based on the Förster theory with polyproline treated as a rigid rod. Interestingly, at donor-acceptor distances less than the Förster radius $R_{0}$, the EET values were lower than expected, whereas at distances comparable to and greater than $R_{0}$, they were higher. Based on simulations, they suggest that at shorter distances the orientation of the dyes is not completely averaged and therefor the orientation factor $\kappa^{2}$ may not equal $2 / 3$. Instead, $\kappa^{2}$ may be smaller than $2 / 3$ and this could be a reason why the efficiency is lower than predicted by the Förster point-dipole approximation. At longer distances the polyproline linker may not be completely rigid and conformers with shorter end-to-end distances contribute causing a higher efficiency than predicted. ${ }^{[59]}$

a)

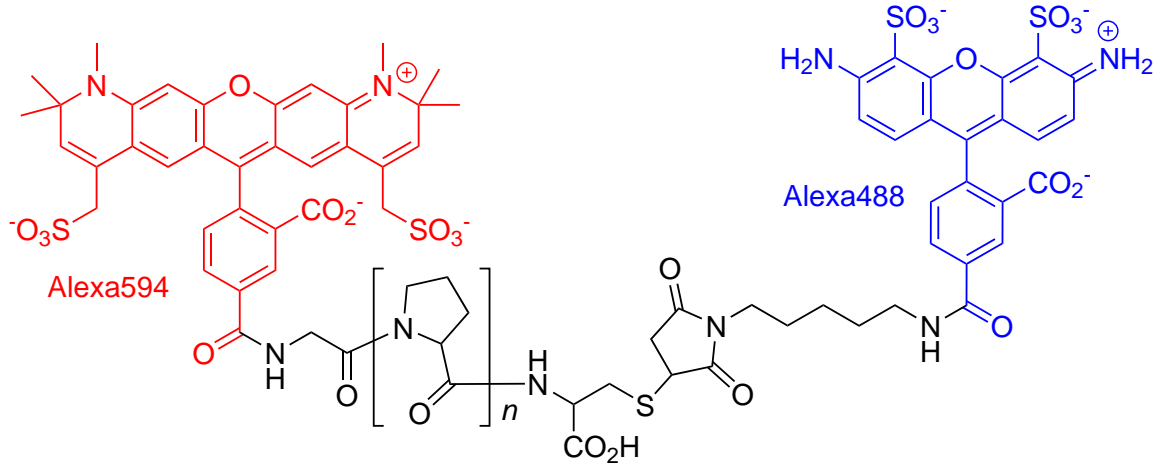

9-nP $(n=6,9,11,12,13,15,20,23,27,33,40)$

b)

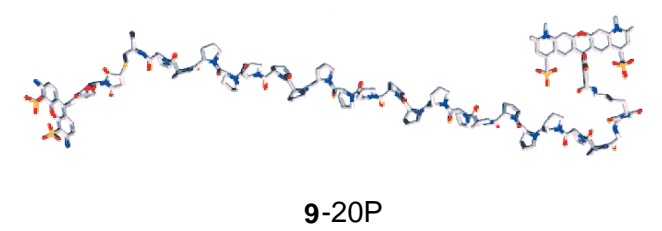

Figure 6: a) Bichromophore 9- $n \mathrm{P}(n=6,9,11,12,13,15,20,23,27,33,40)$ with polyproline based oligoamide linker; b) Energy minimized conformer of 9-20P. ${ }^{[59-61]}$ 


\subsubsection{Bichromophores with linear linkers}

Rigid linear linkers composed of repeating units allow the systematic investigation of the distance between donor and acceptor parts $\left|\vec{R}_{\mathrm{DA}}\right|$, affecting ET and EET processes by changing the number of repeating units. The dependence of the EET rate on $\left|\vec{R}_{\mathrm{DA}}\right|$ allows, for example, to differentiate between a mechanism based on Coulombic interactions (which shows a $\left|\vec{R}_{\mathrm{DA}}\right|^{6}$ dependence according to the Förster approximation; equation 2) and a Dexter type mechanism (which is expected to show an exponential dependence on the distance; equation 8).

Zimmerman and co-workers examined SEET between various chromophores which were connected by rod-like linkers based on one or two bicyclo[2.2.2] octane moieties (figure 7). The observed EET did neither fit the basic Förster nor the classical Dexter electron-exchange model. They draw the conclusion that the EET was promoted by through-bond exchange interactions. ${ }^{[62]}$

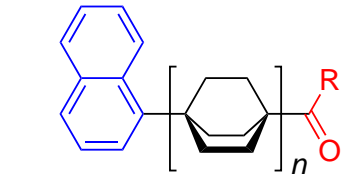

$n=1 ; \mathrm{R}=\mathrm{CH}_{3}, \mathrm{Ph}$, cyclohexyl $n=2 ; \mathrm{R}=\mathrm{CH}_{3}, \mathrm{Ph}$

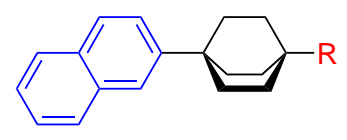

$\mathrm{R}=\mathrm{Ac}, \mathrm{Bz}$, cyclohexanecarbonyl, cis-propenyl

Figure 7: Examples of bichromophoric compounds with rigid linear linkers. ${ }^{[62]}$

Albinsson et al. investigated SEET in two series of zinc/free base porphyrin D-B-A systems. In one series the electronic properties of bridges varied, while their length was kept constant. In another series, the bridge length varied (figure 8). In the first series with a fixed donor-acceptor distance, the authors observed a dependence of the EET rate on the orbital energy of the bridge. Thus, the EET mechanism here cannot be based on Coulombic interactions alone. These findings support the bridge-mediated Dexter exchange mechanism (superexchange), as mentioned in section 1.2.3. As given by equations 10 and 11, the electronic coupling between the chromophores (and thus the attenuation factor $\beta$ ) depends on the orbital energy levels of the bridge as well as the energy gap between the donor and the bridge. ${ }^{[8,20]}$ From these observations, the authors conclude, that both mechanisms based on Coulombic and superexchange interactions contribute to the EET rates. ${ }^{[20]}$ 

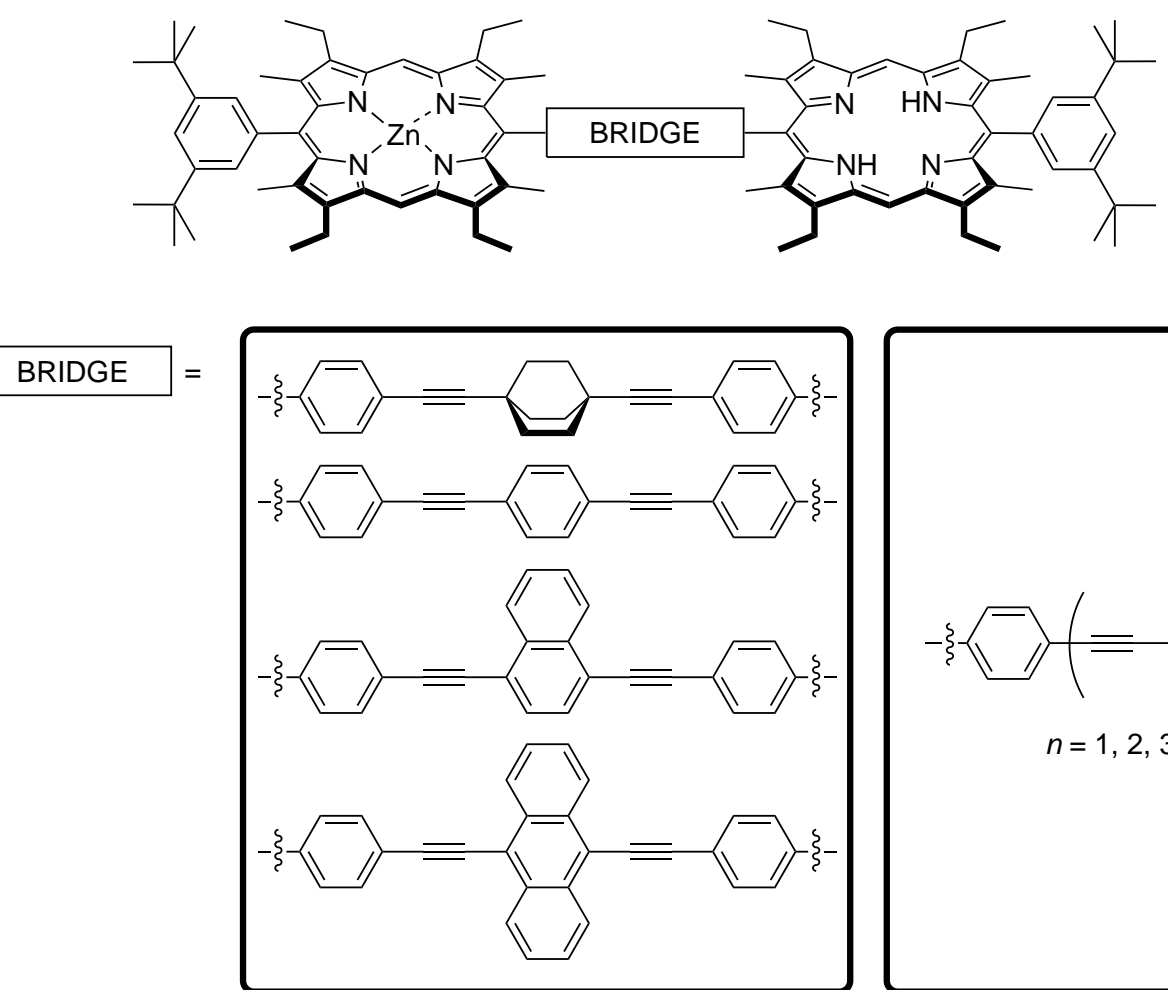

variation of electronic properties

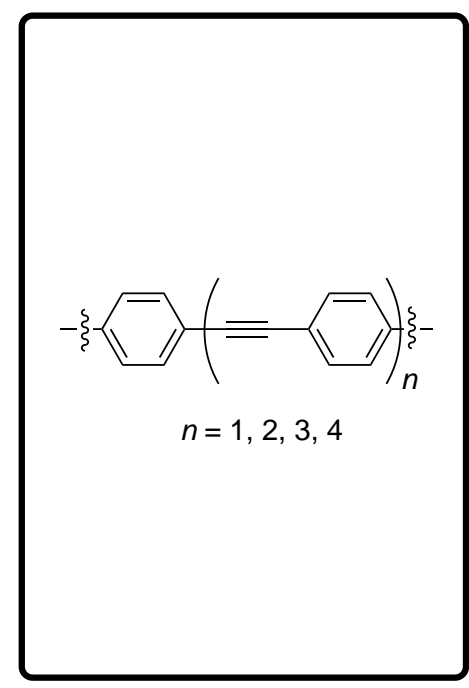

variation of length

Figure 8: Zinc/Zinc-free porphyrin based D-B-A systems with varying electronic properties and length. ${ }^{[20]}$

Langhals et al. synthesized a series of bichromophoric compounds, with two perylene bisimide chromophores which act as energy donor and acceptor (figure 9). ${ }^{[5,63-65]}$ The transition dipole moments of the chromophores are aligned along the long axes of the perylene systems (double-headed arrow in figure 9). By linking the donor side on and the acceptor at the terminal end, the transition dipole moments in the equilibrium geometry should be always oriented perpendicular. Assuming the EET mechanism is exclusively based on dipole-dipole interactions (FRET), energy transfer should be blocked in this particular case. According to equation 4 , the orientation factor $\kappa^{2}$ is 0 , if the transition dipole moments are arranged perpendicularly $\left(\theta_{\mathrm{DA}}=90^{\circ}\right)$ and at least one of them is orthogonal to the interconnecting vector $\vec{R}_{\mathrm{DA}}\left(\theta_{\mathrm{D}}=90^{\circ}\right.$ or $\theta_{\mathrm{A}}=90^{\circ}$, as defined in figure 1). This means that $k_{\mathrm{FRET}}=0$ (equation 2). The linker was varied in length and chemical nature to reveal the energy transfer mechanisms other than Coulombic interactions. However all the bichromophores with pyridinyl moieties and the bichromophore with a tetraphenyl based linker (figure 9) undergo energy 
transfer with $100 \%$ efficiency. No residual fluorescence of the donor was found, despite of the different types of linkers and always the orthogonal chromophore arrangement. For bichromophores with phenyl groups $(\mathrm{X}=\mathrm{CH})$ and the bichromophore with aliphatic bicyclo[2.2.2]octane spacer a lower EET efficiency was found. The lower emission intensities were attributed to electron transfer from the linker to the excited chromophores quenching their emission. ${ }^{[5 d, 63-65]}$

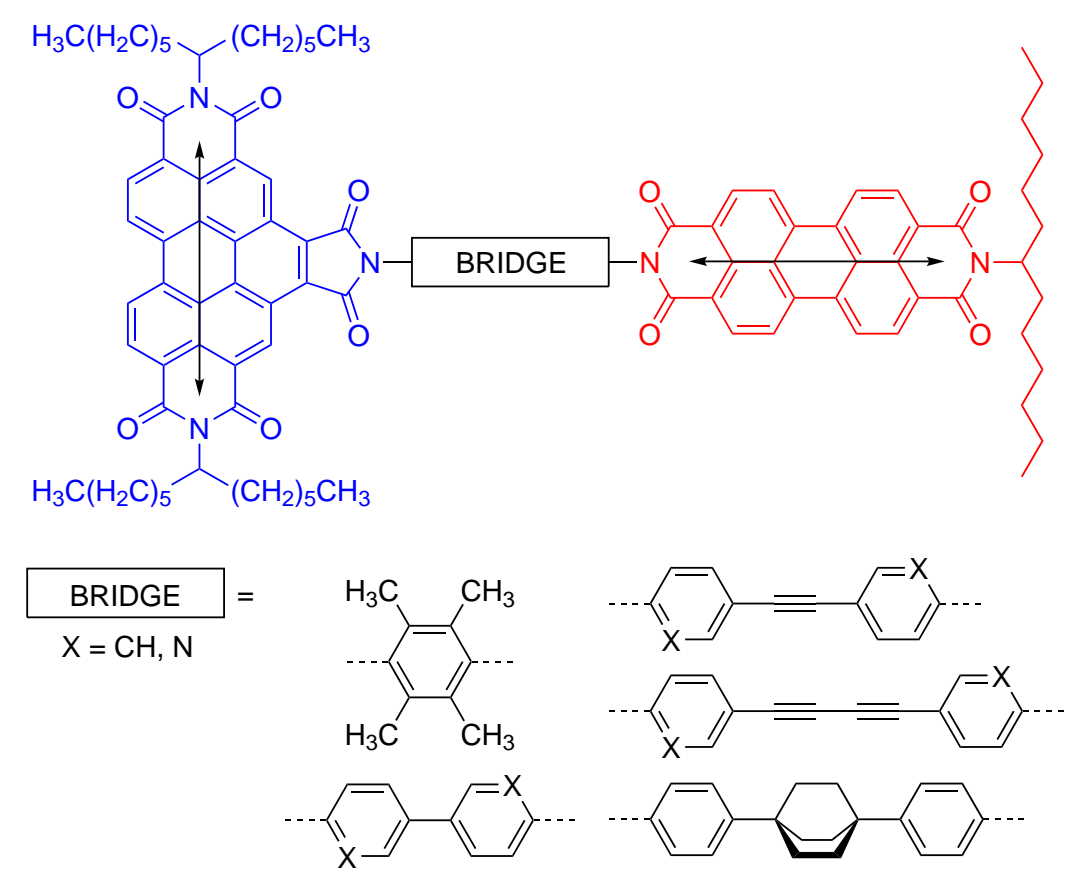

Figure 9: Examples of bichromophores with linear linkers of varying length and chemical nature. Transition dipole moments are shown as double-headed arrows. ${ }^{[5 d, 63-65]}$

The possibility of any Dexter like EET mechanism was excluded by Langhals and co-workers by arguing that the variation of a donor-acceptor distance gave only a weak effect on the EET efficiency. ${ }^{[64]}$ This observation rather suggests a Förster like mechanism which ideally shows a stronger $\left|\vec{R}_{\mathrm{DA}}\right|^{6}$ dependence (equation 2 ) in contrast to a Dexter type mechanism which would decrease exponentially with the distance (equation 8).

Since the orientation factor should be always $\kappa^{2}=0$ in the ground state equilibrium geometry, initially Langhals and co-workers explained a $100 \%$ EET efficiency by low-frequency ground state vibrations, which break the orthogonal geometry and therefor allow FRET. ${ }^{[5 d, 63]}$ In a later publication these au- 


\section{\begin{tabular}{l|l} 
Chapter 2 & BICHROMOPHORIC COMPOUNDS
\end{tabular}}

thors state, that the strict orthogonal dipole arrangement can be broken by environmental fluctuations (solvent fluctuations). They termed this mechanism noise-induced FRET. ${ }^{[64]}$ In a more recent publication Langhals et al. reported a more complex energy transfer mechanism for the bichromophore with the bicyclo[2.2.2] octane linker, which they termed coupled hole-transfer FRET: [65] After excitation of the donor moiety, the main part of molecules transits to two different charge-transfer states by rotation of the whole linker-acceptor moiety. One of the charge-transfer states relaxes back to the ground state, but the transition dipole moment of the other charge-transfer state is aligned parallel to the transition dipole moment of the acceptor. This allows energy transfer to the acceptor via multipole-multipole interactions.

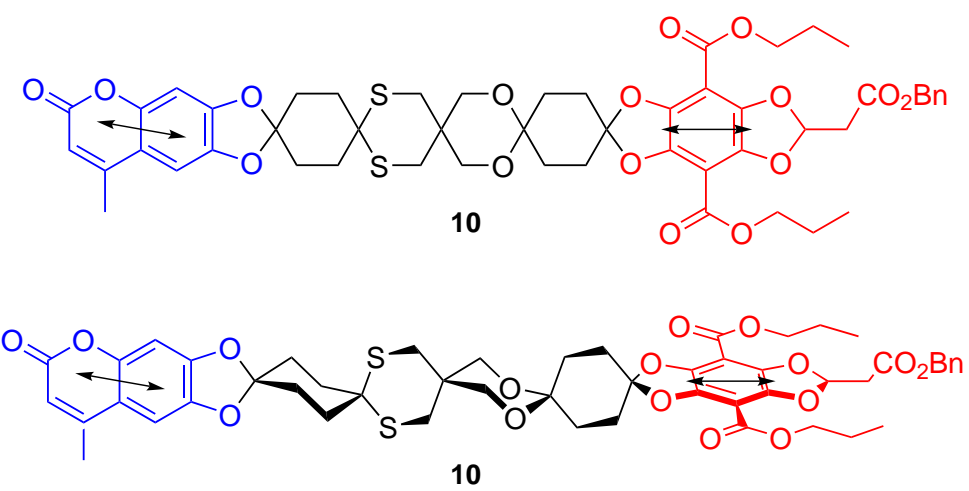

Figure 10: Bichromophore 10 with an oligospirothioketal linker. The lower structure shows one possible conformation. Transition dipole moments are indicated as double-headed arrows. ${ }^{[66]}$

Only in a very few cases, both the distance and the relative orientation of the chromophores is fixed. ${ }^{[67]}$ Wessig et al. developed bichromophore 10 with coumarin and a [1,3]dioxolo[4,5-f][1,3]benzodioxole dyes connected by an oligospirothioketal linker (figure 10). In fact, the chromophores are not able to rotate, but although the observations match the Förster point-dipole approximation the rigidity of the polyspiro alicyclic linker may be questioned. Since the (chair) conformations of the six-membered rings may change, ${ }^{[68]}$ the linker is prone to bending. Besides the conformational changes, the lack of the full symmetry of a coumarin dye results in the not perfectly collinear orientation of the transition dipole moments of the two chromophores. 


\subsubsection{Bichromophores with scaffold linkers}

Scaffold linkers with fixed conformation, such as decalin, ${ }^{[69,70]}$ steroid $^{[24,71-78]}$ or polynorbornyl ${ }^{[67,79-84]}$ systems allow the design of bichromophores with a rather well-defined chromophore distance and in some cases even fixed relative orientation.

Much attention was paid to bichromophores with linkers based on steroid structures. ${ }^{[24,71-78]}$ Vollmer and co-workers synthesized bichromophores with an anthrylthiophene group as energy donor and a bithienylporphyrin part as acceptor. The authors incorporated the steroid epi-androsterone or an $\alpha, \alpha^{\prime}$-oligothiophene as linkers into bichromophores 11 and 12, respectively (figure 11).

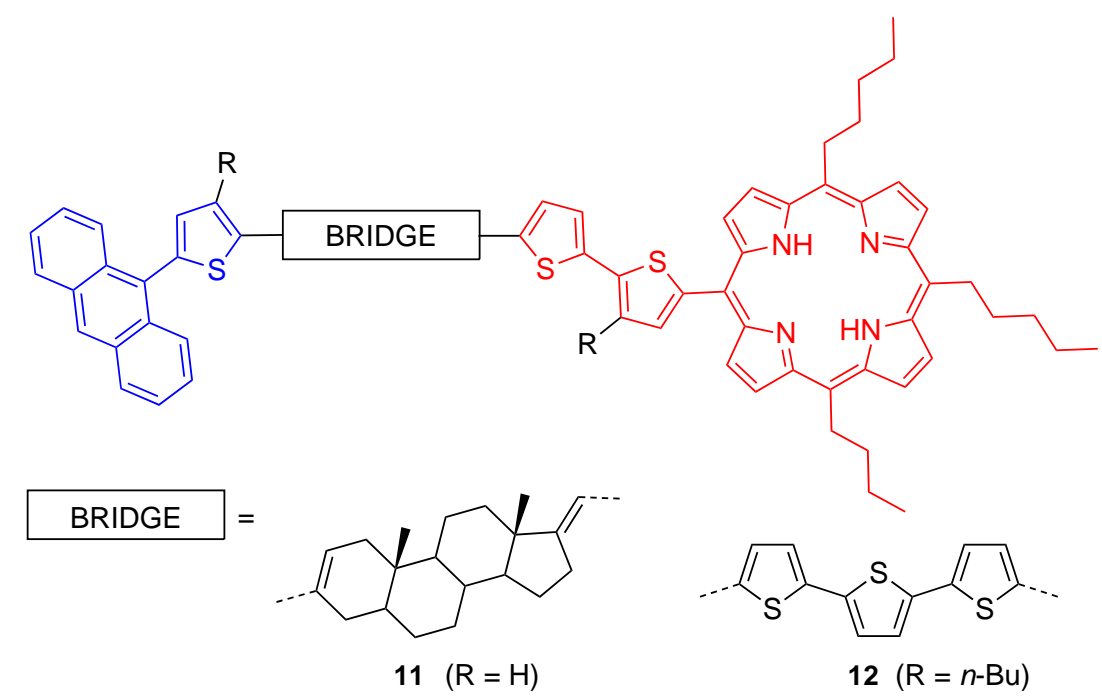

Figure 11: Bichromophores with steroid based (11) and oligothiophene based (12) linkers. ${ }^{[74]}$

The authors observe intramolecular energy transfer from the anthracene to the porphyrin moiety for both compounds (11 and 12). In the case of the oligothiophene linker, the energy transfer is quantitative, whereas in the case of the rigid steroid linker the efficiency is lower, but still in the range of $99 \%$. These results indicate EET via the oligothiophene chain in compound $\mathbf{1 2}$ which is partially interrupted by the androstane linker in compound 11. This interruption leads to some observable donor fluorescence. Since the EET is very efficient even in bichromophore $\mathbf{1 1}$ with a non-conjugated linker, the authors assume that a 


\section{\begin{tabular}{l|l} 
Chapter 2 & BICHROMOPHORIC COMPOUNDS
\end{tabular}}

Förster transfer dominates in both compounds. However, the authors cannot completely rule out through-bond interactions. Besides that, the presence of conformers may lead to different chromophore orientations and influence the FRET efficiency. Furthermore, the distance between the anthracene and porphyrin group is shorter in compound $12(19 \AA)$ compared to $11(21 \AA)$.

Closs and co-workers designed a series of bichromophoric compounds using cyclohexane or decalin as a linker (figure 12), and investigated the influence of the position and stereochemistry of the chromophore attachment to the linker. ${ }^{[85]}$

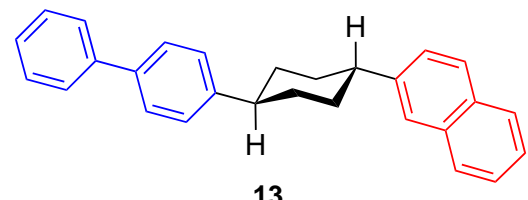

13

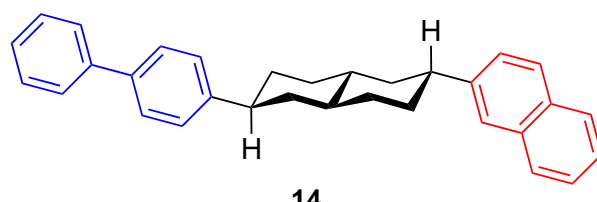

14

Figure 12: Bichromophores with cyclohexane and decalin linkers. ${ }^{[69,85]}$

Initially, these authors studied intramolecular $\mathrm{ET}^{\left[{ }^{[85}\right]}$ but then also intramolecular TEET, in order to investigate similarities between both processes. ${ }^{[69]}$ They found for both processes that the transfer rate falls off exponentially with increasing the number of bonds separating the chromophores. Among stereoisomers the maximum rates were found for the equatorial-equatorial substitution patterns. The logarithmic plots of the transfer rate versus the number of bonds separating the chromophores showed a slope of $\beta=1.15 /$ bond for ET and $\alpha=2.6 /$ bond for TEET. The ratio of $\alpha / \beta \approx 2$ fits the prediction from the rather oversimplified model which treats TEET as two simultaneous occurring ET processes (see section 1.2.2). ${ }^{[69,70]}$

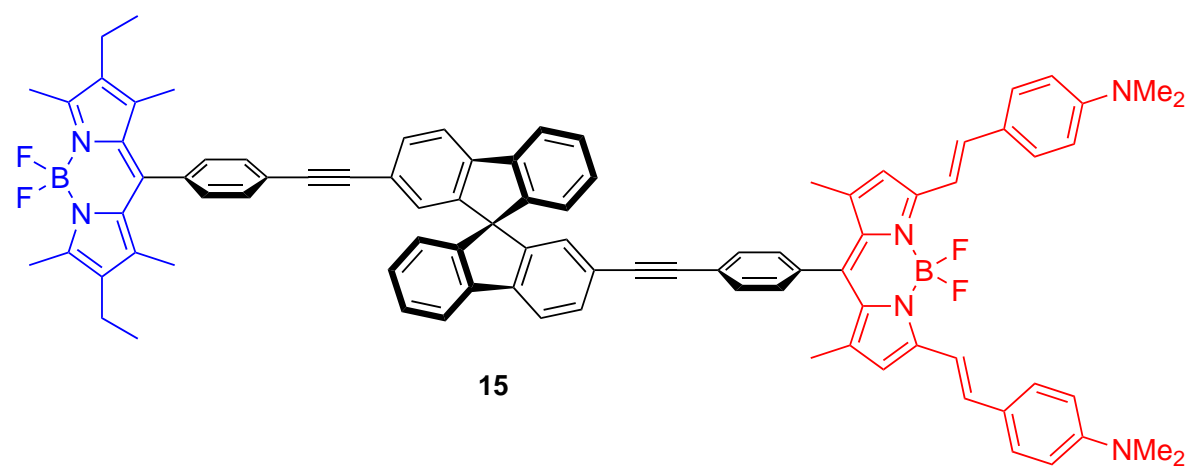

Figure 13: An example of a bichromophore with a scaffold linker and free chromophore rotation. ${ }^{[86]}$ 
Ziessel and co-workers designed compound 15 based on 2,2'-disubstituted 9,9'-spirobifluorene as linker in combination with two boron dipyrromethane (BODIPY) chromophores as shown in figure $13 .{ }^{[86]}$ The authors tried to inhibit through-bond EET by incorporating an orthogonal connection (at the spirocenter) into the linker unit. While the distance was constant $(20 \AA)$, the spectral overlap integral was modified by addition of $\mathrm{HCl}$ creating the mono- and diprotonated forms of the dimethylamino groups: $15\left(\mathrm{H}^{+}\right)$and $15\left(2 \mathrm{H}^{+}\right)$. Experimental values of EET rates were compared with calculated values. For the neutral form of compound 15, the transition dipole moments were approximated as pointdipoles. The authors refrained from applying the point-dipole approximation, in the case of the protonated forms, because the wavefunctions were described as "banana-like" in shape and therefore are unsatisfactorily approximated by pointdipoles. Instead, the transition density cube approach ${ }^{[87]}$ was used to model the Coulombic interactions and to estimate the EET rate. In this method, the ground and excited state wavefunctions of a chromophore (derived from ab initio calculations) are combined to yield a three-dimensional transition density which is called the transition density cube (TDC). The TDCs of two chromophores are interacted to describe the Coulombic coupling between them. ${ }^{[88]}$ While the calculated rates of the neutral compound 15 and the symmetric $15\left(2 \mathrm{H}^{+}\right)$are in good agreement with the experimental values, the rates for $\mathbf{1 5}\left(\mathrm{H}^{+}\right)$with an asymmetric push-pull energy acceptor differ considerably from the experimental values. 


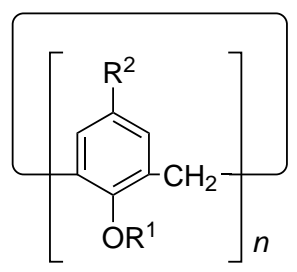

Calix $[n]$ arene

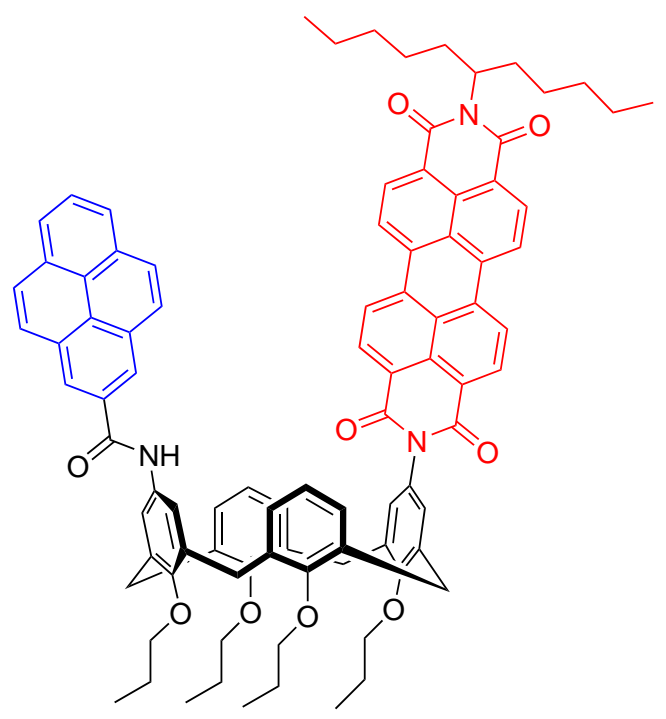

16

Figure 14: General structure of calix[n]arenes and bichromophore 16 with calix[4]arene linker. ${ }^{[89]}$

Anh and co-workers designed compound $\mathbf{1 6}$ based on calix[4]arene. ${ }^{[89]}$ Calix $[n]$ arenes are oligomers composed of para-substituted phenols which are linked by methylene bridges through ortho-positions (figure 14). The authors observed two possible conformers depending on the solvent: in solvents like cyclohexane, toluene or chloroform a conformer with $\pi$-stacking of the two chromophores was observed, while in THF $25 \%$ of the molecules were found to be in an extended conformation with a larger chromophore separation. In the case of the $\pi$-stacked conformation photoexcitation of the perylene bisimide unit leads to ET fom the pyrene chromophore towards the electron-accepting perylene bisimide. In contrast, when exciting the pyrene unit in the extended conformer the calixarene scaffold acts as an electron donor and the pyrene unit acts as electron acceptor. ${ }^{[89]}$ 
a)

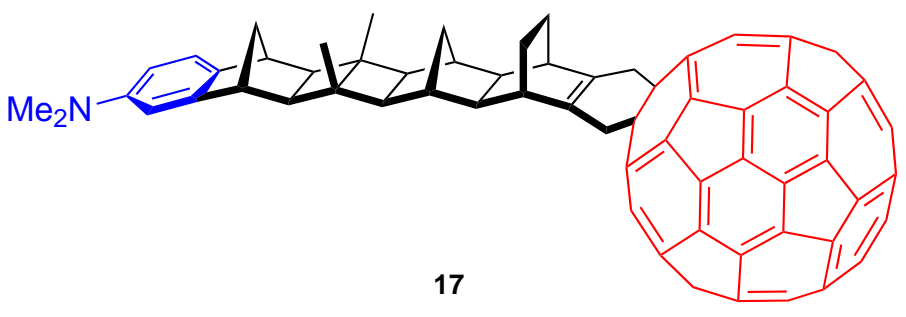

b)

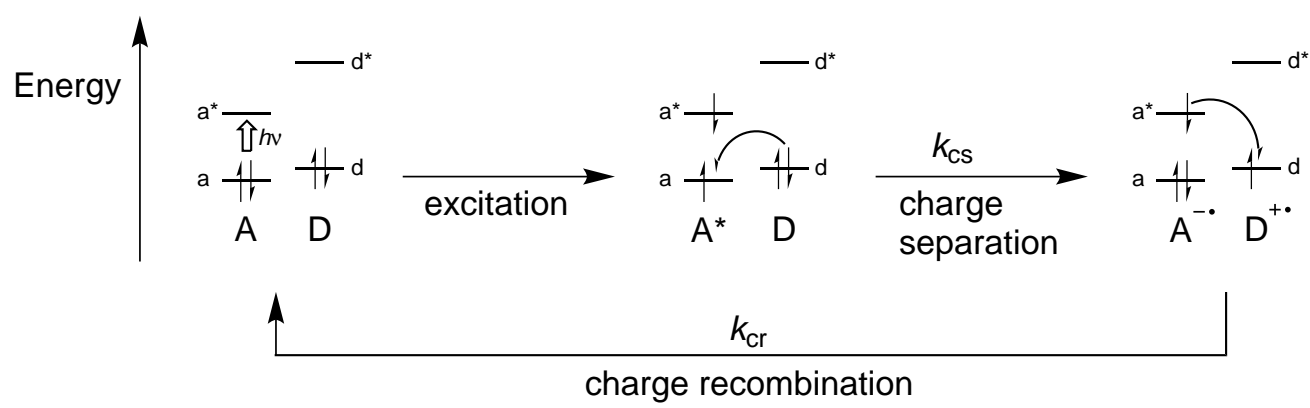

Figure 15: a) Bichromophore 17 with rigid bridge and fixed chromophore orientation. b) Frontier molecular orbital description of the photoinduced electron transfer (ET). HOMOs and LUMOs of electron donor (D) and acceptor (A) are named $\mathrm{d}, \mathrm{d}^{*}$ and $\mathrm{a}, \mathrm{a}^{*}$, respectively. ${ }^{[83,90]}$

Williams and co-workers designed bichromopohore $\mathbf{1 7}$ with a norbornylogous bridge comprising linearly fused norbornane and bicyclo[2.2.0]hexane units connecting a fullerene moiety and a $N, N^{\prime}$-dimethylaminophenyl group (figure 15a). ${ }^{[83]}$ After photoexcitation of the fullerene $C_{60}$ unit, they observed rapid charge separation in a polar solvent that gives a long-lived charge-separated state (with a lifetime of ca. $0.25 \mu \mathrm{s}$ ). Figure 15b shows a simplified frontier molecular orbital description of the photoinduced electron transfer (ET) where the electron acceptor has the lowest excited state energy level. The ratio between the rates of charge separation and charge recombination for this system in is $k_{\mathrm{cs}} / k_{\mathrm{cr}} \approx 1400$. They proposed to use this design of donor-bridge-fullerene (acceptor) systems as molecular building block in optoelectronic devices. ${ }^{[83,90]}$

Scholes and co-workers designed bichromophore 18 in which naphthalene and anthracene are connected by a rigid bis(norbornyl)bicyclo[2.2.0] hexane linker (figure 16a). ${ }^{[80]}$ Although direct Coulombic interactions between the lowestenergy excited states should be forbidden due to orthogonal donor and acceptor transition dipole moments, the authors observed and studied SEET from naph- 


\section{\begin{tabular}{l|l} 
Chapter 2 & BICHROMOPHORIC COMPOUNDS
\end{tabular}}

thalene towards anthracene.

a)

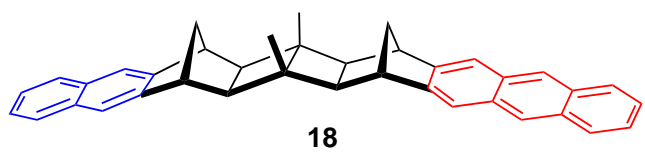

b)
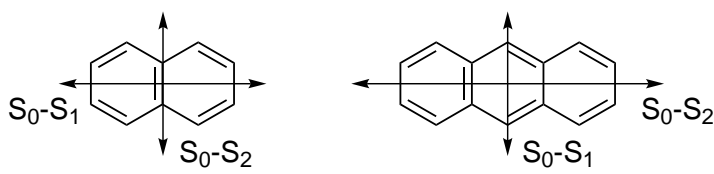

Figure 16: a) Bichromophore 18 with a rigid norbornyl-fused linker, and fixed chromophore orientation; ${ }^{[80]}$ b) Absorption transition dipole moments of naphthalene and anthracene. ${ }^{[6]}$

Normally EET between from naphthalene to anthracene is assumed to occur between the lowest electronically excited states: According to Platt's nomenclature, ${ }^{1} \mathrm{~L}_{b}$ for naphthalene and ${ }^{1} \mathrm{~L}_{a}$ for anthracene. ${ }^{[91]}$ To put it more simply, the relevant transitions are $S_{0} \rightarrow S_{1}$ for naphthalene and $S_{1} \rightarrow S_{0}$ for anthracene. As shown in figure 16b, the corresponding transition dipole moments are oriented along the long and the short axis of naphthalene and anthracene, respectively. Consequently, due to orthogonality of the donor and acceptor transition dipole moments, the direct dipole-dipole coupling between these states should not occur. ${ }^{[81]}$

Belser and co-workers designed a Ru/Os bimetallic complex 19 with a rigid rodlike bridging ligand based on adamantane (figure 17). ${ }^{[92]}$

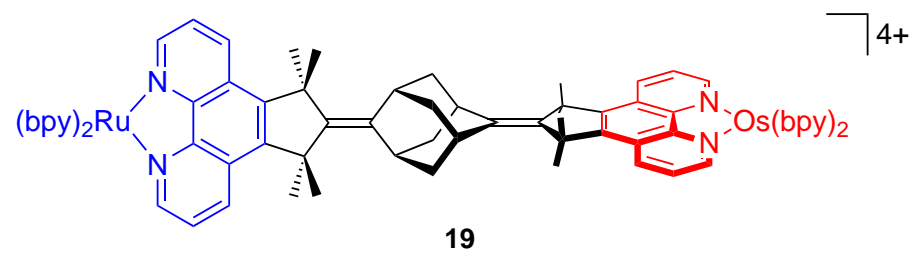

Figure 17: An example of a bichromophore with scaffold linker and fixed chromophore orientation. ${ }^{\left[{ }^{[2]}\right.}$

The ligand posses $C_{2}$ symmetry and leads to bimetallic complexes with a linear arrangement of the linker and the two metals. Upon excitation of the mixedmetal compound $\mathrm{Ru}^{\mathrm{II}} / \mathrm{Os}^{\mathrm{II}}$ at $465 \mathrm{~nm}$, two emission bands were observed, which correspond to the $\mathrm{Ru}^{\mathrm{II}}$ and $\mathrm{Os}^{\mathrm{II}}$ metal centers, respectively. The emission from 
the $\mathrm{Ru}^{\text {II }}$-metal center is strongly reduced, while the luminescense of the Os ${ }^{\mathrm{II}}$ center is enhanced (compared to the $\mathrm{Ru}^{\mathrm{II}} / \mathrm{Ru}^{\mathrm{II}}$ and $\mathrm{Os}{ }^{\mathrm{II}} / \mathrm{Os}^{\mathrm{Il}}$ complexes). The authors conclude an intramolecular energy-transfer process in the mixed $\mathrm{Ru}^{\mathrm{II}} / \mathrm{Os}^{\mathrm{II}}$ complex and determined a transfer rate of $k_{\mathrm{EET}}=5 \cdot 10^{7} \mathrm{~s}^{-1}$.

\subsection{Applications of multichromophoric compounds}

Applications of bi- or multichromophoric compounds have gained immense importance in many areas during the past decades. The following chapter will highlight examples for the application of bichromophores in organic photovoltaics, ${ }^{[4 b]}$ DNA sequencing, ${ }^{[4 e, 93]}$ and super-resolution microscopy. ${ }^{[42]}$

\subsubsection{Artificial photosynthesis}

In natural photosynthesis the sunlight is absorbed by the light-harvesting complexes which represent a network of chromophores. The energy is funneled towards the reaction center where the excitation leads to charge separation. In multiple additional steps the energy is transformed into chemical fuels. ${ }^{[3 a, 3 b]}$ Much research is done to mimic this process. "Molecular funnels" were designed as artificial light-harvesting antennae. ${ }^{[4 \mathrm{~b}]}$ Therefore, multichromophoric compounds, like compound 20, ${ }^{[94]}$ comprise several chromophores which can absorb light of different wavelengths (figure 18). 


\section{\begin{tabular}{l|l} 
Chapter 2 & BICHROMOPHORIC COMPOUNDS
\end{tabular}}

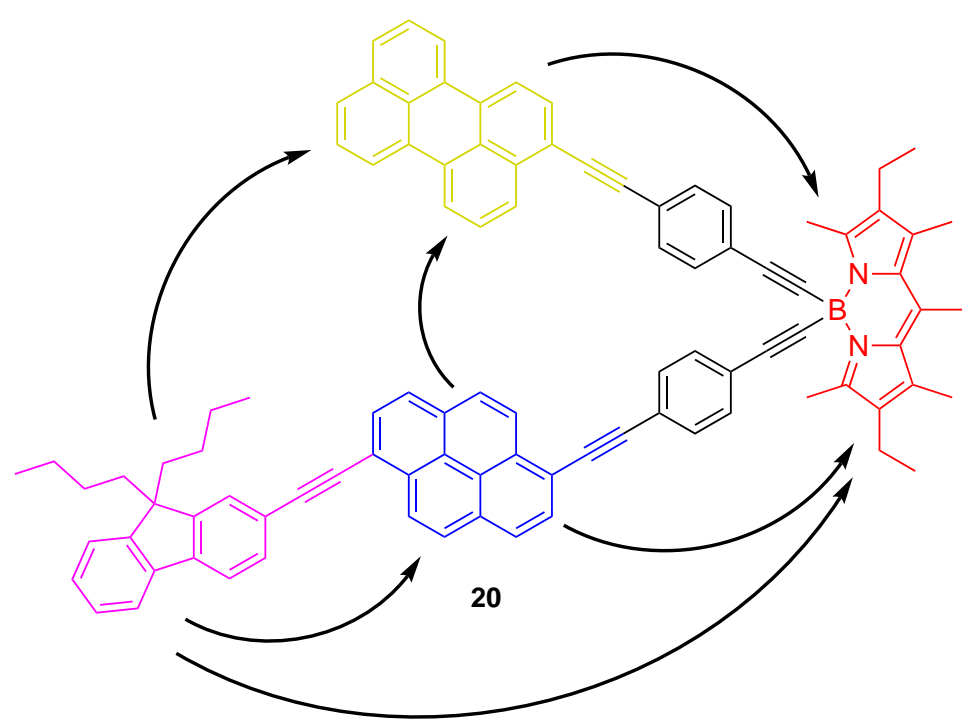

Figure 18: Cascade EET in artificial light-harvesting antennae. The arrows represent sequential EET processes. ${ }^{[94]}$

The excitation energy is subsequently transferred to the BODIPY chromophore with the absorption maximum at the longest wavelength. In other words, excitation energy of a very broad wavelength range can be funneled to the emission wavelength of the BODIPY dye.

\subsubsection{Bichromophores for DNA sequencing}

Automated sequencing of DNA is typically based on the differentiation of fluorescently labeled dideoxy-A, $-\mathrm{T},-\mathrm{G}$, and $-\mathrm{C}$ bases. ${ }^{[42,95,96]}$ The sequencing products are separated by gel electrophoresis and individually detected using a laser induced fluorescence (LIF) detector. It is desirable to excite all four labels simultaneously with a single laser. However, at the same time the fluorescence of the individual labels needs to be spectrally well-separated in order to distinguish between the four nucleotides. Typically, the "big four" dyes Carboxyfluorescein (FAM ${ }^{\mathrm{TM}}$ ), 6-Carboxy-4',5'-dichloro-2',7'-dimethoxyfluorescein ( $\mathrm{JOE}^{\mathrm{TM}}$ ), Tetramethylrhodamine $\left(T_{A M R A^{T M}}\right)$ and Rhodamine-X $\left(\right.$ ROX $\left.^{T M}\right)$ are used for DNA-sequencing. These four dyes are directly excited at $488 \mathrm{~nm}$ with an argon ion laser. A fifth dye is used as an independent internal standard. In order to excite this fifth dye with the same laser and to be able to detect it's emission at longer wavelengths than the "big four" dyes, the fifth dye is a bichromophore with intramolecular EET and large "pseudo" Stokes shift. 
However, instead of four single dyes which are directly excited, also sets of four bichromophores were used for DNA sequencing. For example Nampalli et al. reported the combination of fluorescein as energy donor with four different rhodamine dyes as acceptors (figure 19). ${ }^{[4 \mathrm{e}]}$

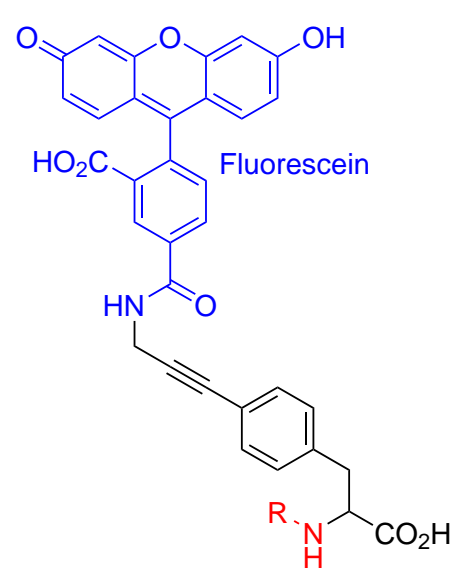

21a: $R=5$-Rhodamine- $X$

21b: $R=5$-Tetramethylrhodamine

21c: $R=5$-Rhodamin-6G

21d: $R=5$-Rhodamin 110
$\mathrm{R}=$

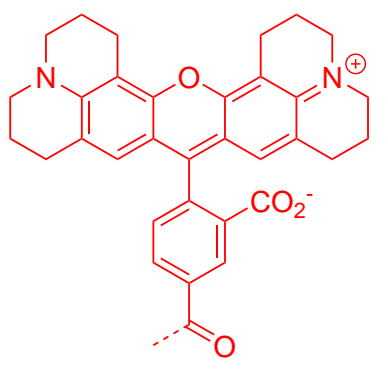

5-Rhodamine-X (ROX ${ }^{\mathrm{TM}}$

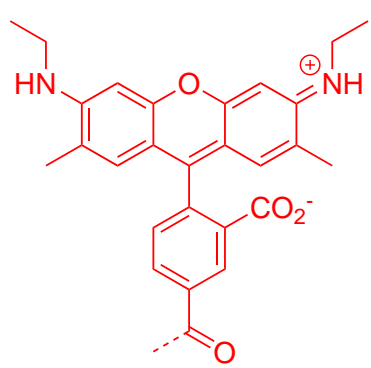

5-Rhodamin-6G<smiles></smiles>

5-Tetramethylrhodamine (TAMRA ${ }^{\mathrm{TM}}$ )<smiles></smiles>

5-Rhodamin 110

Figure 19: Set of four bichromophores based on fluorescein in combination of different rhodamines used for DNA sequencing. ${ }^{[4]]}$

\subsubsection{Bichromophore in super-resolution microscopy}

Kwon et al. exploited the influence of a Cy $3^{T M}$ dye on Alexa $647^{T M}$ for application in super-resolution microscopy. ${ }^{[42]}$ They used bichromophore 22 which consists of $\mathrm{Cy}^{\mathrm{TM}}$ and Alexa $647^{\mathrm{TM}}$ linked by an alkylamide linker (figure 20) as photoswitchable fluorophore for RESOLFT (reversible saturable optical fluorescence transition) microscopy. 


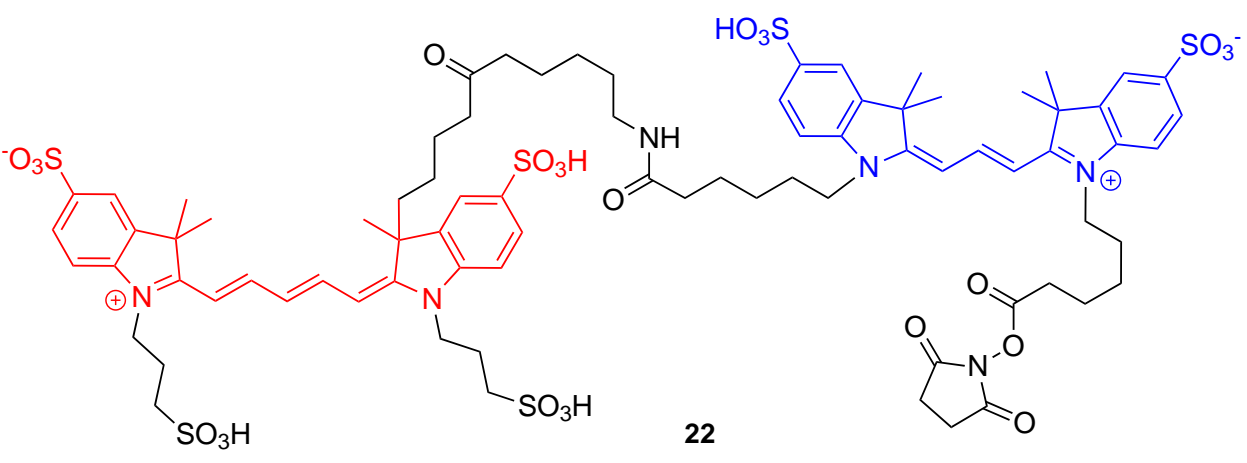

Figure 20: Bichromophore with flexible linker and NHS activated ester for labeling of amine-containing biomolecules for super-resolution microscopy.

The RESOLFT concept is based on reversibly switching of fluorophores between a fluorescent "on" state and a dark "off" state. When cyanine dyes are excited in presence of a thiol, it forms a long-lived non-fluorescent state (the "off state"). The reason for this is an addition of the thiol to the polymethine chain of the cyanine dye and breaking the long conjugation system. Irradiation with light of a wavelength shorter than $550 \mathrm{~nm}$ switches the fluorophore "on" again by restoring the original fluorescent form. The linking of the $\mathrm{Cy}^{\mathrm{TM}}$ dye which absorbs the "restoring" light enhances the "on"-switching efficiency. Thus, Сy $3^{\mathrm{TM}}$ was used as an "activator" for the "reporter" dye Alexa 647 ${ }^{\mathrm{TM}} \cdot{ }^{[42]}$ The NHS-ester allows to use bichromophore $\mathbf{2 2}$ as label, e.g. for the labeling of (secondary) antibodies. Kwon et al. used a conjugate of bichromophore $\mathbf{2 2}$ with a secondary antibody for labeling of mitochondria and microtubule in fixed cells which were incubated with primary antibodies before. 


\section{Experimental investigation of intramolecular EET}

The bichromophores are often designed specifically for the purpose of studying EET processes and are examined by various experimental and theoretical ${ }^{[2,10,97]}$ methods. Besides chemical analysis (MS, NMR, IR, UV/Vis etc.), the experimental methods include the photophysical (fluorescence spectra, fl. lifetime, fl. quantum yield etc.) and electrochemical (e.g. cyclic voltammetry) characterization. In addition to steady state experiments, time-resolved measurements are particularly important to reveal EET dynamics and the underlying EET mechanisms. Among others, transient absorption spectroscopy ${ }^{[47,98]}$ and (time-resolved) fluorescence spectroscopy, ${ }^{[45,99,100]}$ are often applied. Within the scope of this project, time-resolved fluorescence anisotropy ${ }^{[99,101-103]}$ measurements and antibunching experiments ${ }^{[104,105]}$ are particularly relevant and will be described briefly in the following.

\subsection{Steady-state fluorescence anisotropy measurements}

\subsubsection{Photoselection}

Let us consider a diluted fluorophore solution illuminated by linearly polarized light as shown in Scheme 8. The chromophores have randomly oriented absorption transition dipole moments, and each of them may be represented as a superposition of two components with a parallel and perpendicular orientation in respect to the polarization direction of the light source. The chromophores having higher input of the parallel oriented components are excited preferentially (those molecules are depicted as red strokes in figure 8). ${ }^{[106,107]}$ 


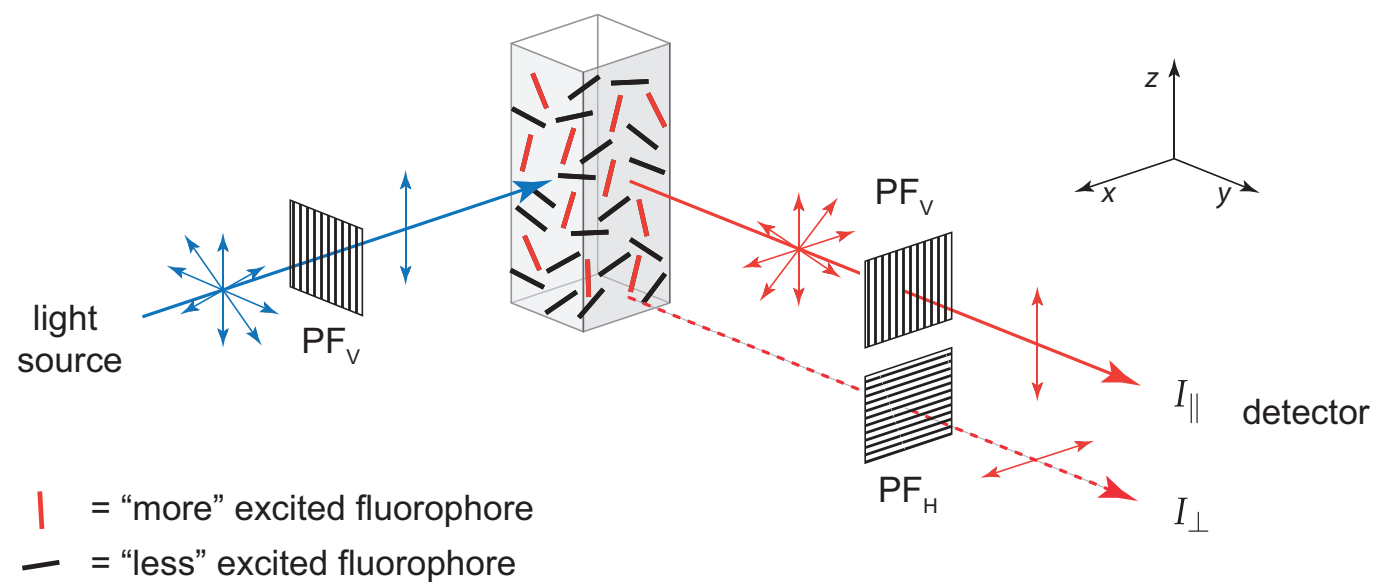

Scheme 8: Fluorescence anisotropy measurement using vertically/horizontally oriented polarization filters $\left(\mathrm{PF}_{\mathrm{V}}\right.$ and $\left.\mathrm{PF}_{\mathrm{H}}\right)$, respectively.

The probability for absorption is proportional to $\cos ^{2}(\theta)$, where $\theta$ denotes the angle between polarization direction and the absorption transition dipole moment (Figure 9). ${ }^{[108]}$ This selective excitation of molecules is called photoselection.
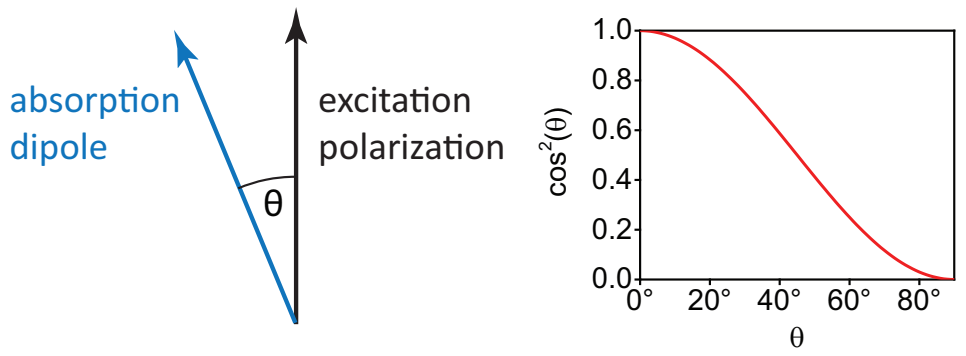

Scheme 9: Photoselection due to angle $\theta$ between direction of excitation polarization and absorption transition dipole (left scheme) and absorption probability proportional to $\cos ^{2}(\theta)$ (right plot).

\subsubsection{Fluorescence polarization ratio and anisotropy}

Measuring the vertically and horizontally polarized components of the emission signal, $I_{\|}$and $I_{\perp}$, using polarization filters $\mathrm{PF}_{V}$ and $\mathrm{PF}_{\mathrm{H}}$, as shown in scheme 8 , gives the fluorescence polarization ratio

$$
P=\frac{I_{\|}-I_{\perp}}{I_{\|}+I_{\perp}},
$$


and fluorescence anisotropy

$$
r=\frac{I_{\|}-I_{\perp}}{I_{\|}+2 I_{\perp}} .
$$

In the definition of the polarization ratio $P$ (equation 12), the denominator represents the fluorescence intensity in the direction of observation, whereas in the case of the fluorescence anisotropy (equation 13), the denominator represents the total fluorescence intensity $I_{\mathrm{TOT}}=I_{\|}+2 I_{\perp}$. In order to explain, why $2 I_{\perp}$ instead of just $I_{\perp}$, let us consider a $x y z$-framework, with the sample placed at the origin. The excitation beam goes along the $x$-axis and is polarized along the $z$-axis. Then the emission is symmetric around the $z$-axis, i.e. the emission along the $x$ - and $y$-axis are equal. The emission $I_{\perp}$ is observed only from along the $y$-axis. In order to take into account also the emission along the $x$-axis, the total intensity is $I_{\mathrm{TOT}}=I_{\|}+2 I_{\perp}$.

The fluorescence polarization ratio or the fluorescence anisotropy can be used to describe the same emission property. However, the fluorescence anisotropy is preferred to the polarization ratio in the following text, because in most cases it leads to simpler theoretical expressions. ${ }^{[6]}$ According to equations 12 and 13 the relationship between the polarization ratio $P$ and the fluorescence anisotropy $r$ is

$$
r=\frac{2 P}{3-P}
$$

Every electronic transition has an associated (absorption or emission) transition dipole moment, which is proper of the chromophore. As described before, the absorption transition dipole determines whether a fluorophore is likely to be excited by polarized light or not (photoselection). The emission transition dipole moment defines the emission anisotropy (and polarization ratio).

If an isotropic solution (i.e. randomly oriented fluorophores) is excited as shown in figure 8 , the emission can be characterized by quantifying the fluorescence polarization ratio $P$ or anisotropy $r$. Mathematically, the polarization has to be between $P=-1$ and $P=1$. The theoretical limits for anisotropy are consequently $r=-1 / 2$ and $r=1$. However, these values could only be reached in 


\section{Chapter 3 EXPERIMENTAL INVESTIGATION OF INTRAMOLECULAR EET}

perfectly aligned emitters (e.g. crystals). In solution the expected limits for the fluorescence anisotropy are -0.2 and 0.4 due to photoselection and the limiting values for the angles between those transition dipole moments. ${ }^{[6]}$ If the fluorescence has the same polarization as the excitation (e.g. parallel absorption and emission transition dipole moments), the value of the anisotropy is $r=0.4$, while a completely perpendicular emission polarization (e.g. due to orthogonal absorption and emission transition dipole moments) gives $r=-0.2$.

\subsubsection{Influences on the fluorescence anisotropy}

The maximum fluorescence anisotropy of randomly oriented fluorophores in solution $r=0.4$, can only be reached if the absorption and emission transition dipoles are parallel. However, the emission transition dipole moment of a fluorophore may or may not be aligned parallel to the absorption transition dipole moment within the molecular framework. ${ }^{[106]}$ An angle $\alpha>0$ between the absorption and emission transition dipole moment reduces the limiting fluorescence anisotropy $r_{0}$, i.e. $r_{0}<0.4$. In addition, if there are changes of $\alpha$ during the fluorescence lifetime, then there will be a transient change in $r$ with the same kinetics. Such changes include internal motion, rotation due to Brownian motion, and EET to another fluorophore with different orientation.

The relationship between the observed fluorescence anisotropy and the angle $\alpha$ between the absorption and emission transition dipole moments is given by ${ }^{[6]}$

$$
\cos \alpha=\sqrt{\frac{5 r+1}{3}} .
$$

Furthermore, due to different excitation intensities, unequal acquisition times or unequal detection inefficiencies for $I_{\|}$and $I_{\perp}$, an instrument and wavelength dependent correction factor $G$ is introduced:

$$
r=\frac{I_{\|}-G \cdot I_{\perp}}{I_{\|}+2 \cdot G \cdot I_{\perp}}
$$

The correction factor $G$ needs to be determined for each setup and wavelength. 


\subsection{Time-resolved fluorescence anisotropy measurements}

Pulsed light sources and time-correlated single photon counting (TCSPC) electronics allow time-resolved anisotropy measurements. Time-resolved fluorescence anisotropy measurements are a powerful tool to investigate processes that change the emission polarization of fluorophores on the time-scale of their fluorescence lifetime. ${ }^{[109]}$

A polarized excitation pulse generates a population of photoselected fluorophores. The fluorescence detected directly after the excitation pulse (limiting anisotropy $r_{0}$ ), shows a high anisotropy if the angle between absorption and emission transition dipoles is small. Due to depolarization processes (e.g. fluorophore rotation or EET to other fluorophores) the anisotropy $r(t)$ decays as time proceeds. Observing the evolution of $r(t)$, after the excitation pulse allows to study the kinetics of these processes.

\subsubsection{Anisotropy decays}

Depolarization processes cause transients in the fluorescence anisotropy $r(t)$. These may be monoexponential but do not have to be. For example, rotational diffusion of an ideal spherical particle causes an monoexponential anisotropy decay characterized by the (limiting) initial anisotropy value $r_{0}$ and the rotational correlation time $\tau_{\mathrm{r}}{ }^{[107]}$

$$
r(t)=r_{0} \exp \left(-t / \tau_{\mathrm{r}}\right)
$$

However, when measuring the time-dependend fluorescence intensities $I_{\|}(t)$ and $I_{\perp}(t)$, a superposition of rotational diffusion and excited state population decay is observed. Because of that, $I_{\|}(t)$ and $I_{\perp}(t)$ are not equal. The pure excited state population decay characterized by the fluorescence life time $\tau_{\mathrm{f}}$ is given by

$$
F(t)=F_{0} \cdot \exp \left(-t / \tau_{\mathrm{f}}\right)
$$




\section{\begin{tabular}{l|l} 
Chapter 3 & EXPERIMENTAL INVESTIGATION OF INTRAMOLECULAR EET
\end{tabular}}

Then the fluorescence intensities decay as ${ }^{[107]}$

$$
\begin{aligned}
I_{\|}(t) & =[1+2 r(t)] \cdot F(t) \\
I_{\perp}(t) & =[1-r(t)] \cdot F(t) .
\end{aligned}
$$

Inserting relations 17 and 18 in $19 \mathrm{a}$ and $19 \mathrm{~b}$, gives ${ }^{[107]}$

$$
\begin{aligned}
& I_{\|}(t)=F_{0}\left[2 r_{0} \exp \left(-t / \frac{\tau_{\mathrm{f}} \tau_{\mathrm{r}}}{\tau_{\mathrm{f}}+\tau_{\mathrm{r}}}\right)+\exp \left(-\frac{t}{\tau_{\mathrm{f}}}\right)\right] \\
& I_{\perp}(t)=F_{0}\left[-r_{0} \exp \left(-t / \frac{\tau_{\mathrm{f}} \tau_{\mathrm{r}}}{\tau_{\mathrm{f}}+\tau_{\mathrm{r}}}\right)+\exp \left(-\frac{t}{\tau_{\mathrm{f}}}\right)\right] .
\end{aligned}
$$

Although, $r_{0}, \tau_{\mathrm{r}}$ and $\tau_{\mathrm{f}}$ in principle could be obtained by fitting the measured $I_{\|}(t)$ or $I_{\perp}(t)$, in practice, $\tau_{\mathrm{f}}$ is usually measured separately (i.e. with polarizes at the magic angle) and fixed when fitting $I_{\|}(t)$ or $I_{\perp}(t)$ in order to obtain reliable values for $r_{0}$ and $\tau_{\mathrm{r}}{ }^{[107]}$

As demonstrated for rotational diffusion with characteristic rotational time $\tau_{r}$, other depolarization processes give expressions analogue to equations $17-20 \mathrm{~b}$. For each case, the $\tau$ in equation 17 is the characteristic time of the process depolarizing the emission (rotational, EET, etc.).

\subsubsection{Anisotropy decay due to excitation energy transfer}

If the energy is transferred from an initially excited fluorophore to another fluorophore - with a different emission dipole orientation - the anisotropy changes.

Based on the pure emission anisotropy of the initially excited fluorophore $r_{0}$ and the angle $\theta$ between the emission transition dipoles of both fluorophores, the pure emission anisotropy of the other fluorophore $r_{1}$ can be calculated: ${ }^{[101]}$

$$
r_{1}=\frac{r_{0}}{2}\left(3 \cos ^{2}(\theta)-1\right)
$$

If the EET may proceed in both directions between the two fluorophores (e.g. between identical fluorophores) than the anisotropy decay can be described as $^{[101,102]}$

$$
r(t)=\frac{r_{0}-r_{1}}{2} \exp \left(-2 k_{\mathrm{t}} t\right)+\frac{r_{0}+r_{1}}{2}
$$


where $k_{\mathrm{t}}$ is the rate of the EET between the fluorophores.

In the case of very slow rotations $\left(\tau_{\mathrm{r}} \gg \tau_{\mathrm{f}}\right)$, for example solutions in highly viscous solvents, the fast decay of the fluorescence intensity can be described neglecting $\tau_{\mathrm{r}}$. Inserting relations 22 and 18 in $19 \mathrm{a}$ and $19 \mathrm{~b}$, gives

$$
\begin{aligned}
I_{\|}(t)=F_{0}\left[\left(1+r_{0}+r_{1}\right)\right. & \exp \left(-\frac{t}{\tau_{\mathrm{f}}}\right) \\
+ & \left.\left(r_{0}-r_{1}\right) \exp \left(-\frac{2 k_{\mathrm{t}} \tau_{\mathrm{f}}+1}{\tau_{\mathrm{f}}} t\right)\right] \\
I_{\perp}(t)=F_{0}\left[\left(1-\frac{r_{0}+r_{1}}{2}\right)\right. & \exp \left(-\frac{t}{\tau_{\mathrm{f}}}\right) \\
& \left.-\frac{r_{0}-r_{1}}{2} \exp \left(-\frac{2 k_{\mathrm{t}} \tau_{\mathrm{f}}+1}{\tau_{\mathrm{f}}} t\right)\right] .
\end{aligned}
$$

\subsection{Antibunching experiments}

Photon antibunching means that there is always a finite temporal separation between the emitted photons of certain (single photon emitting) light sources. ${ }^{[110]}$ In a simplified scheme such light sources (e.g. individual atoms, fluorescent molecules, quantum dots or color centers) represent single two-state quantum emitters which can never emit two photons at the same time. ${ }^{[111]}$ For example an excited fluorophore which decays to the ground state by fluorescence needs some time (fluorescence lifetime) before emitting the next photon, because first it has to get excited again and afterwards it stays for the intrinsic excited state lifetime in the excited state before emitting the next photon. ${ }^{[6]}$

In the following, the Hanbury Brown and Twiss interferometer will be described which can be used to study the temporal correlation between emitted photons.

\subsubsection{Hanbury Brown and Twiss (HBT) interferometer}

To investigate the temporal correlation between photons of a beam, typically a Hanbury Brown and Twiss (HBT) interferometer is used (figure 21a). ${ }^{[112]}$ The incident beam is divided by a 50:50 beam splitter into two parts and both are directed towards separate detectors. The photon correlations, i.e., the conditional probability of detecting a photon at time $t+\tau$ after the detection of the first photon at time $t$ are determined by using time-correlated single photon- 
counting (TCSPC) electronics or fast photon-counting cards. ${ }^{[10]}$

a)

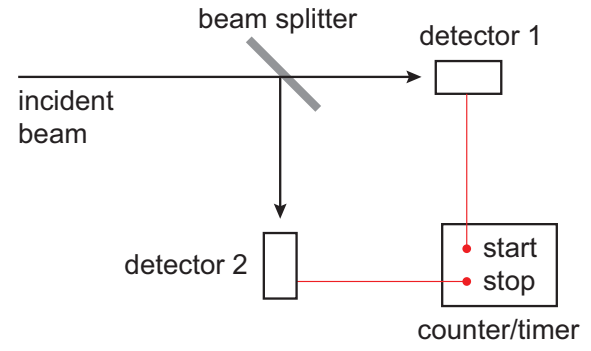

b)

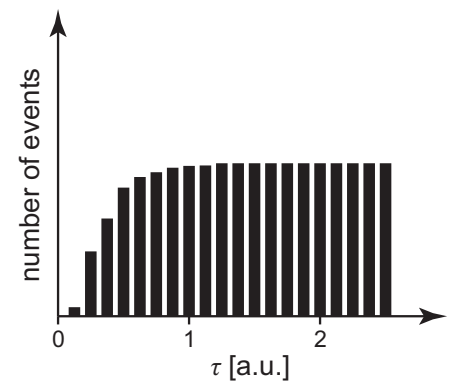

Figure 21: a) Hanbury Brown-Twiss setup for measuring time intervals between individual photons. The pulses of the two single-photon counting detectors are fed into the start/stop inputs of an electronic counter/timer. b) Typical results of such an experiment presented as histogram showing the number of events recorded within a particular time interval. In this case the histogram indicates photon antibunching. This figure was adapted from M. Fox. ${ }^{[113]}$

Figure 21a shows one possibility to determine photon correlations. The time lag between two subsequently detected photons is measured by using the signal of one detector as start-signal and the signal of the other detector as stop-signal for a TCSPC board. "Negative" photon-pair delay times can be measured by delaying the signals from one of the detectors. ${ }^{[110]}$ In this case, the first photon is detected in the stop-channel and causes a delayed signal. During this delay time the second photon is detected in the start-channel and starts the measurement of the photon-pair delay time.

Three scenarios of photon correlations are possible, which can be distinguished using the HBT interferometer: ${ }^{[13]}$

In the case of coherent light, the time intervals between photons are completely independent. The probability to detect a photon at time $t+\tau$ after a first photon at time $t$ is the same for any $\tau$.

The second scenario is termed photon bunching, and means that after detecting a photon at time $t$, there is a higher probability of detecting a second photon within short times than within long times, i.e. the probability to detect a second photon at time $t+\tau$ is larger for small values of $\tau$.

In the third scenario, which is termed photon antibunching, the detected photons are separated in time and two photons are never detected at the same time. In this scenario, the probability of detecting a photon at $t+\tau$ after detecting a 
photon at $t$ is small for small values of $\tau$ and increases with $\tau$.

The results of a measurement using a HBT interferrometer, are typically presented as a histogram as shown in figure $21 \mathrm{~b}$. The histogram shows the number of events at each value of the time $\tau$ between the start- and stop-signal. ${ }^{[113]}$ The example in figure $21 \mathrm{~b}$ shows photon antibunching, as can be seen by a vanishing probability to detect photon-pairs, when the delay time approaches zero $(\tau \approx 0)$.

\subsubsection{Second-order correlation function}

In order to quantify the results, it is helpful to introduce the second-order correlation function $G^{(2)}(\tau)$, which describes the shape of the histogram in figure $21 \mathrm{~b}$ and is defined as ${ }^{[113]}$

$$
G^{(2)}(\tau)=\frac{\langle I(t) I(t+\tau)\rangle}{\langle I(t)\rangle\langle I(t+\tau)\rangle}
$$

with $I(t)$ intensity of a light beam at time $t$, and $\langle\ldots\rangle$ symbols indicate averaging over a long time period.

Coming back to the classification of light, the second-order correlation function of perfectly coherent light is flat and gives $G^{(2)}(\tau)=1$ for any $\tau$. Bunched light shows a second-order correlation function with a maximum at $\tau=0$ and $G^{(2)}(0)>1$. In contrast, antibunched light shows a minimum at $\tau=0$ with $G^{(2)}(0)<1$. Figure 22a shows typical shapes of $G^{(2)}(\tau)$ for the three different photon correlations.

Figure 22b shows $G^{(2)}(\tau)$ for the detected emission of a single emitter which shows ideal antibunching and is excited by a pulsed laser. In this example, the laser has a repetition rate of $10 \mathrm{MHz}$ and accordingly $G^{(2)}(\tau)$ shows peaks with a separation of $100 \mathrm{~ns}$. The missing peak at $\tau=0$ clearly indicates antibunching. ${ }^{[110]}$ However, in real experimental data the peak at $\tau=0$ is small, but usually not completely gone. Reasons for this are the detection of background and the finite laser pulse width (the pulse shape is no $\delta$-function). 

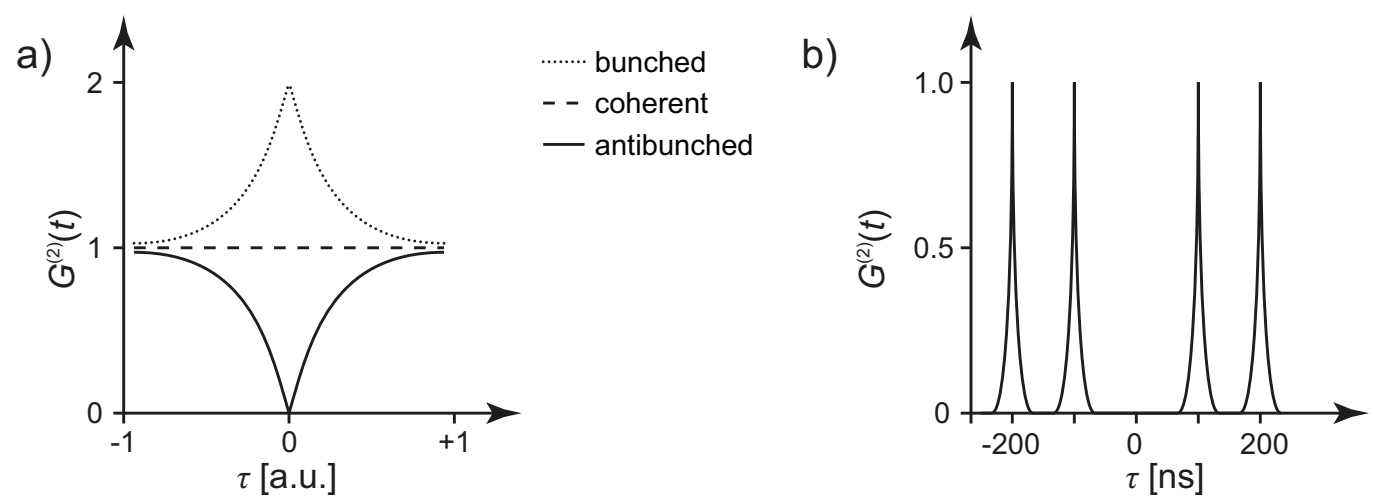

Figure 22: Second-order correlation function $G^{(2)}(\tau)$ versus interphoton time $\tau$ : a) For antibunched, coherent and bunched light (adapted from S. Lukishova); [114] b) (Ideal) antibunched emission of a single emitter excited by a pulsed laser with $10 \mathrm{MHz}$ repetition rate (adapted from K. Grußmayer et al.). ${ }^{[110]}$

\subsubsection{Photon antibunching in single molecule fluorescence spectroscopy}

In order to investigate single emitters, e.g. single molecules, a regular confocal microscope is combined with multiple independent detection channels (figure 23). The excitation beam is focused to a diffraction limited spot and the emission of the sample is collected by the same objective as used for illumination. The emission is separated from the excitation by a dichroic mirror before approaching the detectors. Additional bandpass filters may be placed preferentially before each detector, because upon detection of photons, avalanche photodiodes (APDs) are likely to emit infrared photons. ${ }^{[110]}$ This experimental setup enables measurements of single emitters, since exclusively emitters inside the focal volume contribute to the observed signal.

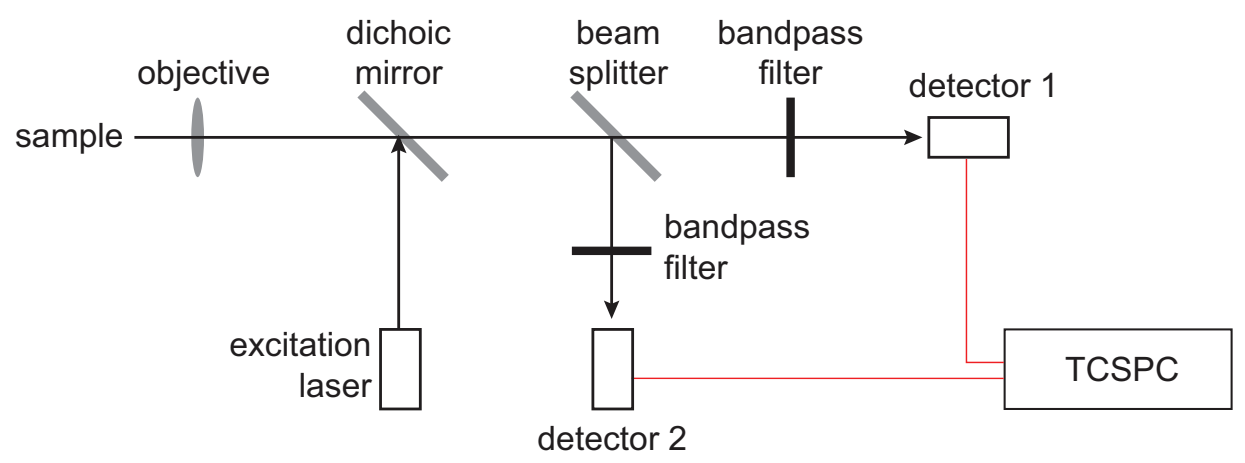

Figure 23: Simplified confocal microscope combined with multiple independent detection channels (adapted from K. Grußmayer et al..). ${ }^{[110]}$ 
As described above, photon antibunching causes a dip in the second-order correlation function at $\tau \approx 0$. In an ideal experiment $G^{(2)}(0)$ goes down to zero for single molecules. However, multiple fluorophores inside the focal volume as well as background photons diminish the dip at $\tau=0 .{ }^{[110]}$

\subsubsection{Determination of emitters per molecule}

The number of emitters per molecule $n$ can be determined by fitting the measured correlation functions as reported by Enderlein and co-workers. ${ }^{[115]}$ In the case of pulsed excitation, the correlation function can be fitted by the model functions

$$
G^{(2)}(\tau \approx 0)=A+B \sum_{j=0}^{\infty} \exp \left(-\frac{\left|\tau-j T_{\text {rep }}\right|}{\tau_{\mathrm{f}}}\right)-C \exp \left(-\frac{\tau}{\tau_{\mathrm{f}}}\right)
$$

for lag time $\tau$ close to zero and

$$
G^{(2)}(\tau \approx \infty)=A^{\prime}+B^{\prime} \sum_{j=0}^{\infty} \exp \left(-\frac{\left|\tau-j T_{\text {rep }}\right|}{\tau_{\mathrm{f}}}\right)
$$

for infinite lag time $\tau$. $T_{\text {rep }}$ is the time between subsequent laser pulses, whereas $A, B, C, A^{\prime}, B^{\prime}$ and $\tau_{\mathrm{f}}$ are fitting parameters. After performing the fitting with both model fuctions (equation 25 and 26) on the same data set, the number of independent emitters/chromophores per molecule is calculated by

$$
n=\frac{B-B^{\prime}}{C}
$$

The number of emitters $n$ given by equation 27 ignores the involvement of other states, for example transitions to triplet states. In order to consider additional states, the fluorescence correlation spectroscopy curves were fitted to model function

$$
G^{(2)}(\tau)=D+\frac{E+F \exp \left(-\frac{t}{t_{1}}\right)+G \exp \left(-\frac{t}{t_{2}}\right)}{(1+t / a) \sqrt{1+t / b}}
$$

with $D, F, G$ and $t_{i}(i=1,2,3$ or 4$)$ as fitting parameters. While $t_{1}$ and $t_{2}$ take fast decay of $G^{(2)}(\tau)$ into account, parameters $a$ and $b$ describe long-time 


\section{\begin{tabular}{l|l} 
Chapter 3 & EXPERIMENTAL INVESTIGATION OF INTRAMOLECULAR EET
\end{tabular}}

decay due to intersystem crossing of fluorophores to a long live triplet-state $\left(\tau \approx 10^{-7} \ldots 10^{-5} \mathrm{~s}\right.$ ) and diffusion of the fluorescent molecules out of the detection volume $\left(\tau \approx 10^{-5} \ldots 1 \mathrm{~s}\right) .{ }^{[116]}$ In the end, the corrected number of independent emitters per molecules is calculated by

$$
n=\frac{B-B^{\prime}}{C} \cdot \frac{D+E+F+G}{D+E} .
$$

\subsubsection{Singlet-singlet annihilation}

One possible reason for a bichromophoric (or multichromophoric) compound acting as a single emitter is singlet-singlet annihilation (scheme 10). ${ }^{[117]}$

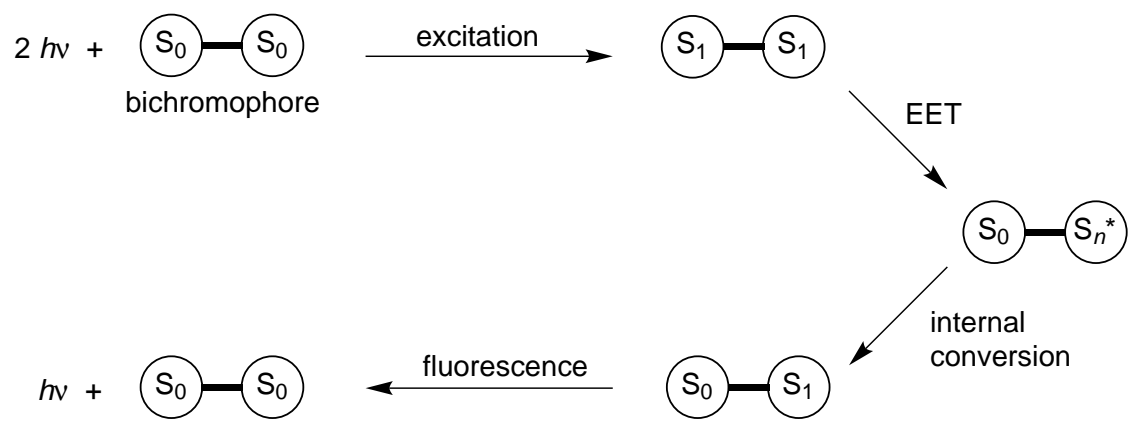

Scheme 10: Singlet-singlet annihilation in a bichromophoric compound.

If both dyes in a bichromophore are excited simultaneously by absorption of two photons, a double excited molecule $\left(\mathrm{S}_{1}-\mathrm{S}_{1}\right)$ can result. In the case of singletsinglet annihilation, the excited state energy from one chromophore is transferred to the other excited chromophore. As a result, one chromophore gets to it's singlet ground state $\left(\mathrm{S}_{0}\right)$ and the other one gets to an vibronically excited singlet state $\left(\mathrm{S}_{n}{ }^{*}\right.$ with $\left.n>1\right)$. The chromophore at high excited singlet state quickly relaxes back to the first singlet state $\left(S_{n}{ }^{*} \rightarrow S_{1}\right)$ by radiationless internal conversion. The resulting mono-excited bichromophore can then emit a single photon. In conclusion, although both chromophores in a bichromophore can be excited simultaneously, in the case of singlet-singlet annihilation it acts as a single emitter. ${ }^{[118]}$

It is noteworthy, that double-excited molecules of type $S_{1}-S_{1}$ are not possible in the other experiments described in this chapter. The excitation rate used in the other experiments is very low and the chances to excite two linked chromophores 
at the same time are virtually zero. In contrast, the excitation intensities in antibunching experiments are very high and the excitation of both chromophores of the same bichromophore is more likely. 



\section{Aim and general strategy of this project}

\subsection{Aim of this project}

Bichromophoric systems of the type "donor-linker-acceptor" are essential for the theoretical and experimental investigation of EET. The properties of the linker are decisive for the properties of the whole assembly determined by the degree of interaction between the donor and acceptor parts. The linker determines the separation and relative orientation of the chromophores, as well as the rigidity of the bichromophore. Many different molecular architectures were utilized for the design of bichromophoric compounds (see chapter 2).

Although most bichromophores provide a rather fixed distance between the chromophores, internal rotation around single bonds often allows a fast changing of the relative orientation. Even in the case of rod-like linkers where rotation around single bonds between aromatic rings neither affects the distance nor the angle between transition dipoles, the latter may suffer from bending at single bonds. ${ }^{[5 \mathrm{~d}, 119]}$ Thus, there is the great demand for rigid bichromophoric systems with fixed distance and relative orientation of chromophores. The perpendicularly oriented bichromophores are of particular interest. They show strongest deviations from common EET theories (e.g. classical Förster or Dexter theory). ${ }^{[5 d, 99]}$ Therefore, this project was aimed at the development and characterization of bichromophores with well-defined geometry in particular with perpendicular oriented transition dipole moments and without chromophore rotation. This goal could be achieved only by applying the rigid adamantane linkers with quaternary carbon atoms which, to great extent, determine the design of fluorophores and the chemistry of their assembling.

\subsection{General strategy}

The fundamental strategy for the design of bichromophores with well-defined geometry is based on the adamantane framework. The unique structural features of adamantane have long been a source of fascination for chemists. ${ }^{[120]}$ The highly symmetric $\sigma$-framework composed of three fused chair cyclohexane rings provides an exceptional rigid but strain-free structure which in this project 
shall serve as linker for the design of rigid bichromophores. The functionalization at the 2 and 6 position as illustrated schematically in figure 24 (left structure) allows the design of bichromophores with fixed relative orientation in desired geometry with high rigidity and no chromophore rotation. As a complement the right structure in figure 24 based on 2-(2-adamantylidene)adamantane is suitable for the design of analogous bichromophores but with parallel orientation of chromophores at larger separation.

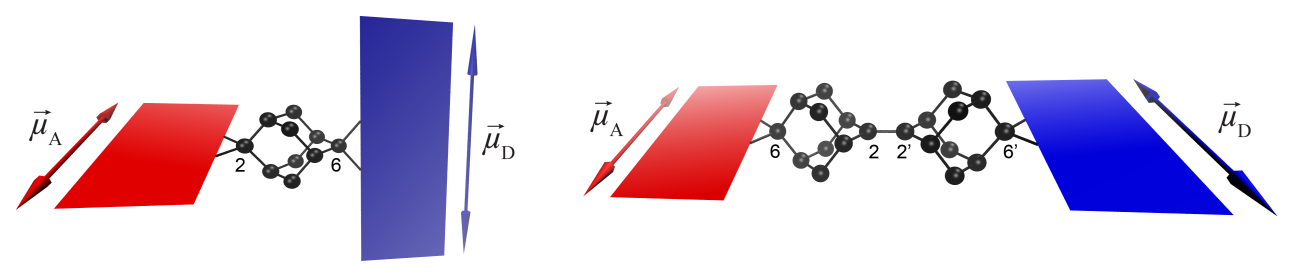

Figure 24: Schematic representation of adamantane (left) and 2-(2-adamantylidene)adamantane (right) as rigid linkers between orthogonally and parallel arranged chromophores, respectively. EET donor and acceptor transition dipole moments $\vec{\mu}_{\mathrm{D}}$ and $\vec{\mu}_{\mathrm{A}}$ are shown as red and blue double-headed arrows, respectively.

According to the FRET point-dipole approximation, the orthogonal transition dipole arrangement forbids and the parallel arrangement favors an energy transfer based on dipole-dipole interactions (see section 1.2.1).

Since the proposed adamantane-based linkers are non-aromatic, electron transfer (ET) processes mediated by conjugated systems are excluded, but also saturated bridges can mediate EET and ET processes ${ }^{[85,121]}$ and the rigidity of the bridge - which provides the desired fixation of the chromophore orientation seems to favor through-bond energy transfer ${ }^{[76]}$ (see chapter 1.2).

As we'll see, adamantane linkers are difficult to incorporate into the structures of "common" fluorescent dyes. Therefore, we had to design and synthesize new "red" fluorophores attachable to adamantane residues. 


\section{Part II}

\section{Results and Discussion}





\section{Strategies to link fluorescent dyes to the adamantane scaffold}

Different methods to link fluorophores to the adamantane skeleton were considered. The most promising approaches are illustrated in scheme 11. For linking of bichromophores, 2,6-adamantanedione (23b) is required, and for model compounds with one dye residue, 2-adamantanone (23a) could be used.

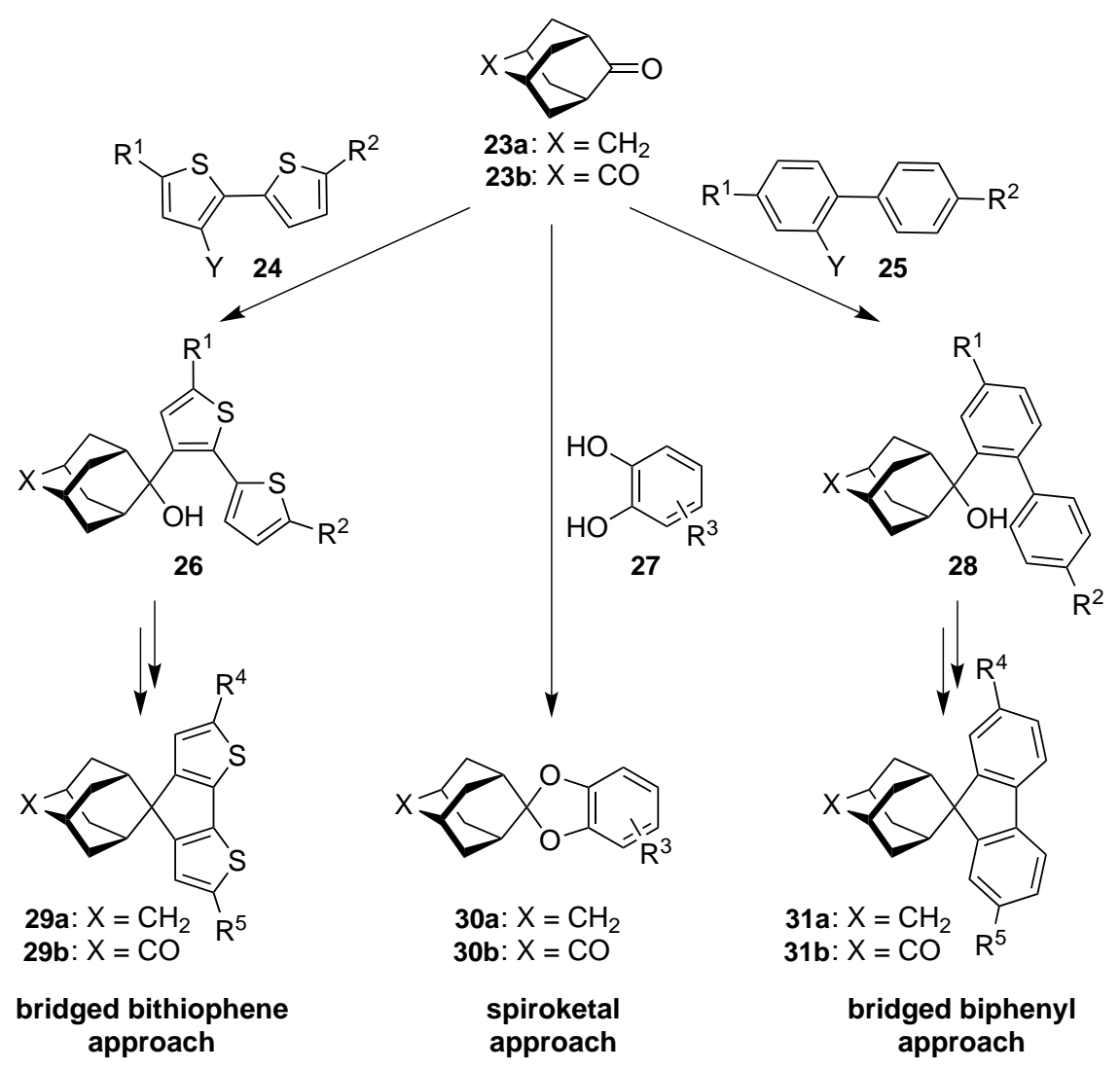

Scheme 11: Strategies to link fluorescent dyes rigidly and without rotation via an adamantane residue; $\mathrm{Y}=\mathrm{Mgl}, \mathrm{MgBr}, \mathrm{MgCl}, \mathrm{Li} ; \mathrm{R}^{1-5}=\mathrm{Ar}$, electron withdrawing/donating group.

One possible option to attach fluorophores to the adamantane structure is based on the formation of spiroketals 30 from $o$-diphenols 27. This can be accomplished by reacting $\boldsymbol{o}$-diphenols $\mathbf{2 7}$ with adamantane derivatives $\mathbf{2 3}$ under DeanStark conditions. ${ }^{[122]}$

An alternative strategy to link chromophores to the adamantane scaffold is the formation of spiro compounds like $\mathbf{2 9}$ or $\mathbf{3 1}$ where a carbonyl carbon 


\section{\begin{tabular}{l|l} 
Chapter 5 & STRATEGIES TO LINK FLUORESCENT DYES TO THE ...
\end{tabular}}

of compound $\mathbf{2 3}$ is transformed into a spiro atom. In general, this may be achieved by the reaction of organometallic biaryl compounds such as $\mathbf{2 4}$ or 25 with 2-adamantanone or 2,6-adamantanedione providing tertiary alcohols 26 or $28,{ }^{[123]}$ respectively. Intramolecular cyclization (dehydration under acidic conditions) is expected to give the desired spiro compounds 29 and 31, ${ }^{[123]}$ respectively. The former has a bridged 2,2'-bithiophene as basic structure, whereas the latter is based on fluorene.

In general, the absorption band of the fluorene core lies at shorter wavelength $\left(261 \mathrm{~nm}^{[124]}\right.$ in $\mathrm{CH}_{2} \mathrm{Cl}_{2}$ ) than in case of bridged bithiophene $(311 \mathrm{~nm}) .{ }^{[125]+}$ The absorption bands may be shifted towards longer wavelengths by attachment of substituents $\mathrm{R}^{4,5}$, such as aromatic systems, which extend the $\pi$-conjugation path, and/or electron donors/acceptors to generate "push/pull" chromophores. Examples of non-bridges analogues show, that the absorption band of the fluorene based chromophore may be shifted only to about $400 \mathrm{~nm},{ }^{[126,127]}$ whereas the absorption of the bithiophene may even be shifted up to about $600 \mathrm{~nm},{ }^{[128,129]}$ depending on the groups $\mathrm{R}^{4,5}$.

Besides the advantageous rigidity of structures 29 and 31, the bridging of the bithiophene or biphenyl unit by the spiro atom forces the chromophore core to adopt a planar conformation and reduces the vulnerability to deformations. Consequently, the fluorescence quantum yield is expected to increase in comparison with the non-bridged analogues. ${ }^{[128-131]}$ This effect is expected to be especially pronounced for red chromophores, which are most sensitive to external influences.

\footnotetext{
${ }^{\dagger}$ Value for $4 H$-cyclopenta[2,1-b:3,4-b']dithiophene; solvent not specified in ref. [125].
} 


\section{Spiroketal compounds}

As illustrated in scheme 11, spiroketals were prepared from o-diphenols and 2-adamantanone (23a) and provided compounds with single fluorophores. Bichromophores with two different chromophoes were prepared by coupling of two different $o$-diphenols with 2,6-adamantanedione (23b).

\subsection{Synthesis of bichromophore 36}

Commercially available 4-methylesculetin (32) and alizarin (34) were chosen as $o$-diphenols. The synthesis was carried out under Dean-Stark conditions, ${ }^{[122]}$ giving model compounds 33, 35 and bichromophore 36 (scheme 12).

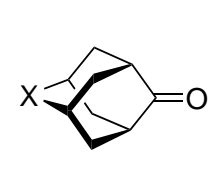

23a: $\mathrm{X}=\mathrm{CH}_{2}$ 23b: $X=\mathrm{CO}$

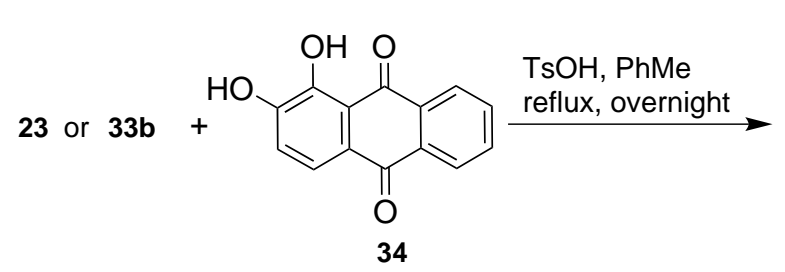

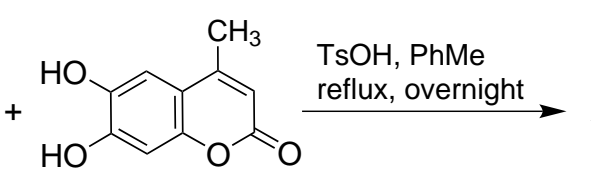

32

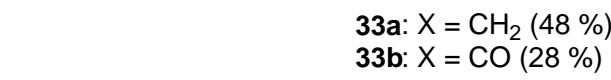

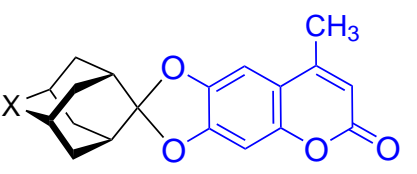

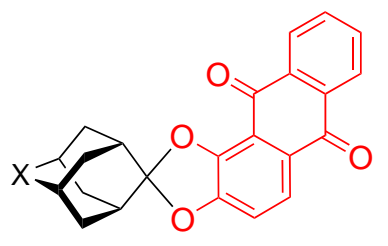

35a: $\mathrm{X}=\mathrm{CH}_{2}(18 \%)$

35b: $X=\mathrm{CO}(44 \%)$

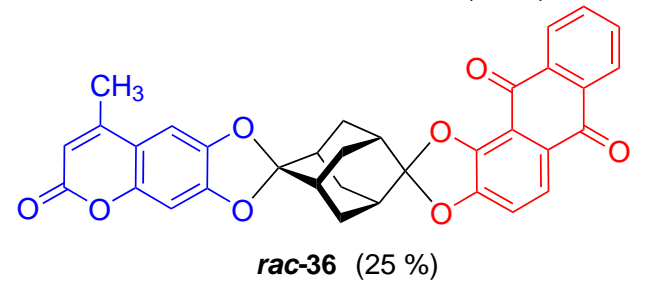

Scheme 12: Spiroketal formation under Dean-Stark conditions based on 4-methylesculetin (32) and alizarin (34).

\subsection{Photophysical properties of model compounds 33a, 35a and bichromophore 36}

The photophysical properties of model compounds $33 \mathbf{a}$ and $\mathbf{3 5 a}$ as well as bichromophore 36 are given in table 1. 


\section{\begin{tabular}{l|l} 
Chapter 6 & SPIROKETAL COMPOUNDS
\end{tabular}}

Table 1: Photophysical properties of model compound 33a, model compound 35a, and compound 36 in $\mathrm{MeCN}$.

\begin{tabular}{lllll}
\hline Compound & $\lambda_{\mathrm{abs}}^{\max } / \mathrm{nm}$ & $\lambda_{\mathrm{em}}^{\max } / \mathrm{nm}$ & $\mathcal{E} / \mathrm{mol} \mathrm{I}^{-1} \mathrm{~cm}^{-1}$ & $\Phi_{\mathrm{f}}{ }^{\mathrm{a}}$ \\
\hline 33a & 345 & 413 & 12,500 & 0.16 \\
35a & 411 & 524 & 6,400 & 0.15 \\
36 & $344,(406)$ & 520 & $12,700(7,000)$ & 0.14 \\
\hline
\end{tabular}

(a) Quantum yields $\Phi_{\mathrm{f}}$ were measured using Coumarin 120 in $\mathrm{MeOH}\left(\Phi_{\mathrm{f}}=0.51\right)^{[132]}$ as a reference for 33a and Coumarin 522 in $\mathrm{EtOH}\left(\Phi_{\mathrm{f}}=0.65\right)^{[133]}$ for compounds 35a and 36.

The absorption spectrum of bichromophore 36 equals the sum of compounds $33 a$ and 35a absorption spectra (figure 25).

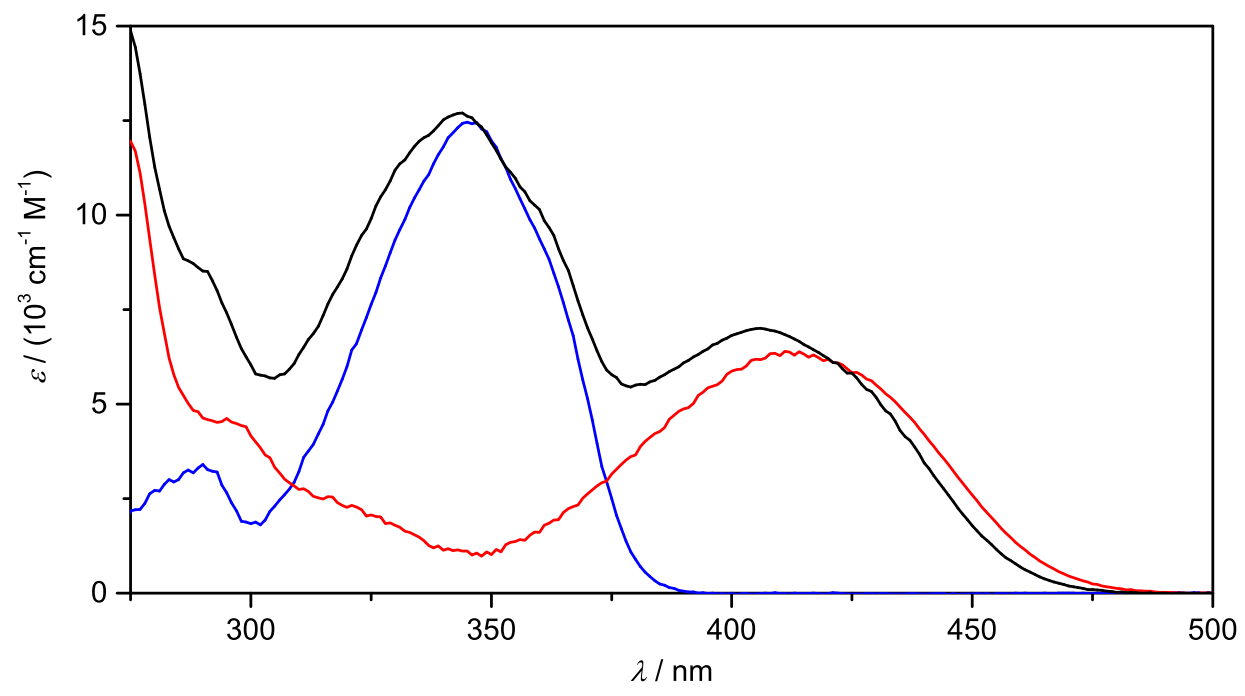

Figure 25: Absorption spectra of model compound 33a (blue line), model compound 35a (red line) and bichromophore 36 (black line) in MeCN.

Figure 26 shows the absorption and emission spectra of model compounds 33a and 35a. The emission maximum of "blue" model compound 33a matches the absorption maximum of the "red" model compound 35a (at $411 \mathrm{~nm})$. The corresponding spectral overlap was calculated to be $J_{\mathrm{DA}}=1.3 \cdot 10^{14} \mathrm{M}^{-1} \mathrm{~cm}^{-1} \mathrm{~nm}^{4}$ (the overlap region is marked as hatched area in figure 26). 


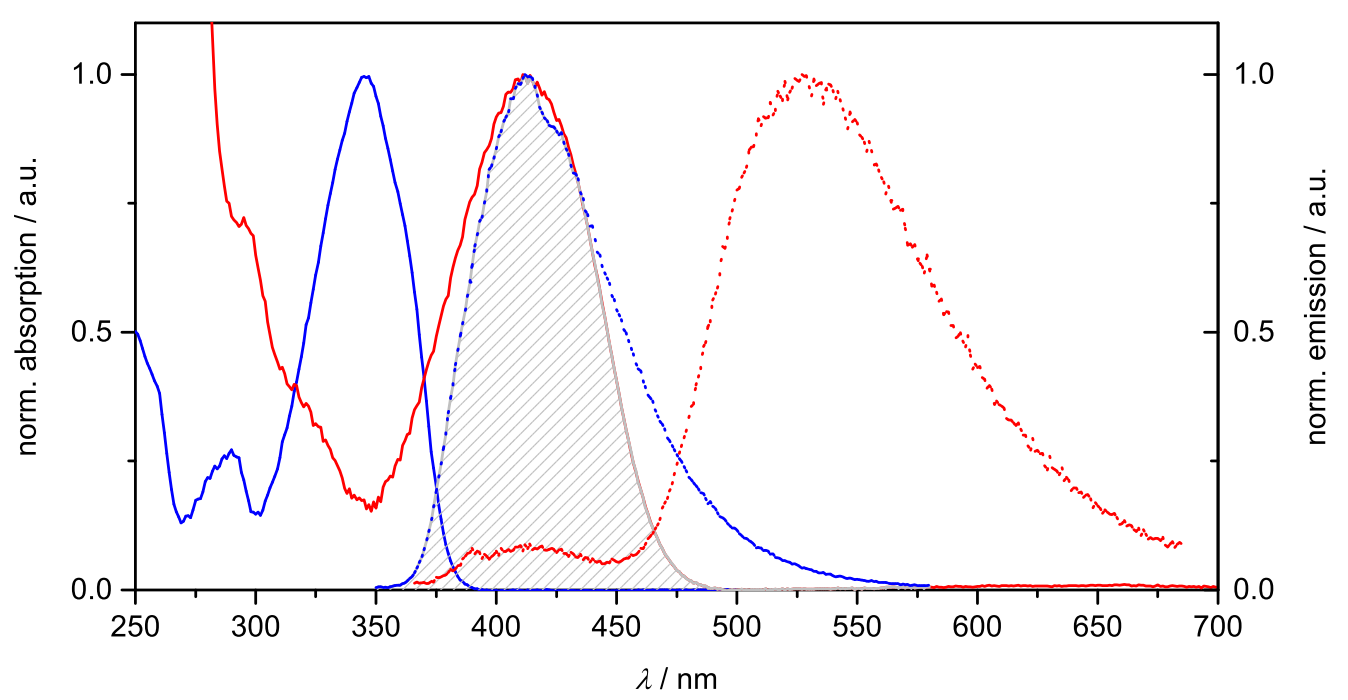

Figure 26: Normalized absorption (solid lines) and emission spectra (dotted lines) of model compounds 33a (blue line) and 35a (red line) in MeCN. Excitation wavelengths were $300 \mathrm{~nm}$ and $350 \mathrm{~nm}$, respectively. Overlapping region of compounds 33a emission and compounds 35a absorption is displayed hatched.

Excitation of bichromophore 36 at $345 \mathrm{~nm}$ (absorption band of the "blue" fluorophore) or at $411 \mathrm{~nm}$ (absorption band of the "red" fluorophore) result in the same emission spectrum as excitation of model compound 35a at $350 \mathrm{~nm}$ (figure 27). This result indicates efficient EET from the "blue" (coumarin) to the "red" (alizarin) fluorophore of bichromophore 36. Some very weak emission at around $410 \mathrm{~nm}$ upon excitation at $345 \mathrm{~nm}$ was observed. Residual emission of the blue fluorophore seems to be unlikely, because the EET is expected to be $100 \%$ due to the big overlap integral, the angle between the chromophores and transition dipole moments (see section 6.3 ) and the short distance $(<1 \mathrm{~nm})$ between the fluorophores. Therefore, the emission at $411 \mathrm{~nm}$ is most likely the result of an impurity, maybe a trace of compound $\mathbf{3 3 b}$ which could be the result of $\mathbf{3 6}$ hydrolysis or remaining from the synthesis of compound 36 . 


\section{\begin{tabular}{l|l} 
Chapter 6 & SPIROKETAL COMPOUNDS
\end{tabular}}

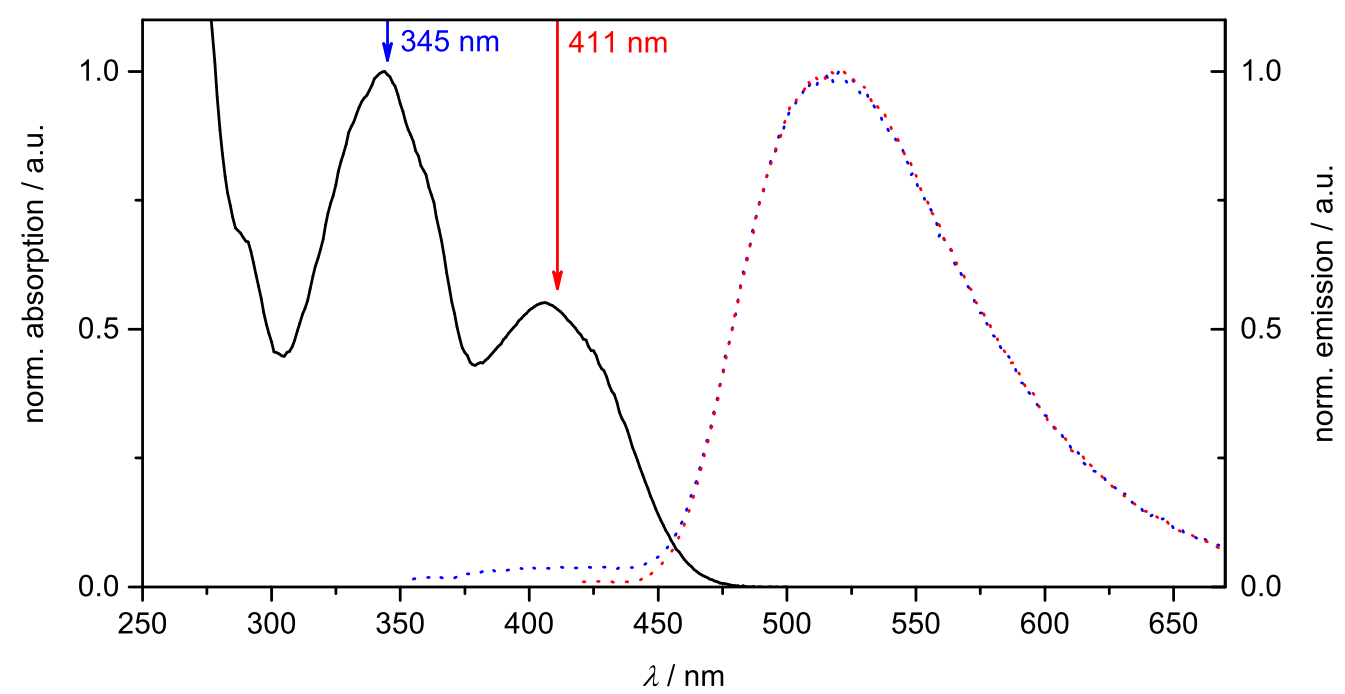

Figure 27: Absorption spectrum (black line) and normalized emission spectra with excitation at $345 \mathrm{~nm}$ (dotted blue line) and at $411 \mathrm{~nm}$ (dotted red line) of bichromophore 36 in $\mathrm{MeCN}$. Excitation wavelengths are marked with arrows.

\subsection{DFT calculations for model compounds 33a, 35a and bichromophore 36}

In order to determine the chromophore arrangement and also the arrangement of corresponding transition dipoles, (time-dependent) density functional calculations were carried out. The geometrical parameters are used later to estimate the energy transfer efficiency based on the Förster point-dipole approximation.

The "blue" and "red" chromophores will act as an energy donor and acceptor in bichromophore 36, respectively. The former will be in the $S_{1}$ state and the latter will be in the $S_{0}$ state geometery, when the EET process takes place. Figure 28 shows the ground state and $S_{1}$ excited state geometry of model compound 33a. 
ground state geometry
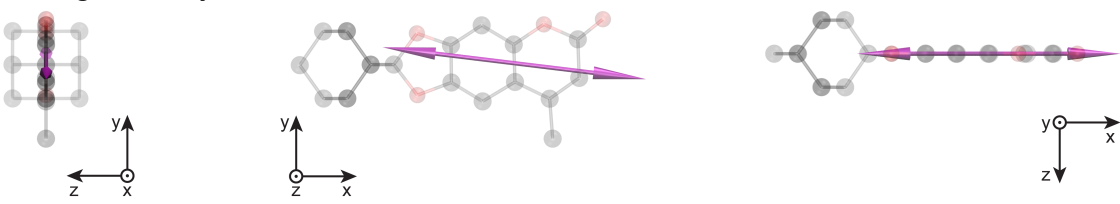

$\mathrm{S}_{1}$ state geometry
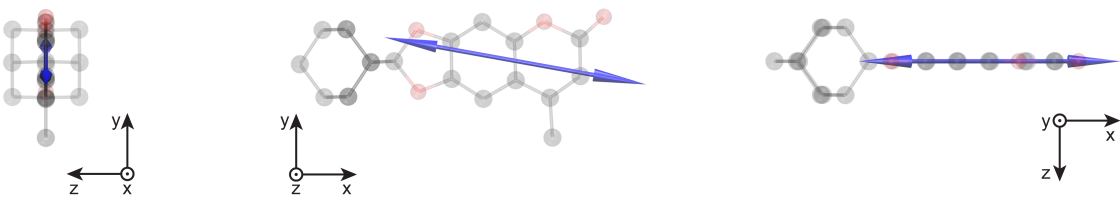

Figure 28: DFT and TD-DFT optimized geometries of model compound 33a and the transition dipole moments shown as double-headed arrows. Top: Ground state geometry with absorption $\left(\mathrm{S}_{0} \rightarrow \mathrm{S}_{1}\right)$ transition dipole moment (purple arrow); bottom: $S_{1}$ state geometry and emission $\left(S_{1} \rightarrow S_{0}\right)$ transition dipole moment (blue arrow). $\mathrm{H}$ atoms are omitted for clarity.

The ground state geometry of the "red" model compound 35a is shown in figure 29.
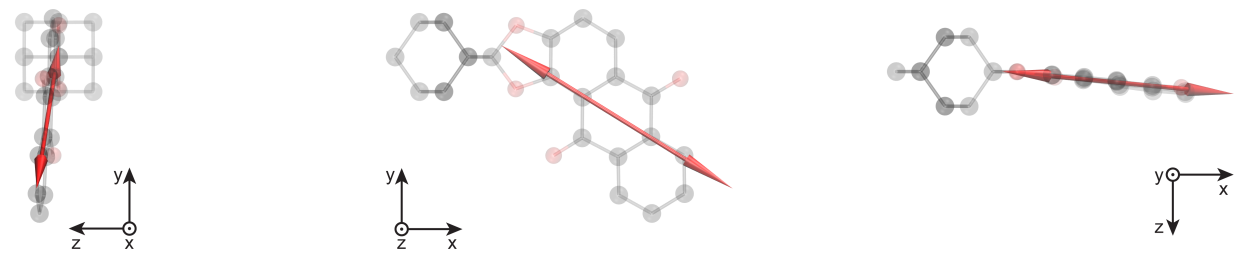

Figure 29: DFT optimized geometry of model compound 35a and absorption $\left(\mathrm{S}_{0} \rightarrow \mathrm{S}_{1}\right)$ transition dipole moment shown as red double-headed arrow. $\mathrm{H}$ atoms are omitted for clarity.

The ground state geometry of bichromophore 36 is shown in figure 30 . An important geometrical parameter for the spiroketal approach is the angle between the plane defined by the chromophore's aromatic system and the axis defined by adamantane's C- 2 and C- 6 atoms. For the design of bichromophores with orthogonally arranged chromophore planes (or transition dipoles) that axis is supposed to be parallel to both chromophore planes. In the ground state, the angle between the axis and the aromatic systems of the "red" and "blue" model compounds are $5.3^{\circ}$ and $2.3^{\circ}$, respectively. The corresponding angles in the bichromophores ground state are $0.2^{\circ}$ and $3.9^{\circ}$. However, in the excited state of the "blue" model compound this angle is $0.5^{\circ}$. The non-zero angles show a major disadvantage of the spiroketal approach. 


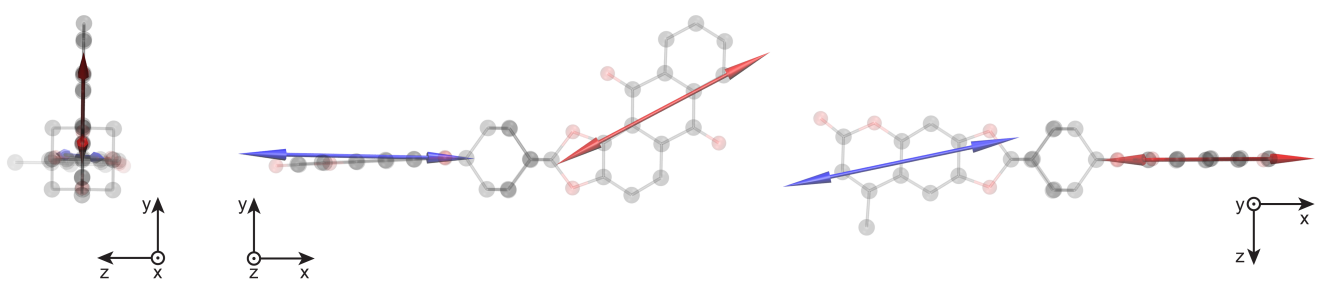

Figure 30: DFT optimized ground state geometry of bichromophore 36. The absorption $\left(\mathrm{S}_{0} \rightarrow \mathrm{S}_{1}\right)$ and emission $\left(\mathrm{S}_{1} \rightarrow \mathrm{S}_{0}\right)$ transition dipole moments are shown as red and blue double-headed arrows, respectively. $\mathrm{H}$ atoms are omitted for clarity.

Time-dependent density functional calculations were carried out to determine the orientation of the absorption/emission transition dipole moments. The absorption transition dipole moment of the "red" chromophore in bichromophore 36 was determined directly for the ground state geometry. The emission transition dipole of the "blue" part in bichromophore 36 was determined by transferring it's orientation from model compound 33a to bichromophore 36 (see chapter 19 for details). The results are shown in figures 28, 29 and 30 as double-headed arrows, placed at the geometrical center of the individual fluorophores.

\subsection{Estimation of energy transfer efficiency based on ideal Förster point-dipole approximation for bichromophore 36}

The geometrical arrangement of the transition dipole moments was used to calculate the orientation factor $\kappa^{2}$ and with the photophysical properties of the model compounds (section 6.2) the energy transfer efficiency based on the ideal Förster point-dipole approximation was estimated.

The angles $\theta_{\mathrm{D}}, \theta_{\mathrm{A}}$ and $\theta_{\mathrm{DA}}$ and distance $\left|\vec{R}_{\mathrm{DA}}\right|$ between the emission and absorption point-dipoles required for applying the FRET approximation are given in table 2 (see section 1.2.1 for angle definitions). Using equation 4, the orientation factor $\kappa^{2}$ was calculated. Taking into account the spectral overlap between compound 33a's emission (donor) and compound 35a's absorption $J_{\mathrm{DA}}=1.3 \cdot 10^{14} \mathrm{M}^{-1} \mathrm{~cm}^{-1} \mathrm{~nm}^{4}$, and the refractive index of the solvent $\left(n_{\mathrm{D}}\left(\mathrm{EtOAc}, 20^{\circ} \mathrm{C}\right)=1.3723\right),{ }^{[134]}$ the Förster radius $R_{0}$ and the FRET efficiency 
$E_{\text {FRET }}$ were calculated (table 2).

Table 2: Angles $\theta_{\mathrm{D}}, \theta_{\mathrm{A}}$ and $\theta_{\mathrm{DA}}$ between transition dipole moments and interconnecting vector $\vec{R}_{\mathrm{DA}}$, orientation factor $\kappa^{2}$, distance between the donor and acceptor $\left|\vec{R}_{\mathrm{DA}}\right|$, Förster radius $R_{0}$, and estimated FRET efficiency $E_{\mathrm{FRET}}$.

\begin{tabular}{llllllll}
\hline Compound & $\theta_{\mathrm{D}}$ & $\theta_{\mathrm{A}}$ & $\theta_{\mathrm{DA}}$ & $\kappa^{2}$ & $\left|\vec{R}_{\mathrm{DA}}\right| / \mathrm{nm}$ & $R_{0} / \mathrm{nm}$ & $E_{\mathrm{FRET}}$ \\
\hline 36 & $30.9^{\circ}$ & $15.0^{\circ}$ & $18.9^{\circ}$ & 3.55 & 1.28 & 3.50 & 1.00 \\
\hline
\end{tabular}

As expected due to the geometrical arrangement of the transition dipole moments and the large spectral overlap, the calculated FRET efficiency is $E_{\mathrm{FRET}}=1.00$.

As a proof of principle, bichromophore 36 shows, that this approach can be used for the design of the rigid bichromophoric compounds without internal rotation of fluorophores. However, this approach has considerable disadvantages. Only few dyes with an $o$-diphenol motif are known. Moreover, for an orthogonal arrangement of transition dipole moments, new chromophores with an $o$-diphenol motif would be necessary. More importantly, density functional calculations showed, that the five-membered ring which is formed by the $o$-diphenol oxygens and the spiro atom of the adamantane scaffold are not planar. In this respect, the alternative approaches based on organometallic biaryl compounds (see chapter 5 ), seemed to be more promising and has been pursued in the following studies. 



\section{Develelopment of spiroadamantylfluorene chromophores}

In this chapter the development of blue fluorescent dyes based on fluorene directly joined with the adamantane scaffold will be described. The general idea was already shown in scheme 11; the fluorene system is joined to the adamantane scaffold via a spiro atom (fluorene C-9 and adamantane C-2). For this reason, the new class of dyes will be termed spiroadamantylfluorenes.

\subsection{Spiroadamantylfluorene core build-up before chromophore modification}

Initially we tried to construct the spiroadamantylfluorene core in a first step and then, in a second step, to introduce aryl substituents in order to shift the absorption bands towards longer wavelengths.

Huang et al. reported the synthesis of spiroadamantylfluoren 43a from 2-aminobiphenyl (37) via 2-iodobiphenyl (38) followed by the reaction with

2-adamantanone (23a) and acid-catalyzed cyclization (scheme13). ${ }^{[123]}$ This method was applied to adamantane-2,6-dione (23b), afforded alcohol 39b, and after cyclization under acidic conditions, the spiro compound $43 \mathbf{b}$. For a bathochromic shift of the absorption band, a pyridyl group was introduced. It extends the aromatic system and could be used later on as an electron acceptor in all push-pull systems after additional modifications. After accomplishing mono-bromination using elemental bromine, bromide $\mathbf{4 2}$ was obtained and reacted with pyridyl stannane $\mathbf{4 1}$ in a Stille cross-coupling reaction (scheme13). 


\section{\begin{tabular}{l|l} 
Chapter 7 & DEVELELOPMENT OF SPIROADAMANTYLFLUORENE...
\end{tabular}}

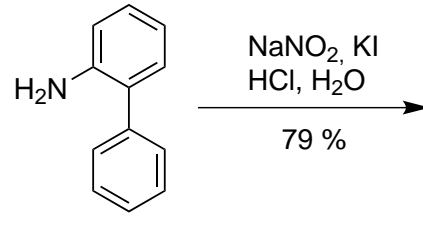

37<smiles>Ic1ccccc1-c1ccccc1</smiles>

38
1) $\mathrm{Mg}, \mathrm{I}_{2}, \mathrm{Et}_{2} \mathrm{O}$

2) $23 a$ or $23 b$

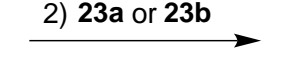

39a: $\mathrm{X}=\mathrm{CH}_{2}$

$39 b: X=C O(47 \%)$
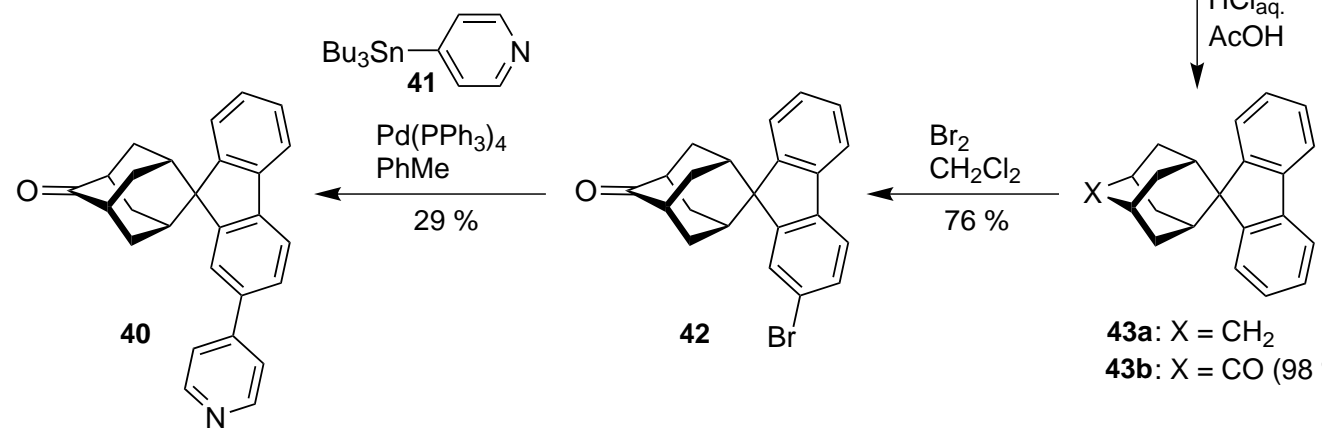

43a: $\mathrm{X}=\mathrm{CH}_{2}$ 43b: $X=C O(98 \%)$

Scheme 13: Synthesis of fluorene related compound 43a with spiroadamantyl linker reported by Huang and co-workers. ${ }^{[123]}$ In an analogous synthesis we obtained carbonyl compound $\mathbf{4 3 b}$ and introduced a pyridyl moiety providing compound 40.

Spiroadamantylfluoren 40 shows an absorption maximum at $\lambda_{\text {abs }}^{\max }=310 \mathrm{~nm}$ and blue fluorescence with maximum at $\lambda_{\mathrm{em}}^{\mathrm{max}}=373 \mathrm{~nm}$ with a quantum yield of $\Phi_{\mathrm{f}}=0.23$ in $\mathrm{MeOH}$. It can be assumed that the obtained spiroadamantylfluorene $\mathbf{4 0}$ has a rigid structure and a favorable geometry. However, we planned to shift the absorption band further towards longer wavelengths, because the absorption at around $310 \mathrm{~nm}$ is still in the UV region and makes experiments with common optics laborious or even impossible.

To shift the absorption band of spiroadamantylfluorene $\mathbf{4 0}$ to longer wavelengths, an electron-donating group (e.g. $N$-morpholinyl substituent) as a counterpart to the electron-withdrawing pyridyl moiety should be installed, in order to generate the push-pull fluorophore 45 (scheme 14). 

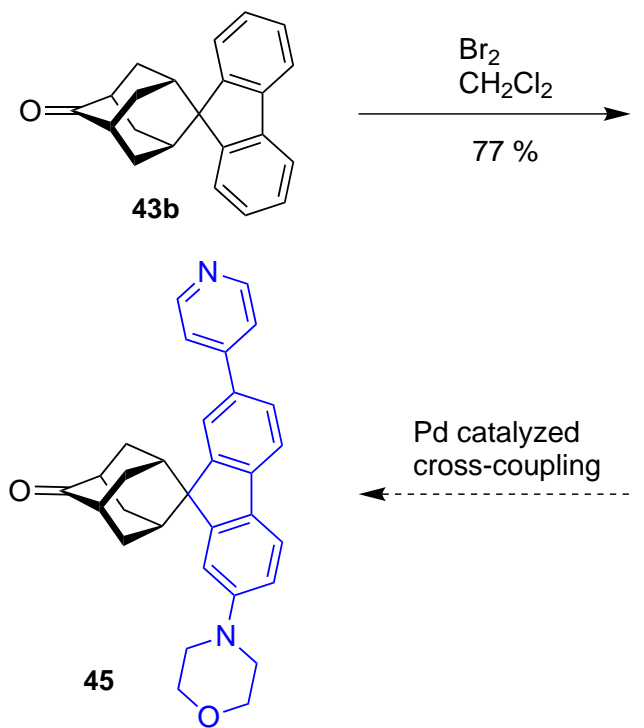

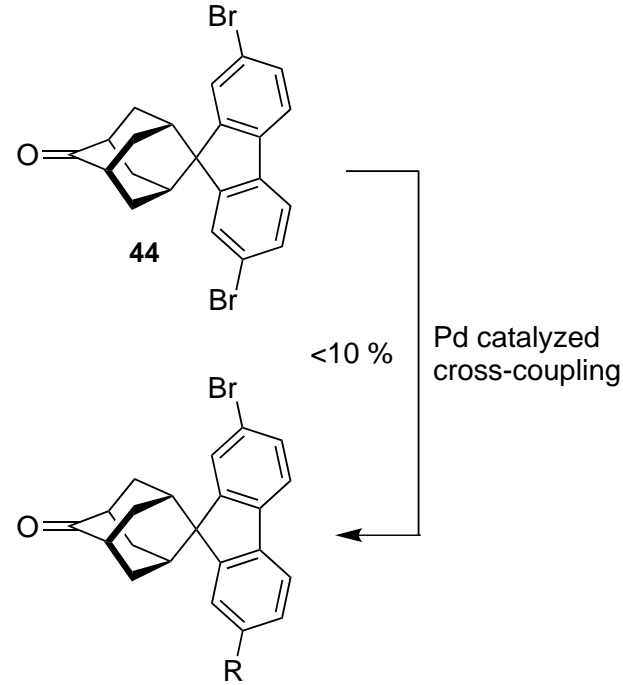

$46: \mathrm{R}=4$-pyridinyl $47: R=4$-morpholinyl

Scheme 14: First synthetic approach to compound 45.

For this purpose, compound $\mathbf{4 3 b}$ was dibrominated to give dibromide 44 (scheme 14). Then the bromine atoms should be replaced sequentially by the pyridyl and morpholinyl groups (or vice versa) using Pd-catalyzed cross-coupling reactions. It turned out, that under common Buchwald-Hartwig conditions only small quantities of the desired mono-substitution coupling product 47 with a $\mathrm{N}$-morpholinyl residue could be obtained (less than $10 \%$ yield, based on equivalents of 44). Also the Stille cross-coupling of dibromide 44 with stannane 41 gave poor yields (also $<10 \%$, regardless the fact, that mono-bromide 42 could be coupled with stannane 41 ). It was concluded, that dibromide 44 possesses weak reactivity in $\mathrm{Pd}$-catalyzed cross-couplings. Consequently, an alternative synthesis route was developed as presented below.

\subsection{Synthesis of substituted spiroadamantylfluorene system from a precursor with already expanded aromatic system}

The introduction of push-pull substituents by Pd-catalyzed reactions of dibromide 44 turned out to be problematic. It was speculated, that the bulky adamantyl group may impede the Pd-catalyzed reactions. For this reason, pre- 


\section{\begin{tabular}{l|l} 
Chapter 7 & DEVELELOPMENT OF SPIROADAMANTYLFLUORENE...
\end{tabular}}

cursor 48 was synthesized, which already included the push-pull substituents. Then compound $\mathbf{4 8}$ was subjected to bromine-lithium exchange and introduced into the reaction with 2,6-adamantanedione (23b) as shown in scheme 15 . In this case, no Pd-catalyzed cross-couplings need to be carried out after spirocyclization with the adamantane residue (scheme 15).

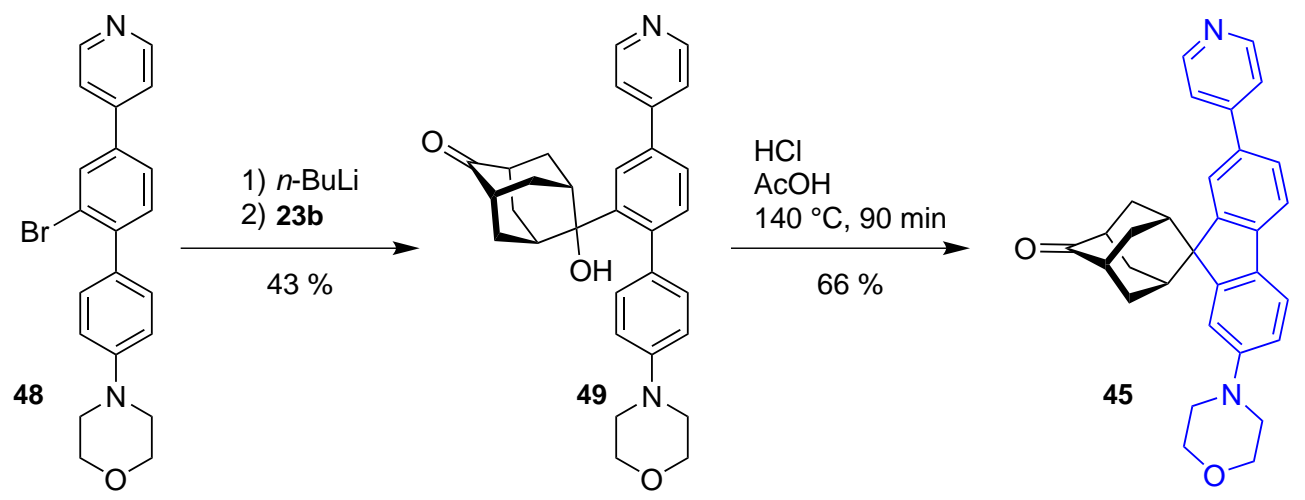

Scheme 15: Preparation of compound 45 based on building block 48.

The tertiary alcohol $\mathbf{4 9}$ was cyclized under acidic conditions to afford spiroadamantylfluorene $\mathbf{4 5}$ as the desired push-pull fluorophore (scheme 15).

The preparation of the aromatic bromide 48 was carried out as given in scheme 16. Firstly, the synthesis of nitrobiphenyl $\mathbf{5 1}$ was accomplished according to literature ${ }^{[135]}$ by nitration of dibromide $\mathbf{5 0}$ followed by Suzuki coupling with 4-pyridylboronic acid (52) to give bromide 54 (scheme 16). The introduction of the morpholinyl group under Buchwald-Hartwig conditions gave the desired product 56. The yield of compound $\mathbf{5 6}$ varied from 30 to $92 \%$, even though the reaction conditions were kept constant. Possibly, the strong base $\mathrm{NaO} t \mathrm{Bu}$ might interact with the electron poor nitrophenyl moiety, e.g. forming a Meisenheimer complex, ${ }^{[136]}$ and in this manner disturb the cross-coupling reaction. To avoid this complication, the nitro compound $\mathbf{5 4}$ was reduced to amine 53 before the cross-coupling reaction. The amino group was then protected with a Bocprotecting group to give compound $\mathbf{5 5}$, in order to avoid the possible formation of a complex between an amino group and $\operatorname{Pd}(0)$. However, also in this case, the Buchwald-Hartwig reaction of compound $\mathbf{5 5}$ gave the coupling product $\mathbf{5 7}$ only in low yield of $20 \%$. This is even worse than the yield in the Pd-coupling in the initial synthesis route. Therefore the initial approach was preferred. The nitro group of compound $\mathbf{5 6}$ was reduced using activated zinc under acidic con- 
ditions to give amine 58 in excellent yield (97\%). Diazotization of amine 58 gave building block 48 in $76 \%$ yield.<smiles>Brc1ccc(-c2ccc(Br)cc2)cc1</smiles>

50

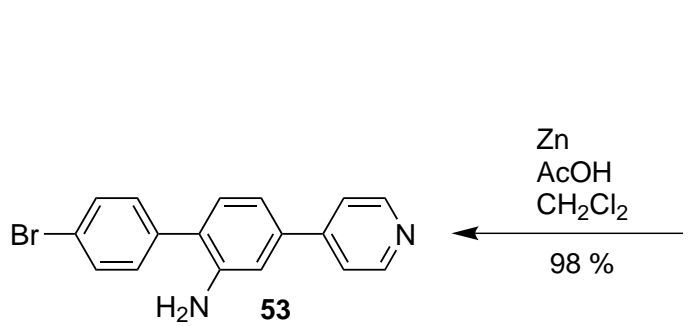

$79 \%$ 1) NaHMDS

2) $\mathrm{Boc}_{2} \mathrm{O}$

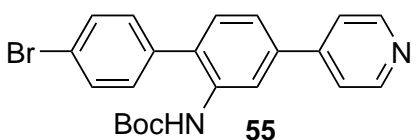

$20 \% \quad \begin{aligned} & \text { morpholine } \\ & \mathrm{Pd}_{2}\left(\mathrm{dba}_{3}, \mathrm{BINAP}\right. \\ & \mathrm{NaOtBu}, \mathrm{PhMe} \\ & 80^{\circ} \mathrm{C}, 24 \mathrm{~h}\end{aligned}$

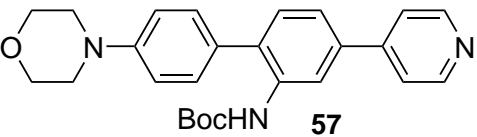

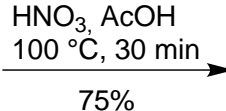

$75 \%$

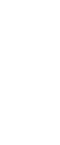

TFA<smiles>O=[N+]([O-])c1cc(Br)ccc1-c1ccc(Br)cc1</smiles>

$51 \mathrm{O}_{2} \mathrm{~N}$

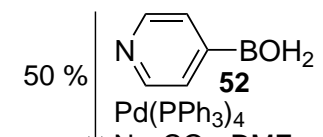

$\checkmark \mathrm{Na}_{2} \mathrm{CO}_{3}, \mathrm{DMF}$
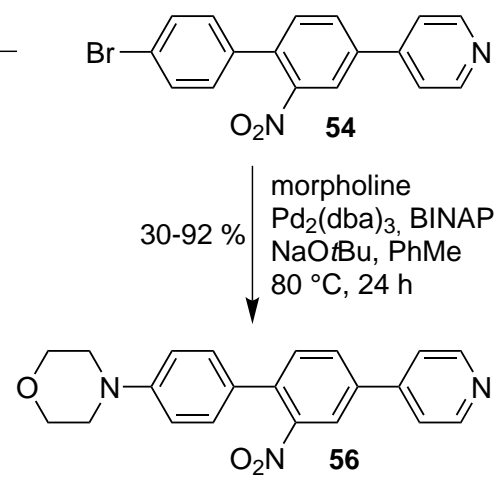

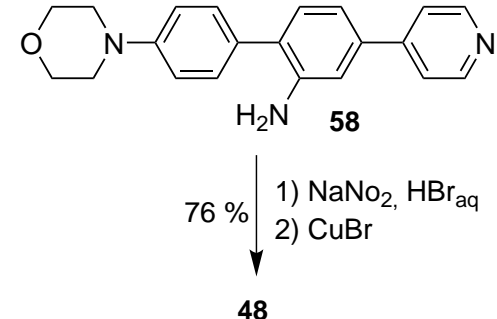

Scheme 16: Synthesis route to biphenyl dye precursor 48. 


\subsection{Photophysical properties of spiroadamantylfluorene 45}

Spiroadamantylfluorene $\mathbf{4 5}$ shows an absorption maximum at $340 \mathrm{~nm}$ with an extinction coefficient of $31,600 \mathrm{~cm}^{-1} \mathrm{M}^{-1}$ and an emission maximum at $452 \mathrm{~nm}$

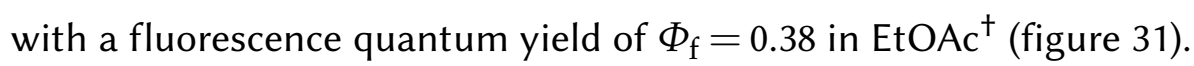

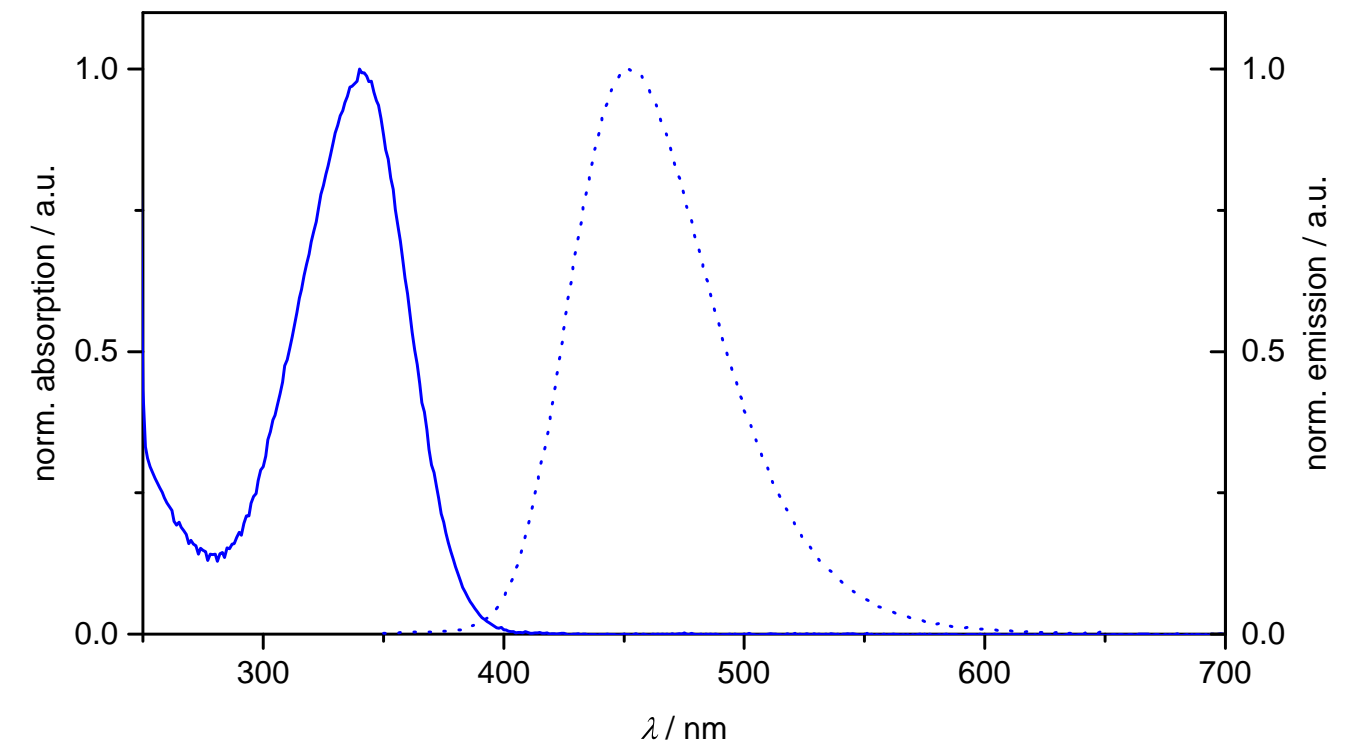

Figure 31: Normalized absorption (solid line) and emission spectra (dashed line) of spiroadamantylfluorene $\mathbf{4 5}$ with excitation at $330 \mathrm{~nm}$ in EtOAc.

\subsection{DFT calculations for model compound 45}

Figure 32 shows the ground and $S_{1}$ excited state geometries of model compound 45 after energy optimization using (time-dependent) density functional methods. The orientations of the absorption and emission transition dipole moments at the ground and excited states, are shown in figure 32 as doubleheaded arrows, respectively (see chapter 19 for details). Both transition dipoles are aligned along the long axis of the conjugated $\pi$-system.

\footnotetext{
${ }^{\dagger}$ The quantum yield was determined of a solution in EtOAc with $1 \% \mathrm{NEt}_{3}$.
} 
ground state geometry
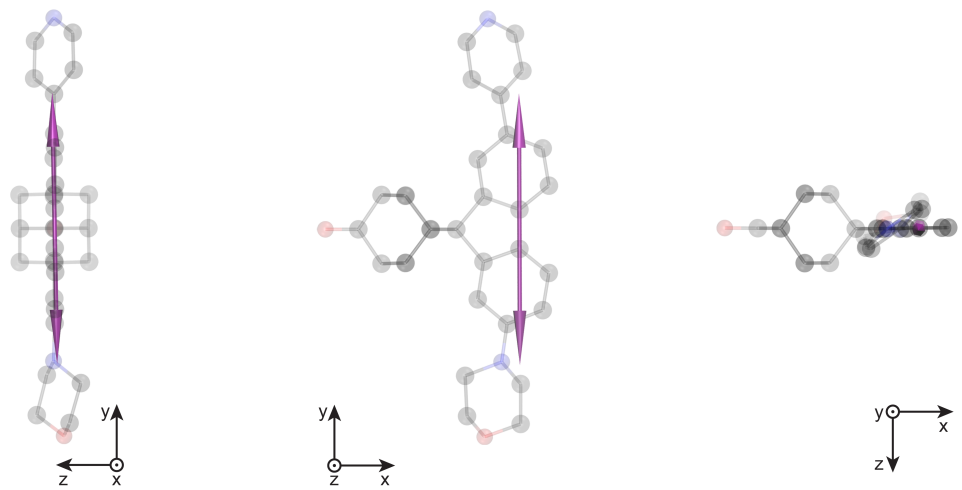

$\mathrm{S}_{1}$ state geometry
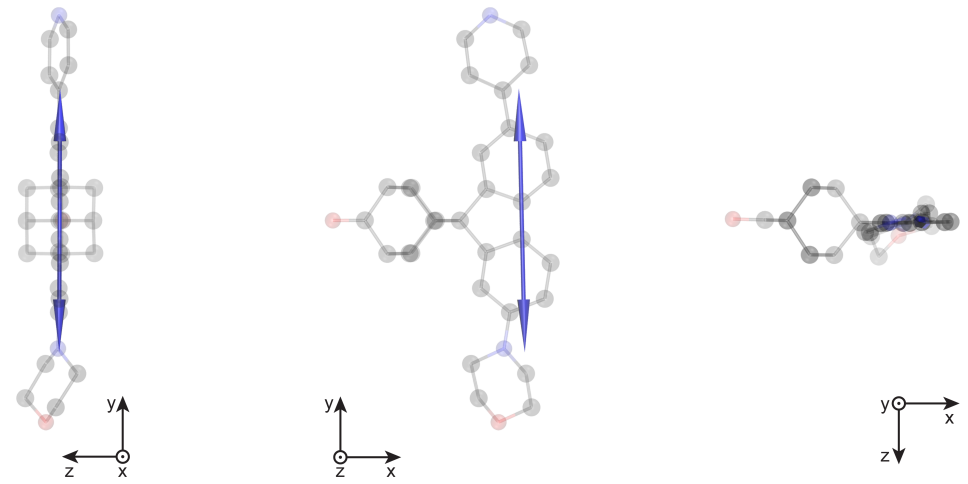

Figure 32: DFT and TD-DFT optimized geometries of model compound 45 and the transition dipole moments shown as double-headed arrows. Top: Ground state geometry with absorption $\left(\mathrm{S}_{0} \rightarrow \mathrm{S}_{1}\right)$ transition dipole moment (purple arrow); bottom: $S_{1}$ state geometry and emission $\left(S_{1} \rightarrow S_{0}\right)$ transition dipole moment (blue arrow). $\mathrm{H}$ atoms are omitted for clarity.

\subsection{Bathochromic modification of spiroadamantylfluorene 45}

In order to test how far the absorption/emission bands of "push-pull" fluorophore $\mathbf{4 5}$ can be shifted to longer wavelengths, the electron acceptor group was modified. The electron withrawing ability was increased by quaternization of the pyridyl nitrogen. While the first reaction of compound 45 with 1,3-propanesultone (60) gave the zwitterionic derivative $\mathbf{6 2}$ in low yield, the use of octafluorocyclopentene (59) in presence of $\mathrm{AcOH}$ and water gave betaine 61 in reasonable yield $(66 \%)$. 


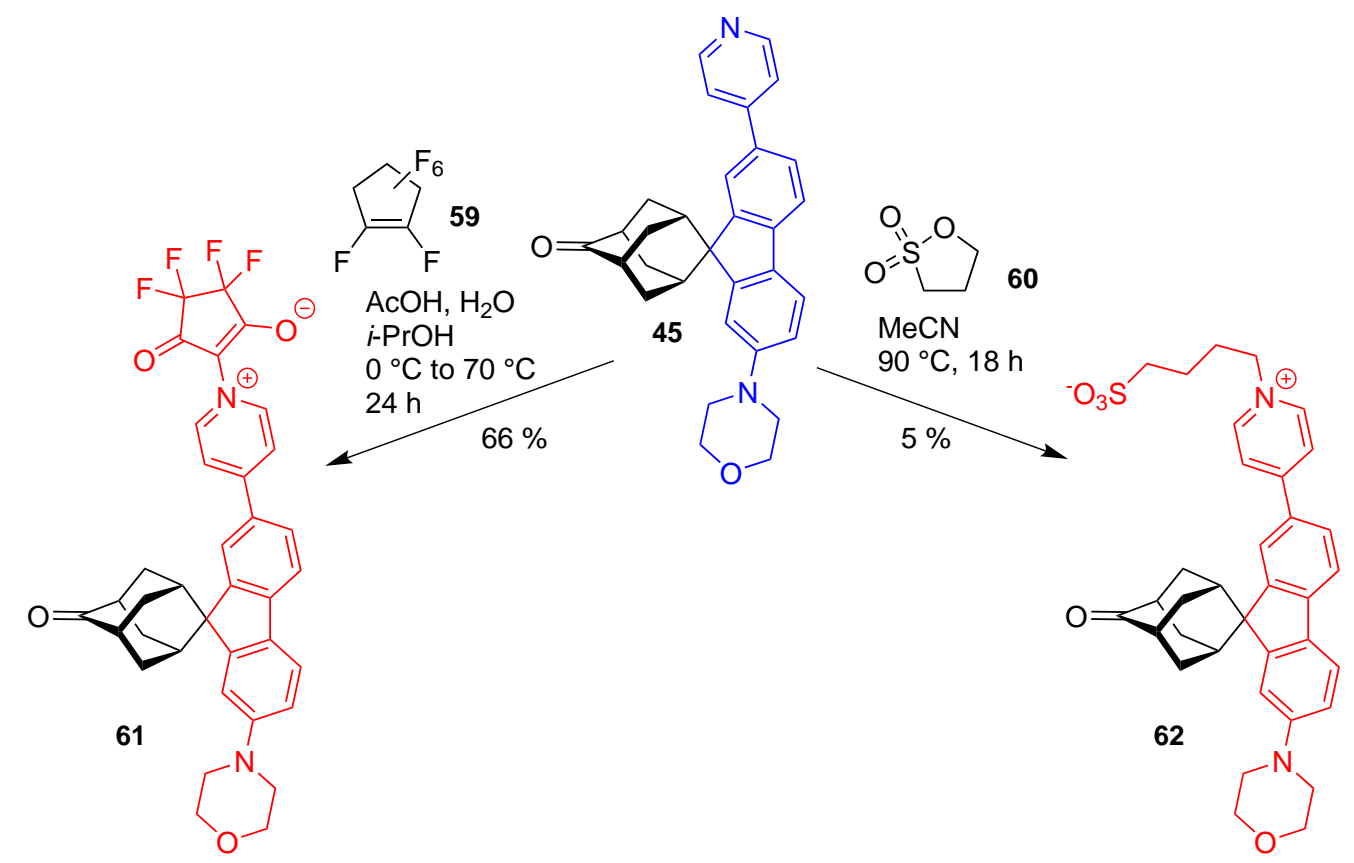

Scheme 17: Modification of compound 45 leads to derivatives with bathochromic shifts of absorption and emission bands.

The optical spectra of compounds $\mathbf{6 1}$ and $\mathbf{6 2}$ are shown in figure 33 and their properties are summarized in table 3. Compared to the initial fluorophore 45, derivatives 61 and $\mathbf{6 2}$ show bathochromic shifts of $91 \mathrm{~nm}$ and $71 \mathrm{~nm}$, respectively. Another common feature is the large Stokes shift of $250 \mathrm{~nm}$ and $324 \mathrm{~nm}$, respectively. Compound $\mathbf{6 2}$ shows a very low solubility in aprotic solvents, and therefore the photophysical properties given in table 3 were determined in methanol. This may be a reason why the emission efficiency of compound $62\left(\Phi_{\mathrm{f}}=0.02\right)$ is substantially lower than of compound $61\left(\Phi_{\mathrm{f}}=0.60\right)$. Fluorescence quantum yields of lipophilic (unpolar) compounds in polar protic solvents (alcohols, water) are generally lower than in unpolar solvents. 


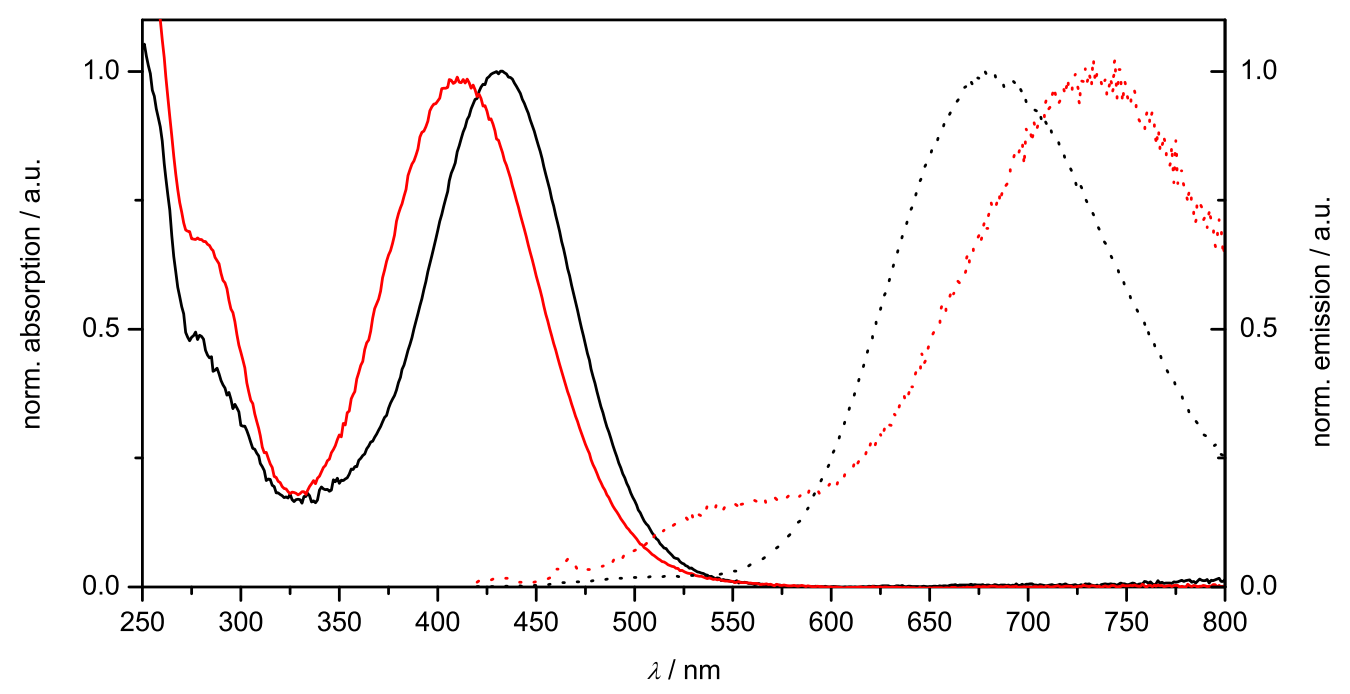

Figure 33: Normalized absorption (solid lines) and emission spectra (dashed lines) of compounds $\mathbf{6 1}$ (black color) and $\mathbf{6 2}$ (red color) with excitation at $410 \mathrm{~nm}$ ethyl acetate and in methanol, respectively.

Table 3: Photophysical properties of compound $\mathbf{6 1}$ in ethyl acetate and 62 in methanol.

\begin{tabular}{lllll}
\hline Compound & $\lambda_{\mathrm{abs}}^{\max } / \mathrm{nm}$ & $\lambda_{\mathrm{em}}^{\max } / \mathrm{nm}$ & $\varepsilon / \mathrm{mol} \mathrm{I}^{-1} \mathrm{~cm}^{-1}$ & $\Phi_{\mathrm{f}}^{\mathrm{a}}$ \\
\hline $\mathbf{6 1}$ & 431 & 681 & 29,000 & 0.60 \\
$\mathbf{6 2}$ & 411 & 735 & n.d. & 0.02 \\
\hline
\end{tabular}

(a) Quantum yields $\Phi_{\mathrm{f}}$ were were measured upon exc. at $410 \mathrm{~nm}$ using Coumarin 153 in $\mathrm{EtOH}\left(\Phi_{\mathrm{f}}=0.55\right)$ as a reference. ${ }^{[137]}$

Compounds $\mathbf{6 1}$ and $\mathbf{6 2}$ show a red emission, and therefore they could be used as "red" dyes in the future assemblies of two dyes. However, since the absorption bands of compound $\mathbf{6 1}$ and $\mathbf{6 2}$ are still in the blue spectral region, combination with "blue" fluorescent dyes would lead to strong overlap of the absorption bands and "cross-excitation". Therefore, fluorophores $\mathbf{6 1}$ and $\mathbf{6 2}$ are not suitable for the design of bichromophores, where the chromophores can be independently excited. The development of spiroadamantylfluorene based red fluorescent dyes was terminated at this point. 



\section{Development of spiroadamantyl bithiophene based chromophores}

For the design of red fluorescent dyes that meet the requirements defined in chapter 4, we have chosen a bithiophene core structure. Genin et al. reported the synthesis of push-pull fluorophores and the systematic tuning of the absorption/emission wavelengths depending on the electron donating and withdrawing groups. ${ }^{[128]}$ Bithiophene dye 63 (see scheme 18a for structure) has the absorption and emission maxima at $541 \mathrm{~nm}$ and $644 \mathrm{~nm}$, respectively, with a fluorescence quantum yield of $\Phi_{\mathrm{f}}=0.09$ in $\mathrm{CHCl}_{3}$. This push-pull fluorophore was used as a template for the design of a "bridged" red fluorescent bithiophene dye. Mierloo et al. developed a method for "bridging" of bithiophenes by using a bromine-lithium exchange of 3-bromo-2,2'-bithiophene (64) followed by the reaction with a ketone and cyclization of an intermediate alcohol 65 to give "bridged" bithiophene 66 (scheme 18b). ${ }^{[138]}$

a)

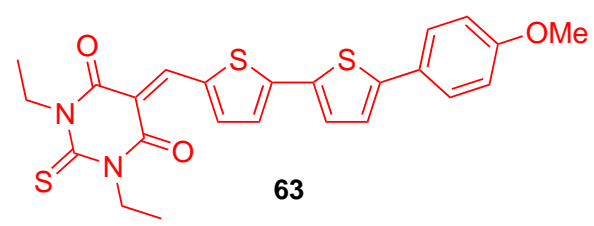

b)<smiles>Brc1ccsc1-c1cccs1</smiles>

64

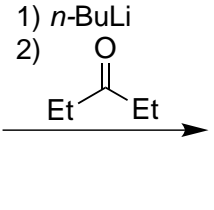

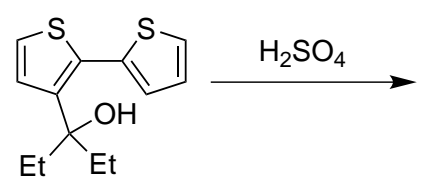

65<smiles>CCC1(CC)c2ccsc2-c2sccc21</smiles>

66

Scheme 18: a) Template for a red-emitting bithiophene dye ${ }^{[128]}$ b) "Bridging" of
bithiophene. ${ }^{[138]}$ Combining the push-pull structure of compound 63 with the bridging methodology reported by Mierloo and co-workers, the adamantylbridged push-pull fluorophore 77 was designed and synthesized (scheme 19). 


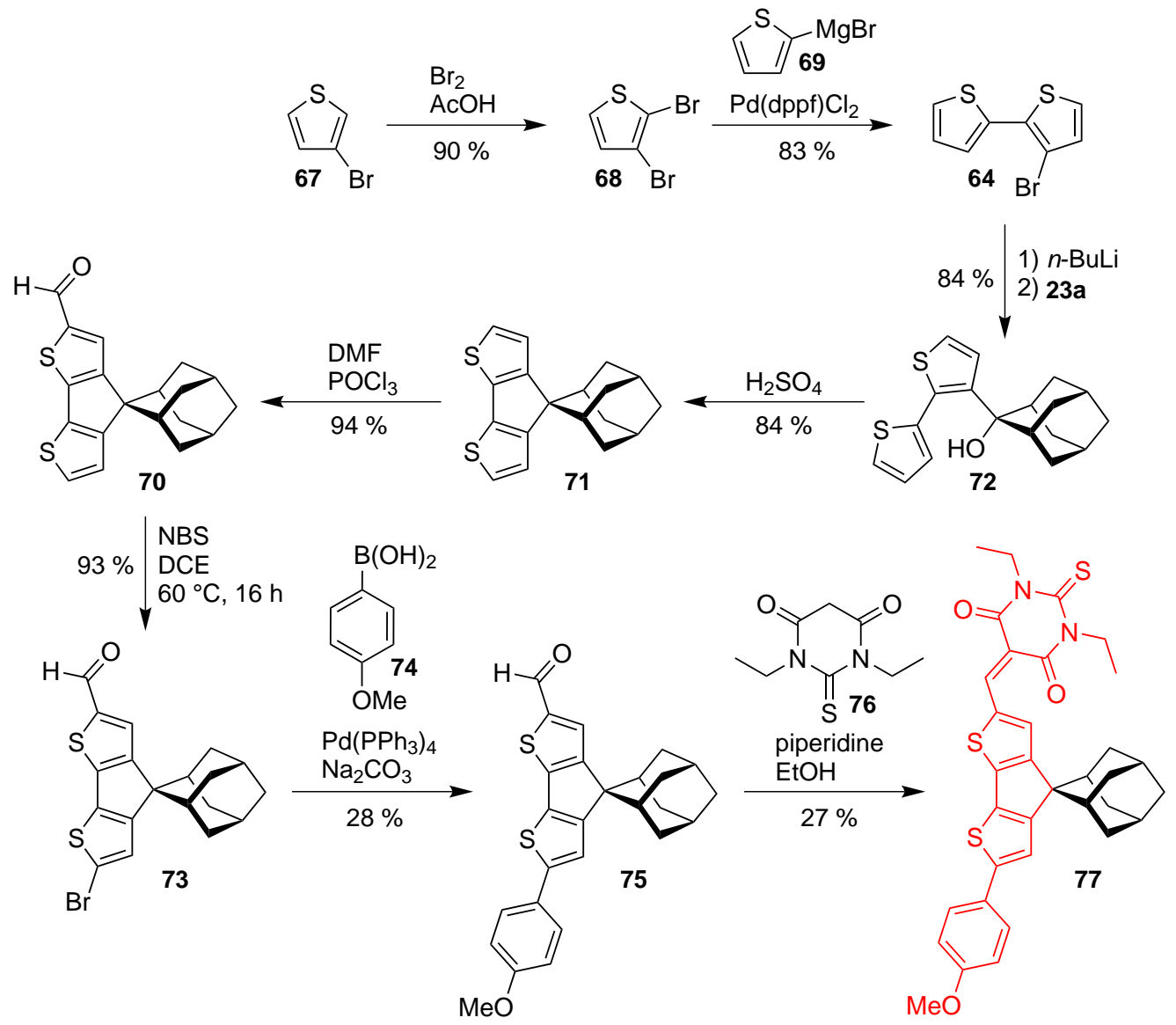

Scheme 19: Synthesis of adamantylbridged bithiophene dye 77.

3-Bromothiophene (67) was brominated selectively and in excellent yield $(90 \%)$ with elementary bromine at C-2. ${ }^{[139]}$ Cross-coupling of 2,3-dibromothiophene (68) with freshly prepared 2-thienylmagnesium bromide (69) in a Kumada reaction gave bithiophene 64 in good yield $(83 \%) .{ }^{[140]}$ Bromine-lithium exchange of compound 64 with $n$-BuLi and subsequent reaction with ketone 23a gave alcohol 72 in good yield (84\%). ${ }^{[38]}$ Acid catalyzed dehydration of alcohol 72 under strong acidic conditions using conc. $\mathrm{H}_{2} \mathrm{SO}_{4}$ gave spiro compound 71 in good yield $(84 \%)$. ${ }^{[138]}$ Formylation of compound 71 in a Vilsmeier-Haak reaction ${ }^{[141]}$ giving compound $\mathbf{7 0}$ and then bromination with NBS giving bromide 73 were accomplished in excellent yields of $94 \%$ and $93 \%$, respectively. ${ }^{[141]}$ Bromide 73 was reacted with boronic acid 74 in a Suzuki cross-coupling reaction to obtain yellow fluorescent compound $75 .^{[142]}$ The aldehyde group in compound $\mathbf{7 5}$ was transformed into a stronger electron withdrawing thiobarbiturate residue in a Knoevenagel condensation ${ }^{[128]}$ with 
thiobarbituric acid 76 Thus, red fluorescent bithiophene dye $\mathbf{7 7}$ was obtained in $27 \%$ yield.

\subsection{Photophysical properties of spiroadamantylbithiophene 77}

As shown in figure 34, bithiophene dye $\mathbf{7 7}$ displayed an absorption maximum at $559 \mathrm{~nm}$ and emitted red light with a maximum at $618 \mathrm{~nm}$ in EtOAc. Although, this structure seems to be promising in respect of rigidity, the fluorescence quantum yield was found to be very low $\left(\Phi_{\mathrm{f}}=0.02\right)$.

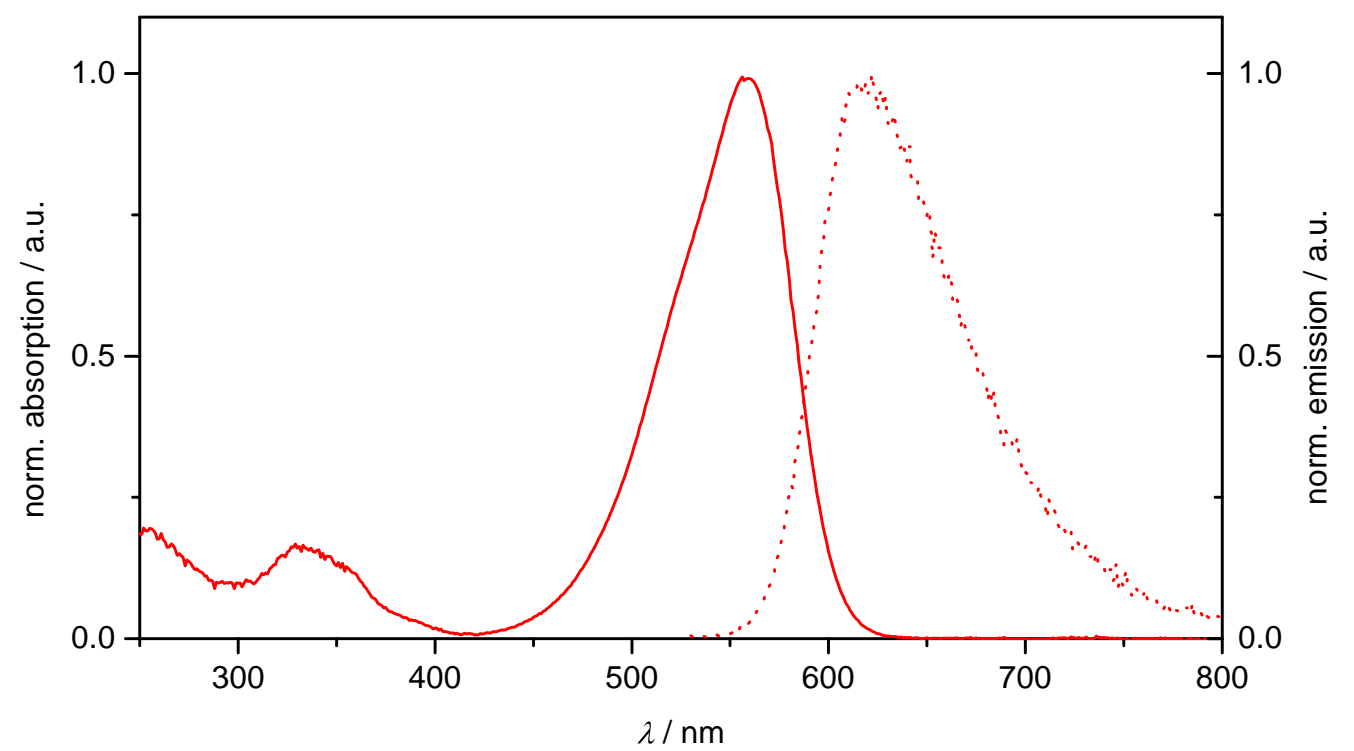

Figure 34: Normalized absorption (solid line) and emission spectra (dashed line) of spiroadamantylbithiophene 77 with excitation at $515 \mathrm{~nm}$ in EtOAc.

\subsection{Revised synthesis strategy of the red bithiophene based fluorophore}

For the design of bichromophoric compounds, the synthesis route leading to the red fluorescent dye $\mathbf{7 7}$ had to be changed for two reasons. On one hand, applying the previous synthesis strategy to blue dye $\mathbf{4 5}$ would most likely result in altering the blue fluorophore. It will undergo bromination or formylation reactions. On the other hand, changing the linear synthesis into a more convergent synthesis can improve the overall efficiency of the synthesis. 


\section{\begin{tabular}{l|l} 
Chapter 8 & DEVELOPMENT OF SPIROADAMANTYL BITHIOPHENE BASED ...
\end{tabular}}

Hence, instead of initial "bridging" of bithiophene with the adamantane structure and then introducing the push-pull substituents, the push-pull substituents were introduced to the bithiophene core prior to coupling with the 2-adamantanone derivative. It should be noted, that the strategy based on performing as many synthesis steps before coupling with an adamantane structure, has been already applied (when building block $\mathbf{4 8}$ was prepared for the synthesis of blue fluorescent dye 45).

The presence of the thiobarbiturate electron acceptor is not compatible with the reaction conditions that are used for attaching the adamantane structure. Therefore, instead of the barbiturate, it's precursor (an aldehyde group) was used (in its protected form as dimethyl acetal) in the design of building block 83 (scheme 20).

Our first attempt to synthesize building block 82 was inspired by the synthesis of 3-bromo-2,2'-bithiophene (64). As shown in scheme 20a, 2-bromothiophene (78) was brominated giving 2,5-dibromothiophene (79) which was cross-coupled with boronic acid $\mathbf{7 4}$ under Suzuki reaction conditions. ${ }^{[143]}$ Then bromide 80 was introduced into the reaction with dibromide 68 under Kumada coupling conditions. However, all attempts to perform this reaction yielded only debromination products instead of bromide $\mathbf{8 2}$.

Fortunately, the synthesis of bromide 82 was successfully accomplished starting from bithiophene derivative $\mathbf{6 4}$ (scheme 20b). Bromide 64 was regioselectively iodinated with NIS giving dihalide 81 in a good yield (88\%). ${ }^{[144]}$ Chemoselective Pd-catalyzed cross-coupling of $\mathbf{8 1}$ with boronic acid $\mathbf{7 4}$ under Suzuki reaction conditions gave bromide $\mathbf{8 2}$ in a good yield (71\%). After that, bromide $\mathbf{8 2}$ was formylated according to Vilsmeier-Haack, ${ }^{[141]}$ and the aldehyde group of compound 84 was protected as dimethyl acetal affording building block 83 in excellent yield (95\%). 
a) Synthesis route $A$<smiles>Brc1cccs1</smiles>

78

79

b) Synthesis route $B$

74

$\mathrm{Pd}\left(\mathrm{PPh}_{3}\right)_{4}$

aq. $\mathrm{NaHCO}_{3}$

DME

$33 \%$
80

1) $\mathrm{Mg}$

2) 68
74

$\mathrm{Pd}\left(\mathrm{PPh}_{3}\right)_{4}$ aq. $\mathrm{NaHCO}_{3}$ DME $19 \mathrm{~h}, 90^{\circ} \mathrm{C}$ $71 \%$ $49 \% \mid \begin{aligned} & \mathrm{DMF}, \mathrm{POCl}_{3} \\ & \mathrm{DCE} \text { to } 90^{\circ} \mathrm{C}, 7 \mathrm{~h} \\ & 0^{\circ} \mathrm{C}\end{aligned}$<smiles>Brc1ccsc1-c1cccs1</smiles>

$\mathrm{MeOH} / \mathrm{AcOH}$ $1 \mathrm{~h}, 60^{\circ} \mathrm{C}$ $88 \%$

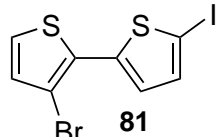<smiles>COc1ccc(-c2ccc(-c3sc(C(OC)OC)cc3Br)s2)cc1</smiles><smiles>CCOC(=O)OCc1ccccc1</smiles><smiles>COc1ccc(-c2ccc(-c3sc(C=O)cc3Br)s2)cc1</smiles>

Scheme 20: Synthesis of building block 83: a) Synthesis route A: coupling of 4-methoxyphenyl group to thiophene before thiophene-thiophene coupling; b) Synthesis route B: thiophene-thiophene coupling before introduction of 4-methoxyphenyl group.

The applicability of building block $\mathbf{8 3}$ for the synthesis of bithiophene fluorophore 77 was successfully proved (scheme 21). Bromine-lithium exchange followed by reaction with 2-adamantanone (23a) gave alcohol 85 in a good yield (84\%). Cyclization of alcohol $\mathbf{8 5}$ and deprotection of the aldehyde function was performed in one step in the course of treating the alcohol 85 with concentrated sulfuric acid. The bithiophene dye precursor 75 was prepared in $71 \%$ yield. 

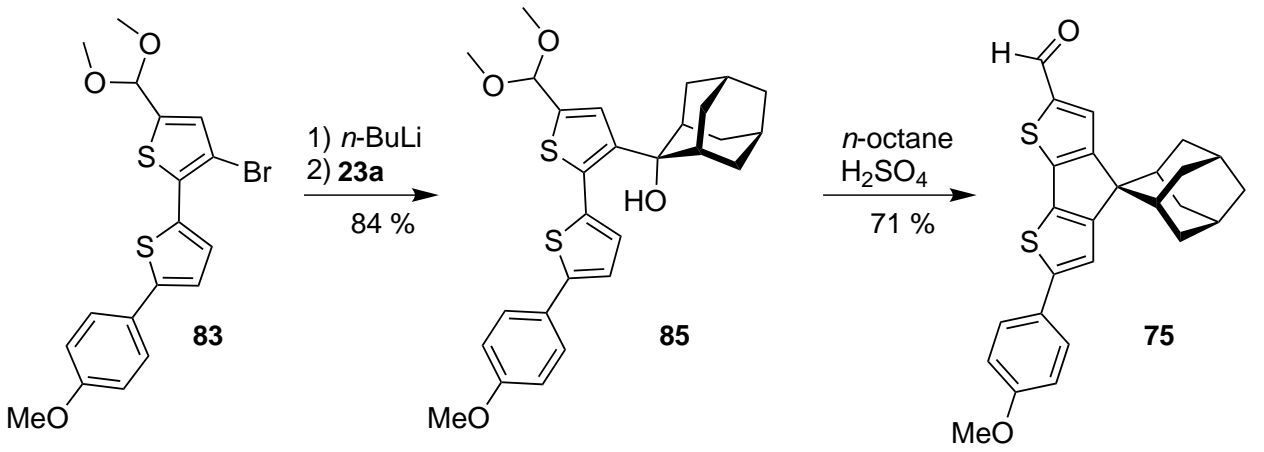

Scheme 21: Building block 83 is applicable for the synthesis of bithiophene dye precursor 75 .

\subsection{DFT calculations for model compound 77}

Figure 35 shows the ground state geometry of model compound 77 after energy optimization using density functional methods. The absorption transition dipole moment is shown as a double-headed arrow (see chapter 19 for details). Most likely, the orientation of the electron accepting thiobarbiturate moiety strongly affects the orientation of the transition dipole moment. Conformational changes are caused by the rotation of the thiobarbiturate group around the single-bond connecting it to the bridged bithiophene. They are likely to change the transition dipole moment orientation. In this respect, a symmetric or non-rotating electron accepting group would be advantageous.
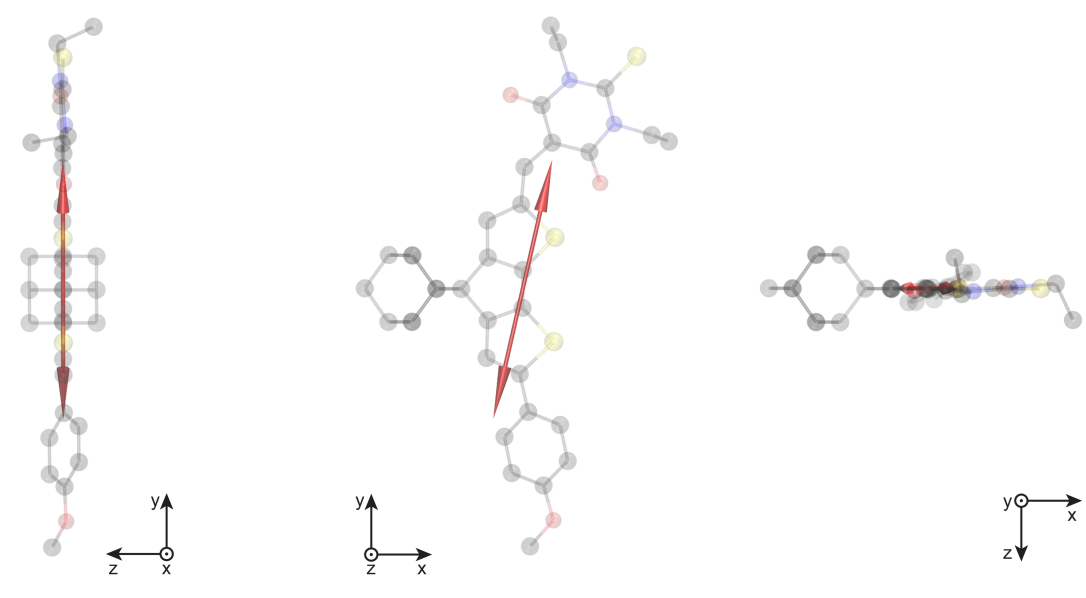

Figure 35: DFT optimized geometry of model compound 77 and absorption $\left(\mathrm{S}_{0} \rightarrow \mathrm{S}_{1}\right)$ transition dipole moment shown as red double-headed arrow. $\mathrm{H}$ atoms are omitted for clarity. 


\section{Bichromophore 87}

The availability of building blocks 48 and 83 enabled the synthesis of bichromophore 87.

\subsection{Synthesis of bichromophore 87}

Attaching of building block 83 to the blue fluorescent dye 45 was carried out using the established methods (scheme 22).

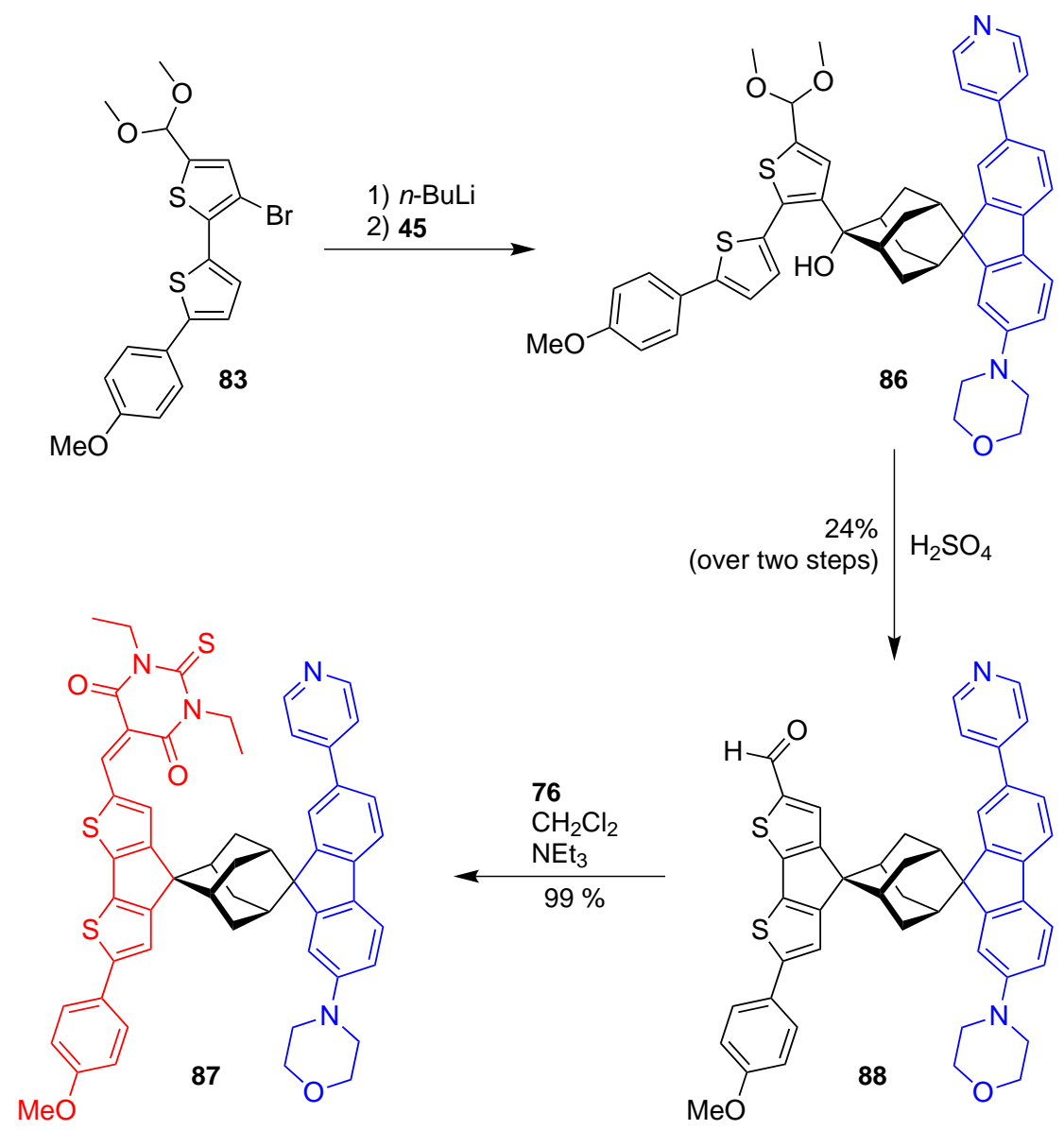

Scheme 22: Synthesis of bichromophore 87.

Bromine-lithium exchange was applied to compound 83 and was followed by the reaction with "blue" biphenyl dye 45 which provided alcohol 86. Cyclization of alcohol $\mathbf{8 6}$ and deprotection of the aldehyde function in one pot were carried out by treatment with conc. $\mathrm{H}_{2} \mathrm{SO}_{4}$. Aldehyde $\mathbf{8 8}$ was obtained in $24 \%$ yield (over two steps). The Knoevenagel reaction used for transformation of the aldehyde 


\section{\begin{tabular}{l|l} 
Chapter 9 & BICHROMOPHORE 87
\end{tabular}}

group into the strong electron withdrawing thiobarbiturate group failed (under conditions used for the synthesis of compound 77). However, when used $\mathrm{CH}_{2} \mathrm{Cl}_{2}$ instead of $\mathrm{EtOH}$ and $\mathrm{NEt}_{3}$ instead of piperidine, the reaction proceeded smoothly and resulted in bichromophore $\mathbf{8 7}$ in almost quantitative yield.

\subsection{Photophysical properties of bichromophore (87)}

The photophysical properties of the target compound 87 were compared with the properties of the blue and red model compounds 45 and 77, respectively.

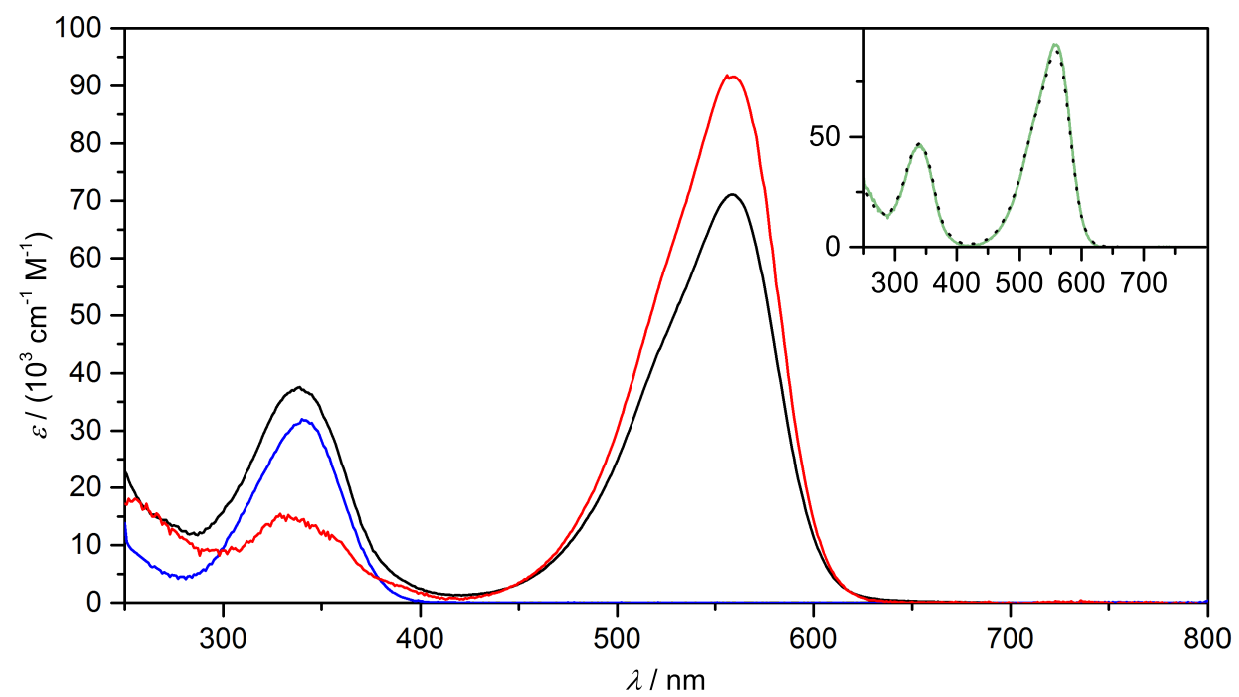

Figure 36: Absorption spectra of model compounds 45 (blue line), 77 (red line), and bichromophore 87 (black line). The inset shows the sum of molar absorptivities of model compounds $\mathbf{4 5}$ and $\mathbf{7 7}$ molar absorbance (dotted line) and the molar absorbance of bichromophore 87 scaled by a factor of $1 / 0.8$ (green line).

The absorption spectrum of bichromophore 87 equals the sum of the absorption spectra of the model compounds and shows the absorption bands of the blue and red fluorophores at about $340 \mathrm{~nm}$ and $560 \mathrm{~nm}$, respectively (figure 36). The measured absorbance of both fluorophores in compound $\mathbf{8 7}$ was determined to be about $25 \%$ lower than the absorbance of the model compounds (figure 36 ). These deviations were attributed to errors in the determination of extinction coefficients (most likely due to remaining silica gel). 


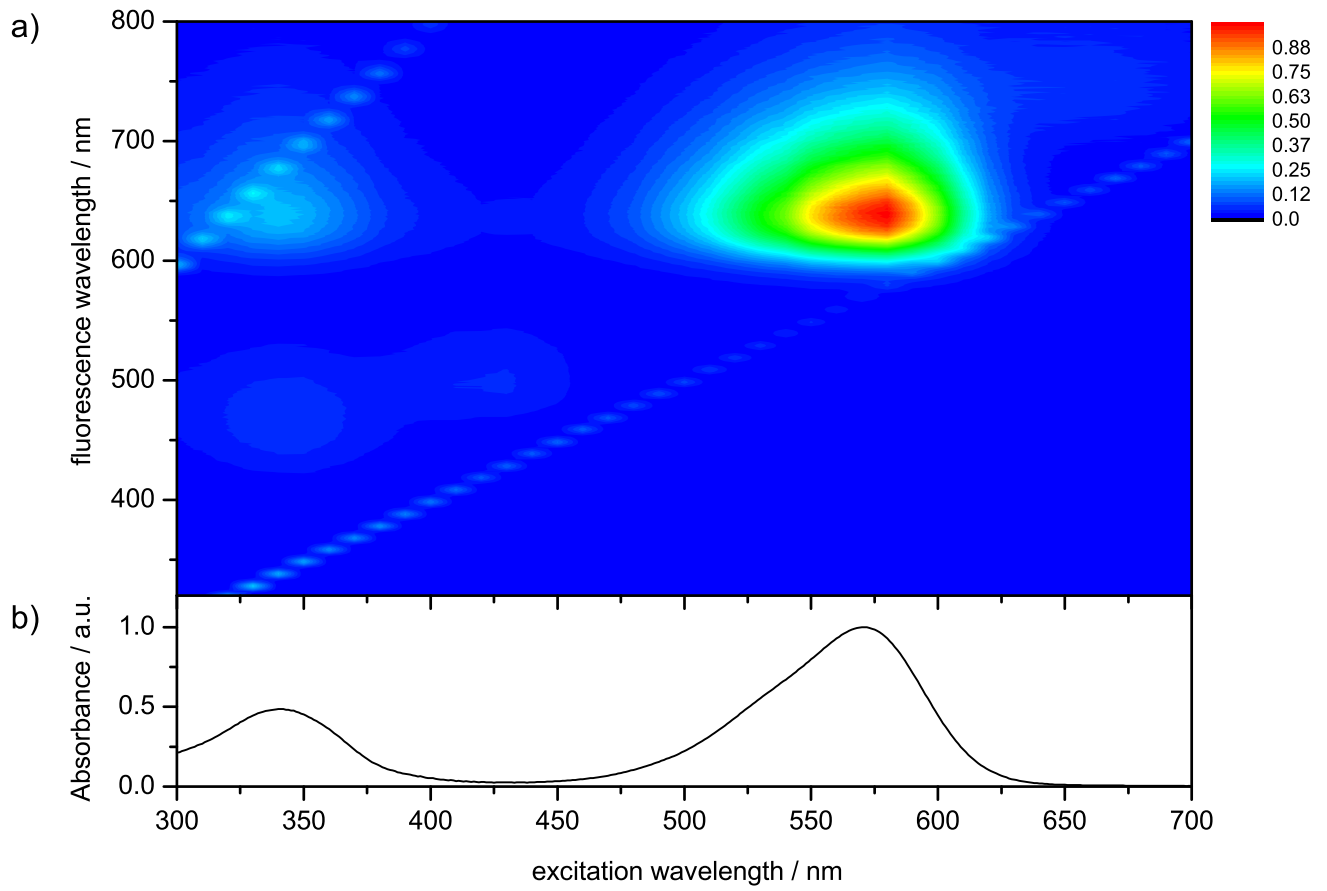

Figure 37: a) Contour plot showing emission intensity (color coded) vs. excitation and fluorescence wavelength for bichromophore 87 in $\mathrm{CH}_{2} \mathrm{Cl}_{2}$. The emission intensity was corrected for wavelength dependence of the detector sensitivity. The traces of the diagonal running emission maxima are caused by the scattered excitation light $\left(n \lambda_{\text {excitation }}=\lambda_{\text {detection }}\right.$ with $\left.n=1,2\right)$; b) absorption spectrum of bichromophore 87 in $\mathrm{CH}_{2} \mathrm{Cl}_{2}$.

Figure 37a shows the emission intensity of bichromophore 87 upon excitation at wavelengths between 300 and $700 \mathrm{~nm}$. In this contour plot the excitation wavelengths ( $x$-coordinate) is plotted versus the fluorescence wavelengths (y-coordinate) while the emission intensity is color coded. In order to correlate the excitation wavelengths with absorption bands, the absorption spectrum of bichromophore $\mathbf{8 7}$ is given in figure 37b. In total, three emission maxima with strongly varying intensities were observed. Excitation at about $340 \mathrm{~nm}$ (absorption band of the "blue" chromophore) gave a very weak emission at ca. $465 \mathrm{~nm}$ and a weak emission at ca. $630 \mathrm{~nm}$. Excitation at about $570 \mathrm{~nm}$ (absorption maximum of the "red" chromophore in $\mathrm{CH}_{2} \mathrm{Cl}_{2}$ ) gave the strongest emission at about $630 \mathrm{~nm}$.

The emission at $630 \mathrm{~nm}$ upon excitation at about $570 \mathrm{~nm}$ can be attributed to direct excitation of the "red" chromophore. The intensity corresponds to a fluo- 


\section{\begin{tabular}{l|l} 
Chapter 9 & BICHROMOPHORE 87
\end{tabular}}

rescence quantum yield of $2 \%$ (same emission quantum yield as the "red" model compound 77). The very weak emission at ca. $465 \mathrm{~nm}$ upon excitation at the absorption band of the "blue" fluorophore corresponds to a fluorescence quantum yield smaller than $0.2 \%$. We assume, this emission is caused by an impurity. If so, then the emission of the "blue" fluorophore is completely quenched. This efficient quenching is most likely a result of intramolecular EET towards the red chromophore, which showed weak emission at about $630 \mathrm{~nm}$. Besides EET towards the red chromophore, this emission may also be the result of direct excitation of the red chromophore's $\mathrm{S}_{0} \rightarrow \mathrm{S}_{2}$ band, which matches the absorption band of the blue chromophore (see figure 36 ).

However, since excitation at about $340 \mathrm{~nm}$ (abs. of the "blue" chromophore) gave only a weak emission of the "red" fluorophore, we cannot exclude the presence of some additional "energy-dissipating" effects. The energy transfer efficiency towards the "red" chromophore might be $100 \%$, but due to the low fluorescence quantum yield of the "red" fluorophore, we cannot say with certainty. To clarify the regularities of the energy-transfer process between the "blue" and "red" fluorophores, we needed to prepare a new bichromophore with brighter "red" fluorophore.

The photophysical properties of bichromophore $\mathbf{8 7}$ and corresponding model compounds $\mathbf{4 5}$ and $\mathbf{7 7}$ are summarized in table 4.

Table 4: Photophysical properties of model compounds 45, 77 and bichromophore 87 in EtOAc.

\begin{tabular}{lllll}
\hline Compound & $\lambda_{\mathrm{abs}}^{\max } / \mathrm{nm}\left(\varepsilon / \mathrm{M}^{-1} \mathrm{~cm}^{-1}\right)$ & $\lambda_{\mathrm{em}}^{\max } / \mathrm{nm}$ & $\Phi_{\mathrm{f}}^{\mathrm{a}}$ & $\tau_{\mathrm{f}} / \mathrm{ns}$ \\
\hline $\mathbf{4 5}$ & $341(32,000)$ & 452 & $0.38^{\mathrm{b}}$ & $1.9 \pm 0.2$ \\
$\mathbf{7 7}$ & $559(92,800)$ & 618 & $0.02^{\mathrm{c}}$ & $1.1 \pm 0.2$ \\
$\mathbf{8 7}$ & $339(38,000)^{\mathrm{d}}$, & n.d., & n.d., & n.d., \\
& $559(71,000)^{\mathrm{d}}$ & 618 & $0.02^{\mathrm{c}}$ & $2.0 \pm 0.2$ \\
\hline
\end{tabular}

(a) Fl. quantum yield $\Phi_{\mathrm{f}}$ was determined using comparative method; (b) exc. at $330 \mathrm{~nm}$, using Coumarin 120 in $\mathrm{MeOH}\left(\Phi_{\mathrm{f}}=0.51\right)$ as a reference; ${ }^{[132]}$ (c) exc. at $550 \mathrm{~nm}$, using Rhodamine 101 in $\mathrm{EtOH}\left(\Phi_{\mathrm{f}}=1.00\right)$ as a reference; ${ }^{[145]}(\mathrm{d})$ assuming a silica content of $20 \%$, the corrected extinction coefficients are 47,000 and $89,000 \mathrm{M}^{-1} \mathrm{~cm}^{-1}$. 


\subsection{DFT calculations for bichromophore 87}

Figure 38 shows the ground state geometry of bichromophore 87 after energy optimization using density functional methods. The emission and absorption transition dipole moments of the "blue" and "red" chromophore were determined using time-dependent density functional calculations and are shown in figure 38 as blue and red double-headed arrows, respectively. The absorption dipole moment of the "red" part in bichromophore $\mathbf{8 7}$ was determined directly for the ground state geometry. The emission transition dipole of the "blue" chromophore was determined by transferring it's orientation from model compound 45 to bichromophore 87 (see chapter 19 for details). Like in the model compound 45, the thiobarbiturate moiety may rotate around the single-bond connecting it to the bridged bithiophene causing a change of the transition dipole moment orientation of this chromophore.
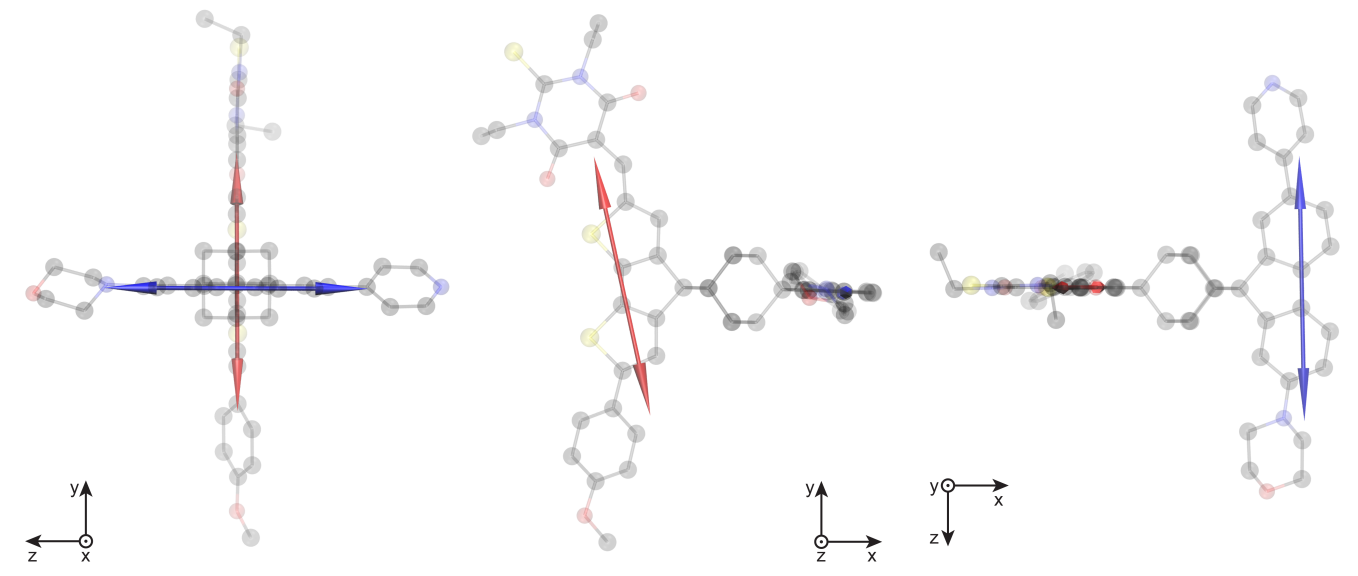

Figure 38: DFT optimized ground state geometry of bichromophore 87 . The absorption $\left(\mathrm{S}_{0} \rightarrow \mathrm{S}_{1}\right)$ and emission $\left(\mathrm{S}_{1} \rightarrow \mathrm{S}_{0}\right)$ transition dipole moments are shown as red and blue double-headed arrows, respectively. $\mathrm{H}$ atoms are omitted for clarity.

\subsection{Estimation of energy transfer efficiency based on ideal Förster point-dipole approximation for bichromophore 87}

The geometrical arrangement of the transition dipole moments was used to determine the orientation factor $\kappa^{2}$ and, having the photophysical properties of the model compounds 77 and 45, the energy transfer efficiency based on the ideal 


\section{\begin{tabular}{l|l} 
Chapter 9 & BICHROMOPHORE 87
\end{tabular}}

Förster point-dipole approximation was estimated.

The angles $\theta_{\mathrm{D}}, \theta_{\mathrm{A}}$ and $\theta_{\mathrm{DA}}$ and distance $\left|\vec{R}_{\mathrm{DA}}\right|$ between the emission and absorption transition dipoles are given in table 5 (see section 1.2.1 for angle definitions). Using equation 4 , the orientation factor $\kappa^{2}$ was calculated (table 5). The spectral overlap of the "blue" model compound's (45) emission and "red" model compound's (77) absorption spectrum (figure 39), was determined to be $J_{\mathrm{DA}}=9.3 \cdot 10^{14} \mathrm{M}^{-1} \mathrm{~cm}^{-1} \mathrm{~nm}^{4}$.

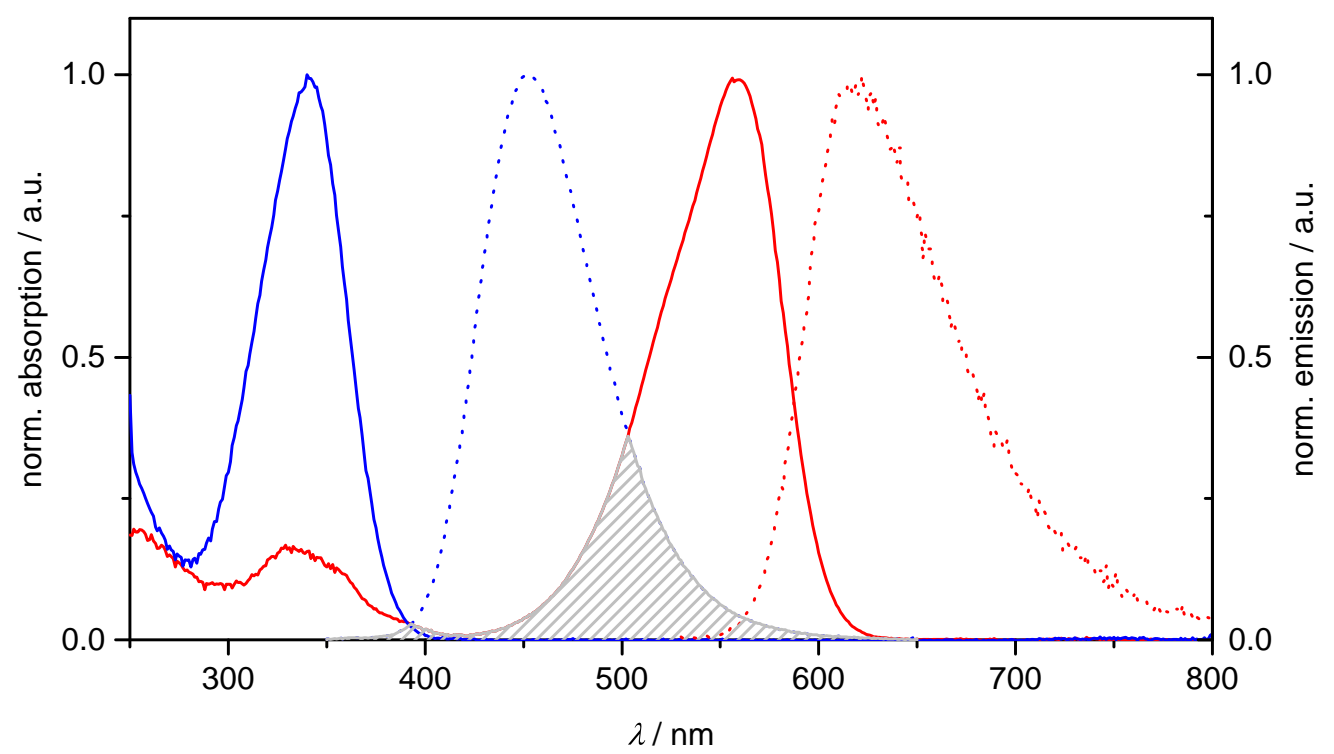

Figure 39: Normalized absorption (solid lines) and emission spectra (dotted lines) of model compounds 45 (blue line) and 77 (red line). The spectral overlap of compound's 45 emission and compound's 77 absorption is displayed, as a hatched area.

Taking into account the spectral overlap $J_{\mathrm{DA}}$ and the refractive index of EtOAc $n_{\mathrm{D}}\left(20^{\circ} \mathrm{C}\right)=1.3723,{ }^{[134]}$ the Förster radius $R_{0}$ and the FRET efficiency $E_{\mathrm{FRET}}$ were calculated (equations 5 and 6). The results are given in table 5.

Table 5: Angles $\theta_{\mathrm{D}}, \theta_{\mathrm{A}}$ and $\theta_{\mathrm{DA}}$ between transition dipole moments and interconnecting vector $\vec{R}_{\mathrm{DA}}$, orientation factor $\kappa^{2}$, distance between the donor and acceptor $\left|\vec{R}_{\mathrm{DA}}\right|$, Förster radius $R_{0}$, and estimated FRET efficiency $E_{\mathrm{FRET}}$.

\begin{tabular}{llllllll}
\hline Compound & $\theta_{\mathrm{D}}$ & $\theta_{\mathrm{A}}$ & $\theta_{\mathrm{DA}}$ & $\kappa^{2}$ & $\left|\vec{R}_{\mathrm{DA}}\right| / \mathrm{nm}$ & $R_{0} / \mathrm{nm}$ & $E_{\mathrm{FRET}}$ \\
\hline $\mathbf{8 7}$ & $90.2^{\circ}$ & $88.6^{\circ}$ & $76.0^{\circ}$ & $4.42 \cdot 10^{-4}$ & 0.84 & 1.25 & 0.92 \\
\hline
\end{tabular}




\section{Advancement of the blue fluorophore}

Despite the significant improvements of bichromophore 87 compared to the first spiroketal approach, bichromophore $\mathbf{8 7}$ has some drawbacks. Further modifications improved the structure of the fluorophores and ruled out their disadvantages.

\subsection{Further development of the blue chromophore}

The blue fluorophore has substituents with electronic "push-pull" effects. The "push" substituent (morpholinyl group as an electron donor), as well as the "pull" substituent (pyridyl group as an electron acceptor), are basic sites. Protonation of these basic groups changes drastically the properties of the fluorophore. For example, protonation inverts the electron donating property of the morpholinyl group. Consequently, the photophysical properties depend on the $\mathrm{pH}$ of the solution. This could explain problems with reproducibility experienced in the course of examination of bichromophore 87. The addition of $\mathrm{NEt}_{3}(0.1$ to $1 \%)$ appeared to improve the reproducibility of the measurements.

Furthermore, if a pair of push-pull fluorophores is incorporated into the structure of bichromopohores, dipole-dipole forces arising from the permanent dipoles of each fluorophore can distort the perpendicular chromophore arrangement as shown in figure 40a. Therefore, if at least one fluorophore is symmetric, the dipole-dipole interactions are less pronounced (figure 40b). 


\section{Chapter 10 $\mid$ ADVANCEMENT OF THE BLUE FLUOROPHORE}

a)

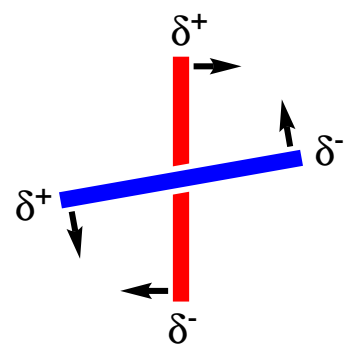

asymmetric blue fluorophore b)

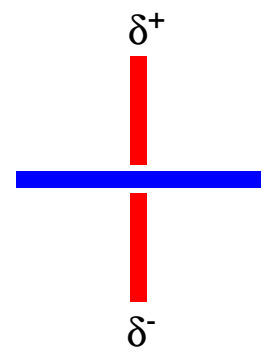

symmetric blue fluorophore (only induced dipole-dipole interactions can occur)

Figure 40: Comparison of bichromophores with a) asymmetric and b) symmetric blue chromophore. Dipole-dipole forces resulting from partial charges $\delta^{+}$and $\delta^{+}$ are depicted as black arrows.

Since a push-pull system as red fluorophore has no alternative, the blue fluorophore was changed to a symmetric structure. The most straight forward solution was to expand the aromatic fluorene system adding two aromatic substituents. According to the literature, ${ }^{[126,146]}$ introduction of two thienyl groups into a similar fluorene system lead to a bathochromic shift (from ca. $304 \mathrm{~nm}$ to $348 \mathrm{~nm}$ ) and increased the Stokes shift (from ca. $2 \mathrm{~nm}$ to $34 \mathrm{~nm}$ ), as well as molar absorptivity. Therefore, 2,7-(dithien-2-yl)fluorene was chosen as base structure for the revised blue fluorophore.

\subsection{Development of model compound 105 and bichromophore 107 with symmetric blue fluorophores}

Novel building block 92 was synthesized as shown in scheme 23. Dibromide 51 was coupled in a Suzuki reaction with 2-thienylboronic acid to give the nitro compound 89 which was reduced to amine 91 with $\mathrm{Zn}$ under acidic conditions. Diazotization of resulting amine $\mathbf{9 1}$ under water-free conditions did not give the desired monobromide 92 but dibromide $\mathbf{9 0}$. A similar reactivity was observed by Xia and co-workers. ${ }^{[147]}$ Adopting their solution using $n$-hexyl nitrite in $\mathrm{CHBr}_{3}$ afforded building block 92 in a yield of $39 \%$ (with some impurities which could not be removed by column chromatography). 

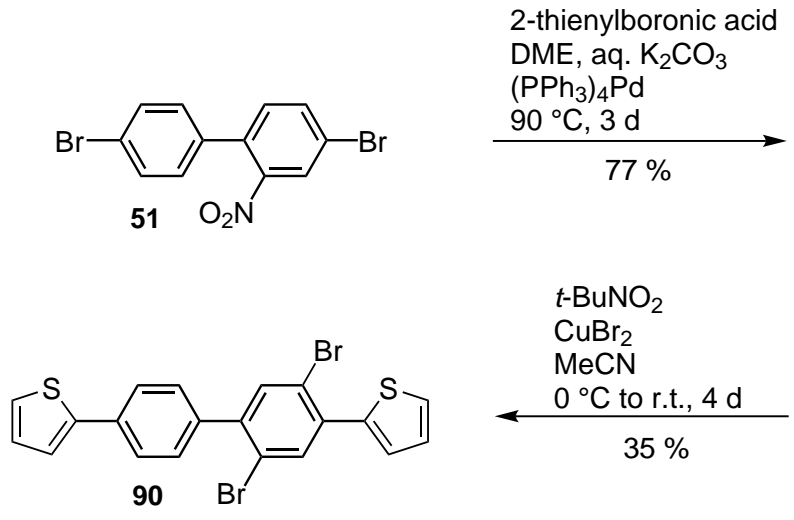

2-thienylboronic acid

DME, aq. $\mathrm{K}_{2} \mathrm{CO}_{3}$

$\left(\mathrm{PPh}_{3}\right)_{4} \mathrm{Pd}$

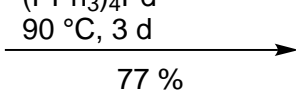

$35 \%$

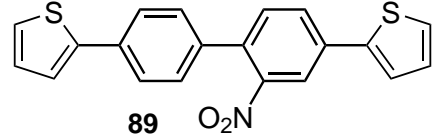

activated $\mathrm{Zn}$ dust $95 \% \mathrm{AcOH}, \mathrm{CH}_{2} \mathrm{Cl}_{2}$ $0^{\circ} \mathrm{C}, 1 \mathrm{~h}$

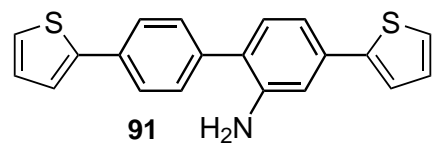

$39 \% \mid \begin{aligned} & n \text {-hexyl nitrite } \\ & \mathrm{CHBr}_{3}, t-\mathrm{BuNO}_{2} \\ & 70{ }^{\circ} \mathrm{C}, 3 \mathrm{~h}\end{aligned}$

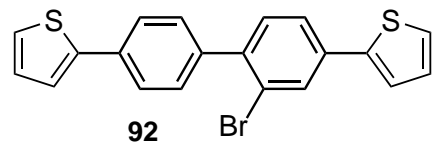

Scheme 23: Synthesis of building block 92.

In order to obtain the pure bromide 92, the synthesis strategy was revised, as shown in scheme 24 . The completely new synthesis strategy not only provided better purity, but has the advantage that diiodide $\mathbf{1 0 2}$ allows the introduction of diverse substituents by $\mathrm{Pd}$-catalyzed reactions in a single step.

Benzidine 98 was prepared as described by Kang and co-workers. ${ }^{[148]}$ Phenyl hydrazine (93) reacted with an excess (5.30 eq.) of $\mathrm{Boc}_{2} \mathrm{O}$ in the presence of catalytic amounts of DMAP. The resulting 1,2,2-tris(t-butyloxycarbonyl)1-phenylhydrazine was converted to compound 94 by addition of $\mathrm{Mg}\left(\mathrm{ClO}_{4}\right)_{2}$ in a one-pot fashion. ${ }^{[149]}$ Hydrazine derivative 94 was coupled to 3-bromo1-iodobenzene (97) giving compound 95 in an Ullmann-type reaction. Compound 97 was prepared from boronic acid 96 according to literature. ${ }^{[150]}$ Bocprotecting group of compound $\mathbf{9 5}$ was cleaved under acidic conditions and $[5,5]$-sigmatropic rearrangement gave benzidine derivative $98 .^{\dagger}$ Diazotization of benzidine 98 gave diiodide 102. Stille cross-coupling with organotin compound 100 gave pure bromide 92.

\footnotetext{
${ }^{\dagger}$ An alternative convenient synthesis of compound 98 was published by Kimura et al. ${ }^{[151]}$
} 


\section{Chapter 10 ADVANCEMENT OF THE BLUE FLUOROPHORE}

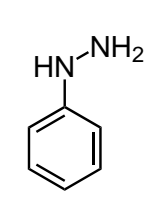

93<smiles>Oc1cccc(Br)c1</smiles>

96

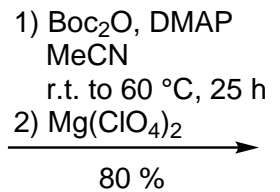

$80 \%$

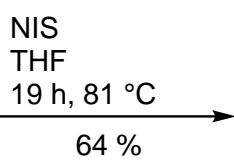

$64 \%$

n-BuLi, THF

$-78^{\circ} \mathrm{C}, 1 \mathrm{~h}$

2) $\mathrm{Bu}_{3} \mathrm{SnCl}$

$\mathrm{H}_{3} \mathrm{C} \longrightarrow$

99

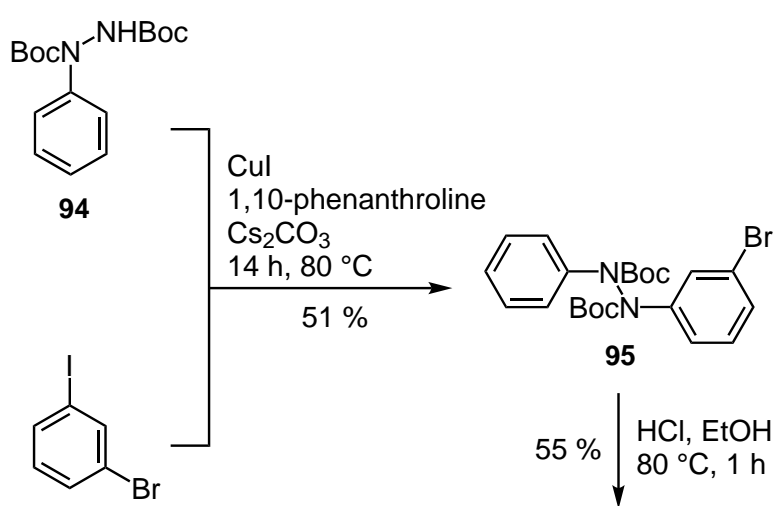

97

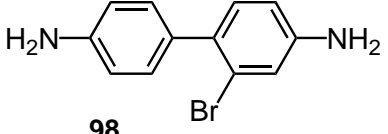

$70 \% \mid \begin{aligned} & \text { 1) } \mathrm{NaNO}_{2} \text {, aq. } \mathrm{HCl} \\ & \mathrm{MeCN}^{\circ}-10{ }^{\circ} \mathrm{C} \\ & \text { 2) } \mathrm{KI} \\ & -10^{\circ} \mathrm{C} \text { to r.t. }\end{aligned}$

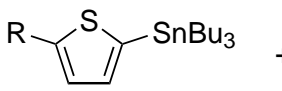

100: $\mathrm{R}=\mathrm{H}$ 101: $\mathrm{R}=\mathrm{CH}_{3}(71 \%)$

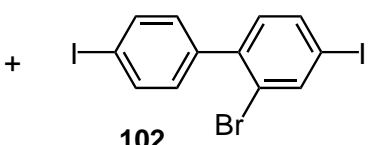

102

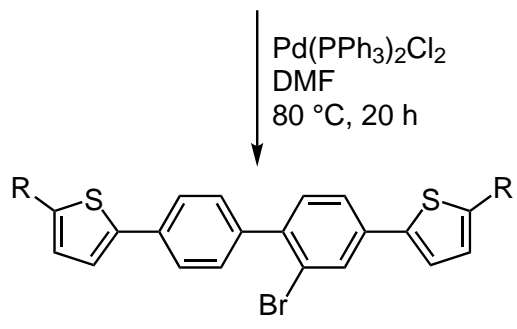

92: $\mathrm{R}=\mathrm{H}(62 \%)$

103: $\mathrm{R}=\mathrm{CH}_{3}(44 \%)$

Scheme 24: Synthesis of building blocks 92 and 103.

Unexpectedly, the coupling of building block 92 to adamantanedione 23b under conditions, which were used for the coupling of building blocks 83 and 48, failed. A possible explanation could be metalation at the thienyl C-5. Although the thiophene's $\mathrm{p} K_{\mathrm{a}} \approx 33^{[152]}$ might contradict this hypothesis, 5-methyl-2-thienyl groups were considered as substituents instead of 2-thienyl residues. Stille cross-coupling ${ }^{[153]}$ of diiodide 102 with organotin compound 101 gave bromide 103 (scheme 24). Gratifyingly, this modification led to success: as shown in scheme 25, lithium-halogen exchange of compound 103 
and subsequent reaction with 2,6-adamantanedione (23b) gave compound 104 and compound 106 depending on the molar ratio of building block 103 and 2,6-adamantanedione (23b). Cyclization gave compounds 105 and 107, respectively.

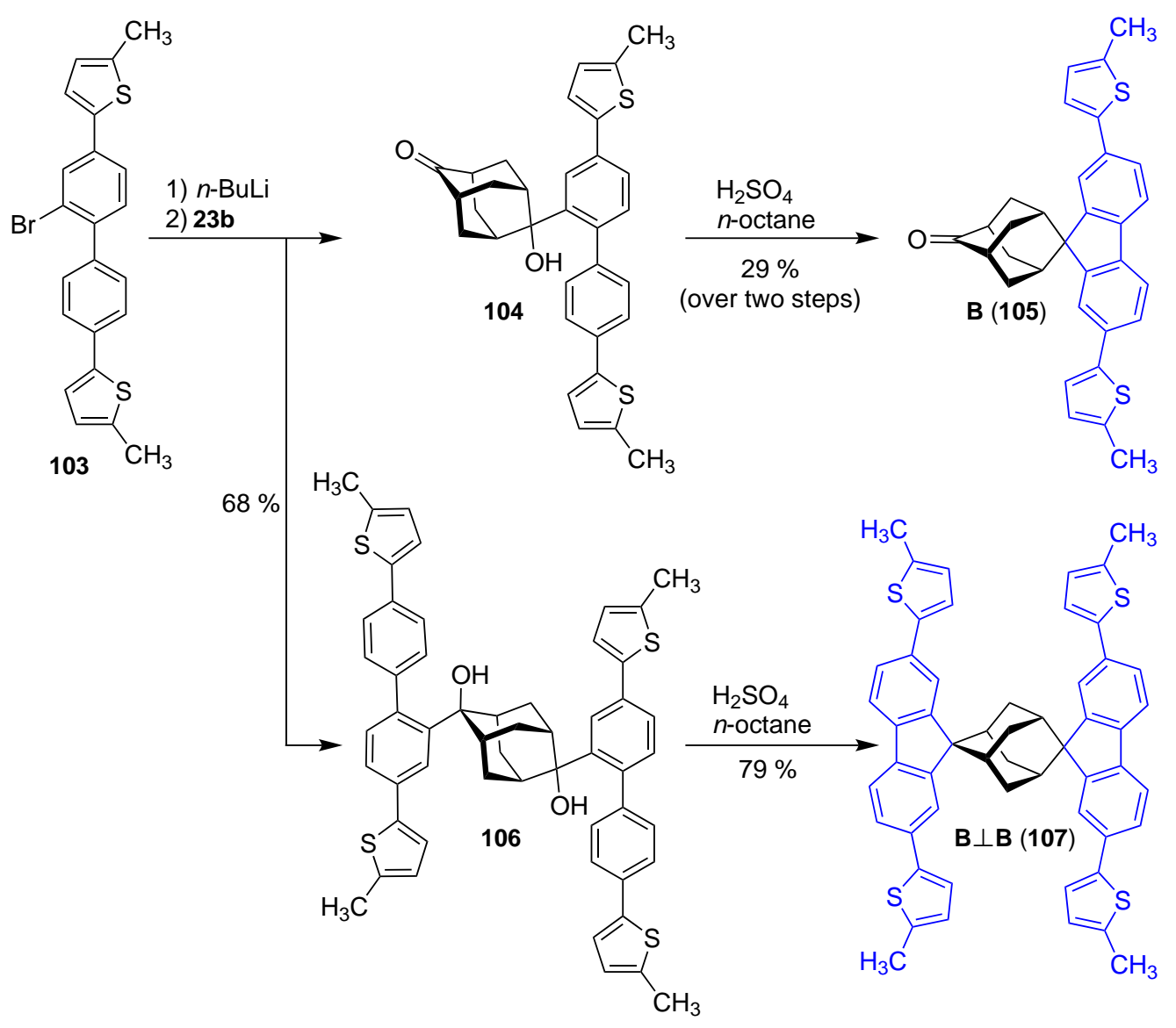

Scheme 25: Synthesis of model compound $\mathbf{B}(\mathbf{1 0 5})$ and bichromophore $\mathbf{B} \perp \mathbf{B}(\mathbf{1 0 7})$.

To simplify the discussion, short and more descriptive compound names are used in the following text. B refers to the blue chromophore in model compound 105 and elsewhere. Later an "improved" red fluorophore will be introduced, which will be termed R. In bichromophores, " $\perp$ " and " ||" indicate the orthogonal or parallel arrangement of the chromophores, respectively. For example bichromophore $\mathbf{1 0 7}$ will be termed $\mathbf{B} \perp \mathbf{B}$. The model compounds themselves are termed $\mathbf{B}$ and $\mathbf{R}$. 


\subsection{Photophysical properties of compounds B (105) and $B \perp B(107)$}

Due to identical chromophore structures, blue fluorescent model compound $\mathbf{B}$ and bichromophore $\mathbf{B} \perp \mathbf{B}$ show similar photophysical properties as expected (table 6). Both dyes show maxima of absorption and emission at $355 \mathrm{~nm}$ and $390 \mathrm{~nm}$ in EtOAc, respectively. The somewhat lower fluorescence quantum yield of $\mathbf{B} \perp \mathbf{B}\left(\Phi_{\mathrm{f}}=0.44\right)$ compared to $\mathbf{B}\left(\Phi_{\mathrm{f}}=0.47\right)$ is within the error of the determination method. The presence of two chromophores within one molecule, causes double molar extinction coefficients. The absorption and emission spectra of $\mathbf{B}$ are shown in figure 41 and the spectra of bichromophore $\mathbf{B} \perp \mathbf{B}$ are shown in figure 48.

Table 6: Photophysical properties of model compound B (105) and bichromophore $\mathbf{B} \perp \mathbf{B}(\mathbf{1 0 7})$ in $\mathrm{CHCl}_{3}$.

\begin{tabular}{lllll}
\hline Compound & $\lambda_{\mathrm{abs}}^{\max } / \mathrm{nm}\left(\varepsilon / \mathrm{M}^{-1} \mathrm{~cm}^{-1}\right)$ & $\lambda_{\mathrm{em}}^{\max } / \mathrm{nm}$ & $\Phi_{\mathrm{f}}{ }^{\mathrm{a}}$ & $\tau_{\mathrm{f}} / \mathrm{ns}$ \\
\hline $\mathbf{B}(\mathbf{1 0 5})$ & $359(50,000)$ & 395 & 0.47 & $1.03 \pm 0.05$ \\
$\mathbf{B} \perp \mathbf{B}(\mathbf{1 0 7})$ & $359(91,000)$ & 396 & 0.44 & $1.02 \pm 0.05$ \\
\hline
\end{tabular}

(a) Fl. quantum yields $\Phi_{\mathrm{f}}$ were measured using Coumarin 120 in $\mathrm{MeOH}\left(\Phi_{\mathrm{f}}=0.51\right)$ as a reference. ${ }^{[132]}$

The spectral overlap of the model compound's emission and absorption spectrum (figure 41), was determined to be $J_{\mathrm{DA}}=6.6 \cdot 10^{13} \mathrm{M}^{-1} \mathrm{~cm}^{-1} \mathrm{~nm}^{4}$. 


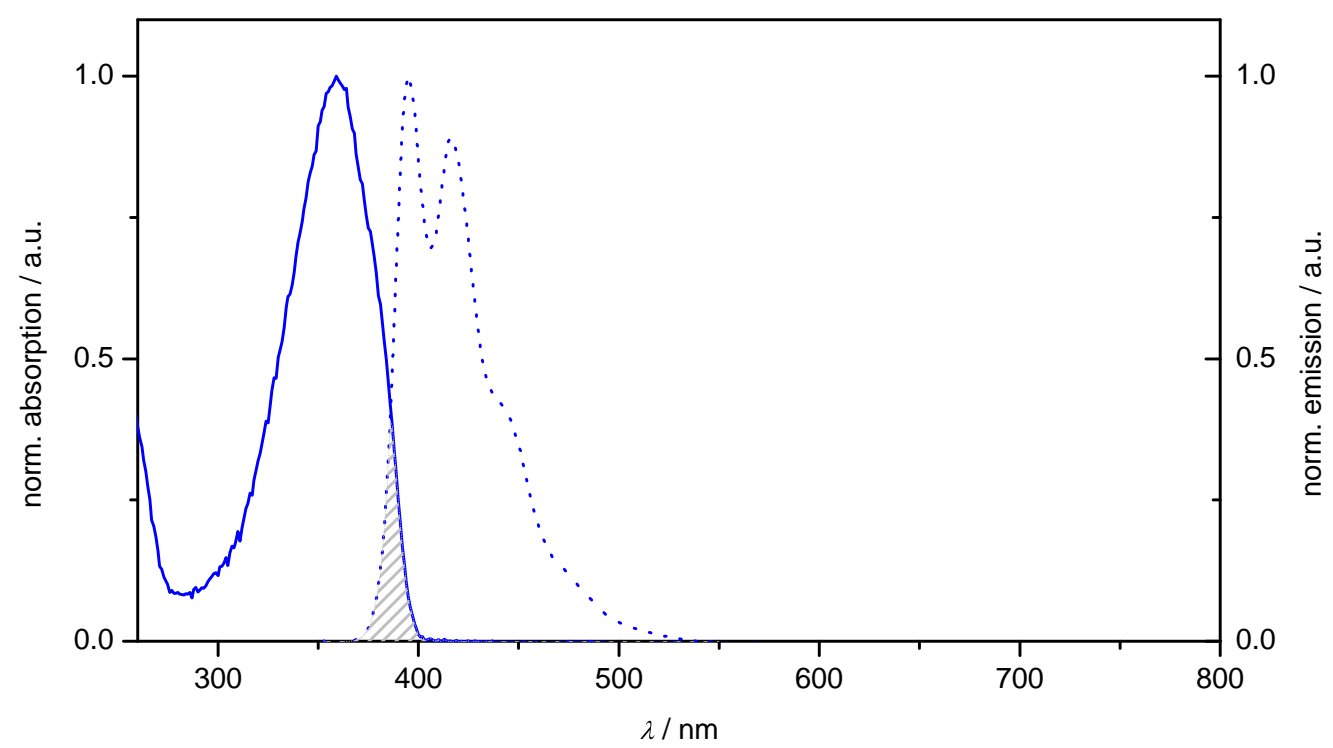

Figure 41: Normalized absorption (solid line) and emission spectrum (dotted line) of model compound $\mathbf{B}$ (105). The overlapping region of emission and absorption is displayed as a hatched area.

\subsection{DFT calculations for model compound B (105) and bichromophore $B \perp B(107)$}

Figure 42 shows the ground and $\mathrm{S}_{1}$ excited state geometry of $\mathbf{B}$ after energy optimization using density functional methods (see chapter 19 for details). According to these calculations, the thienyl groups and the fluorene core are twisted in the ground state but coplanar in the $S_{1}$ excited state. Different conformers are possible due to thienyl group rotation. For the ground state, the energetically most stable conformer was chosen. The absorption $\left(S_{0} \rightarrow S_{1}\right)$ and emission $\left(\mathrm{S}_{1} \rightarrow \mathrm{S}_{0}\right)$ transition dipoles were determined for the ground and excited state geometries, respectively. Both are parallel and aligned along the long axis of the chromophore (figure 42). 
ground state geometry
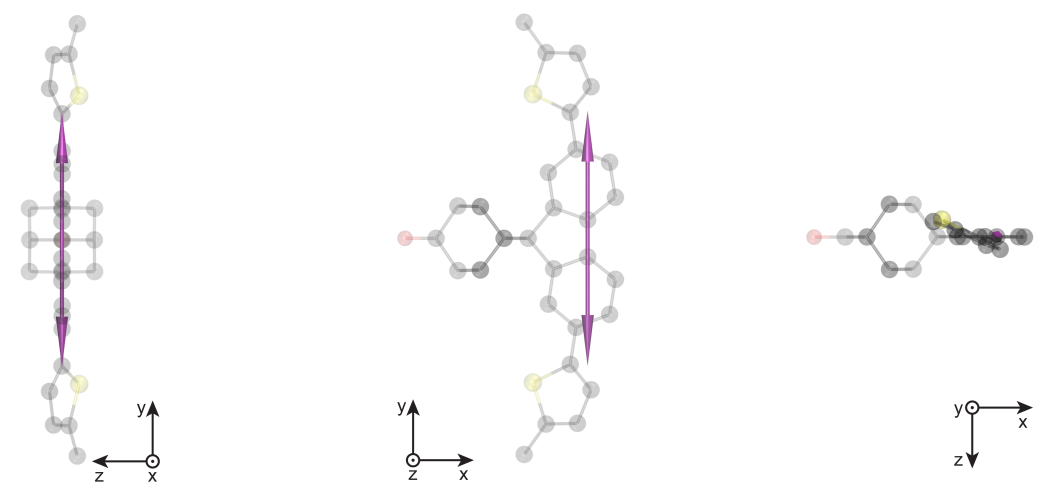

$\mathrm{S}_{1}$ state geometry
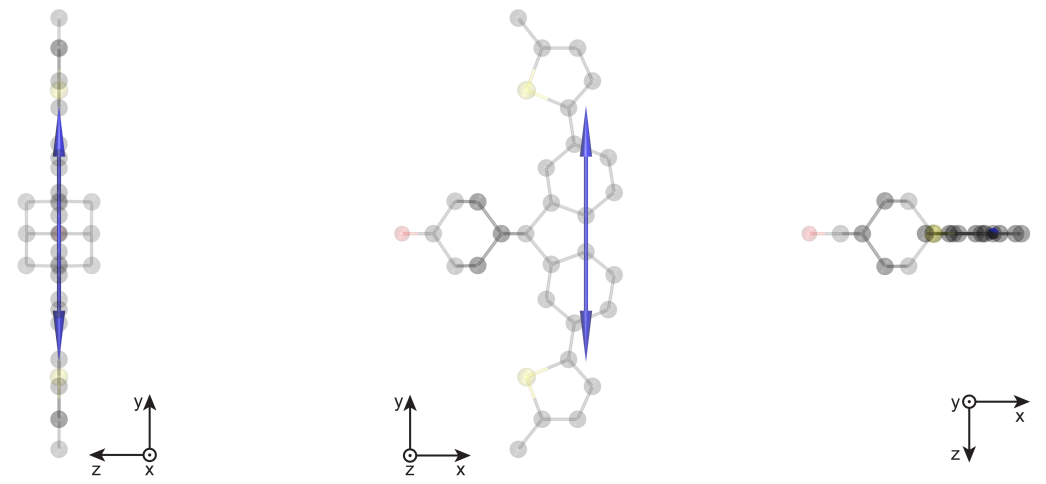

Figure 42: DFT and TD-DFT optimized geometries of model compound B (105) and the transition dipole moments shown as double-headed arrows. Top: Ground state geometry with absorption $\left(\mathrm{S}_{0} \rightarrow \mathrm{S}_{1}\right)$ transition dipole moment (purple arrow); bottom: $\mathrm{S}_{1}$ state geometry and emission $\left(\mathrm{S}_{1} \rightarrow \mathrm{S}_{0}\right)$ transition dipole moment (blue arrow). $\mathrm{H}$ atoms are omitted for clarity.

The ground state geometry of bichromophore $\mathbf{B} \perp \mathbf{B}(\mathbf{1 0 7})$ is shown in figure 43 . Bichromophore $\mathbf{B} \perp \mathbf{B}$ can adopt different conformations due to rotation of the thienyl groups. For both chromophore units of $\mathbf{B} \perp \mathbf{B}$, the same conformation was chosen as for the model compound $\mathbf{B}$ (most stable in the case of $\mathbf{B}$ ). For one chromophore in $\mathbf{B} \perp \mathbf{B}$, the absorption transition dipole and for the other chromophore, the emission transition dipole are shown in figure 43. For that purpose, the emission transition dipole moment of $\mathbf{B}$ was transferred to the ground state geometry of $\mathbf{B} \perp \mathbf{B}$ (see chapter 19 for details). 

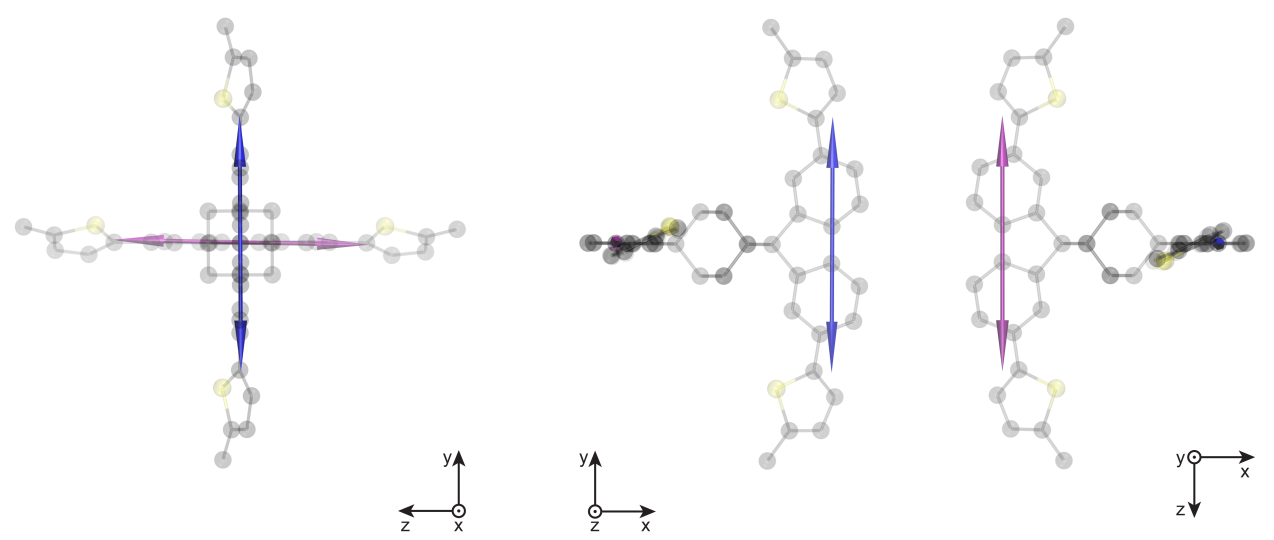

Figure 43: DFT optimized ground state geometry of bichromophore $\mathbf{B} \perp \mathbf{B}$ (107). The absorption $\left(\mathrm{S}_{0} \rightarrow \mathrm{S}_{1}\right)$ and emission $\left(\mathrm{S}_{1} \rightarrow \mathrm{S}_{0}\right)$ transition dipole moments are shown as purple and blue double-headed arrows, respectively. $\mathrm{H}$ atoms are omitted for clarity.

\subsection{Estimation of energy transfer efficiency based on ideal Förster point-dipole approximation for bichromophore $B \perp B(107)$}

The geometry of the transition dipole moments was used to determine the orientation factor $\kappa^{2}$ and, having the photophysical properties of the model compound B (table 6), the energy transfer efficiency based on the ideal Förster pointdipole approximation was estimated.

The angles $\theta_{\mathrm{D}}, \theta_{\mathrm{A}}$ and $\theta_{\mathrm{DA}}$ and distance $\left|\vec{R}_{\mathrm{DA}}\right|$ between the emission and absorption transition dipoles are given in table 7 (see section 1.2.1 for angle definitions). Using equation 4 , the orientation factor $\kappa^{2}$ was calculated (table 7 ).

Taking into account the spectral overlap $J_{\mathrm{DA}}$ and the refractive index of $\mathrm{CHCl}_{3}$ $n_{\mathrm{D}}\left(20^{\circ} \mathrm{C}\right)=1.4459,{ }^{[134]}$ the Förster radius $R_{0}$ and the FRET efficiency $E_{\text {FRET }}$ were calculated (equations 5 and 6). The results are listed in table 7.

Table 7: Angles $\theta_{\mathrm{D}}, \theta_{\mathrm{A}}$ and $\theta_{\mathrm{DA}}$ between transition dipole moments and interconnecting vector $\vec{R}_{\mathrm{DA}}$, orientation factor $\kappa^{2}$, distance between the donor and acceptor $\left|\vec{R}_{\mathrm{DA}}\right|$, Förster radius $R_{0}$, and estimated FRET efficiency $E_{\mathrm{FRET}}$.

\begin{tabular}{llllllll}
\hline Compound & $\theta_{\mathrm{D}}$ & $\theta_{\mathrm{A}}$ & $\theta_{\mathrm{DA}}$ & $\kappa^{2}$ & $\left|\vec{R}_{\mathrm{DA}}\right| / \mathrm{nm}$ & $R_{0} / \mathrm{nm}$ & $E_{\mathrm{FRET}}$ \\
\hline $\mathbf{B} \perp \mathbf{B}(\mathbf{1 0 7})$ & $90.1^{\circ}$ & $90.1^{\circ}$ & $91.4^{\circ}$ & $6.0 \cdot 10^{-4}$ & 0.84 & 0.85 & 0.51 \\
\hline
\end{tabular}





\section{Advancement of the red fluorophore}

The red fluorophore in bichromophore $\mathbf{8 7}$ has a very low fluorescence quantum yield (fl. quantum yield of model compound 77 is $\Phi_{\mathrm{f}}=0.02$ ). For studying intramolecular interactions between the blue and red fluorophores a higher quantum yield would be advantageous.

\subsection{Disadvantages and suggested improvements of previous red chromophore}

The low fluorescence quantum yield of model compound $77\left(\Phi_{\mathrm{f}}=0.02\right)$ is most likely due to the thiobarbiturate electron acceptor group. This hypothesis is supported by the fact that the precursor 75 with an aldehyde group instead of the thiobarbiturate group showed a significantly larger fluorescence quantum yield $\left(\Phi_{\mathrm{f}}=0.73\right)$. Therefore, we intended to use a quaternized pyridyl group with a betaine-like substituent. We did not change the $p$-methoxyphenyl group to a stronger electron donating group such as a dimethylaminophenyl group, because this would introduce an additional protonation site. The resulting red fluorescent compound $\mathbf{1 1 2}$ is shown in scheme 26. In the following text this model compound will be termed $\mathbf{R}$ to help the reader to track the explanations. As mentioned before, in bichromophores, symbols " $\perp$ " and "||" indicate the orthogonal and parallel arrangement of the chromophores, respectively. For example, bichromophore $\mathbf{1 0 7}$ with two perpendicular blue fluorophores is termed $\mathbf{B} \perp \mathbf{B}$.

\subsection{Synthesis of $R(112)$}

Synthesis of model compound $\mathbf{R}$ is shown in scheme 26. lodination of compound 82, was initially carried out using NIS in $\mathrm{MeOH} / \mathrm{AcOH} .{ }^{[144]}$ Full conversion was not observed, unless 10 eq. of NIS were added. Under these conditions, iodide 108 was obtained in $81 \%$ yield. However, the same reaction in THF/AcOH with only 1.20 eq. of NIS ${ }^{[154]}$ easily afforded $87 \%$ yield. Suzuki crosscoupling with 4-pyridyl boronic acid (52) gave bromide 110 which reacted with 2,6-adamantandione (23b) and gave alcohol 109. Cyclization under acidic conditions afforded compound $\mathbf{1 1 1}$. $\mathbf{R}$ was obtained by quaternization using octa- 


\section{Chapter 11 ADVANCEMENT OF THE RED FLUOROPHORE}

fluorocyclopentene (59) in the presence of $\mathrm{AcOH}$ and water using microwave radiation.<smiles>COc1ccc(-c2ccc(-c3sccc3Br)s2)cc1</smiles>

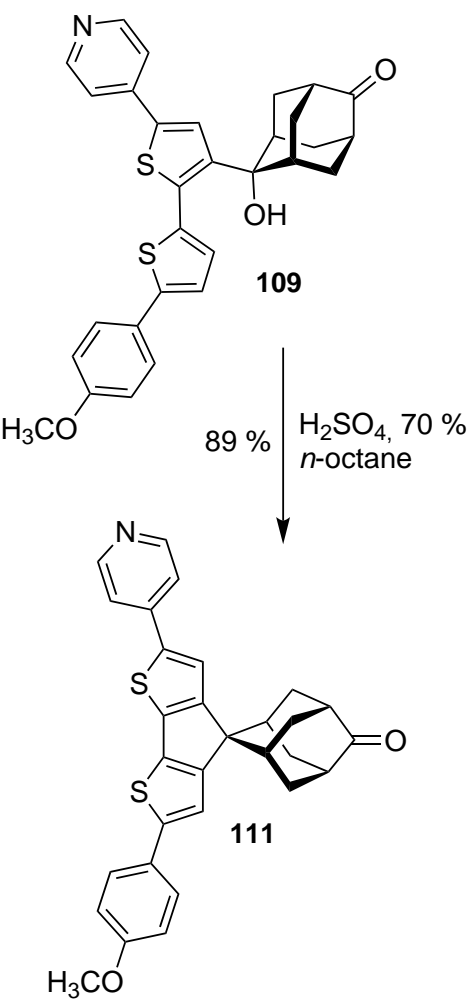

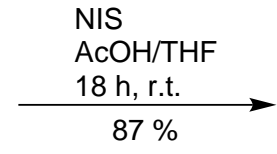

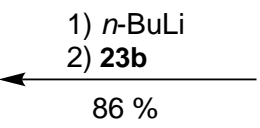<smiles>COc1ccc(-c2ccc(-c3sc(-c4ccncc4)cc3Br)s2)cc1</smiles>
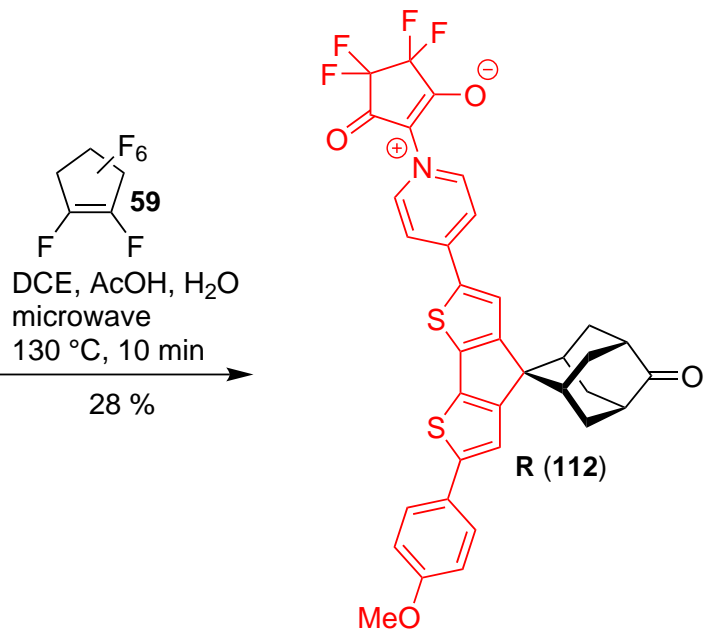

Scheme 26: Synthesis of "red" fluorescent model compound R (112).

\subsection{Photophysical properties of compound R (112)}

The photophysical data of model compound $\mathbf{R}$ are given in table 8 . The absorption maximum of $\mathbf{R}$ is blue-shifted by approximately $45 \mathrm{~nm}$, compared to the previous "red" model compound 77. However, the emission maxima of both red fluorescent model compounds are almost identical: $614 \mathrm{~nm}$ and $618 \mathrm{~nm}$ for $\mathbf{R}$ and compound 77, respectively. The change from the electron accepting thiobarbiturate to the quaternized pyridyl group in $\mathbf{R}$ increased the fluorescence quantum 
yield very strongly from 0.02 to 0.94 . Taking into account the lower extinction coefficient of $\mathbf{R}$ compared to compound $\mathbf{7 7}$, the brightness was increased by factor 39.

Table 8: Photophysical properties of "red" fluorescent model compound $\mathbf{R}$ (112) in $\mathrm{CHCl}_{3}$.

\begin{tabular}{lllll}
\hline Compound & $\lambda_{\mathrm{abs}}^{\max } / \mathrm{nm}\left(\varepsilon / \mathrm{M}^{-1} \mathrm{~cm}^{-1}\right)$ & $\lambda_{\mathrm{em}}^{\max } / \mathrm{nm}$ & $\Phi_{\mathrm{f}}{ }^{\mathrm{a}}$ & $\tau_{\mathrm{f}} / \mathrm{ns}$ \\
\hline $\mathbf{R}(\mathbf{1 1 2})$ & $542(54,000)$ & 614 & 0.94 & $2.5 \pm 0.1$ \\
\hline
\end{tabular}

(a) Fluorescence quantum yield $\Phi_{\mathrm{f}}$ was measured using Rhodamine $6 \mathrm{G}$ in $\mathrm{EtOH}$ $\left(\Phi_{\mathrm{f}}=0.95\right)$ as a reference. ${ }^{[155]}$

\subsection{DFT calculations for model compound R (112)}

Figure 44 shows the ground state geometry of model compound $\mathbf{R}$ calculated upon energy optimization using density functional methods. The absorption transition dipole moment is shown as a double-headed arrow (see chapter 19 for details). The transition dipole moment is slightly tilted relative to the adamantane part of the molecule. The asymmetric structure of the chromophore results in an angle of $84.3^{\circ}$ between the absorption dipole and the axis defined by adamantane's C-2 and C-6 atoms.
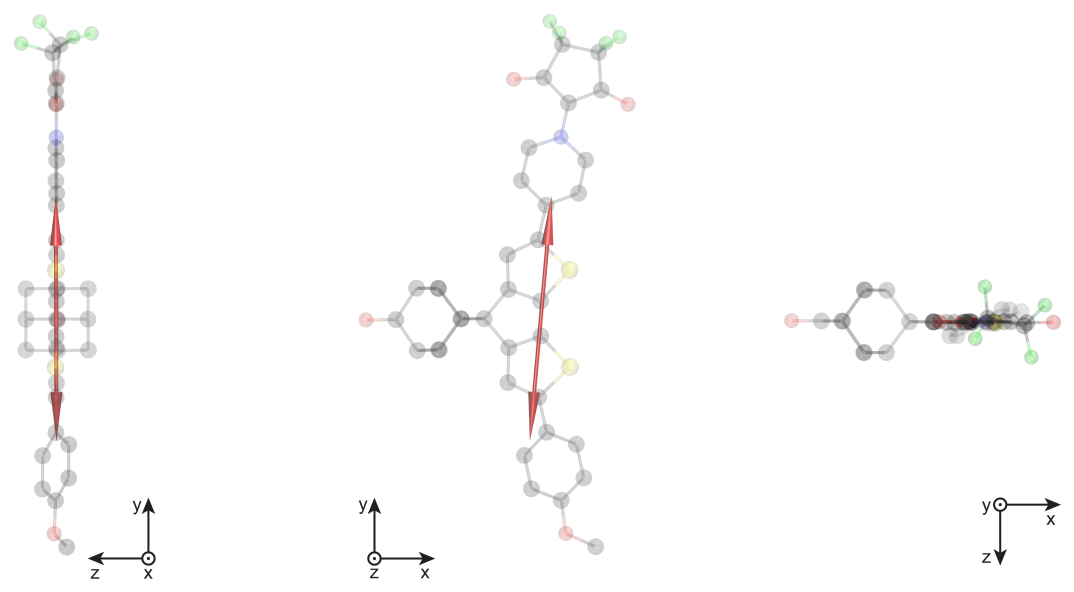

Figure 44: DFT optimized geometry of model compound $\mathbf{R}$ (112) and absorption $\left(\mathrm{S}_{0} \rightarrow \mathrm{S}_{1}\right)$ transition dipole moment shown as red double-headed arrow. $\mathrm{H}$ atoms are omitted for clarity. 



\section{Bichromophore $B \perp R$ (114)}

As defined above, short and descriptive compound names are used in the following text. $\mathbf{B}$ and $\mathbf{R}$ refer to the "blue" and "red" chromophores in model compounds 105, 112, and elsewhere; " $\perp$ " and "||" indicate the orthogonal or parallel arrangement of the chromophores, respectively. For example, bichromophore $\mathbf{1 1 4}$ with blue and red orthogonally arranged chromophores will be termed $\mathbf{B} \perp \mathbf{R}$. The model compounds themselves are termed $\mathbf{B}$ and $\mathbf{R}$, respectively.

Combining the structures of the optimized blue and red fluorescent model compounds $\mathbf{B}$ and $\mathbf{R}$ led to bichromophore $\mathbf{B} \perp \mathbf{R}(\mathbf{1 1 4})$ as shown in scheme 27 . The spectral overlap of $\mathbf{B}$ and $\mathbf{R}$ is shown in figure 45 . The calculated overlap integral is $J_{\mathrm{DA}}=2.4 \cdot 10^{14} \mathrm{M}^{-1} \mathrm{~cm}^{-1} \mathrm{~nm}^{4}$ and is approximately two times lower as for bichromophore 87.

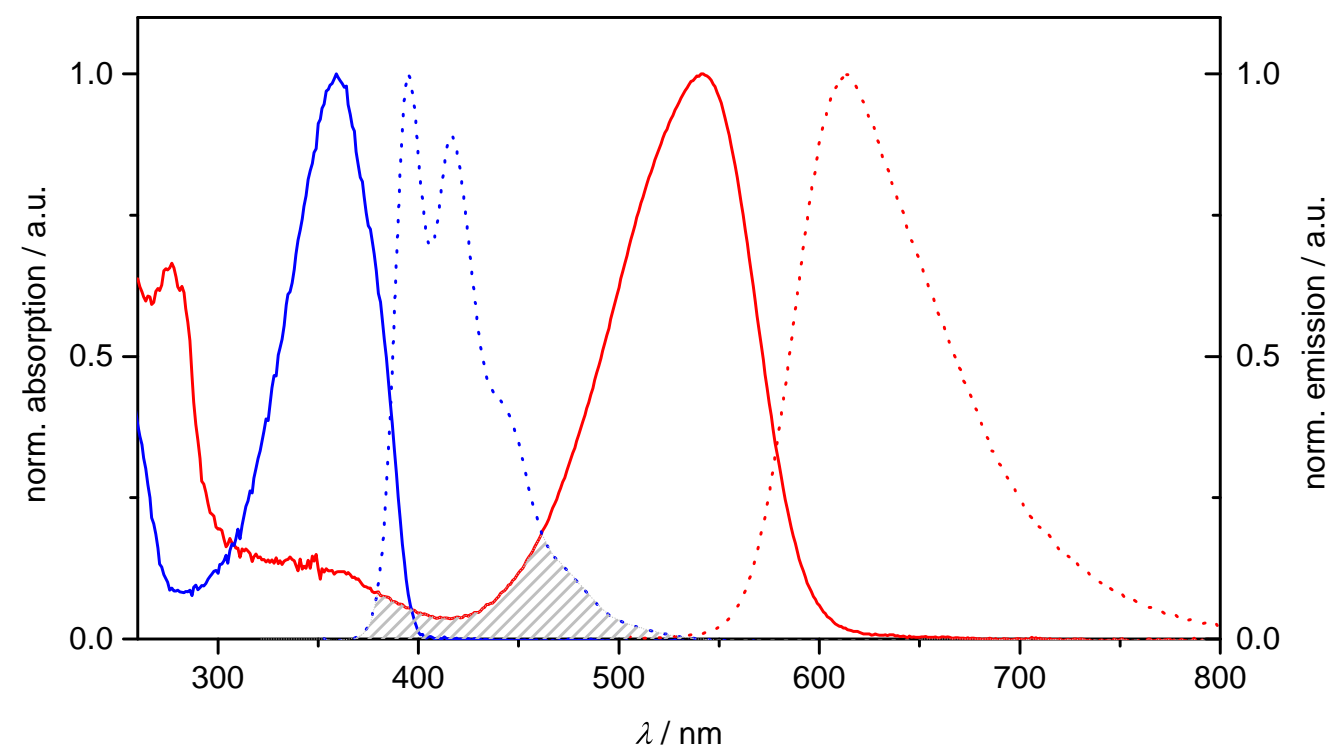

Figure 45: Normalized absorption (solid lines) and emission spectra (dotted lines) of model compounds $\mathbf{B}$ (105, blue curve) and $\mathbf{R}\left(\mathbf{1 1 2}\right.$, red curve) in $\mathrm{CHCl}_{3}$. The overlapping region of $\mathbf{B}$-emission and $\mathbf{R}$-absorption is displayed hatched.

The reduced overlap integral, the fixed orthogonal arrangement of fluorophores, the improved fluorescence quantum yield of the red fluorophore and the symmetric blue fluorophore without basic sites make bichromophore $\mathbf{B} \perp \mathbf{R}$ an interesting candidate for studying intramolecular EET processes. 


\subsection{Synthesis of bichromopohore $B \perp R$ (114)}

As illustrated in scheme 27, reaction of lithiated compound 103 with the red chromophore precursor 111 gave compound 113. Cyclization afforded compound $\mathbf{1 1 5}$ in $35 \%$ yield. Finally, quaternization of the pyridyl group ${ }^{[156-158]}$ using established reaction conditions (microwave assisted synthesis) provided bichromophore $\mathbf{B} \perp \mathbf{R}(\mathbf{1 1 4})$ in $92 \%$ yield.

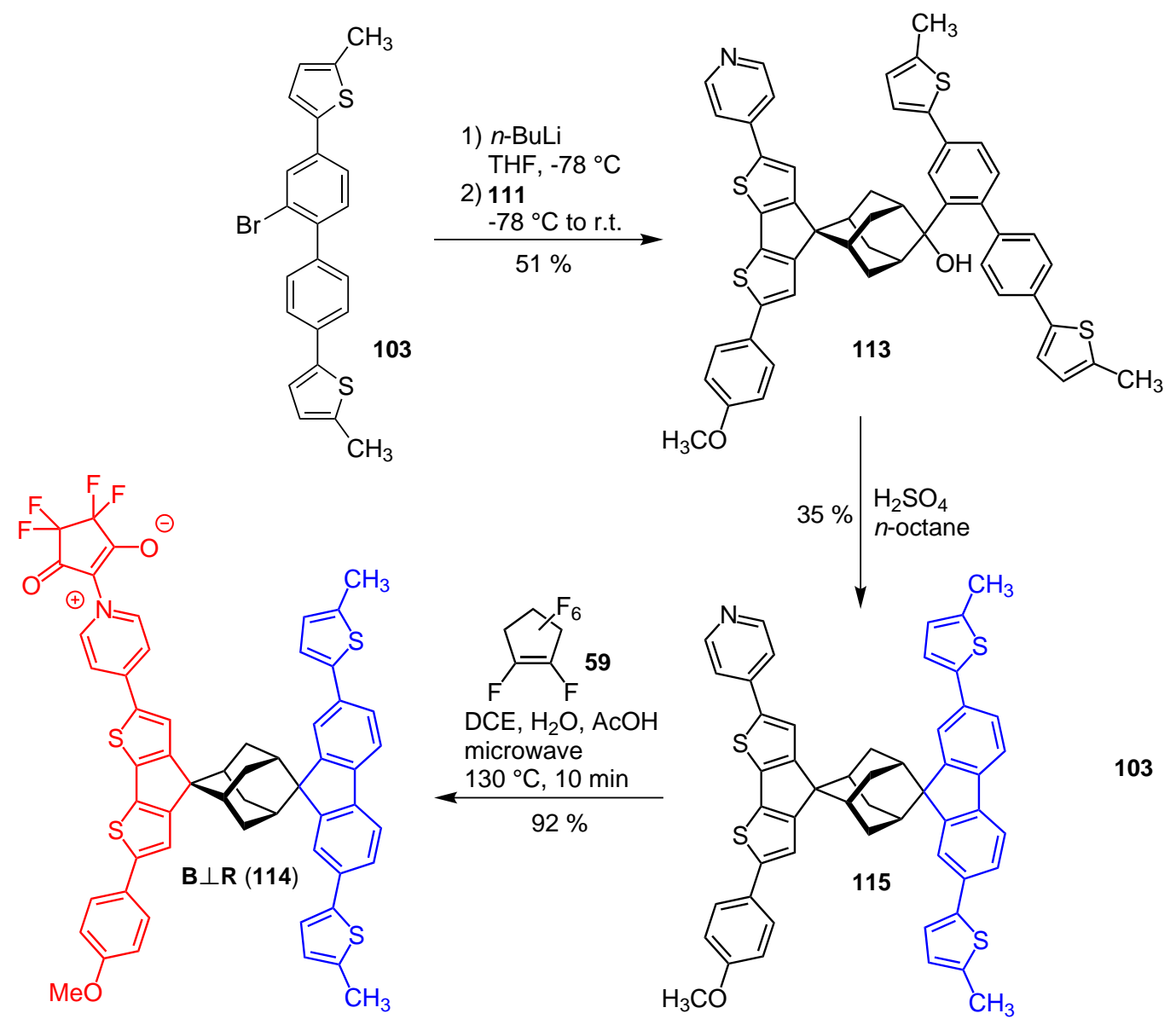

Scheme 27: Synthesis of bichromophore 114. 


\subsection{Photophysical properties of bichromophore $B \perp R(114)$}

As illustrated in figure 46, excitation at the absorption maximum of the "blue" fluorophore causes exclusively "red" emission at around $610 \mathrm{~nm}$. No fluorescence of the "blue" fluorophore (at around $395 \mathrm{~nm}$ ), was detected at all, which indicates efficient EET from the blue towards the red fluorophore.

a)

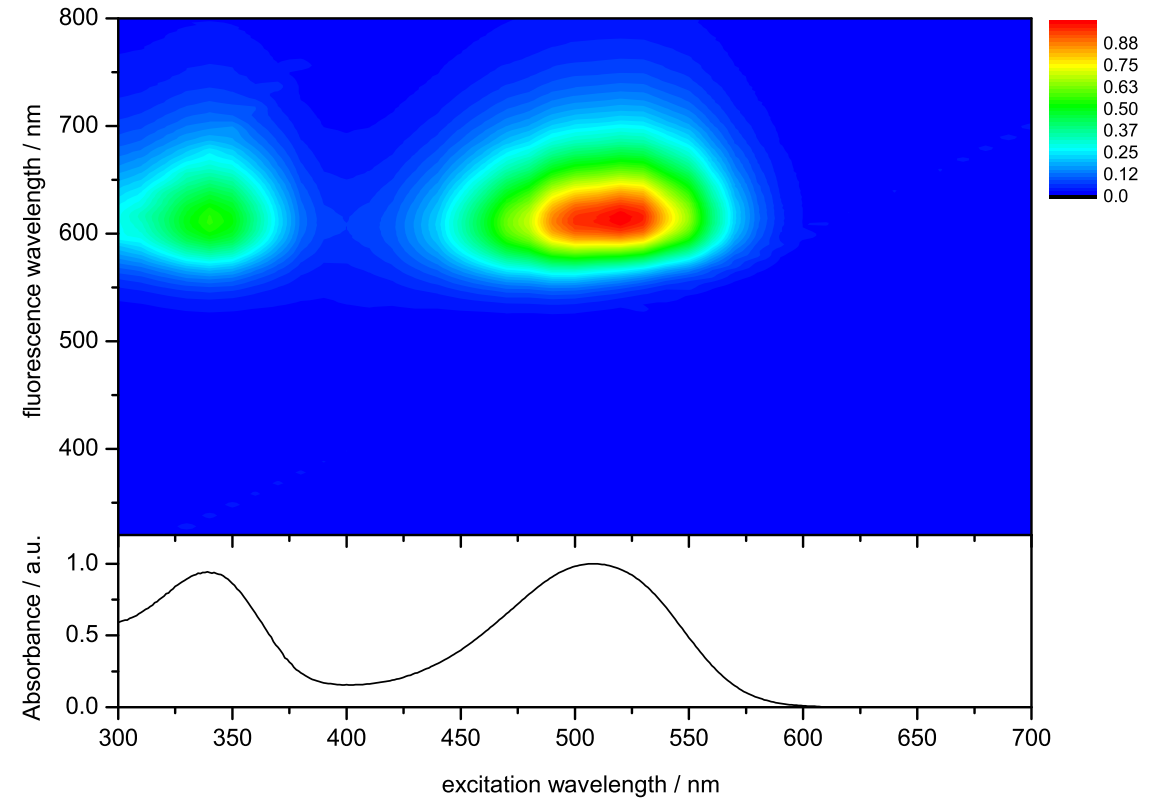

Figure 46: a) Contour plot showing emission intensity (color coded) vs. excitation and fluorescence wavelength for bichromophore $\mathbf{B} \perp \mathbf{R}(\mathbf{1 1 4})$ in EtOAc. The emission intensity was corrected for wavelength dependence of the detector sensitivity; b) absorption spectrum of bichromophore $\mathbf{B} \perp \mathbf{R}(\mathbf{1 1 4})$ in EtOAc.

The photophysical data of $\mathbf{B} \perp \mathbf{R}$ are given in table 9 .

Table 9: Photophysical properties of bichromophore $\mathbf{B} \perp \mathbf{R}(\mathbf{1 1 4})$ in $\mathrm{CHCl}_{3}$.

\begin{tabular}{lllll}
\hline Compound & $\lambda_{\mathrm{abs}}^{\max } / \mathrm{nm}\left(\varepsilon / \mathrm{M}^{-1} \mathrm{~cm}^{-1}\right)$ & $\lambda_{\mathrm{em}}^{\max } / \mathrm{nm}$ & $\Phi_{\mathrm{f}}$ & $\tau_{\mathrm{f}} / \mathrm{ns}$ \\
\hline $\mathbf{B} \perp \mathbf{R}(\mathbf{1 1 4})$ & $546(65,000)$, & 616 & n.d. & $2.3 \pm 0.1$ \\
& $358(51,000)$ & & & \\
\hline
\end{tabular}




\subsection{DFT calculations for bichromophore $B \perp R$ (114)}

Figure 47 shows the ground state geometry of bichromophore $\mathbf{B} \perp \mathbf{R}$ after energy optimization using density functional methods (see chapter 19 for details). For the "blue" chromophore the same conformation was chosen as for model compound B (most stable one for B). For the "red" chromophore, the absorption transition dipole, and for the "blue" chromophore, the emission transition dipole is shown in figure 47 . The emission dipole orientation of model compound $\mathbf{B}$ was used as emission dipole orientation of the "blue" part in bichromophore $\mathbf{B} \perp \mathbf{R}$ (see chapter 19 for details).
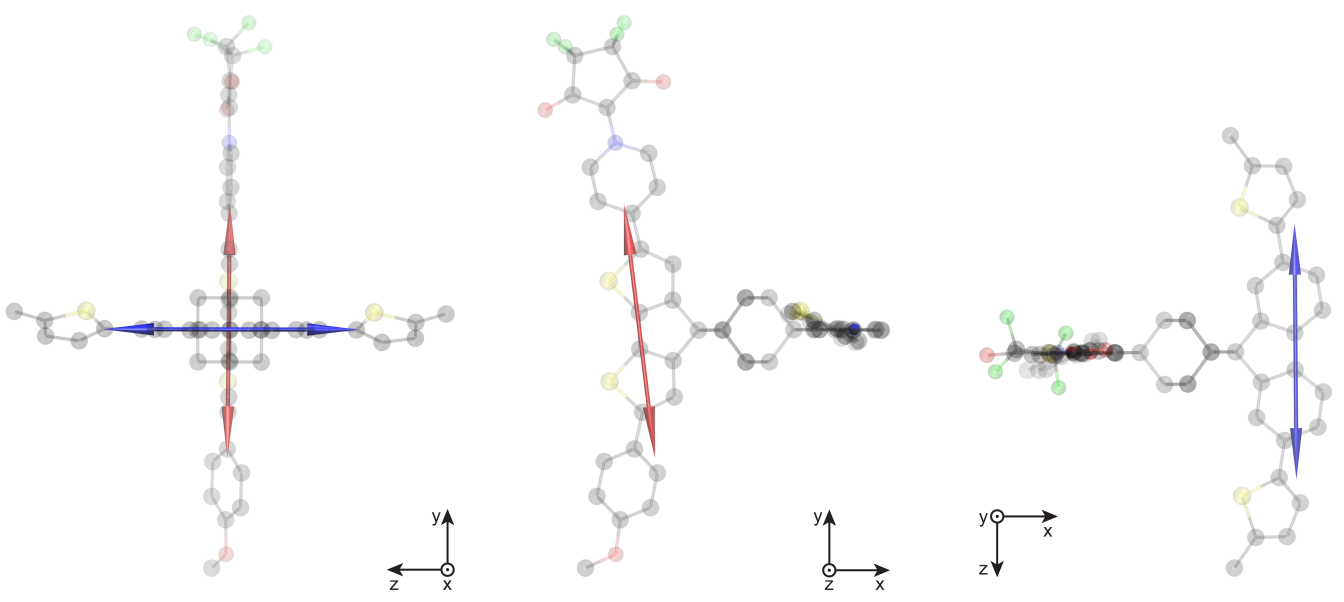

Figure 47: DFT optimized ground state geometry of bichromophore $\mathbf{B} \perp \mathbf{R}$ (114). The absorption $\left(\mathrm{S}_{0} \rightarrow \mathrm{S}_{1}\right)$ and emission $\left(\mathrm{S}_{1} \rightarrow \mathrm{S}_{0}\right)$ transition dipole moments are shown as red and blue double-headed arrows, respectively. $\mathrm{H}$ atoms are omitted for clarity.

\subsection{Estimation of energy transfer efficiency based on ideal Förster point-dipole approximation for bichromophore $B \perp R$ (114)}

The geometric arrangement of the transition dipole moments was used to determine the orientation factor $\kappa^{2}$ and, having the photophysical properties of model compounds 105 and 112 (tables 8 and 6), the energy transfer efficiency based on the ideal Förster point-dipole approximation was estimated.

The angles $\theta_{\mathrm{D}}, \theta_{\mathrm{A}}$ and $\theta_{\mathrm{DA}}$ and distance $\left|\vec{R}_{\mathrm{DA}}\right|$ between the emission and absorption transition dipoles are given in table 10 (see section 1.2.1 for angle defini- 
tions). Using equation 4 , the orientation factor $\kappa^{2}$ was calculated (table 10). Taking into account the spectral overlap J $J_{\mathrm{DA}}$ and the refractive index of the $\mathrm{CHCl}_{3}$ $n_{\mathrm{D}}\left(20^{\circ} \mathrm{C}\right)=1.4459,{ }^{[134]}$ the Förster radius $R_{0}$ and the FRET efficiency $E_{\mathrm{FRET}}$ were calculated (equations 6 and 5). The results are given in table 10.

Table 10: Angles $\theta_{\mathrm{D}}, \theta_{\mathrm{A}}$ and $\theta_{\mathrm{DA}}$ between transition dipole moments and interconnecting vector $\vec{R}_{\mathrm{DA}}$, orientation factor $\kappa^{2}$, distance between the donor and acceptor $\left|\vec{R}_{\mathrm{DA}}\right|$, Förster radius $R_{0}$, and estimated FRET efficiency $E_{\mathrm{FRET}}$.

\begin{tabular}{llllllll}
\hline Compound & $\theta_{\mathrm{D}}$ & $\theta_{\mathrm{A}}$ & $\theta_{\mathrm{DA}}$ & $\kappa^{2}$ & $\left|\vec{R}_{\mathrm{DA}}\right| / \mathrm{nm}$ & $R_{0} / \mathrm{nm}$ & $E_{\mathrm{FRET}}$ \\
\hline $\mathbf{B} \perp \mathbf{R}(\mathbf{1 1 4})$ & $89.9^{\circ}$ & $83.4^{\circ}$ & $90.5^{\circ}$ & $9.54 \cdot 10^{-5}$ & 0.84 & 0.77 & 0.38 \\
\hline
\end{tabular}





\section{Bichromophores with parallel arranged fluorophores}

For comparison, bichromophores with parallel orientation of the two chromophore units bound via 2-(2-adamantylidene)adamantane as a rigid spacer were designed and prepared. As a reference for bichromophore $\mathbf{B} \perp \mathbf{B}$ (107), we used bichromophore B $\| \mathbf{B}$ (116) with two parallel blue fluorophores. Accordingly, for bichromophore $\mathbf{B} \perp \mathbf{R}$ (114), we prepared bichromophore $\mathbf{B} \| \mathbf{R}$ (118) with parallel blue and red fluorophores (scheme 28).

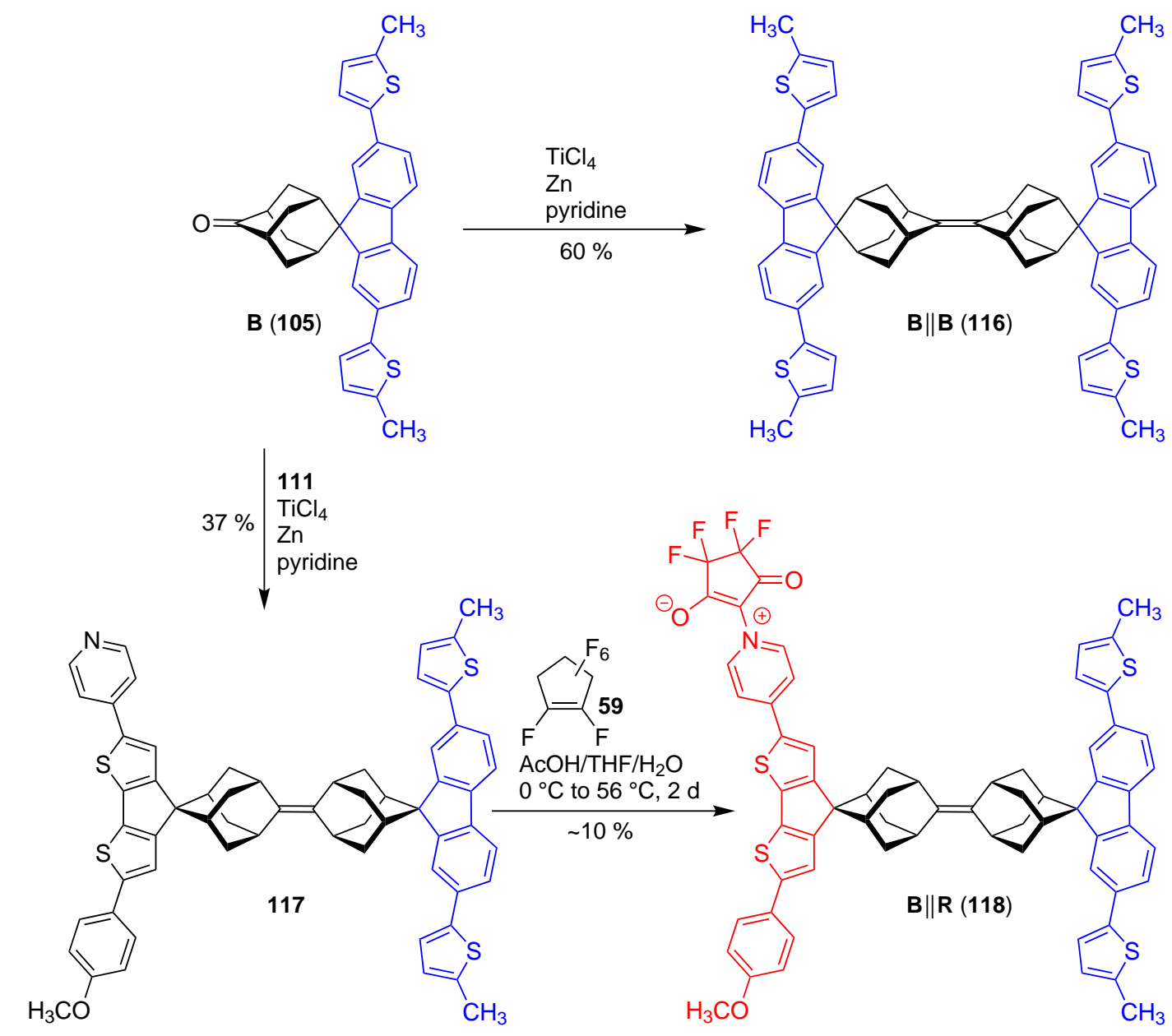

Scheme 28: Synthesis of $\mathbf{B} \| \mathbf{B}(\mathbf{1 1 6})$ and $\mathbf{B} \| \mathbf{R}(\mathbf{1 1 8})$ with parallel orientation of fluorophores. 


\section{Chapter 13 BICHROMOPHORES WITH PARALLEL ARRANGED FLUOROPHORES}

\subsection{Synthesis of compounds with parallel chromophores}

As shown in scheme 28, bichromophore $\mathbf{B} \| \mathbf{B}$ (116) and precursor 117 were prepared from ketones B and $\mathbf{1 1 1}$ in a McMurry reaction, as described by Zhuk and co-workers. ${ }^{[159]}$ The quaternization of the pyridyl nitrogen in $\mathbf{1 1 7}$ turned out to be challenging. Conditions used for the synthesis of $\mathbf{R}$ or $\mathbf{B} \perp \mathbf{R}$ led to complex reaction mixtures. Using wet $\mathrm{AcOH}$ and $\mathrm{THF}$ as a solvent mixture for the reaction, bichromophore $\mathbf{1 1 8}$ was detected in the reaction mixture. Purification by HPLC on a normal phase HPLC gave a pure sample of bichromophore B $\| \mathbf{R}$ (118).

\subsection{Photophysical properties of compounds with parallel chromophores}

The photophysical data of $\mathbf{B} \| \mathbf{B}$ and $\mathbf{B} \| \mathbf{R}$ are given in table 11 .

Table 11: Photophysical properties of bichromophores with parallel chromophores in $\mathrm{CHCl}_{3}$.

\begin{tabular}{lllll}
\hline Compound & $\lambda_{\mathrm{abs}}^{\max } / \mathrm{nm}\left(\varepsilon / \mathrm{M}^{-1} \mathrm{~cm}^{-1}\right)$ & $\lambda_{\mathrm{em}}^{\max } / \mathrm{nm}$ & $\Phi_{\mathrm{f}}$ & $\tau_{\mathrm{f}} / \mathrm{ns}$ \\
\hline $\mathbf{B} \| \mathbf{B}(\mathbf{1 1 6})$ & $357(93,000)$ & 394 & $0.38^{\mathrm{a}}$ & $1.03 \pm 0.05$ \\
$\mathbf{B} \| \mathbf{R}(\mathbf{1 1 8})$ & $548,358$ (n.d.) $)^{\mathrm{b}}$ & 618 & n.d. & $2.6 \pm 0.1$ \\
\hline
\end{tabular}

(a) Fl. quantum yield $\Phi_{\mathrm{f}}$ was measured using Coumarin 120 in $\mathrm{MeOH}\left(\Phi_{\mathrm{f}}=0.51\right)$ as a reference; ${ }^{[132]}$ (b) $\varepsilon$ not determined, ratio of absorbance is 1.00:0.81.

Figure 48 shows the normalized absorption/emission spectra of bichromophores with identical or different chromophores and their combinations with parallel or perpendicular orientations, respectively. As references, the spectra of "blue" and "red" model compounds $\mathbf{B}$ and $\mathbf{R}$, are shown in figure $48 \mathrm{a}$. In figure $48 \mathrm{~b}$, the spectra of bichromophores with two identical chromophores $(\mathbf{B} \perp \mathbf{B}, \mathbf{B} \| \mathbf{B})$ are shown, while spectra of bichromophores with different chromophores $(\mathbf{B} \perp \mathbf{R}$, $\mathbf{B} \| \mathbf{R})$ are shown in figure 48c. Bichromophores with red chromopohores $\mathbf{R} \perp \mathbf{R}$ and $\mathbf{R} \| \mathbf{R}$ were not prepared, because, due to lack of symmetry, the transition dipole moments are most likely not orthogonally oriented in $\mathbf{R} \perp \mathbf{R}$, and therefore also $\mathbf{R} \| \mathbf{R}$ is unimportant. 


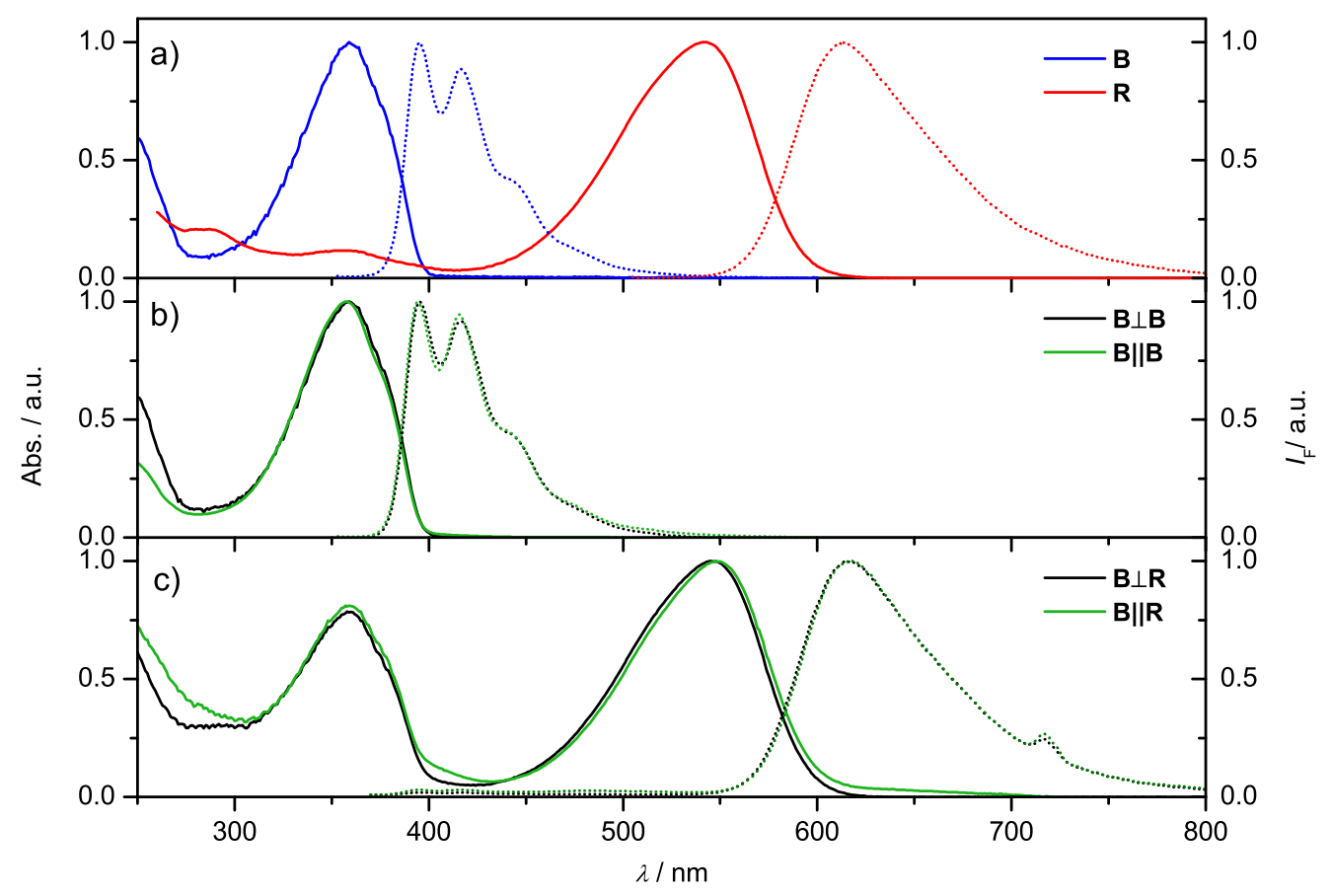

Figure 48: Normalized absorption/emission spectra (solid/dotted curves) in $\mathrm{CHCl}_{3}$ : a) $\mathbf{B}$ (105, blue curve) and $\mathbf{R}(\mathbf{1 1 2}$, red curve); b) $\mathbf{B} \perp \mathbf{B}$ (107, black curve) and $\mathbf{B} \| \mathbf{B}$ (116, green curve); c) $\mathbf{B} \perp \mathbf{R}(\mathbf{1 1 4}$, black curve) and $\mathbf{B} \| \mathbf{R}$ (118, green curve). The excitation wavelength was $348 \mathrm{~nm}$ for 105, 107 and 116, $530 \mathrm{~nm}$ for $\mathbf{1 1 2}$ and $360 \mathrm{~nm}$ for $\mathbf{1 1 4}$ and $\mathbf{1 1 8}$. Emission spectra in (c) show an artefact at $720 \mathrm{~nm}$ due to higher order diffraction of the excitation light. This figure was published in an article with the title "Bichromophoric Compounds with Orthogonally and Parallelly Arranged Chromophores Separated by Rigid Spacers".[160] Copyright Wiley-VCH Verlag GmbH \& Co. KGaA. Reproduced with permission.

As shown in figure 48, the spectra of compounds with blue fluorophores $\mathbf{B}, \mathbf{B} \perp \mathbf{B}$ and $\mathbf{B} \| \mathbf{B}$ are almost identical. The absorption coefficients of bichromophores $\mathbf{B} \perp \mathbf{B}$ and $\mathbf{B} \| \mathbf{B}$ are approximately twice as much as the absorption of $\mathbf{B}$. The emission efficiencies of bichromophoric compounds are slightly reduced compared to the reference compound $\mathbf{B}$. The fluorescence lifetime is identical for all three compounds. A possible reason for the deviations could be aggregation of the bichromophores due to lower solubility. These results indicate that the two fluorophores in $\mathbf{B} \perp \mathbf{B}$ and $\mathbf{B} \| \mathbf{B}$ can be considered as isolated electronic systems without orbital overlap or the interactions can be neglected. Bichromophores $\mathbf{B} \perp \mathbf{R}$ and $\mathbf{B} \| \mathbf{R}$ showed exclusively fluorescence of the red fluorophore upon ex- 
citation of the blue fluorophore. Regardless of bichromophore geometry, highly efficient EET was observed.

\subsection{DFT calculations for bichromophores $B \| B(116)$ and $B \| R$ (118)}

Figure 49 shows the ground state geometry of bichromophore $\mathbf{B} \| \mathbf{B}$ after energy optimization using density functional methods. For both chromophores the same conformation was chosen as for model compound $\mathbf{B}$ (most stable one in the case of B). The absorption and emission dipole moments were calculated using time-dependent density functional methods (see chapter 19 for details). The absorption dipole moment of one chromophore (the chromophore pointing towards the $-x$ direction) was calculated directly for the ground state geometry. The emission transition dipole of the other chromophore (the one pointing towards the $+x$ direction) was determined again by transferring it's orientation from model compound $\mathbf{B}$ to bichromophore $\mathbf{B} \| \mathbf{B}$. In fact, the transition dipole moments are parallel.
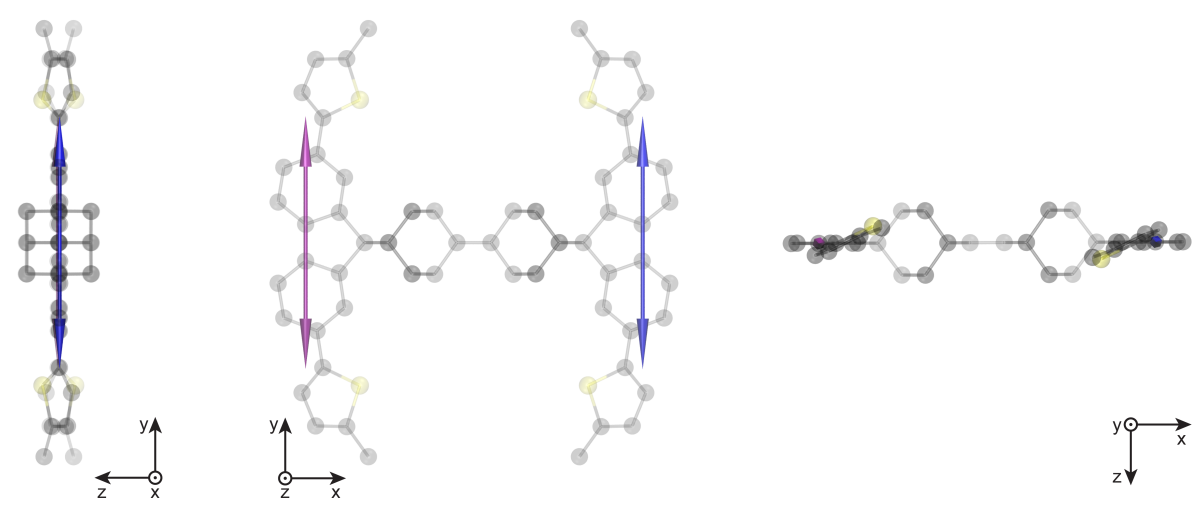

Figure 49: DFT optimized ground state geometry of bichromophore $\mathbf{B} \| \mathbf{B}$ (116). The absorption $\left(\mathrm{S}_{0} \rightarrow \mathrm{S}_{1}\right)$ and emission $\left(\mathrm{S}_{1} \rightarrow \mathrm{S}_{0}\right)$ transition dipole moments are shown as purple and blue double-headed arrows, respectively. $\mathrm{H}$ atoms are omitted for clarity.

The ground state optimized geometry of bichromophore $\mathbf{B} \| \mathbf{R}$ is shown in figure 50. Also here, the same conformation was chosen for the "blue" chromophore as for model compound B (most stable in the case of B). The absorption and emission dipole moments were determined using time-dependent density functional methods (see chapter 19 for details). The absorption dipole moment of the 
"red" chromophore was determined directly for the ground state geometry. The emission transition dipole of the "blue" chromophore was determined again by transferring it's orientation from model compound $\mathbf{B}$ to bichromophore $\mathbf{B} \| \mathbf{R}$.
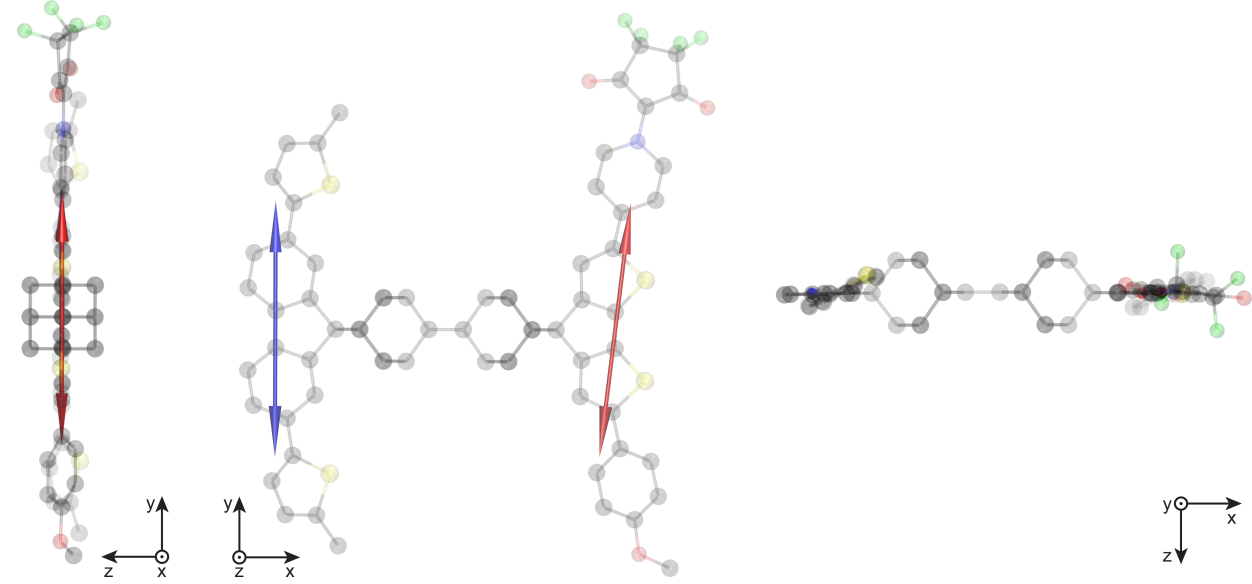

Figure 50: DFT optimized ground state geometry of bichromophore $\mathbf{B} \| \mathbf{R}(\mathbf{1 1 8})$. The absorption $\left(\mathrm{S}_{0} \rightarrow \mathrm{S}_{1}\right)$ and emission $\left(\mathrm{S}_{1} \rightarrow \mathrm{S}_{0}\right)$ transition dipole moments are shown as red and blue double-headed arrows, respectively. $\mathrm{H}$ atoms are omitted for clarity.

\subsection{Estimation of energy transfer efficiency based on ideal Förster point-dipole approximation for bichromophores $B \| B(116)$ and $B \| R$ (118)}

The geometrical arrangement of the transition dipole moments was used to determine the orientation factors $\kappa^{2}$ for bichromophores $\mathbf{B} \| \mathbf{B}$ and $\mathbf{B} \| \mathbf{R}$. Using the photophysical properties of model compounds $\mathbf{B}$ and $\mathbf{R}$, the energy transfer efficiency based on the ideal Förster point-dipole approximation was evaluated.

The angles $\theta_{\mathrm{D}}, \theta_{\mathrm{A}}$ and $\theta_{\mathrm{DA}}$ and distance $\left|\vec{R}_{\mathrm{DA}}\right|$ between the emission and absorption transition dipoles are given in table 12 (see section 1.2.1 for angle definitions). Using equation 4 , the orientation factor $\kappa^{2}$ was calculated (table 12). Taking into account the spectral overlap $J_{\mathrm{DA}}$ and the refractive index of $\mathrm{CHCl}_{3}$ $n_{\mathrm{D}}\left(20^{\circ} \mathrm{C}\right)=1.4459,{ }^{[134]}$ the Förster radii $R_{0}$ and the FRET efficiencies $E_{\mathrm{FRET}}$ were calculated (equations 5 and 6). The results are given in table 12. 
Chapter $13 \mid$ BICHROMOPHORES WITH PARALLEL ARRANGED FLUOROPHORES

Table 12: Angles $\theta_{\mathrm{D}}, \theta_{\mathrm{A}}$ and $\theta_{\mathrm{DA}}$ between transition dipole moments and interconnecting vector $\vec{R}_{\mathrm{DA}}$, orientation factor $\kappa^{2}$, distance between the donor and acceptor $\left|\vec{R}_{\mathrm{DA}}\right|$, Förster radius $R_{0}$, and estimated FRET efficiency $E_{\mathrm{FRET}}$.

\begin{tabular}{llllllll}
\hline Compound & $\theta_{\mathrm{D}}$ & $\theta_{\mathrm{A}}$ & $\theta_{\mathrm{DA}}$ & $\kappa^{2}$ & $\left|\vec{R}_{\mathrm{DA}}\right| / \mathrm{nm}$ & $R_{0} / \mathrm{nm}$ & $E_{\mathrm{FRET}}$ \\
\hline $\mathbf{B} \| \mathbf{B}(\mathbf{1 1 6})$ & $90.0^{\circ}$ & $90.0^{\circ}$ & $0.0^{\circ}$ & 1.00 & 1.33 & 2.93 & 0.99 \\
$\mathbf{B} \| \mathbf{R}(\mathbf{1 1 8})$ & $90.0^{\circ}$ & $83.2^{\circ}$ & $6.9^{\circ}$ & 0.99 & 1.33 & 3.61 & 1.00 \\
\hline
\end{tabular}

These results validate the usefulness of DFT calculations. Indeed, in the case of parallel "blue" and "red" chromophores the expected value of $\kappa^{2}$ is 1 , and the calculated ones are 1.00 and 0.99 for $\mathbf{B} \| \mathbf{R}$ and $\mathbf{B} \| \mathbf{B}$, respectively. As we have seen, in the case of "perpendicular" bichromophores, the calculations predict quite reasonable $\kappa^{2}$ values; In particular, for $\mathbf{B} \perp \mathbf{B}$, the predicted $\kappa^{2}$-value is appreciably higher than that of bichromophore $\mathbf{B} \perp \mathbf{R}$. 


\section{Time-resolved fluorescence anisotropy experiments to study intramolecular EET}

The intramolecular energy transfer in the new bichromophores was studied by (time-resolved) fluorescence anisotropy. These experiments and the data processing were kindly carried out by Dr. Mariano L. Bossi using a custom-made setup and custom-made software (see section 17 for details).

\subsection{EET in bichromophore $B \perp B(107)$}

To prove the presence and the power of intramolecular EET in $\mathbf{B} \perp \mathbf{B}$ between the identical chromophores in the case of nearly perpendicular chromopohore arrangement, time-resolved fluorescence anisotropy experiments were performed. In order to control molecular rotation, the measurements were performed in bis(2-ethylhexyl)phthalate (DEHP) at temperatures between $4{ }^{\circ} \mathrm{C}$ and $60^{\circ} \mathrm{C}$. The high viscosity of DEHP at $4{ }^{\circ} \mathrm{C}(\eta \approx 0.28 \mathrm{Pas})^{[161]}$ almost excludes molecular rotation. The temporal evolution of the fl. anisotropy factor $r(t)$ of compounds $\mathbf{B} \perp \mathbf{B}, \mathbf{B} \| \mathbf{B}$ and $\mathbf{B}$ is shown in figure 51 . 


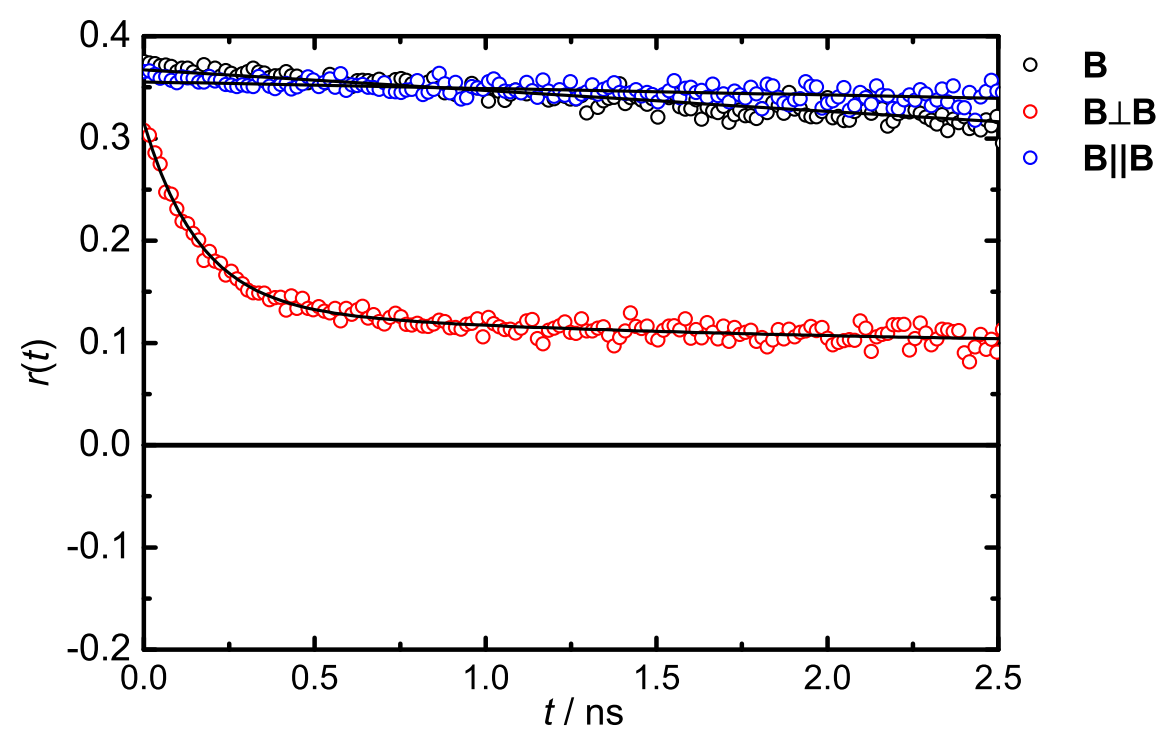

Figure 51: Time-resolved fluorescence anisotropy $r(t)$ of compounds $\mathbf{B} \perp \mathbf{B}(\mathbf{1 0 7}$, red circles), B $\|$ B (116, blue circles) and B (105, black circles) in DEHP at low temperature $\left(4^{\circ} \mathrm{C}\right)$. This figure was published in an article with the title "Bichromophoric Compounds with Orthogonally and Parallelly Arranged Chromophores Separated by Rigid Spacers".[160] Copyright Wiley-VCH Verlag $\mathrm{GmbH} \& \mathrm{Co}$. KGaA. Reproduced with permission.

All three compounds $(\mathbf{B}, \mathbf{B} \| \mathbf{B}$, and $\mathbf{B} \perp \mathbf{B})$ show the same limiting anisotropy $r_{0}$ at $t=0$ (within the error of the measurement), which is close to the theoretical value 0.4 (emission of the photoselected fluorophores and excitation beam have same polarization). As expected for $\mathbf{B}$ and $\mathbf{B} \| \mathbf{B}$, the anisotropy shows almost no decay. The very slow decay can be attributed to remaining rotations (as will be discussed later). In the case of $\mathbf{B} \| \mathbf{B}$ intramolecular EET may occur, but does not affect the fluorescence anisotropy, because both fluorophores have the same orientation $\left(\theta=0^{\circ}\right.$ in equation 21 , and therefore $r_{1}=r_{0}$ in equation 22). In contrast, $\mathbf{B} \perp \mathbf{B}$ shows a fast anisotropy decay with a characteristic time of $163 \mathrm{ps}( \pm 50 \mathrm{ps})$, from an initial value of 0.32 , and convergence towards a nonzero value $\left(r_{\infty} \approx 0.1\right)$. This is a strong indication for EET. The observed behavior can be explained by a fast energy hopping (back and forth) between identical chromophores. In this case the anisotropy evolution $r(t)$ can be described by equation 22. [101,102]

For $\mathbf{B} \perp \mathbf{B}$ with $\theta=90^{\circ}$ and $r_{0}=0.36$ a value of $r_{1} \approx-0.18$ is expected. Directly after excitation, only emission of the initially excited chromophores $\left(r_{0}\right)$ is observed. Then, the energy transfer occurs in both directions, i.e. by energy "hopping" back and forth, resulting in a decrease of anisotropy. After the system 
is well equilibrated $\left(t \gg k_{\mathrm{t}}^{-1}\right)$, an equilibrium anisotropy value $r_{\infty}$ is reached. Equation 22 with $t \rightarrow \infty$ gives

$$
r_{\infty}=\frac{r_{0}+r_{1}}{2}
$$

The observed values for $r_{0} \approx 0.32$ and $r_{\infty} \approx 0.1$ are in fair agreement with the expected ones. Expected for the limiting anisotropy $r_{0}$ is a value close the observed anisotropies for $\mathbf{B}$ and $\mathbf{B} \| \mathbf{B}$, which are both $r_{0} \approx 0.36$. For the equilibrium anisotropy follows an expected value of $r_{\infty} \approx 0.09$ (equation 22 ).

The average anisotropy calculated from the transients is also consistent with steady state anisotropy experiments (figure 52).

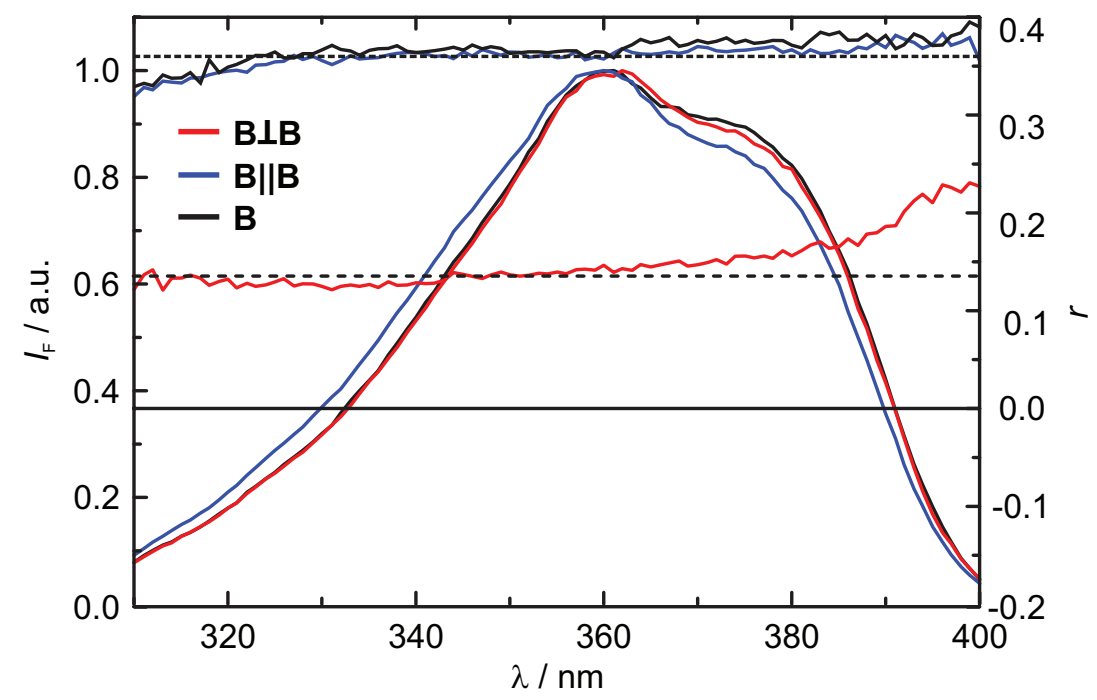

Figure 52: Excitation spectra ( $I_{\mathrm{TOT}}=I_{\|}+2 G I_{\perp}$; dashed lines) and measured steady state anisotropy (solid lines) of $\mathbf{B} \perp \mathbf{B}$ (107) (red curves), $\mathbf{B} \| \mathbf{B}$ (116) (blue curves) and $\mathbf{B}$ (105) (black curves) in DEHP at $4{ }^{\circ} \mathrm{C}$. The average anisotropy calculated from time-resolved anisotropy measurements is plotted as a dotted line for each chromophore. This figure was published in an article with the title "Bichromophoric Compounds with Orthogonally and Parallelly Arranged Chromophores Separated by Rigid Spacers". ${ }^{[160]}$ Copyright WileyVCH Verlag GmbH \& Co. KGaA. Reproduced with permission.

The slow decay in the anisotropy (i.e. $\tau \gg \tau_{\mathrm{fl}}$ ) of these three compounds (figure 51) is due to rotations, which are not completely prevented in DEHP at $4{ }^{\circ} \mathrm{C}$. This was confirmed by measuring the anisotropy decay in DEHP at temperatures between 4 and $60^{\circ} \mathrm{C}$ (see figure 58). 


\section{Chapter 14 TIME-RESOLVED FLUORESCENCE ANISOTROPY EXPERIMENTS ...}

\subsection{EET in bichromophores $B \perp R(114)$ and $B \| R(118)$}

Steady state anisotropy measurements for $\mathbf{B} \perp \mathbf{R}, \mathbf{B} \| \mathbf{R}$ and $\mathbf{R}$ (figures 53,54 and 55) present approximately flat values throughout each spectral region (visible and UV). In the visible region a single absorption band of the red chromophore is observed which corresponds to the first electronic transition $\left(S_{0} \rightarrow S_{1}\right)$. As expected, an anisotropy of 0.35 close to the theoretical maximum of 0.4 was obtained for all three compounds in this band. This indicates that the presence of the blue chromophore does not seem to induce any observable changes in the geometries of the ground and first excited state of the red fluorophore.

In the UV region, (at least) two absorption bands overlap: The second electronic transition $\left(\mathrm{S}_{0} \rightarrow \mathrm{S}_{2}\right)$ of the red fluorophore and the first electronic transition $\left(\mathrm{S}_{0} \rightarrow \mathrm{S}_{1}\right)$ of the blue fluorophore. The overlapping bands in the UV region have very similar shapes and maxima $(350 \mathrm{~nm}$ and $360 \mathrm{~nm}$, respectively; see tables 6 and 8). Accordingly, nearly flat anisotropy in this region is not surprising. However, the observed values of 0.28 for $\mathbf{B} \| \mathbf{R}$, and -0.12 for $\mathbf{B} \perp \mathbf{R}$ are far from the expected ones (approx. 0.35 and -0.16 , respectively).

This can be explained by the contribution from direct excitation of the red chromophore. The red fluorophore can either be directly excited or it can be excited by excitation of the blue fluorophore, followed by EET to the red one. However, the absorption transition dipole moments for these two excitation pathways may have different orientations. As a consequence, the red fluorophores which are excited by EET and the directly excited ones have different orientations due to photoselection. Consequently, the fluorescence anisotropy of the two ensembles of excited red fluorophores are also different.

To extract the pure "through EET" anisotropy, the data were corrected for the contribution of directly excited red fluorophores. For that purpose the wavelength dependent $\mathrm{fl}$. anisotropy $r_{\lambda}$ of red model compound $\mathbf{R}$ was determined (figure 53). 


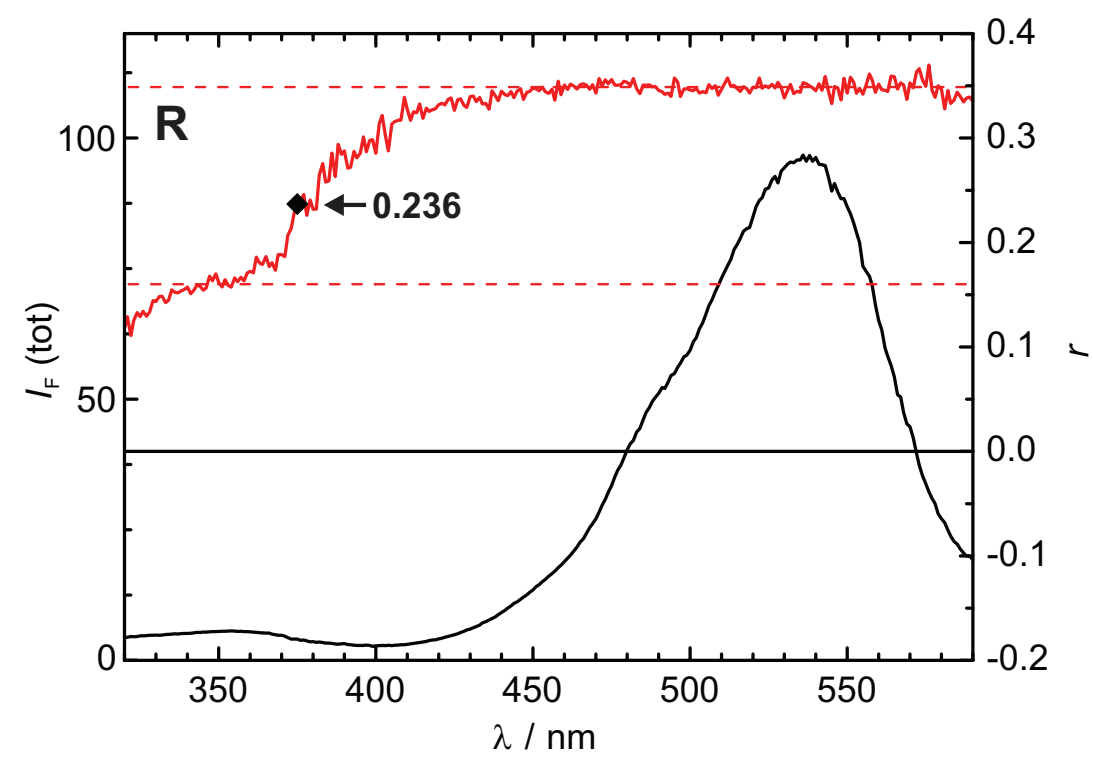

Figure 53: Excitation spectrum $\left(I_{\mathrm{TOT}}=I_{\|}+2 G I_{\perp}\right.$; black lines $)$ and measured steady state anisotropy (red line) of compound $\mathbf{R}(\mathbf{1 1 2})$ in DEHP at $4{ }^{\circ} \mathrm{C}$. The anisotropy value measured with $375 \mathrm{~nm}$ excitation (used for TCSPC experiments) is highlighted. The average anisotropy of the first UV excitation band and the red absorption band is shown with dashed lines. This figure was published in an article with the title "Bichromophoric Compounds with Orthogonally and Parallelly Arranged Chromophores Separated by Rigid Spacers". ${ }^{[160]}$ Copyright Wiley-VCH Verlag GmbH \& Co. KGaA. Reproduced with permission.

When measuring the steady state fluorescence anisotropy $r_{\lambda}^{\text {obs }}$ of bichromophores $\mathbf{B} \perp \mathbf{R}$ or $\mathbf{B} \| \mathbf{R}$, the sum of anisotropies arising from two processes is measured. On one hand, the anisotropy $r^{\mathrm{EET}}$ arising from excitation of the blue fluorophore followed by EET to the red fluorophore and, on the other hand, ${ }_{r}^{R}$ which is the anisotropy of the direct excitation of the red fluorophore to it's $\mathrm{S}_{2}$ state followed by internal conversion to $\mathrm{S}_{1}$. The observed anisotropy $r_{\lambda}^{\text {obs }}$ correspond to the weighted sum of both anisotropies ${ }^{[6]}$

$$
r_{\lambda}^{\mathrm{obs}}=x_{\lambda} r_{\lambda}^{\mathrm{R}}+\left(1-x_{\lambda}\right) r_{\lambda}^{\mathrm{EET}}
$$

where $x_{\lambda}$ defines the fraction of absorbed photons which leads to direct excitation of the red fluorophore, while $1-x_{\lambda}$ corresponds to the fraction of photons which are absorbed by the blue fluorophore and will cause excitation of the red fluorophore via EET. As measure for direct excitation $x_{\lambda}$ was calculated from 
Chapter 14 TIME-RESOLVED FLUORESCENCE ANISOTROPY EXPERIMENTS ...

the excitation spectrum of the bichromophore $\left(I_{\lambda}^{\mathrm{B} \perp \mathrm{R}}\right.$ or $\left.I_{\lambda}^{\mathrm{B} \| \mathrm{R}}\right)$ and of model compound $\left(I_{\lambda}^{\mathrm{R}}\right)$ :

$$
x_{\lambda}=\frac{I_{\lambda}^{\mathrm{R}}}{I_{\lambda}^{\mathrm{B} \perp \mathrm{R}}} \text { or } \frac{I_{\lambda}^{\mathrm{R}}}{I_{\lambda}^{\mathrm{B} \| \mathrm{R}}}
$$

The excitation spectrum of $\mathbf{R}$ is shown in figure 53 , whereas the excitation spectra of $\mathbf{B} \perp \mathbf{R}$ and $\mathbf{B} \| \mathbf{R}$ as well as the uncorrected anisotropies $r_{\lambda}^{\text {obs }}$ for the two latter compounds are shown in figure 54. 

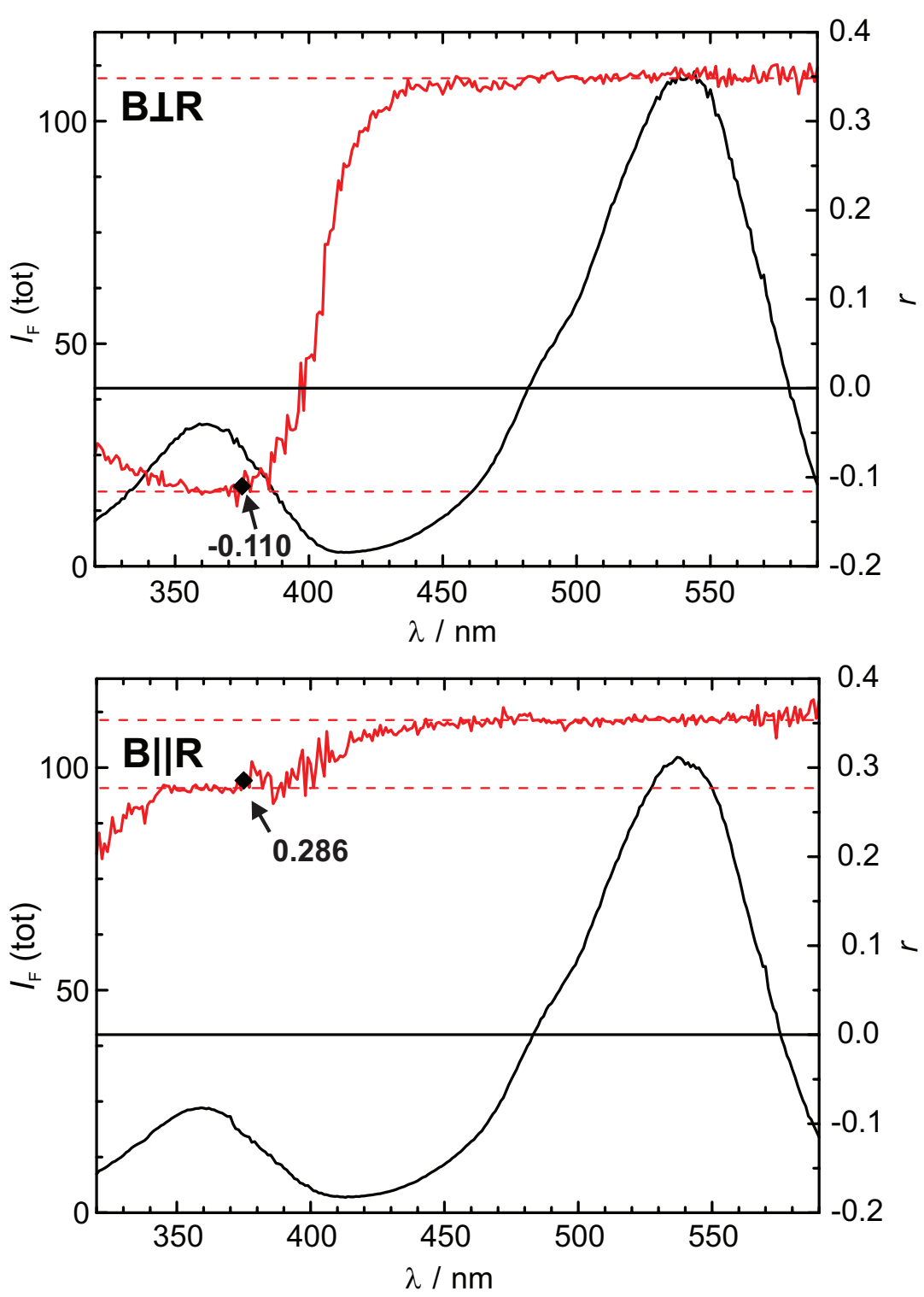

Figure 54: Excitation spectrum $\left(I_{\mathrm{TOT}}=I_{\|}+2 G I_{\perp}\right.$; black lines $)$ and measured steady state anisotropy (red lines) of compounds $\mathbf{B} \perp \mathbf{R}(\mathbf{1 1 4})$ and $\mathbf{B} \| \mathbf{R}(\mathbf{1 1 8})$ in DEHP at $4{ }^{\circ} \mathrm{C}$. The anisotropy value measured with $375 \mathrm{~nm}$ excitation (used for TCSPC experiments) is highlighted. The average anisotropy of the first UV excitation band and the red absorption band is shown with dashed lines for each chromophore. This figure was published in an article with the title "Bichromophoric Compounds with Orthogonally and Parallelly Arranged Chromophores Separated by Rigid Spacers".[160] Copyright Wiley-VCH Verlag $\mathrm{GmbH} \&$ Co. KGaA. Reproduced with permission.

Using equation 31, the pure "through EET" anisotropy $r_{\lambda}^{\mathrm{EET}}$ was determined ac- 


\section{Chapter 14 TIME-RESOLVED FLUORESCENCE ANISOTROPY EXPERIMENTS ...}

cording to

$$
r_{\lambda}^{\mathrm{EET}}=\frac{r_{\lambda}^{\mathrm{obs}}-x_{\lambda} r_{\lambda}^{\mathrm{R}}}{1-x_{\lambda}} .
$$

The effect of direct red-chromophore excitation was subtracted, and the pure "through EET" anisotropy $r(\lambda)$ was calculated for $\mathbf{B} \perp \mathbf{R}$ and $\mathbf{B} \| \mathbf{R}$ (figure 55). The anisotropies observed at the absorption maxima of the blue chromophore $(-0.18$ and 0.31 at $360 \mathrm{~nm}$, respectively) are close to the expected ones, and confirm the perpendicular and parallel orientation between the chromophores of $\mathbf{B} \perp \mathbf{R}$ and $\mathbf{B} \| \mathbf{R}$, respectively.

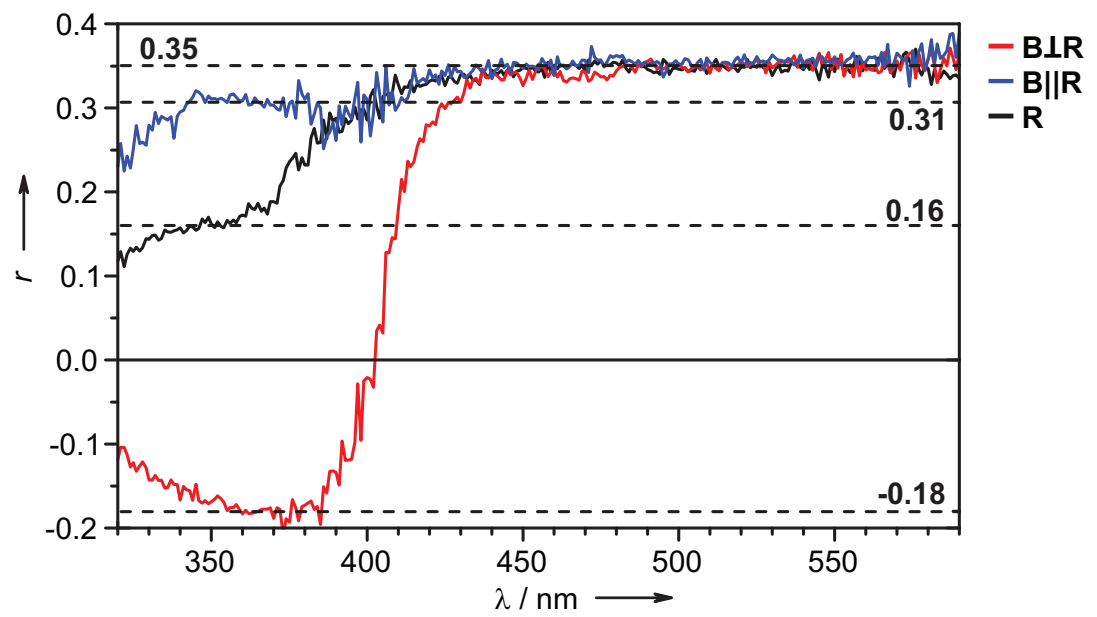

Figure 55: Corrected anisotropy $r$ (anisotropy of the directly excited red chromophore $\mathbf{R}$ (112) was subtracted) of compounds $\mathbf{B} \perp \mathbf{R}$ (114, red curve), $\mathbf{B} \| \mathbf{R}(\mathbf{1 1 8}$, blue curve) and anisotropy of $\mathbf{R}\left(\mathbf{1 1 2}\right.$, black curve) in DEHP at $4{ }^{\circ} \mathrm{C}$. The average anisotropy at the wavelengths of the first UV excitation band of each chromophore is marked with dashed lines at $-0.18\left(\mathbf{B} \perp \mathbf{R}, \mathrm{S}_{0} \rightarrow \mathrm{S}_{1}\right.$ blue fluorophore), 0.31 (B $\| \mathbf{R}, S_{0} \rightarrow S_{1}$ blue fluorophore) and $0.16\left(\mathbf{R}, S_{0} \rightarrow S_{2}\right.$ red fluorophore), as well as at 0.35 for excitation of the red fluorophore $\left(S_{0} \rightarrow S_{1}\right)$ for these three compounds. This figure was published in an article with the title "Bichromophoric Compounds with Orthogonally and Parallelly Arranged Chromophores Separated by Rigid Spacers".[160] Copyright Wiley-VCH Verlag $\mathrm{GmbH} \& \mathrm{Co}$. KGaA. Reproduced with permission.

The time-resolved measurements of red fluorophore containing compounds $\mathbf{R}$, $\mathbf{B} \perp \mathbf{B}, \mathbf{B} \| \mathbf{R}$ are fully consistent with the stationary measurements. Figure 56 shows the time-resolved anisotropy of the red fluorescence after excitation at $375 \mathrm{~nm}$ (left plots) and $515 \mathrm{~nm}$ (rigth plots). 
As expected, all three compounds show a limiting anisotropy of $0.34 \pm 0.01$ when the red fluorophores are excited at $515 \mathrm{~nm}\left(S_{0} \rightarrow S_{1}\right.$ transition), which is a typical value for the nearly parallel absorption/emission transition dipoles (figure 56, left).

After excitation at $375 \mathrm{~nm}$, the three compounds display various anisotropies of the red fluorescence. When model compound $\mathbf{R}$ is excited at $375 \mathrm{~nm}$, the observed limiting anisotropy value of about 0.18 shows that the $S_{0} \rightarrow S_{2}$ absorption and the emission dipole moments are not parallel but tilted by an angle of about $37^{\circ}$ according to equation 15 (figure 56, top right). 

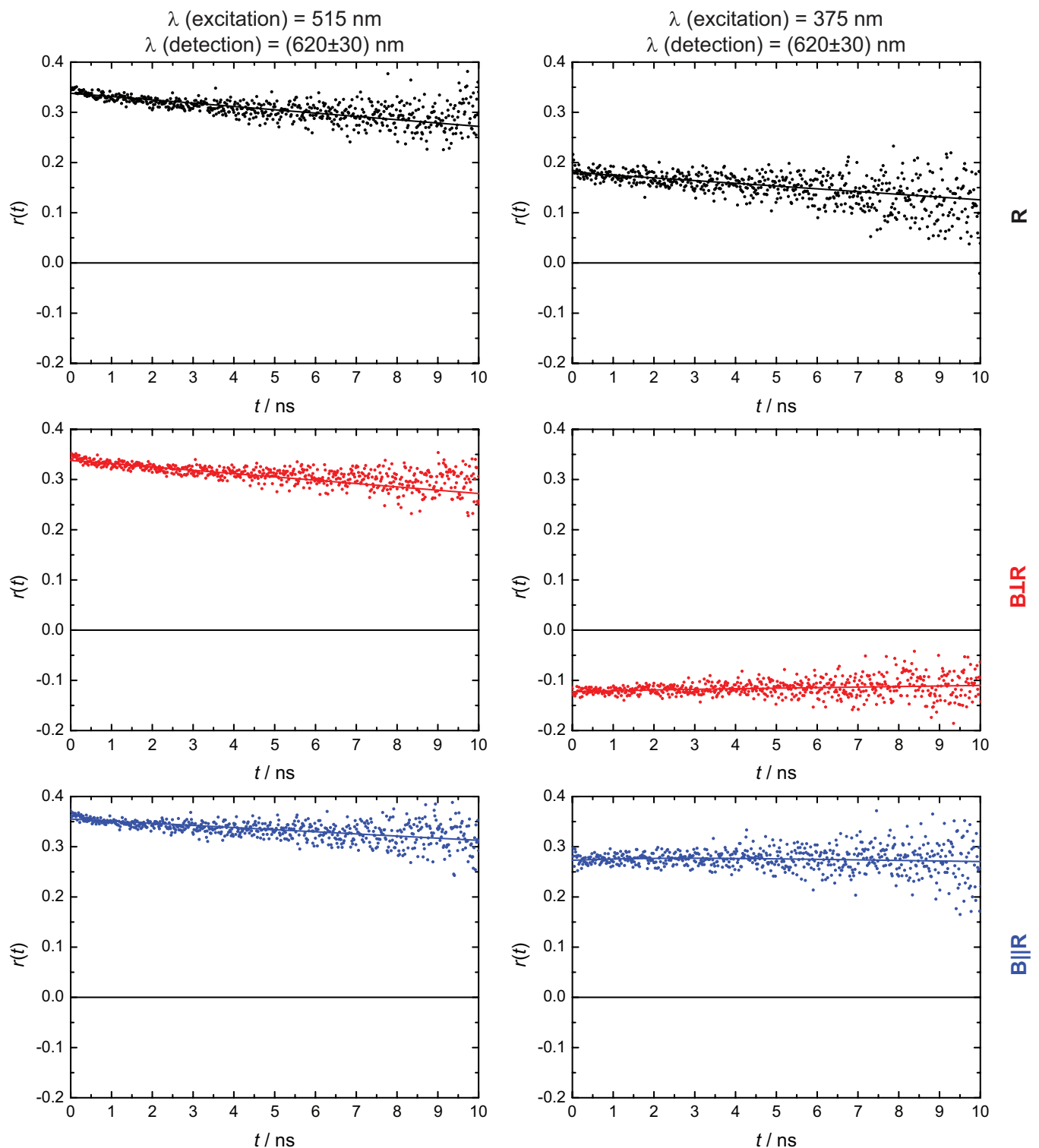

Figure 56: Time-resolved anisotropy decays for compounds $\mathbf{R}$ (112, top plots), $\mathbf{B} \perp \mathbf{R}\left(\mathbf{1 1 4}\right.$, middle plots) and $\mathbf{B} \| \mathbf{R}\left(\mathbf{1 1 8}\right.$, bottom plots) in DEHP at $4{ }^{\circ} \mathrm{C}$ with excitation at $515 \mathrm{~nm}$ (left plots), $375 \mathrm{~nm}$ (right plots) and with detection at $620 \mathrm{~nm}( \pm 30 \mathrm{~nm})$ in all cases (near the emission maximum of the red fluorophore). Dots represent experimental values, and the lines represent the best fits to the corresponding decays with deconvolution of the instrument response function (IRF). This figure was published in an article with the title "Bichromophoric Compounds with Orthogonally and Parallelly Arranged Chromophores Separated by Rigid Spacers".[160] Copyright Wiley-VCH Verlag $\mathrm{GmbH} \& \mathrm{Co}$. KGaA. Reproduced with permission.

When compound $\mathbf{B} \perp \mathbf{R}$ is excited in the blue region, the anisotropy at $t \approx 0$ has 
a value of -0.13 (figure 56 , middle right), which indicated nearly perpendicular alignment of the emitting red fluorophores relative to the initially excited blue fluorophores (the theoretical value would be -0.2 for perpendicular geometry). As in the steady state anisotropy measurements, the observed value is slightly increased due to a contribution of directly excited red fluorophores $\left(\mathrm{S}_{0} \rightarrow \mathrm{S}_{2}\right.$ transition). Unlike in the case of $\mathbf{B} \perp \mathbf{B}$, compound $\mathbf{B} \perp \mathbf{R}$ did not show a fast decay after excitation of the blue fluorophore.

Excitation of $\mathbf{B} \| \mathbf{R}$ at $375 \mathrm{~nm}$ results in a limiting anisotropy of 0.28 (figure 56, bottom right). This value shows the close to parallel orientation of the transition dipole moments of blue absorption and red emission. The derivation from the theoretical value of 0.4 is again attributed to the contribution of directly excited red fluorophores $\left(\mathrm{S}_{0} \rightarrow \mathrm{S}_{2}\right.$ transition).

The unidirectional EET in bichromophores $\mathbf{B} \perp \mathbf{R}$ and $\mathbf{B} \| \mathbf{R}$ originating from the excitation of the blue fluorophore and resulting in the emission of the red one is fast enough to observe an anisotropy value of -0.13 already at $t \approx 0$. This process has a quantum efficiency $\Phi_{\mathrm{EET}} \approx 1$, as confirmed by the absence of emission from the donor at ca. $400 \mathrm{~nm}$ in compounds $\mathbf{B} \perp \mathbf{R}$ and $\mathbf{B} \| \mathbf{R}$.

The slow anisotropy decay at a timescale much longer than the fluorescence lifetime of the red chromophore ( $2.3 \mathrm{~ns})$ is consistent with depolarization caused by residual rotational motion at $4{ }^{\circ} \mathrm{C}$. The slopes of such quasi-linear decays follow the expected order $\mathbf{R}>\mathbf{B} \perp \mathbf{R}>\mathbf{B} \| \mathbf{R}$, i.e. inverse to the size of the chromophores $(\mathbf{B} \| \mathbf{R}>\mathbf{B} \perp \mathbf{R}>\mathbf{R})$, and agree with the observed slopes for $\mathbf{B} \| \mathbf{B}$, $\mathbf{B} \perp \mathbf{B}$ and $\mathbf{B}$. The temperature dependence of the depolarization due to rotational motion was used to calculate the effective hydrodynamic volumes, as will be described below. 


\subsection{Effective hydrodynamic volumes}

The longest characteristic time of rotational anisotropy decays (rotational correlation time) $\tau_{\text {rot }}$ for compounds $\mathbf{B}(\mathbf{1 0 5}), \mathbf{B} \perp \mathbf{B}(\mathbf{1 0 7})$ and $\mathbf{B} \| \mathbf{B}(\mathbf{1 1 6})$ was determined at different temperatures (figure 57). For $\mathbf{B}$ and $\mathbf{B} \| \mathbf{B}$ the anisotropy decays were fitted by an monoexponential fit (depolarization only due to rotation), whereas for $\mathbf{B} \perp \mathbf{B}$ a biexponential fit was used.
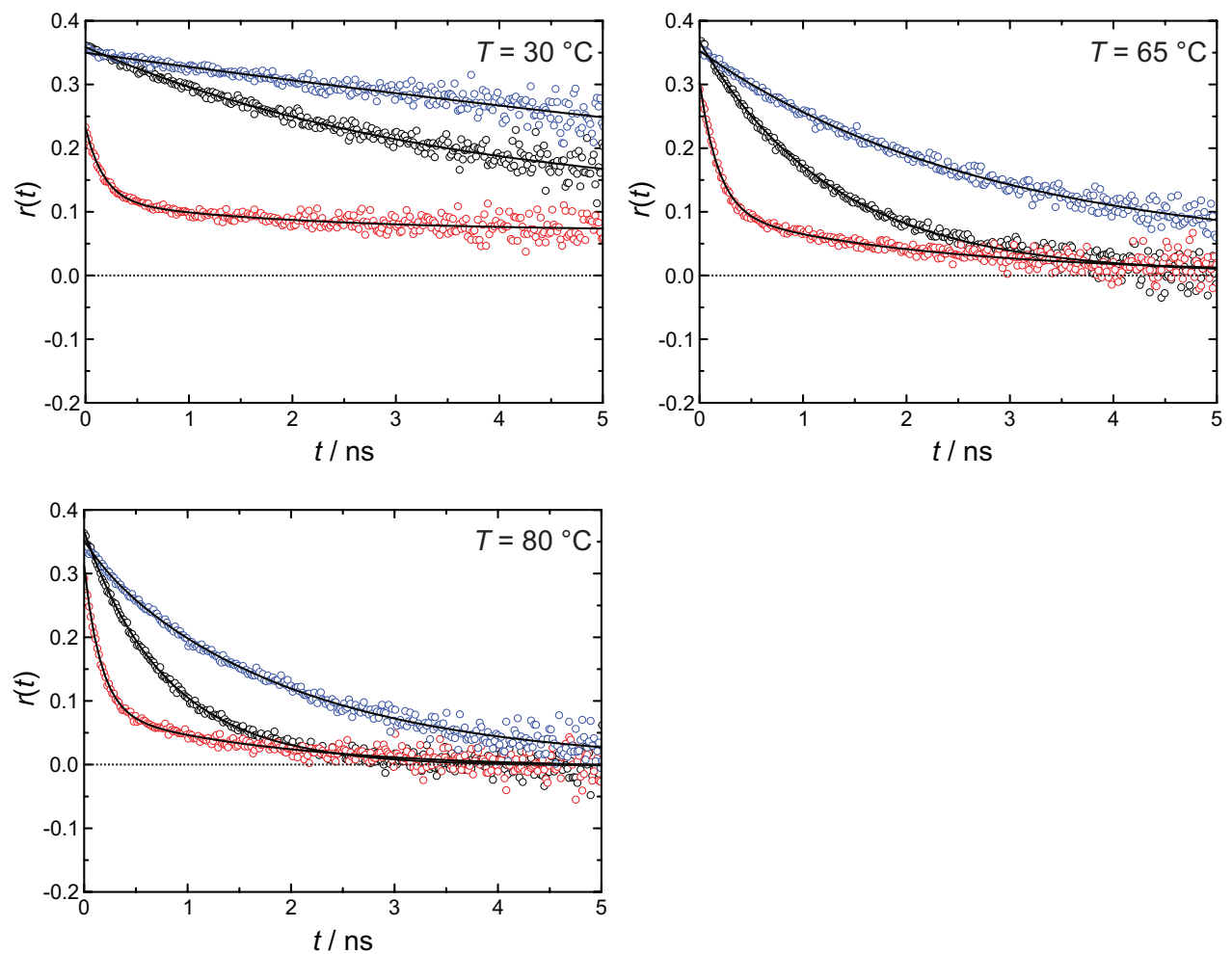

Figure 57: Anisotropy decays of $\mathbf{B} \perp \mathbf{B}$ (107, red symbols), $\mathbf{B} \| \mathbf{B}$ (116, blue symbols) and B (105, black symbols) in DEHP at different temperatures. Black lines represent the best monoexponential $(\mathbf{B}, \mathbf{B} \| \mathbf{B})$ and biexponential $(\mathbf{B} \perp \mathbf{B})$ fits. This figure was published in an article with the title "Bichromophoric Compounds with Orthogonally and Parallelly Arranged Chromophores Separated by Rigid Spacers". ${ }^{[160]}$ Copyright Wiley-VCH Verlag GmbH \& Co. KGaA. Reproduced with permission.

In order to calculate the effective hydrodynamic volume $V_{\mathrm{h}}$ for each compound, the rotational correlation time $\tau_{\text {rot }}$ was plotted against $\eta / k_{\mathrm{B}} T$. Where $\eta$ is the viscosity of the solvent (DEHP) ${ }^{[161]}$ at the corresponding temperature $T$ and $k_{\mathrm{B}}$ is the Boltzmann constant (figure 58). 


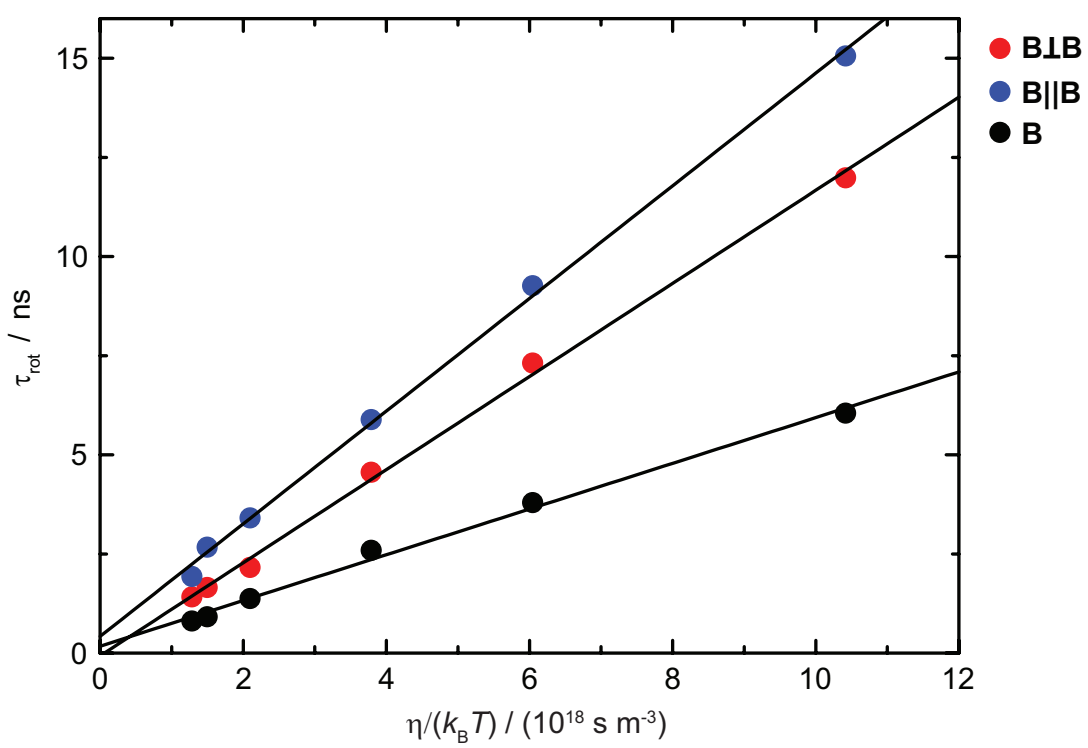

Figure 58: Rotational (longest) characteristic time of anisotropy decays $\tau_{\text {rot }}$ of $\mathbf{B}$ (105), $\mathbf{B} \perp \mathbf{B}(\mathbf{1 0 7})$ and $\mathbf{B} \| \mathbf{B}(\mathbf{1 1 6})$ in DEHP as a function of solvent viscosity. The lines represent the best linear fits. This figure was published in an article with the title "Bichromophoric Compounds with Orthogonally and Parallelly Arranged Chromophores Separated by Rigid Spacers".[160] Copyright WileyVCH Verlag GmbH \& Co. KGaA. Reproduced with permission.

According to the relation ${ }^{[162]}$

$$
\tau_{\mathrm{rot}}=\frac{\eta V_{\mathrm{h}}}{k_{\mathrm{B}} T}
$$

the slope equals $V_{\mathrm{h}}$. The resulting effective hydrodynamic volumes $V_{\mathrm{h}}$ and radii of spheres $r_{\text {sphere }}$ with these volumes are given in table 13 .

Table 13: Calculated hydrodynamic volumes $V_{\mathrm{h}}$ and corresponding sphere radii $r_{\text {sphere. }}$.

\begin{tabular}{lll}
\hline compound & $V_{\mathrm{h}} / \mathrm{nm}^{3}$ & $r_{\text {sphere }} / \mathrm{nm}$ \\
\hline $\mathbf{B}(\mathbf{1 0 5})$ & 6.86 & 1.18 \\
$\mathbf{B} \perp \mathbf{B}(\mathbf{1 0 7})$ & 12.0 & 1.42 \\
$\mathbf{B} \| \mathbf{B}(\mathbf{1 1 6})$ & 13.6 & 1.48 \\
\hline
\end{tabular}

Figure 59 shows ground state energy minimized DFT structures of compounds $\mathbf{B}, \mathbf{B} \perp \mathbf{B}$ and $\mathbf{B} \| \mathbf{B}$ drawn inside spheres which correspond to the hydrodynamic volume (table 13). Thus, theoretical calculations and experimental rotational 


\section{Chapter 14 TIME-RESOLVED FLUORESCENCE ANISOTROPY EXPERIMENTS ...}

correlation times extracted from time-resolved anisotropy measurements are in good agreement.
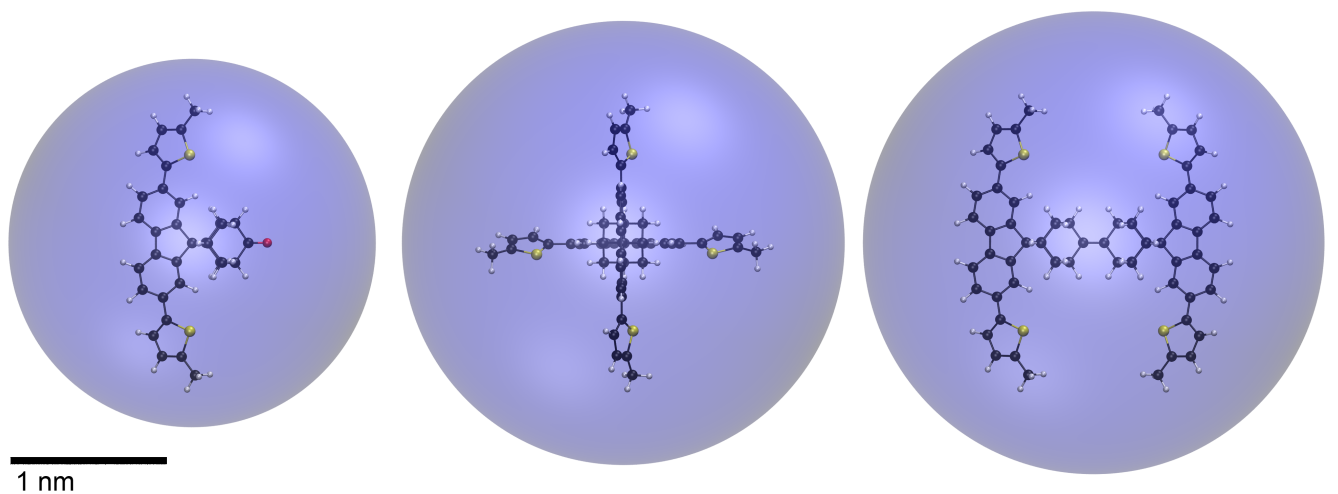

Figure 59: Orthographic projections of DFT structures of $\mathbf{B}(\mathbf{1 0 5}), \mathbf{B} \perp \mathbf{B}(\mathbf{1 0 7})$ and B $\| \mathbf{B}$ (116) drawn inside spheres corresponding to the effective hydrodynamic volumes. This figure was published in an article with the title "Bichromophoric Compounds with Orthogonally and Parallelly Arranged Chromophores Separated by Rigid Spacers".[160] Copyright Wiley-VCH Verlag $\mathrm{GmbH} \&$ Co. KGaA. Reproduced with permission. 


\section{Evidence for intramolecular EET by antibunching experiments}

We also addressed intramolecular EET of bichromophores $\mathbf{B} \| \mathbf{B}$ (116) and $\mathbf{B} \perp \mathbf{B}(\mathbf{1 0 7})$ by fluorescence antibunching experiments in solution, allowing to determine the number of emitters per bichromophore (see chapter 3.3). ${ }^{[111,115,163]}$ These experiments and the data processing were kindly carried out by Dr. Haisen Ta using a custom-made setup and custom-made software (see section 18 for details).

With very short excitation pulses ( $\sim 70 \mathrm{ps} \ll \tau_{\mathrm{f}}=1.0 \mathrm{~ns}$ ), every chromophore can be excited only once and emit not more than one photon (antibunching). Multiple photons detected within a short period of time $(<25 \mathrm{~ns})$, can result from either multiple fluorescent molecules diffusing through the detection volume or multiple emitters in the same molecule. The former contribution can be quantified by cross correlation of the two independent detection channels at large lag time $t \approx 1 \mathrm{~s}$ (figure 60 ). Interestingly, the cross correlation has the same amplitude as at $t \approx 0$ (i.e. the occurrence of multiple photon events is the same), indicating that none of the bichromophore compounds emits two photons at the same time. By fitting the measured autocorrelation function, we found that the numbers of emitters for compounds $\mathbf{B} \perp \mathbf{B}$ and $\mathbf{B} \| \mathbf{B}$ and $\mathbf{B}$ are all very similar $\left(1.18 \pm 0.12,1.00 \pm 0.05\right.$ and $1.22 \pm 0.08$, respectively). ${ }^{[117]}$ This means that these compounds behave as single quantum systems. Highly efficient EET with nearly quantitative quantum yield enabling singlet-singlet annihilation ${ }^{[117,164]}$ is suggested as a most probable explanation (see section 3.3.5). 


\section{Chapter 15 EVIDENCE FOR INTRAMOLECULAR EET BY ANTIBUNCHING ...}
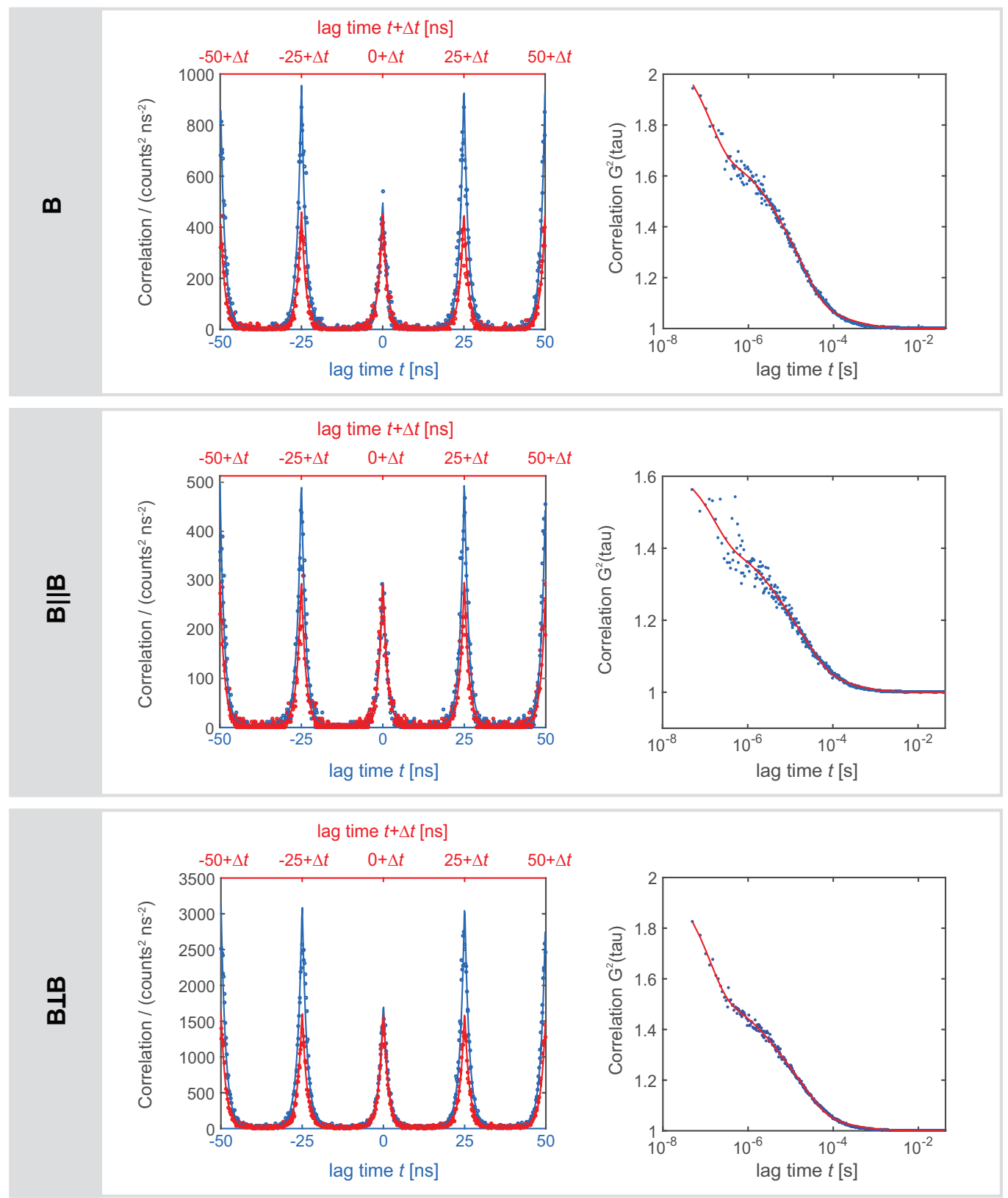

Figure 60: Measured cross correlation functions of antibunching experiments. Left: Magnification at zero lag time $t \approx 0$ (blue curve) and at large lag time $t+\Delta t$ where $\Delta t$ was chosen to be $1 \mathrm{~s}$ (red curve); Right: Cross correlation function normalized by its value at $t=\infty$; Dots correspond to the measured values of the cross correlation and solid lines correspond to fitting of the cross-correlation function to the experimental data (model functions 25 and 26). This figure was published in an article with the title "Bichromophoric Compounds with Orthogonally and Parallelly Arranged Chromophores Separated by Rigid Spacers". ${ }^{[160]}$ Copyright Wiley-VCH Verlag GmbH \& Co. KGaA. Reproduced with permission. 
Part III

\section{Conclusion and Outlook}



In this thesis a new approach for the design of bichromophoric systems with well-defined geometry was developed. As shown in figure 61, application of adamantane and 2-(2-adamantylidene)adamantane as rigid bridges between two chromophores led to assemblies with perpendicular and parallel oriented chromophores, respectively. Linking the chromophores by spiro junctions to both sides of the bridge provides rigid bichromophoric systems with fixed relative orientation and precisely defined distance between the chromophores.

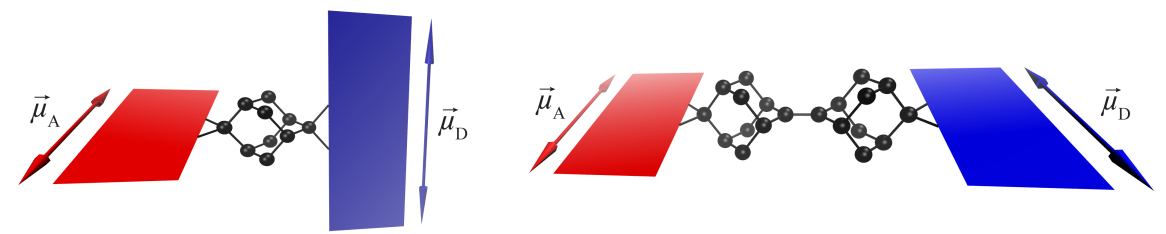

Figure 61: Adamantane and 2-(2-adamantylidene)adamantane as rigid linkers between two chromophores provide an access to assemblies with perpendicular and parallel arranged chromophores.

The fluorophores cannot be varied freely and need to meet several requirements: First, they need to be capable of being attached to the adamantane scaffold. As shown in scheme 29 , this was achieved by metal-halogen exchange of biaromatic halogenides 24 and 25 (chromophore precursors). The resulting alcohol was cyclized under acidic conditions to give spiro compounds 29 or 31.

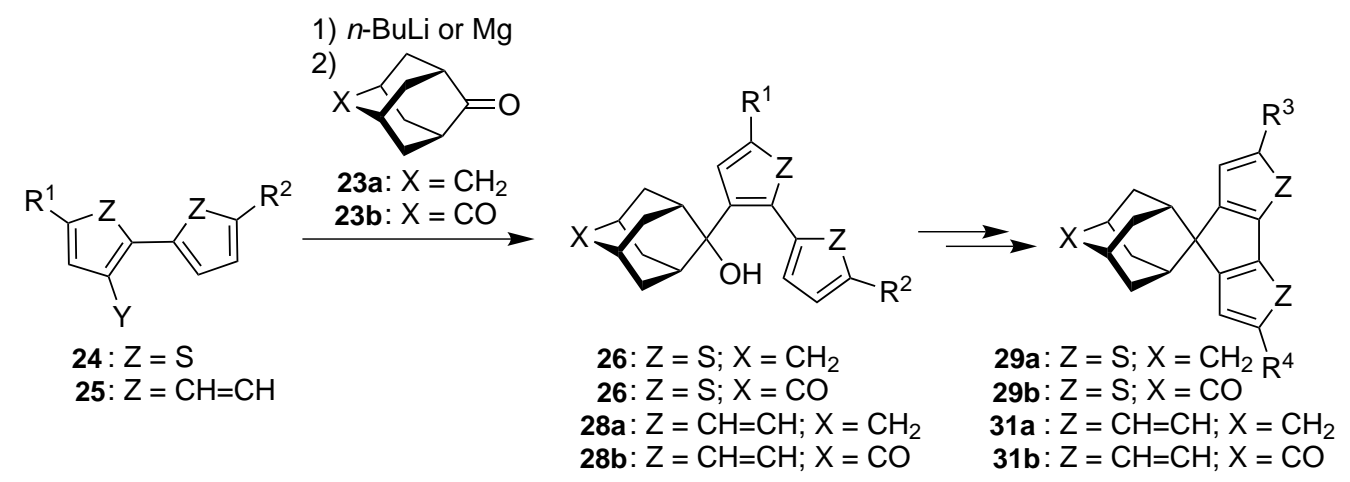

Scheme 29: Attaching dye precursors $\mathbf{2 4}$ or $\mathbf{2 5}$ to adamantane derivatives 23a and 23b; $\mathrm{Y}=\mathrm{Br}, \mathrm{I} ; \mathrm{R}^{1-4}=\mathrm{Ar}$, electron withdrawing/donating group.

For spectroscopic studies, a decent brightness (product of extinction coefficient and fluorescence quantum yield) is required. Furthermore, a pair of two fluorophores with spectrally separated absorption/emission bands is necessary for selective excitation of individual fluorophores and independent observation of 
their emission. A series of new fluorophores were developed in the course of improving their properties. Finally, a pair of "blue" and "red" fluorophores was obtained; these were used for the design of model compounds and bichromophores shown in figure 62.
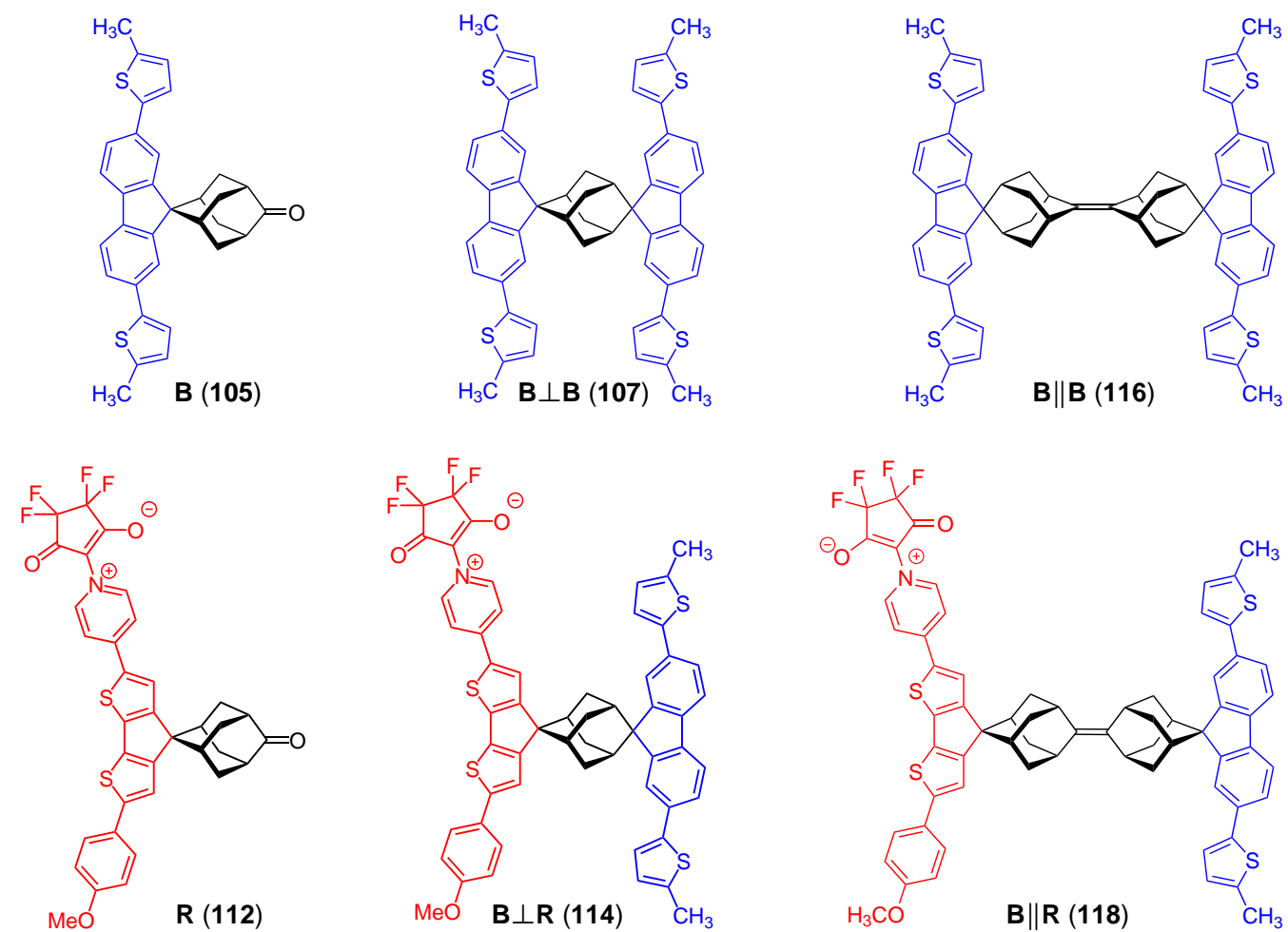

Figure 62: Bichromophoric compounds with rigid linkers arranged in orthogonal $(\mathbf{B} \perp \mathbf{B}, \mathbf{B} \perp \mathbf{R})$ and parallel geometry $(\mathbf{B}\|\mathbf{B}, \mathbf{B}\| \mathbf{R})$, as well as model compounds $\mathbf{B}$ and $\mathbf{R}$.

To characterize the photophysical properties of the fluorophores, model compounds $\mathbf{B}$ and $\mathbf{R}$ were synthesized (figure 62). Then, bichromophores with two identical (blue/blue) and different fluorophores (blue/red) were synthesized. Bichromophores $\mathbf{B} \perp \mathbf{B}$ and $\mathbf{B} \perp \mathbf{R}$ with perpendicular arranged chromophores as well as $\mathbf{B} \| \mathbf{B}$ and $\mathbf{B} \| \mathbf{R}$ with the parallel arrangement were prepared.

The novel bichromophoric systems are perfectly suited for the study of intramolecular electronic energy transfer (EET) between chromophores at welldefined geometry. The high rigidity and well-defined geometry of the systems could be advantageous relative to most systems reported up to now, which are less rigid and prone to rotation and bending of single bonds distorting the molecular geometry. 
The common approach for explaining through-space EET is the Förster theory, where the EET is described as resonance energy transfer between two pointdipoles. According to Förster's theory, the resonance energy transfer between perpendicularly oriented chromophores should not occur. However, an important requirement for this approximation is a sufficiently large distance between the fluorophores. This distance has to be much larger than the dimensions of the fluorophores. Therefore it becomes clear, that Förster's theory may not correctly describe the EET in the bichromophores presented here. The distances between the chromophores are about 0.8 and $1.3 \mathrm{~nm}$ for the perpendicular and parallel geometry, respectively. This separation equals roughly the dimension of the chromophores. Therefore it is no surprise to observe deviations from the FRET theory.

All studied bichromophoric molecules showed efficient EET, independently of the relative chromophore orientation. Steady-state absorption and emission spectroscopy of $\mathbf{B} \perp \mathbf{R}$ and $\mathbf{B} \| \mathbf{R}$ revealed EET with near quantitative transfer efficiency due to the absence of the donor fluorescence. In bichromophore $\mathbf{B} \perp \mathbf{B}$ very fast EET was proven by time-resolved fluorescence anisotropy measurements. The results showed very fast anisotropy decay to a non-zero anisotropy value. This was explained by a fast back and forth energy transfer between two fluorophores. To address the energy transfer between the chromophores in $\mathbf{B} \| \mathbf{B}$, fluorescence antibunching experiments were carried out. The results confirmed, that the bichromophores behave as single emitters, i.e. both parts of the same bichromophore never emit photons at the same time. We assume, that singletsinglet annihilation (SSA) is accountable for this behavior. SSA provides evidence for fast intramolecular EET between two simultaneously excited chromophores. Having in mind, that Förtster's dipole-dipole approximation may not be valid at these short distances, it was examined whether FRET may explain the efficient EET observed for the bichromophores introduced here. The quantum yield, the fluorescence lifetime and the spectral overlap were taken from the model compounds $\mathbf{B}$ and $\mathbf{R}$. To determine the orientation factor $\kappa^{2}$, the relative orientation of the emission and absorption transition dipole moments and the distance between the chromophores were determined using density functional theory (DFT) and time-dependend density functional theory (TD-DFT). The results are summarized in table 14. 
Table 14: Calculated distance between chromophores $\left|\vec{R}_{\mathrm{DA}}\right|$, orientation factor $\kappa^{2}$, Förster radius $R_{0}$ and resulting FRET efficiency $E_{\text {FRET }}$ for bichromophores based on photophysical properties of model compounds $\mathbf{B}$ and $\mathbf{R}$ and DFT/TD-DFT calculations.

\begin{tabular}{lllll}
\hline Compound & $\kappa^{2}$ & $\left|\vec{R}_{\mathrm{DA}}\right| / \mathrm{nm}$ & $R_{0} / \mathrm{nm}$ & $E_{\mathrm{FRET}}$ \\
\hline $\mathbf{B} \perp \mathbf{B}(\mathbf{1 0 7})$ & $6.0 \cdot 10^{-4}$ & 0.84 & 0.85 & 0.51 \\
$\mathbf{B} \| \mathbf{B}(\mathbf{1 1 6})$ & 1.0 & 1.33 & 2.93 & 0.99 \\
$\mathbf{B} \perp \mathbf{R}(\mathbf{1 1 4})$ & $1.0 \cdot 10^{-4}$ & 0.84 & 0.77 & 0.38 \\
$\mathbf{B} \| \mathbf{R}(\mathbf{1 1 8})$ & 1.0 & 1.33 & 3.61 & 1.00 \\
\hline
\end{tabular}

Geometries and orientation of transition dipole moments were calculated using DFT and TD-DFT methods, see chapter 19 for details.

Since all DFT studies were performed in gas-phase (neglecting any solventeffects) the results should be interpreted with caution. Nonetheless, the results indicate that even at these short interchromophoric distances the FRET approximation gives a FRET efficiency for compounds $\mathbf{B} \perp \mathbf{B}$ and $\mathbf{B} \perp \mathbf{R}$ much smaller 1 . The calculation results do not completely explain the efficient EET in the bichromophores.

Additionally, the Förster point-dipole approximation may be not valid at these short distances due to multipole-multipole Coulombic interactions. At the same time, the classical Dexter mechanism seems to be unlikely due to the vanishingly small orbital overlap of the two $\pi$-systems, which are separated by a bulky saturated hydrocarbon spacer. However, the rigidity of the $\sigma$-framework may enhance a through-bond (superexchange) interactions. Finally, despite the rigidity of the adamantane linkers, deviations from the equilibrium geometry caused by vibrations in the excited state cannot be completely disregarded. To identify the EET mechanism further experiments with bichromophoric compounds possessing longer rigid linkers are required. 


\section{Part IV}

Experimental Part 



\section{General remarks}

\subsection{Solvents and reagents}

The used solvents had pro analysi grade. Anhydrous solvents were dried over $3 \AA$ or $4 \AA$ molecular sieves. Commercially available substances were used without further purification. 2,6-adamantanedione was purchased from Ambinter (a trademark of Greenpharma S.A.S), Orléans, France.

\subsection{Preparative methods}

If not stated otherwise, reactions were carried out with magnetic stirring under an argon atmosphere. For reactions at low temperatures, an acetone/dry ice bath was used. Reactions at $0{ }^{\circ} \mathrm{C}$ were carried out using an ice-water bath. Microwaveassisted organic synthesis were performed in a closed vessel microwave device (Discover series) manufactured by CEM Corporation.

\subsubsection{Thin layer chromatography (TLC)}

Analytical TLC was performed on ready-to-use plates. For TLC on silica gel, ALUGRAM SIL G/UV254 silica gel 60 (MACHEREY-NAGEL GmbH \& Co. KG) was used. For TLC on reversed phase, silica gel 60 RP-18 F254 s (MERCK KGaA) was used. UV-active substances were detected by a UV Iamp $(\lambda=254$ or $366 \mathrm{~nm})$. If necessary, the TLC plates were stained using reagents given in Table 15

\subsubsection{Column chromatography}

Silica gel 60 with different particle sizes was used: $0.015-0.040 \mathrm{~mm}, 0.040-$ $0.063 \mathrm{~mm}$, and $0.05-0.2 \mathrm{~mm}$ (MACHEREY-NAGEL GmbH \& Co. KG). 
Table 15: Stains used for developing TLC plates.

\begin{tabular}{|c|c|}
\hline Stain & Composition \\
\hline cerium molybdate (CAM) & $\begin{array}{l}5 \mathrm{~g}\left(\mathrm{NH}_{4}\right)_{6} \mathrm{Mo}_{7} \mathrm{O}_{24}, \\
2 \mathrm{~g} \mathrm{Ce}\left(\mathrm{NH}_{4}\right)_{2}\left(\mathrm{NO}_{3}\right)_{4}, \\
60 \mathrm{ml} \text { water, } \\
20 \mathrm{ml} \text { conc. } \mathrm{H}_{2} \mathrm{SO}_{4}\end{array}$ \\
\hline potassium permangante & $\begin{array}{l}0.5 \mathrm{~g} \mathrm{KMnO}_{4} \\
100 \mathrm{ml} 1.0 \mathrm{M} \mathrm{NaOH}\end{array}$ \\
\hline ninhydrin & $\begin{array}{l}0.30 \mathrm{~g} \text { ninhydrin, } \\
100 \mathrm{ml} \text { 1-butanol, } \\
3.0 \mathrm{ml} \mathrm{AcOH}\end{array}$ \\
\hline phosphomolybdic acid (PMA) & $\begin{array}{l}4 \mathrm{~g} 12 \mathrm{MoO}_{3} \cdot \mathrm{H}_{3} \mathrm{PO}_{4} \\
100 \mathrm{ml} \mathrm{EtOH}\end{array}$ \\
\hline
\end{tabular}

\subsection{Instrumental analytics}

\subsubsection{Steady-state absorption and emission spectroscopy}

UV/Vis absorption spectra were recorded on a Varian Cary 4000 UV/Vis spectrophotometer, and fluorescence spectra were recorded on a Varian Cary Eclipse fluorescence spectrophotometer. All measurements were carried out in sealed quartz cuvettes with $1.00 \mathrm{~cm}$ path length.

\subsubsection{Nuclear magnetic resonance (NMR)}

NMR spectra were recorded on an Agilent $400 M R$ spectrometer. All spectra are referenced to tetramethylsilane as an internal standard $(\delta=0 \mathrm{ppm})$ using the signals of the residual protons of $\mathrm{CHCl}_{3}$ (7.26 ppm) in $\mathrm{CDCl}_{3}, \mathrm{CHD}_{2} \mathrm{OD}$ (3.31 ppm) in $\mathrm{CD}_{3} \mathrm{OD}$ or DMSO- $\mathrm{d}_{5}(2.50 \mathrm{ppm})$ in $\mathrm{DMSO}-\mathrm{d}_{6}$. Multiplicities of signals are described as follows: $s=$ singlet, $s_{b r}=$ broad singlet, $d=$ doublet, $d_{b r}=$ broad doublet, $\mathrm{t}=$ triplet, $\mathrm{q}=$ quartet, $\mathrm{m}=$ multiplet. In the case of diastereotopic protons, $\mathrm{a}$ marks the high-field shifted proton and ${ }_{b}$ the low-field shifted signal. Coupling constants ${ }^{n} J_{x, y}$ are given in $\mathrm{Hz}$, where $n$ is the number of bonds between the two coupling nuclei $x$ and $y$. Proton and carbon resonances were assigned with the aid of COSY, HSQC, and $\mathrm{HMBC}{ }^{1} \mathrm{H}-{ }^{1} \mathrm{H}$ and ${ }^{1} \mathrm{H}-{ }^{13} \mathrm{C}$ correlations. 


\subsubsection{Mass spectrometry (MS)}

Low resolution mass spectra (ESI) were obtained using a Varian $500 \mathrm{MS}$. High resolution mass spectra were obtained on a Bruker APEX IV (ESI-HRMS) or Bruker Autoflex Speed (MALDI-TOF HRMS) spectrometer.

\subsubsection{High-performance liquid chromatography (HPLC)}

Analytical and preparative HPLC were performed on a Knauer HPLC system: Smartline pump $1000(2 \times)$, UV detector 2500 , column thermostat 4000 , mixing chamber, injection valve with $20 \mu$ l loop for the analytical column and $200 \mu$ loop for the preparative column; 6-port-3 channel switching valve; analytical column: Eurospher $100 \mathrm{C} 18,5 \mu \mathrm{m}, 250 \times 4 \mathrm{~mm}$; preparative column: Eurospher-100 C18, $5 \mu \mathrm{m}, 8 \times 250 \mathrm{~mm}, 200 \mu \mathrm{l}$ loop; flow rate: $1.2 \mathrm{ml} / \mathrm{min}$ (or $5 \mathrm{ml} / \mathrm{min}$ in the preparative mode); solvent $A:$ water $+0.1 \% \mathrm{v} / \mathrm{v}$ TFA, solvent $\mathrm{B}: \mathrm{MeCN}+0.1 \% \mathrm{v} / \mathrm{v}$ TFA; gradient $A / B: 70: 30$ to $0: 100$ in 25 min (unless stated otherwise). 



\section{Time-resolved fluorescence anisotropy measurements}

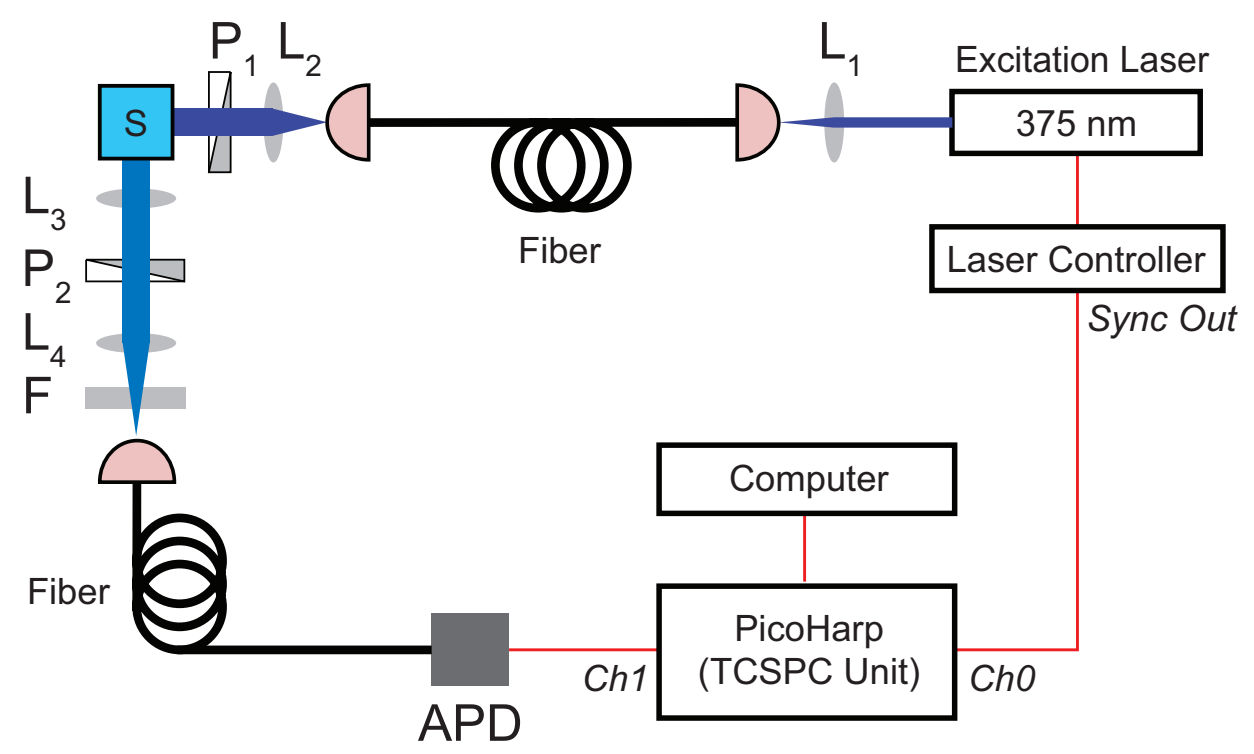

Scheme 30: Setup for time-dependent fluorescenece anisotropy measurements. APD: avalanche photodiode; $F$ : fluorescence band pass filter; $L_{1,4}$ : focusing lenses; $L_{2}$ : collimating lens, $L_{3}$ : collecting lens; $P_{1}$ : excitation polarizer; $\mathrm{P}_{2}$ : emission polarizer; $\mathrm{S}$ : sample solution in a cuvette, placed inside a thermostat.

TCSPC measurements were performed in a custom-made setup consisting of a LDH-P-C-375 (blue chromophores excitation) or a LDH-P-C-510 (red chromophores excitation) picosecond pulsed laser (PicoQuant $\mathrm{GmbH}$, Berlin, Germany), running on its internal clock at a frequency of $40 \mathrm{MHz}$, an ID100-50 (ID Quantique SA, Geneva, Switzerland) SPAD detector, and a PicoHarp 300 standalone TCSPC module. The sync signal used to compute the photon arrival times was provided by the laser driver. Count rates on the detector were adjusted to values below $10^{4} \mathrm{~Hz}$ in all cases (typically ca. $10^{3} \mathrm{~Hz}$ ). An "L-shape" geometry was used with two Glan-Thompson polarizers (B. Halle Nachfl. GmbH, Berlin, Germany), a 440/40 nm or a 620/60 nm bandpass filter (AHF Analysentechnik, Tübingen, Germany) was placed in front of the detector, and diverse lenses to collimate the excitation light and to collect and focus the emission onto the detector were used. The temperature of the sample was controlled with a single Peltier cell holder (Varian Inc., Australia). The internal response function (IRF) was collected with a dispersant in identical conditions as sample's measurements, except for 
Chapter 17 TIME-RESOLVED FLUORESCENCE ANISOTROPY MEASUREMENTS

the bandpass filter which was removed. Fluorescence decays were deconvoluted and fitted using custom made routines in MATLAB software. 


\section{Antibunching experiments}

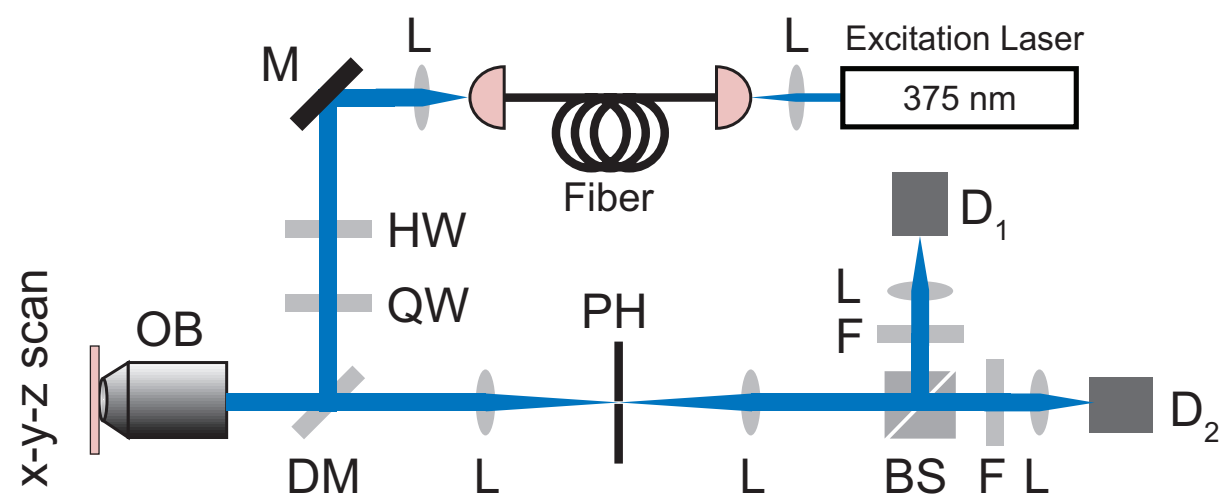

Scheme 31: Confocal microscope combined with two independent detection channels. BS: beam splitter; $\mathrm{D}_{1,2}$ : avalanche photodiodes; DM: dichroic mirrors; F: fluorescence band pass filter; HW: half-wave plate; L: lens; M: Mirror; OB: objective; PH: pinhole; QW: quarter-wave plate. This scheme was published in an article with the title "Bichromophoric Compounds with Orthogonally and Parallelly Arranged Chromophores Separated by Rigid Spacers".[160] Copyright Wiley-VCH Verlag GmbH \& Co. KGaA. Reproduced with permission.

A $375 \mathrm{~nm}$ diode laser (LDH-P-C-375; PicoQuant GmbH, Berlin, Germany) was fed to an optical fiber (PM-S405-XP; Thorlabs GmbH, Dachau/Munich, Germany) to generate a Gaussian beam. Circular polarization was generated, after the excitation light passed a pair of half- and quarter-wave plates. Excitation light was then reflected by a hot ${ }^{\dagger}$ mirror (M254H00; Thorlabs) into an objective (HCX-PL-APO 100x/1.4-0.7 OIL CS; Leica Microsystems, Wetzlar, Germany) and the fluorescence was collected by the same objective and transmitted through the hot mirror. After a tube lens $(f=200 \mathrm{~mm})$, the fluorescence went through a $50 \mu \mathrm{m}$ pinhole (correspond to ca. 1 airy disk diameter) and was collimated by another lens $(f=100 \mathrm{~mm})$, before it was split into two identical detection channels. Detection filters of type 460/60 (F39-46; AHF Analysentechnik AG, Tübingen, Germany) were used with single photon counting modules (COUNT @blue; Laser Components GmbH, Olching, Germany) as detectors. The excitation repetition rate was $40 \mathrm{MHz}$ and the laser power was ca. $21 \mu \mathrm{W}$. The detected signal was transferred through a time-correlated single photon counting card (DPC 230; Becker \& Hickl GmbH, Berlin, Germany) and stored by a PC for post

\footnotetext{
${ }^{\dagger}$ reflecting infrared (heat) radiation, while allowing visible light to pass
} 
Chapter 18 ANTIBUNCHING EXPERIMENTS

processing. Experimental control, data acquisition and analysis were performed with custom made MATLAB software. 


\section{DFT and TD-DFT calculations}

To estimate the EET efficiency based on the Förster point-dipole approximation (see section 1.2.1), the orientation of the absorption and emission transition dipole moments were calculated using density functional methods. All calculations were carried out using B3YLP method ${ }^{[165]}$ with $6-31 G(d, p)$ basis set in gas phase with Gaussian $09^{[166]}$ software.

The method may be summarized as follows: First of all, the ground state geometry was optimized. To determine the absorption transition dipole moment, the electronic transitions were calculated for the ground state geometry using TDDFT. ${ }^{[167]}$ For determination of the emission transition dipole moment, the geometry of the $S_{1}$ state was optimized first, since that geometry is present when the photon is emitted (vertical transition). Then the electronic transitions (and transition dipole moments) between the ground and excited states were calculated for the geometry of the excited state. The resulting emission transition dipole moment was obtained for the geometry of the excited state. To transfer it to the ground state geometry the adamantane structure was used as a reference system. The absorption and emission transition dipole moments obtained in this way were placed at the geometrical centers of the fluorophores' aromatic cores allowing to determine the distance between the transition dipoles and the orientation factor $\kappa^{2}$.

\subsection{Ground state geometry optimization}

For all molecules studied using DFT methods, the ground state geometry was optimized first. The ground state geometry of the model compounds was optimized for all relevant conformers. The conformer with lowest energy was chosen for all further calculations. The bichromophore conformers were chosen to match the conformation of the corresponding model compounds.

The following code fragment of an input file root section was used typically for ground state geometry optimizations (opt keyword) followed by a frequency analysis (Freq keyword): 


\section{Chapter 19 DFT AND TD-DFT CALCULATIONS}

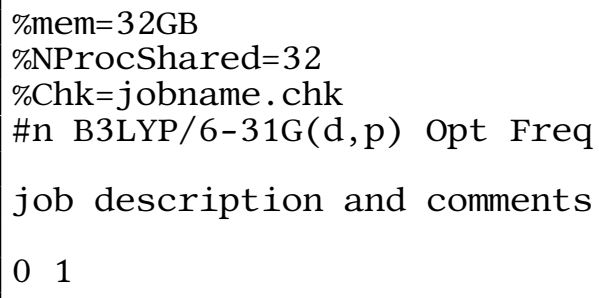

In some cases the optimization did not converge. In these cases the number of self-consistent field optimization cycles was increased using the keyword $\operatorname{scf}=($ maxcycle $=2000$ ). The frequency analysis was performed to verify that there are no significant imaginary frequencies, i.e. to check that the optimized geometry represents a minimum of the potential energy surface. In case of imaginary frequencies, the optimization was repeated with stricter convergence criteria using option opt=tight or opt=verytight.

\subsection{Excited state geometry optimization}

TD-DFT was used for geometry optimization of the $S_{1}$ state. ${ }^{[167]}$ This was done by adding the option TD(NStates=10, Root=1) to the root section of the geometry optimization, where NStates $=10$ gives the number of calculated states and Root=1 gives the number of the calculated state. In some cases the geometry optimization of the excited state failed, most likely due to too big steps taken during the optimization process. In that case, the step size was reduced from 0.3 to 0.1 Bohr radius using the option opt $=($ MaxStep $=10)$.

\subsection{Electronic transitions}

The electronic ground to excited state transitions including the transition dipole moments were calculated for the ground state geometry (absorption) and excited state geometry (emission) using TD-DFT. [167]

The following code fragment of an input file root section was used typically for calculation of the electronic transitions: 


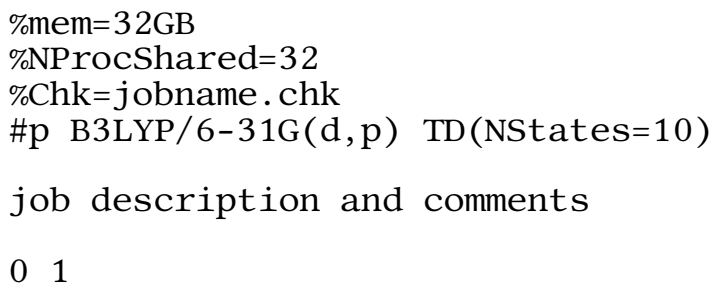

The individual transitions were assigned to fluorophores by their orientations, oscillator strengths and energies.

\subsection{Transferring transition dipoles from excited to ground state geometries}

The emission transition dipole moment was determined for the excited state geometry $\left(\vec{\mu}^{\prime}\right)$. In order to transfer it to the ground state geometry $(\vec{\mu})$, the adamantane structure was used as a reference system. Therefore, the transition moment was expressed as linear combination of unit vectors defined by the adamantane carbon atoms (figure 63): $\vec{\mu}^{\prime}=a \vec{n}_{1}^{\prime}+b \vec{n}_{2}^{\prime}+c \vec{n}_{3}^{\prime}$. The resulting parameters $a, b$, and $c$ were used with the corresponding unit vectors of the ground state geometry to determine the emission transition dipole in the ground state geometry: $\vec{\mu}=a \vec{n}_{1}+b \vec{n}_{2}+c \vec{n}_{3}$.

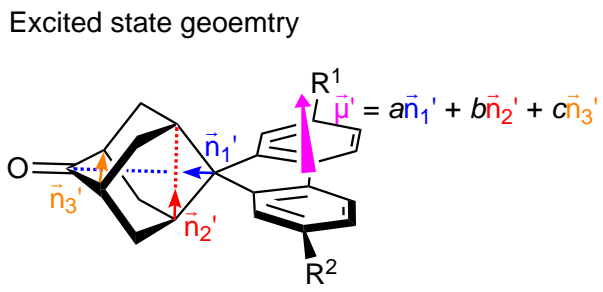
Ground state geoemtry

Figure 63: Transferring the transition dipole moment from the excited state geometry $\left(\vec{\mu}^{\prime}\right)$ to the ground state geometry $(\vec{\mu})$ by expressing it as linear combination of unit vectors associated by the adamantane structure. This figure was published in an article with the title "Bichromophoric Compounds with Orthogonally and Parallelly Arranged Chromophores Separated by Rigid Spacers". ${ }^{160]}$ Copyright Wiley-VCH Verlag GmbH \& Co. KGaA. Reproduced with permission. 
Chapter 19 DFT AND TD-DFT CALCULATIONS

\subsection{Placement of the transition dipoles within the molecular framework}

For calculation of the geometrical parameters, the position of the point dipoles were assumed to be in the middle between $\mathrm{C}-4 \mathrm{a}$ and $\mathrm{C}-8 \mathrm{a}$ of the coumarin moiety, and in the middle of the benzoquinone system of the alizarine moiety, respectively (figure 64).

a)

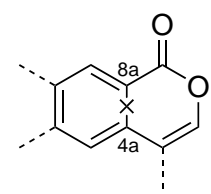

c)

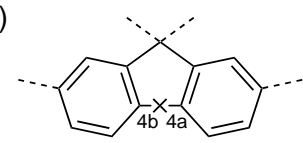

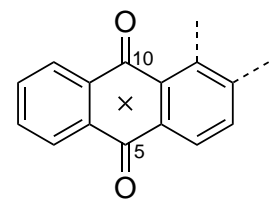

d)

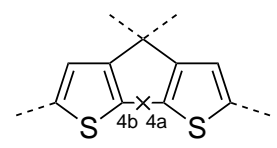

Figure 64: Assumed positions of transition dipole moments (marked as $\times$ ) for point dipole approximation. Illustrated for a) coumarin, b) alizarin, c) bridged biphenyl and d) bridged bithiophene based chromophores. 


\section{Estimation of FRET efficiency based on density functional calculations and model compound properties}

Absorption and emission transition dipole moments were calculated using TDDFT (see chapter 19 for details). According to the Franck-Condon principle, the absorption $\left(\mathrm{S}_{0} \rightarrow \mathrm{S}_{1}\right)$ transition dipole moments were calculated at the optimized $\mathrm{S}_{0}$ state geometries, whereas the emission $\left(\mathrm{S}_{1} \rightarrow \mathrm{S}_{0}\right)$ transition dipole moments were calculated at the optimized $\mathrm{S}_{1}$ state geometry. In order to reduce the computational load, the emission transition dipole moments of the energy donors were calculated exclusively for the corresponding model compounds. The position and orientation was transferred to the blue fluorophores of the individual bichromophores in their ground state optimized geometry using the adamantane scaffold as reference system (see section 19.4).

The transition dipole moments are shown as double-headed arrows with arbitrary length of $10 \AA$ (see section 19.5 for details).

\subsection{Calculation of orientation factor $\kappa^{2}$}

From the angles between the transition dipole moments of the donor $\mu_{\mathrm{D}}$ and acceptor $\mu_{\mathrm{A}}$ and interconnecting vector $\vec{R}_{\mathrm{DA}}$ (see figure 1 for definition of angles), the orientation factor $\kappa^{2}$ was calculated according to equation 4.

\subsection{Determination of spectral overlap integral $J_{D A}$}

The spectral overlap integral $J_{\mathrm{DA}}$ was calculated for the combinations of blue/blue and blue/red chromophores based on the absorption and emission spectra of the model compounds according to equation 3.

\subsection{Calculation of Förster radius $R_{0}$}

The Förster radius $R_{0}$ was calculated using the photophysical data of model compounds with the refractive index of the solvent according to equation 6 and 
Chapter 20 ESTIMATION OF FRET EFFICIENCY BASED ON DENSITY ...

the quantum yield of the donor model compound.

\subsection{Calculation of FRET efficiency}

The FRET efficiency $E_{\text {FRET }}$ was calculated according to equation 7 with $\left|\vec{R}_{\mathrm{DA}}\right|$ based on the assumed positions of the transition dipole moments as described in section 19.5 . 


\section{General procedures}

\subsection{GP1: General procedure for formation of spiroketals}

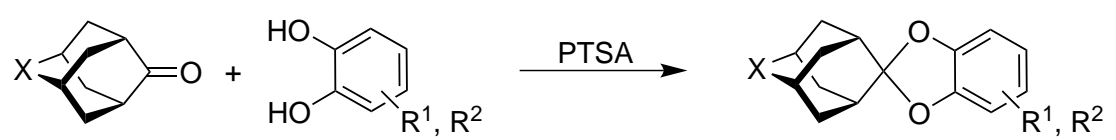

The catechol and 2-adamantanone derivatives were suspended with $p$-toluenesulfonic acid monohydrate $\left(\mathrm{TsOH} \cdot \mathrm{H}_{2} \mathrm{O}\right)$ in dry toluene inside a Dean-Stark apparatus equipped with a reflux condenser and a water trap filled with molecular sieves $(4 \AA)$. The mixture was heated to $135-140^{\circ} \mathrm{C}$ (oil bath temperature) for $17-$ $65 \mathrm{~h}$. After cooling to room temperature, the reaction mixture was mixed with $5 \%$ aq. $\mathrm{NaHCO}_{3}$ (ca. the double volume of the reaction mixture) and extracted with EtOAc $(3 \times$ ca. the same volume as aq. layer $)$. The combined organic solutions were dried over $\mathrm{Na}_{2} \mathrm{SO}_{4}$ and concentrated in vacuo. The product was isolated by column chromatography on silica gel.

\subsection{GP2: General procedure for coupling of aryl bromide to adamantane scaffold}

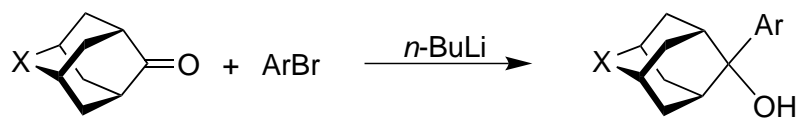

The aryl bromide was dissolved in dry THF, and the solution was cooled to $-78^{\circ} \mathrm{C}$ using an ace-tone/dry ice bath. 1.6 or $2.5 \mathrm{M} \mathrm{n}$-BuLi in $n$-hexane was added dropwise over a period of $1-2 \mathrm{~min}$. After $10-15 \mathrm{~min}$ at $-78^{\circ} \mathrm{C}$, either a solution of the ketone in dry THF was added, or the solution of the lithiated aryl was transferred to a solution of the ketone in dry THF. In both cases, the addition was carried out dropwise at $-78^{\circ} \mathrm{C}$ over a period of $1-2 \mathrm{~min}$. After stirring at $-78^{\circ} \mathrm{C}$ for $6-10 \mathrm{~min}$, the cooling bath was removed and the reaction mixture was let to warm-up to room temperature and stirred at room temperature for additional $0.75-4 \mathrm{~h}$. The reaction mixture was quenched by addition of sat. aq. $\mathrm{NH}_{4} \mathrm{Cl}$ and/or water and extracted with organic solvent. The combined organic solutions were dried over $\mathrm{Na}_{2} \mathrm{SO}_{4}$ and concentrated in vacuo. The product was isolated by column chromatography on silica gel. 


\subsection{GP3: General procedure for cyclization of tertiary alcohols}

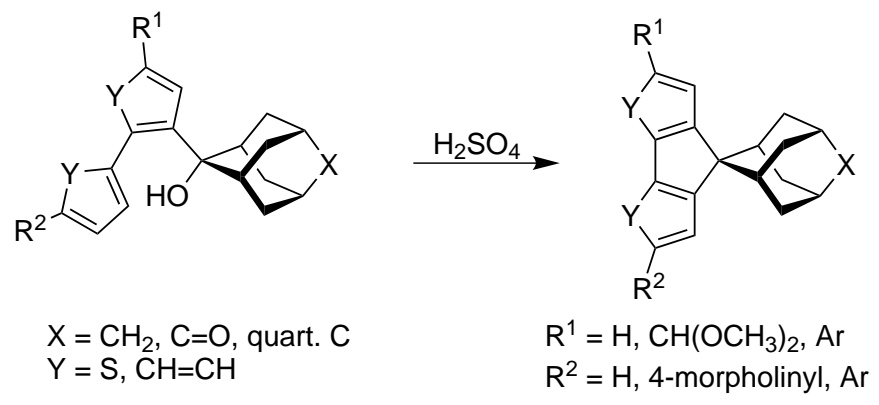

Acid-catalyzed Friedel-Crafts dehydrations of tertiary alcohols were carried out according to literature methods. ${ }^{[138]}$ Alcohol was suspended in $n$-octane using an ultrasonic bath. $60-98 \%$ aq. $\mathrm{H}_{2} \mathrm{SO}_{4}$ was added dropwise at room temperature over a period of $1 \mathrm{~min}$, while stirring vigorously. After stirring at room temperature for 15-120 min, the reaction was quenched by addition of aq. $\mathrm{NaOH}$ or aq. $\mathrm{Na}_{2} \mathrm{CO}_{3}$. The mixture was extracted with $\mathrm{CH}_{2} \mathrm{Cl}_{2}$ (often brine was added to achieve a better phase separation), and the combined organic solutions were dried over $\mathrm{Na}_{2} \mathrm{SO}_{4}$. Volatiles were removed in vacuo and the crude product was purified by column chromatography on silica gel. 


\section{Compounds}

\subsection{Compound 33a}

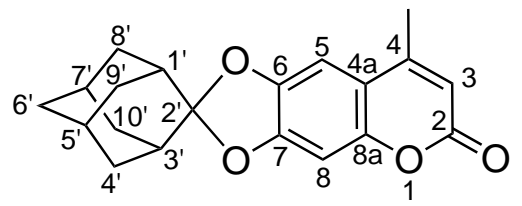

According to GP1, 6,7-dihydroxy-4-methylcoumarin ( $83 \mathrm{mg}, 0.43 \mathrm{mmol}, 1.0 \mathrm{eq}$ ), 2-adamantanone $(50 \mathrm{mg}, 0.33 \mathrm{mmol}, 1.3 \mathrm{eq})$ and $\mathrm{TsOH} \cdot \mathrm{H}_{2} \mathrm{O}(8.0 \mathrm{mg}, 43 \mu \mathrm{mol}$, $0.13 \mathrm{eq})$ reacted in dry toluene $(5.0 \mathrm{ml})$. An aq. work-up after $17 \mathrm{~h}$ gave a grey solid. The crude product was purified by column chromatography on silica gel (two columns: first one with cyclohexane/EtOAc $=2: 1$; the second one with cyclohexane/EtOAc $=3: 1)$ yielding $26 \mathrm{mg}(80 \mu \mathrm{mol}, 24 \%)$ of the title compound as colorless solid.

${ }^{1} \mathbf{H}$ NMR $\left(400 \mathrm{MHz}, \mathrm{CDCl}_{3}\right): \delta=1.74-1.84\left(\mathrm{~m}, 6 \mathrm{H}, 4^{\prime}-\mathrm{H}_{\mathrm{a}}, 6^{\prime}-\mathrm{H}_{2}, 8^{\prime}-\mathrm{H}_{\mathrm{a}}, 9^{\prime}-\mathrm{H}_{\mathrm{a}}\right.$, $\left.10^{\prime}-\mathrm{H}_{\mathrm{a}}\right), 1.87-1.94\left(\mathrm{~m}, 2 \mathrm{H}, 5^{\prime}-\mathrm{H}, 7^{\prime}-\mathrm{H}\right), 2.06-2.14\left(\mathrm{~m}, 4 \mathrm{H}, 4^{\prime}-\mathrm{H}_{\mathrm{b}}, 8^{\prime}-\mathrm{H}_{\mathrm{b}}, 9^{\prime}-\mathrm{H}_{\mathrm{b}}\right.$, $\left.10^{\prime}-\mathrm{H}_{\mathrm{b}}\right), 2.16-2.22\left(\mathrm{~m}, 2 \mathrm{H}, 1^{\prime}-\mathrm{H}, 3^{\prime}-\mathrm{H}\right), 2.34\left(\mathrm{~d},{ }^{4} J_{\mathrm{H}, \mathrm{H}}=1.3 \mathrm{~Hz}, 3 \mathrm{H}, \mathrm{CH}_{3}\right), 6.14(\mathrm{~d}$, $\left.{ }^{4} J_{\mathrm{H}, \mathrm{H}}=1.3 \mathrm{~Hz}, 1 \mathrm{H}, 3-\mathrm{H}\right), 6.81(\mathrm{~s}, 1 \mathrm{H}, 8-\mathrm{H}), 6.96(\mathrm{~s}, 1 \mathrm{H}, 5-\mathrm{H}) \mathrm{ppm}$.

${ }^{13} \mathbf{C}$ NMR $\left(101 \mathrm{MHz}, \mathrm{CDCl}_{3}\right): \delta=19.3\left(\mathrm{CH}_{3}\right), 26.6,27.0(\mathrm{C}-5$ ', C-7'), 34.3, 34.5 (C-4', C-8', C-9', C-10'), 36.9 (C-1', C-3'), 37.0 (C-6'), 98.1 (C-8), 101.7 (C-5), 111.7 (C-3), 113.3 (C-4a), 124.0 (C-2'), 145.2, 150.5, 151.4, 152.8 (C-4, C-6, C-7, C-8a), $161.7(\mathrm{C}-2) \mathrm{ppm}$.

MS (ESI, pos.): $m / z(\%)=325.2(100)[\mathrm{M}+\mathrm{H}]^{+}$.

HRMS (ESI): $m / z$ calc. for $\mathrm{C}_{20} \mathrm{H}_{21} \mathrm{O}_{4}[\mathrm{M}+\mathrm{H}]^{+}$325.1434; found 325.1434.

$\operatorname{TLC}\left(\mathrm{SiO}_{2}\right): R_{\mathrm{f}}=0.47($ cyclohexane $/$ EtOAc $=1: 1)$.

\subsection{Compound 33b}

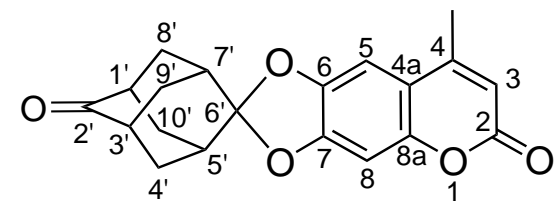

According to GP1, 6,7-dihydroxy-4-methylcoumarin (59 mg, $0.30 \mathrm{mmol}, 1.0 \mathrm{eq}$ ), 


\section{Chapter 22 $\mid$ COMPOUNDS}

2,6-adamantanedione $(50 \mathrm{mg}, 0.30 \mathrm{mmol}, \quad 1.0 \mathrm{eq})$ and $\mathrm{TsOH} \cdot \mathrm{H}_{2} \mathrm{O}(27 \mathrm{mg}$, $0.14 \mathrm{mmol}, 0.47 \mathrm{eq})$ were reacted in dry toluene $(10 \mathrm{ml})$. Aq. work-up after $18 \mathrm{~h}$ gave $69 \mathrm{mg}$ of a greenish solid. The crude product was purified by column chromatography on silica gel $\left(\mathrm{CH}_{2} \mathrm{Cl}_{2} / \mathrm{EtOAc}=1: 0 \rightarrow 10: 1\right)$ and afforded $29 \mathrm{mg}$ $(86 \mu \mathrm{mol}, 28 \%)$ of the title compound as colorless solid.

${ }^{1} \mathbf{H}$ NMR $\left(400 \mathrm{MHz}, \mathrm{CDCl}_{3}\right): \delta=1.99-2.08\left(\mathrm{~m}, 4 \mathrm{H}, 4^{\prime}-\mathrm{H}_{\mathrm{a}}, 8^{\prime}-\mathrm{H}_{\mathrm{a}}, 9^{\prime}-\mathrm{H}_{\mathrm{a}}, 10^{\prime}-\mathrm{H}_{\mathrm{a}}\right)$, $2.31-2.35\left(\mathrm{~m}, 2 \mathrm{H}, 5^{\prime}-\mathrm{H}, 7^{\prime}-\mathrm{H}\right), 2.36\left(\mathrm{~d},{ }^{4} J_{\mathrm{H}, \mathrm{H}}=1.2 \mathrm{~Hz}, 3 \mathrm{H}, \mathrm{CH}_{3}\right), 2.39-2.49(\mathrm{~m}$, $\left.4 \mathrm{H}, 4^{\prime}-\mathrm{H}_{\mathrm{b}}, 8^{\prime}-\mathrm{H}_{\mathrm{b}}, 9^{\prime}-\mathrm{H}_{\mathrm{b}}, 10^{\prime}-\mathrm{H}_{\mathrm{b}}\right), 2.54-2.60\left(\mathrm{~m}, 2 \mathrm{H}, 1^{\prime}-\mathrm{H}, 3^{\prime}-\mathrm{H}\right), 6.14\left(\mathrm{~d},{ }^{4} J_{\mathrm{H}, \mathrm{H}}=\right.$ $1.3 \mathrm{~Hz}, 1 \mathrm{H}, 3-\mathrm{H}), 6.81(\mathrm{~s}, 1 \mathrm{H}, 8-\mathrm{H}), 6.96(\mathrm{~s}, 1 \mathrm{H}, 5-\mathrm{H}) \mathrm{ppm}$.

${ }^{13}$ C NMR $\left(101 \mathrm{MHz}, \mathrm{CDCl}_{3}\right): \delta=19.3\left(\mathrm{CH}_{3}\right), 35.2,35.3\left(\mathrm{C}-4^{\prime}, \mathrm{C}-8^{\prime}, \mathrm{C}-9{ }^{\prime}, \mathrm{C}-10^{\prime}\right)$, 36.2 (C-5', C-7'), 44.7 (C-1', C-3'), 98.6 (C-8), 102.3 (C-5), 112.2 (C-3), 113.8 (C-4a), 121.0 (C-6'), 144.8, 150.6, 150.8 (C-6, C-7, C-8a), 152.6 (C-4), 161.5 (C-2), 214.9 $\left(\mathrm{C}-2^{\prime}\right) \mathrm{ppm}$.

MS (ESI, pos.): $m / z(\%)=339.1(100)[\mathrm{M}+\mathrm{H}]^{+}$.

$\operatorname{TLC}\left(\mathrm{SiO}_{2}\right): R_{\mathrm{f}}=0.13\left(\mathrm{CH}_{2} \mathrm{Cl}_{2}\right)$.

\subsection{Compound 35a}

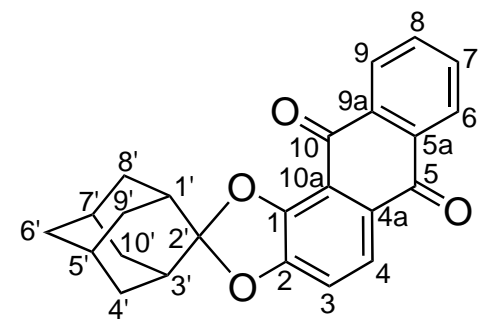

According to GP1, 1,2-dihydroxyanthraquinone (107 mg, $444 \mu \mathrm{mol}, 1.3 \mathrm{eq}$ ), 2-adamantanone (50 mg, $0.33 \mathrm{mmol}, 1.0 \mathrm{eq})$ and $\mathrm{TsOH} \cdot \mathrm{H}_{2} \mathrm{O}(34 \mathrm{mg}, 0.18 \mathrm{mmol}$, $0.53 \mathrm{eq})$ reacted in dry toluene $(10 \mathrm{ml})$. Aq. work-up after $17 \mathrm{~h}$ gave $150 \mathrm{mg}$ of a brown solid. The crude product was purified by column chromatography on silica gel (two columns: first one with cyclohexane/EtOAc $=7: 3$; the second one with $\mathrm{CH}_{2} \mathrm{Cl}_{2}$ /cyclohexane $\left.=1: 1\right)$ and afforded $22 \mathrm{mg}(60 \mu \mathrm{mol}, 18 \%)$ of the title compound as yellow solid.

${ }^{1} \mathbf{H}$ NMR $\left(400 \mathrm{MHz}, \mathrm{CDCl}_{3}\right): \delta=1.77-1.90\left(\mathrm{~m}, 6 \mathrm{H}, 4^{\prime}-\mathrm{H}_{\mathrm{a}}, 8^{\prime}-\mathrm{H}_{\mathrm{a}}, 9^{\prime}-\mathrm{H}_{\mathrm{a}}, 10^{\prime}-\mathrm{H}_{\mathrm{a}}\right.$, $\left.6^{\prime}-\mathrm{H}_{2}\right), 1.91-2.02\left(\mathrm{~m}, 2 \mathrm{H}, 5^{\prime}-\mathrm{H}, 7^{\prime}-\mathrm{H}\right), 2.10-2.19\left(\mathrm{~m}, 2 \mathrm{H},\left[4^{\prime}-\mathrm{H}_{\mathrm{b}}, 9^{\prime}-\mathrm{H}_{\mathrm{b}}\right]\right.$ or $\left[8^{\prime}-\mathrm{H}_{\mathrm{b}}\right.$, 
$\left.\left.10^{\prime}-\mathrm{H}_{\mathrm{b}}\right]\right), 2.24-2.31\left(\mathrm{~m}, 2 \mathrm{H}, 1^{\prime}-\mathrm{H}, 3^{\prime}-\mathrm{H}\right), 2.31-2.40\left(\mathrm{~m}, 2 \mathrm{H},\left[8^{\prime}-\mathrm{H}_{\mathrm{b}}, 10^{\prime}-\mathrm{H}_{\mathrm{b}}\right]\right.$ or $\left.\left[4^{\prime}-\mathrm{H}_{\mathrm{b}}, 9^{\prime}-\mathrm{H}_{\mathrm{b}}\right]\right), 7.05\left(\mathrm{~d},{ }^{3} \mathrm{~J}_{\mathrm{H}, \mathrm{H}}=8.1 \mathrm{~Hz}, 1 \mathrm{H}, 3-\mathrm{H}\right), 7.72-7.78(\mathrm{~m}, 2 \mathrm{H}, 7-\mathrm{H}, 8-\mathrm{H})$, $7.92\left(\mathrm{~d},{ }^{3} J_{\mathrm{H}, \mathrm{H}}=8.1 \mathrm{~Hz}, 1 \mathrm{H}, 4-\mathrm{H}\right), 8.24-8.32(\mathrm{~m}, 2 \mathrm{H}, 6-\mathrm{H}, 9-\mathrm{H}) \mathrm{ppm}$.

${ }^{13} \mathrm{C}$ NMR $\left(101 \mathrm{MHz}, \mathrm{CDCl}_{3}\right): \delta=26.6,26.6$ (C-5', C-7'), 34.4, 34.5 (C-4', C-8', C-9', C-10'), 36.9 (C-6'), 37.2 (C-1', C-3'), 112.2 (C-3), 116.5, 123.6 (C-4), 126.0, 126.8 (C-4a, C-10a), 126.9, 127.3 (C-6, C-9), 133.8, 133.8 (C-8, C-7), 134.0, 134.3 (C-5a, C-9a), 148.6 (C-1), 154.5 (C-2), 181.9, 182.0 (C-5, C-10) ppm.

MS (ESI, pos.): $m / z(\%)=373.1(100)[\mathrm{M}+\mathrm{H}]^{+}, 395.2(45)[\mathrm{M}+\mathrm{Na}]^{+}$.

$\operatorname{TLC}\left(\mathrm{SiO}_{2}\right): R_{\mathrm{f}}=0.49($ cyclohexane$/$ EtOAc $=7: 3)$.

\subsection{Compound 35b}

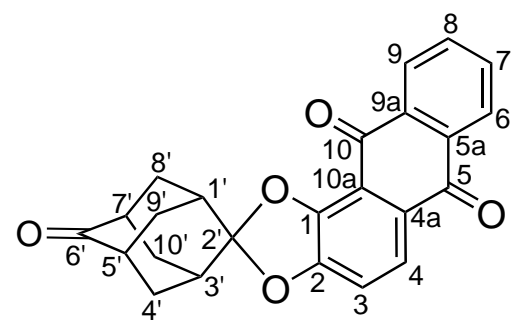

According to GP1, 1,2-dihydroxyanthraquinone ( $151 \mathrm{mg}, 609 \mu \mathrm{mol}, 1.00 \mathrm{eq})$, 2,6-adamantanedione $(100 \mathrm{mg}, 609 \mu \mathrm{mol}, 1.00 \mathrm{eq})$ and $\mathrm{TsOH} \cdot \mathrm{H}_{2} \mathrm{O}(58 \mathrm{mg}$, $0.31 \mathrm{mmol}, 0.50 \mathrm{eq})$ were reacted in dry toluene $(10 \mathrm{ml})$. Aq. work-up after $65 \mathrm{~h}$ gave a brown solid. The crude product was purified by column chromatography on silica gel $\left(\mathrm{CH}_{2} \mathrm{Cl}_{2} / \mathrm{EtOAc}=30: 1\right)$ and afforded $104 \mathrm{mg}(270 \mu \mathrm{mol}, 44 \%)$ of the title compound as yellow solid.

${ }^{1} \mathbf{H}$ NMR $\left(400 \mathrm{MHz}, \mathrm{CDCl}_{3}\right): \delta=2.05-2.14\left(\mathrm{~m}, 4 \mathrm{H}, 4^{\prime}-\mathrm{H}_{\mathrm{a}}, 8^{\prime}-\mathrm{H}_{\mathrm{a}}, 9^{\prime}-\mathrm{H}_{\mathrm{a}}, 10^{\prime}-\mathrm{H}_{\mathrm{a}}\right)$, $2.38-2.52\left(\mathrm{~m}, 4 \mathrm{H}, 1^{\prime}-\mathrm{H}, 3^{\prime}-\mathrm{H},\left[4^{\prime}-\mathrm{H}_{\mathrm{b}}, 9^{\prime}-\mathrm{H}_{\mathrm{b}}\right]\right.$ or $\left.\left[8^{\prime}-\mathrm{H}_{\mathrm{b}}, 10^{\prime}-\mathrm{H}_{\mathrm{b}}\right]\right), 2.58-2.73(\mathrm{~m}$, $4 \mathrm{H}, 5^{\prime}-\mathrm{H}, 7^{\prime}-\mathrm{H},\left[8^{\prime}-\mathrm{H}_{\mathrm{b}}, 10^{\prime}-\mathrm{H}_{\mathrm{b}}\right]$ or $\left.\left[4^{\prime}-\mathrm{H}_{\mathrm{b}}, 9^{\prime}-\mathrm{H}_{\mathrm{b}}\right]\right), 7.13\left(\mathrm{~d},{ }^{3} J_{\mathrm{H}, \mathrm{H}}=8.2 \mathrm{~Hz}, 1 \mathrm{H}, 3-\mathrm{H}\right)$, $7.73-7.82(\mathrm{~m}, 2 \mathrm{H}, 7-\mathrm{H}, 8-\mathrm{H}), 7.97\left(\mathrm{~d},{ }^{3} \mathrm{~J}_{\mathrm{H}, \mathrm{H}}=8.2 \mathrm{~Hz}, 1 \mathrm{H}, 4-\mathrm{H}\right), 8.23-8.34(\mathrm{~m}, 2 \mathrm{H}$, 6-H, 9-H) ppm.

${ }^{13}$ C NMR $\left(101 \mathrm{MHz}, \mathrm{CDCl}_{3}\right): \delta=35.3,35.3\left(\mathrm{C}-4^{\prime}, \mathrm{C}-8^{\prime}, \mathrm{C}-9{ }^{\prime}, \mathrm{C}-10^{\prime}\right), 36.4\left(\mathrm{C}-1^{\prime}\right.$, C-3'), 44.6, 44.7 (C-5', C-7'), 112.7 (C-3), 117.0 (C-10a), 122.8 (C-2'), 123.9 (C-4), 126.9 (C-6 or C-9), 127.3 (C-4a), 127.4 (C-9 or C-6), 133.8 (C-5a or C-9a), 134.0, 134.1 (C-7, C-8), 134.1 (C-9a or C-5a), 147.9 (C-1), 153.8 (C-2), 181.8, 181.9 (C-5, 


\section{Chapter 22 COMPOUNDS}

C-10), 214.8 (C-6') ppm.

MS (ESI, pos.): $m / z(\%)=409.1(95)[\mathrm{M}+\mathrm{Na}]^{+}, 441.1(100)[\mathrm{M}+\mathrm{MeOH}+\mathrm{Na}]^{+}, 795.1$

(44) $[2 \mathrm{M}+\mathrm{Na}]^{+}$.

HRMS (ESI): $m / z$ calc. for $\mathrm{C}_{24} \mathrm{H}_{18} \mathrm{NaO}_{5}[\mathrm{M}+\mathrm{Na}]^{+}$409.1046; found 409.1033.

$\operatorname{TLC}\left(\mathrm{SiO}_{2}\right): R_{\mathrm{f}}=0.17\left(\mathrm{CH}_{2} \mathrm{Cl}_{2} / \mathrm{EtOAc}=30: 1\right)$.

\subsection{Compound 36}

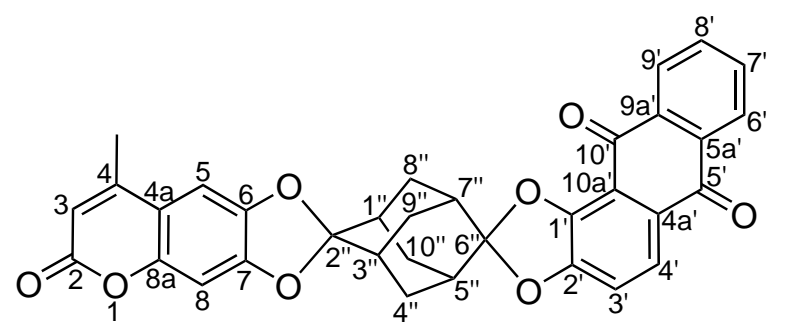

According to GP1, 1,2-dihydroxyanthraquinone ( $22 \mathrm{mg}, 93 \mu \mathrm{mol}, 1.3 \mathrm{eq})$, ketone 33b $(24 \mathrm{mg}, 72 \mu \mathrm{mol}, 1.0 \mathrm{eq})$ and $\mathrm{TsOH} \cdot \mathrm{H}_{2} \mathrm{O}(7.5 \mathrm{mg}, 40 \mu \mathrm{mol}, 0.54 \mathrm{eq})$ reacted in dry toluene $(10 \mathrm{ml})$. After $23 \mathrm{~h}$, additional $\mathrm{TsOH} \cdot \mathrm{H}_{2} \mathrm{O}(21 \mathrm{mg}, 40 \mu \mathrm{mol}$, $1.5 \mathrm{eq}$ ) was added and the reaction mixture was heated to reflux for additional $24 \mathrm{~h}$. Aq. work-up according to GP1 gave a brown solid. The crude product was purified by column chromatography on silica gel $\left(\mathrm{CH}_{2} \mathrm{Cl}_{2} / \mathrm{EtOAc}=35: 1 \rightarrow 10: 1\right)$ and afforded $10 \mathrm{mg}(18 \mu \mathrm{mol}, 25 \%)$ of the title compound as yellow solid.

${ }^{1} \mathbf{H}$ NMR $\left(400 \mathrm{MHz}, \mathrm{CDCl}_{3}\right): \delta=2.19-2.49\left(\mathrm{~m}, 12 \mathrm{H}, 1^{\prime \prime}-\mathrm{H}, 3\right.$ "- $\mathrm{H}, 4$ "- $\mathrm{H}_{2}, 5$ "-H, 7"-H, 8"- $\left.\mathrm{H}_{2}, 9 "-\mathrm{H}_{2}, 10^{\prime \prime}-\mathrm{H}_{2}, \mathrm{CH}_{3}\right), 6.16\left(\mathrm{~d},{ }^{4} \mathrm{~J}_{\mathrm{H}, \mathrm{H}}=1.3 \mathrm{~Hz}, 1 \mathrm{H}, 3-\mathrm{H}\right), 6.80(\mathrm{~s}, 1 \mathrm{H}$, $5-\mathrm{H}$ or $8-\mathrm{H}), 6.94(\mathrm{~s}, 1 \mathrm{H}, 8-\mathrm{H}$ or $5-\mathrm{H}), 7.10\left(\mathrm{~d},{ }^{3} J_{\mathrm{H}, \mathrm{H}}=8.2 \mathrm{~Hz}, 1 \mathrm{H}, 3^{\prime}-\mathrm{H}\right), 7.74-7.80$ $\left(\mathrm{m}, 2 \mathrm{H}, 7^{\prime}-\mathrm{H}, 8^{\prime}-\mathrm{H}\right), 7.96\left(\mathrm{~d},{ }^{3} \mathrm{~J}_{\mathrm{H}, \mathrm{H}}=8.2 \mathrm{~Hz}, 1 \mathrm{H}, 4^{\prime}-\mathrm{H}\right), 8.23-8.34\left(\mathrm{~m}, 2 \mathrm{H}, 6^{\prime}-\mathrm{H}\right.$, 9'-H) ppm.

MS (ESI, pos.): $m / z(\%)=561.2(100)[\mathrm{M}+\mathrm{H}]^{+}, 583.2(80)[\mathrm{M}+\mathrm{Na}]^{+}$.

HRMS (ESI): $m / z$ calc. for $\mathrm{C}_{34} \mathrm{H}_{24} \mathrm{O}_{8}[\mathrm{M}+\mathrm{H}]^{+} 583.1362$; found 583.1363.

$\operatorname{TLC}\left(\mathrm{SiO}_{2}\right): R_{\mathrm{f}}=0.19\left(\mathrm{CH}_{2} \mathrm{Cl}_{2} / \mathrm{EtOAc}=30: 1\right)$. 


\subsection{Compound $38^{[123]}$}

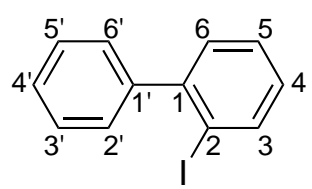

Hydrochloric acid (37\%, $14.6 \mathrm{ml}, 175 \mathrm{mmol}, 5.92 \mathrm{eq})$ was added to 2-aminobiphenyl ( $5.00 \mathrm{~g}, 29.5 \mathrm{mmol}, 1.00 \mathrm{eq})$. To the resulting suspension dropwise $2.1 \mathrm{M}$ aq. solution of sodium nitrite $(16.8 \mathrm{ml}, 35.2 \mathrm{mmol}, 1.19 \mathrm{eq})$, was added at $0{ }^{\circ} \mathrm{C}$ over a period of $15 \mathrm{~min}$. The yellow reaction mixture was stirred at $0{ }^{\circ} \mathrm{C}$ for $15 \mathrm{~min}$ and then poured into a solution of potassium iodide $(18.1 \mathrm{~g}, 109 \mathrm{mmol}$, $3.69 \mathrm{eq})$ in water $(61 \mathrm{ml})$ at $0{ }^{\circ} \mathrm{C}$. The mixture was allowed to warm up to r.t. overnight. The brown mixture was extracted with EtOAc $(3 \times 25 \mathrm{ml})$, and the combined organic solutions were washed with water $(3 \times 25 \mathrm{ml})$. The organic solution was dried over $\mathrm{Na}_{2} \mathrm{SO}_{4}$ and concentrated in vacuo. The crude product was purified by column chromatography on silica gel ( $n$-hexane) and afforded $6.56 \mathrm{~g}(23.4 \mathrm{mmol}, 79 \%)$ of the title compound as red oil. Spectral data were in agreement with literature values. ${ }^{[168]}$

${ }^{1} \mathbf{H}$ NMR $\left(400 \mathrm{MHz}, \mathrm{CDCl}_{3}\right): \delta=7.05\left(\mathrm{ddd},{ }^{3} J_{\mathrm{H}, \mathrm{H}}=8.0,7.3 \mathrm{~Hz},{ }^{4} J_{\mathrm{H}, \mathrm{H}}=1.8 \mathrm{~Hz}, 1 \mathrm{H}\right.$, $4-\mathrm{H}), 7.30-7.48$ (m, $\left.7 \mathrm{H}, 5-\mathrm{H}, 6-\mathrm{H}, 2^{\prime}-\mathrm{H}, 3^{\prime}-\mathrm{H}, 4^{\prime}-\mathrm{H}, 5^{\prime}-\mathrm{H}, 66^{\prime}-\mathrm{H}\right), 7.98\left(\mathrm{dd},{ }^{3} J_{\mathrm{H}, \mathrm{H}}=\right.$ $\left.8.0 \mathrm{~Hz},{ }^{4} J_{\mathrm{H}, \mathrm{H}}=1.2 \mathrm{~Hz}, 1 \mathrm{H}, 3-\mathrm{H}\right) \mathrm{ppm}$.

${ }^{13} \mathrm{C}$ NMR $\left(101 \mathrm{MHz}, \mathrm{CDCl}_{3}\right): \delta=98.8(\mathrm{C}-2), 127.8\left(\mathrm{C}-4^{\prime}\right), 128.1\left(\mathrm{C}-2^{\prime}, \mathrm{C}-6\right.$ '), 128.2 (C-5 or C-6), 128.9 (C-4), 129.4 (C-3', C-5'), 130.2 (C-6 or C-5), 139.6 (C-3), 144.3 (C-1'), 146.8 (C-1) ppm.

GC-MS (EI): $m / z(\%)=278(100)^{+}\left[\mathrm{M}^{+\cdot}\right]$.

$\operatorname{TLC}\left(\mathrm{SiO}_{2}\right): R_{\mathrm{f}}=0.29$ ( $n$-hexane). 


\section{Chapter 22 COMPOUNDS}

\subsection{Compound 39b}

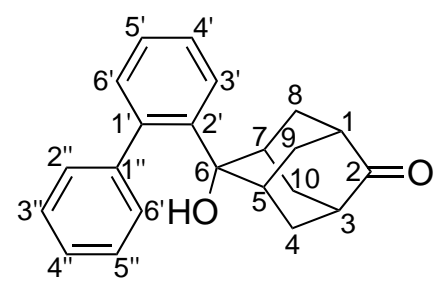

Magnesium swarf (252 mg, $10.4 \mathrm{mmol}, 1.70 \mathrm{eq}$ ) was stirred under nitrogen atmosphere. lodine ( $46 \mathrm{mg}, 0.18 \mathrm{mmol}, 0.03 \mathrm{eq}$ ) was added and the mixture was heated to $60^{\circ} \mathrm{C}$. Dry THF $\left(5.0 \mathrm{ml}\right.$ ) was added and the mixture was heated to $88^{\circ} \mathrm{C}$ (oil bath temperature). A solution of 2-iodobiphenyl $(2.22 \mathrm{~g}, 7.92 \mathrm{mmol}, 1.30 \mathrm{eq})$ in dry THF $(25 \mathrm{ml})$ was added over a period of $2.5 \mathrm{~h}$ via syringe pump while heating at reflux. The resulting suspension was heated at reflux for $1 \mathrm{~h}$. The mixture was cooled to r.t. and the solution was collected using a syringe. The Grignard solution was added dropwise to a solution of 2,6-adamantanedione (1.00 g, $6.09 \mathrm{mmol}, 1.00 \mathrm{eq})$ in dry THF $(30 \mathrm{ml})$ at r.t. over a period of $8 \mathrm{~min}$. The reaction mixture was stirred at r.t. for $25 \mathrm{~min}$ and heated at reflux for $3 \mathrm{~h}$. After cooling to r.t., the reaction mixture was poured into $3.0 \mathrm{M}$ aq. $\mathrm{HCl}(200 \mathrm{ml})$ at $0{ }^{\circ} \mathrm{C}$ and extracted with $\mathrm{Et}_{2} \mathrm{O}(6 \times 100 \mathrm{ml})$. The combined organic solutions were dried over $\mathrm{Na}_{2} \mathrm{SO}_{4}$ and concentrated in vacuo giving $1.86 \mathrm{~g}$ of brown solid. Column chromatography on silica gel (two columns: first one with cyclohexane/EtOAc $=4: 1$; the second one with cyclohexane $\left./ \mathrm{CH}_{2} \mathrm{Cl}_{2} / \mathrm{EtOAc}=5: 5: 2\right)$ gave $962 \mathrm{mg}(3.02 \mathrm{mmol}$, $50 \%$ ) of the title compound as colorless solid.

${ }^{1} \mathbf{H}$ NMR $\left(400 \mathrm{MHz}, \mathrm{CDCl}_{3}\right): \delta=1.66-1.73\left(\mathrm{~m}, 2 \mathrm{H},\left[4-\mathrm{H}_{\mathrm{a}}, 10-\mathrm{H}_{\mathrm{a}}\right]\right.$ or $\left[8-\mathrm{H}_{\mathrm{a}}\right.$, 9- $\left.\left.\mathrm{H}_{\mathrm{a}}\right]\right), 1.76-1.84\left(\mathrm{~m}, 2 \mathrm{H},\left[8-\mathrm{H}_{\mathrm{a}}, 9-\mathrm{H}_{\mathrm{a}}\right]\right.$ or $\left.\left[4-\mathrm{H}_{\mathrm{a}}, 10-\mathrm{H}_{\mathrm{a}}\right]\right), 1.94\left(\mathrm{~s}_{\mathrm{br}}, 1 \mathrm{H}, \mathrm{OH}\right), 1.96$ - $2.05\left(\mathrm{~m}, 2 \mathrm{H},\left[4-\mathrm{H}_{\mathrm{b}}, 10-\mathrm{H}_{\mathrm{b}}\right]\right.$ or $\left.\left[8-\mathrm{H}_{\mathrm{b}}, 9-\mathrm{H}_{\mathrm{b}}\right]\right), 2.30-2.42(\mathrm{~m}, 4 \mathrm{H}, 1-\mathrm{H}, 3-\mathrm{H}, 5-\mathrm{H}$, $7-\mathrm{H}), 2.49-2.58\left(\mathrm{~m}, 2 \mathrm{H},\left[8-\mathrm{H}_{\mathrm{b}}, 9-\mathrm{H}_{\mathrm{b}}\right]\right.$ or $\left.\left[4-\mathrm{H}_{\mathrm{b}}, 10-\mathrm{H}_{\mathrm{b}}\right]\right), 7.18\left(\mathrm{dd},{ }^{3} J_{\mathrm{H}, \mathrm{H}}=7.5 \mathrm{~Hz}\right.$, $\left.{ }^{4} J_{\mathrm{H}, \mathrm{H}}=1.7 \mathrm{~Hz}, 1 \mathrm{H}, 3^{\prime}-\mathrm{H}\right), 7.30-7.43\left(\mathrm{~m}, 7 \mathrm{H}, 4^{\prime}-\mathrm{H}, 5^{\prime}-\mathrm{H}, 2^{\prime \prime}-\mathrm{H}, 3^{\prime \prime}-\mathrm{H}, 4^{\prime \prime}-\mathrm{H}, 5^{\prime \prime}-\mathrm{H}\right.$, $\left.6^{\prime \prime}-\mathrm{H}\right), 7.68\left(\mathrm{dd},{ }^{3} J_{\mathrm{H}, \mathrm{H}}=8.1 \mathrm{~Hz},{ }^{4} J_{\mathrm{H}, \mathrm{H}}=1.3 \mathrm{~Hz}, 1 \mathrm{H}, 66^{\prime}-\mathrm{H}\right) \mathrm{ppm}$.

${ }^{13} \mathrm{C}$ NMR $\left(101 \mathrm{MHz}, \mathrm{CDCl}_{3}\right): \delta=34.3$ ([C-4, C-10] or [C-8, C-9]), 34.7 (C-5, C-7), 36.2 ([C-8, C-9] or [C-4, C-10]), 44.9 (C-1 or C-3), 45.4 (C-3 or C-1), 76.5 (C-6), 126.8 (C-6'), 127.3 (C-4", C-4' or C-5'), 127.6 (C-5' or C-4'), 128.1 (C-3", C-5"), 129.1 (C-2", C-6"), 134.1 (C-3'), 140.6 (C-2'), 142.6 (C-1'), 144.4 (C-1"), 217.7 (C-2) ppm. MS (ESI, pos.): $m / z(\%)=319(100)[\mathrm{M}+\mathrm{H}]^{+}$. 
$\operatorname{TLC}\left(\mathrm{SiO}_{2}\right): R_{\mathrm{f}}=0.20($ cyclohexane/EtOAc $=7: 3)$.

\subsection{Compound 40}

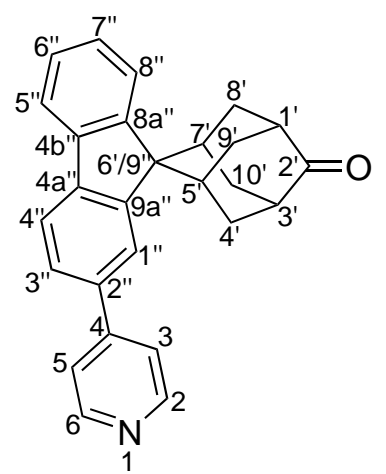

Compound 42 (78\% purity based on ${ }^{1} \mathrm{H}$ NMR, $\left.10 \mathrm{mg}, 26 \mu \mathrm{mol}, 1.0 \mathrm{eq}\right)$, was dissolved in dry toluene $(0.90 \mathrm{ml})$ in a screw cap pressure tube and a slow stream of nitrogen was bubbled through the solution for $10 \mathrm{~min}$. Then $\mathrm{Pd}\left(\mathrm{PPh}_{3}\right)_{2} \mathrm{Cl}_{2}$ (3.0 mg, $4.0 \mu \mathrm{mol}, 0.15 \mathrm{eq}$ ) and 2-(tributylstannyl)pyridine (17 mg, $44 \mu \mathrm{mol}, 1.7 \mathrm{eq}$ ) was added. The reaction vessel was sealed, and the solution was heated to $110^{\circ} \mathrm{C}$ for $19 \mathrm{~h}$. After cooling to room temperature, the black reaction mixture was diluted with $\mathrm{CH}_{2} \mathrm{Cl}_{2}(5 \mathrm{ml})$ and filtered through a pad of Celite ${ }^{\mathrm{TM}}(\varnothing=30 \mathrm{~mm}$, $h=6 \mathrm{~mm})$. The filter cake was rinsed with $\mathrm{CH}_{2} \mathrm{Cl}_{2}(2 \times 20 \mathrm{ml})$, and the combined filtrates were concentrated in vacuo. Column chromatography on silica gel $\left(\mathrm{CH}_{2} \mathrm{Cl}_{2} / \mathrm{MeOH}=30: 1\right)$ gave $3.4 \mathrm{mg}(7.5 \mu \mathrm{mol}, 29 \%)$ of the title compound as colorless solid.

${ }^{1} \mathbf{H}$ NMR $\left(400 \mathrm{MHz}, \mathrm{CDCl}_{3}\right): \delta=1.80-1.86\left(\mathrm{~m}, 2 \mathrm{H}, 5^{\prime}-\mathrm{H}, 7^{\prime}-\mathrm{H}\right), 1.96-2.10(\mathrm{~m}$, $\left.4 \mathrm{H}, 4^{\prime}-\mathrm{H}_{\mathrm{a}}, 8^{\prime}-\mathrm{H}_{\mathrm{a}}, 9^{\prime}-\mathrm{H}_{\mathrm{a}}, 10^{\prime}-\mathrm{H}_{\mathrm{a}}\right), 2.87-2.97\left(\mathrm{~m}, 2 \mathrm{H}, 1^{\prime}-\mathrm{H}, 3^{\prime}-\mathrm{H}\right), 3.10-3.28(\mathrm{~m}$, $\left.4 \mathrm{H}, 4^{\prime}-\mathrm{H}_{\mathrm{a}}, 8^{\prime}-\mathrm{H}_{\mathrm{a}}, 9^{\prime}-\mathrm{H}_{\mathrm{a}}, 10^{\prime}-\mathrm{H}_{\mathrm{a}}\right), 7.40\left(\mathrm{td},{ }^{3} J_{\mathrm{H}, \mathrm{H}}=7.7 \mathrm{~Hz},{ }^{4} J_{\mathrm{H}, \mathrm{H}}=1.5 \mathrm{~Hz}, 1 \mathrm{H}, 7{ }^{\prime \prime}-\mathrm{H}\right)$, $7.48\left(\mathrm{td},{ }^{3} J_{\mathrm{H}, \mathrm{H}}=7.7 \mathrm{~Hz},{ }^{4} J_{\mathrm{H}, \mathrm{H}}=1.0 \mathrm{~Hz}, 1 \mathrm{H}, 6 "-\mathrm{H}\right), 7.70-7.76(\mathrm{~m}, 3 \mathrm{H}, 3-\mathrm{H}, 5-\mathrm{H}$, $3 "-\mathrm{H}), 7.90\left(\mathrm{dd},{ }^{3} J_{\mathrm{H}, \mathrm{H}}=7.7 \mathrm{~Hz},{ }^{4} J_{\mathrm{H}, \mathrm{H}}=1.5 \mathrm{~Hz}, 1 \mathrm{H}, 5^{\prime \prime}-\mathrm{H}\right), 7.97\left(\mathrm{~d},{ }^{3} J_{\mathrm{H}, \mathrm{H}}=7.9 \mathrm{~Hz}\right.$, $\left.1 \mathrm{H}, 4^{\prime \prime}-\mathrm{H}\right), 8.13\left(\mathrm{~d},{ }^{3} J_{\mathrm{H}, \mathrm{H}}=7.7 \mathrm{~Hz}, 1 \mathrm{H}, 8 "-\mathrm{H}\right), 8.33\left(\mathrm{~d},{ }^{4} J_{\mathrm{H}, \mathrm{H}}=1.5 \mathrm{~Hz}, 1 \mathrm{H}, 1 "-\mathrm{H}\right)$, $8.71-8.76(\mathrm{~m}, 2 \mathrm{H}, 2-\mathrm{H}, 6-\mathrm{H}) \mathrm{ppm}$.

${ }^{13}$ C NMR $\left(101 \mathrm{MHz}, \mathrm{CDCl}_{3}\right): \delta=34.2\left(\mathrm{C}-5^{\prime}, \mathrm{C}-7^{\prime}\right), 34.4,34.9$ (C-4', C-8', C-9', C-10'), 45.3, 45.3 (C-1', C-3'), 57.2 (C-6'/9"), 120.7 (C-5"), 120.8 (C-4"), 122.7 (C-3, C-5), 127.3 (C-3"), 127.5 (C-7"), 128.0 (C-1", C-6"), 129.4 (C-8"), 135.3 (C-2"), 140.0, 143.2 (C-4a", C-4b"), 147.4 (C-2, C-6), 150.3 (C-8a" or C-9a"), 150.6 (C-4) , 151.0 


\section{Chapter 22 COMPOUNDS}

(C-9a" or C-8a"), 215.67 (C-2') ppm.

MS (ESI, pos.): $m / z(\%)=378(100)[\mathrm{M}+\mathrm{H}]^{+}$.

$\operatorname{TLC}\left(\mathrm{SiO}_{2}\right): R_{\mathrm{f}}=0.48\left(\mathrm{CH}_{2} \mathrm{Cl}_{2} / \mathrm{EtOAc}=10: 1\right)$.

Fluorescence $(\mathrm{MeOH}$, exc. at $290 \mathrm{~nm}): \lambda_{\mathrm{em}}^{\max }=373 \mathrm{~nm} ; \Phi_{\mathrm{f}}=0.23 .^{\dagger}$

\subsection{Compound 42}

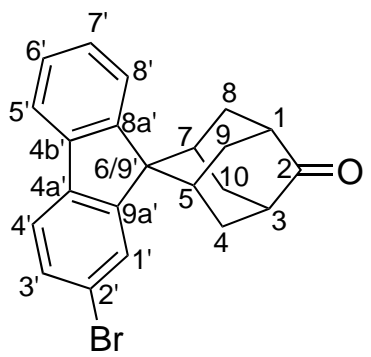

Compound 43b (100 mg, $333 \mu \mathrm{mol}, 1.00 \mathrm{eq})$ was dissolved in dry $\mathrm{CH}_{2} \mathrm{Cl}_{2}(5 \mathrm{ml})$ in a screw cap pressure tube. A solution of bromine in $\mathrm{CH}_{2} \mathrm{Cl}_{2}(0.25 \mathrm{M}, 1.6 \mathrm{ml}$, $0.40 \mathrm{mmol}, 1.2 \mathrm{eq}$ ) was added and the reaction vessel was sealed. The solution was stirred at room temperature for $17 \mathrm{~h}$. Monitoring by ${ }^{1} \mathrm{H}$ NMR spectroscopy detected the starting material $\mathbf{4 3 b}$, the desired product $\mathbf{4 2}$ and the dibrominated compound 44 in a molar ratio of 15:75:10. Stirring at $40{ }^{\circ} \mathrm{C}$ for $27 \mathrm{~h}$ resulted in a reaction mixture with 6:79:16 molar ratios of the products. The reaction was quenched by addition of sat. aq. sodium thiosulfate $(5 \mathrm{ml})$ and water $(5 \mathrm{ml})$. The mixture was extracted with EtOAc $(3 \times 15 \mathrm{ml})$, and the combined organic solutions were dried over $\mathrm{Na}_{2} \mathrm{SO}_{4}$. Concentration in vacuo gave $133 \mathrm{mg}$ of brownish solid consisting of the desired product $42(79 \% \mathrm{w} / \mathrm{w})$, dibromo derivative 44 (16\% $\mathrm{w} / \mathrm{w})$ and compound $\mathbf{4 3 b}(6 \% \mathrm{w} / \mathrm{w})$. This crude product contained $105 \mathrm{mg}$ of compound 42 ( $276 \mu \mathrm{mol}, 83 \%$ yield) and was used without further purification.

${ }^{1} \mathbf{H}$ NMR $\left(400 \mathrm{MHz}, \mathrm{CDCl}_{3}\right): \delta=1.76(\mathrm{~s}, 2 \mathrm{H}, 5-\mathrm{H}, 7-\mathrm{H}), 1.92-2.03\left(\mathrm{~m}, 4 \mathrm{H}, 4-\mathrm{H}_{\mathrm{a}}\right.$, $\left.8-\mathrm{H}_{\mathrm{a}}, 9-\mathrm{H}_{\mathrm{a}}, 10-\mathrm{H}_{\mathrm{a}}\right), 2.84-2.93(\mathrm{~m}, 2 \mathrm{H}, 1-\mathrm{H}, 3-\mathrm{H}), 3.03-3.26\left(\mathrm{~m}, 4 \mathrm{H}, 4-\mathrm{H}_{\mathrm{b}}, 8-\mathrm{H}_{\mathrm{b}}\right.$, $\left.9-\mathrm{H}_{\mathrm{b}}, 10-\mathrm{H}_{\mathrm{b}}\right), 7.35\left(\mathrm{td},{ }^{3} J_{\mathrm{H}, \mathrm{H}}=7.7 \mathrm{~Hz},{ }^{4} J_{\mathrm{H}, \mathrm{H}}=1.5 \mathrm{~Hz}, 1 \mathrm{H}, 7^{\prime}-\mathrm{H}\right), 7.43\left(\mathrm{td},{ }^{3} J_{\mathrm{H}, \mathrm{H}}=\right.$ $\left.7.7 \mathrm{~Hz},{ }^{4} J_{\mathrm{H}, \mathrm{H}}=1.2 \mathrm{~Hz}, 1 \mathrm{H}, 66^{\prime}-\mathrm{H}\right), 7.56\left(\mathrm{dd},{ }^{3} J_{\mathrm{H}, \mathrm{H}}=8.1 \mathrm{~Hz},{ }^{4} J_{\mathrm{H}, \mathrm{H}}=1.7 \mathrm{~Hz}, 1 \mathrm{H}, 3^{\prime}-\mathrm{H}\right)$, $7.69\left(\mathrm{~d},{ }^{3} J_{\mathrm{H}, \mathrm{H}}=8.1 \mathrm{~Hz}, 1 \mathrm{H}, 4^{\prime}-\mathrm{H}\right), 7.79\left(\mathrm{dd},{ }^{3} J_{\mathrm{H}, \mathrm{H}}=7.7 \mathrm{~Hz},{ }^{4} J_{\mathrm{H}, \mathrm{H}}=1.2 \mathrm{~Hz}, 1 \mathrm{H}, 8^{\prime}-\mathrm{H}\right)$, $8.07\left(\mathrm{~d},{ }^{3} J_{\mathrm{H}, \mathrm{H}}=7.7 \mathrm{~Hz}, 1 \mathrm{H}, 5^{\prime}-\mathrm{H}\right), 8.21\left(\mathrm{~d},{ }^{3} J_{\mathrm{H}, \mathrm{H}}=1.7 \mathrm{~Hz}, 1 \mathrm{H}, 1^{\prime}-\mathrm{H}\right) \mathrm{ppm}$.

\footnotetext{
${ }^{\dagger}$ 7-methoxycoumarin in $\mathrm{MeOH}$ as a fl. quantum yield standard with $\Phi_{\mathrm{f}}=0.05^{[169]}$
} 
MS (ESI, pos.): $m / z(\%)=389 / 381(100)[\mathrm{M}+\mathrm{H}]^{+}$.

$\operatorname{TLC}\left(\mathrm{SiO}_{2}\right): R_{\mathrm{f}}=0.33($ cyclohexane $/$ EtOAc $=7: 3)$.

\subsection{Compound 43b}

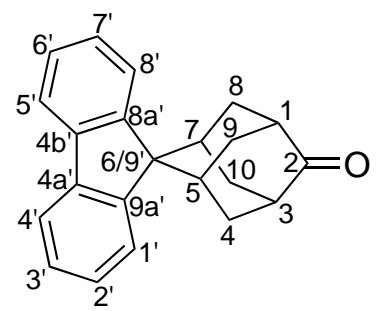

A mixture of acetic acid and $37 \%$ aq. $\mathrm{HCl}(70: 1,10.8 \mathrm{ml})$ was added to compound $39 \mathrm{~b}(613 \mathrm{mg}, 1.93 \mathrm{mmol})$ in a screw cap pressure tube, and the reaction vessel was sealed. The reaction mixture was heated at $140^{\circ} \mathrm{C}$ for $1 \mathrm{~h}$ while stirring. After cooling to r.t., water $(100 \mathrm{ml})$ was added, and a colorless precipitate formed. The suspension was extracted with $\mathrm{CH}_{2} \mathrm{Cl}_{2}(3 \times 50 \mathrm{ml})$, and the combined organic solutions were washed with water $(50 \mathrm{ml})$. The aq. layer was extracted with $\mathrm{CH}_{2} \mathrm{Cl}_{2}(20 \mathrm{ml})$ and the combined organic solutions were dried over $\mathrm{Na}_{2} \mathrm{SO}_{4}$. Concentration in vacuo gave $568 \mathrm{mg}(1.89 \mathrm{mmol}, 98 \%)$ of the title compound as colorless solid.

${ }^{1} \mathbf{H}$ NMR $\left(400 \mathrm{MHz}, \mathrm{CDCl}_{3}\right): \delta=1.73-1.78(\mathrm{~m}, 2 \mathrm{H}, 5-\mathrm{H}, 7-\mathrm{H}), 1.97\left(\mathrm{~d},{ }^{2} J_{\mathrm{H}, \mathrm{H}}=\right.$ $\left.13.0 \mathrm{~Hz}, 4 \mathrm{H}, 4-\mathrm{H}_{\mathrm{a}}, 8-\mathrm{H}_{\mathrm{a}}, 9-\mathrm{H}_{\mathrm{a}}, 10-\mathrm{H}_{\mathrm{a}}\right), 2.85-2.90(\mathrm{~m}, 2 \mathrm{H}, 1-\mathrm{H}, 3-\mathrm{H}), 3.22\left(\mathrm{~d},{ }^{2} J_{\mathrm{H}, \mathrm{H}}\right.$ $\left.=13.0 \mathrm{~Hz}, 4 \mathrm{H}, 4-\mathrm{H}_{\mathrm{b}}, 8-\mathrm{H}_{\mathrm{b}}, 9-\mathrm{H}_{\mathrm{b}}, 10-\mathrm{H}_{\mathrm{b}}\right), 7.33\left(\mathrm{td},{ }^{3} J_{\mathrm{H}, \mathrm{H}}=7.7 \mathrm{~Hz},{ }^{4} J_{\mathrm{H}, \mathrm{H}}=1.4 \mathrm{~Hz}\right.$, $\left.2 \mathrm{H}, 2^{\prime}-\mathrm{H}, 7^{\prime}-\mathrm{H}\right), 7.43\left(\mathrm{td},{ }^{3} J_{\mathrm{H}, \mathrm{H}}=7.7 \mathrm{~Hz},{ }^{4} J_{\mathrm{H}, \mathrm{H}}=0.9 \mathrm{~Hz}, 2 \mathrm{H}, 3^{\prime}-\mathrm{H}, 66^{\prime}-\mathrm{H}\right), 7.84(\mathrm{dd}$, $\left.{ }^{3} J_{\mathrm{H}, \mathrm{H}}=7.7 \mathrm{~Hz},{ }^{4} J_{\mathrm{H}, \mathrm{H}}=1.4 \mathrm{~Hz}, 2 \mathrm{H}, 4^{\prime}-\mathrm{H}, 5^{\prime}-\mathrm{H}\right), 8.09\left(\mathrm{~d}_{\mathrm{br}},{ }^{3} J_{\mathrm{H}, \mathrm{H}}=7.7 \mathrm{~Hz}, 2 \mathrm{H}, 1^{\prime}-\mathrm{H}\right.$, 8'-H) ppm.

${ }^{13}$ C NMR $\left(101 \mathrm{MHz}, \mathrm{CDCl}_{3}\right): \delta=34.2$ (C-5, C-7), 34.6 (C-4, C-8, C-9, C-10), 45.4 (C-1, C-3), 56.9 (C-6/9'), 120.0 (C-4', C-5'), 126.5 (C-2', C-7'), 127.7 (C-3', C-6'), 129.1 (C-1', C-8'), 141.1 (C-4a', C-4b'), 149.8 (C-8a', C-9a'), 216.5 (C-2) ppm.

GC-MS (EI): $m / z(\%)=300(100)\left[\mathrm{M}^{+\cdot}\right]$.

$\operatorname{TLC}\left(\mathrm{SiO}_{2}\right): R_{\mathrm{f}}=0.37($ cyclohexane/EtOAc $=7: 3)$. 


\section{Chapter 22 COMPOUNDS}

\subsection{Compound 44}

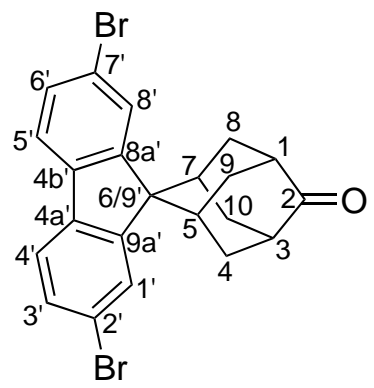

Bromination of compound $\mathbf{4 3 b}$ was carried out with elementary bromine in the presence of catalytic amounts of iodine. ${ }^{[170]}$

To compound 43b (125 mg, $416 \mu \mathrm{mol}, 1.00 \mathrm{eq})$ in $\mathrm{CH}_{2} \mathrm{Cl}_{2}(4.0 \mathrm{ml})$, iodine ( $1 \mathrm{mg}$, $4 \mu \mathrm{mol}, 0.01 \mathrm{eq})$ was added at room temperature. The solution was cooled to $0{ }^{\circ} \mathrm{C}$ and stirred in the dark. A solution of bromine in $\mathrm{CH}_{2} \mathrm{Cl}_{2}(0.25 \mathrm{M}, 4.2 \mathrm{ml}, 1.0 \mathrm{mmol}$, $2.5 \mathrm{eq}$ ) was added dropwise at $0{ }^{\circ} \mathrm{C}$ over a period of $2 \mathrm{~min}$. The reaction mixture was stirred at room temperature for $17 \mathrm{~h}$ and then at $40{ }^{\circ} \mathrm{C}$ for $8 \mathrm{~h}$. The reaction was monitored by ${ }^{1} \mathrm{H}$ NMR spectroscopy; small samples of the reaction mixture were worked-up. Additional solution of bromine in $\mathrm{CH}_{2} \mathrm{Cl}_{2}(0.25 \mathrm{M}, 0.33 \mathrm{ml}$, $83 \mu \mathrm{mol}, 0.20 \mathrm{eq}$ ) was added dropwise at $0^{\circ} \mathrm{C}$, and the reaction mixture was stirred at $40{ }^{\circ} \mathrm{C}$ for additional $3 \mathrm{~d}$. The reaction mixture was quenched by adding aq. sodium thiosulfate $(1.0 \mathrm{M}, 40 \mathrm{ml})$ and extracted with $\mathrm{CH}_{2} \mathrm{Cl}_{2}(3 \times 40 \mathrm{ml})$. The combined organic solutions were dried over $\mathrm{Na}_{2} \mathrm{SO}_{4}$. Concentration in vacuo gave $184 \mathrm{mg}(401 \mu \mathrm{mol}, 97 \%)$ of the pure title compound as light yellow solid.

${ }^{1} \mathbf{H}$ NMR $\left(400 \mathrm{MHz}, \mathrm{CDCl}_{3}\right): \delta=1.71-1.76(\mathrm{~m}, 2 \mathrm{H}, 5-\mathrm{H}, 7-\mathrm{H}), 1.93-2.03(\mathrm{~m}$, $\left.4 \mathrm{H}, 4-\mathrm{H}_{\mathrm{a}}, 8-\mathrm{H}_{\mathrm{a}}, 9-\mathrm{H}_{\mathrm{a}}, 10-\mathrm{H}_{\mathrm{a}}\right), 2.85-2.92(\mathrm{~m}, 2 \mathrm{H}, 1-\mathrm{H}, 3-\mathrm{H}), 3.01-3.12(\mathrm{~m}, 4 \mathrm{H}$, $\left.4-\mathrm{H}_{\mathrm{b}}, 8-\mathrm{H}_{\mathrm{b}}, 9-\mathrm{H}_{\mathrm{b}}, 10-\mathrm{H}_{\mathrm{b}}\right), 7.55\left(\mathrm{dd},{ }^{3} J_{\mathrm{H}, \mathrm{H}}=8.1 \mathrm{~Hz},{ }^{4} J_{\mathrm{H}, \mathrm{H}}=1.6 \mathrm{~Hz}, 2 \mathrm{H}, 3^{\prime}-\mathrm{H}, 66^{\prime}-\mathrm{H}\right)$, $7.63\left(\mathrm{~d},{ }^{3} J_{\mathrm{H}, \mathrm{H}}=8.1 \mathrm{~Hz}, 2 \mathrm{H}, 4^{\prime}-\mathrm{H}, 5^{\prime}-\mathrm{H}\right), 8.18\left(\mathrm{~d},{ }^{4} J_{\mathrm{H}, \mathrm{H}}=1.6 \mathrm{~Hz}, 2 \mathrm{H}, 1^{\prime}-\mathrm{H}, 8^{\prime}-\mathrm{H}\right) \mathrm{ppm}$.

${ }^{13}$ C NMR $\left(101 \mathrm{MHz}, \mathrm{CDCl}_{3}\right): \delta=33.9$ (C-5, C-7), 34.4 (C-4, C-8, C-9, C-10), 45.0 (C-1, C-3), 57.5 (C-6/9'), 120.8 (C-2', C-7'), 121.2 (C-4', C-5'), 131.0 (C-3', C-6'), 132.2 (C-1', C-8'), 139.0 (C-4a', C-4b') 151.3 (C-8a', C-9a'), 215.4 (C-2) ppm.

$\operatorname{TLC}\left(\mathrm{SiO}_{2}\right): R_{\mathrm{f}}=0.51($ cyclohexane $/$ EtOAc $=7: 3)$. 


\subsection{Compound 45}

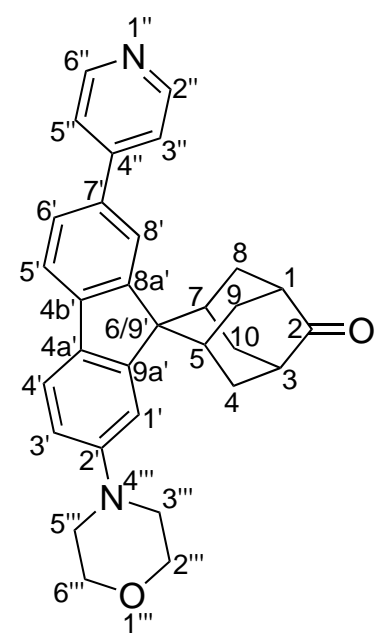

According to GP3, alcohol 49 (49 mg, $0.10 \mathrm{mmol}, 1.0 \mathrm{eq})$ was treated with conc. $\mathrm{H}_{2} \mathrm{SO}_{4}(0.13 \mathrm{ml})$ in $n$-octane $(2.4 \mathrm{ml})$ and stirred for $60 \mathrm{~min}$. The aq. work-up included quenching with $1.0 \mathrm{M}$ aq. $\mathrm{Na}_{2} \mathrm{CO}_{3}(40 \mathrm{ml})$ followed by extraction with $\mathrm{CH}_{2} \mathrm{Cl}_{2}(6 \times 20 \mathrm{ml})$. Concentration in vacuo left $44 \mathrm{mg}$ of brown solid. The crude product was dissolved in a 1:1 mixture of $\mathrm{CHCl}_{3}$ and EtOAc $(4 \mathrm{ml})$ and loaded onto a silica gel column. Column chromatography on silica gel $\left(\mathrm{EtOAc} / \mathrm{CHCl}_{3}=\right.$ $\left.9: 1+1 \% \mathrm{NEt}_{3}\right)$ gave $38 \mathrm{mg}(81 \mu \mathrm{mol}, 82 \%)$ of the title compound as light brown solid.

NOTE: The product is poorly soluble in neat EtOAc; sufficient dilution during the chromatography is recommended in order to avoid precipitation.

${ }^{1} \mathbf{H}$ NMR $\left(400 \mathrm{MHz}, \mathrm{CDCl}_{3}\right): \delta=1.80-1.88(\mathrm{~m}, 2 \mathrm{H}, 5-\mathrm{H}, 7-\mathrm{H}), 1.94-2.10(\mathrm{~m}$, $\left.4 \mathrm{H}, 4-\mathrm{H}_{\mathrm{a}}, 8-\mathrm{H}_{\mathrm{a}}, 9-\mathrm{H}_{\mathrm{a}}, 10-\mathrm{H}_{\mathrm{a}}\right), 2.86-2.99(\mathrm{~m}, 2 \mathrm{H}, 1-\mathrm{H}, 3-\mathrm{H}), 3.14-3.32(\mathrm{~m}, 8 \mathrm{H}$,

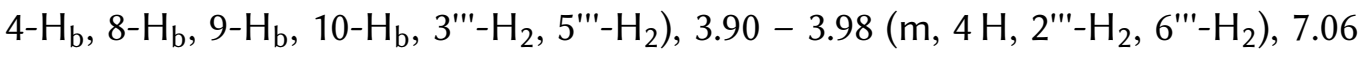
$\left(\mathrm{dd},{ }^{3} J_{\mathrm{H}, \mathrm{H}}=8.4 \mathrm{~Hz},{ }^{4} J_{\mathrm{H}, \mathrm{H}}=2.1 \mathrm{~Hz}, 1 \mathrm{H}, 3^{\prime}-\mathrm{H}\right), 7.65\left(\mathrm{~d},{ }^{4} J_{\mathrm{H}, \mathrm{H}}=2.1 \mathrm{~Hz}, 1 \mathrm{H}, 1^{\prime}-\mathrm{H}\right), 7.67$ - $7.80\left(\mathrm{~m}, 4 \mathrm{H}, 4^{\prime}-\mathrm{H}, 6^{\prime}-\mathrm{H}, 3^{\prime \prime}-\mathrm{H}, 5^{\prime \prime}-\mathrm{H}\right), 7.84\left(\mathrm{~d},{ }^{3} J_{\mathrm{H}, \mathrm{H}}=7.9 \mathrm{~Hz}, 1 \mathrm{H}, 5^{\prime}-\mathrm{H}\right), 8.29(\mathrm{~s}$, $\left.1 \mathrm{H}, 8^{\prime}-\mathrm{H}\right), 8.66-8.79\left(\mathrm{~m}, 2 \mathrm{H}, 2^{\prime \prime}-\mathrm{H}, 6^{\prime \prime}-\mathrm{H}\right) \mathrm{ppm}$.

${ }^{13}$ C NMR (101 MHz, CDCl 3$): \delta=34.1$ (C-5, C-7), 34.3, 34.9 (C-4, C-8, C-9, C-10), 45.2 (C-1, C-3), 50.2 (C-3"', C-5"'), 57.2 (C-6/9'), 67.0 (C-2'", C-6"'), 116.0 (C-3'), 118.0 (C-1'), 119.9 (C-5'), 121.1 (C-4'), 122.5 (C-3", C-5"), 127.3 (C-6'), 128.0 (C-8'), 132.5 (C-4a'), 133.7 (C-7'), 143.5 (C-4b'), 147.1 (C-2", C-6"), 150.4 (C-8a'), 151.2 (C-2'), 151.7 (C-9a'), 152.3 (C-4"), 215.5 (C-2) ppm. 


\section{Chapter 22 COMPOUNDS}

MS (ESI, pos.): $m / z(\%)=463.2(100)[\mathrm{M}+\mathrm{H}]^{+}$.

HRMS (ESI): $m / z$ calc. for $\mathrm{C}_{31} \mathrm{H}_{31} \mathrm{~N}_{2} \mathrm{O}_{2}[\mathrm{M}+\mathrm{H}]^{+}$463.2380; found 463.2384

$\operatorname{TLC}\left(\mathrm{SiO}_{2}\right): R_{\mathrm{f}}=0.19\left(\mathrm{EtOAc} / \mathrm{CHCl}_{3}=9: 1+1 \% \mathrm{NEt}_{3}\right)$.

UV/Vis (EtOAc): $\lambda_{\text {abs }}^{\max }=341 \mathrm{~nm}, \varepsilon=32000 \mathrm{M}^{-1} \mathrm{~cm}^{-1}$.

Fluorescence $\left(\mathrm{EtOAc}+1 \% \mathrm{NEt}_{3}\right.$, exc. at $\left.315 \mathrm{~nm}\right): \lambda_{\mathrm{em}}^{\max }=452 \mathrm{~nm} ; \Phi_{\mathrm{f}}=0.52 .^{\dagger}$

\subsection{4-[2'-Bromo-4'-(4-pyridyl)-4-biphenylyl]- morpholine (48)}

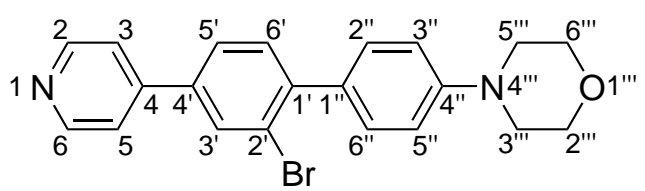

To a suspension of amine 58 (50 mg, $0.15 \mathrm{mmol}, 1.0 \mathrm{eq})$ in water $(0.13 \mathrm{ml}), 48 \%$ aq. $\mathrm{HBr}(0.20 \mathrm{ml})$ was added. The clear yellow solution was cooled to $-5^{\circ} \mathrm{C}$ and $2.8 \mathrm{M}$ aq. sodium nitrite $(83 \mu \mathrm{l}, 0.23 \mathrm{mmol}, 1.6 \mathrm{eq})$ was added dropwise. After stirring at $0{ }^{\circ} \mathrm{C}$ for $1 \mathrm{~h}$, the reaction mixture was added to a pre-cooled $1.0 \mathrm{~m}$ solution of copper $(\mathrm{I})$ bromide in $48 \%$ aq. $\mathrm{HBr}(0.20 \mathrm{ml})$ at $0{ }^{\circ} \mathrm{C}$. The brown slurry was allowed to warm up to room temperature. After stirring for $2 \mathrm{~d}$, the reaction mixture was diluted with water $(5 \mathrm{ml})$, and the clear red solution was extracted with EtOAc $(5 \mathrm{ml})$. The aq. layer was neutralized with aq. sat. $\mathrm{NaHCO}_{3}(10 \mathrm{ml})$ and extracted with EtOAc $(2 \times 10 \mathrm{ml})$ and $\mathrm{CH}_{2} \mathrm{Cl}_{2}(2 \times 10 \mathrm{ml})$. The combined organic solutions were dried over $\mathrm{Na}_{2} \mathrm{SO}_{4}$ and concentrated in vacuo to give $49 \mathrm{mg}$ of yellow foam. Column chromatography on silica gel $\left(\mathrm{CH}_{2} \mathrm{Cl}_{2} / \mathrm{EtOAc}=4: 1+1 \% \mathrm{NEt}_{3}\right)$ gave $45 \mathrm{mg}(0.12 \mathrm{mmol}, 77 \%)$ of the title compound as yellow solid.

${ }^{1} \mathbf{H}$ NMR $\left(400 \mathrm{MHz}, \mathrm{CDCl}_{3}\right): \delta=3.22-3.28\left(\mathrm{~m}, 4 \mathrm{H}, 3^{\prime \prime}-\mathrm{H}_{2}, 5^{\prime \prime}-\mathrm{H}_{2}\right), 3.86-3.92$

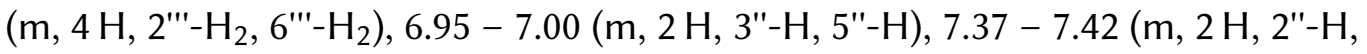
6"-H), $7.44\left(\mathrm{~d},{ }^{3} \mathrm{~J}_{\mathrm{H}, \mathrm{H}}=8.0 \mathrm{~Hz}, 1 \mathrm{H}, 6^{\prime}-\mathrm{H}\right), 7.51-7.55(\mathrm{~m}, 2 \mathrm{H}, 3-\mathrm{H}, 5-\mathrm{H}), 7.61$ (dd, $\left.{ }^{3} J_{\mathrm{H}, \mathrm{H}}=8.0 \mathrm{~Hz},{ }^{4} J_{\mathrm{H}, \mathrm{H}}=1.9 \mathrm{~Hz}, 1 \mathrm{H}, 5^{\prime}-\mathrm{H}\right), 7.64\left(\mathrm{~d},{ }^{4} J_{\mathrm{H}, \mathrm{H}}=1.9 \mathrm{~Hz}, 1 \mathrm{H}, 3^{\prime}-\mathrm{H}\right), 8.66-$ $8.71(\mathrm{~m}, 2 \mathrm{H}, 2-\mathrm{H}, 6-\mathrm{H}) \mathrm{ppm}$.

${ }^{13}$ C NMR $\left(101 \mathrm{MHz}, \mathrm{CDCl}_{3}\right): \delta=48.9$ (C-3"', C-5"'), 67.0 (C-2"', C-6"'), 114.8 (C-3", C-5"), 121.6 (C-3, C-5), 123.7 (C-2'), 126.0 (C-5'), 130.4 (C-2", C-6"), 131.5 (C-3'),

\footnotetext{
${ }^{+}$Coumarin 120 in $\mathrm{MeOH}$ as a fl. quantum yield standard with $\Phi_{\mathrm{f}}=0.51^{[132]}$
} 
131.8 (C-6'), 132.0 (C-1"), 138.2 (C-4'), 143.1 (C-1'), 146.9 (C-4), 150.2 (C-2, C-6), $150.9(\mathrm{C}-4 ") \mathrm{ppm}$.

MS (ESI, pos.): $m / z(\%)=395.1 / 397.1(100)[\mathrm{M}+\mathrm{H}]^{+}$.

HRMS (ESI): $m / z$ calc. for $\mathrm{C}_{21} \mathrm{H}_{20} \mathrm{~N}_{2} \mathrm{OBr}[\mathrm{M}+\mathrm{H}]^{+}$395.0754; found 395.0753.

$\operatorname{TLC}\left(\mathrm{SiO}_{2}\right): R_{\mathrm{f}}=0.17\left(\mathrm{CH}_{2} \mathrm{Cl}_{2} / \mathrm{EtOAc}=4: 1+1 \% \mathrm{NEt}_{3}\right)$.

\subsection{Compound 49}

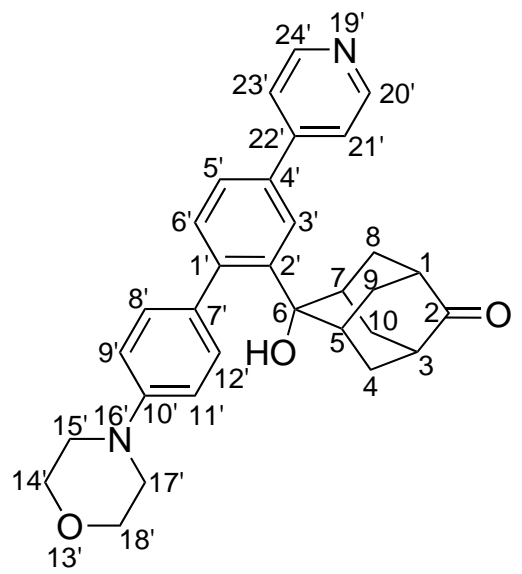

According to GP2, bromide 48 ( $100 \mathrm{mg}, 253 \mu \mathrm{mol}, 1.00 \mathrm{eq}$ ) was lithiated with $2.5 \mathrm{M}$ $n$-BuLi in $n$-hexane $(0.15 \mathrm{ml}, 0.38 \mathrm{mmol}, 1.5 \mathrm{eq})$ in dry THF $(3.0 \mathrm{ml})$. The solution of lithiated 48 was transferred via syringe to a solution of 2,6-adamantanedione $(83 \mathrm{mg}, 0.51 \mathrm{mmol}, 2.0 \mathrm{eq})$ in dry THF $(2.0 \mathrm{ml})$. The reaction mixture was stirred at $-78{ }^{\circ} \mathrm{C}$ for $10 \mathrm{~min}$ and at ambient temperature for $4 \mathrm{~h}$. Aq. work-up included addition of sat. aq. $\mathrm{NH}_{4} \mathrm{Cl}(10 \mathrm{ml})$ and water $(10 \mathrm{ml})$ followed by extraction with $\mathrm{CH}_{2} \mathrm{Cl}_{2}(5 \times 10 \mathrm{ml})$ and gave $177 \mathrm{mg}$ of brown solid. Purification of the crude product by flash column chromatography on silica gel $\left(\mathrm{EtOAc} / \mathrm{CHCl}_{3}=9: 1+1 \%\right.$ $\left.\mathrm{NEt}_{3}\right)$ gave $85 \mathrm{mg}(0.18 \mathrm{mmol}, 70 \%)$ of the title compound as yellow solid.

${ }^{1} \mathbf{H}$ NMR $\left(400 \mathrm{MHz}, \mathrm{CDCl}_{3}\right): \delta=1.70-1.77\left(\mathrm{~m}, 2 \mathrm{H},\left[4-\mathrm{H}_{\mathrm{a}}, 10-\mathrm{H}_{\mathrm{a}}\right]\right.$ or $\left[8-\mathrm{H}_{\mathrm{a}}\right.$, $\left.\left.9-\mathrm{H}_{\mathrm{a}}\right]\right), 1.79-1.87\left(\mathrm{~m}, 2 \mathrm{H},\left[8-\mathrm{H}_{\mathrm{a}}, 9-\mathrm{H}_{\mathrm{a}}\right]\right.$ or $\left.\left[4-\mathrm{H}_{\mathrm{a}}, 10-\mathrm{H}_{\mathrm{a}}\right]\right), 1.92-2.03\left(\mathrm{~m}, 2 \mathrm{H},\left[8-\mathrm{H}_{\mathrm{b}}\right.\right.$, 9- $\left.\mathrm{H}_{\mathrm{b}}\right]$ or $\left.\left[4-\mathrm{H}_{\mathrm{b}}, 10-\mathrm{H}_{\mathrm{b}}\right]\right), 2.30-2.36(\mathrm{~m}, 1 \mathrm{H}, 1-\mathrm{H}$ or $3-\mathrm{H}), 2.37-2.42(\mathrm{~m}, 1 \mathrm{H}, 3-\mathrm{H}$ or $1-\mathrm{H}), 2.52-2.63\left(\mathrm{~m}, 4 \mathrm{H},\left[4-\mathrm{H}_{\mathrm{b}}, 10-\mathrm{H}_{\mathrm{b}}\right]\right.$ or $\left.\left[8-\mathrm{H}_{\mathrm{b}}, 9-\mathrm{H}_{\mathrm{b}}\right], 5-\mathrm{H}, 7-\mathrm{H}\right), 3.19-3.24$ $\left(\mathrm{m}, 4 \mathrm{H}, 15^{\prime}-\mathrm{H}_{2}, 17^{\prime}-\mathrm{H}_{2}\right), 3.86-3.91\left(\mathrm{~m}, 4 \mathrm{H}, 14^{\prime}-\mathrm{H}_{2}, 18^{\prime}-\mathrm{H}_{2}\right), 4.80\left(\mathrm{~s}_{\mathrm{br}}, 1 \mathrm{H}, \mathrm{OH}\right)$, $6.90-6.96\left(\mathrm{~m}, 2 \mathrm{H}, 9^{\prime}-\mathrm{H}, 11^{\prime}-\mathrm{H}\right), 7.28-7.35$ (m, 3 H, 6'-H, 8'-H, 12'-H), $7.54-7.62$ $\left(\mathrm{m}, 3 \mathrm{H}, 5^{\prime}-\mathrm{H}, 21^{\prime}-\mathrm{H}, 23^{\prime}-\mathrm{H}\right), 7.86\left(\mathrm{~d},{ }^{4} J_{\mathrm{H}, \mathrm{H}}=1.9 \mathrm{~Hz}, 1 \mathrm{H}, 3^{\prime}-\mathrm{H}\right), 8.57-8.70(\mathrm{~m}, 2 \mathrm{H}$, 


\section{Chapter 22 $\mid$ COMPOUNDS}

$\left.20^{\prime}-\mathrm{H}, 24^{\prime}-\mathrm{H}\right) \mathrm{ppm}$.

${ }^{13}$ C NMR $\left(101 \mathrm{MHz}, \mathrm{CDCl}_{3}\right): \delta=34.3$ ([C-4, C-10] or [C-8, C-9]), $34.6(\mathrm{C}-5, \mathrm{C}-7)$, 36.2 ([C-8, C-9] or [C-4, C-10]), 44.9, 45.4 (C-1, C-3), 49.1 (C-15', C-17'), 66.9 (C-14', C-18'), 76.4 (C-6), 115.0 (C-9', C-11'), 122.13 (C-21', C-23'), 125.7 (C-5'), 126.0 (C-3'), 129.8 (C-8', C-12'), 134.7 (C-1'), 135.5 (C-6'), 136.1 (C-4'), 142.0 (C-2'), 144.2 (C-1'), 148.6 (C-20', C-24'), 149.9 (C-22'), 150.5 (C-10'), 217.3 (C-2) ppm.

MS (ESI, pos.): $m / z(\%)=481.2(100)[\mathrm{M}+\mathrm{H}]^{+}$.

HRMS (ESI): $m / z$ calc. for $\mathrm{C}_{31} \mathrm{H}_{33} \mathrm{~N}_{2} \mathrm{O}_{3}[\mathrm{M}+\mathrm{H}]^{+}$481.2486; found 481.2486.

$\operatorname{TLC}\left(\mathrm{SiO}_{2}\right): R_{\mathrm{f}}=0.30\left(\mathrm{EtOAc}+1 \% \mathrm{NEt}_{3}\right)$.

\section{$22.154,4^{\prime}$-Dibromo-2-nitrobiphenyl (51) ${ }^{[171]}$}

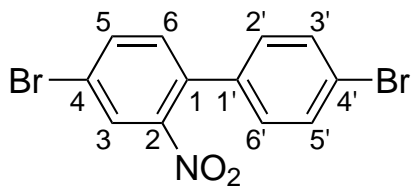

4,4'-Dibromobiphenyl ( $40.0 \mathrm{~g}, 128 \mathrm{mmol}, 1.00 \mathrm{eq})$ was suspended in glacial acetic acid $(600 \mathrm{ml})$ and heated to $100^{\circ} \mathrm{C}$. A mixture of fuming nitric acid $(185 \mathrm{ml})$ and water $(15.0 \mathrm{ml})$ was added dropwise to the resulting colorless clear solution at $100^{\circ} \mathrm{C}$ over a period of $10 \mathrm{~min}$. The reaction mixture was stirred at $100^{\circ} \mathrm{C}$ for $2.5 \mathrm{~h}$. After cooling to $5^{\circ} \mathrm{C}$, the reaction mixture was poured onto ice $(300 \mathrm{~g})$, and a yellow precipitate formed. The precipitate was removed by filtration, washed with water $(2 \times 200 \mathrm{ml})$ and dried in a desiccator over aluminum silicate (Drying pearls Orange) overnight. The yellow solid was crystallized from EtOH. It was dissolved in refluxing $\mathrm{EtOH}(630 \mathrm{ml})$, allowed to cool down to room temperature, cooled to $0^{\circ} \mathrm{C}$ for $1 \mathrm{~h}$, vacuum-filtered and washed with ice-cold EtOH $(50 \mathrm{ml})$. The residue was dried in vacuo, affording $34.5 \mathrm{~g}(96.5 \mathrm{mmol}, 75 \%)$ of the title compound as yellow powder.

${ }^{1} \mathbf{H}$ NMR $\left(400 \mathrm{MHz}, \mathrm{CDCl}_{3}\right): \delta=7.12-7.19\left(\mathrm{~m}, 2 \mathrm{H}, 2^{\prime}-\mathrm{H}, 6^{\prime}-\mathrm{H}\right), 7.29\left(\mathrm{~d},{ }^{3} \mathrm{~J}_{\mathrm{H}, \mathrm{H}}=\right.$ $8.2 \mathrm{~Hz}, 1 \mathrm{H}, 6-\mathrm{H}), 7.52-7.59\left(\mathrm{~m}, 2 \mathrm{H}, 3^{\prime}-\mathrm{H}, 5^{\prime}-\mathrm{H}\right), 7.75\left(\mathrm{dd},{ }^{3} J_{\mathrm{H}, \mathrm{H}}=8.2 \mathrm{~Hz},{ }^{4} J_{\mathrm{H}, \mathrm{H}}=\right.$ $2.0 \mathrm{~Hz}, 1 \mathrm{H}, 5-\mathrm{H}), 8.03\left(\mathrm{~d},{ }^{4} J_{\mathrm{H}, \mathrm{H}}=2.0 \mathrm{~Hz}, 1 \mathrm{H}, 3-\mathrm{H}\right) \mathrm{ppm}$.

${ }^{13}$ C NMR $\left(101 \mathrm{MHz}, \mathrm{CDCl}_{3}\right): \delta=121.95$ (C-4), 123.18 (C-4'), 127.38 (C-3), 129.53 (C-2', C-6'), 132.14 (C-3', C-5'), 133.14 (C-6), 134.23 (C-1), 135.41 (C-1'), 135.68 
(C-5), 149.36 (C-2) ppm.

GC-MS (EI): $m / z(\%)=355 / 357 / 359(30)\left[\mathrm{M}^{+\cdot}\right]$.

HRMS (EI): $m / z$ calc. for $\mathrm{C}_{12} \mathrm{H}_{7} \mathrm{Br}_{2} \mathrm{NO}_{2}\left[\mathrm{M}^{+\cdot}\right] 356.8823$; found 356.8829 .

\subsection{4'-Bromo-4-(4-pyridyl)-2-biphenylylamine (53)}

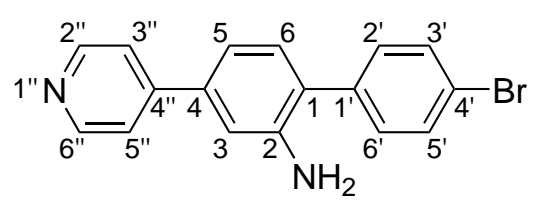

Activated zinc dust ${ }^{\dagger}(4.10 \mathrm{~g}, 63.1 \mathrm{mmol}, 60.0 \mathrm{eq})$ was suspended in a solution of nitro compound 54 (300 mg, $845 \mu \mathrm{mol}, 1.00 \mathrm{eq})$ in dry $\mathrm{CH}_{2} \mathrm{Cl}_{2}(15 \mathrm{ml})$. The suspension was cooled to $0^{\circ} \mathrm{C}$ and $\mathrm{AcOH}(2.1 \mathrm{ml}, 2.2 \mathrm{~g}, 37 \mathrm{mmol}, 44 \mathrm{eq})$ was added dropwise at $0{ }^{\circ} \mathrm{C}$ over a period of $1 \mathrm{~min}$. After $1 \mathrm{~h}$ at $0^{\circ} \mathrm{C}$, the mixture was filtered through Celite ${ }^{\mathrm{TM}}(\varnothing=60 \mathrm{~mm}, h=10 \mathrm{~mm})$. The filter cake was washed with EtOAc $(5 \times 10 \mathrm{ml})$. The combined organic solutions were concentrated in vacuo to give $571 \mathrm{mg}$ of yellow oil. The oil was dissolved in $\mathrm{CH}_{2} \mathrm{Cl}_{2}(25 \mathrm{ml})$, washed with $5 \% \mathrm{NaHCO}_{3}(25 \mathrm{ml})$ and the aq. layer was extracted with $\mathrm{CHCl}_{3}(2 \times 25 \mathrm{ml})$. The combined organic solutions were dried over $\mathrm{Na}_{2} \mathrm{SO}_{4}$ and concentrated in vacuo to give $259 \mathrm{mg}$ of a yellow solid. Column chromatography on silica gel $\left(\mathrm{CH}_{2} \mathrm{Cl}_{2} / \mathrm{EtOAc}=3: 2+1 \% \mathrm{NEt}_{3}\right)$ gave $232 \mathrm{mg}(713 \mu \mathrm{mol}, 84 \%)$ of the title compound as yellow solid.

${ }^{1} \mathbf{H}$ NMR $\left(400 \mathrm{MHz}, \mathrm{DMSO}-\mathrm{d}_{6}\right): \delta=5.08\left(\mathrm{~s}_{\mathrm{br}}, 2 \mathrm{H}, \mathrm{NH}_{2}\right), 7.04\left(\mathrm{dd},{ }^{3} J_{\mathrm{H}, \mathrm{H}}=7.9 \mathrm{~Hz}\right.$, $\left.{ }^{4} J_{\mathrm{H}, \mathrm{H}}=1.9 \mathrm{~Hz}, 1 \mathrm{H}, 5-\mathrm{H}\right), 7.12\left(\mathrm{~d},{ }^{3} J_{\mathrm{H}, \mathrm{H}}=7.9 \mathrm{~Hz}, 1 \mathrm{H}, 6-\mathrm{H}\right), 7.18\left(\mathrm{~d},{ }^{4} J_{\mathrm{H}, \mathrm{H}}=1.9 \mathrm{~Hz}\right.$, $1 \mathrm{H}, 3-\mathrm{H}), 7.40-7.45$ (m, $\left.2 \mathrm{H}, 2^{\prime}-\mathrm{H}, 6^{\prime}-\mathrm{H}\right), 7.59-7.62$ (m, $\left.2 \mathrm{H}, 3^{\prime \prime}-\mathrm{H}, 5^{\prime \prime}-\mathrm{H}\right), 7.62-$ $7.66\left(\mathrm{~m}, 2 \mathrm{H}, 3^{\prime}-\mathrm{H}, 5^{\prime}-\mathrm{H}\right), 8.60-8.64$ (m, $2 \mathrm{H}, 2^{\prime \prime}-\mathrm{H}, 6$ "'-H) ppm.

${ }^{13}$ C NMR $\left(101 \mathrm{MHz}, \mathrm{DMSO}-\mathrm{d}_{6}\right): \delta=113.4(\mathrm{C}-3), 115.1$ (C-5), $120.2(\mathrm{C}-4$ '), 120.9 (C-3", C-5"), 125.3 (C-1), 130.8, 130.8 (C-6, C-3', C-5'), 131.7 (C-3", C-5"), 137.3 (C-4), 138.2 (C-1'), 145.8 (C-2), 147.3 (C-4"), 150.1 (C-2", C-6") ppm.

MS (ESI, pos.): $m / z(\%)=325 / 327(100)[\mathrm{M}+\mathrm{H}]^{+}$.

HRMS (EI): $m / z$ calc. for $\mathrm{C}_{17} \mathrm{H}_{13} \mathrm{BrN}_{2}\left[\mathrm{M}^{+\cdot}\right]$ 324.0262; found 324.0261.

\footnotetext{
${ }^{\dagger} \mathrm{Zn}$ was activated by stirring with $2 \%$ aq. $\mathrm{HCl}$, washing with water, ethanol, acetone and dry diethyl ether before drying in vacuo, according to ref. [172].
} 


\section{Chapter 22 COMPOUNDS}

$\operatorname{TLC}\left(\mathrm{SiO}_{2}\right): R_{\mathrm{f}}=0.20\left(\mathrm{CH}_{2} \mathrm{Cl}_{2} / \mathrm{EtOAc}=1: 1\right)$.

\subsection{4-(4'-Bromo-2-nitro-4-biphenylyl)pyridine (54)}

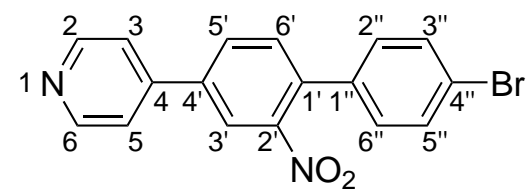

Palladium-catalyzed Suzuki cross-coupling of dibromide 51 and 4-pyridinylboronic acid was carried out according to literature method. ${ }^{[135]}$

To a mixture of 4,4'-dibromo-2-nitro-biphenyl (51, $8.00 \mathrm{~g}, 22.4 \mathrm{mmol}, 1.00 \mathrm{eq}$ ), 4-pyridylboronic acid $(2.75 \mathrm{~g}, 22.4 \mathrm{mmol}, 1.00 \mathrm{eq})$, and $\left[\mathrm{Pd}\left(\mathrm{PPh}_{3}\right)_{4}\right](1.81 \mathrm{~g}$, $1.57 \mathrm{mmol}, 0.07 \mathrm{eq})$ under an argon atmosphere, aqueous $1.0 \mathrm{M} \mathrm{Na} \mathrm{CO}_{3}(20 \mathrm{ml}$, $20 \mathrm{mmol}, 0.89 \mathrm{eq})$ and DMF (72 ml) were added. The mixture was deoxygenated by bubbling argon through the mixture for 5 min. After sealing, the mixture was stirred at $100^{\circ} \mathrm{C}$ for $21 \mathrm{~h}$. After concentrating under reduced pressure, the residue was suspended in a mixture of $\mathrm{CH}_{2} \mathrm{Cl}_{2} / \mathrm{EtOAc}=3: 1(100 \mathrm{ml}$, ultrasonic bath) and loaded onto a silica gel column. Flash column chromatography $\left(\mathrm{CH}_{2} \mathrm{Cl}_{2} / \mathrm{EtOAc}\right.$ $=3: 1)$ yielded a yellow solid which was recrystallized from toluene $(33 \mathrm{ml}$, reflux temperature to $\left.-10{ }^{\circ} \mathrm{C}\right)$ to give $3.96 \mathrm{~g}(11.1 \mathrm{mmol}, 50 \%)$ of the title compound as light yellow crystals.

m.p. $197-203^{\circ} \mathrm{C}$.

${ }^{1}$ H NMR $(400 \mathrm{MHz}$, toluene-d 8$): \delta=6.76-6.81(\mathrm{~m}, 4 \mathrm{H}, 3-\mathrm{H}, 5-\mathrm{H}, 2$ "- $\mathrm{H}, 6$ "-H), $6.85\left(\mathrm{~d},{ }^{3} J_{\mathrm{H}, \mathrm{H}}=8.0 \mathrm{~Hz}, 1 \mathrm{H}, 66^{\prime}-\mathrm{H}\right), 7.10\left(\mathrm{dd},{ }^{3} J_{\mathrm{H}, \mathrm{H}}=8.0 \mathrm{~Hz},{ }^{4} J_{\mathrm{H}, \mathrm{H}}=1.9 \mathrm{~Hz}, 1 \mathrm{H}, 5^{\prime}-\mathrm{H}\right)$, $7.17-7.22\left(\mathrm{~m}, 2 \mathrm{H}, 3^{\prime \prime}-\mathrm{H}, 5^{\prime \prime}-\mathrm{H}\right), 7.63\left(\mathrm{~d},{ }^{4} J_{\mathrm{H}, \mathrm{H}}=1.9 \mathrm{~Hz}, 1 \mathrm{H}, 6^{\prime}-\mathrm{H}\right), 8.53-8.57(\mathrm{~m}$, $2 \mathrm{H}, 2-\mathrm{H}, 6-\mathrm{H}) \mathrm{ppm}$.

${ }^{13} \mathrm{C}$ NMR $\left(101 \mathrm{MHz}\right.$, toluene- $\mathrm{d}_{8}, \mathrm{C}-2$ ' not detected): $\delta=121.06$ (C-3, C-5), 122.53 (C-3'), 123.21 (C-4"), 129.65 (C-2", C-6"), 129.94 (C-5'), 132.19 (C-3", C-5"), 132.22 (C-6'), 135.24 (C-1'), 135.90 (C-1"), 138.98 (C-4'), 144.55 (C-4), 151.03 (C-2, C-6) ppm.

MS (ESI, pos.): $m / z(\%)=355 / 357(100)[\mathrm{M}+\mathrm{H}]^{+}$.

HRMS (ESI): $m / z$ calc. for $\mathrm{C}_{17} \mathrm{H}_{12} \mathrm{~N}_{2} \mathrm{O}_{2} \mathrm{Br}[\mathrm{M}+\mathrm{H}]^{+} 355.0077$; found 355.0076 .

$\operatorname{TLC}\left(\mathrm{SiO}_{2}\right): R_{\mathrm{f}}=0.43\left(\mathrm{CH}_{2} \mathrm{Cl}_{2} / \mathrm{MeOH}=95: 5\right)$. 


\subsection{4'-Bromo-4-(4-pyridyl)-2 biphenylylamino-2,2-dimethylpropionate (55)}

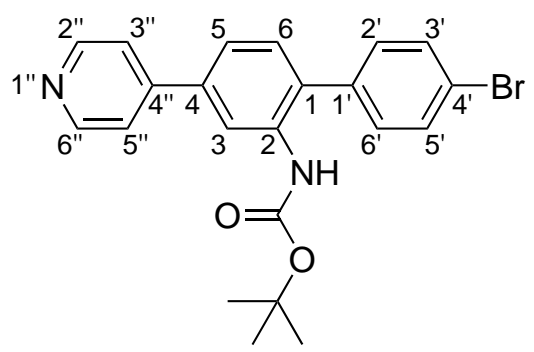

Boc-protection of amine $\mathbf{5 3}$ was prepared by formation of the sodium salt of amine 53 followed by the reaction with di-tert-butylpyrocarbonate (in order to avoid bis-carbamoylation and urea formation). ${ }^{[173]}$

Amine 53 (50 mg, $0.15 \mathrm{mmol}, 1.0 \mathrm{eq})$ was dissolved in dry THF $(0.50 \mathrm{ml})$ and added dropwise a $1.0 \mathrm{M}$ solution of NaHMDS in THF $(0.34 \mathrm{ml}, 0.34 \mathrm{mmol}, 2.2 \mathrm{eq})$ at room temperature over a period of $1 \mathrm{~min}$. The dark brown reaction mixture was stirred at room temperature for $60 \mathrm{~min}$. After cooling to $0{ }^{\circ} \mathrm{C}$, di-tert-butylpyrocarbonate ( $39 \mu \mathrm{l}, 37 \mathrm{mg}, 0.17 \mathrm{mmol}, 1.1 \mathrm{eq}$ ) was added dropwise. The reaction mixture was allowed to warm up to room temperature over a period of $2 \mathrm{~h}$. After stirring for additional $60 \mathrm{~min}$ at room temperature, the reaction was quenched by addition of $0.1 \mathrm{M} \mathrm{HCl}(5.0 \mathrm{ml})$. The mixture was extracted with $\mathrm{CH}_{2} \mathrm{Cl}_{2}(3 \times 5 \mathrm{ml})$. The aq. layer was neutralized by addition of sat. aq. $\mathrm{NaHCO}_{3}(5 \mathrm{ml})$ and extracted with $\mathrm{CH}_{2} \mathrm{Cl}_{2}(2 \times 5 \mathrm{ml})$. The combined organic solutions were dried over $\mathrm{Na}_{2} \mathrm{SO}_{4}$ and concentrated in vacuo to give $68 \mathrm{mg}$ of a yellow solid. The crude product was purified by flash column chromatography on silica gel $(n$-hexane/EtOAc $=1: 1$ ) and gave $52 \mathrm{mg}(0.12 \mathrm{mmol}, 79 \%)$ of the title compound as colorless solid.

${ }^{1} \mathbf{H}$ NMR $\left(400 \mathrm{MHz}, \mathrm{CDCl}_{3}\right): \delta=1.49\left(\mathrm{~s}, 9 \mathrm{H}, 3 \times \mathrm{CH}_{3}\right), 6.52\left(\mathrm{~s}_{\mathrm{br}}, 1 \mathrm{H}, \mathrm{NH}\right), 7.25$ $-7.31\left(\mathrm{~m}, 3 \mathrm{H}, 6-\mathrm{H}, 2^{\prime}-\mathrm{H}, 66^{\prime}-\mathrm{H}\right), 7.39\left(\mathrm{dd},{ }^{3} \mathrm{~J}_{\mathrm{H}, \mathrm{H}}=7.9 \mathrm{~Hz},{ }^{4} J_{\mathrm{H}, \mathrm{H}}=1.6 \mathrm{~Hz}, 1 \mathrm{H}, 5-\mathrm{H}\right)$, $7.63-7.69\left(\mathrm{~m}, 4 \mathrm{H}, 3^{\prime}-\mathrm{H}, 5^{\prime}-\mathrm{H}, 3^{\prime \prime}-\mathrm{H}, 5^{\prime \prime}-\mathrm{H}\right), 6.53\left(\mathrm{~d},{ }^{4} J_{\mathrm{H}, \mathrm{H}}=1.6 \mathrm{~Hz}, 1 \mathrm{H}, 3-\mathrm{H}\right), 8.66$ - 8.69 (m, 2 H, 2"-H, 6"-H) ppm.

${ }^{13} \mathrm{C}$ NMR $\left(101 \mathrm{MHz}, \mathrm{CDCl}_{3}\right): \delta=28.4\left(3 \times \mathrm{CH}_{3}\right), 81.3\left(\underline{\mathrm{C}}\left(\mathrm{CH}_{3}\right)_{3}\right), 118.5(\mathrm{C}-3), 121.7$ (C-5), 122.2 (C-3", C-5"), 122.7 (C), 130.9 (C-2', C-6'), 131.0 (C-6), 132.6 (C-3', C-5'), 136.3 (C), 136.5 (C), 138.1 (C), 148.7 (C-2", C-6"), 149.6 (C-4"), 152.8 (C=O) ppm. MS (ESI, pos.): $m / z(\%)=425 / 427(100)[\mathrm{M}+\mathrm{H}]^{+}$. 


\section{Chapter 22 COMPOUNDS}

HRMS (ESI): $m / z$ calc. for $\mathrm{C}_{22} \mathrm{H}_{22} \mathrm{BrN}_{2} \mathrm{O}_{2}[\mathrm{M}+\mathrm{H}]^{+} 425.0859$; found 425.0853 .

$\operatorname{TLC}\left(\mathrm{SiO}_{2}\right): R_{\mathrm{f}}=0.19(n$-hexane/EtOAc $=1: 1)$.

\subsection{4-[2'-Nitro-4'-(4-pyridyl)-4-biphenylyl]- morpholine (56)}

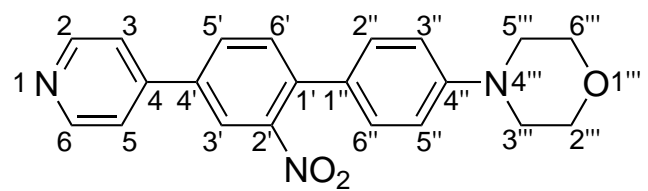

Morpholine reacted with bromide $\mathbf{5 4}$ under Buchwald-Hartwig reaction conditions. ${ }^{[174]}$

Argon was bubbled through a suspension of BINAP ( $26 \mathrm{mg}, 42 \mu \mathrm{mol}, 0.03 \mathrm{eq})$ in dry toluene $(2.0 \mathrm{ml})$ inside a screw cap pressure tube for $2 \mathrm{~min}$. In order to dissolve BINAP, the suspension was heated to $80^{\circ} \mathrm{C}$ for $2 \mathrm{~min}$. After cooling to room temperature, $\mathrm{Pd}_{2}(\mathrm{dba})_{3}(13 \mathrm{mg}, 14 \mu \mathrm{mol}, 0.01 \mathrm{eq})$ was added. Then bromide 54 (500 mg, $1.41 \mathrm{mmol}, 1.00 \mathrm{eq})$, morpholine $(0.15 \mathrm{ml}, 0.15 \mathrm{~g}, 1.7 \mathrm{mmol}, 1.2 \mathrm{eq})$ and $\mathrm{NaOt}$-Bu (190 mg, $1.97 \mathrm{mmol}, 1.40 \mathrm{eq}$ ) were added, and the flask wall was rinsed with deoxygenated dry toluene $(1.0 \mathrm{ml})$. The dark red reaction mixture was heated in the sealed reaction vessel at $80^{\circ} \mathrm{C}$ for $22 \mathrm{~h}$. The reaction mixture was cooled to room temperature, diluted with EtOAc $(10 \mathrm{ml})$ and filtered through a pad of Celite ${ }^{\mathrm{TM}}(\varnothing=30 \mathrm{~mm}, h=10 \mathrm{~mm})$. The filter cake was washed with EtOAc $(4 \times 10 \mathrm{ml})$. The combined filtrates were concentrated in vacuo to give a brown oil which solidified after coevaporation with EtOAc. The brown residue was suspended in a mixture of $\mathrm{CH}_{2} \mathrm{Cl}_{2} /$ EtOAc $=1: 1+1 \% \mathrm{NEt}_{3}(5 \mathrm{ml})$. Solids were separated by decantation and the liquid was loaded directly onto a silica gel column. Flash column chromatography on silica gel $\left(\mathrm{CH}_{2} \mathrm{Cl}_{2} / \mathrm{EtOAc}=1: 1+\right.$ $\left.1 \% \mathrm{NEt}_{3}\right)$ gave $445 \mathrm{mg}(1.23 \mathrm{mmol}, 87 \%)$ of the title compound as orange solid.

${ }^{1} \mathbf{H}$ NMR $\left(400 \mathrm{MHz}, \mathrm{CDCl}_{3}\right): \delta=3.19-3.26\left(\mathrm{~m}, 4 \mathrm{H}, 3^{\prime \prime}-\mathrm{H}_{2}, 5^{\prime \prime}-\mathrm{H}_{2}\right), 3.83-3.89$ (m, 4 H, 2"'- $\mathrm{H}_{2}, 6$ '"- $\left.\mathrm{H}_{2}\right), 6.91-6.98$ (m, $2 \mathrm{H}, 3$ "-H, 5"-H), $7.22-7.31$ (m, $2 \mathrm{H}, 2$ "-H, $\left.6^{\prime \prime}-\mathrm{H}\right), 7.50-7.60\left(\mathrm{~m}, 3 \mathrm{H}, 3-\mathrm{H}, 5-\mathrm{H}, 66^{\prime}-\mathrm{H}\right), 7.83\left(\mathrm{dd},{ }^{3} J_{\mathrm{H}, \mathrm{H}}=8.0 \mathrm{~Hz},{ }^{4} J_{\mathrm{H}, \mathrm{H}}=2.0 \mathrm{~Hz}\right.$, $\left.1 \mathrm{H}, 5^{\prime}-\mathrm{H}\right), 8.04\left(\mathrm{~d},{ }^{4} \mathrm{~J}_{\mathrm{H}, \mathrm{H}}=2.0 \mathrm{~Hz}, 1 \mathrm{H}, 3^{\prime}-\mathrm{H}\right), 8.68-8.76(\mathrm{~m}, 2 \mathrm{H}, 2-\mathrm{H}, 6-\mathrm{H}) \mathrm{ppm}$. MS (ESI, pos.): $m / z(\%)=362.2(100)[\mathrm{M}+\mathrm{H}]^{+}$. 
HRMS (ESI): $m / z$ calc. for $\mathrm{C}_{21} \mathrm{H}_{20} \mathrm{~N}_{3} \mathrm{O}_{3}[\mathrm{M}+\mathrm{H}]^{+}$362.1499; found 362.1501.

$\operatorname{TLC}\left(\mathrm{SiO}_{2}\right): R_{\mathrm{f}}=0.17\left(\mathrm{CH}_{2} \mathrm{Cl}_{2} / \mathrm{EtOAc}=9: 1+1 \% \mathrm{NEt}_{3}\right)$.

\subsection{4'-Morpholino-4-(4-pyridyl)-2-biphenylyl- amine (58)}

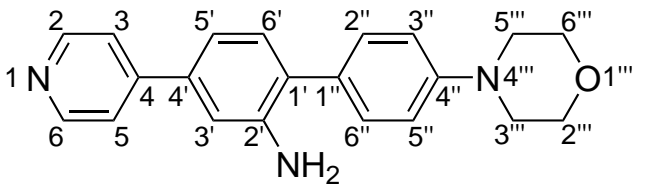

Activated zinc dust ${ }^{\dagger}(4.10 \mathrm{~g}, 63.1 \mathrm{mmol}, 60.0 \mathrm{eq})$ was suspended in a solution of nitro compound $56(380 \mathrm{mg}, 1.05 \mathrm{mmol}, 1.00 \mathrm{eq})$ in dry $\mathrm{CH}_{2} \mathrm{Cl}_{2}(19 \mathrm{ml})$. The suspension was cooled to $0{ }^{\circ} \mathrm{C}$ and $\mathrm{AcOH}(2.7 \mathrm{ml}, 2.8 \mathrm{~g}, 46 \mathrm{mmol}, 44 \mathrm{eq})$ was added dropwise at $0{ }^{\circ} \mathrm{C}$ over a period of $5 \mathrm{~min}$. After $1 \mathrm{~h}$ at $0{ }^{\circ} \mathrm{C}$ the mixture was filtered through Celite ${ }^{\mathrm{TM}}(\varnothing=60 \mathrm{~mm}, h=10 \mathrm{~mm})$. The filter cake was washed with EtOAc $(4 \times 10 \mathrm{ml})$. The combined organic solutions were concentrated in vacuo; the residue was dissolved in $\mathrm{CHCl}_{3}(50 \mathrm{ml})$ and washed with a mixture of $5 \%$ aq. $\mathrm{NaHCO}_{3}(50 \mathrm{ml})$ and brine $(50 \mathrm{ml})$. The aq. layer was extracted with $\mathrm{CHCl}_{3}(2 \times 50 \mathrm{ml})$. The combined organic solutions were dried over $\mathrm{Na}_{2} \mathrm{SO}_{4}$ and concentrated in vacuo to give $383 \mathrm{mg}$ of brown solid. The crude product was dissolved in hot $\mathrm{CHCl}_{3}(30 \mathrm{ml})$, adsorbed on silica gel $(4.0 \mathrm{~g})$ and loaded onto a prepacked column with silica gel. Column chromatography $\left(\mathrm{CH}_{2} \mathrm{Cl}_{2} / \mathrm{MeOH}=\right.$ 98:2 + 1\% NEt 3 ) gave $312 \mathrm{mg}(941 \mu \mathrm{mol}, 90 \%)$ of the title compound as brown solid.

${ }^{1} \mathbf{H}$ NMR $\left(400 \mathrm{MHz}, \mathrm{CDCl}_{3}\right): \delta=3.20-3.26\left(\mathrm{~m}, 4 \mathrm{H}, 3^{\prime \prime}-\mathrm{H}_{2}, 5^{\prime \prime}-\mathrm{H}_{2}\right), 3.87-3.92(\mathrm{~m}$,

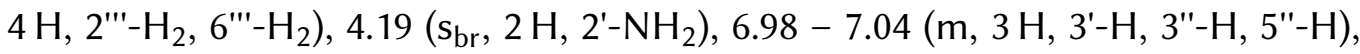
$7.09\left(\mathrm{dd},{ }^{3} J_{\mathrm{H}, \mathrm{H}}=7.8 \mathrm{~Hz},{ }^{4} J_{\mathrm{H}, \mathrm{H}}=1.9 \mathrm{~Hz}, 1 \mathrm{H}, 5^{\prime}-\mathrm{H}\right), 7.23\left(\mathrm{~d},{ }^{3} J_{\mathrm{H}, \mathrm{H}}=7.8 \mathrm{~Hz}, 1 \mathrm{H}, 6{ }^{\prime}-\mathrm{H}\right)$, $7.38-7.44$ (m, 2 H, 2"-H, 6"-H), $7.50-7.58$ (m, 2 H, 3-H, 5-H), $8.62-8.66$ (m, 2 H, 2-H, 6-H) ppm.

${ }^{13}$ C NMR $\left(101 \mathrm{MHz}^{\mathrm{C}} \mathrm{CDCl}_{3}\right): \delta=49.2(\mathrm{C}-3$ "', C-5"'), 67.0 (C-2"', C-6'"'), 113.8 (C-3'), 115.9 (C-3", C-5"), 117.4 (C-5'), 121.8 (C-3, C-5), 128.8 (C-1'), 129.9 (C-2", C-6"), 131.3 (C-6'), 137.5 (C-4'), 144.6 (C-2'), 149.2 (C-2, C-6), 149.5 (C-4), 150.7

\footnotetext{
${ }^{\dagger} \mathrm{Zn}$ was activated by stirring with $2 \%$ aq. $\mathrm{HCl}$, washing with water, ethanol, acetone and dry diethyl ether before drying in vacuo, according to ref. [172].
} 


\section{Chapter 22 COMPOUNDS}

(C-4") ppm.

MS (ESI, pos.): $m / z(\%)=332.2(100)[\mathrm{M}+\mathrm{H}]^{+}$.

HRMS (ESI): $m / z$ calc. for $\mathrm{C}_{21} \mathrm{H}_{22} \mathrm{~N}_{3} \mathrm{O}[\mathrm{M}+\mathrm{H}]^{+} 332.1757$; found 332.1762.

$\operatorname{TLC}\left(\mathrm{SiO}_{2}\right): R_{\mathrm{f}}=0.25\left(\mathrm{CH}_{2} \mathrm{Cl}_{2} / \mathrm{MeOH}=1: 1+1 \% \mathrm{NEt}_{3}\right)$.

\subsection{Compound 61}

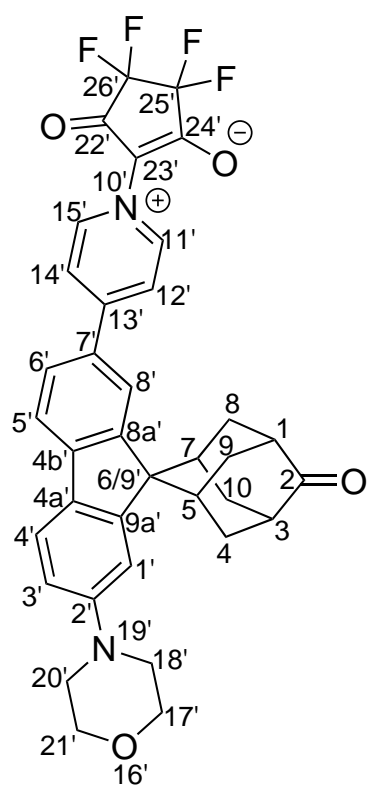

Compound 45 (11 mg, $23 \mu \mathrm{mol}, 1.0 \mathrm{eq})$ was suspended in $i-\operatorname{PrOH}(0.80 \mathrm{ml})$ in a screw cap pressure tube and heated to reflux for $1 \mathrm{~min}$. After cooling to $0{ }^{\circ} \mathrm{C}$, perfluorocyclopentene $(18 \mu \mathrm{l}, 28 \mathrm{mg}, 0.13 \mathrm{mmol}, 5.8 \mathrm{eq})$ was added using a cold syringe. Water $(80 \mu \mathrm{l})$ and $\mathrm{AcOH}(5 \mu \mathrm{l})$ were added and the sealed reaction tube was heated to $70^{\circ} \mathrm{C}$. The reaction was monitored by $\mathrm{TLC}\left(\mathrm{SiO}_{2}\right.$ with EtOAc as eluent) and after $2 \mathrm{~h}$ showed uncomplete conversion. Additional $i-\mathrm{PrOH}(5.2 \mathrm{ml})$ and $\mathrm{AcOH}(20 \mu \mathrm{l})$ were added at $0{ }^{\circ} \mathrm{C}$ and the sealed reaction vessel was heated to $70^{\circ} \mathrm{C}$. After $17 \mathrm{~h}$, TLC still displayed remaining starting material. Additional perfluorocyclopentene $(36 \mu \mathrm{l}, 57 \mathrm{mg}, 0.27 \mathrm{mmol}, 12 \mathrm{eq})$ was added at $0{ }^{\circ} \mathrm{C}$ and the reaction mixture was stirred at $70^{\circ} \mathrm{C}$ in the sealed vessel for additional $5 \mathrm{~h}$. The crude product was precipitated by diluting the reaction mixture with water $(10 \mathrm{ml})$ and sat. aq. $\mathrm{NaHCO}_{3}(1.0 \mathrm{ml})$. The orange precipitate was separated by centrifugation, and the pellet was washed with water (ca. $5 \mathrm{ml})$. The pellet of the second centrifugation was suspended in $\mathrm{CH}_{2} \mathrm{Cl}_{2}(12 \mathrm{ml})$ and concentrated 
in vacuo giving $12 \mathrm{mg}$ of orange solid. Column chromatography on silica gel (EtOAc) gave $9.6 \mathrm{mg}(15 \mu \mathrm{mol}, 66 \%)$ of the title compound as orange solid.

${ }^{1} \mathbf{H}$ NMR $(400 \mathrm{MHz}$, DMSO-d 6 ): $\delta=1.63-1.70(\mathrm{~m}, 2 \mathrm{H}, 5-\mathrm{H}, 7-\mathrm{H}), 1.85-1.98$ $\left(\mathrm{m}, 4 \mathrm{H}, 4-\mathrm{H}_{\mathrm{a}}, 8-\mathrm{H}_{\mathrm{a}}, 9-\mathrm{H}_{\mathrm{a}}, 10-\mathrm{H}_{\mathrm{a}}\right), 2.76-2.86(\mathrm{~m}, 2 \mathrm{H}, 1-\mathrm{H}, 3-\mathrm{H}), 3.05-3.21(\mathrm{~m}$, $\left.4 \mathrm{H}, 4-\mathrm{H}_{\mathrm{b}}, 8-\mathrm{H}_{\mathrm{b}}, 9-\mathrm{H}_{\mathrm{b}}, 10-\mathrm{H}_{\mathrm{b}}\right), 3.23-3.30\left(\mathrm{~m}, 4 \mathrm{H}, 18^{\prime}-\mathrm{H}_{2}, 20^{\prime}-\mathrm{H}_{2}\right), 3.77-3.83(\mathrm{~m}$, $\left.4 \mathrm{H}, 17^{\prime}-\mathrm{H}_{2}, 21^{\prime}-\mathrm{H}_{2}\right), 7.15\left(\mathrm{dd},{ }^{3} J_{\mathrm{H}, \mathrm{H}}=8.5 \mathrm{~Hz},{ }^{4} J_{\mathrm{H}, \mathrm{H}}=2.0 \mathrm{~Hz}, 1 \mathrm{H}, 3^{\prime}-\mathrm{H}\right), 7.62-7.67$ $\left(\mathrm{m}, 1 \mathrm{H}, 1^{\prime}-\mathrm{H}\right), 7.97\left(\mathrm{~d},{ }^{3} \mathrm{~J}_{\mathrm{H}, \mathrm{H}}=8.5 \mathrm{~Hz}, 1 \mathrm{H}, 4^{\prime}-\mathrm{H}\right), 8.08\left(\mathrm{~d},{ }^{3} J_{\mathrm{H}, \mathrm{H}}=8.2 \mathrm{~Hz}, 1 \mathrm{H}, 5^{\prime}-\mathrm{H}\right)$, $8.13\left(\mathrm{dd},{ }^{3} J_{\mathrm{H}, \mathrm{H}}=8.2 \mathrm{~Hz},{ }^{4} J_{\mathrm{H}, \mathrm{H}}=1.4 \mathrm{~Hz}, 1 \mathrm{H}, 6^{\prime}-\mathrm{H}\right), 8.54-8.61\left(\mathrm{~m}, 3 \mathrm{H}, 12^{\prime}-\mathrm{H}, 14^{\prime}-\mathrm{H}\right.$, 8 '-H), $9.20-9.24$ (m, $\left.2 \mathrm{H}, 11^{\prime}-\mathrm{H}, 15^{\prime}-\mathrm{H}\right) \mathrm{ppm}$.

${ }^{19}$ F NMR $\left(376.4 \mathrm{MHz}, \mathrm{DMSO}-\mathrm{d}_{6}\right): \delta=-126.0 \mathrm{ppm}$.

MS (ESI, pos.): $m / z(\%)=631.4(100)[\mathrm{M}+\mathrm{H}]^{+}$.

HRMS (ESI): $m / z$ calc. for $\mathrm{C}_{36} \mathrm{H}_{30} \mathrm{~F}_{4} \mathrm{KN}_{2} \mathrm{O}_{4}[\mathrm{M}+\mathrm{K}]^{+}$669.1773; found 669.1770.

$\operatorname{TLC}\left(\mathrm{SiO}_{2}\right): R_{\mathrm{f}}=0.38(\mathrm{EtOAc})$.

UV/Vis (EtOAc): $\lambda_{\text {abs }}^{\max }=431 \mathrm{~nm}, \varepsilon=28800 \mathrm{M}^{-1} \mathrm{~cm}^{-1}$.

Fluorescence (EtOAc, exc. at $410 \mathrm{~nm}): \lambda_{\mathrm{em}}^{\max }=681 \mathrm{~nm} ; \Phi_{\mathrm{f}}=0.60 .^{\dagger}$

\subsection{Compound 62}

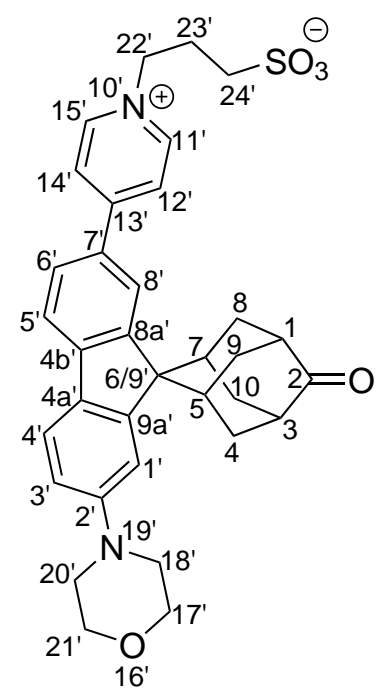

A suspension of pyridine derivative $45(27 \mathrm{mg}, 58 \mu \mathrm{mol}, 1.0 \mathrm{eq})$ and 1,3-propanesultone $(28 \mathrm{mg}, 0.23 \mathrm{mmol}, 4.0 \mathrm{eq})$ in $\mathrm{MeCN}(0.80 \mathrm{ml})$ was heated

\footnotetext{
${ }^{\dagger}$ Coumarin 153 in EtOH as a fl. quantum yield standard with $\Phi_{\mathrm{f}}=0.55^{[137]}$
} 


\section{Chapter 22 $\mid$ COMPOUNDS}

in a sealed screw cap pressure tube to $90^{\circ} \mathrm{C}$ for $18 \mathrm{~h}$. The dark red mixture was cooled to r.t. and diluted with $\mathrm{CH}_{2} \mathrm{Cl}_{2}(3 \mathrm{ml})$ to give a brown suspension. Addition of $\mathrm{MeOH}(0.1 \mathrm{ml})$ gave a clear brown solution which was loaded directly onto a silica gel column. Column chromatography $\left(\mathrm{CH}_{2} \mathrm{Cl}_{2} / \mathrm{MeOH}=\right.$ 4:1) gave $16 \mathrm{mg}$ of crude product. Purification by preparative HPLC (RP column: Kinex $5 \mu \mathrm{m} \mathrm{C18}$; solvent system: MeCN/water 30:70 $\rightarrow$ 100:0 with 0.05\% TFA in $20 \mathrm{~min})$ yielded $1.6 \mathrm{mg}(2.7 \mu \mathrm{mol}, 5 \%)$ of the title compound as brown solid.

${ }^{1} \mathbf{H}$ NMR $\left(400 \mathrm{MHz}, \mathrm{CD}_{3} \mathrm{OD}\right): \delta=1.50-1.57(\mathrm{~m}, 2 \mathrm{H}, 5-\mathrm{H}, 7-\mathrm{H}), 1.99-2.15(\mathrm{~m}$, $\left.4 \mathrm{H}, 4-\mathrm{H}_{\mathrm{a}}, 8-\mathrm{H}_{\mathrm{a}}, 9-\mathrm{H}_{\mathrm{a}}, 10-\mathrm{H}_{\mathrm{a}}\right), 2.39-2.55\left(\mathrm{~m}, 4 \mathrm{H}, 1-\mathrm{H}, 3-\mathrm{H}, 23^{\prime}-\mathrm{H}_{2}\right), 2.78-2.87(\mathrm{~m}$, $\left.4 \mathrm{H}, 4-\mathrm{H}_{\mathrm{b}}, 8-\mathrm{H}_{\mathrm{b}}, 9-\mathrm{H}_{\mathrm{b}}, 10-\mathrm{H}_{\mathrm{b}}\right), 2.92\left(\mathrm{t},{ }^{3} J_{\mathrm{H}, \mathrm{H}}=6.8 \mathrm{~Hz}, 2 \mathrm{H}, 24^{\prime}-\mathrm{H}_{2}\right), 3.26-3.30(\mathrm{~m}$, $\left.4 \mathrm{H}, 18^{\prime}-\mathrm{H}_{2}, 20^{\prime}-\mathrm{H}_{2}\right), 3.89-3.94\left(\mathrm{~m}, 4 \mathrm{H}, 17^{\prime}-\mathrm{H}_{2}, 21^{\prime}-\mathrm{H}_{2}\right), 4.82\left(\mathrm{t},{ }^{3} J_{\mathrm{H}, \mathrm{H}}=7.2 \mathrm{~Hz}, 2 \mathrm{H}\right.$, $\left.22^{\prime}-\mathrm{H}_{2}\right), 7.14\left(\mathrm{dd},{ }^{3} J_{\mathrm{H}, \mathrm{H}}=8.5 \mathrm{~Hz},{ }^{4} J_{\mathrm{H}, \mathrm{H}}=2.2 \mathrm{~Hz}, 1 \mathrm{H}, 3^{\prime}-\mathrm{H}\right), 7.77\left(\mathrm{~d},{ }^{4} J_{\mathrm{H}, \mathrm{H}}=2.2 \mathrm{~Hz}\right.$, $\left.1 \mathrm{H}, 1^{\prime}-\mathrm{H}\right), 7.85\left(\mathrm{~d},{ }^{3} J_{\mathrm{H}, \mathrm{H}}=8.5 \mathrm{~Hz}, 1 \mathrm{H}, 4^{\prime}-\mathrm{H}\right), 7.96\left(\mathrm{~d},{ }^{3} J_{\mathrm{H}, \mathrm{H}}=8.1 \mathrm{~Hz}, 1 \mathrm{H}, 5^{\prime}-\mathrm{H}\right), 8.01$ $\left(\mathrm{dd},{ }^{3} J_{\mathrm{H}, \mathrm{H}}=8.1 \mathrm{~Hz},{ }^{4} J_{\mathrm{H}, \mathrm{H}}=1.5 \mathrm{~Hz}, 1 \mathrm{H}, 6^{\prime}-\mathrm{H}\right), 8.37-8.43\left(\mathrm{~m}, 2 \mathrm{H}, 12^{\prime}-\mathrm{H}, 14^{\prime}-\mathrm{H}\right), 8.62$ $\left(\mathrm{d},{ }^{4} J_{\mathrm{H}, \mathrm{H}}=1.5 \mathrm{~Hz}, 1 \mathrm{H}, 8^{\prime}-\mathrm{H}\right), 8.91-8.95\left(\mathrm{~m}, 2 \mathrm{H}, 11^{\prime}-\mathrm{H}, 15^{\prime}-\mathrm{H}\right) \mathrm{ppm}$.

MS (ESI, pos.): $m / z(\%)=585.5(100)[\mathrm{M}+\mathrm{H}]^{+}$.

HRMS (ESI): $m / z$ calc. for $\mathrm{C}_{34} \mathrm{H}_{36} \mathrm{KN}_{2} \mathrm{O}_{5} \mathrm{~S}[\mathrm{M}+\mathrm{K}]^{+}$623.1977; found 623.1971.

$\operatorname{TLC}\left(\mathrm{SiO}_{2}\right): R_{\mathrm{f}}=0.20\left(\mathrm{CH}_{2} \mathrm{Cl}_{2} / \mathrm{MeOH}=4: 1\right)$.

\subsection{3-Bromo-2,2'-bithiophene $(64)^{[140]}$}

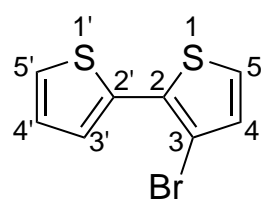

Magnesium swarf $(0.83 \mathrm{~g}, 34 \mathrm{mmol}, 1.9 \mathrm{eq})$ was stirred under nitrogen atmosphere. Several $\mathrm{mg}$ of iodine were added, and the mixture was heated to $40^{\circ} \mathrm{C}$. Dry $\mathrm{Et}_{2} \mathrm{O}(6 \mathrm{ml})$ and a solution of 2-bromothiophene $(2.1 \mathrm{ml}, 3.5 \mathrm{~g}, 21 \mathrm{mmol}$, $1.2 \mathrm{eq})$ in dry $\mathrm{Et}_{2} \mathrm{O}(25 \mathrm{ml})$ were added dropwise while heating at reflux over a period of $30 \mathrm{~min}$. The resulting suspension was heated at reflux for $45 \mathrm{~min}$ and diluted with dry $\mathrm{Et}_{2} \mathrm{O}(10 \mathrm{ml})$. The mixture was cooled to r.t. and the solution was drawn into a syringe. The Grignard solution was added dropwise to a mixture of $[\mathrm{Pd}(\mathrm{dppf})] \mathrm{Cl}_{2} \cdot \mathrm{CH}_{2} \mathrm{Cl}_{2}$ (131 mg, $\left.179 \mu \mathrm{mol}, 0.01 \mathrm{eq}\right)$ and 2,3-dibromothiophene

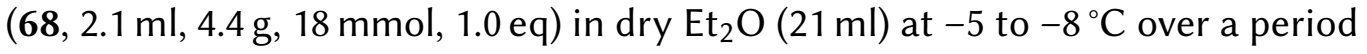


of $1 \mathrm{~h}$ using a syringe pump. The reaction mixture was stirred at $0^{\circ} \mathrm{C}$ for $3 \mathrm{~h}$ and then quenched by addition of $\mathrm{MeOH}(2 \mathrm{ml})$. The mixture was filtered through a double layer of $\mathrm{Na}_{2} \mathrm{SO}_{4}(1.5 \mathrm{~cm})$ and silica gel $(1.5 \mathrm{~cm})$. The residue was washed with $\mathrm{Et}_{2} \mathrm{O}(3 \times 15 \mathrm{ml})$ and the combined filtrates were concentrated in vacuo giving $4.40 \mathrm{~g}$ of yellow oil. The crude product was dissolved in a mixture of $n$-hexane $(3 \mathrm{ml})$ and $\mathrm{CCl}_{4}(1 \mathrm{ml})$. The homogeneous solution was loaded onto a silica gel column. Column chromatography (pure $n$-hexane as eluent) gave $3.7 \mathrm{~g} \mathrm{(15} \mathrm{mmol,}$ $83 \%$ ) of the title compound as greenish oil.

${ }^{1} \mathbf{H}$ NMR $\left(400 \mathrm{MHz}, \mathrm{CDCl}_{3}\right): \delta=7.02\left(\mathrm{~d},{ }^{3} J_{\mathrm{H}, \mathrm{H}}=5.4 \mathrm{~Hz}, 1 \mathrm{H}, 4-\mathrm{H}\right), 7.09\left(\mathrm{dd},{ }^{3} \mathrm{~J}_{\mathrm{H}, \mathrm{H}}\right.$ $\left.=5.2 \mathrm{~Hz},{ }^{4} J_{\mathrm{H}, \mathrm{H}}=3.7 \mathrm{~Hz}, 1 \mathrm{H}, 4^{\prime}-\mathrm{H}\right), 7.19\left(\mathrm{~d},{ }^{3} J_{\mathrm{H}, \mathrm{H}}=5.4 \mathrm{~Hz}, 1 \mathrm{H}, 5-\mathrm{H}\right), 7.36\left(\mathrm{dd},{ }^{3} J_{\mathrm{H}, \mathrm{H}}\right.$ $\left.=5.2 \mathrm{~Hz},{ }^{4} J_{\mathrm{H}, \mathrm{H}}=1.2 \mathrm{~Hz}, 1 \mathrm{H}, 5^{\prime}-\mathrm{H}\right), 7.43\left(\mathrm{dd},{ }^{3} J_{\mathrm{H}, \mathrm{H}}=3.7 \mathrm{~Hz},{ }^{4} J_{\mathrm{H}, \mathrm{H}}=1.2 \mathrm{~Hz}, 1 \mathrm{H}\right.$, 3'-H) ppm.

${ }^{13}$ C NMR $\left(101 \mathrm{MHz}, \mathrm{CDCl}_{3}\right): \delta=108.0(\mathrm{C}-3), 124.3(\mathrm{C}-5), 126.3\left(\mathrm{C}-5^{\prime}\right), 126.9\left(\mathrm{C}-3^{\prime}\right)$, 127.4 (C-4'), 132.0 (C-4), 132.4 (C-2), 134.5 (C-2') ppm.

GC-MS (EI): $m / z(\%)=244 / 246(100)\left[\mathrm{M}^{+\cdot}\right]$.

$\operatorname{TLC}\left(\mathrm{SiO}_{2}\right): R_{\mathrm{f}}=0.31$ (cyclohexane).

\subsection{2,3-Dibromothiophene (68) ${ }^{[139]}$}<smiles>Brc1ccsc1Br</smiles>

To a solution of 3-bromothiophene $(67,4.7 \mathrm{ml}, 8.2 \mathrm{~g}, 50 \mathrm{mmol}, 1.0 \mathrm{eq})$ in glacial acetic acid $(10 \mathrm{ml})$ a solution of bromine $(2.7 \mathrm{ml}, 8.4 \mathrm{~g}, 53 \mathrm{mmol}, 1.1 \mathrm{eq})$ in glacial acetic acid $(5.0 \mathrm{ml})$ was added dropwise at room temperature over a period of $10 \mathrm{~min}$. After stirring for $29 \mathrm{~h}$ at room temperature, the reaction mixture was poured onto ice $(50 \mathrm{~g})$. The mixture was extracted with $\mathrm{Et}_{2} \mathrm{O}(3 \times 50 \mathrm{ml})$. The combined organic solutions were washed with $2.0 \mathrm{M} \mathrm{NaOH}(50 \mathrm{ml})$ and dried over $\mathrm{Na}_{2} \mathrm{SO}_{4}$. Concentration in vacuo gave $11.0 \mathrm{~g}$ of yellow oil. Vacuum distillation $\left(98^{\circ} \mathrm{C}, 13 \mathrm{mbar}\right)$ gave $8.9 \mathrm{~g}(4.1 \mathrm{mmol}, 74 \%)$ of the title compound as colorless oil.

${ }^{1} \mathbf{H}$ NMR $\left(400 \mathrm{MHz}, \mathrm{CDCl}_{3}\right): \delta=6.92\left(\mathrm{~d},{ }^{3} J_{\mathrm{H}, \mathrm{H}}=5.8 \mathrm{~Hz}, 1 \mathrm{H}, 4-\mathrm{H}\right), 7.26\left(\mathrm{~d},{ }^{3} J_{\mathrm{H}, \mathrm{H}}=\right.$ $5.8 \mathrm{~Hz}, 1 \mathrm{H}, 5-\mathrm{H}) \mathrm{ppm}$.

${ }^{13}$ C NMR $\left(101 \mathrm{MHz}, \mathrm{CDCl}_{3}\right): \delta=111.4(\mathrm{C}-2), 114.3(\mathrm{C}-3), 127.2(\mathrm{C}-5), 130.4$ 


\section{Chapter 22 $\mid$ COMPOUNDS}

(C-4) ppm.

GC-MS (EI): $m / z(\%)=318 / 320 / 322 / 324(100)\left[\mathrm{M}^{+\cdot}\right]$.

$\operatorname{TLC}\left(\mathrm{SiO}_{2}\right): R_{\mathrm{f}}=0.46(n$-hexane $)$.

\subsection{Compound 70}

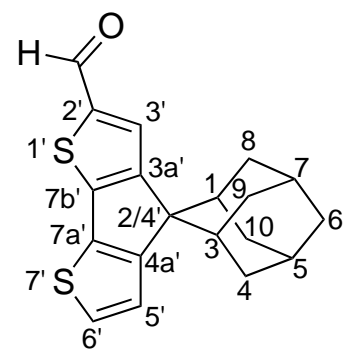

Formylation of spiro compound $\mathbf{7 1}$ according to Vilsmeier-Haack was carried out as described. ${ }^{[141]}$

Spiro compound 71 (80 mg, $0.27 \mu \mathrm{mol}, 1.0 \mathrm{eq})$ was dissolved in 1,2-dichloroethane $(2.7 \mathrm{ml})$, and then DMF ( $82 \mu \mathrm{l}, 78 \mathrm{mg}, 1.1 \mathrm{mmol}, 4.0 \mathrm{eq})$ was added. The solution was cooled to $0{ }^{\circ} \mathrm{C}$, and $\mathrm{POCl}_{3}(38 \mu \mathrm{l}, 62 \mathrm{mg}, 0.40 \mathrm{mmol}, 1.5 \mathrm{eq})$ was added. After $5 \mathrm{~min}$ at $0{ }^{\circ} \mathrm{C}$, the mixture was heated to $70{ }^{\circ} \mathrm{C}$ and kept for $1 \mathrm{~h}$. The reaction mixture was poured into an ice-cold solution of $1.0 \mathrm{M}$ aq. $\mathrm{NaOH}(10 \mathrm{ml})$. The aq. layer was separated and extracted with $\mathrm{CH}_{2} \mathrm{Cl}_{2}(4 \times 10 \mathrm{ml})$. The combined organic solutions were dried over $\mathrm{Na}_{2} \mathrm{SO}_{4}$ and concentrated in vacuo to give $82 \mathrm{mg}$ $(0.25 \mathrm{mmol}, 94 \%)$ of the title compound as orange-yellow solid.

${ }^{1} \mathbf{H}$ NMR $\left(400 \mathrm{MHz}, \mathrm{CDCl}_{3}\right): \delta=1.55-1.61(\mathrm{~m}, 2 \mathrm{H}, 1-\mathrm{H}, 3-\mathrm{H}), 1.81-1.96(\mathrm{~m}$, $\left.6 \mathrm{H}, 4-\mathrm{H}_{\mathrm{a}}, 6-\mathrm{H}_{2}, 8-\mathrm{H}_{\mathrm{a}}, 9-\mathrm{H}_{\mathrm{a}}, 10-\mathrm{H}_{\mathrm{a}}\right), 2.13-2.22(\mathrm{~m}, 2 \mathrm{H}, 5-\mathrm{H}, 7-\mathrm{H}), 2.45-2.57(\mathrm{~m}$, $\left.4 \mathrm{H}, 4-\mathrm{H}_{\mathrm{b}}, 8-\mathrm{H}_{\mathrm{b}}, 9-\mathrm{H}_{\mathrm{b}}, 10-\mathrm{H}_{\mathrm{b}}\right), 7.37\left(\mathrm{~d},{ }^{3} J_{\mathrm{H}, \mathrm{H}}=5.0 \mathrm{~Hz}, 1 \mathrm{H}, 5^{\prime}-\mathrm{H}\right.$ or $\left.6{ }^{\prime}-\mathrm{H}\right), 7.45(\mathrm{~d}$, ${ }^{3} J_{\mathrm{H}, \mathrm{H}}=5.0 \mathrm{~Hz}, 1 \mathrm{H}, 6^{\prime}-\mathrm{H}$ or $\left.5^{\prime}-\mathrm{H}\right), 8.03\left(\mathrm{~s}, 1 \mathrm{H}, 3^{\prime}-\mathrm{H}\right), 9.87(\mathrm{~s}, 1 \mathrm{H}, \mathrm{CHO}) \mathrm{ppm}$.

${ }^{13}$ C NMR $\left(101 \mathrm{MHz}, \mathrm{CDCl}_{3}\right): \delta=27.3$ (C-5, C-7), 34.3 (C-1, C-3), 34.7, 35.5 (C-4, C-8, C-9, C-10), 39.6 (C-6), 58.8 (C-2/4'), 127.2, 127.6 (C-5', C-6'), 135.6 (C-3'), 136.2, 141.6, 148.0, 157.7, 162.1 (C-2', C-3a', C-4a', C-7a', C-7b'), 182.9 (CHO) ppm.

HRMS (EI): $m / z$ calc. for $\mathrm{C}_{19} \mathrm{H}_{18} \mathrm{OS}_{2}\left[\mathrm{M}^{+\cdot}\right]$ 326.0799; found 326.0803 .

$\operatorname{TLC}\left(\mathrm{SiO}_{2}\right): R_{\mathrm{f}}=0.28($ cyclohexane $/$ EtOAc $=9: 1)$. 


\subsection{Compound 71}

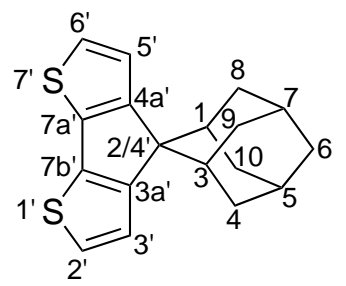

According to GP3, alcohol $72(206 \mathrm{mg}, 650 \mu \mathrm{mol}, 1.00 \mathrm{eq})$ was treated with conc. $\mathrm{H}_{2} \mathrm{SO}_{4}(0.54 \mathrm{ml})$ in $n$-octane $(11 \mathrm{ml})$ and stirred for $60 \mathrm{~min}$. The aq. work-up included quenching with $1.0 \mathrm{M}$ aq. $\mathrm{NaOH}(10 \mathrm{ml})$ followed by extraction with $\mathrm{CH}_{2} \mathrm{Cl}_{2}(7 \times 10 \mathrm{ml})$ and gave $171 \mathrm{mg}$ of green solid. Column chromatography on silica gel ( $n$-hexane) gave $162 \mathrm{mg}(543 \mu \mathrm{mol}, 84 \%)$ of the title compound as colorless solid.

${ }^{1} \mathbf{H}$ NMR $\left(400 \mathrm{MHz}, \mathrm{CDCl}_{3}\right): \delta=1.52-1.58(\mathrm{~m}, 2 \mathrm{H}, 1-\mathrm{H}, 3-\mathrm{H}), 1.79-1.88(\mathrm{~m}$, $\left.4 \mathrm{H}, 4-\mathrm{H}_{\mathrm{a}}, 8-\mathrm{H}_{\mathrm{a}}, 9-\mathrm{H}_{\mathrm{a}}, 10-\mathrm{H}_{\mathrm{a}}\right), 1.89-1.94\left(\mathrm{~m}, 2 \mathrm{H}, 6-\mathrm{H}_{2}\right), 2.11-2.17(\mathrm{~m}, 2 \mathrm{H}, 5-\mathrm{H}$, $7-\mathrm{H}), 2.51-2.60\left(\mathrm{~m}, 4 \mathrm{H}, 4-\mathrm{H}_{\mathrm{b}}, 8-\mathrm{H}_{\mathrm{b}}, 9-\mathrm{H}_{\mathrm{b}}, 10-\mathrm{H}_{\mathrm{b}}\right), 7.12\left(\mathrm{~d},{ }^{3} J_{\mathrm{H}, \mathrm{H}}=5.0 \mathrm{~Hz}, 2 \mathrm{H}\right.$, $\left.3^{\prime}-\mathrm{H}, 5^{\prime}-\mathrm{H}\right), 7.40\left(\mathrm{~d},{ }^{3} J_{\mathrm{H}, \mathrm{H}}=5.0 \mathrm{~Hz}, 2 \mathrm{H}, 2^{\prime}-\mathrm{H}, 6{ }^{\prime}-\mathrm{H}\right) \mathrm{ppm}$.

${ }^{13}$ C NMR $\left(101 \mathrm{MHz}, \mathrm{CDCl}_{3}\right): \delta=27.5$ (C-5, C-7), 34.3 (C-1, C-3), 35.1 (C-4, C-8, C-9, C-10), 39.8 (C-6), 58.6 (C-2/4'), 122.6 (C-3', C-5'), 127.1 (C-2', C-6'), 137.1 (C-7a', C-7b'), 157.9 (C-3a', C-4a') ppm.

MS (ESI, pos.): $m / z(\%)=299(100)[\mathrm{M}+\mathrm{H}]^{+}$.

HRMS (EI): $m / z$ calc. for $\mathrm{C}_{18} \mathrm{H}_{18} \mathrm{~S}_{2}\left[\mathrm{M}^{+\cdot}\right] 298.0850$; found 298.0855 .

$\operatorname{TLC}\left(\mathrm{SiO}_{2}\right): R_{\mathrm{f}}=0.59\left(n\right.$-hexane $\left./ \mathrm{Et}_{2} \mathrm{O}=10: 1\right)$.

\subsection{Compound 72}

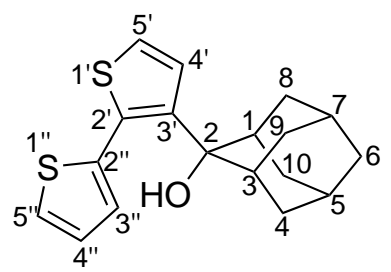

Bromide 64 was subjected to lithium-halogen exchange followed by the reaction with 2-adamantanone (23a). ${ }^{[138]}$ In contrast to GP2, the aromatic bromide was added slowly to a solution of $n$-BuLi, but not vice versa. 


\section{Chapter 22 $\mid$ COMPOUNDS}

A solution of $1.6 \mathrm{M} n$-BuLi in $n$-hexane $(1.0 \mathrm{ml}, 1.6 \mathrm{mmol}, 1.0 \mathrm{eq})$ was diluted with dry $\mathrm{Et}_{2} \mathrm{O}(28 \mathrm{ml})$ at $-78^{\circ} \mathrm{C}$. To this solution, a solution of bromide $64(400 \mathrm{mg}$, $1.63 \mathrm{mmol}, 1.00 \mathrm{eq})$ in dry $\mathrm{Et}_{2} \mathrm{O}(28 \mathrm{ml})$ was added at $-78^{\circ} \mathrm{C}$ over a period of $2 \mathrm{~h}$ using a syringe pump. Then a solution of 2-adamantanone (23a, $245 \mathrm{mg}$, $1.63 \mathrm{mmol}, 1.00 \mathrm{eq})$ in $\mathrm{dry} \mathrm{Et}_{2} \mathrm{O}(1.0 \mathrm{ml})$ was added at $-78^{\circ} \mathrm{C}$ over a period of $5 \mathrm{~min}$. The reaction mixture was allowed to warm up very slowly to $4^{\circ} \mathrm{C}$ within $20 \mathrm{~h}$. The reaction was quenched by addition of sat. aq. $\mathrm{NH}_{4} \mathrm{Cl}(10 \mathrm{ml})$ and water $(18 \mathrm{ml})$. The aq. layer was extracted with $\mathrm{Et}_{2} \mathrm{O}(2 \times 15 \mathrm{ml})$. All organic solutions were combined and dried over $\mathrm{Na}_{2} \mathrm{SO}_{4}$. Concentration in vacuo gave $529 \mathrm{mg}$ of yellow oil which solidified in several minutes. Column chromatography on silica gel ( $n$-hexane $\left./ \mathrm{Et}_{2} \mathrm{O}=15: 1\right)$ gave $432 \mathrm{mg}(1.36 \mathrm{mmol}, 84 \%)$ of the title compound as colorless solid.

${ }^{1} \mathbf{H}$ NMR $\left(400 \mathrm{MHz}, \mathrm{CDCl}_{3}\right): \delta=1.51-1.82\left(\mathrm{~m}, 10 \mathrm{H}, 4-\mathrm{H}_{\mathrm{a}}, 5-\mathrm{H}, 6-\mathrm{H}_{2}, 7-\mathrm{H}, 8-\mathrm{H}_{\mathrm{a}}\right.$, $9-\mathrm{H}_{\mathrm{a}}, 10-\mathrm{H}_{\mathrm{a}},\left[8-\mathrm{H}_{\mathrm{b}}, 9-\mathrm{H}_{\mathrm{b}}\right]$ or $\left.\left[4-\mathrm{H}_{\mathrm{b}}, 10-\mathrm{H}_{\mathrm{b}}\right]\right), 1.90(\mathrm{~s}, 1 \mathrm{H}, \mathrm{OH}), 2.23-2.35(\mathrm{~m}, 4 \mathrm{H}$, $1-\mathrm{H}, 3-\mathrm{H},\left[4-\mathrm{H}_{\mathrm{b}}, 10-\mathrm{H}_{\mathrm{b}}\right]$ or $\left.\left[8-\mathrm{H}_{\mathrm{b}}, 9-\mathrm{H}_{\mathrm{b}}\right]\right), 7.02\left(\mathrm{dd},{ }^{3} \mathrm{~J}_{\mathrm{H}, \mathrm{H}}=5.2,3.5 \mathrm{~Hz}, 1-\mathrm{H}, 4 "-\mathrm{H}\right)$, $7.17\left(\mathrm{~d},{ }^{3} J_{\mathrm{H}, \mathrm{H}}=5.5 \mathrm{~Hz}, 1 \mathrm{H}, 4^{\prime}-\mathrm{H}\right), 7.21\left(\mathrm{dd},{ }^{3} J_{\mathrm{H}, \mathrm{H}}=3.5 \mathrm{~Hz},{ }^{4} J_{\mathrm{H}, \mathrm{H}}=1.2 \mathrm{~Hz}, 1 \mathrm{H}\right.$, $\left.3^{\prime \prime}-\mathrm{H}\right), 7.27\left(\mathrm{~d},{ }^{3} J_{\mathrm{H}, \mathrm{H}}=5.5 \mathrm{~Hz}, 1 \mathrm{H}, 5^{\prime}-\mathrm{H}\right), 7.35\left(\mathrm{dd},{ }^{3} J_{\mathrm{H}, \mathrm{H}}=5.2 \mathrm{~Hz},{ }^{4} J_{\mathrm{H}, \mathrm{H}}=1.2 \mathrm{~Hz}\right.$, $1 \mathrm{H}, 5 "-\mathrm{H}) \mathrm{ppm}$.

${ }^{13} \mathrm{C}$ NMR $\left(101 \mathrm{MHz}, \mathrm{CDCl}_{3}\right): \delta=26.8,27.3(\mathrm{C}-5, \mathrm{C}-7), 33.0,35.3(\mathrm{C}-4, \mathrm{C}-8, \mathrm{C}-9$, C-10), 36.1 (C-1, C-3), 37.7 (C-6), 75.9 (C-2), 124.9 (C-5'), 127.0 (C-5"), 127.1 (C-4"), 128.2 (C-4'), 123.0 (C-3"), 132.4 (C-2'), 136.3 (C-2"), 144.2 (C-3') ppm.

GC-MS (EI): $m / z(\%)=316(100)\left[\mathrm{M}^{+\cdot}\right]$.

HRMS (EI): $m / z$ calc. for $\mathrm{C}_{18} \mathrm{H}_{20} \mathrm{OS}_{2}\left[\mathrm{M}^{+\cdot}\right]$ 316.0956; found 316.0948.

$\operatorname{TLC}\left(\mathrm{SiO}_{2}\right): R_{\mathrm{f}}=0.32($ cyclohexane $/$ EtOAc $=9: 1)$. 


\subsection{Compound 73}

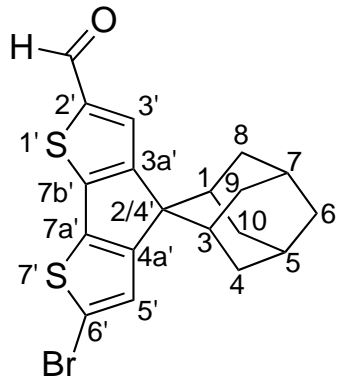

Bromination of aldehyde $\mathbf{7 0}$ was carried out using NBS as a reagent. [141]

Aldehyde 70 (22 mg, $69 \mu \mathrm{mol}, 1.0 \mathrm{eq})$ was suspended in $\mathrm{CCl}_{4}(0.46 \mathrm{ml})$ in a screw cap pressure tube. NBS ( $12 \mathrm{mg}, 69 \mu \mathrm{mol}, 1.0 \mathrm{eq})$ was added to the dark green mixture, the screw cap pressure tube was sealed and heated for $16 \mathrm{~h}$ to $75^{\circ} \mathrm{C}$. The reaction mixture was cooled to $0^{\circ} \mathrm{C}$ and quenched by addition of sat. aq. $\mathrm{NaHCO}_{3}$ $(5.0 \mathrm{ml})$. The mixture was extracted with $\mathrm{CH}_{2} \mathrm{Cl}_{2}(5 \times 5 \mathrm{ml})$ and the combined organic solutions were dried over $\mathrm{Na}_{2} \mathrm{SO}_{4}$. Concentration in vacuo gave $31 \mathrm{mg}$ of brown solid. Column chromatography on silica gel (cyclohexane/EtOAc $=15: 1$ ) gave $27 \mathrm{~g}(67 \mu \mathrm{mol}, 97 \%)$ of the title compound as yellow solid.

${ }^{1} \mathbf{H}$ NMR $\left(400 \mathrm{MHz} \mathrm{CDCl}_{3}\right): \delta=1.54-1.60(\mathrm{~m}, 2 \mathrm{H}, 1-\mathrm{H}, 3-\mathrm{H}), 1.82-1.94(\mathrm{~m}$, $\left.6 \mathrm{H}, 4-\mathrm{H}_{\mathrm{a}}, 6-\mathrm{H}_{2}, 8-\mathrm{H}_{\mathrm{a}}, 9-\mathrm{H}_{\mathrm{a}}, 10-\mathrm{H}_{\mathrm{a}}\right), 2.12-2.21(\mathrm{~m}, 2 \mathrm{H}, 5-\mathrm{H}, 7-\mathrm{H}), 2.37-2.51(\mathrm{~m}$, $\left.4 \mathrm{H}, 4-\mathrm{H}_{\mathrm{b}}, 8-\mathrm{H}_{\mathrm{b}}, 9-\mathrm{H}_{\mathrm{b}}, 10-\mathrm{H}_{\mathrm{b}}\right), 7.47\left(\mathrm{~s}, 1 \mathrm{H}, 3^{\prime}-\mathrm{H}\right.$ or $\left.5^{\prime}-\mathrm{H}\right), 8.01$ (s, $1 \mathrm{H}, 5^{\prime}-\mathrm{H}$ or 3'-H), $9.86(\mathrm{~s}, 1 \mathrm{H}, \mathrm{CHO}) \mathrm{ppm}$.

${ }^{13}$ C NMR $\left(101 \mathrm{MHz}, \mathrm{CDCl}_{3}\right): \delta=27.1(\mathrm{C}-5, \mathrm{C}-7), 34.2(\mathrm{C}-1, \mathrm{C}-3), 34.6,35.4$ (C-4, C-8, C-9, C-10), 39.5 (C-6), 59.6 (C-2/4'), 114.5 (C-6'), 130.2, 135.3 (C-3', C-5'), 136.6, 141.9, 146.9, 156.8, 160.7 (C-2', C-3a', C-4a', C-7a', C-7b'), 182.8 (CHO) ppm.

MS (ESI, pos.): $m / z(\%)=405 / 407(100)[\mathrm{M}+\mathrm{H}]^{+}$.

HRMS (EI): $m / z$ calc. for $\mathrm{C}_{19} \mathrm{H}_{17} \mathrm{BrOS}_{2}\left[\mathrm{M}^{+\cdot}\right]$ 403.9904; found 403.9881 .

$\operatorname{TLC}\left(\mathrm{SiO}_{2}\right): R_{\mathrm{f}}=0.25($ cyclohexane/EtOAc $=9: 1)$. 


\subsection{4-Methoxyphenylboronic acid (74)}<smiles>COc1ccc(B(O)O)cc1</smiles>

74<smiles>COc1ccc(B2OB(c3ccc(OC)cc3)OB(c3ccc(OC)cc3)O2)cc1</smiles>

anhydride of $\mathbf{7 4}$

The boronic acid 74 was prepared from the corresponding aryl bromide. ${ }^{[150]}$

A solution of 4-bromoanisol $(5.2 \mathrm{ml}, 7.9 \mathrm{~g}, 42 \mathrm{mmol}, 1.0 \mathrm{eq})$ dry THF $(120 \mathrm{ml})$ was cooled to $-78^{\circ} \mathrm{C} .2 .5 \mathrm{M} n$-BuLi in $n$-hexane $(16.8 \mathrm{ml}, 42.0 \mathrm{mmol}, 1.00 \mathrm{eq})$ was added dropwise over a period of $5 \mathrm{~min}$ and a colorless precipitate formed. The mixture was warmed up to $35^{\circ} \mathrm{C}$, and the precipitate dissolved. Then the solution was cooled down to $-78{ }^{\circ} \mathrm{C}$ again and stirred for $1 \mathrm{~h}$. Triisopropyl borate $(9.7 \mathrm{ml}, 7.9 \mathrm{~g}$, $42 \mathrm{mmol}, 1.0 \mathrm{eq}$ ) was added dropwise over a period of $10 \mathrm{~min}$ and the reaction mixture was stirred at $-78^{\circ} \mathrm{C}$ for $1 \mathrm{~h}$. After warming to room temperature, the product was precipitated by slow addition of water $(40 \mathrm{ml})$ and separated by filtration. The filter cake was washed with water $(20 \mathrm{ml})$ dissolving much product. The filter cake and filtrate were combined, sat. aq. $\mathrm{NH}_{4} \mathrm{Cl}(15 \mathrm{ml})$ was added and the mixture was extracted with $\mathrm{Et}_{2} \mathrm{O}(3 \times 20 \mathrm{ml})$. The combined organic solutions were washed with sat. aq. $\mathrm{NH}_{4} \mathrm{Cl}(50 \mathrm{ml})$ and dried over $\mathrm{Na}_{2} \mathrm{SO}_{4}$. Concentration in vacuo gave $5.78 \mathrm{~g}$ of a colorless solid. Recrystallization from $n$-hexane/ $\mathrm{Et}_{2} \mathrm{O}$ $=1: 3\left(60 \mathrm{ml}\right.$, reflux to $\left.0{ }^{\circ} \mathrm{C}\right)$ gave $4.4 \mathrm{~g}(29 \mathrm{mmol}, 69 \%)$ of the title compound as mixture with corresponding anhydride as colorless solid. The anhydride was detected by UV/VIS spectroscopy. The absorption spectrum of the anhydride shows an absorption maximum at $254 \mathrm{~nm}$, while the free boronic acid shows an absorption maximum at approximately $237 \mathrm{~nm}$. ${ }^{[175]}$

${ }^{1} \mathbf{H}$ NMR $\left(400 \mathrm{MHz}, \mathrm{CD}_{3} \mathrm{OD}\right): \delta=3.77\left(\mathrm{~s}, 3 \mathrm{H}, \mathrm{OCH}_{3}\right), 6.86-6.91(\mathrm{~m}, 2 \mathrm{H}, 3-\mathrm{H}$, 5-H), $7.53-7.59$ (m, 2 H, 2-H, 6-H) ppm.

${ }^{13}$ C NMR (101 MHz, CD 3 OD): $\delta=55.4\left(\mathrm{OCH}_{3}\right), 114.0(\mathrm{C}-3, \mathrm{C}-5), 136.6(\mathrm{C}-2, \mathrm{C}-6)$, $162.9(\mathrm{C}-4) \mathrm{ppm}$.

UV/Vis $(\mathrm{MeCN}): \lambda_{\mathrm{abs}}^{\max }=254 \mathrm{~nm}$. UV/Vis $\left(\mathrm{MeCN} / \mathrm{H}_{2} \mathrm{O}=99: 1\right): \lambda_{\mathrm{abs}}^{\max }=237 \mathrm{~nm}$. 


\subsection{Compound $75^{[142]}$}

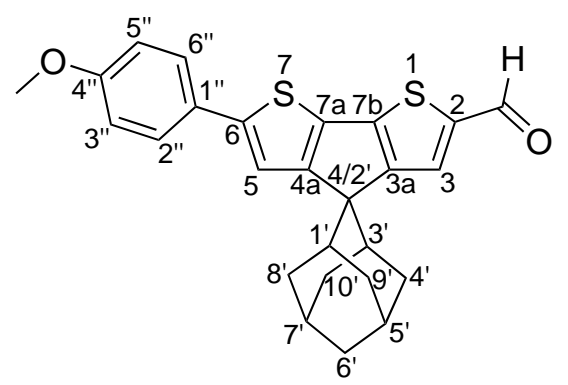

Arylbromide 73 (55 mg, $0.23 \mathrm{mmol}, 1.0 \mathrm{eq})$ was suspended in DME $(5.2 \mathrm{ml})$ inside a screw cap pressure tube. Boronic acid $74(40 \mathrm{mg}, 0.27 \mathrm{mmol}, 1.2 \mathrm{eq})$ and $2.0 \mathrm{M}$ aq. $\mathrm{Na}_{2} \mathrm{CO}_{3}(0.18 \mathrm{ml}, 0.35 \mathrm{mmol}, 1.6 \mathrm{eq})$ were added and argon was bubbled through the suspension for $10 \mathrm{~min}$. [Pd( $\left.\left(\mathrm{PPh}_{3}\right)_{4}\right](26 \mathrm{mg}, 22 \mu \mathrm{mol}, 0.10 \mathrm{eq})$ was added and the sealed reaction vessel was heated at $88^{\circ} \mathrm{C}$. After $49 \mathrm{~h}$, TLC showed the presence of the starting bromide 73. An additional amount of boronic acid $\mathbf{7 4}$ (14 mg, $92 \mu \mathrm{mol}, 0.42 \mathrm{eq})$ and $\left[\mathrm{Pd}\left(\mathrm{PPh}_{3}\right)_{4}\right](17 \mathrm{mg}, 15 \mu \mathrm{mol}, 0.07 \mathrm{eq})$ were added, and the reaction mixture was heated at $88^{\circ} \mathrm{C}$ for $25 \mathrm{~h}$. The reaction mixture was cooled to room temperature, diluted with brine $(30 \mathrm{ml})$ and extracted with EtOAc $(6 \times 20 \mathrm{ml})$. The combined organic solutions were dried over $\mathrm{Na}_{2} \mathrm{SO}_{4}$ and concentrated in vacuo to give $167 \mathrm{mg}$ of brown oil. Column chromatography on silica gel (cyclohexane/ $i-\operatorname{Pr}_{2} \mathrm{O}=12: 1 \rightarrow 1: 1$ ) gave $110 \mathrm{mg}$ of the crude product as brown solid. The crude product was dissolved in $\mathrm{CH}_{2} \mathrm{Cl}_{2}(10 \mathrm{ml})$ and the organic solution washed with $1.0 \mathrm{M} \mathrm{NaOH}(5 \mathrm{ml})$. The aq. layer was extracted with $\mathrm{CH}_{2} \mathrm{Cl}_{2}$ $(2 \times 5 \mathrm{ml})$, and the combined organic solutions were dried over $\mathrm{Na}_{2} \mathrm{SO}_{4}$. Concentration in vacuo gave $92 \mathrm{mg}$ of brown solid. Column chromatography on silica gel $\left(\mathrm{CH}_{2} \mathrm{Cl}_{2} / n\right.$-hexane $\left.=2: 1\right)$ gave $36 \mathrm{mg}(81 \mu \mathrm{mol}, 37 \%)$ of the title compound as brown solid.

${ }^{1} \mathbf{H}$ NMR $\left(400 \mathrm{MHz}, \mathrm{CDCl}_{3}\right): \delta=1.61-1.68(\mathrm{~m}, 2 \mathrm{H}, 1-\mathrm{H}, 3-\mathrm{H}), 1.79-2.02(\mathrm{~m}$, $\left.6 \mathrm{H}, 4-\mathrm{H}_{\mathrm{a}}, 6-\mathrm{H}_{2}, 8-\mathrm{H}_{\mathrm{a}}, 9-\mathrm{H}_{\mathrm{a}}, 10-\mathrm{H}_{\mathrm{a}}\right), 2.12-2.27(\mathrm{~m}, 2 \mathrm{H}, 5-\mathrm{H}, 7-\mathrm{H}), 2.44-2.65(\mathrm{~m}$, $\left.4 \mathrm{H}, 4-\mathrm{H}_{\mathrm{b}}, 8-\mathrm{H}_{\mathrm{b}}, 9-\mathrm{H}_{\mathrm{b}}, 10-\mathrm{H}_{\mathrm{b}}\right), 3.86\left(\mathrm{~s}, 3 \mathrm{H}, \mathrm{OCH}_{3}\right), 6.84-7.06(\mathrm{~m}, 2 \mathrm{H}, 3 "-\mathrm{H}, 5$ "-H), $7.45-7.65$ (m, 3 H, 5-H, 2"-H, 6"-H), 8.02 (s, 1 H, 3-H), 9.86 (s, $1 \mathrm{H}, \mathrm{CHO})$ ppm.

${ }^{13} \mathrm{C}$ NMR $\left(101 \mathrm{MHz}, \mathrm{CDCl}_{3}\right): \delta=27.2,27.2\left(\mathrm{C}-5^{\prime}, \mathrm{C}-7^{\prime}\right), 34.3\left(\mathrm{C}-1^{\prime}, \mathrm{C}-3^{\prime}\right), 34.7,35.5$ (C-4', C-8', C-9', C-10'), 39.6 (C-6'), $55.6\left(\mathrm{OCH}_{3}\right), 59.1$ (C-4/2'), 114.6 (C-3", C-5"), 122.4 (C-5), 127.3 (C-2", C-6"), 127.4, 134.5, 135.4, 141.2, 147.3, 148.4, 156.9, 159.8, 163.0 (C-2, C-3, C-3a, C-4a, C-6, C-7a, C-7b, C-1", C-4"), 182.6 (CHO) ppm. 


\section{Chapter 22 COMPOUNDS}

MS (ESI, pos.): $m / z(\%)=433.2(100)[\mathrm{M}+\mathrm{H}]^{+}$.

HRMS (EI): $m / z$ calc. for $\mathrm{C}_{26} \mathrm{H}_{24} \mathrm{O}_{2} \mathrm{~S}_{2}\left[\mathrm{M}^{+\cdot}\right]$ 432.1218; found 432.1221.

$\operatorname{TLC}\left(\mathrm{SiO}_{2}\right): R_{\mathrm{f}}=0.23($ cyclohexane/EtOAc $=5: 1)$.

\subsection{Compound 77}

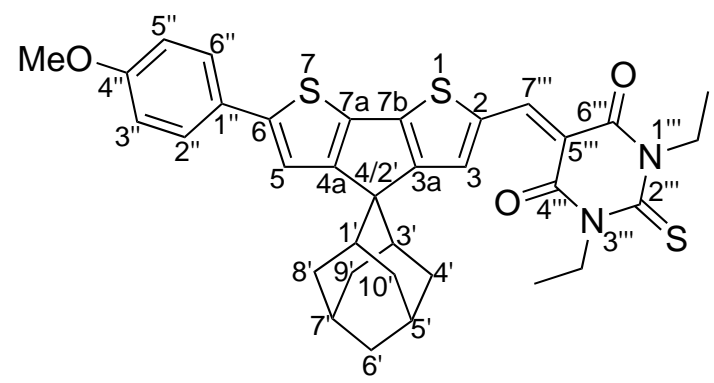

Knoevenagel condensation of aldehyde 75 with 1,3-diethyl-2-thiobarbituric acid (76) was carried out as described. ${ }^{[128]}$

Aldehyde 75 (34 mg, $56 \mu \mathrm{mol}, 1.0 \mathrm{eq}$ ) was suspended in a solution of 1,3-diethyl2-thiobarbituric acid (76, $14 \mathrm{mg}, 67 \mu \mathrm{mol}, 1.2 \mathrm{eq})$ in anhydrous $\mathrm{EtOH}(3.5 \mathrm{ml})$ with piperidine $(13 \mu \mathrm{l})$ inside a screw cap pressure tube. The reaction mixture was heated to $90^{\circ} \mathrm{C}$ for $15 \mathrm{~h}$. The brown suspension was diluted with $\mathrm{CH}_{2} \mathrm{Cl}_{2}(5 \mathrm{ml})$, adsorbed on silica and loaded onto a prepacked silica gel column. Column chromatography ( $n$-hexane/ $\mathrm{Et}_{2} \mathrm{O} / \mathrm{CH}_{2} \mathrm{Cl}_{2}=10: 1: 0 \rightarrow 10: 1: 10$ ) gave $33 \mathrm{mg}$ of crude product as black solid. Second purification on silica gel $(n$-hexane/EtOAc $=10: 1)$ gave $24 \mathrm{mg}(39 \mu \mathrm{mol}, 70 \%)$ of the pure title compound as black powder.

${ }^{1} \mathbf{H}$ NMR $\left(400 \mathrm{MHz}, \mathrm{CDCl}_{3}\right): \delta=1.33\left(\mathrm{t},{ }^{3} J_{\mathrm{H}, \mathrm{H}}=7.1 \mathrm{~Hz}, 3 \mathrm{H}, \mathrm{CH}_{2} \mathrm{C}_{3}\right), 1.37\left(\mathrm{t},{ }^{3} J_{\mathrm{H}, \mathrm{H}}\right.$ $\left.=7.1 \mathrm{~Hz}, 3 \mathrm{H}, \mathrm{CH}_{2} \mathrm{C}_{3}\right), 1.63-1.69\left(\mathrm{~m}, 2 \mathrm{H}, 1^{\prime}-\mathrm{H}, 3^{\prime}-\mathrm{H}\right), 1.85-1.99\left(\mathrm{~m}, 6 \mathrm{H}, 4^{\prime}-\mathrm{H}_{\mathrm{a}}\right.$, $\left.6^{\prime}-\mathrm{H}_{2}, 8^{\prime}-\mathrm{H}_{\mathrm{a}}, 9^{\prime}-\mathrm{H}_{\mathrm{a}}, 10^{\prime}-\mathrm{H}_{\mathrm{a}}\right), 2.17-2.25\left(\mathrm{~m}, 2 \mathrm{H}, 5^{\prime}-\mathrm{H}, 7^{\prime}-\mathrm{H}\right), 2.44-2.59(\mathrm{~m}, 4 \mathrm{H}$, 4'- $\left.\mathrm{H}_{\mathrm{b}}, 8^{\prime}-\mathrm{H}_{\mathrm{b}}, 9^{\prime}-\mathrm{H}_{\mathrm{b}}, 10^{\prime}-\mathrm{H}_{\mathrm{b}}\right), 3.87\left(\mathrm{~s}, 3 \mathrm{H}, \mathrm{OCH}_{3}\right), 4.57-4.68\left(\mathrm{~m}, 4 \mathrm{H}, 2 \times \mathrm{C}_{2} \mathrm{CH}_{3}\right)$, $6.93-6.99$ (m, 2 H, 3"-H, 5"-H), 7.56 - 7.63 (m, 3 H, 5-H, 2"-H, 6"-H), 8.07 (s, $1 \mathrm{H}$, $3-\mathrm{H}$ or 7 "'-H), 8.70 (s, $1 \mathrm{H}, 7^{\prime \prime}-\mathrm{H}$ or 3-H) ppm.

${ }^{13} \mathrm{C}$ NMR $\left(101 \mathrm{MHz}, \mathrm{CDCl}_{3}\right): \delta=12.6,12.7\left(2 \times \mathrm{CH}_{2} \underline{\mathrm{CH}}_{3}\right), 27.1,27.2(\mathrm{C}-5$ ', C-7'), 34.4 (C-1', C-3'), 34.5, 35.6 (C-4', C-8', C-9', C-10'), 39.5 (C-6'), 43.2, $44.0\left(2 \times \mathrm{CH}_{2} \mathrm{CH}_{3}\right), 55.6\left(\mathrm{OCH}_{3}\right), 58.9\left(\mathrm{C}-4 / 2^{\prime}\right), 106.8(\mathrm{C}), 110.1(\mathrm{C}), 114.8(\mathrm{C}-3 "$, C-5"), 122.5 (C-5), 127.1 (C), 127.6 (C-2", C-6"), 135.3 (C), 137.3 (C), 144.1, 149.3 (C-3, C-7"'), 151.5 (C), 158.0 (C), 160.4 (C), 160.5 (C), 161.5 (C), 166.2 (C), 178.7 
(C-2"') ppm.

HRMS (ESI): $m / z$ calc. for $\mathrm{C}_{34} \mathrm{H}_{35} \mathrm{~N}_{2} \mathrm{O}_{3} \mathrm{~S}_{3}\left[\mathrm{M}^{+\cdot}\right]$ 615.1804; found 615.1793.

$\operatorname{TLC}\left(\mathrm{SiO}_{2}\right): R_{\mathrm{f}}=0.27($ cyclohexane $/$ EtOAc $=9: 1)$.

\subsection{2,5-Dibromothiophene (79)}

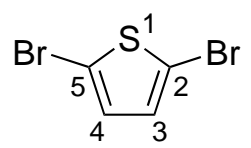

Direct bromination of 2-bromothiophene $\mathbf{7 8}$ was carried out according to the published procedure. ${ }^{[176]}$

2-Bromothiophene $(6.0 \mathrm{ml}, 10 \mathrm{~g}, 61 \mathrm{mmol}, 1.0 \mathrm{eq})$ was dissolved in a mixture of $\mathrm{CHCl}_{3}(20 \mathrm{ml})$ and glacial acetic acid $(20 \mathrm{ml})$. NBS (12.0 g, $\left.67.5 \mathrm{mmol}, 1.1 \mathrm{eq}\right)$ was added in 12 portions at $30^{\circ} \mathrm{C}$ over a period of $1 \mathrm{~h}$. The reaction mixture was heated to $85^{\circ} \mathrm{C}$ (oil bath temperature) for $30 \mathrm{~min}$, cooled to room temperature and diluted with water $(40 \mathrm{ml})$. The aq. layer was extracted with $\mathrm{CHCl}_{3}(20 \mathrm{ml})$, and the combined organic solutions were washed with $2.0 \mathrm{M}$ aq. $\mathrm{NaOH}(40 \mathrm{ml})$. The aq. layer was extracted with $\mathrm{CHCl}_{3}(20 \mathrm{ml})$, and the combined organic solutions were dried over $\mathrm{Na}_{2} \mathrm{SO}_{4}$. Concentration in vacuo $\left(45^{\circ} \mathrm{C}, 10 \mathrm{mbar}\right)$ gave $14 \mathrm{~g}$ (57 mmol, $93 \%$ ) of the title compound as colorless oil.

${ }^{1} \mathbf{H}$ NMR $\left.\left(400 \mathrm{MHz}^{\mathrm{CDCl}}\right)_{3}\right): \delta=6.84(\mathrm{~s}, 2 \mathrm{H}, 3-\mathrm{H}, 4-\mathrm{H}) \mathrm{ppm}$.

${ }^{13}$ C NMR $\left(101 \mathrm{MHz}, \mathrm{CDCl}_{3}\right): \delta=111.7$ (C-2, C-5), 130.5 (C-3, C-4) ppm.

GC-MS (EI): $m / z(\%)=240 / 242 / 244(100)\left[\mathrm{M}^{+\cdot}\right]$.

$\operatorname{TLC}\left(\mathrm{SiO}_{2}\right): R_{\mathrm{f}}=0.78$ (cyclohexane).

\subsection{2-Bromo-5-(4-methoxyphenyl)- thiophene $(80)^{[143]}$}<smiles>COc1ccc(-c2ccc(Br)s2)cc1</smiles>

Dibromide 79 (724 mg, $2.99 \mathrm{mmol}, 1.00 \mathrm{eq})$ and 4-methoxyphenylboronic acid 


\section{Chapter 22 COMPOUNDS}

(74) were suspended in a mixture of sat. aq. $\mathrm{NaHCO}_{3}(8.2 \mathrm{ml})$ and DME $(8.2 \mathrm{ml})$. Argon was bubbled through the suspension for $5 \mathrm{~min}$, and $\left[\mathrm{Pd}\left(\mathrm{PPh}_{3}\right)_{4}\right](70 \mathrm{mg}$, $60 \mu \mathrm{mol}, 0.02 \mathrm{eq})$ was added. The sealed reaction vessel was heated to $90^{\circ} \mathrm{C}$ for $3 \mathrm{~h}$. The reaction mixture was cooled to room temperature and diluted with water $(15 \mathrm{ml})$. The brown suspension was extracted with $\mathrm{CHCl}_{3}(4 \times 20 \mathrm{ml})$. The combined organic solutions were dried over $\mathrm{Na}_{2} \mathrm{SO}_{4}$ and concentrated in vacuo to give a black solid. The crude product was purified by flash column chromatography on silica gel (two columns: first one with $n$-hexane $/ \mathrm{CH}_{2} \mathrm{Cl}_{2} / \mathrm{EtOAc}=60: 1: 0$ $\rightarrow$ 10:0:1; the second one with $n$-hexane $\left./ i-\operatorname{Pr}_{2} \mathrm{O}=15: 1\right)$ gave $262 \mathrm{mg}(973 \mu \mathrm{mol}$, $33 \%$ ) of the title compound as colorless solid.

${ }^{1} \mathbf{H}$ NMR $\left(400 \mathrm{MHz}, \mathrm{CDCl}_{3}\right): \delta=3.83\left(\mathrm{~s}, 3 \mathrm{H}, \mathrm{OCH}_{3}\right), 8.88-6.94(\mathrm{~m}, 3 \mathrm{H}, 4-\mathrm{H}$, $\left.3^{\prime}-\mathrm{H}, 5^{\prime}-\mathrm{H}\right), 6.99\left(\mathrm{~d},{ }^{3} J_{\mathrm{H}, \mathrm{H}}=3.9 \mathrm{~Hz}, 1 \mathrm{H}, 3-\mathrm{H}\right), 7.41-7.46\left(\mathrm{~m}, 2 \mathrm{H}, 2^{\prime}-\mathrm{H}, 6^{\prime}-\mathrm{H}\right) \mathrm{ppm}$.

${ }^{13} \mathrm{C}$ NMR $\left(101 \mathrm{MHz}, \mathrm{CDCl}_{3}\right): \delta=55.5\left(\mathrm{OCH}_{3}\right), 110.3(\mathrm{C}-5), 114.5\left(\mathrm{C}-3^{\prime}, \mathrm{C}-5^{\prime}\right), 122.3$ (C-4), 126.7 (C-1'), 127.1 (C-2', C-6'), 130.9 (C-3), 146.0 (C-2), 159.6 (C-4') ppm.

GC-MS (EI): $m / z(\%)=268 / 270(100)\left[\mathrm{M}^{+\cdot}\right]$.

HRMS (EI): $m / z$ calc. for $\mathrm{C}_{11} \mathrm{H}_{9} \mathrm{BrOS}\left[\mathrm{M}^{+\cdot}\right] 267.9557$; found 267.9552.

$\operatorname{TLC}\left(\mathrm{SiO}_{2}\right): R_{\mathrm{f}}=0.29\left(n\right.$-hexane $\left./ i-\operatorname{Pr}_{2} \mathrm{O}=15: 1\right)$.

\subsection{3-Bromo-5'-iodo-2,2'-bithienyl (81)}

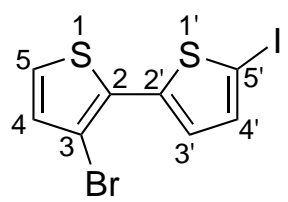

Regioselective iodination of bithiophene derivative 64 was carried out under conditions given in ref. [144].

To a solution of 3-bromo-2,2'-bithiophene $(64,2.15 \mathrm{~g}, 8.77 \mathrm{mmol}, 1.00 \mathrm{eq})$ in dry $\mathrm{MeOH}(194 \mathrm{ml}), \mathrm{N}$-iodosuccinimide $(8.29 \mathrm{~g}, 36.8 \mathrm{mmol}, 4.20 \mathrm{eq})$ was added at room temperature. To the clear yellow solution $\mathrm{AcOH}(2.2 \mathrm{ml}, 2.3 \mathrm{~g}, 38 \mathrm{mmol}$, $1.0 \mathrm{eq}$ ) was added. The solution was heated at $60^{\circ} \mathrm{C}$ for $60 \mathrm{~min}$. TLC of the clear brown reaction mixture on $\mathrm{RP} \mathrm{SiO}_{2}-\mathrm{C}_{18}$ with $\mathrm{MeCN}$ as eluent displayed almost full consumption of compound 64 . Then $1.0 \mathrm{M}$ aq. $\mathrm{Na}_{2} \mathrm{~S}_{2} \mathrm{O}_{3}(200 \mathrm{ml})$ was added. The mixture was extracted with $\mathrm{CH}_{2} \mathrm{Cl}_{2}(3 \times 100 \mathrm{ml})$; combined organic solu- 
tions were dried over $\mathrm{Na}_{2} \mathrm{SO}_{4}$ and concentrated in vacuo to give $4.16 \mathrm{~g}$ of brown solids suspended in a yellow oil. Column chromatography on silica gel (150 g $\mathrm{SiO}_{2}$, cyclohexane) gave $2.81 \mathrm{~g}(7.57 \mathrm{mmol}, 86 \%)$ of the title compound as yellow oil.

${ }^{1} \mathbf{H}$ NMR $\left(400 \mathrm{MHz}, \mathrm{CDCl}_{3}\right): \delta=7.00\left(\mathrm{~d},{ }^{3} \mathrm{~J}_{\mathrm{H}, \mathrm{H}}=5.4 \mathrm{~Hz}, 1 \mathrm{H}, 4-\mathrm{H}\right), 7.05\left(\mathrm{~d},{ }^{3} \mathrm{~J}_{\mathrm{H}, \mathrm{H}}\right.$ $\left.=3.8 \mathrm{~Hz}, 1 \mathrm{H}, 3^{\prime}-\mathrm{H}\right), 7.19\left(\mathrm{~d},{ }^{3} J_{\mathrm{H}, \mathrm{H}}=5.4 \mathrm{~Hz}, 1 \mathrm{H}, 5-\mathrm{H}\right), 7.22\left(\mathrm{~d},{ }^{3} J_{\mathrm{H}, \mathrm{H}}=3.8 \mathrm{~Hz}, 1 \mathrm{H}\right.$, 4'-H) ppm.

${ }^{13} \mathrm{C}$ NMR $\left(101 \mathrm{MHz}, \mathrm{CDCl}_{3}\right): \delta=74.7\left(\mathrm{C}-5^{\prime}\right), 108.5(\mathrm{C}-3), 124.9(\mathrm{C}-5), 128.1\left(\mathrm{C}-3^{\prime}\right)$, 131.6 (C-2'), 132.0 (C-4), 137.1 (C-4'), 140.4 (C-2) ppm.

GC-MS (EI): $m / z(\%)=370 / 372(100)\left[\mathrm{M}^{+\cdot}\right]$.

HRMS (EI): $m / z$ calc. for $\mathrm{C}_{8} \mathrm{H}_{4} \mathrm{BrIS}_{2}\left[\mathrm{M}^{+\cdot}\right]$ 369.7982; found 369.7978 .

$\operatorname{TLC}\left(\mathrm{SiO}_{2}\right): R_{\mathrm{f}}=0.34$ (cyclohexane).

$\operatorname{TLC}\left(\mathrm{RP} \mathrm{SiO}_{2}-\mathrm{C}_{18}\right): R_{\mathrm{f}}=0.42(\mathrm{MeCN})$.

\subsection{3-Bromo-5'-(p-methoxyphenyl)-2,2'-bi- thienyl (82)}

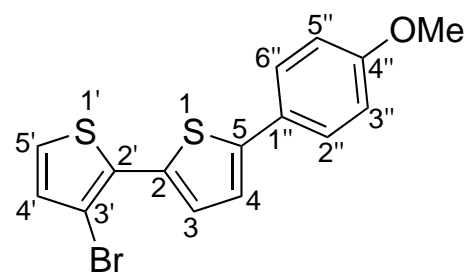

3'-Bromo-5-iodo-2,2'-bithiophene (81, $3.01 \mathrm{~g}, 7.57 \mathrm{mmol}), 1.00 \mathrm{eq}), 4$-methoxyphenylboronic acid $(74,1.38 \mathrm{~g}, 9.08 \mathrm{mmol}, 1.20 \mathrm{eq})$, 1,2-dimethoxyethane $(21.0 \mathrm{ml})$, and sat. aq. $\mathrm{NaHCO}_{3}(21 \mathrm{ml})$ were mixed and argon was bubbled through the mixture for $10 \mathrm{~min}$. [Pd( $\left.\left(\mathrm{PPh}_{3}\right)_{4}\right](0.44 \mathrm{~g}, 0.38 \mathrm{mmol}, 0.05 \mathrm{eq})$ was added and the yellow suspension was stirred at $90^{\circ} \mathrm{C}$ for $16 \mathrm{~h}$. The reaction mixture was diluted with water $(100 \mathrm{ml})$ and extracted with $\mathrm{CH}_{2} \mathrm{Cl}_{2}(3 \times 50 \mathrm{ml})$. The combined organic solutions were dried over $\mathrm{Na}_{2} \mathrm{SO}_{4}$ and concentrated in vacuo to give $3.35 \mathrm{~g}$ of a yellow oil which solidified upon standing at room temperature. The crude product was recrystallized from a mixture of $\mathrm{CHCl}_{3} / \mathrm{MeOH}(3: 4)$. Column chromatography on silica gel $\left(100 \mathrm{~g} \mathrm{SiO}_{2}\right.$, cyclohexane/EtOAc $\left.=9: 1\right)$ gave $1.56 \mathrm{~g}(4.46 \mathrm{mmol}, 59 \%)$ of the title compound as yellow solid. 


\section{Chapter 22 COMPOUNDS}

NOTE: The product is poorly soluble in non-chlorinated solvents; sufficient dilution during the chromatography is recommended in order to avoid precipitation.

${ }^{1} \mathbf{H}$ NMR $\left(400 \mathrm{MHz} \mathrm{CDCl}_{3}\right): \delta=3.84\left(\mathrm{~s}, 3 \mathrm{H}, \mathrm{CH}_{3}\right), 6.90-6.95(\mathrm{~m}, 2 \mathrm{H}, 3$ "- $\mathrm{H}$, $\left.5^{\prime \prime}-\mathrm{H}\right), 7.02\left(\mathrm{~d},{ }^{3} J_{\mathrm{H}, \mathrm{H}}=5.4 \mathrm{~Hz}, 1 \mathrm{H}, 4^{\prime}-\mathrm{H}\right), 7.16\left(\mathrm{~d},{ }^{3} J_{\mathrm{H}, \mathrm{H}}=3.8 \mathrm{~Hz}, 1 \mathrm{H}, 4-\mathrm{H}\right), 7.18(\mathrm{~d}$, $\left.{ }^{3} J_{\mathrm{H}, \mathrm{H}}=5.4 \mathrm{~Hz}, 1 \mathrm{H}, 5^{\prime}-\mathrm{H}\right), 7.37\left(\mathrm{~d},{ }^{3} J_{\mathrm{H}, \mathrm{H}}=3.8 \mathrm{~Hz}, 1 \mathrm{H}, 3-\mathrm{H}\right), 7.54-7.58(\mathrm{~m}, 2 \mathrm{H}$, 2"-H, 6"-H) ppm.

${ }^{13}$ C NMR $\left(101 \mathrm{MHz}, \mathrm{CDCl}_{3}\right): \delta=55.5\left(\mathrm{CH}_{3}\right), 107.6(\mathrm{C}-3$ '), $114.5(\mathrm{C}-3 ", \mathrm{C}-5 "), 122.3$ (C-4), 124.2 (C-5'), 126.9 (C-1"), 127.2 (C-2", C-6"), 127.7 (C-3), 132.1 (C-4'), 132.6, 132.7 (C-2, C-2'), 145.0 (C-5), 159.6 (C-4") ppm.

GC-MS (EI): $m / z(\%)=350 / 352(100)\left[\mathrm{M}^{+\cdot}\right]$.

HRMS (EI): $m / z$ calc. for $\mathrm{C}_{15} \mathrm{H}_{11} \mathrm{BrOS}_{2}\left[\mathrm{M}^{+\cdot}\right]$ 349.9435; found 349.9435 .

$\operatorname{TLC}\left(\mathrm{SiO}_{2}\right): R_{\mathrm{f}}=0.26($ cyclohexane/EtOAc $=9: 1)$.

$\operatorname{TLC}\left(\mathrm{RP} \mathrm{SiO}_{2}-\mathrm{C}_{18}\right): R_{\mathrm{f}}=0.47(\mathrm{MeCN})$.

\subsection{3-Bromo-5-(dimethoxymethyl)-5'-(p-methoxy- phenyl)-2,2'-bithienyl (83)}<smiles>COc1ccc(-c2ccc(-c3sc(C(OC)OC)cc3Br)s2)cc1</smiles>

Aldehyde 84 (50 mg, $0.13 \mathrm{mmol}, 1.0 \mathrm{eq}$ ) was loaded into a screw cap pressure tube and suspended in dry $\mathrm{MeOH}(5.0 \mathrm{ml})$ using an ultrasonic bath. A solution of $5.0 \mathrm{M}$ $\mathrm{HCl}$ in $\mathrm{EtOAc}(0.13 \mathrm{ml}, 0.66 \mathrm{mmol}, 5.0 \mathrm{eq})$ was added. The screw cap pressure tube was sealed, and the brown suspension was stirred at room temperature. The suspension became slowly clearer but after about $60 \mathrm{~min}$, a colorless precipitate formed. After stirring for $3.5 \mathrm{~h}$ at r.t., the reaction mixture was transferred into aq. sat. $\mathrm{NaHCO}_{3}(10 \mathrm{ml})$. The mixture was extracted with EtOAc $(3 \times 8 \mathrm{ml})$. The combined organic solutions were dried over $\mathrm{Na}_{2} \mathrm{SO}_{4}$ and concentrated in vacuo to give $54 \mathrm{mg}(0.17 \mathrm{mmol}, 96 \%)$ of the title compound as red oil which solidified at $0{ }^{\circ} \mathrm{C}$ overnight to give an orange solid. 
${ }^{1} \mathbf{H}$ NMR $\left(400 \mathrm{MHz}, \mathrm{CD}_{2} \mathrm{Cl}_{2}\right): \delta=3.37\left(\mathrm{~s}, 6 \mathrm{H}, \mathrm{CH}\left(\mathrm{OCH}_{3}\right)_{2}\right), 3.83\left(\mathrm{~s}, 3 \mathrm{H}, 4\right.$ "- $\left.\mathrm{OCH}_{3}\right)$, $5.56\left(\mathrm{~d},{ }^{4} J_{\mathrm{H}, \mathrm{H}}=0.9 \mathrm{~Hz}, 1 \mathrm{H}, 5^{\prime}-\mathrm{CH}\left(\mathrm{OCH}_{3}\right)_{2}\right), 6.91-6.96(\mathrm{~m}, 2 \mathrm{H}, 3$ "-H, 5"-H), $7.00(\mathrm{~d}$, $\left.{ }^{4} J_{\mathrm{H}, \mathrm{H}}=0.9 \mathrm{~Hz}, 1 \mathrm{H}, 4^{\prime}-\mathrm{H}\right), 7.19\left(\mathrm{~d},{ }^{3} J_{\mathrm{H}, \mathrm{H}}=3.8 \mathrm{~Hz}, 1 \mathrm{H}, 4-\mathrm{H}\right), 7.37\left(\mathrm{~d},{ }^{3} J_{\mathrm{H}, \mathrm{H}}=3.8 \mathrm{~Hz}\right.$, $1 \mathrm{H}, 3-\mathrm{H}), 7.54-7.60$ (m, $2 \mathrm{H}, 2 "-\mathrm{H}, 6$ "-H) ppm.

${ }^{13} \mathrm{C}$ NMR $\left(101 \mathrm{MHz}, \mathrm{CD}_{2} \mathrm{Cl}_{2}\right): \delta=53.2\left(\mathrm{CH}\left(\mathrm{OCH}_{3}\right)_{2}\right), 55.9\left(4 "-\mathrm{OCH}_{3}\right), 99.9$ $\left(\underline{\mathrm{CH}}\left(\mathrm{OCH}_{3}\right)_{2}\right), 107.0$ (C-3'), 114.9 (C-3", C-5"), 122.8 (C-4), 127.1 (C-1"), 127.6 (C-2", C-6"), 128.2 (C-3), 130.4 (C-4'), 133.1 (C-2), 133.3 (C-2'), 140.8 (C-5'), 145.5 (C-5), $160.2(\mathrm{C}-4 ")$ ppm.

MS (ESI, pos.): $m / z(\%)=247 / 249(100)[\mathrm{M}+\mathrm{Na}]^{+}$.

HRMS (EI): $m / z$ calc. for $\mathrm{C}_{18} \mathrm{H}_{17} \mathrm{BrO}_{3} \mathrm{~S}_{2}\left[\mathrm{M}^{+\cdot}\right]$ 423.9800; found 423.9802 .

$\operatorname{TLC}\left(\mathrm{SiO}_{2}\right): R_{\mathrm{f}}=0.56\left(\right.$ cyclohexane/EtOAc $\left.=1: 1+1 \% \mathrm{NEt}_{3}\right)$.

\subsection{3-Bromo-5'-(p-methoxyphenyl)-2,2'-bithienyl- 5-carbaldehyde (84)}

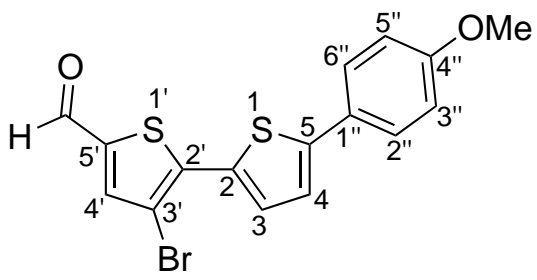

Formylation of bithiophene derivative $\mathbf{8 2}$ was carried out under common Vilsmeier Haack conditions. ${ }^{[141]}$

To a solution of 3'-bromo-5-(4-methoxyphenyl)-2,2'-bithiophene (82, $502 \mathrm{mg}$, $1.43 \mathrm{mmol}, 1.00 \mathrm{eq})$ in dry 1,2-dichloroethane (12 ml), DMF (1.3 ml, $1.3 \mathrm{~g}, 17 \mathrm{mmol}$, $12 \mathrm{eq)}$ was added under an argon atmosphere at room temperature. The solution was cooled to $0{ }^{\circ} \mathrm{C}$, and $\mathrm{POCl}_{3}(0.60 \mathrm{ml}, 0.99 \mathrm{~g}, 4.5 \mathrm{eq})$ was added dropwise over a period of $20 \mathrm{~min}$. The mixture was stirred at $0{ }^{\circ} \mathrm{C}$ for $10 \mathrm{~min}$ and then heated at $90{ }^{\circ} \mathrm{C}$ for $7 \mathrm{~h}$. The reaction was monitored by TLC on regular phase $\mathrm{SiO}_{2}$ plates with cyclohexane/EtOAc $=9: 1$ as eluent. After full conversion of the starting material, the reaction mixture was cooled to $0^{\circ} \mathrm{C}$, transferred into pre-cooled $1.0 \mathrm{M}$ aq. $\mathrm{NaOH}(100 \mathrm{ml})$ at $0{ }^{\circ} \mathrm{C}$ and mixed vigorously. The mixture was extracted with $\mathrm{CHCl}_{3}(3 \times 50 \mathrm{ml})$. The combined organic solutions were dried over $\mathrm{Na}_{2} \mathrm{SO}_{4}$ and concentrated in vacuo to give $592 \mathrm{mg}$ of brown solid. Column chromatography on 


\section{Chapter 22 $\mid$ COMPOUNDS}

silica gel (cyclohexane $\left./ \mathrm{CHCl}_{3} / \mathrm{EtOAc}=16: 4: 1\right)$ afforded $347 \mathrm{mg}(915 \mu \mathrm{mol}, 64 \%)$ of the title compound as brown solid.

${ }^{1} \mathbf{H}$ NMR $\left(400 \mathrm{MHz}_{\mathrm{CDCl}}\right): \delta=3.85\left(\mathrm{~s}, 3 \mathrm{H}, \mathrm{CH}_{3}\right), 6.90-6.96(\mathrm{~m}, 2 \mathrm{H}, 3$ "- $\mathrm{H}$, 5"-H), $7.21\left(\mathrm{~d},{ }^{3} J_{\mathrm{H}, \mathrm{H}}=3.8 \mathrm{~Hz}, 1 \mathrm{H}, 4-\mathrm{H}\right), 7.54-7.61\left(\mathrm{~m}, 3 \mathrm{H}, 3-\mathrm{H}, 2^{\prime \prime}-\mathrm{H}, 6\right.$ "-H), 7.65 (s, $\left.1 \mathrm{H}, 4^{\prime}-\mathrm{H}\right), 9.80\left(\mathrm{~s}, 1 \mathrm{H}, 5^{\prime}-\mathrm{CHO}\right) \mathrm{ppm}$.

${ }^{13}$ C NMR $\left(101 \mathrm{MHz}, \mathrm{CDCl}_{3}\right): \delta=55.5\left(\mathrm{CH}_{3}\right), 107.8(\mathrm{C}-3$ '), $114.6(\mathrm{C}-3 ", \mathrm{C}-5 "), 122.8$ (C-4), 126.3 (C-1"), 127.5 (C-2", C-6"), 129.9 (C-3), 131.6 (C-5), 139.5 (C-5'), 140.5 (C-4'), 142.3 (C-2'), 147.8 (C-2), 160.1 (C-4'), 181.6 (5'-CHO) ppm.

GC-MS (EI): $m / z(\%)=378 / 380(100)\left[\mathrm{M}^{+\cdot}\right]$.

HRMS (EI): $m / z$ calc. for $\mathrm{C}_{16} \mathrm{H}_{11} \mathrm{BrO}_{2} \mathrm{~S}_{2}\left[\mathrm{M}^{+\cdot}\right]$ 377.9384; found 377.9389 .

$\operatorname{TLC}\left(\mathrm{SiO}_{2}\right): R_{\mathrm{f}}=0.15($ cyclohexane/EtOAc $=9: 1)$.

\subsection{Compound 85}

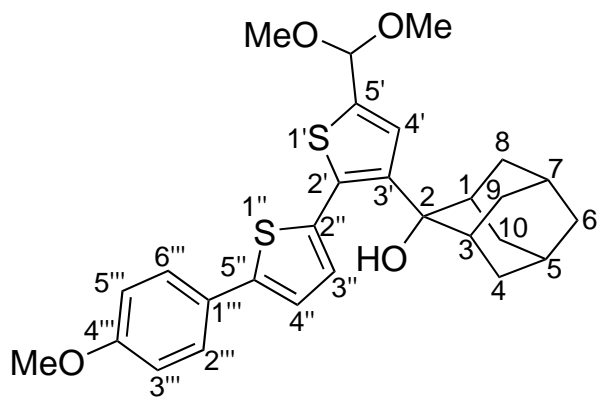

According to GP2, bromide 83 (22 mg, $51 \mu \mathrm{mol}, 1.0 \mathrm{eq})$ was lithiated with $1.6 \mathrm{M}$ $n$-BuLi in $n$-hexane $(41 \mu \mathrm{l}, 66 \mu \mathrm{mol}, 1.3 \mathrm{eq})$ in dry THF $(0.58 \mathrm{ml})$. After addition of 2-adamantanone $(15 \mathrm{mg}, 0.10 \mathrm{mmol}, 2.0 \mathrm{eq})$ in dry THF $(0.10 \mathrm{ml})$, the reaction mixture was stirred at $-78^{\circ} \mathrm{C}$ for $10 \mathrm{~min}$, and the reaction mixture was stirred at ambient temperature for additional $30 \mathrm{~min}$. Aq. work-up included addition of water $(5 \mathrm{ml})$ followed by extraction with $\mathrm{CH}_{2} \mathrm{Cl}_{2}(5 \times 4 \mathrm{ml})$ and gave $27 \mathrm{mg}$ of yellow oil. Column chromatography on silica gel $(n$-hexane/EtOAc $=1: 1+1 \%$ $\left.\mathrm{NEt}_{3}\right)$ gave $17 \mathrm{mg}(35 \mu \mathrm{mol}, 68 \%)$ of the title compound as yellow foam.

${ }^{1} \mathbf{H}$ NMR $\left(400 \mathrm{MHz}, \mathrm{CD}_{2} \mathrm{Cl}_{2}\right): \delta=1.52-1.60\left(\mathrm{~m}, 2 \mathrm{H},\left[4-\mathrm{H}_{\mathrm{a}}, 10-\mathrm{H}_{\mathrm{a}}\right]\right.$ or $\left[8-\mathrm{H}_{\mathrm{a}}\right.$, $\left.\left.9-\mathrm{H}_{\mathrm{a}}\right]\right), 1.62-1.81\left(\mathrm{~m}, 6 \mathrm{H}, 5-\mathrm{H}, 7-\mathrm{H},\left[8-\mathrm{H}_{2}, 9-\mathrm{H}_{2}\right]\right.$ or $\left.\left[4-\mathrm{H}_{2}, 10-\mathrm{H}_{2}\right]\right), 2.21-2.32$ $\left(\mathrm{m}, 2 \mathrm{H},\left[4-\mathrm{H}_{\mathrm{b}}, 10-\mathrm{H}_{\mathrm{b}}\right]\right.$ or $\left.\left[8-\mathrm{H}_{\mathrm{b}}, 9-\mathrm{H}_{\mathrm{b}}\right]\right), 2.36-2.43(\mathrm{~m}, 2 \mathrm{H}, 1-\mathrm{H}, 3-\mathrm{H}), 3.37(\mathrm{~s}, 6$ $\left.\mathrm{H}, \mathrm{CH}\left(\mathrm{OC}_{3}\right)_{2}\right), 3.83\left(\mathrm{~s}, 3 \mathrm{H}, 4 "-\mathrm{OCH}_{3}\right), 5.55\left(\mathrm{~d},{ }^{4} \mathrm{~J}_{\mathrm{H}, \mathrm{H}}=0.9 \mathrm{~Hz}, 1 \mathrm{H}, \mathrm{C} \underline{\mathrm{H}}\left(\mathrm{OCH}_{3}\right)_{2}\right)$, 
$6.90-6.95\left(\mathrm{~m}, 2 \mathrm{H}, 3^{\prime \prime \prime}-\mathrm{H}, 5^{\prime \prime \prime}-\mathrm{H}\right), 7.12\left(\mathrm{~d},{ }^{4} J_{\mathrm{H}, \mathrm{H}}=0.9 \mathrm{~Hz}, 1 \mathrm{H}, 4^{\prime}-\mathrm{H}\right), 7.14\left(\mathrm{~d},{ }^{3} J_{\mathrm{H}, \mathrm{H}}\right.$ $=3.7 \mathrm{~Hz}, 1 \mathrm{H}, 4 "-\mathrm{H}), 7.18\left(\mathrm{~d},{ }^{3} J_{\mathrm{H}, \mathrm{H}}=3.7 \mathrm{~Hz}, 1 \mathrm{H}, 3^{\prime \prime}-\mathrm{H}\right), 7.52-7.57(\mathrm{~m}, 2 \mathrm{H}, 2 \mathrm{\prime}-\mathrm{H}$, 6"'-H) ppm.

${ }^{13} \mathrm{C}$ NMR $\left(101 \mathrm{MHz}, \mathrm{CD}_{2} \mathrm{Cl}_{2}\right): \delta=27.4,27.9(\mathrm{C}-5, \mathrm{C}-7), 33.3,35.8$ (C-4, C-8, C-9, $\mathrm{C}-10), 36.5(\mathrm{C}-1, \mathrm{C}-3), 38.1(\mathrm{C}-6), 53.2\left(\mathrm{CH}\left(\mathrm{OCH}_{3}\right)_{2}\right), 55.9\left(\mathrm{OCH}_{3}\right), 76.2(\mathrm{C}-2), 100.7$ ( $\left.\underline{\mathrm{CH}}\left(\mathrm{OCH}_{3}\right)_{2}\right), 114.9$ (C-3", C-5"), 122.5 (C-4"), 127.1 (C-4'), 127.3 (C-1'"), 127.4 (C-2"', C-6"'), 130.5 (C-3"), 133.3, 134.9, 140.9, 144.2 (C-2', C-3', C-5', C-2"), 146.0 (C-5"), $160.1(\mathrm{C}-4 " ')$ ppm.

HRMS (EI): $m / z$ calc. for $\mathrm{C}_{28} \mathrm{H}_{32} \mathrm{BrO}_{4} \mathrm{~S}_{2}\left[\mathrm{M}^{+\cdot}\right]$ 496.1742; found 496.1736.

$\operatorname{TLC}\left(\mathrm{SiO}_{2}\right): R_{\mathrm{f}}=0.17(n$-hexane/EtOAc $=1: 1)$.

\subsection{Compound 86}

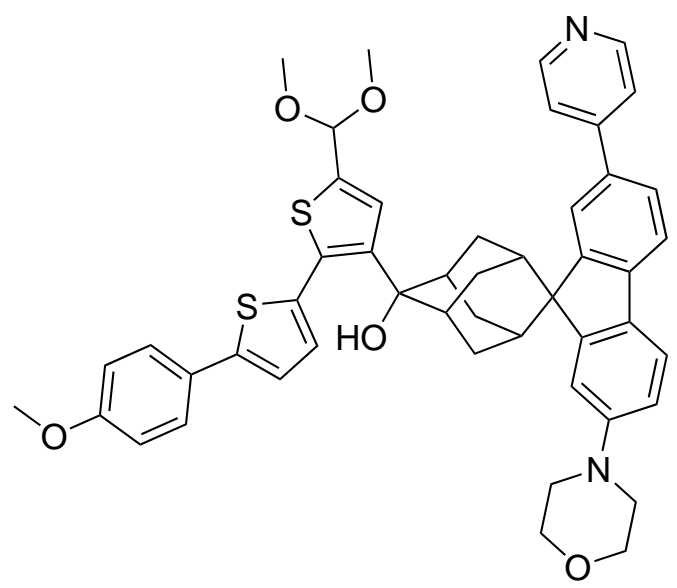

According to GP2, bromide 83 (174 mg, $410 \mu \mathrm{mol}, 2.67 \mathrm{eq})$ was lithiated with $2.5 \mathrm{M} n$-BuLi in $n$-hexane $(0.18 \mathrm{ml}, 0.46 \mathrm{mmol}, 3.0 \mathrm{eq})$ in dry THF $(2.0 \mathrm{ml})$. A precooled suspension of ketone 45 (71 $\mathrm{mg}, 0.15 \mathrm{mmol}, 1.0 \mathrm{eq})$ in dry THF $(2.0 \mathrm{ml})$ was added. Residual suspension of compound $\mathbf{4 5}$ was diluted with additional dry THF $(1.0 \mathrm{ml})$ and added dropwise to the reaction mixture at $-78^{\circ} \mathrm{C}$ over a period of $1 \mathrm{~min}$. The reaction mixture was stirred at $-78^{\circ} \mathrm{C}$ for $10 \mathrm{~min}$ and at ambient temperature for additional $5 \mathrm{~h}$. Aq. work-up included addition of water $(25 \mathrm{ml})$ followed by extraction with $\mathrm{CH}_{2} \mathrm{Cl}_{2}(6 \times 20 \mathrm{ml})$ and gave $228 \mathrm{mg}$ of brown solid. Column chromatography on silica gel $\left(\mathrm{CH}_{2} \mathrm{Cl}_{2} / \mathrm{EtOAc}=1: 1+1 \%\right.$ $\mathrm{NEt}_{3}$ ) gave $79 \mathrm{mg}$ of crude alcohol 86 (content of 86 was ca. $50 \%$, based on ${ }^{1} \mathrm{H}$ NMR spectroscopy) as an orange solid. Crude alcohol 86 was directly used in 


\section{Chapter 22 COMPOUNDS}

the next step without further purification.

MS (ESI, pos.): $m / z(\%)=809(100)[\mathrm{M}+\mathrm{H}]^{+}$.

$\operatorname{TLC}\left(\mathrm{SiO}_{2}\right): R_{\mathrm{f}}=0.19\left(\mathrm{CH}_{2} \mathrm{Cl}_{2} / \mathrm{EtOAc}=1: 1+1 \% \mathrm{NEt}_{3}\right)$.

\subsection{Compound 87}

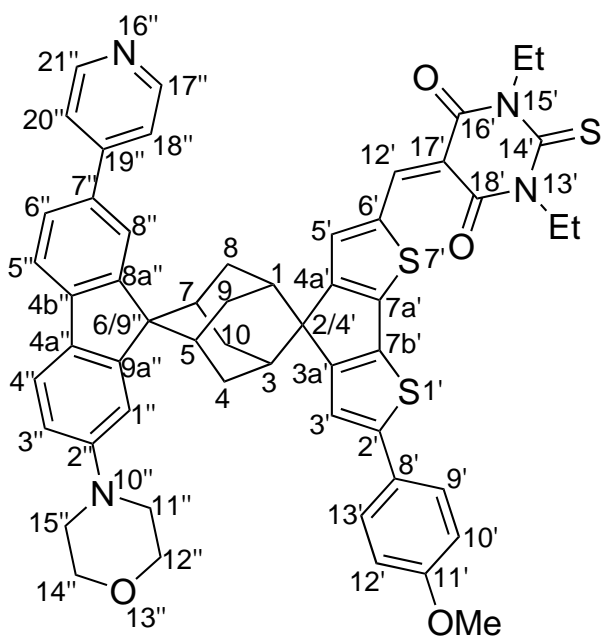

Aldehyde $88(11 \mathrm{mg}, 12 \mu \mathrm{mol}, 1.0 \mathrm{eq})$ and 1,3-diethyl-2-thiobarbituric acid ( $4.7 \mathrm{mg}, 23 \mu \mathrm{mol}, 1.3 \mathrm{eq}$ ) were dissolved in a $4.0 \mathrm{mM}$ solution of $\mathrm{NEt}_{3}$ in $\mathrm{CH}_{2} \mathrm{Cl}_{2}$ $(0.58 \mathrm{ml}, 2.3 \mu \mathrm{mol}, 0.10 \mathrm{eq})$ inside a screw cap pressure tube. The sealed reaction vessel was heated to $50^{\circ} \mathrm{C}$ for $4 \mathrm{~h}$. The mixture was cooled to r.t. and diluted with $\mathrm{CH}_{2} \mathrm{Cl}_{2}(10 \mathrm{ml})$ and water $(10 \mathrm{ml})$. The aq. layer was extracted with $\mathrm{CH}_{2} \mathrm{Cl}_{2}$ $(2 \times 10 \mathrm{ml})$, and the combined organic solutions were dried over $\mathrm{Na}_{2} \mathrm{SO}_{4}$. Concentration in vacuo gave $16 \mathrm{mg}$ of a black solid. Column chromatography on silica gel (two columns: first one with $\mathrm{CH}_{2} \mathrm{Cl}_{2} / \mathrm{EtOAc} / \mathrm{NEt}_{3}=75: 25: 1$; the second one with $\mathrm{CH}_{2} \mathrm{Cl}_{2} / \mathrm{EtOAc} / \mathrm{NEt}_{3}=80: 20: 0 \rightarrow$ 80:20:0.1) gave $11 \mathrm{mg}(12 \mu \mathrm{mol}, 99 \%)$ of racemic mixture of the title compound as black solid.

${ }^{1} \mathbf{H}$ NMR $\left(400 \mathrm{MHz}, \mathrm{CD}_{2} \mathrm{Cl}_{2}\right): \delta=1.27\left(\mathrm{t},{ }^{3} J_{\mathrm{H}, \mathrm{H}}=7.0 \mathrm{~Hz}, 3 \mathrm{H}, \mathrm{CH}_{2} \mathrm{CH}_{3}\right), 1.33(\mathrm{t}$, $\left.{ }^{3} J_{\mathrm{H}, \mathrm{H}}=7.0 \mathrm{~Hz}, 3 \mathrm{H}, \mathrm{CH}_{2} \mathrm{C}_{3}\right), 1.95-2.11(\mathrm{~m}, 4 \mathrm{H}, 1-\mathrm{H}, 3-\mathrm{H}, 5-\mathrm{H}, 7-\mathrm{H}), 2.57-2.76$ $\left(\mathrm{m}, 4 \mathrm{H}, 4-\mathrm{H}_{\mathrm{a}}, 8-\mathrm{H}_{\mathrm{a}}, 9-\mathrm{H}_{\mathrm{a}}, 10-\mathrm{H}_{\mathrm{a}}\right), 3.10-3.23\left(\mathrm{~m}, 8 \mathrm{H}, 4-\mathrm{H}_{\mathrm{b}}, 8-\mathrm{H}_{\mathrm{b}}, 9-\mathrm{H}_{\mathrm{b}}, 10-\mathrm{H}_{\mathrm{b}}\right.$, $\left.11 "-\mathrm{H}_{2}, 15 "-\mathrm{H}_{2}\right), 3.82-3.87\left(\mathrm{~m}, 7 \mathrm{H}, 12 "-\mathrm{H}_{2}, 14 "-\mathrm{H}_{2}, \mathrm{OCH}_{3}\right), 4.51-4.64(\mathrm{~m}, 4 \mathrm{H}$, $\left.2 \times \mathrm{C}_{2} \mathrm{CH}_{3}\right), 6.93-6.99\left(\mathrm{~m}, 2 \mathrm{H}, 10^{\prime}-\mathrm{H}, 12^{\prime}-\mathrm{H}\right), 7.06\left(\mathrm{dd},{ }^{3} J_{\mathrm{H}, \mathrm{H}}=8.5 \mathrm{~Hz},{ }^{4} J_{\mathrm{H}, \mathrm{H}}=\right.$ $1.8 \mathrm{~Hz}, 1 \mathrm{H}, 3 "-\mathrm{H}), 7.55-7.59$ (m, $\left.2 \mathrm{H}, 18^{\prime \prime}-\mathrm{H}, 20^{\prime \prime}-\mathrm{H}\right), 7.61-7.66\left(\mathrm{~m}, 2 \mathrm{H}, 9^{\prime}-\mathrm{H}\right.$, $\left.13^{\prime}-\mathrm{H}\right), 7.69\left(\mathrm{dd},{ }^{3} J_{\mathrm{H}, \mathrm{H}}=8.0 \mathrm{~Hz},{ }^{4} J_{\mathrm{H}, \mathrm{H}}=0.8 \mathrm{~Hz}, 1 \mathrm{H}, 6{ }^{\prime \prime}-\mathrm{H}\right), 7.74-7.77\left(\mathrm{~m}, 2 \mathrm{H}, 3^{\prime}-\mathrm{H}\right.$, 
1"-H), $7.79\left(\mathrm{~d},{ }^{3} J_{\mathrm{H}, \mathrm{H}}=8.5 \mathrm{~Hz}, 1 \mathrm{H}, 4\right.$ "-H), $7.86\left(\mathrm{~d},{ }^{3} J_{\mathrm{H}, \mathrm{H}}=8.0 \mathrm{~Hz}, 1 \mathrm{H}, 5^{\prime \prime}-\mathrm{H}\right), 8.30(\mathrm{~s}$, $\left.1 \mathrm{H}, 5^{\prime}-\mathrm{H}\right), 8.41\left(\mathrm{~d},{ }^{4} J_{\mathrm{H}, \mathrm{H}}=0.8 \mathrm{~Hz}, 1 \mathrm{H}, 8{ }^{\prime \prime}-\mathrm{H}\right), 8.58-8.63\left(\mathrm{~m}, 2 \mathrm{H}, 17^{\prime \prime}-\mathrm{H}, 21^{\prime \prime}-\mathrm{H}\right)$, $8.71\left(\mathrm{~s}, 1 \mathrm{H}, 12^{\prime}-\mathrm{H}\right) \mathrm{ppm}$.

${ }^{13} \mathrm{C}$ NMR $\left(101 \mathrm{MHz}, \mathrm{CD}_{2} \mathrm{Cl}_{2}\right): \delta=12.7,12.8\left(2 \times \mathrm{CH}_{2} \underline{\mathrm{CH}_{3}}\right), 30.1,30.6,31.1$, 31.6, (C-4, C-8, C-9, C-10), 34.1, 34.1, 34.7, 34.8 (C-1, C-3, C-5, C-7), 43.5, 44.2 $\left(2 \times \underline{\mathrm{CH}}_{2} \mathrm{CH}_{3}\right), 50.8\left(\mathrm{C}-11^{\prime \prime}, \mathrm{C}-15^{\prime \prime}\right), 56.0\left(11^{\prime}-\mathrm{OCH}_{3}\right), 59.4,61.0\left(\mathrm{C}-2 / 4^{\prime}, \mathrm{C}-6 / 9^{\prime \prime}\right)$, 67.4 (C-12", C-14"), 107.6 (C-17'), 115.1 (C-10', C-12'), 116.2 (C-3"), 118.8 (C-1"), 119.8 (C-5"), 121.0 (C-4"), 122.4 (C-18", C-20"), 123.2 (C-3'), 127.2 (C-6"), 127.4 (C-8'), 127.9 (C-9', C-13'), 128.7 (C-8"), 133.2 (C-4a"), 135.2 (C-7"), 136.2 (C-7b'), 137.7 (C-6'), 143.1 (C-4b"), 144.8 (C-5'), 149.5 (C-12'), 150.1 (C-19"), 150.1 (C-17", C-21"), 150.9 (C-8a"), 151.6, 151.7 (C-2', C-2"), 152.3 (C-9a"), 157.3, 160.0 (C-4a', C-7a'), 160.8, 160.9, 161.8 (C-11', C-16', C-18'), 165.3 (C-3a'), 179.3 (C-14') ppm.

MS (ESI, pos.): $m / z(\%)=927(100)[\mathrm{M}+\mathrm{H}]^{+}$.

HRMS (ESI): $m / z$ calc. for $\mathrm{C}_{55} \mathrm{H}_{51} \mathrm{~N}_{4} \mathrm{O}_{4} \mathrm{~S}_{3}[\mathrm{M}+\mathrm{H}]^{+}$927.3067; found 927.3031.

$\operatorname{TLC}\left(\mathrm{SiO}_{2}\right): R_{\mathrm{f}}=0.36\left(\mathrm{CH}_{2} \mathrm{Cl}_{2} / \mathrm{EtOAc}=4: 1\right)$.

\subsection{Compound 88}

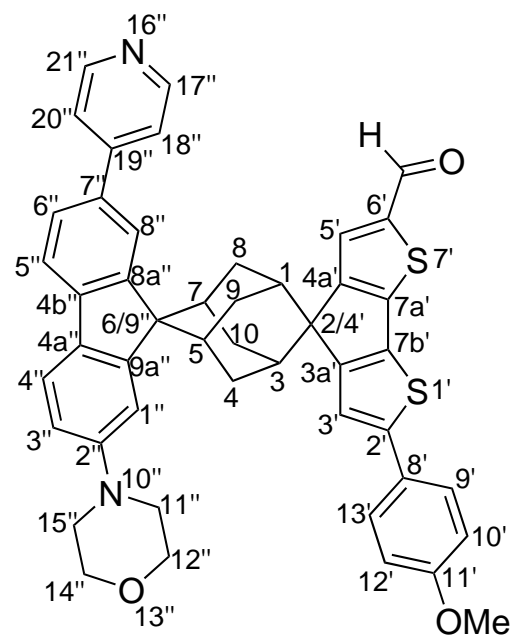

According to GP3, crude alcohol 86 (79 mg, content ca. $50 \%$, based on ${ }^{1} \mathrm{H}$ NMR spectroscopy) was treated with conc. $\mathrm{H}_{2} \mathrm{SO}_{4}(0.17 \mathrm{ml})$ in $n$-octane $(3.3 \mathrm{ml})$ and stirred for $60 \mathrm{~min}$. The aq. work-up included quenching with $0.6 \mathrm{M}$ aq. $\mathrm{Na}_{2} \mathrm{CO}_{3}$ $(35 \mathrm{ml})$ followed by extraction with $\mathrm{CH}_{2} \mathrm{Cl}_{2}(10 \times 25 \mathrm{ml})$ and gave $94 \mathrm{mg}$ of yellow solid. Column chromatography on silica gel (two columns: first one with 


\section{Chapter 22 COMPOUNDS}

$\mathrm{CH}_{2} \mathrm{Cl}_{2} /$ EtOAc $=3: 1+1 \% \mathrm{NEt}_{3}$; the second one with $\mathrm{CH}_{2} \mathrm{Cl}_{2} / E t O A c=4: 1 \rightarrow$ $2: 1+1 \% \mathrm{NEt}_{3}$ ) gave $18 \mathrm{mg}$ of the desired product in $88 \%$ purity. Purification by preparative HPLC (normal phase column: Nucleosil 100-7 OH; solvent system: iso-octane/EtOAc 50:50 $\rightarrow$ 15:85 over $15 \mathrm{~min}$; flow: $8.0 \mathrm{ml} / \mathrm{min}$ ) yielded $14 \mathrm{mg}$ ( $19 \mu \mathrm{mol}, 12 \%$ over two steps) of the title compound as yellow solid.

${ }^{1} \mathbf{H}$ NMR $\left(400 \mathrm{MHz}, \mathrm{CD}_{2} \mathrm{Cl}_{2}\right): \delta=1.92-2.04(\mathrm{~m}, 4 \mathrm{H}, 1-\mathrm{H}, 3-\mathrm{H}, 5-\mathrm{H}, 7-\mathrm{H}), 2.57-$ $2.77\left(\mathrm{~m}, 4 \mathrm{H}, 4-\mathrm{H}_{\mathrm{a}}, 8-\mathrm{H}_{\mathrm{a}}, 9-\mathrm{H}_{\mathrm{a}}, 10-\mathrm{H}_{\mathrm{a}}\right), 3.05-3.27\left(\mathrm{~m}, 8 \mathrm{H}, 4-\mathrm{H}_{\mathrm{b}}, 8-\mathrm{H}_{\mathrm{b}}, 9-\mathrm{H}_{\mathrm{b}}, 10-\mathrm{H}_{\mathrm{b}}\right.$, $\left.11 "-\mathrm{H}_{2}, 15 "-\mathrm{H}_{2}\right), 3.81-3.88\left(\mathrm{~m}, 7 \mathrm{H}, 12 "-\mathrm{H}_{2}, 14 "-\mathrm{H}_{2}, \mathrm{OCH}_{3}\right), 6.91-6.96(\mathrm{~m}, 2 \mathrm{H}$, $\left.10^{\prime}-\mathrm{H}, 12^{\prime}-\mathrm{H}\right), 7.04\left(\mathrm{dd},{ }^{3} J_{\mathrm{H}, \mathrm{H}}=8.5 \mathrm{~Hz},{ }^{4} \mathrm{~J}_{\mathrm{H}, \mathrm{H}}=1.9 \mathrm{~Hz}, 1 \mathrm{H}, 3 "-\mathrm{H}\right), 7.51-7.54(\mathrm{~m}$, $\left.2 \mathrm{H}, 18^{\prime \prime}-\mathrm{H}, 20^{\prime \prime}-\mathrm{H}\right), 7.56-7.61\left(\mathrm{~m}, 2 \mathrm{H}, 9^{\prime}-\mathrm{H}, 13^{\prime}-\mathrm{H}\right), 7.67\left(\mathrm{dd},{ }^{3} J_{\mathrm{H}, \mathrm{H}}=8.0 \mathrm{~Hz},{ }^{4} J_{\mathrm{H}, \mathrm{H}}\right.$ $\left.=1.2 \mathrm{~Hz}, 1 \mathrm{H}, 6^{\prime \prime}-\mathrm{H}\right), 7.71\left(\mathrm{~s}, 1 \mathrm{H}, 3^{\prime}-\mathrm{H}\right), 7.77\left(\mathrm{~d},{ }^{4} J_{\mathrm{H}, \mathrm{H}}=1.8 \mathrm{~Hz}, 1 \mathrm{H}, 1^{\prime \prime}-\mathrm{H}\right), 7.77(\mathrm{~d}$, $\left.{ }^{3} J_{\mathrm{H}, \mathrm{H}}=8.4 \mathrm{~Hz}, 1 \mathrm{H}, 4^{\prime \prime}-\mathrm{H}\right), 7.83\left(\mathrm{~d},{ }^{3} J_{\mathrm{H}, \mathrm{H}}=8.0 \mathrm{~Hz}, 1 \mathrm{H}, 5 "-\mathrm{H}\right), 8.17\left(\mathrm{~s}, 1 \mathrm{H}, 5^{\prime}-\mathrm{H}\right)$, $8.41\left(\mathrm{~d},{ }^{4} J_{\mathrm{H}, \mathrm{H}}=1.2 \mathrm{~Hz}, 1 \mathrm{H}, 8^{\prime \prime}-\mathrm{H}\right), 8.68-8.61\left(\mathrm{~m}, 2 \mathrm{H}, 17^{\prime \prime}-\mathrm{H}, 21 "-\mathrm{H}\right), 9.85$ (s, $1 \mathrm{H}$, 6'-CHO) ppm.

${ }^{13}$ C NMR $\left(101 \mathrm{MHz}, \mathrm{CD}_{2} \mathrm{Cl}_{2}\right): \delta=30.2,30.7,31.0,31.5$ (C-4, C-8, C-9, C-10), 34.0, 34.8 (C-1, C-3, C-5, C-7), 50.8 (C-11", C-15"), $55.9\left(\mathrm{OCH}_{3}\right), 59.5$ (C-6/9"), 61.3 (C-2/4'), 67.4 (C-12", C-14"), 115.0 (C-10', C-12'), 116.2 (C-6"), 118.7 (C-1"), 119.8 (C-5"), 120.9 (C-4"), 122.1 (C-18", C-20"), 123.1 (C-3'), 127.1 (C-6"), 127.6 (C-9', C-13', C-8'), 128.7 (C-8"), 133.2 (C-4a"), 135.4, 135.4 (C-7b', C-7"), 136.09 (C-5'), 141.9 (C-6'), 142.9 (C-4b"), 147.7 (C-2'), 148.7 (C-7a'), 149.5 (C-19"), 150.8 (C-17", C-21"), 151.0 (C-8a"), 151.5 (C-2"), 152.3 (C-9a"), 156.3 (C-4a'), 160.4 (C-11'), 162.2 (C-3a'), 182.9 (6'-CHO) ppm.

MS (ESI, pos.): $m / z(\%)=745(100)[\mathrm{M}+\mathrm{H}]^{+}$.

HRMS (ESI): $m / z$ calc. for $\mathrm{C}_{47} \mathrm{H}_{41} \mathrm{~N}_{2} \mathrm{O}_{3} \mathrm{~S}_{2}[\mathrm{M}+\mathrm{H}]^{+}$745.2553; found 745.2515.

$\operatorname{TLC}\left(\mathrm{SiO}_{2}\right): R_{\mathrm{f}}=0.31\left(\mathrm{CH}_{2} \mathrm{Cl}_{2} / \mathrm{EtOAc}=3: 1+1 \% \mathrm{NEt}_{3}\right)$.

\subsection{Compound 89}

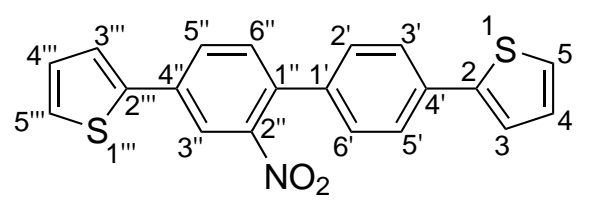

Suzuki cross-coupling of aryldibromide 51 and 2-thienylboronic acid was carried out according to literature method. ${ }^{[177]}$ 
Aryldibromide 51 (1.00 g, $2.80 \mathrm{mmol}, 1.00 \mathrm{eq})$ and 2-thienylboronic acid (1.25 g, $9.80 \mathrm{mmol}, 3.50 \mathrm{eq})$ were dissolved in a mixture of 1,2-dimethoxyethane $(45 \mathrm{ml})$ and $2.0 \mathrm{M}$ aq. $\mathrm{K}_{2} \mathrm{CO}_{3}(5.6 \mathrm{ml}, 11 \mathrm{mmol}, 4.0 \mathrm{eq})$. Argon was bubbled through the clear yellow solution over a period of $10 \mathrm{~min}$. After addition of $\left[\mathrm{Pd}\left(\mathrm{PPh}_{3}\right)_{4}\right]$ $(0.32 \mathrm{~g}, 0.28 \mathrm{mmol}, 0.10 \mathrm{eq})$, the reaction mixture was heated at $90^{\circ} \mathrm{C}$ for $3 \mathrm{~d}$. The reaction mixture was diluted with water $(50 \mathrm{ml})$, and the grey suspension extracted with EtOAc $(5 \times 100 \mathrm{ml})$. The combined organic solutions were dried over $\mathrm{Na}_{2} \mathrm{SO}_{4}$ and concentrated in vacuo to give $1.32 \mathrm{~g}$ of brown solid. Column chromatography on silica gel (cyclohexane $\left./ \mathrm{CH}_{2} \mathrm{CH}_{2}=2: 1\right)$ gave $782 \mathrm{mg}(2.15 \mathrm{mmol}$, $77 \%$ ) of the title compound as yellow solid.

${ }^{1} \mathbf{H}$ NMR $\left(400 \mathrm{MHz}, \mathrm{CDCl}_{3}\right): \delta=7.11\left(\mathrm{dd},{ }^{3} J_{\mathrm{H}, \mathrm{H}}=5.1,3.6 \mathrm{~Hz}, 1 \mathrm{H}, 4-\mathrm{H}\right), 7.15(\mathrm{dd}$, $\left.{ }^{3} J_{\mathrm{H}, \mathrm{H}}=5.1,3.6 \mathrm{~Hz}, 1 \mathrm{H}, 4^{\prime \prime}-\mathrm{H}\right), 7.32\left(\mathrm{dd},{ }^{3} J_{\mathrm{H}, \mathrm{H}}=5.1 \mathrm{~Hz},{ }^{4} J_{\mathrm{H}, \mathrm{H}}=1.6 \mathrm{~Hz}, 1 \mathrm{H}, 5-\mathrm{H}\right)$, $7.34-7.38\left(\mathrm{~m}, 3 \mathrm{H}, 3-\mathrm{H}, 2^{\prime}-\mathrm{H}, 6^{\prime}-\mathrm{H}\right), 7.40\left(\mathrm{dd},{ }^{3} J_{\mathrm{H}, \mathrm{H}}=5.1 \mathrm{~Hz},{ }^{4} J_{\mathrm{H}, \mathrm{H}}=1.2 \mathrm{~Hz}, 1 \mathrm{H}\right.$, $\left.5^{\prime \prime \prime-H}\right), 7.44\left(\mathrm{dd},{ }^{3} J_{\mathrm{H}, \mathrm{H}}=3.6 \mathrm{~Hz},{ }^{4} J_{\mathrm{H}, \mathrm{H}}=1.2 \mathrm{~Hz}, 1 \mathrm{H}, 3^{\prime \prime \prime}-\mathrm{H}\right), 7.48\left(\mathrm{~d},{ }^{3} J_{\mathrm{H}, \mathrm{H}}=5.1 \mathrm{~Hz}\right.$, $\left.1 \mathrm{H}, 6^{\prime \prime}-\mathrm{H}\right), 7.66-7.70\left(\mathrm{~m}, 2 \mathrm{H}, 3^{\prime}-\mathrm{H}, 5^{\prime}-\mathrm{H}\right), 7.83\left(\mathrm{dd},{ }^{3} J_{\mathrm{H}, \mathrm{H}}=8.0 \mathrm{~Hz},{ }^{4} J_{\mathrm{H}, \mathrm{H}}=1.9 \mathrm{~Hz}\right.$, $\left.1 \mathrm{H}, 5^{\prime \prime}-\mathrm{H}\right), 8.08\left(\mathrm{~d},{ }^{4} J_{\mathrm{H}, \mathrm{H}}=1.9 \mathrm{~Hz}, 1 \mathrm{H}, 3^{\prime \prime}-\mathrm{H}\right) \mathrm{ppm}$.

${ }^{13}$ C NMR $\left(101 \mathrm{MHz}, \mathrm{CDCl}_{3}\right): \delta=121.3(\mathrm{C}-3 "), 123.8$ (C-3), 124.9 (C 3"'-), 125.5 (C-5), 126.4 (C-3', C-5'), 126.7 (C-5"), 128.3 (C-4), 128.6 (C-2', C-6'), 128.6 (C-4'"'), 129.4 (C-5"), 132.5 (C-6"), 134.3 (C-1"), 134.6 (C-4'), 135.1 (C-4"), 136.0 (C-1'), 141.3 (C-2"'), 143.7 (C-2), 149.7 (C-2") ppm.

HRMS (EI): $m / z$ calc. for $\mathrm{C}_{20} \mathrm{H}_{13} \mathrm{NO}_{2} \mathrm{~S}_{2}\left[\mathrm{M}^{+\cdot}\right]$ 363.0388; found 363.0387.

$\operatorname{TLC}\left(\mathrm{SiO}_{2}\right): R_{\mathrm{f}}=0.28$ (cyclohexane $\left./ \mathrm{CH}_{2} \mathrm{Cl}_{2}=2: 1\right)$.

\subsection{2-[2',5'-Dibromo-4'-(2-thienyl)-4-biphenylyl]- thiophene (90)}

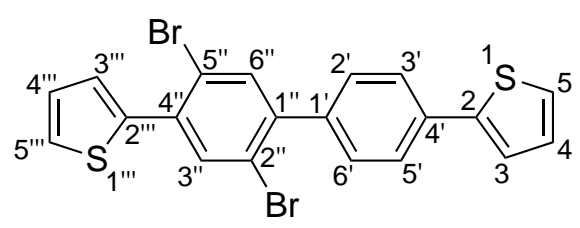

tert-Butyl nitrite $(11 \mu \mathrm{l}, 9.3 \mathrm{mg}, 90 \mu \mathrm{mol}, 1.5 \mathrm{eq})$ was added to a suspension of copper(II) bromide ( $16 \mathrm{mg}, 72 \mu \mathrm{mol}, 1.2 \mathrm{eq})$ in dry $\mathrm{MeCN}(0.61 \mathrm{ml})$ at $0{ }^{\circ} \mathrm{C}$. After stirring for $5 \mathrm{~min}$, amine 91 (20 mg, $60 \mu \mathrm{mol}, 1.0 \mathrm{eq})$ was added in one portion. The suspension was allowed to warm up to r.t. slowly by melting ice in an ice-water 


\section{Chapter 22 $\mid$ COMPOUNDS}

bath and leaving the reaction flask inside it. After $4 \mathrm{~d}$, the solvent evaporated. The light brown residue was suspended in $1.0 \mathrm{M}$ aq. $\mathrm{HCl}(6 \mathrm{ml})$ and extracted with $\mathrm{Et}_{2} \mathrm{O}(3 \times 6 \mathrm{ml})$. The combined organic solutions were washed with brine $(10 \mathrm{ml})$, dried over $\mathrm{Na}_{2} \mathrm{SO}_{4}$ and concentrated in vacuo to give $28 \mathrm{mg}$ of brown solid. Column chromatography on silica gel (cyclohexane) gave $10 \mathrm{mg}(21 \mu \mathrm{mol}, 35 \%)$ of the title compound as yellowish solid.

${ }^{1} \mathbf{H}$ NMR $\left(400 \mathrm{MHz}, \mathrm{CDCl}_{3}\right): \delta=7.12\left(\mathrm{dd},{ }^{3} J_{\mathrm{H}, \mathrm{H}}=5.1,3.7 \mathrm{~Hz}, 1 \mathrm{H}, 4-\mathrm{H}\right), 7.14(\mathrm{dd}$, $\left.{ }^{3} J_{\mathrm{H}, \mathrm{H}}=5.2,3.7 \mathrm{~Hz}, 1 \mathrm{H}, 4{ }^{\prime \prime}-\mathrm{H}\right), 7.33\left(\mathrm{dd},{ }^{3} J_{\mathrm{H}, \mathrm{H}}=5.1 \mathrm{~Hz},{ }^{3} J_{\mathrm{H}, \mathrm{H}}=1.2 \mathrm{~Hz}, 1 \mathrm{H}, 5-\mathrm{H}\right)$, $7.37\left(\mathrm{dd},{ }^{3} J_{\mathrm{H}, \mathrm{H}}=3.7 \mathrm{~Hz},{ }^{4} J_{\mathrm{H}, \mathrm{H}}=1.2 \mathrm{~Hz}, 1 \mathrm{H}, 3^{\prime \prime \prime}-\mathrm{H}\right), 7.39\left(\mathrm{dd},{ }^{3} J_{\mathrm{H}, \mathrm{H}}=3.7 \mathrm{~Hz},{ }^{4} J_{\mathrm{H}, \mathrm{H}}=\right.$ $1.2 \mathrm{~Hz}, 1 \mathrm{H}, 3-\mathrm{H}), 7.43$ - 7.48 (m, 3 H, 2'-H, 6'-H, 5'"-H), 7.67 (s, 1 H, 6"-H), 7.68 $7.72\left(\mathrm{~m}, 2 \mathrm{H}, 3^{\prime}-\mathrm{H}, 5^{\prime}-\mathrm{H}\right), 7.81\left(\mathrm{~s}, 1 \mathrm{H}, 3^{\prime \prime}-\mathrm{H}\right) \mathrm{ppm}$.

${ }^{13}$ C NMR $\left(101 \mathrm{MHz}, \mathrm{CDCl}_{3}\right): \delta=121.4,121.6(\mathrm{C}-2 ", \mathrm{C}-5 "), 123.7$ (C-3), 125.4 (C-5), 125.8 (C-3', C-5'), 127.0 (C-5'"), 127.3 (C-4'"'), 128.3 (C-4), 128.5 (C-3"'), 130.0 (C-2', C-6'), 134.5 (C-4'), 135.7 (C-6"), 135.8 (C-3"), 135.9 (C-4"), 138.3 (C-1'), 140.0 (C-2'"), 142.6 (C-1'), $143.8(\mathrm{C}-2) \mathrm{ppm}$.

HRMS (EI): $m / z$ calc. for $\mathrm{C}_{20} \mathrm{H}_{12} \mathrm{Br}_{2} \mathrm{~S}_{2}\left[\mathrm{M}^{+\cdot}\right]$ 473.8747; found 473.8747 .

$\operatorname{TLC}\left(\mathrm{SiO}_{2}\right): R_{\mathrm{f}}=0.29$ (cyclohexane).

\subsection{4,4'-Bis(2-thienyl)-2-biphenylylamine (91)}

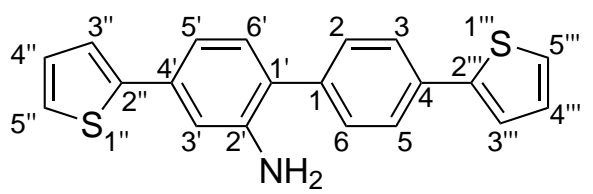

Activated zinc dust ${ }^{\dagger}(0.54 \mathrm{~g}, 8.3 \mathrm{mmol}, 60 \mathrm{eq})$ was added to a solution of nitro compound 89 (50 mg, $0.14 \mathrm{mmol}, 1.0 \mathrm{eq})$ in dry $\mathrm{CH}_{2} \mathrm{Cl}_{2}(10 \mathrm{ml})$. The suspension was cooled to $0^{\circ} \mathrm{C}$ and $\mathrm{AcOH}(0.35 \mathrm{ml}, 0.36 \mathrm{~g}, 6.1 \mathrm{mmol}, 44 \mathrm{eq})$ was added dropwise at $0^{\circ} \mathrm{C}$ over a period of $5 \mathrm{~min}$. After $1 \mathrm{~h}$ at $0{ }^{\circ} \mathrm{C}$, the mixture was filtered through Celite ${ }^{\mathrm{TM}}(\varnothing=30 \mathrm{~mm}, h=5 \mathrm{~mm})$. The filter cake was washed with $\mathrm{CH}_{2} \mathrm{Cl}_{2}(5 \times 10 \mathrm{ml})$, and the combined organic solutions were concentrated in vacuo to give $53 \mathrm{mg}$ of colorless solid. The crude compound was dissolved in hot $\mathrm{CHCl}_{3}($ ca. $3 \mathrm{ml})$ and loaded onto a prepacked silica gel column. Column chro-

\footnotetext{
${ }^{\dagger} \mathrm{Zn}$ was activated by stirring with $2 \%$ aq. $\mathrm{HCl}$, washing with water, ethanol, acetone and dry diethyl ether before drying in vacuo, according to ref. [172].
} 
matography (cyclohexane $\left./ \mathrm{CH}_{2} \mathrm{Cl}_{2}=1: 1\right)$ gave $44 \mathrm{mg}(0.13 \mathrm{mmol}, 95 \%)$ of the title compound as colorless solid.

${ }^{1} \mathbf{H}$ NMR $\left(400 \mathrm{MHz}, \mathrm{CDCl}_{3}\right): \delta=3.91\left(\mathrm{~s}_{\mathrm{br}}, 2 \mathrm{H}, \mathrm{NH}_{2}\right), 7.04\left(\mathrm{~d},{ }^{4} J_{\mathrm{H}, \mathrm{H}}=1.7 \mathrm{~Hz}, 1 \mathrm{H}\right.$, 3'-H), $7.07-7.14$ (m, 3 H, 4"-H, 4'"-H, 5'-H), 7.18 (d, $\left.{ }^{3} J_{\mathrm{H}, \mathrm{H}}=7.9 \mathrm{~Hz}, 1 \mathrm{H}, 66^{\prime}-\mathrm{H}\right), 7.28$ $\left(\mathrm{dd},{ }^{3} J_{\mathrm{H}, \mathrm{H}}=5.1 \mathrm{~Hz},{ }^{4} J_{\mathrm{H}, \mathrm{H}}=1.1 \mathrm{~Hz}, 1 \mathrm{H}, 5^{\prime \prime}-\mathrm{H}\right.$ or $\left.5^{\prime \prime \prime-H}\right), 7.30-7.33\left(\mathrm{~m}, 2 \mathrm{H}, 5{ }^{\prime \prime}-\mathrm{H}\right.$ or $5^{\prime \prime}-\mathrm{H}, 3$ "-H or $\left.3 " '-\mathrm{H}\right), 7.36\left(\mathrm{dd},{ }^{3} J_{\mathrm{H}, \mathrm{H}}=3.6 \mathrm{~Hz},{ }^{4} J_{\mathrm{H}, \mathrm{H}}=1.2 \mathrm{~Hz}, 1 \mathrm{H}, 3^{\prime \prime \prime-}-\mathrm{H}\right)$ or $3 "-\mathrm{H}$, $7.49-7.53(\mathrm{~m}, 2 \mathrm{H}, 2-\mathrm{H}, 6-\mathrm{H}), 7.69-7.73(\mathrm{~m}, 2 \mathrm{H}, 3-\mathrm{H}, 5-\mathrm{H}) \mathrm{ppm}$.

${ }^{13}$ C NMR $\left(101 \mathrm{MHz}, \mathrm{CDCl}_{3}\right): \delta=113.1\left(\mathrm{C}-3^{\prime}\right), 116.8\left(\mathrm{C}-5^{\prime}\right), 123.2,123.3\left(\mathrm{C}-3^{\prime \prime}\right.$, C-3"'), 124.8, 125.1 (C-5", C-5"'), 126.5 (C-1'), 126.5 (C-3, C-5), 128.1, 128.3 (C-4", C-4'"'), 129.6 (C-2, C-6), 131.0 (C-6'), 133.5 (C-4), 134.9 (C-4'), 138.3 (C-1), 144.0, 144.1, 144.4 (C-2', C-2", C-2"') ppm.

MS (ESI, pos.): $m / z(\%)=334.15(100)[\mathrm{M}+\mathrm{H}]^{+}$.

HRMS (ESI): $m / z$ calc. for $\mathrm{C}_{20} \mathrm{H}_{16} \mathrm{NS}_{2}[\mathrm{M}+\mathrm{H}]^{+} 334.0719$; found 334.0713.

$\operatorname{TLC}\left(\mathrm{SiO}_{2}\right): R_{\mathrm{f}}=0.15$ (cyclohexane $\left./ \mathrm{CH}_{2} \mathrm{Cl}_{2}=1: 1\right)$.

\subsection{2-[2'-Bromo-4'-(2-thienyl)-4-biphenylyl]- thiophene (92)}

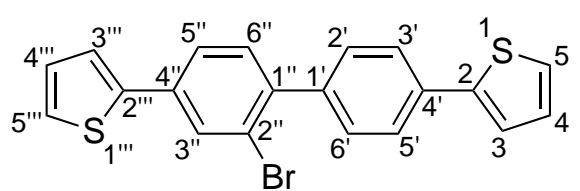

Synthesis from amine $\mathbf{9 1}$ was adapted with slight modifications from literature: ${ }^{[178,179]}$

n-Hexyl nitrite ( $54 \mu \mathrm{l}, 47 \mathrm{mg}, 0.36 \mathrm{mmol}, 1.2 \mathrm{eq})$ was diluted with $\mathrm{CHBr}_{3}(0.60 \mathrm{ml})$ and heated to $70^{\circ} \mathrm{C}$. A suspension of amine 91 (100 mg, $\left.300 \mu \mathrm{mol}, 1.00 \mathrm{eq}\right)$ in $\mathrm{CHBr}_{3}(1.0 \mathrm{ml})$ was added dropwise at $70^{\circ} \mathrm{C}$ over a period of $15 \mathrm{~min}$. After $3 \mathrm{~h}$, the dark-brown reaction mixture was cooled to r.t., diluted with $\mathrm{CH}_{2} \mathrm{Cl}_{2}$ and loaded directly onto a prepacked silica gel column. Column chromatography ( $n$-hexane $\left./ i-\mathrm{Pr}_{2} \mathrm{O}=95: 5\right)$ gave $46 \mathrm{mg}(0.12 \mathrm{mmol}, 39 \%)$ of the title compound as yellow solid.

Synthesis from diiodide 102: 


\section{Chapter 22 $\mid$ COMPOUNDS}

Tributyl(2-thienyl)stannane $(23 \mu \mathrm{l}, 27 \mathrm{mg}, 72 \mu \mathrm{mol}, 2.0 \mathrm{eq})$ was added to a solution of diiodide 102 ( $80 \%$ content, $17 \mathrm{mg}, 29 \mu \mathrm{mol}, 1.0 \mathrm{eq})$ in dry DMF $(0.50 \mathrm{ml})$ in a screw cap pressure tube, and a slow stream of argon was bubbled through the solution for $2 \mathrm{~min}$. Then [ $\left.\mathrm{Pd}\left(\mathrm{PPh}_{3}\right)_{4}\right](2.5 \mathrm{mg}, 3.6 \mu \mathrm{mol}, 0.10 \mathrm{eq})$ was added. The reaction mixture was heated at $80^{\circ} \mathrm{C}$ for $20 \mathrm{~h}$. After cooling to r.t., the reaction mixture was diluted with $\mathrm{Et}_{2} \mathrm{O}(6 \mathrm{ml})$ and $1.0 \mathrm{M}$ aq. $\mathrm{KF}(6 \mathrm{ml})$. The mixture was stirred vigorously for $20 \mathrm{~min}$. The aq. layer was separated and extracted with $\mathrm{Et}_{2} \mathrm{O}(2 \times 6 \mathrm{ml})$. The combined organic solutions were washed with sat. aq. $\mathrm{NH}_{4} \mathrm{Cl}(2 \times 10 \mathrm{ml})$, brine $(2 \times 10 \mathrm{ml})$, dried over $\mathrm{Na}_{2} \mathrm{SO}_{4}$ and concentrated in vacuo to give $33 \mathrm{mg}$ of yellow solid. Column chromatography on silica gel (cyclohexane $\left./ \mathrm{CH}_{2} \mathrm{Cl}_{2}=1: 1\right)$ gave $7.1 \mathrm{mg}(18 \mu \mathrm{mol}, 62 \%$ ) of the title compound as yellow solid.

${ }^{1} \mathbf{H}$ NMR $\left(400 \mathrm{MHz}, \mathrm{CDCl}_{3}\right): \delta=7.09-7.14\left(\mathrm{~m}, 2 \mathrm{H}, 4-\mathrm{H}, 44^{\prime \prime}-\mathrm{H}\right), 7.31\left(\mathrm{dd},{ }^{3} \mathrm{~J}_{\mathrm{H}, \mathrm{H}}=\right.$ $\left.5.1 \mathrm{~Hz},{ }^{4} J_{\mathrm{H}, \mathrm{H}}=1.1 \mathrm{~Hz}, 1 \mathrm{H}, 5-\mathrm{H}\right), 7.33-7.40(\mathrm{~m}, 4 \mathrm{H}, 3-\mathrm{H}, 6$ "-H, 3"'-H, 5"'-H), 7.44 $-7.50\left(\mathrm{~m}, 2 \mathrm{H}, 2^{\prime}-\mathrm{H}, 6^{\prime}-\mathrm{H}\right), 7.60\left(\mathrm{dd},{ }^{3} J_{\mathrm{H}, \mathrm{H}}=8.0 \mathrm{~Hz},{ }^{4} J_{\mathrm{H}, \mathrm{H}}=1.9 \mathrm{~Hz}, 1 \mathrm{H}, 5^{\prime \prime}-\mathrm{H}\right), 7.67$ - $7.72\left(\mathrm{~m}, 2 \mathrm{H}, 3^{\prime}-\mathrm{H}, 5^{\prime}-\mathrm{H}\right), 7.94\left(\mathrm{~d},{ }^{4} J_{\mathrm{H}, \mathrm{H}}=1.9 \mathrm{~Hz}, 1 \mathrm{H}, 3^{\prime \prime}-\mathrm{H}\right) \mathrm{ppm}$.

${ }^{13}$ C NMR $\left(101 \mathrm{MHz}, \mathrm{CDCl}_{3}\right): \delta=123.1$ (C-2"), 123.5 (C-3), 124.1 (C-3"'), 125.1 (C-5"), 125.2 (C-5), 125.7 (C-3', C-5'), 125.8 (C-5'"), 128.2, 128.4 (C-4, C-4"'), 130.1 (C-2', C-6'), 130.5 (C-3"), 131.6 (C-6"), 134.0 (C-4'), 135.3 (C-4"), 139.8 (C-1'), 140.9 (C-1"), 142.4 (C-2"'), $144.1(\mathrm{C}-2) \mathrm{ppm}$.

$\operatorname{TLC}\left(\mathrm{SiO}_{2}\right): R_{\mathrm{f}}=0.52\left(n\right.$-hexane $\left./ i-\mathrm{Pr}_{2} \mathrm{O}=1: 1\right)$.

\subsection{1,2-Bis(tert-butoxycarbonyl)-1-phenyl- hydrazine (94)}

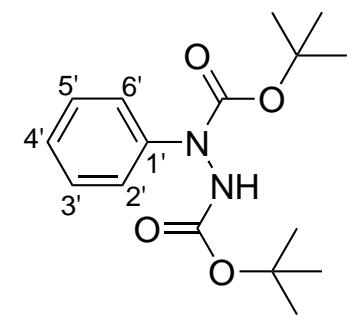

Compound 94 was prepared according to modified published methods. The procedure of Loog et al. ${ }^{[149]}$ was supplemented by addition of catalytic amounts of DMAP. 
$\mathrm{Boc}_{2} \mathrm{O}(240 \mathrm{~g}, 1.10 \mathrm{~mol}, 6.00 \mathrm{eq})$ was dissolved in dry $\mathrm{MeCN}(140 \mathrm{ml})$ and phenyl hydrazine $(18.2 \mathrm{ml}, 20.0 \mathrm{~g}, 185 \mathrm{mmol}, 1.00 \mathrm{eq})$ was added over a period of $2 \mathrm{~min}$ causing intense gas evolution. The reaction mixture was stirred at r.t. for $30 \mathrm{~min}$ and at $60{ }^{\circ} \mathrm{C}$ for $45 \mathrm{~min}$. DMAP (566 mg, $4.63 \mathrm{mmol}, 0.025 \mathrm{eq}$ ) was added in portions causing very intense gas evolution and the reaction mixture was stirred at $60{ }^{\circ} \mathrm{C}$ for $23 \mathrm{~h} . \mathrm{Mg}\left(\mathrm{ClO}_{4}\right)_{2}(8.26 \mathrm{~g}, 37.0 \mathrm{mmol}, 0.20 \mathrm{eq})$ was added, causing intense gas evolution. The reaction mixture was stirred for $90 \mathrm{~min}$ and poured into a mixture of brine $(1.00 \mathrm{I})$ and $1.0 \mathrm{M}$ aq. $\mathrm{KHSO}_{4}(1.00 \mathrm{I})$. The mixture was extracted with $\mathrm{Et}_{2} \mathrm{O}(4 \times 600 \mathrm{ml})$. The combined organic solutions were washed with brine $(3 \times 600 \mathrm{ml})$ and the combined aqueous solutions were extracted with $\mathrm{Et}_{2} \mathrm{O}(3 \times 300 \mathrm{ml})$. The combined organic solutions were dried over $\mathrm{Na}_{2} \mathrm{SO}_{4}$ and concentrated in vacuo to give an orange oil. Addition of petroleum ether (bp 40$60^{\circ} \mathrm{C}$, ca. $0.10 \mathrm{ml}$ ) initiated crystallization. The colorless crystalls were washed with cold $\left(0^{\circ} \mathrm{C}\right)$ petroleum ether $\left(\mathrm{bp} 40-60^{\circ} \mathrm{C}, 2 \times 10 \mathrm{ml}\right)$ and dried in vacuo to give $45.7 \mathrm{~g}$ ( $148 \mathrm{mmol}, 80 \%)$ of the title compound as yellow solid.

${ }^{1} \mathbf{H}$ NMR $\left(400 \mathrm{MHz}, \mathrm{CDCl}_{3}, 2\right.$ rotamers $): \delta=1.49\left(\mathrm{~s}, 18 \mathrm{H}, 6 \times \mathrm{CH}_{3}\right), 6.58\left(\mathrm{~s}_{\mathrm{br}}, 1 \mathrm{H}\right.$, $\mathrm{NH}), 6.82\left(\mathrm{~s}_{\mathrm{br}}, 1 \mathrm{H}, \mathrm{NH}\right), 7.12-7.18\left(\mathrm{~m}, 1 \mathrm{H}, 4^{\prime}-\mathrm{H}\right), 7.28-7.34\left(\mathrm{~m}, 2 \mathrm{H}, 3^{\prime}-\mathrm{H}, 5^{\prime}-\mathrm{H}\right)$, $7.36-7.46\left(\mathrm{~m}, 2 \mathrm{H}, 2^{\prime}-\mathrm{H}, 6^{\prime}-\mathrm{H}\right) \mathrm{ppm}$.

${ }^{13} \mathrm{C}$ NMR $\left(101 \mathrm{MHz}, \quad \mathrm{CDCl}_{3}\right): \quad \delta=28.3, \quad 28.3\left(2 \times \mathrm{C}\left(\underline{\mathrm{CH}}_{3}\right)_{3}\right), \quad 81.6, \quad 82.4$ $\left(2 \times \underline{\mathrm{C}}\left(\mathrm{CH}_{3}\right)_{3}\right), 123.8\left(\mathrm{C}-2^{\prime}, \mathrm{C}^{\prime} 6^{\prime}\right), 125.7\left(\mathrm{C}-4^{\prime}\right), 128.5$ (C-3', C-5'), 142.3 (C-1'), 153.7, $155.5(2 \times \mathrm{N}(\mathrm{CO}) \mathrm{O}) \mathrm{ppm}$.

HRMS (ESI): $m / z$ calc. for $\mathrm{C}_{16} \mathrm{H}_{24} \mathrm{~N}_{2} \mathrm{NaO}_{4}[\mathrm{M}+\mathrm{Na}]^{+}$331.1628; found 331.1628.

$\operatorname{TLC}\left(\mathrm{SiO}_{2}\right): R_{\mathrm{f}}=0.24(n$-hexane $/$ EtOAc $=5: 1)$.

m.p. $109-110^{\circ} \mathrm{C}$. 


\section{Chapter 22 $\mid$ COMPOUNDS}

\subsection{Di-tert-butyl-1-(3-bromophenyl)-2-phenyl- hydrazine-1,2-dicarboxylate (95)}

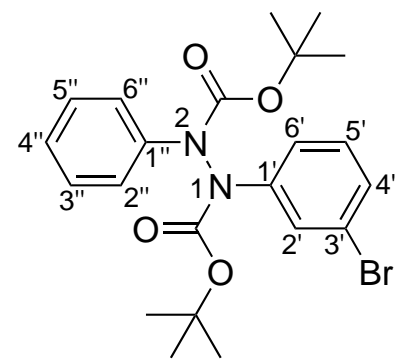

Compound 95 was prepared by an Ullmann reaction according to the modified literature method. ${ }^{[148]}$ The procedure of Cho et al. ${ }^{[170]}$ was modified by replacing $\mathrm{Cs}_{2} \mathrm{CO}_{3}$ with $\mathrm{K}_{2} \mathrm{CO}_{3}$ and using a smaller catalyst loading.

Under argon atmosphere compound $94(10.0 \mathrm{~g}, 32.4 \mathrm{mmol}, 1.00 \mathrm{eq})$, 3-iodo1-bromobenzene $(\mathbf{9 7}, 9.17 \mathrm{~g}, 4.11 \mathrm{ml}, 32.4 \mathrm{mmol}, 1.00 \mathrm{eq})$, copper(I) iodide (1.54 g, $8.11 \mathrm{mmol}, 0.25 \mathrm{eq}), 1,10$-phenanthroline $(2.9 \mathrm{~g}, 16 \mathrm{mmol}, 0.50 \mathrm{eq})$ and $\mathrm{K}_{2} \mathrm{CO}_{3}$ $(6.72 \mathrm{~g}, 48.6 \mathrm{mmol}, 1.50 \mathrm{eq})$ were combined with dry DMF $(50 \mathrm{ml})$ in a $250 \mathrm{ml}$ three-neck round-bottom flask equipped with a large magnetic stirrer bar and reflux condensor. Argon was bubbled through the reaction mixture for $10 \mathrm{~min}$. Then the mixture was heated at $80^{\circ} \mathrm{C}$ for $24 \mathrm{~h}$. The reaction mixture was diluted with EtOAc $(100 \mathrm{ml})$ and filtered through a plug of silica gel $(\varnothing=60 \mathrm{~mm}$, $h=20 \mathrm{~mm})$. The silica gel was washed with EtOAc $(3 \times 30 \mathrm{ml})$. The combined filtrates were concentrated in vacuo $\left(5 \mathrm{mbar}, 50^{\circ} \mathrm{C}, 1 \mathrm{~h}\right)$ to give an orange oil. The oil was dissolved in EtOAc $(200 \mathrm{ml})$ and washed with water $(100 \mathrm{ml})$ and then brine $(2 \times 100 \mathrm{ml})$. All aq. solutions were combined and extracted with EtOAc $(100 \mathrm{ml})$. The combined organic solutions were dried over $\mathrm{Na}_{2} \mathrm{SO}_{4}$ and concentrated in vacuo. Column chromatography on silica gel (cyclohexane $/ i-\operatorname{Pr}_{2} \mathrm{O}=7: 1$ ) gave $7.16 \mathrm{~g}$ ( $15.5 \mathrm{mmol}, 48 \%)$ of the title compound as yellow oil.

${ }^{1} \mathbf{H}$ NMR $\left(400 \mathrm{MHz}, \mathrm{CDCl}_{3}\right): \delta=1.53\left(\mathrm{~s}, 18 \mathrm{H}, 6 \times \mathrm{CH}_{3}\right), 7.15-7.20(\mathrm{~m}, 2 \mathrm{H}), 7.27$ $-7.45(\mathrm{~m}, 6 \mathrm{H}), 7.60-7.74(\mathrm{~m}, 1 \mathrm{H}) \mathrm{ppm}$.

${ }^{13} \mathrm{C}$ NMR $\left(101 \mathrm{MHz}, \quad \mathrm{CDCl}_{3}\right): \quad \delta=28.3, \quad 28.3\left(2 \times \mathrm{C}\left(\underline{\mathrm{CH}}_{3}\right)_{3}\right), \quad 82.8, \quad 83.0$ $\left(2 \times \underline{\mathrm{C}}\left(\mathrm{CH}_{3}\right)_{3}\right), 120.8,122.2,122.5,125.9,128.5,128.8,129.9,134.4,141.0(\mathrm{C}-1 ")$, $142.6\left(\mathrm{C}-1^{\prime}\right), 152.9,153.1(2 \times \mathrm{C}=\mathrm{O}) \mathrm{ppm}$.

HRMS (EI): $m / z$ calc. for $\mathrm{C}_{22} \mathrm{H}_{27} \mathrm{BrN}_{2} \mathrm{NaO}_{4}\left[\mathrm{M}^{+\cdot}\right] 483.7820$; found 483.7829 . 
$\operatorname{TLC}\left(\mathrm{SiO}_{2}\right): R_{\mathrm{f}}=0.31$ (cyclohexane $\left./ i-\mathrm{Pr}_{2} \mathrm{O}=7: 1\right)$.

\subsection{3-lodobromobenzene $(97)^{[150]}$}<smiles>Brc1cccc(I)c1</smiles>

3-Bromophenylboronic acid (2.00 g, $9.96 \mathrm{mmol}, 1.00 \mathrm{eq})$ was suspended in dry $\mathrm{MeCN}(50 \mathrm{ml})$ and NIS $(3.36 \mathrm{~g}, 14.9 \mathrm{mmol}, 1.50 \mathrm{eq})$ was added in one portion at room temperature. The reaction mixture was stirred at $81^{\circ} \mathrm{C}$ for $19 \mathrm{~h}$. After cooling to r.t., the mixture was extracted with $n$-hexane $(5 \times 50 \mathrm{ml})$. The combined organic solutions were washed with water $(100 \mathrm{ml}), 1.0 \mathrm{M} \mathrm{NaSO}{ }_{3}(100 \mathrm{ml}), 1.0 \mathrm{M}$ $\mathrm{NaHCO}_{3}(100 \mathrm{ml})$ and again with water $(100 \mathrm{ml})$. The organic layer was separated and dried over $\mathrm{Na}_{2} \mathrm{SO}_{4}$ and concentrated in vacuo to give $1.81 \mathrm{~g}(6.40 \mathrm{mmol}$, $64 \%$ ) of the title compound as colorless oil.

${ }^{1} \mathbf{H}$ NMR $\left(400 \mathrm{MHz}, \mathrm{CDCl}_{3}\right): \delta=6.97\left(\mathrm{dd},{ }^{3} J_{\mathrm{H}, \mathrm{H}}=8.0,8.0 \mathrm{~Hz}, 1 \mathrm{H}, 5-\mathrm{H}\right), 7.47(\mathrm{ddd}$, $\left.{ }^{3} J_{\mathrm{H}, \mathrm{H}}=8.0 \mathrm{~Hz},{ }^{4} J_{\mathrm{H}, \mathrm{H}}=1.8,0.9 \mathrm{~Hz}, 1 \mathrm{H}, 6-\mathrm{H}\right), 7.63\left(\mathrm{ddd},{ }^{3} J_{\mathrm{H}, \mathrm{H}}=8.0 \mathrm{~Hz},{ }^{4} J_{\mathrm{H}, \mathrm{H}}=1.8\right.$, $0.9 \mathrm{~Hz}, 1 \mathrm{H}, 4-\mathrm{H}), 7.87\left(\mathrm{dd},{ }^{4} J_{\mathrm{H}, \mathrm{H}}=1.8,1.8 \mathrm{~Hz}, 1 \mathrm{H}, 2-\mathrm{H}\right) \mathrm{ppm}$.

${ }^{13} \mathrm{C}$ NMR $\left(101 \mathrm{MHz}, \mathrm{CDCl}_{3}\right): \delta=94.6(\mathrm{C}-3), 123.2(\mathrm{C}-1), 131.0(\mathrm{C}-6), 131.5(\mathrm{C}-5)$, 136.2 (C-4), 139.9 (C-2) ppm.

$\operatorname{TLC}\left(\mathrm{SiO}_{2}\right): R_{\mathrm{f}}=0.68(n-$ hexane/EtOAc $=1: 1)$.

\subsection{2-Bromobiphenyl-4,4'-diamine $(98)^{[148]}$}

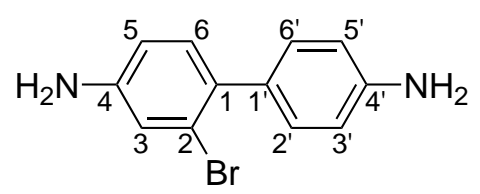

Under argon atmosphere, compound 95 (10.7 g, $23.0 \mathrm{mmol}, 1.00 \mathrm{eq})$ was dissolved in anhydrous $\mathrm{EtOH}(300 \mathrm{ml})$, and conc. $\mathrm{HCl}(10.6 \mathrm{ml}, 123 \mathrm{mmol}, 5.37 \mathrm{eq})$ was added at room temperature over a period of $2 \mathrm{~min}$. The reaction mixture was heated at $80^{\circ} \mathrm{C}$ for $90 \mathrm{~min}$. The reaction mixture was cooled to $0^{\circ} \mathrm{C}$ and transferred into sat. aq. $\mathrm{NaHCO}_{3}(600 \mathrm{ml})$ at $0{ }^{\circ} \mathrm{C}$. The mixture was extracted with $\mathrm{CH}_{2} \mathrm{Cl}_{2}(3 \times 400 \mathrm{ml})$. The combined organic solutions were dried over $\mathrm{Na}_{2} \mathrm{SO}_{4}$ and concentrated in vacuo to give a yellow oil. Column chromatography on silica 


\section{Chapter 22 $\mid$ COMPOUNDS}

gel $\left(\mathrm{CH}_{2} \mathrm{Cl}_{2} / \mathrm{EtOAc}=10: 1\right)$ gave $3.40 \mathrm{~g}(12.9 \mathrm{mmol}, 56 \%)$ of the title compound as yellow oil.

${ }^{1} \mathbf{H}$ NMR $\left(400 \mathrm{MHz} \mathrm{CDCl}_{3}\right): \delta=3.69\left(\mathrm{~s}_{\mathrm{br}}, 4 \mathrm{H}, 2 \times \mathrm{NH}_{2}\right), 6.64\left(\mathrm{dd},{ }^{3} J_{\mathrm{H}, \mathrm{H}}=8.0 \mathrm{~Hz}\right.$, $\left.{ }^{4} J_{\mathrm{H}, \mathrm{H}}=2.4 \mathrm{~Hz}, 1 \mathrm{H}, 5-\mathrm{H}\right), 6.68-6.74\left(\mathrm{~m}, 2 \mathrm{H}, 3^{\prime}-\mathrm{H}, 5^{\prime}-\mathrm{H}\right), 6.98\left(\mathrm{~d},{ }^{4} J_{\mathrm{H}, \mathrm{H}}=2.4 \mathrm{~Hz}\right.$, $1 \mathrm{H}, 3-\mathrm{H}), 7.09\left(\mathrm{~d},{ }^{3} J_{\mathrm{H}, \mathrm{H}}=8.0 \mathrm{~Hz}, 1 \mathrm{H}, 6-\mathrm{H}\right), 7.16-7.22\left(\mathrm{~m}, 2 \mathrm{H}, 2^{\prime}-\mathrm{H}, 6^{\prime}-\mathrm{H}\right) \mathrm{ppm}$.

${ }^{13}$ C NMR (101 MHz, CDCl $): \delta=114.4(\mathrm{C}-5), 114.6\left(\mathrm{C}-3^{\prime}, \mathrm{C}^{-5}{ }^{\prime}\right), 119.1$ (C-3), 123.4 (C-2), 130.7 (C-2', C-6'), 131.7 (C-1'), 131.9 (C-6), 132.8 (C-1), 145.5 (C-4'), 146.3 (C-4) ppm.

MS (ESI, pos.): $m / z(\%)=263 / 265(90)[\mathrm{M}+\mathrm{H}]^{+}, 285 / 287(100)[\mathrm{M}+\mathrm{Na}]^{+}$.

HRMS (ESI): $m / z$ calc. for $\mathrm{C}_{12} \mathrm{H}_{12} \mathrm{BrN}_{2}\left[\mathrm{M}^{+\cdot}\right]$ 263.0178; found 263.0181.

$\operatorname{TLC}\left(\mathrm{SiO}_{2}\right): R_{\mathrm{f}}=0.42\left(\mathrm{CH}_{2} \mathrm{Cl}_{2} / \mathrm{EtOAc}=10: 1\right)$.

\subsection{5-Methyl-2-(tributylstannyl)- thiophene $(101)^{[153]}$}

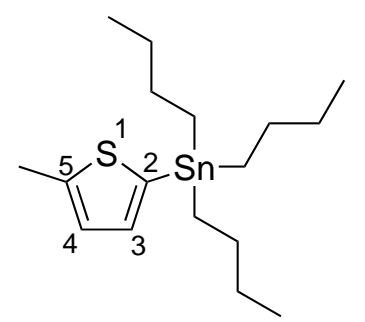

A solution of 2-methylthiophene $(4.0 \mathrm{ml}, 4.1 \mathrm{~g}, 42 \mathrm{mmol}, 1.0 \mathrm{eq})$ in dry THF $(100 \mathrm{ml})$ was cooled to $-78^{\circ} \mathrm{C} .1 .6 \mathrm{M} n$-BuLi in $n$-hexane $(28.6 \mathrm{ml}, 45.7 \mathrm{mmol}$, $1.10 \mathrm{eq}$ ) was added slowly at $-78^{\circ} \mathrm{C}$ over a period of $6 \mathrm{~min}$. After stirring for $1 \mathrm{~h}$, tributytin chloride $(12.4 \mathrm{ml}, 14.9 \mathrm{~g}, 45.7 \mathrm{mmol}, 1.10 \mathrm{eq})$ was added dropwise at $-78^{\circ} \mathrm{C}$ over a period of $4 \mathrm{~min}$. The reaction mixture was allowed to warm up to room temperature slowly. After $18 \mathrm{~h}$, ice-cold water $(200 \mathrm{ml})$ was added and the mixture was extracted with $n$-hexane $(3 \times 200 \mathrm{ml})$. The combined organic solutions were dried over $\mathrm{Na}_{2} \mathrm{SO}_{4}$ and concentrated in vacuo to give $17.5 \mathrm{~g}$ of a yellow oil. Vacuum distillation $\left(140-162^{\circ} \mathrm{C}, 2.0 \mathrm{mbar}\right)$ gave $11 \mathrm{~g}(30 \mathrm{mmol}, 71 \%)$ of the title compound as colorless oil.

${ }^{1} \mathbf{H} \quad \mathbf{N M R}\left(400 \mathrm{MHz}, \quad \mathrm{CDCl}_{3}\right): \quad \delta=0.92\left(\mathrm{t}, \quad{ }^{3} J_{\mathrm{H}, \mathrm{H}}=7.3 \mathrm{~Hz}, \quad 9 \mathrm{H}\right.$, $\left.\mathrm{Sn}\left(\mathrm{CH}_{2} \mathrm{CH}_{2} \mathrm{CH}_{2} \underline{\mathrm{C}}_{3}\right)_{3}\right), 1.01-1.19\left(\mathrm{~m}, 6 \mathrm{H}, \mathrm{Sn}\left(\mathrm{C}_{2} \mathrm{CH}_{2} \mathrm{CH}_{2} \mathrm{CH}_{3}\right)_{3}\right), 1.30-$ 
$1.42\left(\mathrm{~m}, 6 \mathrm{H}, \mathrm{Sn}\left(\mathrm{CH}_{2} \mathrm{CH}_{2} \mathrm{CH}_{2} \mathrm{CH}_{3}\right)_{3}\right), 1.46-1.69\left(\mathrm{~m}, 6 \mathrm{H}, \mathrm{Sn}\left(\mathrm{CH}_{2} \mathrm{C}_{2} \mathrm{CH}_{2} \mathrm{CH}_{3}\right)_{3}\right)$,

$2.56\left(\mathrm{~d},{ }^{4} J_{\mathrm{H}, \mathrm{H}}=1.0 \mathrm{~Hz}, 3 \mathrm{H}, 5-\mathrm{CH}_{3}\right), 6.91\left(\mathrm{dd},{ }^{3} J_{\mathrm{H}, \mathrm{H}}=3.1 \mathrm{~Hz},{ }^{4} J_{\mathrm{H}, \mathrm{H}}=1.0 \mathrm{~Hz}, 1 \mathrm{H}\right.$, $4-\mathrm{H}), 6.99\left(\mathrm{~d},{ }^{3} J_{\mathrm{H}, \mathrm{H}}=3.1 \mathrm{~Hz}, 1 \mathrm{H}, 3-\mathrm{H}\right) \mathrm{ppm}$.

${ }^{13} \mathbf{C}$ NMR $\left(101 \mathrm{MHz}, \quad \mathrm{CDCl}_{3}\right): \quad \delta=10.9\left(\mathrm{Sn}\left(\mathrm{CH}_{2} \mathrm{CH}_{2} \mathrm{CH}_{2} \mathrm{CH}_{3}\right)_{3}\right), \quad 13.8$ $\left(\mathrm{Sn}\left(\mathrm{CH}_{2} \mathrm{CH}_{2} \mathrm{CH}_{2} \underline{\mathrm{CH}}\right)_{3}\right), \quad 15.2 \quad\left(5-\mathrm{CH}_{3}\right), \quad 27.4 \quad\left(\mathrm{Sn}\left(\mathrm{CH}_{2} \mathrm{CH}_{2} \underline{\mathrm{C}} \mathrm{H}_{2} \mathrm{CH}_{3}\right)_{3}\right), \quad 29.1$ $\left(\mathrm{Sn}\left(\mathrm{CH}_{2} \mathrm{CH}_{2} \mathrm{CH}_{2} \mathrm{CH}_{3}\right)_{3}\right), 126.8$ (C-4), 134.4 (C-2), 135.6 (C-3), 145.4 (C-5) ppm.

\subsection{2-Bromo-4,4'-diiodobiphenyl (102)}

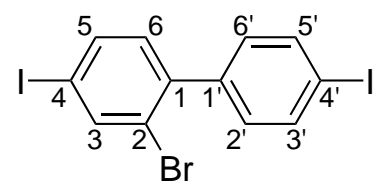

Compound $\mathbf{1 0 2}$ was prepared by a Sandmeyer reaction ${ }^{[180]}$ from 2-bromobenzidine (98).

Compound 98 (1.00 g, $3.80 \mathrm{mmol}, 1.00 \mathrm{eq})$ was dissolved in dry $\mathrm{MeCN}(61 \mathrm{ml})$ and $2.0 \mathrm{M} \mathrm{HCl}(61 \mathrm{ml}, 0.12 \mathrm{~mol}, 32 \mathrm{eq})$ was added at room temperature. The colorless clear solution was cooled to $-10^{\circ} \mathrm{C}$ (controlled by an internal themometer). A solution of $\mathrm{NaNO}_{2}(658 \mathrm{mg}, 9.54 \mathrm{mmol}, 2.51 \mathrm{eq})$ in water $(20 \mathrm{ml})$ was added slowly over a period of $6 \mathrm{~min}$ keeping the temperature of the reaction mixture below $-5^{\circ} \mathrm{C}$. The orange-brown reaction mixture was stirred at $-10^{\circ} \mathrm{C}$ for $30 \mathrm{~min}$. Then $\mathrm{KI}(5.49 \mathrm{~g}, 33.1 \mathrm{mmol}, 8.71 \mathrm{eq})$ was added at $-10^{\circ} \mathrm{C}$ in portions over a period of $5 \mathrm{~min}$. The cooling-bath was removed and the reaction mixture was stirred at room temperature for $16 \mathrm{~h}$. The brown suspension was diluted with water $(150 \mathrm{ml})$ and neutralized by addition of sat. aq. $\mathrm{NaHCO}_{3}(170 \mathrm{ml})$. The mixture was extracted with $\mathrm{Et}_{2} \mathrm{O}(3 \times 200 \mathrm{ml})$. The dark brown organic solutions were combined and washed with $1.0 \mathrm{M}$ aq. $\mathrm{Na}_{2} \mathrm{~S}_{2} \mathrm{O}_{3}(3 \times 100 \mathrm{ml})$. The combined aqueous solutions were extracted with $\mathrm{Et}_{2} \mathrm{O}(100 \mathrm{ml})$. All organic solutions were combined, dried over $\mathrm{Na}_{2} \mathrm{SO}_{4}$ and concentrated in vacuo to give a yellow solid which was dissolved in hot cylohexane (ca. $40 \mathrm{ml}$ ), and loaded onto a silica gel column. Column chromatography ( $150 \mathrm{~g} \mathrm{SiO}_{2}$, cyclohexane) gave $1.29 \mathrm{~g}$ (2.66 mmol, $70 \%$ ) of the title compound as colorless solid.

${ }^{1} \mathbf{H}$ NMR $\left(400 \mathrm{MHz}, \mathrm{CDCl}_{3}\right): \delta=7.01\left(\mathrm{~d},{ }^{3} \mathrm{~J}_{\mathrm{H}, \mathrm{H}}=8.1 \mathrm{~Hz}, 1 \mathrm{H}, 6-\mathrm{H}\right), 7.09-7.14(\mathrm{~m}$, $\left.2 \mathrm{H}, 2^{\prime}-\mathrm{H}, 66^{\prime}-\mathrm{H}\right), 7.68\left(\mathrm{dd},{ }^{3} J_{\mathrm{H}, \mathrm{H}}=8.1 \mathrm{~Hz},{ }^{4} J_{\mathrm{H}, \mathrm{H}}=1.8 \mathrm{~Hz}, 1 \mathrm{H}, 3-\mathrm{H}\right), 7.74-7.79(\mathrm{~m}$, 


\section{Chapter 22 COMPOUNDS}

$\left.2 \mathrm{H}, 3^{\prime}-\mathrm{H}, 5^{\prime}-\mathrm{H}\right), 8.02\left(\mathrm{~d},{ }^{4} \mathrm{~J}_{\mathrm{H}, \mathrm{H}}=1.8 \mathrm{~Hz}, 1 \mathrm{H}, 3-\mathrm{H}\right) \mathrm{ppm}$.

${ }^{13} \mathrm{C}$ NMR $\left(101 \mathrm{MHz}, \mathrm{CDCl}_{3}\right): \delta=93.4(\mathrm{C}-4), 94.1(\mathrm{C}-4$ '), $123.3(\mathrm{C}-2), 131.1$ (C-2', C-6'), 132.4 (C-6), 136.8 (C-5), 137.4 (C-3', C-5'), 139.6 (C-1'), 141.1 (C-1), 141.3 (C-3) ppm.

GC-MS (EI): $m / z(\%)=484 / 486(30)\left[\mathrm{M}^{+\cdot}\right]$.

HRMS (EI): $m / z$ calc. for $\mathrm{C}_{12} \mathrm{H}_{7} \mathrm{Brl}_{2}\left[\mathrm{M}^{+\cdot}\right]$ 483.7820; found 483.7829 .

$\operatorname{TLC}\left(\mathrm{SiO}_{2}\right): R_{\mathrm{f}}=0.48$ (cyclohexane).

\subsection{2-[2'-Bromo-4'-(5-methyl-2-thienyl)- 4-biphenylyl]-5-methylthiophene (103)}

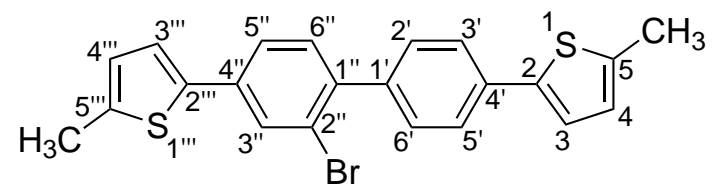

Compound $101(7.00 \mathrm{~g}, 18.1 \mathrm{mmol}, 2.20 \mathrm{eq})$ was added to a solution of compound $102(3.98 \mathrm{~g}, 8.22 \mathrm{mmol}, 1.00 \mathrm{eq})$ in dry toluene $(60 \mathrm{ml})$ in a screw cap pressure tube, and a slow stream of argon was bubbled through the solution for $5 \mathrm{~min}$. Then $\left[\mathrm{Pd}\left(\mathrm{PPh}_{3}\right)_{4}\right](0.95 \mathrm{~g}, 0.82 \mathrm{mmol}, 0.10 \mathrm{eq})$ was added. The sealed reaction vessel was heated to $110^{\circ} \mathrm{C}$ for $20 \mathrm{~h}$. The reaction mixture was cooled to r.t. and diluted with $\mathrm{CHCl}_{3}(240 \mathrm{ml})$ followed by washing with $1.0 \mathrm{M}$ aq. $\mathrm{KF}$ $(3 \times 200 \mathrm{ml})$. The organic solution was dried over $\mathrm{Na}_{2} \mathrm{SO}_{4}$ and concentrated in vacuo to give $9.81 \mathrm{~g}$ of greenish solid. The crude product was adsorbed on silica (ca. $20 \mathrm{~g}$ ) and loaded onto a silica gel column. Column chromatography (150 g $\mathrm{SiO}_{2}$, cyclohexane $\left./ \mathrm{CH}_{2} \mathrm{Cl}_{2}=2: 1\right)$ gave $1.72 \mathrm{~g}(4.04 \mathrm{mmol}, 49 \%)$ of the title compound as colorless solid.

${ }^{1} \mathbf{H}$ NMR $\left(400 \mathrm{MHz}, \mathrm{CDCl}_{3}\right): \delta=2.53\left(\mathrm{~s}, 6 \mathrm{H}, 2 \times \mathrm{CH}_{3}\right), 6.73-6.77(\mathrm{~m}, 2 \mathrm{H}, 4-\mathrm{H}$, $\left.4^{\prime \prime \prime}-\mathrm{H}\right), 7.15\left(\mathrm{~d},{ }^{3} J_{\mathrm{H}, \mathrm{H}}=3.5 \mathrm{~Hz}, 1 \mathrm{H}, 3^{\prime \prime \prime}-\mathrm{H}\right), 7.17\left(\mathrm{~d},{ }^{3} J_{\mathrm{H}, \mathrm{H}}=3.5 \mathrm{~Hz}, 1 \mathrm{H}, 3-\mathrm{H}\right), 7.32(\mathrm{~d}$, $\left.{ }^{3} J_{\mathrm{H}, \mathrm{H}}=8.0 \mathrm{~Hz}, 1 \mathrm{H}, 6^{\prime \prime}-\mathrm{H}\right), 7.41-7.46\left(\mathrm{~m}, 2 \mathrm{H}, 2^{\prime}-\mathrm{H}, 6^{\prime}-\mathrm{H}\right), 7.52\left(\mathrm{dd},{ }^{3} J_{\mathrm{H}, \mathrm{H}}=8.0 \mathrm{~Hz}\right.$, $\left.{ }^{4} J_{\mathrm{H}, \mathrm{H}}=1.9 \mathrm{~Hz}, 1 \mathrm{H}, 5^{\prime \prime}-\mathrm{H}\right), 7.59-7.63\left(\mathrm{~m}, 2 \mathrm{H}, 3^{\prime}-\mathrm{H}, 5^{\prime}-\mathrm{H}\right), 7.86\left(\mathrm{~d},{ }^{4} J_{\mathrm{H}, \mathrm{H}}=1.9 \mathrm{~Hz}\right.$, $1 \mathrm{H}, 3$ "-H) ppm.

${ }^{13} \mathrm{C}$ NMR $\left(101 \mathrm{MHz}, \mathrm{CDCl}_{3}\right): \delta=15.7\left(2 \times \mathrm{CH}_{3}\right), 123.0(\mathrm{C}-2 "), 123.3(\mathrm{C}-3), 123.9$ (C-3"'), 124.6 (C-5"), 125.1 (C-3', C-5'), 126.4, 126.6 (C-4, C-4"'), 130.0 (C-3"), 130.0 
(C-2', C-6'), 131.6 (C-6"), 134.2 (C-4'), 135.6 (C-4"), 139.3 (C-1'), 139.9, 139.9, 140.4, 140.7 (C-5, C-1", C-2"', C-5"'), 141.6 (C-2) ppm.

HRMS (EI): $m / z$ calc. for $\mathrm{C}_{22} \mathrm{H}_{17} \mathrm{BrS}_{2}\left[\mathrm{M}^{+\cdot}\right]$ 423.9955; found 423.9938 .

$\operatorname{TLC}\left(\mathrm{SiO}_{2}\right): R_{\mathrm{f}}=0.53(n$-hexane $/ \mathrm{EtOAc}=4: 1)$.

\subsection{Compound 104}

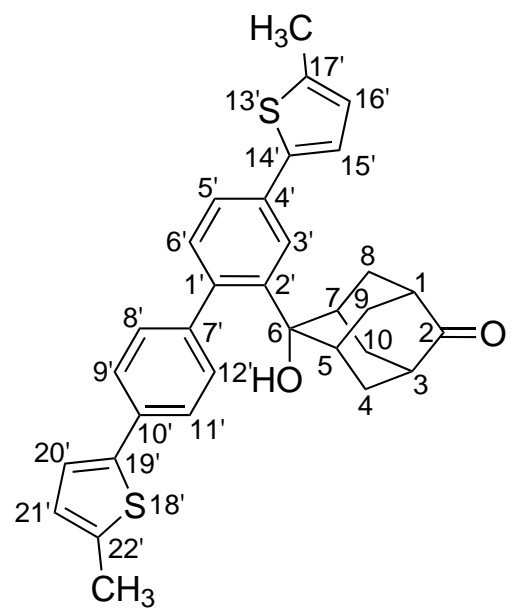

According to GP2, aromatic bromide $103(306 \mathrm{mg}, 719 \mu \mathrm{mol}, 1.00 \mathrm{eq})$ was dissolved in dry THF $(23 \mathrm{ml})$ and subjected to bromine-lithium exchange with $1.6 \mathrm{M}$ $n$-BuLi in $n$-hexane $(0.49 \mathrm{ml}, 0.79 \mathrm{mmol}, 1.1 \mathrm{eq})$. The solution of the lithiated 103 was added dropwise to a solution of 2,6-adamantanedione $(236 \mathrm{mg}, 1.44 \mathrm{mmol}$, $2.00 \mathrm{eq})$ in dry THF $(12 \mathrm{ml})$. The reaction mixture was stirred at $-78^{\circ} \mathrm{C}$ for $10 \mathrm{~min}$ and at room temperature for additional $4 \mathrm{~h}$. The reaction was quenched by addition of sat. aq. $\mathrm{NH}_{4} \mathrm{Cl}(40 \mathrm{ml})$ followed by water $(40 \mathrm{ml})$ and extracted with $\mathrm{CHCl}_{3}$ $(4 \times 50 \mathrm{ml})$. Drying of the combined organic solutions over $\mathrm{Na}_{2} \mathrm{SO}_{4}$ and concentration in vacuo gave $479 \mathrm{mg}$ of a brownish solid. Column chromatography on silica gel $\left(40 \mathrm{~g} \mathrm{SiO}_{2}, \mathrm{CHCl}_{3}\right)$ gave $411 \mathrm{mg}$ of the title compound (with residual 2,6-adamantanedione) as yellowish solid. The product was used without further purification.

${ }^{1} \mathbf{H}$ NMR $\left(400 \mathrm{MHz}, \mathrm{CDCl}_{3}\right): \delta=1.68-1.76\left(\mathrm{~m}, 2 \mathrm{H},\left[4-\mathrm{H}_{\mathrm{a}}, 10-\mathrm{H}_{\mathrm{a}}\right]\right.$ or $\left[8-\mathrm{H}_{\mathrm{a}}\right.$, $\left.\left.9-\mathrm{H}_{\mathrm{a}}\right]\right), 1.79-1.87\left(\mathrm{~m}, 2 \mathrm{H},\left[8-\mathrm{H}_{\mathrm{a}}, 9-\mathrm{H}_{\mathrm{a}}\right]\right.$ or $\left.\left[4-\mathrm{H}_{\mathrm{a}}, 10-\mathrm{H}_{\mathrm{a}}\right]\right), 1.98-2.07(\mathrm{~m}, 2 \mathrm{H}$, $\left[8-\mathrm{H}_{\mathrm{b}}, 9-\mathrm{H}_{\mathrm{b}}\right]$ or $\left.\left[4-\mathrm{H}_{\mathrm{b}}, 10-\mathrm{H}_{\mathrm{b}}\right]\right), 2.31-2.41(\mathrm{~m}, 2 \mathrm{H}, 1-\mathrm{H}, 3-\mathrm{H}), 2.48-2.60(\mathrm{~m}, 10 \mathrm{H}$, $\left[4-\mathrm{H}_{\mathrm{b}}, 10-\mathrm{H}_{\mathrm{b}}\right]$ or $\left.\left[8-\mathrm{H}_{\mathrm{b}}, 9-\mathrm{H}_{\mathrm{b}}\right], 5-\mathrm{H}, 7-\mathrm{H}, 2 \times \mathrm{CH}_{3}\right), 6.72-6.77(\mathrm{~m}, 2 \mathrm{H}, 16-\mathrm{H}$, $\left.21^{\prime}-\mathrm{H}\right), 7.12\left(\mathrm{~d},{ }^{3} J_{\mathrm{H}, \mathrm{H}}=3.5 \mathrm{~Hz}, 1 \mathrm{H}, 15^{\prime}-\mathrm{H}\right.$ or $\left.20^{\prime}-\mathrm{H}\right), 7.16\left(\mathrm{~d},{ }^{3} J_{\mathrm{H}, \mathrm{H}}=3.5 \mathrm{~Hz}, 1 \mathrm{H}\right.$, 


\section{Chapter 22 $\mid$ COMPOUNDS}

$20^{\prime}-\mathrm{H}$ or $\left.15^{\prime}-\mathrm{H}\right), 7.17\left(\mathrm{~d},{ }^{3} \mathrm{~J}_{\mathrm{H}, \mathrm{H}}=7.8 \mathrm{~Hz}, 1 \mathrm{H}, 6^{\prime}-\mathrm{H}\right), 7.35-7.40\left(\mathrm{~m}, 2 \mathrm{H}, 8^{\prime}-\mathrm{H}, 12^{\prime}-\mathrm{H}\right)$, $7.49\left(\mathrm{dd},{ }^{3} J_{\mathrm{H}, \mathrm{H}}=7.8 \mathrm{~Hz},{ }^{4} J_{\mathrm{H}, \mathrm{H}}=1.8 \mathrm{~Hz}, 1 \mathrm{H}, 5^{\prime}-\mathrm{H}\right), 7.58-7.54\left(\mathrm{~m}, 2 \mathrm{H}, 9^{\prime}-\mathrm{H}, 11^{\prime}-\mathrm{H}\right)$, $7.82\left(\mathrm{~d},{ }^{4} J_{\mathrm{H}, \mathrm{H}}=1.8 \mathrm{~Hz}, 1 \mathrm{H}, 3^{\prime}-\mathrm{H}\right) \mathrm{ppm}$.

${ }^{13} \mathrm{C}$ NMR $\left(101 \mathrm{MHz}, \mathrm{CDCl}_{3}\right): \delta=15.6\left(2 \times \mathrm{CH}_{3}\right), 34.3([\mathrm{C}-4, \mathrm{C}-10]$ or $[\mathrm{C}-8, \mathrm{C}-9])$, 34.8 (C-5, C-7), 36.2 ([C-8, C-9] or [C-4, C-10]), 45.0, 45.4 (C-1, C-3), 76.4 (C-6), 123.1, 123.3 (C-9', C-20'), 124.2, 124.3 (C-3', C-5'), 125.0 (C-9', C-11'), 126.4, 126.5 (C-16', C-21'), 129.7 (C-8', C-12'), 133.6 (C-10'), 134.0 (C-4'), 134.7 (C-6'), 139.9, 140.1 (C-17', C-22'), 140.9 (C-1'), 141.1 (C-7'), 141.5, 141.6 (C-14', C-19'), 142.7 (C-7'), 217.5 (C-2) ppm.

MS (ESI, pos.): $m / z(\%)=533.2(100)[\mathrm{M}+\mathrm{Na}]^{+}$.

HRMS (ESI): $m / z$ calc. for $\mathrm{C}_{32} \mathrm{H}_{30} \mathrm{NaO}_{2} \mathrm{~S}_{2}[\mathrm{M}+\mathrm{Na}]^{+}$533.1579; found 533.1566.

$\operatorname{TLC}\left(\mathrm{SiO}_{2}\right): R_{\mathrm{f}}=0.24\left(\mathrm{CHCl}_{3}\right)$.

\subsection{Compound 105}

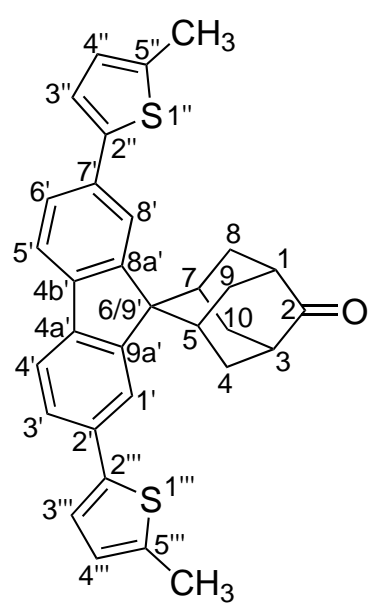

According to GP3, alcohol 104 (363 mg with residual 2,6-adamantanedione) was suspended in $n$-octane $(10 \mathrm{ml})$ and $75 \%$ aq. $\mathrm{H}_{2} \mathrm{SO}_{4}(5.0 \mathrm{ml})$ was added. After stirring at r.t. for $30 \mathrm{~min}$, aq. work-up with $3.0 \mathrm{M}$ aq. $\mathrm{NaOH}(100 \mathrm{ml})$ and extraction with $\mathrm{CH}_{2} \mathrm{Cl}_{2}(5 \times 200 \mathrm{ml}) 320 \mathrm{mg}$ of a colorless solid were isolated. Column chromatography on silica gel ( $50 \mathrm{~g} \mathrm{SiO}{ }_{2}$, cyclohexane $\left./ \mathrm{CH}_{2} \mathrm{Cl}_{2}=1: 1\right)$ gave $186 \mathrm{mg}$ ( $378 \mu \mathrm{mol}, 53 \%$, over two steps) of the title compound as colorless solid.

${ }^{1} \mathbf{H}$ NMR $\left(400 \mathrm{MHz}^{\mathrm{C}} \mathrm{CDCl}_{3}\right): \delta=1.81-1.86(\mathrm{~m}, 2 \mathrm{H}, 5-\mathrm{H}, 7-\mathrm{H}), 1.97-2.05(\mathrm{~m}$, $\left.4 \mathrm{H}, 4-\mathrm{H}_{\mathrm{a}}, 8-\mathrm{H}_{\mathrm{a}}, 9-\mathrm{H}_{\mathrm{a}}, 10-\mathrm{H}_{\mathrm{a}}\right), 2.54\left(\mathrm{~s}, 6 \mathrm{H}, 2 \times \mathrm{CH}_{3}\right), 2.90-2.95(\mathrm{~m}, 2 \mathrm{H}, 1-\mathrm{H}, 3-\mathrm{H})$, 
$3.19-3.29\left(\mathrm{~m}, 4 \mathrm{H}, 4-\mathrm{H}_{\mathrm{b}}, 8-\mathrm{H}_{\mathrm{b}}, 9-\mathrm{H}_{\mathrm{b}}, 10-\mathrm{H}_{\mathrm{b}}\right), 6.76-6.79(\mathrm{~m}, 2 \mathrm{H}, 4 "-\mathrm{H}, 4$ "'-H), $7.13\left(\mathrm{~d}^{3}{ }^{3} \mathrm{H}_{\mathrm{H}}=3.5 \mathrm{~Hz}, 2 \mathrm{H}, 3^{\prime \prime}-\mathrm{H}, 3^{\prime \prime \prime}-\mathrm{H}\right), 7.61\left(\mathrm{~d}^{3}{ }^{3} \mathrm{H}_{\mathrm{H}}=7.9 \mathrm{~Hz}, 2 \mathrm{H}, 3^{\prime}-\mathrm{H}, 6^{\prime}-\mathrm{H}\right), 7.77$ $\left(\mathrm{d}^{3}{ }^{3} \mathrm{H}_{\mathrm{H}, \mathrm{H}}=7.9 \mathrm{~Hz}, 2 \mathrm{H}, 4^{\prime}-\mathrm{H}, 5^{\prime}-\mathrm{H}\right), 8.24\left(\mathrm{~s}, 2 \mathrm{H}, 1^{\prime}-\mathrm{H}, 8^{\prime}-\mathrm{H}\right) \mathrm{ppm}$.

${ }^{13} \mathrm{C}$ NMR $\left(101 \mathrm{MHz}, \mathrm{CDCl}_{3}\right): \delta=15.6\left(2 \times \mathrm{CH}_{3}\right), 34.2(\mathrm{C}-5, \mathrm{C}-7), 34.6(\mathrm{C}-4, \mathrm{C}-8$, C-9, C-10), 45.4 (C-1, C-3), 56.9 (C-6/9'), 120.2 (C-4', C-5'), 123.0 (C-3", C-3"'), 125.4 (C-3', C-6'), 126.5 (C-4", C-4'"), 126.7 (C-1', C-8'), 133.0 (C-2', C-7'), 139.8 (C-4a', C-4b', C-5", C-5'"), 142.6 (C-2", C-2"'), 150.5 (C-8a, C-9a), 216.1 (C-2) ppm.

HRMS (EI): $m / z$ calc. for $\mathrm{C}_{32} \mathrm{H}_{28} \mathrm{OS}_{2}\left[\mathrm{M}^{+\cdot}\right]$ 492.1582; found 492.1575 .

$\operatorname{TLC}\left(\mathrm{SiO}_{2}\right): R_{\mathrm{f}}=0.65\left(\mathrm{CHCl}_{3}\right)$.

UV/Vis $\left(\right.$ EtOAc): $\lambda_{\text {abs }}^{\max }=357 \mathrm{~nm}, \varepsilon=54300 \mathrm{M}^{-1} \mathrm{~cm}^{-1}$. UV/Vis $\left(\mathrm{CHCl}_{3}\right)$ : $\lambda_{\mathrm{abs}}^{\max }=359 \mathrm{~nm}, \varepsilon=50400 \mathrm{M}^{-1} \mathrm{~cm}^{-1}$.

Fluorescence $\left(\mathrm{CHCl}_{3}\right.$, exc. at $\left.348 \mathrm{~nm}\right): \lambda_{\mathrm{em}}^{\max }=395,(417) \mathrm{nm} ; \Phi_{\mathrm{f}}=0.47 .^{\dagger}$

\subsection{Compound rac-106}

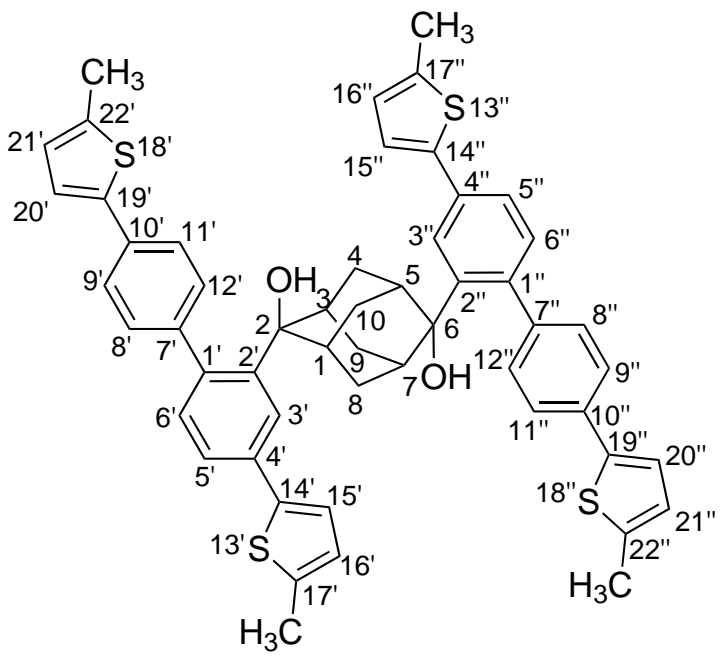

According to GP2, bromide 103 (50 mg, $0.12 \mathrm{mmol}, 2.0 \mathrm{eq}$ ) was dissolved in dry THF $(3.0 \mathrm{ml})$ and lithiated with $1.6 \mathrm{M} n$-BuLi in $n$-hexane ( $81 \mu \mathrm{l}, 0.13 \mu \mathrm{mol}, 2.2 \mathrm{eq})$. After $10 \mathrm{~min}$, a solution of 2,6-adamantanedione $(9.6 \mathrm{mg}, 59 \mu \mathrm{mol}, 1.0 \mathrm{eq})$ in dry THF $(1.0 \mathrm{ml})$ was added at $-78^{\circ} \mathrm{C}$ over a period of $10 \mathrm{~min}$. The mixture was stirred at $-78^{\circ} \mathrm{C}$ for $15 \mathrm{~min}$ and at ambient temperature for additional $2 \mathrm{~h}$. Aq. work-up with sat. aq. $\mathrm{NH}_{4} \mathrm{Cl}(5 \mathrm{ml})$ and water $(5 \mathrm{ml})$, followed by extraction with $\mathrm{CH}_{2} \mathrm{Cl}_{2}$

\footnotetext{
${ }^{\dagger}$ Coumarin 120 in $\mathrm{MeOH}$ as a fl. quantum yield standard with $\Phi_{\mathrm{f}}=0.51^{[132]}$
} 


\section{Chapter 22 $\mid$ COMPOUNDS}

$(3 \times 5 \mathrm{ml})$, drying over $\mathrm{Na}_{2} \mathrm{SO}_{4}$ and concentration in vacuo gave $86 \mathrm{mg}$ of a yellowish solid. Column chromatography on silica gel (two columns, $2 \times 15 \mathrm{~g} \mathrm{SiO}_{2}$, $\left.\mathrm{CHCl}_{3}\right)$ gave $34 \mathrm{mg}(40 \mu \mathrm{mol}, 68 \%)$ of the title compound rac-106 as colorless solid.

${ }^{1} \mathbf{H}$ NMR $\left(400 \mathrm{MHz}, \mathrm{CDCl}_{3}\right): \delta=1.36-1.55\left(\mathrm{~m}, 4 \mathrm{H}, \mathrm{CH}_{2}, 2 \times \mathrm{CH}_{\mathrm{a}}\right), 1.79(\mathrm{~s}, 2 \mathrm{H}$, 2-OH, 6-OH), $1.97-2.31\left(\mathrm{~m}, 8 \mathrm{H}, \mathrm{CH}_{2}, 2 \times \mathrm{CH}_{\mathrm{b}}, 1-\mathrm{H}, 3-\mathrm{H}, 5-\mathrm{H}, 7-\mathrm{H}\right), 2.50-2.52$ $\left(\mathrm{m}, 12 \mathrm{H}, 4 \times \mathrm{CH}_{3}\right), 6.70-6.75\left(\mathrm{~m}, 4 \mathrm{H}, 16^{\prime}-\mathrm{H}, 21^{\prime}-\mathrm{H}, 16^{\prime \prime}-\mathrm{H}, 21^{\prime \prime}-\mathrm{H}\right), 7.09\left(\mathrm{~d},{ }^{3} J_{\mathrm{H}, \mathrm{H}}\right.$ $=3.6 \mathrm{~Hz}, 2 \mathrm{H},\left[15^{\prime}-\mathrm{H}, 15^{\prime \prime}-\mathrm{H}\right]$ or $\left.\left[20^{\prime}-\mathrm{H}, 20^{\prime \prime}-\mathrm{H}\right]\right), 7.09\left(\mathrm{~d},{ }^{3} J_{\mathrm{H}, \mathrm{H}}=7.9 \mathrm{~Hz}, 2 \mathrm{H}, 6^{\prime}-\mathrm{H}\right.$, $\left.6^{\prime \prime}-\mathrm{H}\right), 7.12\left(\mathrm{~d},{ }^{3} J_{\mathrm{H}, \mathrm{H}}=3.6 \mathrm{~Hz}, 2 \mathrm{H},\left[20^{\prime}-\mathrm{H}, 20^{\prime \prime}-\mathrm{H}\right]\right.$ or [15'-H, 15"-H]), $7.28-7.35(\mathrm{~m}$, $\left.4 \mathrm{H}, 8^{\prime}-\mathrm{H}, 12^{\prime}-\mathrm{H}, 8^{\prime \prime}-\mathrm{H}, 12 "-\mathrm{H}\right), 7.43\left(\mathrm{dd},{ }^{3} J_{\mathrm{H}, \mathrm{H}}=7.9 \mathrm{~Hz},{ }^{4} J_{\mathrm{H}, \mathrm{H}}=1.8 \mathrm{~Hz}, 2 \mathrm{H}, 5^{\prime}-\mathrm{H}\right.$, $\left.5^{\prime \prime}-\mathrm{H}\right), 7.47\left(\mathrm{~m}, 4 \mathrm{H}, 9^{\prime}-\mathrm{H}, 11^{\prime}-\mathrm{H}, 9^{\prime \prime}-\mathrm{H}, 11^{\prime \prime}-\mathrm{H}\right), 7.73\left(\mathrm{~d},{ }^{4} J_{\mathrm{H}, \mathrm{H}}=1.8 \mathrm{~Hz}, 2 \mathrm{H}, 3^{\prime}-\mathrm{H}\right.$, 3"-H) ppm.

${ }^{13}$ C NMR $\left(101 \mathrm{MHz}, \mathrm{CDCl}_{3}\right): \delta=15.6\left(4 \times \mathrm{CH}_{3}\right), 28.2\left(\mathrm{CH}_{2}\right), 30.0\left(2 \times \mathrm{CH}_{2}\right), 31.6$ $\left(\mathrm{CH}_{2}\right), 33.9,34.1$ (C-1, C-3, C-5, C-7), 76.9 (C-2, C-6), 123.0, 123.1 (C-15', C-15", C-20', C-20"), 123.7 (C-5', C-5"), 124.5 (C-3', C-3"), 124.8 (C-9', C-11', C-9", C-11"), 126.3, 126.4 (C-16', C-21', C-16", C-21"), 129.6 (C-8', C-12', C-8", C-12"), 133.3 (C-19', C-19"), 133.7 (C-14', C-14"), 134.2 (C-6', C-6"), 139.6, 139.7, 141.7, 141.8, 141.9, 143.1 (C-1', C-4', C-7', C-10',C-17', C-22', C-1", C-4", C-7", C-10", C-17", C-22") ppm.

HRMS (ESI): $m / z$ calc. for $\mathrm{C}_{54} \mathrm{H}_{48} \mathrm{NaO}_{2} \mathrm{~S}_{4}[\mathrm{M}+\mathrm{Na}]^{+}$879.2429; found 879.2378. $\operatorname{TLC}\left(\mathrm{SiO}_{2}\right): R_{\mathrm{f}}=0.41\left(\mathrm{CH}_{2} \mathrm{Cl}_{2}\right)$. 


\subsection{Compound 107}

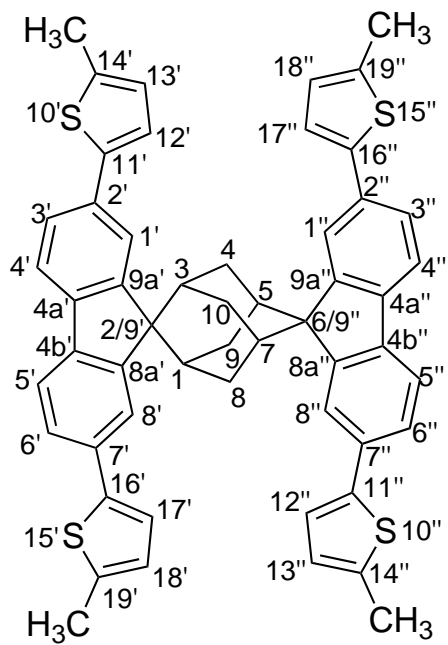

According to GP3, alcohol $106(34 \mathrm{mg}, 40 \mu \mathrm{mol}, 1.0 \mathrm{eq})$ was treated with aq. $\mathrm{H}_{2} \mathrm{SO}_{4}(75 \%, 1.0 \mathrm{ml})$ in $n$-octane $(2.0 \mathrm{ml})$ and stirred for $60 \mathrm{~min}$. Aq. work-up included quenching with $3.0 \mathrm{M}$ aq. $\mathrm{NaOH}(10 \mathrm{ml})$, as well as extraction with $\mathrm{CH}_{2} \mathrm{Cl}_{2}$ $(5 \times 15 \mathrm{ml})$, and gave $27 \mathrm{mg}$ of yellowish solid. The crude product was purified by column chromatography on silica gel $\left(15 \mathrm{~g} \mathrm{SiO}_{2}\right.$, cyclohexane $/ \mathrm{CHCl}_{3}=5: 1 \rightarrow$ 4:1); $26 \mathrm{mg}$ ( $31 \mu \mathrm{mol}, 79 \%$ ) of the title compound were obtained as colorless solid.

${ }^{1} \mathbf{H}$ NMR $\left(400 \mathrm{MHz}, \mathrm{CDCl}_{3}\right): \delta=2.09-2.16(\mathrm{~m}, 4 \mathrm{H}, 1-\mathrm{H}, 3-\mathrm{H}, 5-\mathrm{H}, 7-\mathrm{H}), 2.48(\mathrm{~d}$, $\left.{ }^{4} J_{\mathrm{H}, \mathrm{H}}=1.1 \mathrm{~Hz}, 12 \mathrm{H}, 4 \times \mathrm{CH}_{3}\right), 3.07-3.17\left(\mathrm{~m}, 8 \mathrm{H}, 4-\mathrm{H}_{2}, 8-\mathrm{H}_{2}, 9-\mathrm{H}_{2}, 10-\mathrm{H}_{2}\right), 6.69-$ $6.74\left(\mathrm{~m}, 4 \mathrm{H}, 13^{\prime}-\mathrm{H}, 18^{\prime}-\mathrm{H}, 13^{\prime \prime}-\mathrm{H}, 18^{\prime \prime}-\mathrm{H}\right), 7.08\left(\mathrm{~d},{ }^{3} J_{\mathrm{H}, \mathrm{H}}=3.6 \mathrm{~Hz}, 4 \mathrm{H}, 12^{\prime}-\mathrm{H}, 17^{\prime}-\mathrm{H}\right.$, $\left.12^{\prime \prime}-\mathrm{H}, 17^{\prime \prime}-\mathrm{H}\right), 7.60\left(\mathrm{dd},{ }^{3} J_{\mathrm{H}, \mathrm{H}}=7.9 \mathrm{~Hz},{ }^{4} J_{\mathrm{H}, \mathrm{H}}=1.2 \mathrm{~Hz}, 4 \mathrm{H}, 3^{\prime}-\mathrm{H}, 6^{\prime}-\mathrm{H}, 3^{\prime \prime}-\mathrm{H}, 6{ }^{\prime \prime}-\mathrm{H}\right)$, $7.78\left(\mathrm{~d},{ }^{3} J_{\mathrm{H}, \mathrm{H}}=8.0 \mathrm{~Hz}, 4 \mathrm{H}, 4^{\prime}-\mathrm{H}, 66^{\prime}-\mathrm{H}, 4^{\prime \prime}-\mathrm{H}, 66^{\prime \prime}-\mathrm{H}\right), 8.46\left(\mathrm{~d},{ }^{4} J_{\mathrm{H}, \mathrm{H}}=1.2 \mathrm{~Hz}, 4 \mathrm{H}, 1^{\prime}-\mathrm{H}\right.$, $\left.8^{\prime}-\mathrm{H}, 1^{\prime \prime}-\mathrm{H}, 8^{\prime \prime}-\mathrm{H}\right) \mathrm{ppm}$.

${ }^{13} \mathrm{C}$ NMR $\left(101 \mathrm{MHz}, \mathrm{CDCl}_{3}\right): \delta=15.6\left(4 \times \mathrm{CH}_{3}\right), 28.9(\mathrm{C}-4, \mathrm{C}-8, \mathrm{C}-9, \mathrm{C}-10), 34.6$ (C-1, C-3, C-5, C-7), 59.7 (C-2/9', C-6/9"), 119.9 (C-4', C-6', C-4", C-6"), 122.8 (C-12', C-17', C-12", C-17"), 125.1 (C-3', C-6', C-3", C-6"), 126.4 (C-13', C-18', C-13", C-18"), 127.4 (C-1', C-8', C-1", C-8"), 132.7 (C-2', C-7', C-2", C-7"), 139.4 (C-14', C-14", C-19', C-19"), 140.1 (C-4a', C-4b', C-4a", C-4b"), 143.1 (C-11', C-16', C-11", C-16"), 151.1 (C-8a', C-9a', C-8a", C-9a") ppm.

HRMS (MALDI-TOF): $m / z$ calc. for $\mathrm{C}_{54} \mathrm{H}_{44} \mathrm{~S}_{4}\left[\mathrm{M}^{+\cdot}\right]$ 820.2320; found 820.2331.

$\operatorname{TLC}\left(\mathrm{SiO}_{2}\right): R_{\mathrm{f}}=0.21\left(\right.$ cyclohexane $\left./ \mathrm{CHCl}_{3}=5: 1\right)$.

UV/Vis $\left(\mathrm{CHCl}_{3}\right): \lambda_{\mathrm{abs}}^{\max }=359 \mathrm{~nm}, \varepsilon=91300 \mathrm{M}^{-1} \mathrm{~cm}^{-1}$. 


\section{Chapter 22 COMPOUNDS}

Fluorescence $\left(\mathrm{CHCl}_{3}\right.$, exc. at $\left.348 \mathrm{~nm}\right): \lambda_{\mathrm{em}}^{\max }=396,(416) \mathrm{nm} ; \Phi_{\mathrm{f}}=0.45 .^{\dagger}$

\subsection{3-Bromo-5-iodo-5'-(p-methoxyphenyl)-2,2'-bi- thienyl (108)}

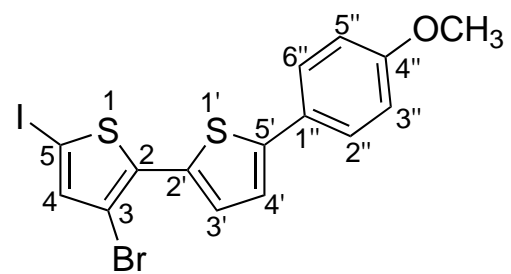

Regioselective iodination of bithiophene derivative $\mathbf{8 2}$ was carried out under mild iodination conditions. ${ }^{[154]}$

To a solution of compound $82(1.00 \mathrm{~g}, 2.85 \mathrm{mmol}, 1.00 \mathrm{eq})$ in dry THF $(8.0 \mathrm{ml})$, $\mathrm{N}$-iodosuccinimide (769 mg, $3.42 \mathrm{mmol}, 1.20 \mathrm{eq}$ ) was added at room temperature. To the clear yellow solution, $\mathrm{AcOH}(6.0 \mathrm{ml})$ was added. The reaction mixture was stirred at room temperature for $24 \mathrm{~h}$ giving a yellowish suspension. TLC of the reaction mixture on $\mathrm{RP} \mathrm{SiO}_{2}-\mathrm{C}_{18}$ with $\mathrm{MeCN}$ as eluent showed almost full consumption of the starting material. Then $1.0 \mathrm{M}$ aq. $\mathrm{Na}_{2} \mathrm{~S}_{2} \mathrm{O}_{3}(80 \mathrm{ml})$ was added, and the mixture was extracted with $\mathrm{CH}_{2} \mathrm{Cl}_{2}(3 \times 80 \mathrm{ml})$. The combined organic solutions were dried over $\mathrm{Na}_{2} \mathrm{SO}_{4}$ and concentrated in vacuo to give $1.76 \mathrm{~g}$ of light brown solid. Column chromatography on silica gel $\left(200 \mathrm{~g} \mathrm{SiO}_{2}\right.$, cyclohexane $\left./ \mathrm{CHCl}_{3}=5: 1 \rightarrow 3: 1\right)$ gave $1.19 \mathrm{~g}(2.49 \mathrm{mmol}, 87 \%)$ of the title compound as yellowish solid.

${ }^{1} \mathbf{H}$ NMR $\left(400 \mathrm{MHz} \mathrm{CDCl}_{3}\right): \delta=3.84\left(\mathrm{~s}, 3 \mathrm{H}, \mathrm{OCH}_{3}\right), 6.90-6.95\left(\mathrm{~m}, 2 \mathrm{H}, 3^{\prime \prime}-\mathrm{H}\right.$, $\left.5^{\prime \prime}-\mathrm{H}\right), 7.14\left(\mathrm{~d},{ }^{3} J_{\mathrm{H}, \mathrm{H}}=3.9 \mathrm{~Hz}, 1 \mathrm{H}, 4^{\prime}-\mathrm{H}\right), 7.14(\mathrm{~s}, 1 \mathrm{H}, 4-\mathrm{H}), 7.29\left(\mathrm{~d},{ }^{3} J_{\mathrm{H}, \mathrm{H}}=3.9 \mathrm{~Hz}\right.$, $\left.1 \mathrm{H}, 3^{\prime}-\mathrm{H}\right), 7.52-7.57$ (m, $\left.2 \mathrm{H}, 2^{\prime \prime}-\mathrm{H}, 6^{\prime \prime}-\mathrm{H}\right) \mathrm{ppm}$.

${ }^{13} \mathrm{C}$ NMR $\left(101 \mathrm{MHz}, \mathrm{CDCl}_{3}\right): \delta=55.5\left(\mathrm{OCH}_{3}\right), 71.5(\mathrm{C}-5), 107.3(\mathrm{C}-3), 114.5(\mathrm{C}-3 "$, C-5"), 122.3 (C-4'), 126.7 (C-1"), 127.3 (C-2", C-6"), 128.1 (C-3'), 131.6 (C-2), 138.7 (C-2'), 140.6 (C-5), 145.6 (C-5'), 159.7 (C-4") ppm.

MS (ESI, pos.): $m / z(\%)=476 / 478(100)\left[\mathrm{M}^{+\cdot}\right]$.

HRMS (EI): $m / z$ calc. for $\mathrm{C}_{15} \mathrm{H}_{10} \mathrm{BrIOS}_{2}\left[\mathrm{M}^{+\cdot}\right] 475.8401$; found 475.8413 .

$\operatorname{TLC}\left(\mathrm{SiO}_{2}\right): R_{\mathrm{f}}=0.47\left(\right.$ cyclohexane $\left./ \mathrm{CHCl}_{3}=5: 1\right)$.

\footnotetext{
${ }^{\dagger}$ Coumarin 120 in $\mathrm{MeOH}$ as a fl. quantum yield standard with $\Phi_{\mathrm{f}}=0.51^{[132]}$
} 
$\operatorname{TLC}\left(\mathrm{RP} \mathrm{SiO}_{2}-\mathrm{C}_{18}\right): R_{\mathrm{f}}=0.31(\mathrm{MeCN})$.

\subsection{Compound 109}

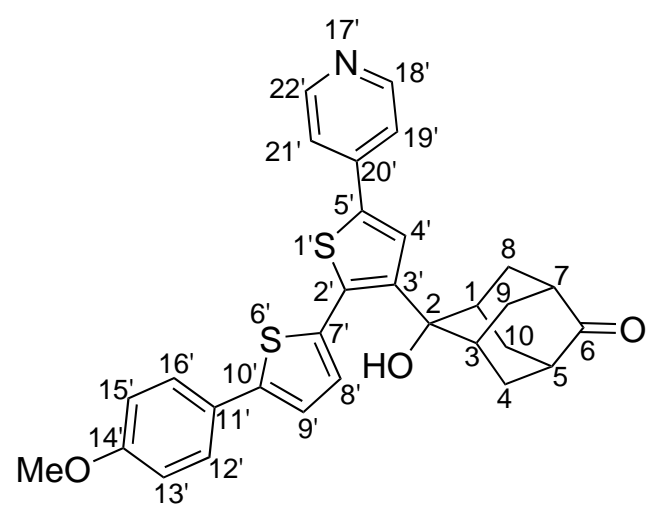

According to GP2, bromide 110 ( $152 \mathrm{mg}, 354 \mu \mathrm{mol}, 1.00 \mathrm{eq}$ ) was dissolved in dry THF $(7.5 \mathrm{ml})$ and lithiated with $1.6 \mathrm{M} \mathrm{n}$-BuLi in $n$-hexane $(0.27 \mathrm{ml}, 0.43 \mathrm{mmol}$, $1.2 \mathrm{eq})$. The red-brown solution of the lithiated $\mathbf{1 1 0}$ was added dropwise to a solution of 2,6-adamantanedione $(175 \mathrm{mg}, 1.06 \mathrm{mmol}, 3.00 \mathrm{eq})$ in dry THF $(5.0 \mathrm{ml})$. The reaction mixture was stirred at $-78^{\circ} \mathrm{C}$ for $10 \mathrm{~min}$ and then at room temperature for $1.5 \mathrm{~h}$. Sat. aq. $\mathrm{NH}_{4} \mathrm{Cl}(25 \mathrm{ml})$ was added followed by water $(25 \mathrm{ml})$. Extraction with EtOAc $(3 \times 25 \mathrm{ml})$, drying of the combined organic solutions over $\mathrm{Na}_{2} \mathrm{SO}_{4}$ and concentration in vacuo gave $305 \mathrm{mg}$ of orange solid. Column chromatography on silica gel $\left(45 \mathrm{~g} \mathrm{SiO}_{2}, \mathrm{CH}_{2} \mathrm{Cl}_{2} / \mathrm{EtOAc}=1: 1+0.1 \% \mathrm{NEt}_{3}\right)$ gave $156 \mathrm{mg}(304 \mu \mathrm{mol}, 86 \%)$ of the title compound as yellow solid.

${ }^{1} \mathbf{H}$ NMR $\left(400 \mathrm{MHz}, \mathrm{CDCl}_{3}\right): \delta=1.77-1.85\left(\mathrm{~m}, 2 \mathrm{H},\left[4-\mathrm{H}_{\mathrm{a}}, 10-\mathrm{H}_{\mathrm{a}}\right]\right.$ or $\left[8-\mathrm{H}_{\mathrm{a}}\right.$, $\left.\left.9-\mathrm{H}_{\mathrm{a}}\right]\right), 1.89-1.98\left(\mathrm{~m}, 2 \mathrm{H},\left[8-\mathrm{H}_{\mathrm{a}}, 9-\mathrm{H}_{\mathrm{a}}\right]\right.$ or $\left.\left[4-\mathrm{H}_{\mathrm{a}}, 10-\mathrm{H}_{\mathrm{a}}\right]\right), 2.03-2.12(\mathrm{~m}, 2 \mathrm{H}$, $\left[8-\mathrm{H}_{\mathrm{b}}, 9-\mathrm{H}_{\mathrm{b}}\right]$ or $\left.\left[4-\mathrm{H}_{\mathrm{b}}, 10-\mathrm{H}_{\mathrm{b}}\right]\right), 2.36-2.41(\mathrm{~m}, 1 \mathrm{H}, 5-\mathrm{H}$ or $7-\mathrm{H}), 2.45-2.50(\mathrm{~m}$, $1 \mathrm{H}, 7-\mathrm{H}$ or $5-\mathrm{H}), 2.59-2.65(\mathrm{~m}, 2 \mathrm{H}, 1-\mathrm{H}, 3-\mathrm{H}), 2.71-2.80\left(\mathrm{~m}, 2 \mathrm{H},\left[4-\mathrm{H}_{\mathrm{b}}, 10-\mathrm{H}_{\mathrm{b}}\right]\right.$ or $\left.\left[8-\mathrm{H}_{\mathrm{b}}, 9-\mathrm{H}_{\mathrm{b}}\right]\right), 3.40\left(\mathrm{~s}_{\mathrm{br}}, 1 \mathrm{H}, 2-\mathrm{OH}\right), 3.84\left(\mathrm{~s}, 3 \mathrm{H}, \mathrm{OCH}_{3}\right), 6.89-6.98(\mathrm{~m}, 2 \mathrm{H}$, $\left.13^{\prime}-\mathrm{H}, 15^{\prime}-\mathrm{H}\right), 7.16\left(\mathrm{~d},{ }^{3} \mathrm{~J}_{\mathrm{H}, \mathrm{H}}=3.7 \mathrm{~Hz}, 1 \mathrm{H}, 9^{\prime}-\mathrm{H}\right), 7.30-7.36\left(\mathrm{~m}, 3 \mathrm{H}, 8^{\prime}-\mathrm{H}, 19^{\prime}-\mathrm{H}\right.$, $\left.21^{\prime}-\mathrm{H}\right), 7.38$ (s, 1 H, 4'-H), $7.52-7.60$ (m, 2 H, 12'-H, 16'-H), $8.45-8.52$ (m, $2 \mathrm{H}$, $\left.18^{\prime}-\mathrm{H}, 22^{\prime}-\mathrm{H}\right) \mathrm{ppm}$.

${ }^{13} \mathrm{C}$ NMR $\left(101 \mathrm{MHz}, \mathrm{CDCl}_{3}\right): \delta=34.1$ ([C-8, C-9] or [C-4, C-10]), 35.5 (C-1, C-3), 36.5 ([C-4, C-10] or [C-8, C-9]), 45.2, 45.3 (C-5, C-7), $55.5\left(\mathrm{OCH}_{3}\right), 74.1$ (C-2), 114.5 (C-13', C-15'), 119.5 (C-19', C-21'), 122.4 (C-9'), 126.1 (C-4'), 126.6 (C-11'), 127.2 (C-12', C-16'), 130.6 (C-8'), 133.0 (C-7'), 135.9 (C-2'), 139.4 (C-5'), 140.8 (C-20'), 


\section{Chapter 22 $\mid$ COMPOUNDS}

143.6 (C-3'), 146.8 (C-10'), 150.3 (C-18', C-22'), 159.8 (C-14'), 217.2 (C-6) ppm.

MS (ESI, pos.): $m / z(\%)=514.4(100)[\mathrm{M}+\mathrm{H}]^{+}$.

HRMS (ESI): $m / z$ calc. for $\mathrm{C}_{30} \mathrm{H}_{28} \mathrm{NO}_{3} \mathrm{~S}_{2}[\mathrm{M}+\mathrm{H}]^{+}$514.1505; found 514.1515.

$\operatorname{TLC}\left(\mathrm{SiO}_{2}\right): R_{\mathrm{f}}=0.27\left(\mathrm{CH}_{2} \mathrm{Cl}_{2} / \mathrm{EtOAc}=1: 1+0.1 \% \mathrm{NEt}_{3}\right)$.

\subsection{3-Bromo-5'-(p-methoxyphenyl)-5-(4-pyridyl)- 2,2'-bithienyl (110)}

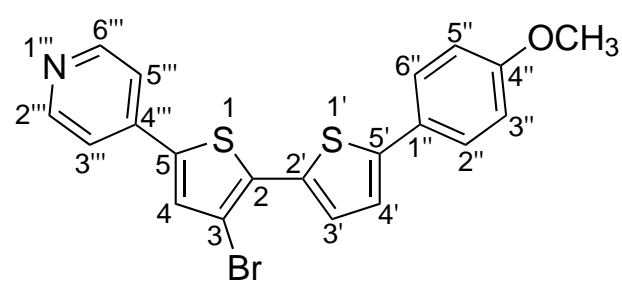

Compound 108 (618 mg, $1.30 \mathrm{mmol}, 1.00 \mathrm{eq})$, 4-pyridylboronic acid (52, $159 \mathrm{mg}$, $1.30 \mathrm{mmol}$, $1.00 \mathrm{eq}), 1,4$-dioxane $(8.4 \mathrm{ml})$, and $2.0 \mathrm{M} \mathrm{K}_{3} \mathrm{PO}_{4}(2.4 \mathrm{ml})$ were mixed in a screw cap pressure tube, and argon was bubbled through the mixture for $10 \mathrm{~min} . \mathrm{Pd}\left(\mathrm{PPh}_{3}\right)_{2} \mathrm{Cl}_{2}(91 \mathrm{mg}, 0.13 \mathrm{mmol}, 0.10 \mathrm{eq})$ was added, and the yellow suspension was stirred at $70^{\circ} \mathrm{C}$ for $22 \mathrm{~h}$. The reaction mixture was diluted with EtOAc $(30 \mathrm{ml})$ and filtered through Celite ${ }^{\mathrm{TM}}(\varnothing=30 \mathrm{~mm}, h=6 \mathrm{~mm})$. The filter cake was washed with EtOAc $(100 \mathrm{ml})$ and then $\mathrm{CH}_{2} \mathrm{Cl}_{2}(120 \mathrm{ml})$. The filtrates were combined and concentrated in vacuo to give $625 \mathrm{mg}$ of orange solid. Column chromatography on silica gel $\left(40 \mathrm{~g} \mathrm{SiO}_{2}, \mathrm{CH}_{2} \mathrm{Cl}_{2} / \mathrm{EtOAc}=4: 1+0.1 \% \mathrm{NEt}_{3}\right)$ gave $280 \mathrm{mg}$ ( $654 \mu \mathrm{mol}, 50 \%)$ of the title compound as orange-yellow solid.

${ }^{1} \mathbf{H}$ NMR $\left(400 \mathrm{MHz}, \mathrm{CDCl}_{3}\right): \delta=3.85\left(\mathrm{~s}, 3 \mathrm{H}, 4\right.$ "- $\left.\mathrm{OCH}_{3}\right), 6.91-6.96(\mathrm{~m}, 2 \mathrm{H}, 3$ "--H, 5"-H), $7.18\left(\mathrm{~d},{ }^{3} J_{\mathrm{H}, \mathrm{H}}=3.9 \mathrm{~Hz}, 1 \mathrm{H}, 4^{\prime}-\mathrm{H}\right), 7.39-7.43\left(\mathrm{~m}, 3 \mathrm{H}, 4-\mathrm{H}, 3^{\prime \prime \prime-H, ~ 5 " '-H), ~} 7.45\right.$ $\left(\mathrm{d},{ }^{3} \mathrm{~J}_{\mathrm{H}, \mathrm{H}}=3.9 \mathrm{~Hz}, 1 \mathrm{H}, 3^{\prime}-\mathrm{H}\right), 7.54-7.59\left(\mathrm{~m}, 2 \mathrm{H}, 2^{\prime \prime}-\mathrm{H}, 6^{\prime \prime}-\mathrm{H}\right), 8.60-8.64$ (m, $2 \mathrm{H}$, 2"'-H, 6"'-H) ppm.

${ }^{13} \mathrm{C}$ NMR $\left(101 \mathrm{MHz}, \mathrm{CDCl}_{3}\right): \delta=55.5\left(\mathrm{OCH}_{3}\right), 108.3(\mathrm{C}-3), 114.6(\mathrm{C}-3 ", \mathrm{C}-5 ")$, 119.4 (C-3"', C-5"'), 122.5 (C-4'), 126.6 (C-1"), 127.3 (C-2", C-6"), 128.3 (C-3'), 130.0 (C-4), 132.2 (C-2), 134.3 (C-5), 138.4 (C-2'), 140.0 (C-4'"'), 145.9 (C-5'), 150.6 (C-2'", C-6"'), 159.8 (C-4") ppm.

MS (ESI, pos.): $m / z(\%)=428 / 430(100)[\mathrm{M}+\mathrm{H}]^{+}$. 
HRMS (EI): $m / z$ calc. for $\mathrm{C}_{20} \mathrm{H}_{14} \mathrm{BrNOS}_{2}\left[\mathrm{M}^{+\cdot}\right]$ 426.9700; found 426.9703.

$\operatorname{TLC}\left(\mathrm{SiO}_{2}\right): R_{\mathrm{f}}=0.24\left(\mathrm{CH}_{2} \mathrm{Cl}_{2} / \mathrm{EtOAc}=1: 1+0.1 \% \mathrm{NEt}_{3}\right)$.

\subsection{Compound 111}

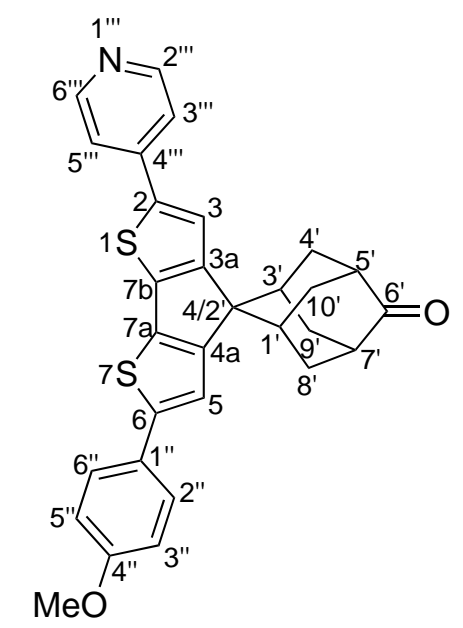

According to GP3, alcohol 109 (105 mg, $205 \mu \mathrm{mol}, 1.00 \mathrm{eq})$ was suspended in $n$-octane $(10 \mathrm{ml})$ using an ultrasonic bath and $70 \%$ aq. $\mathrm{H}_{2} \mathrm{SO}_{4}(1.0 \mathrm{ml})$ was added. After vigorous stirring for $15 \mathrm{~min}$, the reaction was quenched by addition of $4.0 \mathrm{M}$ aq. $\mathrm{NaOH}(70 \mathrm{ml})$ and extracted with $\mathrm{CH}_{2} \mathrm{Cl}_{2}(5 \times 50 \mathrm{ml})$. The combined organic solutions were dried over $\mathrm{Na}_{2} \mathrm{SO}_{4}$ and concentrated in vacuo to give $91 \mathrm{mg}$ $(0.18 \mathrm{mmol}, 89 \%)$ of the title compound as yellow-orange solid.

${ }^{1} \mathbf{H}$ NMR $\left(400 \mathrm{MHz}, \mathrm{CDCl}_{3}\right): \delta=1.80-1.83\left(\mathrm{~m}, 2 \mathrm{H}, 1^{\prime}-\mathrm{H}, 3^{\prime}-\mathrm{H}\right), 2.05-2.16(\mathrm{~m}$, $\left.4 \mathrm{H}, 4-\mathrm{H}_{\mathrm{a}}, 8-\mathrm{H}_{\mathrm{a}}, 9-\mathrm{H}_{\mathrm{a}}, 10-\mathrm{H}_{\mathrm{a}}\right), 2.81-2.94\left(\mathrm{~m}, 6 \mathrm{H}, 4-\mathrm{H}_{\mathrm{b}}, 8-\mathrm{H}_{\mathrm{b}}, 9-\mathrm{H}_{\mathrm{b}}, 10-\mathrm{H}_{\mathrm{b}}, 5-\mathrm{H}\right.$, 7'-H), 3.86 (s, $\left.3 \mathrm{H}, \mathrm{OCH}_{3}\right), 6.92-6.98$ (m, $\left.2 \mathrm{H}, 3 "-\mathrm{H}, 5 "-\mathrm{H}\right), 7.45-7.52$ (m, $3 \mathrm{H}, 5-\mathrm{H}$, 3"'-H, 5"'-H), 7.53 - 7.59 (m, 2 H, 2"-H, 6"-H), 7.80 (s, 1 H, 3-H), 8.55 - 8.61 (m, 2 H, 2"'-H, 6"'-H) ppm.

${ }^{13}$ C NMR $\left(101 \mathrm{MHz}, \mathrm{CDCl}_{3}\right): \delta=33.5\left(\mathrm{C}-1^{\prime}, \mathrm{C}-3^{\prime}\right), 35.6,36.0$ (C-4', C-8', C-9', C-10'), $45.4\left(\mathrm{C}-5^{\prime}, \mathrm{C}-7^{\prime}\right), 55.6\left(\mathrm{OCH}_{3}\right), 57.6\left(\mathrm{C}-2^{\prime} / 4\right), 114.7$ (C-3", C-5"), $119.2\left(\mathrm{C}-3^{\prime \prime \prime}\right.$, C-5"'), 121.8 (C-5), 124.5 (C-3), 127.1 (C-2", C-6"), 127.5 (C-1"), 135.7 (C-7a), 139.1 (C-2), 140.2 (C-7b), 142.1 (C-4"'), 145.1 (C-6), 150.5 (C-2"', C-6"'), 156.2 (C-3a), 157.9 (C-4a), 159.6 (C-4"), 216.1 (C-6') ppm.

MS (ESI, pos.): $m / z(\%)=469.4(100)[\mathrm{M}+\mathrm{H}]^{+}$.

HRMS (ESI): $m / z$ calc. for $\mathrm{C}_{30} \mathrm{H}_{26} \mathrm{NO}_{2} \mathrm{~S}_{2}[\mathrm{M}+\mathrm{H}]^{+} 496.1399$; found 496.1399 . 


\section{Chapter 22 COMPOUNDS}

$\operatorname{TLC}\left(\mathrm{SiO}_{2}\right): R_{\mathrm{f}}=0.18\left(\mathrm{EtOAc}+0.1 \% \mathrm{NEt}_{3}\right)$.

UV/Vis (EtOAc): $\lambda_{\text {abs }}^{\max }=407 \mathrm{~nm}, \varepsilon=40400 \mathrm{M}^{-1} \mathrm{~cm}^{-1}$.

Fluorescence $($ EtOAc, exc. at $400 \mathrm{~nm}): \lambda_{\mathrm{em}}^{\max }=485 \mathrm{~nm} ; \Phi_{\mathrm{f}}=0.41 .^{\dagger}$

\subsection{Compound 112}

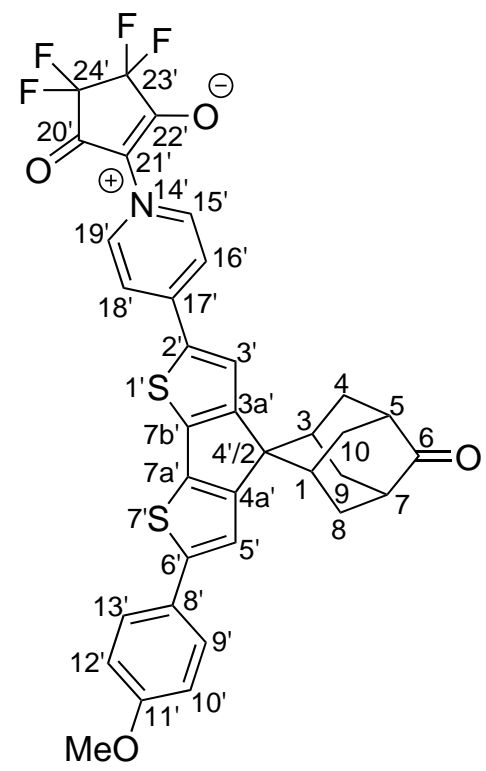

To a solution of compound $111(12 \mathrm{mg}, 25 \mu \mathrm{mol}, 1.0 \mathrm{eq})$ in 1,2-dichloroethane $(4.0 \mathrm{ml})$, water $(44 \mu \mathrm{l})$ was added followed by $\mathrm{AcOH}(45 \mu \mathrm{l})$. The mixture was cooled to $0{ }^{\circ} \mathrm{C}$, and perfluorocyclopentene $(0.44 \mathrm{ml}, 0.70 \mathrm{~g}, 3.3 \mathrm{mmol}$, ca. $130 \mathrm{eq})$ was added using a cold syringe. The sealed rection vessel was placed into a microwave cavity and the reaction mixture was irradiated ( $150 \mathrm{~W}$ max. power) at $130^{\circ} \mathrm{C}$ for $10 \mathrm{~min}$. The dark purple reaction mixture was cooled to r.t. and diluted with $\mathrm{CH}_{2} \mathrm{Cl}_{2}(25 \mathrm{ml})$ and sat. aq. $\mathrm{Na}_{2} \mathrm{CO}_{3}(25 \mathrm{ml})$. The aq. layer was separated and extracted with $\mathrm{CH}_{2} \mathrm{Cl}_{2}(25 \mathrm{ml})$. The combined organic solutions were dried over $\mathrm{Na}_{2} \mathrm{SO}_{4}$ and concentrated in vacuo to give $12 \mathrm{mg}$ of a purple black solid. Column chromatography on silica gel ( $\left.10 \mathrm{~g} \mathrm{SiO}_{2}, \mathrm{CH}_{2} \mathrm{CH}_{2} / \mathrm{MeOH}=25: 1\right)$ gave $4.6 \mathrm{mg}(6.9 \mu \mathrm{mol}, 28 \%)$ of the title compound as black solid.

${ }^{1} \mathbf{H}$ NMR $(400 \mathrm{MHz}$, DMSO-d 6 ): $\delta=1.66-1.72(\mathrm{~m}, 2 \mathrm{H}, 1-\mathrm{H}, 3-\mathrm{H}), 1.92-2.05(\mathrm{~m}$, $\left.4 \mathrm{H}, 4-\mathrm{H}_{\mathrm{a}}, 8-\mathrm{H}_{\mathrm{a}}, 9-\mathrm{H}_{\mathrm{a}}, 10-\mathrm{H}_{\mathrm{a}}\right), 2.65-2 .(\mathrm{m}, 2 \mathrm{H}, 5-\mathrm{H}, 7-\mathrm{H}), 2.82-2.94\left(\mathrm{~m}, 4 \mathrm{H}, 4-\mathrm{H}_{\mathrm{b}}\right.$, $\left.8-\mathrm{H}_{\mathrm{b}}, 9-\mathrm{H}_{\mathrm{b}}, 10-\mathrm{H}_{\mathrm{b}}\right), 3.82\left(\mathrm{~s}, 3 \mathrm{H}, \mathrm{OCH}_{3}\right), 7.01-7.07\left(\mathrm{~m}, 2 \mathrm{H}, 10^{\prime}-\mathrm{H}, 12^{\prime}-\mathrm{H}\right), 7.75-$

\footnotetext{
${ }^{\dagger}$ Coumarin 30 in EtOAc as a fl. quantum yield standard with $\Phi_{\mathrm{f}}=0.56^{[181]}$
} 
$7.82\left(\mathrm{~m}, 2 \mathrm{H}, 9^{\prime}-\mathrm{H}, 13^{\prime}-\mathrm{H}\right), 8.09$ (s, $\left.1 \mathrm{H}, 5^{\prime}-\mathrm{H}\right), 8.36-8.42\left(\mathrm{~m}, 2 \mathrm{H}, 16^{\prime}-\mathrm{H}, 18^{\prime}-\mathrm{H}\right), 8.82$ (s, $\left.1 \mathrm{H}, 3^{\prime}-\mathrm{H}\right), 8.95-9.01\left(\mathrm{~m}, 2 \mathrm{H}, 15^{\prime}-\mathrm{H}, 19^{\prime}-\mathrm{H}\right) \mathrm{ppm}$.

${ }^{19}$ F NMR $(376.4 \mathrm{MHz}$, DMSO-d 6 ): $\delta=-126.0 \mathrm{ppm}$.

MS (ESI, neg.): $m / z(\%)=698.6(100)[\mathrm{M}+\mathrm{Cl}]^{-}$.

HRMS (ESI): $m / z$ calc. for $\mathrm{C}_{35} \mathrm{H}_{25} \mathrm{~F}_{4} \mathrm{NO}_{4} \mathrm{~S}_{2}[\mathrm{M}+\mathrm{H}]^{+}$664.1234; found 664.1221.

$\operatorname{TLC}\left(\mathrm{SiO}_{2}\right): R_{\mathrm{f}}=0.59\left(\mathrm{EtOAc}+0.1 \% \mathrm{NEt}_{3}\right)$.

UV/Vis $\left(\right.$ EtOAc): $\lambda_{\mathrm{abs}}^{\max }=514 \mathrm{~nm}, \varepsilon=47000 \mathrm{M}^{-1} \mathrm{~cm}^{-1}$. UV/Vis $\left(\mathrm{CHCl}_{3}\right)$ : $\lambda_{\text {abs }}^{\max }=542 \mathrm{~nm}, \varepsilon=54000 \mathrm{M}^{-1} \mathrm{~cm}^{-1}$.

Fluorescence $\left(\mathrm{CHCl}_{3}\right.$, exc. at $\left.500 \mathrm{~nm}\right): \lambda_{\mathrm{em}}^{\max }=614 \mathrm{~nm} ; \Phi_{\mathrm{f}}=0.94 .^{\dagger}$

\subsection{Compound 113}

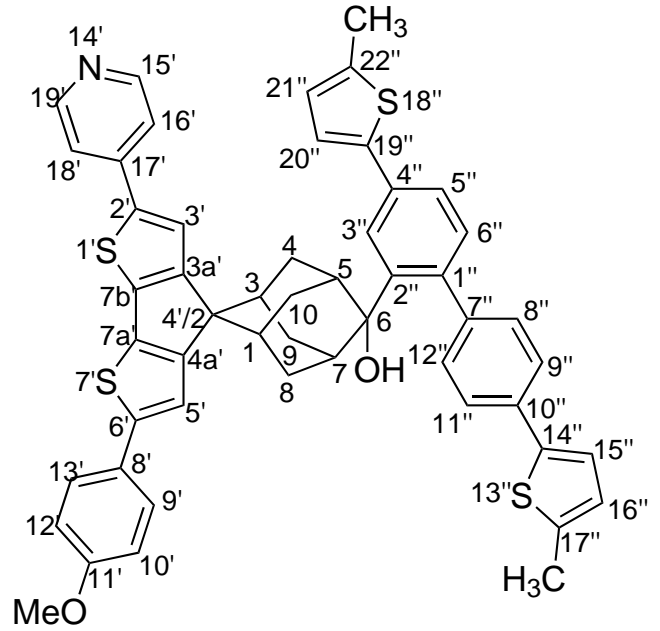

According to GP2, bromide 103 (31 mg, $73 \mu \mathrm{mol}$, $1.2 \mathrm{eq})$ was dissolved in dry THF $(3.0 \mathrm{ml})$ and lithiated with $1.6 \mathrm{M} n$-BuLi in $n$-hexane ( $45 \mu \mathrm{l}, 73 \mu \mathrm{mol}, 1.2 \mathrm{eq})$. The solution of lithiated $\mathbf{1 0 3}$ was added dropwise to a solution of ketone $\mathbf{1 1 1}$ (30 mg, $61 \mathrm{mmol}, 1.0 \mathrm{eq})$ in dry THF $(7.0 \mathrm{ml})$. The reaction mixture was stirred at $-78^{\circ} \mathrm{C}$ for $10 \mathrm{~min}$ and then $5 \mathrm{~h}$ at room temperature. Then sat. aq. $\mathrm{NH}_{4} \mathrm{Cl}(3 \mathrm{ml})$ was added followed by water $(3 \mathrm{ml})$. The mixture was extracted with $\mathrm{CH}_{2} \mathrm{Cl}_{2}$ $(5 \times 8 \mathrm{ml})$, and the combined organic solutions were dried over $\mathrm{Na}_{2} \mathrm{SO}_{4}$. Concentration in vacuo gave $60 \mathrm{mg}$ of orange solid. Column chromatography on silica gel $\left(16 \mathrm{~g} \mathrm{SiO}_{2}, \mathrm{CH}_{2} \mathrm{Cl}_{2} / \mathrm{EtOAc}=1: 1+0.1 \% \mathrm{NEt}_{3}\right)$ gave $26 \mathrm{mg}(31 \mu \mathrm{mol}, 51 \%)$ of

\footnotetext{
${ }^{\dagger}$ Rhodamine $6 \mathrm{G}$ in $\mathrm{EtOH}$ as a fl. quantum yield standard with $\Phi_{\mathrm{f}}=0.95^{[155]}$
} 


\section{Chapter 22 $\mid$ COMPOUNDS}

the title compound as orange solid.

${ }^{1} \mathbf{H}$ NMR $\left(400 \mathrm{MHz} \mathrm{CDCl}_{3}\right): \delta=1.34-1.41(\mathrm{~m}, 1 \mathrm{H}, 1-\mathrm{H}$ or $3-\mathrm{H}), 1.51-1.57(\mathrm{~m}$, $1 \mathrm{H}, 3-\mathrm{H}$ or $1-\mathrm{H}), 1.64-1.89\left(\mathrm{~m}, 2 \mathrm{H}, 2 \times \mathrm{CH}_{\mathrm{a}}\right), 2.11-2.77\left(\mathrm{~m}, 14 \mathrm{H}, 2 \times \mathrm{CH}_{\mathrm{b}}\right.$, $\left.2 \times \mathrm{CH}_{2}, 5-\mathrm{H}, 7-\mathrm{H}, 2 \times \mathrm{CH}_{3}\right), 3.85\left(\mathrm{~s}, 3 \mathrm{H}, \mathrm{OCH}_{3}\right), 6.73-6.76(\mathrm{~m}, 1 \mathrm{H}, 16 "-\mathrm{H}$ or 21"-H), $6.78-6.81$ (m, 1 H, 21"-H or 16"-H), $6.92-6.97$ (m, $\left.2 \mathrm{H}, 10^{\prime}-\mathrm{H}, 12^{\prime}-\mathrm{H}\right), 7.12$ $\left(\mathrm{d},{ }^{3} J_{\mathrm{H}, \mathrm{H}}=3.4 \mathrm{~Hz}, 1 \mathrm{H}, 15^{\prime \prime}-\mathrm{H}\right.$ or $\left.20^{\prime \prime}-\mathrm{H}\right), 7.11\left(\mathrm{~d},{ }^{3} J_{\mathrm{H}, \mathrm{H}}=7.9 \mathrm{~Hz}, 1 \mathrm{H}, 6^{\prime \prime}-\mathrm{H}\right), 7.21(\mathrm{~d}$, ${ }^{3} J_{\mathrm{H}, \mathrm{H}}=3.4 \mathrm{~Hz}, 1 \mathrm{H}, 20$ "-H or 15"-H), $7.38-7.48$ (m, 4 H, 5'-H, 5"-H, 2"-H, 12"-H), $7.48-7.51$ (m, 2 H, 16'-H, 18'-H), 7.53 - 7.57 (m, 2 H, 9'-H, 13'-H), 7.58 - 7.62 (m, $\left.2 \mathrm{H}, 9 "-\mathrm{H}, 11^{\prime \prime}-\mathrm{H}\right), 7.73-7.78\left(\mathrm{~m}, 1 \mathrm{H}, 3^{\prime}-\mathrm{H}\right), 7.87\left(\mathrm{~d},{ }^{4} J_{\mathrm{H}, \mathrm{H}}=1.8 \mathrm{~Hz}, 1 \mathrm{H}, 3^{\prime \prime}-\mathrm{H}\right), 8.50$ -8.55 (m, $\left.2 \mathrm{H}, 15^{\prime}-\mathrm{H}, 19^{\prime}-\mathrm{H}\right) \mathrm{ppm}$.

MS (ESI, pos.): $m / z(\%)=842.3(11)[\mathrm{M}+\mathrm{H}]^{+}$.

HRMS (ESI): $m / z$ calc. for $\mathrm{C}_{52} \mathrm{H}_{44} \mathrm{NO}_{2} \mathrm{~S}_{4}[\mathrm{M}+\mathrm{H}]^{+}$842.2249; found 842.2213.

\subsection{Compound 114}

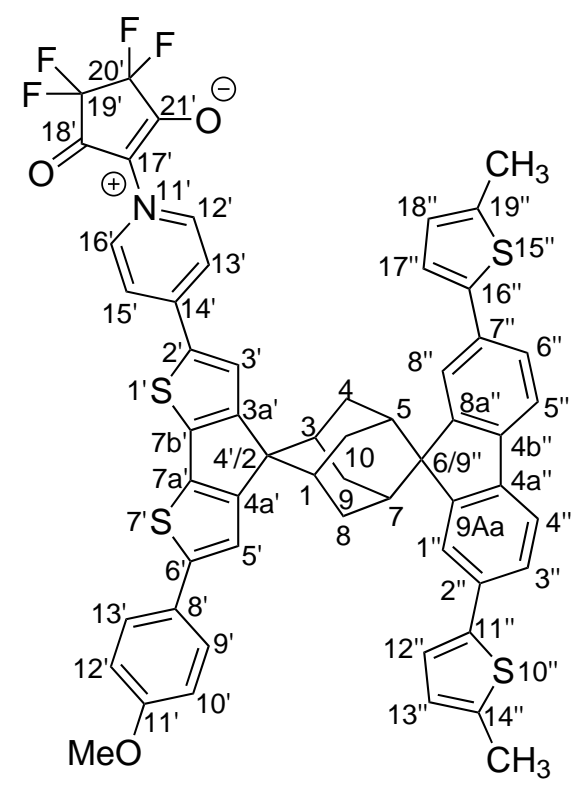

To a solution of compound $115(9.8 \mathrm{mg}, 10 \mu \mathrm{mol}, 1.0 \mathrm{eq})$ in 1,2-dichloroethane $(4.0 \mathrm{ml})$, water $(44 \mu \mathrm{l})$ and $\mathrm{AcOH}(45 \mu \mathrm{l})$ were added. The mixture was cooled to $0{ }^{\circ} \mathrm{C}$ and perfluorocyclopentene $(0.44 \mathrm{ml}, 0.70 \mathrm{~g}, 3.3 \mathrm{mmol}$, ca. $320 \mathrm{eq})$ was added using a cold syringe. The sealed reaction vessel was placed into a microwave cavity, and the reaction mixture was irradiated (150 W max. power) at $130^{\circ} \mathrm{C}$ for $10 \mathrm{~min}$. The dark purple reaction mixture was cooled down to r.t., di- 
luted with $\mathrm{CH}_{2} \mathrm{Cl}_{2}(25 \mathrm{ml})$, and then sat. aq. $\mathrm{Na}_{2} \mathrm{CO}_{3}(25 \mathrm{ml})$ was added. The aq. layer was separated and extracted with $\mathrm{CH}_{2} \mathrm{Cl}_{2}(25 \mathrm{ml})$. The combined organic solutions were dried over $\mathrm{Na}_{2} \mathrm{SO}_{4}$ and concentrated in vacuo to give $11 \mathrm{mg}$ $(9.3 \mu \mathrm{mol}, 92 \%)$ of a purple black solid. An analytical sample was obtained by normal phase HPLC (ProntoSIL ${ }^{\mathrm{TM}} 120-5$ Amino E, $5 \mu \mathrm{m}, \varnothing=16 \mathrm{~mm}, l=25 \mathrm{~cm}$; iso-octane $/ \mathrm{CHCl}_{3}=55: 45 ; 11 \mathrm{ml} / \mathrm{min}$ flow; 6.9 min retention time). Purity was confirmed by analytical HPLC (figure 65).

${ }^{1} \mathbf{H}$ NMR $(400 \mathrm{MHz}$, DMSO-d 6 ): $\delta=1.76-1.95(\mathrm{~m}, 4 \mathrm{H}, 1-\mathrm{H}, 3-\mathrm{H}, 5-\mathrm{H}, 7-\mathrm{H}), 2.32$ $\left(\mathrm{s}, 6 \mathrm{H}, 2 \times \mathrm{CH}_{3}\right) 2.63-2.75\left(\mathrm{~m}, 2 \mathrm{H}, 4-\mathrm{H}_{\mathrm{a}}, 8-\mathrm{H}_{\mathrm{a}}, 9-\mathrm{H}_{\mathrm{a}}, 10-\mathrm{H}_{\mathrm{a}}\right), 2.86-3.04(\mathrm{~m}, 4 \mathrm{H}$, $\left.4-\mathrm{H}_{\mathrm{b}}, 8-\mathrm{H}_{\mathrm{b}}, 9-\mathrm{H}_{\mathrm{b}}, 10-\mathrm{H}_{\mathrm{b}}\right), 3.71\left(\mathrm{~s}, 3 \mathrm{H}, \mathrm{OCH}_{3}\right), 6.69-6.72\left(\mathrm{~m}, 2 \mathrm{H}, 13^{\prime \prime}-\mathrm{H}, 18 "-\mathrm{H}\right)$, $6.87-6.93$ (m, 2 H, 10'-H, 12'-H), 7.20 - 7.24 (m, 2 H, 12"-H, 17"-H), 7.56 - 7.63 (m, 4 H, 9'-H, 13'-H, 3"-H, 6"-H), 7.86 - 7.92 (m, $\left.2 \mathrm{H}, 4^{\prime \prime}-\mathrm{H}, 5^{\prime \prime}-\mathrm{H}\right), 7.95$ (s, $1 \mathrm{H}, 5$ '-H), $8.17-8.24$ (m, 2 H, 16'-H, 18'-H), 8.27 (s, 2 H, 1"-H, 8"-H), 8.71 (s, 1 H, 3'-H), 8.84 $-8.90\left(\mathrm{~m}, 2 \mathrm{H}, 15^{\prime}-\mathrm{H}, 19^{\prime}-\mathrm{H}\right) \mathrm{ppm}$.

${ }^{13}$ C NMR $\left(101 \mathrm{MHz}, \mathrm{DMSO}-\mathrm{d}_{6}\right.$, some quaternary carbon atoms not detected): $\delta=14.9\left(2 \times \mathrm{CH}_{3}\right), 29.1,30.0$ (C-4, C-8, C-9, C-10), 32.9, 33.9 (C-1, C-3, C-5, C-7), $55.1\left(\mathrm{OCH}_{3}\right), 58.3,60.5\left(\mathrm{C}-4^{\prime} / 2, \mathrm{C}-6 / 9^{\prime \prime}\right), 114.5$ (C-10', C-12'), 119.8 (C-16', C-18'), 120.4 (C-4", C-5"), 122.7 (C-5'), 123.2 (C-12", C-17"), 124.6 ([C-3", C-6"] or [C-9', C-13']), 125.7 (C-1", C-8"), 126.4, 126.8 (C-13", C-18"), 126.9 ([C-9', C-13'] or [C-3", C-6"]), 131.2 (C-3'), 131.9, 133.8, 134.7, 138.8, 139.2, 140.8 (C-15', C-19'), 141.6, $146.6,147.0,147.5,150.5,157.7,159.3,161.4 \mathrm{ppm}$.

${ }^{19}$ F NMR $\left(376.4 \mathrm{MHz}, \mathrm{DMSO}-\mathrm{d}_{6}\right): \delta=-125.9 \mathrm{ppm}$.

HRMS (ESI): $m / z$ calc. for $\mathrm{C}_{57} \mathrm{H}_{42} \mathrm{~F}_{4} \mathrm{NO}_{3} \mathrm{~S}_{4}[\mathrm{M}+\mathrm{H}]^{+}$992.1978; found 992.1978.

$\operatorname{TLC}\left(\mathrm{SiO}_{2}\right): R_{\mathrm{f}}=0.76\left(\mathrm{CH}_{2} \mathrm{Cl}_{2} / \mathrm{MeOH}=50: 1\right)$.

UV/Vis $\left(\mathrm{CHCl}_{3}\right): \lambda_{\mathrm{abs}}^{\max }=546,(358) \mathrm{nm}, \varepsilon=65000,(51000) \mathrm{M}^{-1} \mathrm{~cm}^{-1}$.

Fluorescence $\left(\mathrm{CHCl}_{3}\right.$, exc. at $\left.360 \mathrm{~nm}\right): \lambda_{\mathrm{em}}^{\max }=616 \mathrm{~nm}$. 


\section{Chapter 22 $\quad$ COMPOUNDS}
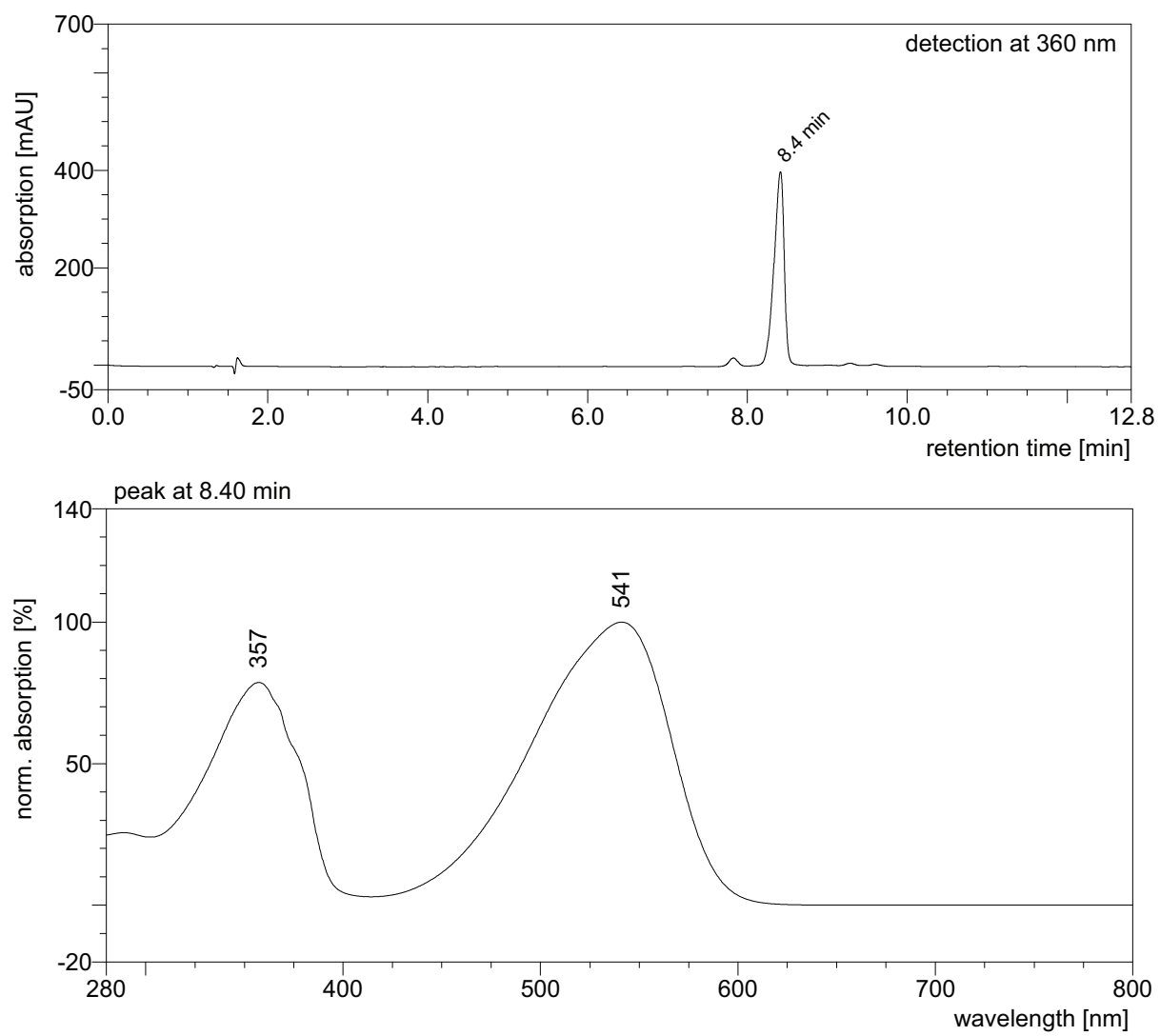

Figure 65: HPLC trace of compound 114 after purification by preparative HPLC (ProntoSIL ${ }^{\mathrm{TM}}$ 120-5 Amino $\mathrm{E}, 5 \mu \mathrm{m}, \varnothing=4 \mathrm{~mm}, l=25 \mathrm{~cm}$; iso-octane $/ \mathrm{CHCl}_{3}$ $=80: 20$ to $0: 100$ in $20 \mathrm{~min} ; 1.5 \mathrm{ml} / \mathrm{min}$ flow); top: chromatogram; bottom: UV/Vis absorption spectrum at $8.4 \mathrm{~min}$ retention time. 


\subsection{Compound 115}

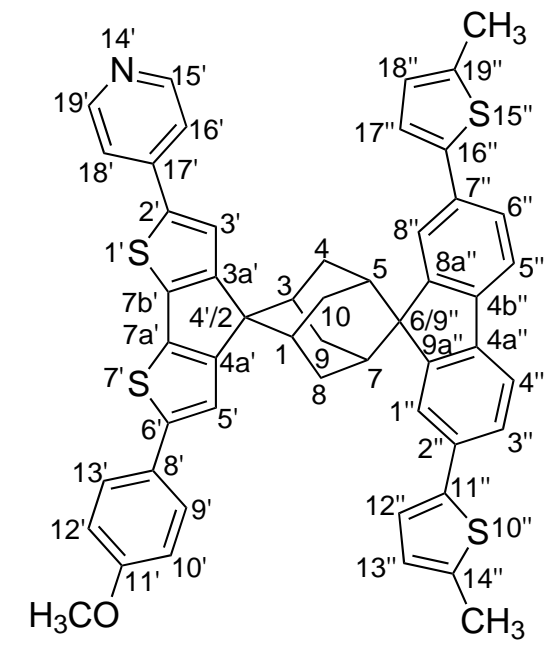

According to GP3, alcohol 113 (15 mg, $17 \mu \mathrm{mol}, 1.0 \mathrm{eq})$ was suspended in $n$-octane $(1.0 \mathrm{ml})$ using an ultrasonic bath, and $60 \%$ aq. $\mathrm{H}_{2} \mathrm{SO}_{4}(0.20 \mathrm{ml})$ was added. After stirring for $120 \mathrm{~min}$, the reaction was quenched by addition of $2.0 \mathrm{M}$ aq. $\mathrm{NaOH}$ $(10 \mathrm{ml})$ and extracted with $\mathrm{CH}_{2} \mathrm{Cl}_{2}(3 \times 10 \mathrm{ml})$. The combined organic solutions were dried over $\mathrm{Na}_{2} \mathrm{SO}_{4}$ and concentrated in vacuo to give $14 \mathrm{mg}$ of yellow solid. Column chromatography on silica gel $\left(6 \mathrm{~g} \mathrm{SiO}_{2}, \mathrm{CH}_{2} \mathrm{Cl}_{2} / \mathrm{EtOAc}=5: 1+0.1 \%\right.$ $\left.\mathrm{NEt}_{3}\right)$ gave $6.0 \mathrm{mg}(7.3 \mu \mathrm{mol}, 42 \%)$ of the title compound as orange solid.

${ }^{1} \mathbf{H}$ NMR $\left(400 \mathrm{MHz} \mathrm{CDCl}_{3}\right): \delta=1.98-2.06(\mathrm{~m}, 2 \mathrm{H}, 5-\mathrm{H}, 7-\mathrm{H}), 2.08-2.12(\mathrm{~m}, 2 \mathrm{H}$, 1-H, 3-H), $2.48\left(\mathrm{~s}, 6 \mathrm{H}, 2 \times \mathrm{CH}_{3}\right), 2.56-2.78\left(\mathrm{~m}, 4 \mathrm{H}, 4-\mathrm{H}_{\mathrm{a}}, 8-\mathrm{H}_{\mathrm{a}}, 9-\mathrm{H}_{\mathrm{a}}, 10-\mathrm{H}_{\mathrm{a}}\right), 3.09$ - $3.23\left(\mathrm{~m}, 4 \mathrm{H}, 4-\mathrm{H}_{\mathrm{b}}, 8-\mathrm{H}_{\mathrm{b}}, 9-\mathrm{H}_{\mathrm{b}}, 10-\mathrm{H}_{\mathrm{b}}\right), 3.85\left(\mathrm{~s}, 3 \mathrm{H}, \mathrm{OCH}_{3}\right), 6.72-6.74(\mathrm{~m}, 2 \mathrm{H}$, $\left.13^{\prime \prime}-\mathrm{H}, 18^{\prime \prime}-\mathrm{H}\right), 6.93-6.98\left(\mathrm{~m}, 2 \mathrm{H}, 10^{\prime}-\mathrm{H}, 12^{\prime}-\mathrm{H}\right), 7.10\left(\mathrm{~d},{ }^{3} J_{\mathrm{H}, \mathrm{H}}=3.6 \mathrm{~Hz}, 2 \mathrm{H}, 12^{\prime \prime}-\mathrm{H}\right.$, 17"-H), $7.55-7.59$ (m, 2 H, 9'-H, 13'-H), 7.63 (d, ${ }^{3} J_{\mathrm{H}, \mathrm{H}}=7.8 \mathrm{~Hz}, 2^{\prime}-\mathrm{H}, 3^{\prime \prime}-\mathrm{H}, 6$ "'-H), $7.65\left(\mathrm{~s}, 1 \mathrm{H}, 5^{\prime}-\mathrm{H}\right), 7.69-7.73\left(\mathrm{~m}, 2 \mathrm{H}, 16^{\prime}-\mathrm{H}, 18^{\prime}-\mathrm{H}\right), 7.80\left(\mathrm{~d},{ }^{3} \mathrm{~J}_{\mathrm{H}, \mathrm{H}}=7.8 \mathrm{~Hz}, 2 \mathrm{H}\right.$, 4"-H, 5"-H), 8.12 (s, 1 H, 3'-H), 8.35 (s, 2 H, 1"-H, 8"-H), 8.46 - 8.49 (m, 2 H, 15'-H, $\left.19^{\prime}-\mathrm{H}\right)$ ppm.

${ }^{13} \mathbf{C}$ NMR $\left(101 \mathrm{MHz}, \mathrm{CDCl}_{3}\right.$, some quaternary carbon atoms not detected): $\delta=15.6\left(2 \times \mathrm{CH}_{3}\right), 30.1,31.1(\mathrm{C}-4, \mathrm{C}-8, \mathrm{C}-9, \mathrm{C}-10), 33.6,34.2(\mathrm{C}-1, \mathrm{C}-3, \mathrm{C}-5$, $\mathrm{C}-7), 55.6\left(\mathrm{OCH}_{3}\right), 58.7,61.4\left(\mathrm{C}-4^{\prime} / 2, \mathrm{C}-6 / 9^{\prime \prime}\right), 114.8$ (C-10', C-12'), $119.5(\mathrm{C}-16$ ', C-18'), 120.1 (C-4", C-5"), 122.8 (C-5'), 122.9 (C-12", C-17"), 125.2 (C-3", C-6"), 126.5 (C-13", C-18"), 127.0 (C-1", C-8"), 127.3 (C-8'), 127.3 (C-9', C-13'), 132.8 (C-2", C-7"), 134.9 (C-7a'), 135.0 (C-2'), 139.7 (C-14", C-19"), 140.0 (C-4a", C-4b"), 142.9 (C-11", C-16"), 143.2 (C-15', C-19'), 145.5 (C-7b'), 150.8 (C-8a", C-9a"), 157.5 


\section{Chapter 22 COMPOUNDS}

(C-3a'), 159.9 (C-11'), 160.7 (C-4a') ppm.

MS (ESI, pos.): $m / z(\%)=824.4(100)[\mathrm{M}+\mathrm{H}]^{+}$.

HRMS (ESI): $m / z$ calc. for $\mathrm{C}_{52} \mathrm{H}_{42} \mathrm{NOS}_{4}[\mathrm{M}+\mathrm{H}]^{+}$824.2144; found 824.2134.

$\operatorname{TLC}\left(\mathrm{SiO}_{2}\right): R_{\mathrm{f}}=0.37\left(\mathrm{CH}_{2} \mathrm{Cl}_{2} / \mathrm{EtOAc}=5: 1+0.1 \% \mathrm{NEt}_{3}\right)$.

UV/Vis $\left(\mathrm{CHCl}_{3}\right): \lambda_{\mathrm{abs}}^{\max }=363(413) \mathrm{nm}, \varepsilon=57000 \mathrm{M}^{-1} \mathrm{~cm}^{-1}$.

\subsection{Compound 116}

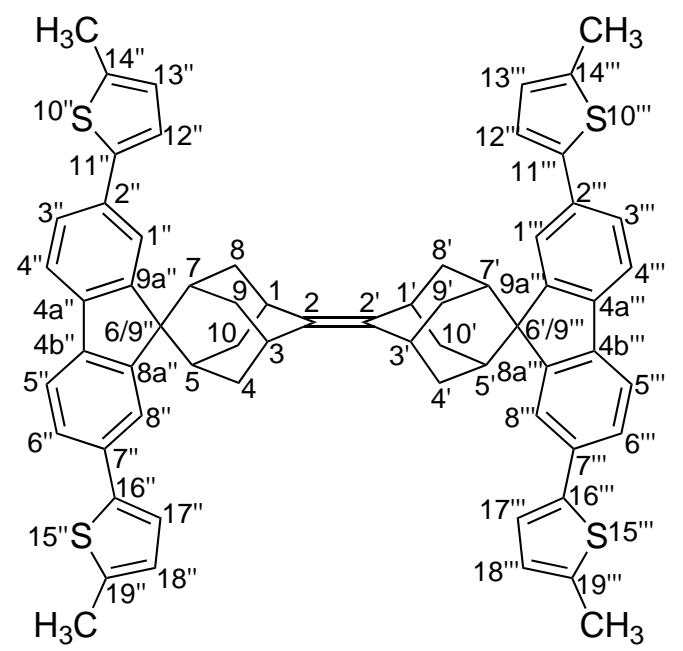

To a freshly prepared solution of $\mathrm{TiCl}_{4}(18 \mu \mathrm{l}, 31 \mathrm{mg}, 0.16 \mathrm{mmol}, 4.6 \mathrm{eq})$ in dry THF $(0.43 \mathrm{ml})$, zinc dust $(<10 \mu \mathrm{m}$ particle size, $22 \mathrm{mg}, 0.33 \mathrm{mmol}, 9.3 \mathrm{eq})$ was added at $0{ }^{\circ} \mathrm{C}$ in one portion. The reaction vessel was purged with argon and sealed. The reaction mixture was heated at $80^{\circ} \mathrm{C}$ (oil bath temperature) for $1 \mathrm{~h}$ to give a brown suspension. After cooling to room temperature, a solution of pyridine $(6.6 \mu \mathrm{l}, 6.5 \mathrm{mg}, 82 \mu \mathrm{mol}, 2.3 \mathrm{eq})$ in dry THF $(93 \mu \mathrm{l})$, followed by the solid compound 105 (35 mg, $71 \mu \mathrm{mol}, 2.0 \mathrm{eq})$ were added. The reaction vessel was purged with argon, sealed and heated to $80^{\circ} \mathrm{C}$ (oil bath temperature) for $16 \mathrm{~h}$. The reaction mixture was cooled to $0{ }^{\circ} \mathrm{C}$ and quenched with $10 \%$ aq. $\mathrm{K}_{2} \mathrm{CO}_{3}(5 \mathrm{ml})$. The mixture was stirred at room temperature for $15 \mathrm{~min}$ and filtered through a pad of Celite $^{\mathrm{TM}}(\varnothing=30 \mathrm{~mm}, h=5 \mathrm{~mm})$. Some product remained as yellow solid on the Celite ${ }^{\mathrm{TM}}$. The Celite ${ }^{\mathrm{TM}}$ was refluxed with $\mathrm{CHCl}_{3}(100 \mathrm{ml})$ for $3 \mathrm{~min}$, filtered hot and washed with hot $\mathrm{CHCl}_{3}(2 \times 30 \mathrm{ml})$. The combined filtrates were dried over $\mathrm{Na}_{2} \mathrm{SO}_{4}$ and concentrated in vacuo to give $33 \mathrm{mg}$ of a yellowish solid with low solubility in common organic solvents including EtOAc, $\mathrm{CH}_{2} \mathrm{Cl}_{2}$ and $n$-hexane. The 
crude product was recrystallized from EtOAc (ca. $100 \mathrm{ml})$ to give $20 \mathrm{mg}(21 \mu \mathrm{mol}$, $60 \%$ ) of the title compound as yellowish solid.

${ }^{1} \mathbf{H}$ NMR $\left(400 \mathrm{MHz}, \mathrm{CDCl}_{3}\right): \delta=1.77-1.91\left(\mathrm{~m}, 12 \mathrm{H}, 4-\mathrm{H}_{\mathrm{a}}, 8-\mathrm{H}_{\mathrm{a}}, 9-\mathrm{H}_{\mathrm{a}}, 10-\mathrm{H}_{\mathrm{a}}\right.$, $4^{\prime}-\mathrm{H}_{\mathrm{a}}, 8^{\prime}-\mathrm{H}_{\mathrm{a}}, 9^{\prime}-\mathrm{H}_{\mathrm{a}}, 10^{\prime}-\mathrm{H}_{\mathrm{a}},\left[1-\mathrm{H}, 3-\mathrm{H}, 1^{\prime}-\mathrm{H}, 3^{\prime}-\mathrm{H}\right]$ or [5-H, 7-H, 5'-, $\left.\left.7^{\prime}-\mathrm{H}\right]\right), 2.56(\mathrm{~d}$, $\left.{ }^{4} J_{\mathrm{H}, \mathrm{H}}=1.2 \mathrm{~Hz}, 4 \times \mathrm{CH}_{3}\right), 3.08-3.19\left(\mathrm{~m}, 8 \mathrm{H}, 4-\mathrm{H}_{\mathrm{b}}, 8-\mathrm{H}_{\mathrm{b}}, 9-\mathrm{H}_{\mathrm{b}}, 10-\mathrm{H}_{\mathrm{b}}, 4^{\prime}-\mathrm{H}_{\mathrm{b}}, 8-\mathrm{H}_{\mathrm{b}}\right.$, $\left.9^{\prime}-\mathrm{H}_{\mathrm{b}}, 10^{\prime}-\mathrm{H}_{\mathrm{b}}\right), 3.43-3.51\left(\mathrm{~m}, 4 \mathrm{H},\left[5-\mathrm{H}, 7-\mathrm{H}, 5^{\prime}-\mathrm{H}, 7^{\prime}-\mathrm{H}\right]\right.$ or [1-H, 3- $\left.\left.\mathrm{H}, 1^{\prime}-\mathrm{H}, 3^{\prime}-\mathrm{H}\right]\right)$, $6.78\left(\mathrm{dd},{ }^{3} J_{\mathrm{H}, \mathrm{H}}=3.6 \mathrm{~Hz},{ }^{4} J_{\mathrm{H}, \mathrm{H}}=1.2 \mathrm{~Hz}, 4 \mathrm{H}, 13^{\prime \prime}-\mathrm{H}, 18^{\prime \prime}-18 " \mathrm{H}, 13^{\prime \prime \prime-H, ~} 18^{\prime \prime \prime-H}\right), 7.17$ $\left(\mathrm{d},{ }^{3} J_{\mathrm{H}, \mathrm{H}}=3.5 \mathrm{~Hz}, 4 \mathrm{H}, 12^{\prime \prime}-\mathrm{H}, 17^{\prime \prime-H}, 122^{\prime \prime}-\mathrm{H}, 17^{\prime \prime \prime-H}\right), 7.61\left(\mathrm{dd},{ }^{3} J_{\mathrm{H}, \mathrm{H}}=7.9 \mathrm{~Hz},{ }^{4} J_{\mathrm{H}, \mathrm{H}}=\right.$ $1.3 \mathrm{~Hz}, 4 \mathrm{H}, 3$ "-H, 6"-H, 3"'-H, 6"'-H), 7.76 (d, ${ }^{3} \mathrm{JH}_{\mathrm{H}, \mathrm{H}}=7.9 \mathrm{~Hz}, 4 \mathrm{H}, 4$ "-H, 5"-H, 4'"-H, $\left.5^{\prime \prime \prime-H}\right), 8.38\left(\mathrm{~d},{ }^{4} \mathrm{~J}_{\mathrm{H}, \mathrm{H}}=1.3 \mathrm{~Hz}, 4 \mathrm{H}, 1\right.$ "-H, 8"-H, 1 "'-H, 8"'-H ppm.

HRMS (MALDI-TOF): $m / z$ calc. for $\mathrm{C}_{64} \mathrm{H}_{56} \mathrm{~S}_{4}\left[\mathrm{M}^{+\cdot}\right]$ 952.3259; found 952.3260 .

$\operatorname{TLC}\left(\mathrm{SiO}_{2}\right): R_{\mathrm{f}}=0.80\left(\mathrm{CH}_{2} \mathrm{Cl}_{2}\right)$.

UV/Vis $\left(\mathrm{CHCl}_{3}\right): \lambda_{\mathrm{abs}}^{\max }=357 \mathrm{~nm}, \varepsilon=92600 \mathrm{M}^{-1} \mathrm{~cm}^{-1}$.

Fluorescence $\left(\mathrm{CHCl}_{3}\right.$, exc. at $\left.348 \mathrm{~nm}\right): \lambda_{\mathrm{em}}^{\max }=394,(416) \mathrm{nm} ; \Phi_{\mathrm{f}}=0.38{ }^{\dagger}$

\subsection{Compound 117}

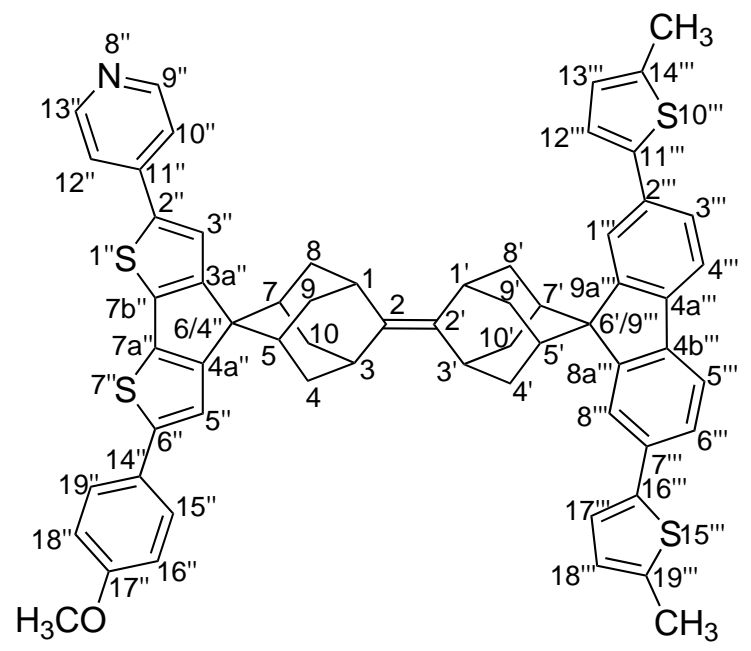

To a freshly prepared solution of $\mathrm{TiCl}_{4}(31 \mu \mathrm{l}, 53 \mathrm{mg}, 0.28 \mathrm{mmol}, 4.6 \mathrm{eq})$ in dry THF $(0.73 \mathrm{ml})$, zinc dust ( $<10 \mu \mathrm{m}$ particle size, $37 \mathrm{mg}, 0.57 \mu \mathrm{mol}, 9.3 \mathrm{eq})$ was added in one portion at $0{ }^{\circ} \mathrm{C}$. The reaction vessel was purged with argon and sealed. The reaction mixture was heated to $80^{\circ} \mathrm{C}$ (oil bath temperature) for $1 \mathrm{~h}$ to give

\footnotetext{
${ }^{\dagger}$ Coumarin 120 in $\mathrm{MeOH}$ as a fl. quantum yield standard with $\Phi_{\mathrm{f}}=0.51^{[132]}$
} 


\section{Chapter 22 $\mid$ COMPOUNDS}

a brown suspension. After cooling to room temperature, a solution of pyridine (11 mg, $11 \mu \mathrm{l}, 0.14 \mathrm{mmol}, 2.3 \mathrm{eq})$ in dry THF $(0.19 \mathrm{ml})$ followed by compound 111 (30 mg, $61 \mu \mathrm{mol}, 1.0 \mathrm{eq})$ and compound 105 (30 mg, $61 \mu \mathrm{mol}, 1.0 \mathrm{eq})$ were added sequentially. The reaction vessel was purged with argon, sealed and heated to $80{ }^{\circ} \mathrm{C}$ (oil bath temperature) for $23 \mathrm{~h}$. The reaction mixture was cooled to $0{ }^{\circ} \mathrm{C}$ and quenched by addition of $10 \%$ aq. $\mathrm{K}_{2} \mathrm{CO}_{3}(10 \mathrm{ml})$. The mixture was diluted with water $(100 \mathrm{ml})$ and brine $(100 \mathrm{ml})$ and extracted with $\mathrm{CHCl}_{3}(10 \times 40 \mathrm{ml})$. The combined organic solutions were dried over $\mathrm{Na}_{2} \mathrm{SO}_{4}$ and concentrated in vacuo to give $61 \mathrm{mg}$ of orange solid. The crude product was purified by column chromatography on silica gel $\left(15 \mathrm{~g} \mathrm{SiO}_{2}, \mathrm{CHCl}_{3}\right)$ and $21 \mathrm{mg}(22 \mu \mathrm{mol}, 37 \%)$ of the title compound were isolated as colorless solid.

NOTE: Compound 116 is poorly soluble in acetone, $\mathrm{CH}_{2} \mathrm{Cl}_{2}$, benzene or THF and sensitive to acids. Dissolving in $\mathrm{CDCl}_{3}$ (containing traces of phosgen and $\mathrm{HCl}$ $(\mathrm{DCl})$ ) yielded a black suspension; therefore $\mathrm{CDCl}_{3}$ was filtered through basic $\mathrm{Al}_{2} \mathrm{O}_{3}$ before preparing NMR samples.

${ }^{1} \mathbf{H}$ NMR $\left(400 \mathrm{MHz}, \mathrm{CDCl}_{3}\right): \delta=1.73-1.98(\mathrm{~m}, 12 \mathrm{H}, 5-\mathrm{H}, 7-\mathrm{H}, 5$ '- $\mathrm{H}, 7$ '-H, $\left.8 \times \mathrm{CH}_{\mathrm{a}}\right), 2.55\left(\mathrm{~s}, 6 \mathrm{H}, 2 \times \mathrm{CH}_{3}\right), 2.67-2.81\left(\mathrm{~m}, 4 \mathrm{H}, 4 \times \mathrm{CH}_{\mathrm{b}}\right), 3.08-3.18(\mathrm{~m}$, $\left.4 \mathrm{H}, 4 \times \mathrm{CH}_{\mathrm{b}}\right), 3.35-3.46\left(\mathrm{~s}, 4 \mathrm{H}, 1-\mathrm{H}, 3-\mathrm{H}, 1^{\prime}-\mathrm{H}, 3^{\prime}-\mathrm{H}\right), 3.87\left(\mathrm{~s}, 3 \mathrm{H}, \mathrm{OCH}_{3}\right), 6.76$ - 6.80 (m, $2 \mathrm{H}, 13$ "'-H, 18'"-H), $6.94-7.00$ (m, $\left.2 \mathrm{H}, 16 "-\mathrm{H}, 18^{\prime \prime}-\mathrm{H}\right), 7.16\left(\mathrm{~d},{ }^{3} \mathrm{~J}_{\mathrm{H}, \mathrm{H}}=\right.$ $3.3 \mathrm{~Hz}, 2 \mathrm{H}, 12$ "'-H, 17"'-H), $7.46-7.51$ (m, 2 H, 10"-H, 12"-H), 7.55 - 7.63 (m, 5 H, 15"-H, 19"-H, 3'"-H, 6"'-H, 5"-H), $7.75-7.79$ (m, 2 H, 4"'-H, 5"'-H), 7.88 (s, 1 H, 3"-H), 8.36 (s, 2 H, 1"'-H, 8"'-H), 8.56 - 8.62 (m, 2 H, 9"-H, 13"-H) ppm.

${ }^{13} \mathrm{C}$ NMR $\left(101 \mathrm{MHz}, \mathrm{CDCl}_{3}\right): \delta=15.7\left(2 \times \mathrm{CH}_{3}\right), 30.6,30.8(\mathrm{C}-1, \mathrm{C}-3, \mathrm{C}-1$ ', C-3'), 34.6, 35.2 (C-5, C-7, C-5', C-7'), 35.9, 36.5, 36.9 (C-4, C-8, C-9, C-10, C-4', C-8', C-9', C-10'), $55.6\left(\mathrm{OCH}_{3}\right), 58.0,59.0$ (C-6/4", C-6'/9"'), 114.7 (C-16", C-18"), 119.2 (C-10", C-12"), 119.9 (C-4"', C-5"'), 122.5 (C-5"), 122.8 (C-12"', C-17"'), 124.8 (C-3"', C-6"'), 125.2 (C-3"), 126.5 (C-13"', C-18"'), 127.1 (C-15", C-19"), 127.2 (C-1"', C-8"'), 127.9 (C-14"), 132.4 (C-2 or C-2'), 132.6 (C-2'", C-7"'), 135.1 (C-2' or C-2), 135.3 (C-7a"), 138.3 (C-2"), 139.4 (C-14"', C-19"'), 139.9 (C-4a"', C-4b"'), 142.6 (C-11"), 143.2 (C-11"', C-16"'), 144.2 (C-7b"), 150.4 (C-9", C-13"), 151.8 (C-8a"', C-9a"'), 157.8 (C-3a"), 159.4, 159.5 (C-4a", C-17") ppm.

HRMS (ESI): $m / z$ calc. for $\mathrm{C}_{62} \mathrm{H}_{54} \mathrm{NOS}_{4}[\mathrm{M}+\mathrm{H}]^{+}$956.3083; found 956.3077 .

MS (ESI, pos.): $m / z(\%)=956.6(100)[\mathrm{M}+\mathrm{H}]^{+}$. 
$\operatorname{TLC}\left(\mathrm{SiO}_{2}\right): R_{\mathrm{f}}=0.49\left(\mathrm{EtOAc}+0.1 \% \mathrm{NEt}_{3}\right)$.

\subsection{Compound 118}

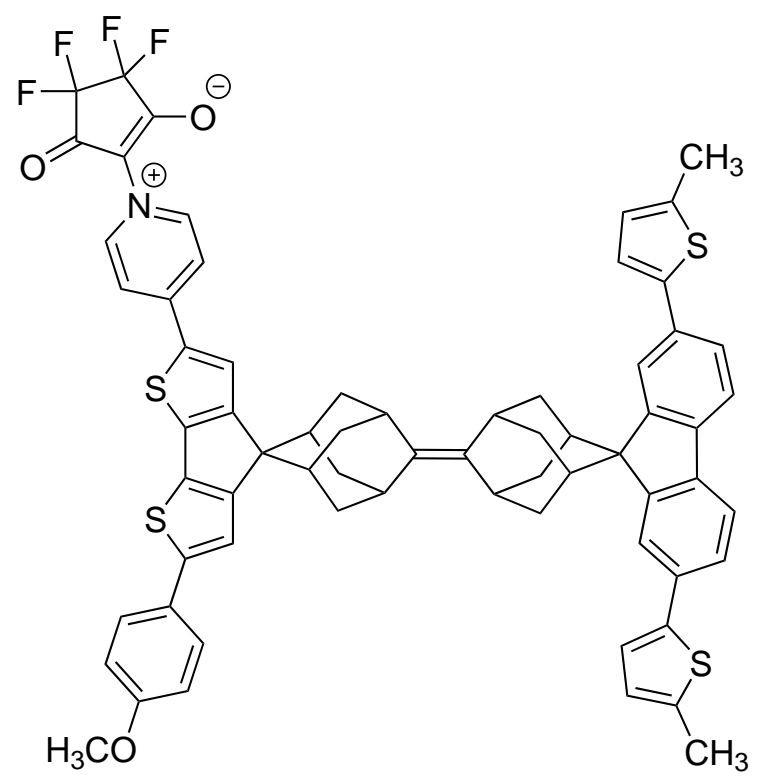

To a suspension of compound 117 (ca. $1 \mathrm{mg}$, ca. $1 \mu \mathrm{mol}, 1 \mathrm{eq})$ in $\mathrm{AcOH}(450 \mu \mathrm{l})$, water $(10 \mu \mathrm{l})$ was added. The mixture was cooled to $0^{\circ} \mathrm{C}$ and perfluorocyclopentene $(50 \mu \mathrm{l}, 79 \mathrm{mg}, 0.37 \mathrm{mmol}$, ca. $370 \mathrm{eq})$ was added using a cold syringe. The sealed reaction vessel was stirred at r.t. for $22 \mathrm{~h}$. TLC $\left(\mathrm{SiO}_{2}\right.$, EtOAc) showed unreacted starting material. After addition of THF $(0.46 \mathrm{ml})$ and perfluorocyclopentene ( $50 \mu \mathrm{l}, 79 \mathrm{mg}, 0.37 \mathrm{mmol}$, ca. $370 \mathrm{eq}$ ) followed by stirring at r.t. for $18 \mathrm{~h}$ and at $56^{\circ} \mathrm{C}$ for $8 \mathrm{~h}$, more perfluorocyclopentene $(50 \mu \mathrm{l}, 79 \mathrm{mg}, 0.37 \mathrm{mmol}$, ca. $370 \mathrm{eq})$ was added, and the reaction mixture was stirred at $56^{\circ} \mathrm{C}$ for $14 \mathrm{~h}$. The dark purple reaction mixture was cooled to room temperature. TLC showed almost full conversion and a complex mixture of products. Ca. $0.1 \mathrm{mg}(0.1 \mu \mathrm{mol}, 10 \%)$ of the title compound was isolated by normal phase HPLC (Knauer Nucleosil $100 \mathrm{OH}, 7 \mu \mathrm{m}$, $\varnothing=10 \mathrm{~mm}, l=25 \mathrm{~cm}$; iso-octane $/ \mathrm{CHCl}_{3}=60: 40 \rightarrow 0: 100$ in $15 \mathrm{~min} ; 5 \mathrm{ml} / \mathrm{min}$ flow; 6.3 min retention time) as dark purple solid. The purity was evaluated by analytical HPLC (figure 66).

${ }^{19}$ F NMR (376.4 MHz, $\left.\mathrm{CD}_{2} \mathrm{Cl}_{2}\right): \delta=-127.4 \mathrm{ppm}$.

HRMS (MALDI-TOF): $m / z$ calc. for $\mathrm{C}_{67} \mathrm{H}_{53} \mathrm{~F}_{4} \mathrm{NO}_{3} \mathrm{~S}_{4}\left[\mathrm{M}^{+\cdot}\right]$ 1123.2844; found 1123.2861. 


\section{Chapter 22 $\mid$ COMPOUNDS}

MS (ESI, pos.): $m / z(\%)=1124.4(100)[\mathrm{M}+\mathrm{H}]^{+}$.

$\operatorname{TLC}\left(\mathrm{SiO}_{2}\right): R_{\mathrm{f}}=0.62\left(\mathrm{CH}_{2} \mathrm{CH}_{2} / \mathrm{MeOH}=100: 1\right)$.

UV/Vis $\left(\mathrm{CHCl}_{3}\right): \lambda_{\mathrm{abs}}^{\max }=548,(357) \mathrm{nm}$.

Fluorescence $\left(\mathrm{CHCl}_{3}\right.$, exc. at $\left.360 \mathrm{~nm}\right): \lambda_{\mathrm{em}}^{\max }=618 \mathrm{~nm}$.
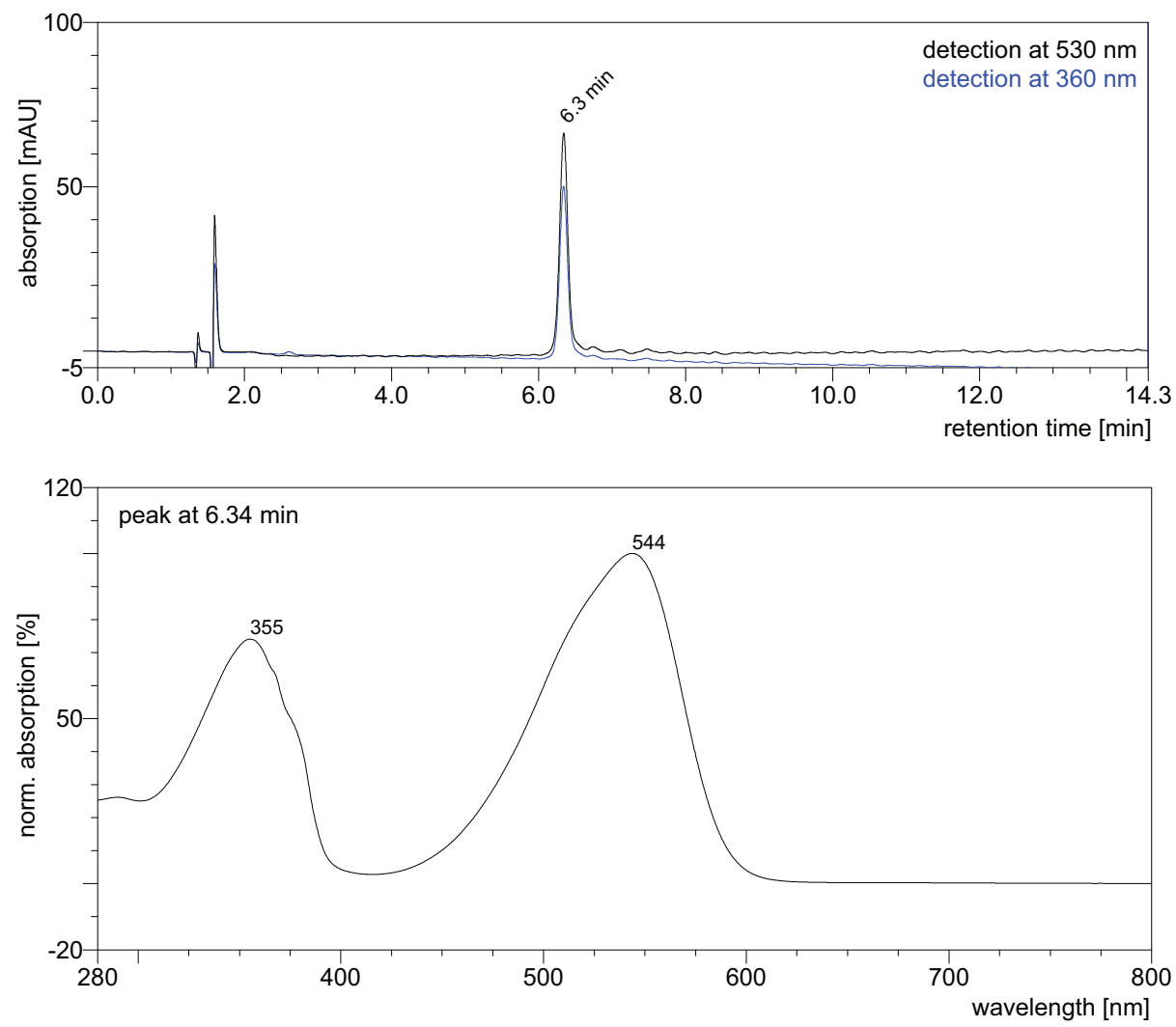

Figure 66: HPLC trace of compound 118 after purification by preparative HPLC (ProntoSIL ${ }^{\mathrm{TM}}$ 120-5 Amino E, $5 \mu \mathrm{m}, \varnothing=4 \mathrm{~mm}, l=25 \mathrm{~cm}$; iso-octane $/ \mathrm{CHCl}_{3}$ $=70: 30$ to $0: 100$ in $15 \mathrm{~min} ; 1.5 \mathrm{ml} / \mathrm{min}$ flow); top: chromatogram; bottom: UV/Vis absorption spectrum at $6.3 \mathrm{~min}$ retention time. 


\section{Part V}

Appendix 



\section{List of abbreviations}

$\begin{array}{ll}\text { aq. } & \text { aqueous } \\ \text { Ac } & \text { acetyl } \\ \text { BINAP } & \text { 2,2'-bis(diphenylphosphino)-1,1'-binaphthyl } \\ \text { Boc } & \text { tert-Butyloxycarbonyl } \\ \text { Bu } & \text { butyl } \\ \text { bp } & \text { boiling point } \\ \text { bpy } & \text { 2,2'-bipyridine } \\ \text { ca. } & \text { circa } \\ \text { conc. } & \text { concentrated } \\ \text { dba } & \text { dibenzylideneacetone } \\ \text { DCE } & \text { 1,2-dichloroethane } \\ \text { DEHP } & \text { bis(2-ethylhexyl) phthalate } \\ \text { DFT } & \text { density functional theory } \\ \text { DME } & \text { 1,2-dimethoxyethane } \\ \text { DMF } & \text { dimethylformamide } \\ \text { DMAP } & \text { 4-dimethylaminopyridine } \\ \text { DMSO } & \text { dimethyl sulfoxide } \\ \text { DNA } & \text { deoxyribonucleic acid } \\ \text { dppf } & \text { 1,1'-bis(diphenylphosphino)ferrocene } \\ \text { EI } & \text { electron ionization } \\ \text { ESI } & \text { electrospray ionisation } \\ \text { Et } & \text { ethyl } \\ \text { ET } & \text { electron transfer } \\ \text { EET } & \text { excitation energy transfer } \\ \text { FRET } & \text { Förster resonance energy transfer } \\ \text { eq } & \text { equivalent } \\ \text { GC } & \text { gas chromatography } \\ \text { GP } & \text { general procedure } \\ \text { HBT } & \text { Hanbury Brown and Twiss } \\ \text { HMDS } & \text { hexamethyldisilazane } \\ \text { HPLC } & \text { high-performance liquid chromatography } \\ \text { HRMS } & \text { high-resolution mass spectrometry } \\ \text { IR } & \text { infrared spectroscopy } \\ \text { IRF } & \text { instrument response function }\end{array}$




\section{\begin{tabular}{l|l} 
Appendix & LIST OF ABBREVIATIONS
\end{tabular}}

MALDI matrix-assisted laser desorption ionization

Me methyl

MS mass spectrometry

NBS N-Bromosuccinimide

NHS $\quad N$-Hydroxysuccinimide

n.d. not determined

NIS $\quad N$-iodosuccinimide

NMR nuclear magnetic resonance

OLED organic light emitting diode

$\mathrm{Ph} \quad$ phenyl

$\operatorname{Pr} \quad$ propyl

quant. quantitative

RESOLFT reversible saturable optical fluorescence transition

RP reversed phase

r.t. room temperature

sat. saturated

SEET singlet excitation energy transfer

TCSPC time-correlated single photon counting

TD time-dependent

TEET triplet excitation energy transfer

TFA trifluoroacetic acid

THF tetrahydrofuran

TLC thin-layer chromatography

TOF time-of-flight

Ts $\quad p$-toluenesulfonyl

UV/Vis ultraviolet-visible 


\section{List of symbols}

\section{Greek letters}

$\beta$

$\varepsilon_{0}$

$\varepsilon_{\mathrm{A}}$

$\varepsilon_{\mathrm{D}}$

$\eta$

$\kappa^{2}$

$\lambda$

$\mu_{\mathrm{A}}$

$\mu_{\mathrm{D}}$

$\tau_{\mathrm{f}}$

$\tau_{\text {rot }}$

$\Phi$

$\Phi_{\mathrm{f}}$ attenuation factor (mediating ability of a bridge)

vacuum permittivity

molar absorption of acceptor

molar absorption of donor

viscosity

orientation factor

wavelength

acceptor transition dipole

donor transition dipole

fluorescence lifetime

rotational characteristic time of anisotropy decay

EET quantum yield

fluorescence quantum yield

\section{Latin letters}

$\begin{array}{ll}f_{\mathrm{D}}(\lambda) & \text { donor emission at wavelength } \lambda \\ G^{(2)} & \text { second-order correlation function } \\ h & \text { Planck's constant } \\ I_{\|} & \text {vertically polarized fluorescence intensity } \\ I_{\perp} & \text { horizontally polarized fluorescence intensity } \\ J_{\mathrm{DA}} & \text { overlap integral of donor emission and acceptor absorption } \\ k_{\ldots} & \text { rate constant of process } \ldots \\ k_{\mathrm{B}} & \text { Boltzmann constant } \\ n & \text { refractive index } \\ N_{\mathrm{A}} & \text { Avogadro's number } \\ P & \text { fluorescence polarization } \\ q_{\mathrm{A}} & \text { acceptor electronic charge } \\ q_{\mathrm{D}} & \text { donor electronic charge } \\ r & \text { fluorescence anisotropy } \\ R_{0} & \text { Förster radius } \\ R_{\mathrm{f}} & \text { retardation factor }\end{array}$


\begin{tabular}{l|l} 
Appendix & LIST OF SYMBOLS
\end{tabular}

$\vec{R}_{\mathrm{DA}} \quad$ interconnecting vector between donor and acceptor chromophores

$\left|\vec{R}_{\mathrm{DA}}\right| \quad$ donor-acceptor distance

$r_{\text {sphere }} \quad$ radius of a sphere corresponding to the hydrodynamic volume

$V_{\text {Coulomb }} \quad$ Coulomb coupling potential

$V_{\mathrm{h}} \quad$ hydrodynamic volume 


\section{List of figures}

1 Relative orientation of energy donor and acceptor transition dipole moments . . . . . . . . . . . . . . . 9

2 Bichromophores for studying through-bond SEET via a rigid polynorbornyl system . . . . . . . . . . . . . . . . . 11

3 Bichromophoric compounds with flexible polymethylene linkers . 16

4 Bichromophore with a flexible peptide linker . . . . . . . . . . 16

5 Bichromophores with a quinoline based oligoamide linker forming rigid helices . . . . . . . . . . . . . . . . 17

6 Bichromophores with polyproline based oligoamide linker . . . . 18

7 Bichromophoric compounds with rigid linear linkers . . . . . . 19

8 Zinc/Zinc-free porphyrin based bichromophores . . . . . . . . . 20

9 Bichromophores with linear linkers . . . . . . . . . . . . 21

10 Bichromophore with an oligospirothioketal linker . . . . . . . 22

11 Bichromophores with steroid based and oligothiophene based linkers . . . . . . . . . . . . . . . . . . . 23

12 Bichromophores with cyclohexane and decalin linkers . . . . . . . 24

13 Example of a bichromophore with a scaffold linker and free chromophore rotation . . . . . . . . . . . . . . . 24

14 Calix[n]arene structure and bichromophore with a calix[4]arene linker ........................ 26

15 Bichromophore with a rigid bridge and fixed chromophore orientation and frontier molecular orbital description of photoinduced electron transfer . . . . . . . . . . . . . . . . . 27

16 Bichromophore with a rigid norbornyl-fused linker, fixed chromophore orientation and absorption transition dipole moments of naphthalene and anthracene . . . . . . . . . . . 28

17 Bichromophore with scaffold linker and fixed chromophore orientation . . . . . . . . . . . . . . . . . 28

18 Cascade EET in artificial light-harvesting antennae . . . . . . . 30

19 Set of four bichromophores used for DNA sequencing . . . . . . . 31

20 Bichromophore with flexible linker and NHS activated ester for labeling of amino biomolecules for super-resolution microscopy . 32 


\section{\begin{tabular}{l|l} 
Appendix & LIST OF FIGURES
\end{tabular}}

21 Hanbury Brown-Twiss setup . . . . . . . . . . . . . . . 40

22 Second-order correlation function versus interphoton time . . . . 42

23 Simplified confocal microscope combined with multiple independent detection channels . . . . . . . . . . . . . . . . 42

24 Schematic representation of adamantane and 2-(2-adamantylidene)adamantane as rigid linkers between orthogonally and parallel arranged chromophores, respectively . . . . . . . . . . 48

25 Absorption spectra of model compounds 33a, 35a and bichromophore $36 \ldots \ldots \ldots \ldots$

26 Absorption and emission spectra of model compounds 33a and 35a with highlighted spectral overlap . . . . . . . . 55

27 Absorption and emission spectra of bichromophore 36 . . . . . 56

28 DFT and TD-DFT optimized geometries of model compound 33a and the transition dipole moments . . . . . . . . . . 57

29 DFT optimized geometry of model compound 35a and absorption transition dipole moment . . . . . . . . . . . . 57

30 DFT optimized ground state geometry of bichromophore 36 and transition dipole moments $\ldots \ldots \ldots \ldots$

31 Absorption and emission spectra of spiroadamantylfluorene 45 . 66

32 DFT and TD-DFT optimized geometries of model compound 45 and the transition dipole moments . . . . . . . . . 67

33 Absorption and emission spectra of compounds 61 and 62 . . . 69

34 Absorption and emission spectra of spiroadamantylbithiophene $77 \quad 73$

35 DFT optimized geometry of model compound 77 and absorption transition dipole moment . . . . . . . . . . 76

36 Absorption spectra of model compounds 45, 77 and bichro-

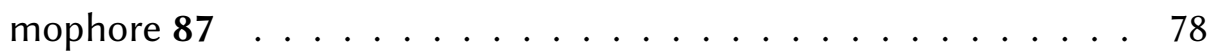

37 Contour plot showing emission intensity vs. excitation and emission wavelength for bichromophore $87 \ldots \ldots$

38 DFT optimized ground state geometry of bichromophore 87 and the transition dipole moments . . . . . . . . . . . . 81 
39 Absorption and emission spectra of model compounds $\mathbf{4 5}$ and 77 with highlighted spectral overlap . . . . . . . . . 82

40 Comparison of bichromophores with asymmetric and symmetric blue chromophore . . . . . . . . . . . . . . . . . . . . 84

41 Absorption and emission spectra of model compound B (105) with highlighted spectral overlap . . . . . . . . . . . . . 89

42 DFT and TD-DFT optimized geometries of model compound B (105) and transition dipole moments . . . . . . . . . . 90

43 DFT optimized ground state geometry of bichromophore $\mathbf{B} \perp \mathbf{B}(\mathbf{1 0 7})$ and the transition dipole moments . . . . . . . . . . 91

44 DFT optimized geometry of model compound R (112) and absorption $\left(\mathrm{S}_{0} \rightarrow \mathrm{S}_{1}\right)$ transition dipole moment shown as red doubleheaded arrow. $\mathrm{H}$ atoms are omitted for clarity. . . . . . . . . 95

45 Absorption and emission spectra of model compounds B (105) and $\mathbf{R}(\mathbf{1 1 2}) \ldots \ldots \ldots \ldots$. . . . . . . . . . . . . . . . 97

46 Contour plot showing emission intensity vs. excitation and emission wavelength for bichromophore $\mathbf{B} \perp \mathbf{R}(\mathbf{1 1 4}) \ldots \ldots$

47 DFT optimized ground state geometry of bichromophore $\mathbf{B} \perp \mathbf{R}(\mathbf{1 1 4})$ and the transition dipole moments . . . . . . . . . 100

48 Absorption and emission spectra of $\mathbf{B}(\mathbf{1 0 5}), \mathbf{R}(\mathbf{1 1 2}), \mathbf{B} \perp \mathbf{B}(\mathbf{1 0 7})$, $\mathbf{B} \| \mathbf{B}(\mathbf{1 1 6}), \mathbf{B} \perp \mathbf{R}(\mathbf{1 1 4})$ and $\mathbf{B} \| \mathbf{R}(\mathbf{1 1 8}) \ldots \ldots \ldots 5$

49 DFT optimized ground state geometry of bichromophore B $\| \mathbf{B}(\mathbf{1 1 6})$ and the transition dipole moments . . . . . . . . . 106

50 DFT optimized ground state geometry of bichromophore B $\| \mathbf{R}(\mathbf{1 1 8})$ and the transition dipole moments . . . . . . . . 107

51 Time-resolved fluorescence anisotropy of compounds $\mathbf{B} \perp \mathbf{B}$ (107), B $\| \mathbf{B}(\mathbf{1 1 6})$ and $\mathbf{B}(\mathbf{1 0 5})$ in DEHP at low tem-

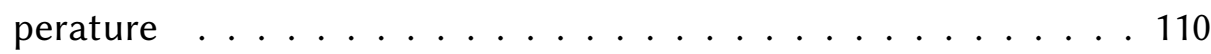

52 Excitation spectra and steady state anisotropy of $\mathbf{B} \perp \mathbf{B}$ (107), B $\| \mathbf{B}(\mathbf{1 1 6})$ and $\mathbf{B}(\mathbf{1 0 5}) \ldots \ldots . \ldots 111$

53 Excitation spectrum and steady state anisotropy of compound R (112) . . . . . . . . . . . . . . . . . . . . . . . 113 


\section{\begin{tabular}{l|l} 
Appendix & LIST OF FIGURES
\end{tabular}}

54 Excitation spectrum and steady state anisotropy of compounds $\mathbf{B} \perp \mathbf{R}(\mathbf{1 1 4})$ and $\mathbf{B} \| \mathbf{R}(\mathbf{1 1 8}) \ldots \ldots \ldots$

55 Corrected anisotropy of compounds $\mathbf{B} \perp \mathbf{R}(\mathbf{1 1 4}), \mathbf{B} \| \mathbf{R}(\mathbf{1 1 8})$ and anisotropy of $\mathbf{R}(\mathbf{1 1 2}) \ldots \ldots \ldots$. . . . . . . . . . 116

56 Time-resolved anisotropy decays for compounds $\mathbf{R}$ (112), $\mathbf{B} \| \mathbf{R}(\mathbf{1 1 8})$ and $\mathbf{B} \perp \mathbf{R}(\mathbf{1 1 4})$ in DEHP . . . . . . . . . . 118

57 Anisotropy decays of $\mathbf{B} \perp \mathbf{B}$ (107), $\mathbf{B} \| \mathbf{B}$ (116) and $\mathbf{B}(\mathbf{1 0 5})$ in DEHP at different temperatures . . . . . . . . . . . . 120

58 Rotational (longest) characteristic time of anisotropy decays of $\mathbf{B}$ (105), $\mathbf{B} \perp \mathbf{B}(\mathbf{1 0 7})$ and $\mathbf{B} \| \mathbf{B}(\mathbf{1 1 6})$ as a function of solvent viscosity 121

59 DFT structures of $\mathbf{B}(\mathbf{1 0 5}), \mathbf{B} \perp \mathbf{B}(\mathbf{1 0 7})$ and $\mathbf{B} \| \mathbf{B}(\mathbf{1 1 6})$ drawn inside spheres corresponding to the effective hydrodynamic volumes 122

60 Measured cross correlation functions of antibunching experiments 124

61 Adamantane and 2-(2-adamantylidene)adamantane as rigid linkers between two chromophores . . . . . . . . . . . . . . 127

62 Bichromophoric compounds with rigid linkers arranged in orthogonal $(\mathbf{B} \perp \mathbf{B}, \mathbf{B} \perp \mathbf{R})$ and parallel geometry $(\mathbf{B}\|\mathbf{B}, \mathbf{B}\| \mathbf{R})$, as well as model compounds B and $\mathbf{R} \ldots \ldots \ldots$. . . . . . . 128

63 Transferring the transition dipole moment from the excited to the ground state geometry . . . . . . . . . . . . . . . 143

64 Assumed positions of transition dipole moments . . . . . . . . . . 144 


\section{List of Schemes}

1 Radiative and nonradiative energy transfer processes. . . . . . . 4

2 Sequential and coherent energy transfer processes . . . . . . . . 4

3 Singlet and triplet excitation energy transfer . . . . . . . . . 5

4 Interactions enabling EET between independent and linked chromophores . . . . . . . . . . . . . . . . 6

5 Singlet excitation energy transfer via Förster resonance energy transfer ...................... 7

6 Singlet and triplet excitation energy transfer via Dexter electron exchange mechanism . . . . . . . . . . . . . 10

$7 \quad$ McConnell superexchange model . . . . . . . . . . . . . 13

8 Fluorescence anisotropy measurement using vertically/horizontally oriented polarization filters . . . . . . . . . . . 34

9 Photoselection . . . . . . . . . . . . . . . . . 34

10 Singlet-singlet annihilation in a bichromophoric compound . . . . 44

11 Strategies to link fluorescent dyes rigidly and without rotation via an adamantane residue . . . . . . . . . . . . 51

12 Spiroketal formation under Dean-Stark conditions . . . . . . . . . 53

13 Synthesis of fluorene related compounds with spiroadamantyl linkers . . . . . . . . . . . . . . . . . . . 62

14 First synthetic approach to compound $45 \ldots 63$

15 Preparation of compound $45 \ldots$. . . . . . . . . . . . 64

16 Synthesis route to biphenyl dye precursor $48 \ldots \ldots 65$

17 Modification of compound $45 \ldots \ldots$. . . . . . . . . 68

18 Template for red fluorescent bithiophene dye and methodology for bridging of bithiophene . . . . . . . . . . . . . 71

19 Synthesis of adamantylbridged bithiophene dye $77 \ldots 72$

20 Synthesis of building block $83 \ldots \ldots$. . . . . . . . . 75

21 Building block 83 is applicable for the synthesis of bithiophene dye precursor $75 \ldots \ldots \ldots \ldots \ldots 76 \ldots \ldots \ldots$ 


\section{\begin{tabular}{l|l} 
Appendix & LIST OF SCHEMES
\end{tabular}}

22 Synthesis of bichromophore $87 \ldots \ldots$. . . . . . . . 77

23 Synthesis of building block $92 \ldots \ldots$. . . . . . . . . . 85

24 Synthesis of building blocks 92 and $103 \ldots \ldots$. . . . . . 86

25 Synthesis of model compound B (105) and bichromophore B $\perp$ B (107) . . . . . . . . . . . . . . . . . . . 87

26 Synthesis of "red" fluorescent model compound R (112) . . . . . 94

27 Synthesis of bichromophore $114 \ldots \ldots$. . . . . . . 98

28 Synthesis of $\mathbf{B} \| \mathbf{B}(\mathbf{1 1 6})$ and $\mathbf{B} \| \mathbf{R}(\mathbf{1 1 8})$ with parallel orientation of fluorophores . . . . . . . . . . . . . . 103

29 Attaching dye precursors $\mathbf{2 4}$ or $\mathbf{2 5}$ to adamantane derivatives 23a and 23b . . . . . . . . . . . . . . . . . . . . . . . 127

30 Setup for time-dependent fluorescenece anisotropy measurements 137

31 Confocal microscope combined with two independent detection channels . . . . . . . . . . . . . . . . . . 139 


\section{List of tables}

1 Photophysical properties of model compounds 33a and 35a, as well as compound $36 \ldots \ldots \ldots$. . . . . . . . . . . 54

2 Angles between transition dipole moments and interconnecting vector between donor and acceptor, orientation factor, distance between the donor and acceptor, Förster radius, and estimated FRET efficiency of compound $36 \ldots \ldots$. . . . . . . . . . 59

3 Photophysical properties of compounds 61 and $62 \ldots \ldots 9$

4 Photophysical properties of model compounds 45, 77 and bichromophore $87 \ldots \ldots \ldots$. . . . . . . . . . . 80

5 Angles between transition dipole moments and interconnecting vector between donor and acceptor, orientation factor, distance between the donor and acceptor, Förster radius, and estimated FRET efficiency of compound $87 \ldots \ldots$. . . . . . . . 82

6 Photophysical properties of model compound B (105) and bichromophore $\mathbf{B} \perp \mathbf{B}(\mathbf{1 0 7}) \ldots \ldots$. . . . . . . . . . . 88

7 Angles between transition dipole moments and interconnecting vector between donor and acceptor, orientation factor, distance between the donor and acceptor, Förster radius, and estimated FRET efficiency of $\mathbf{B} \perp \mathbf{B}(\mathbf{1 0 7}) \ldots \ldots . \ldots . \ldots 91$

8 Photophysical properties of "red" fluorescent model compound R (112) . . . . . . . . . . . . . . . . . . . . . . 95

9 Photophysical properties of bichromophore $\mathbf{B} \perp \mathbf{R}(\mathbf{1 1 4}) \ldots \ldots 9$

10 Angles between transition dipole moments and interconnecting vector between donor and acceptor, orientation factor, distance between the donor and acceptor, Förster radius, and estimated FRET efficiency of $\mathbf{B} \perp \mathbf{R}(\mathbf{1 1 4}) \ldots \ldots \ldots 10 \ldots$

11 Photophysical properties of bichromophores with parallel chromophores . . . . . . . . . . . . . . . . . . . 104 


\section{Appendix $\mid$ LIST OF TABLES}

12 Angles between transition dipole moments and interconnecting vector between donor and acceptor, orientation factor, distance between the donor and acceptor, Förster radius, and estimated FRET efficiency of $\mathbf{B} \| \mathbf{B}(\mathbf{1 1 6})$ and $\mathbf{B} \| \mathbf{R}(\mathbf{1 1 8}) \ldots \ldots . \ldots 108$

13 Calculated hydrodynamic volumes and corresponding sphere radii 121

14 Calculated distance between chromophores, orientation factor, Förster radius and resulting FRET efficiency for bichromophores . 130

15 Stains used for developing TLC plates . . . . . . . . . . . 134 


\section{References}

[1] R. Coughlan, LIFE 1954, 37, 61-65.

[2] Z.-Q. You, C.-P. Hsu, Int. J. Quant. Chem. 2014, 114, 102-115.

[3] a) R. Croce, H. van Amerongen, Nat. Chem. Biol. 2014, 10, 492-501; b) M. Sener, J. Strümpfer, J. Hsin, D. Chandler, S. Scheuring, C. N. Hunter, K. Schulten, ChemPhysChem 2011, 12, 518-531; c) C. König, J. Neugebauer, ChemPhysChem 2012, 13, 386-425; d) D.-G. Xu, T. M. Nordlund, Biophys. J. 2000, 78, 1042-1058; e) C. T. Middleton, K. de La Harpe, C. Su, Y. K. Law, C. E. Crespo-Hernández, B. Kohler, Annu. Rev. Phys. Chem. 2009, 60, 217-239.

[4] a) H. Nakanotani, T. Higuchi, T. Furukawa, K. Masui, K. Morimoto, M. Numata, H. Tanaka, Y. Sagara, T. Yasuda, C. Adachi, Nat. Commun. 2014, 5; b) R. Ziessel, A. Harriman, Chem. Commun. 2011, 47, 611-631; c) A. R. S. Kandada, G. Grancini, A. Petrozza, S. Perissinotto, D. Fazzi, S. S. K. Raavi, G. Lanzani, Sci. Rep. 2013, 3, 2073; d) H. Yersin in Transition Metal and Rare Earth Compounds: Excited States, Transitions, Interactions III, Springer Berlin Heidelberg, Berlin, Heidelberg, 2004, pp. 1-26; e) S. Nampalli, M. Khot, S. Kumar, Tetrahedron Lett. 2000, 41, 8867-8871; f) M. Aparna, K. Pankaj, M. N. Kamalasanan, C. Subhas, Semicond. Sci. Technol. 2006, 21, R35.

[5] a) Y. R. Khan, T. E. Dykstra, G. D. Scholes, Chem. Phys. Lett. 2008, 461, 305-309; b) A. Muñoz-Losa, C. Curutchet, B. P. Krueger, L. R. Hartsell, B. Mennucci, Biophys. J. 2009, 96, 4779-4788; c) W. Barford, J. Phys. Chem. A 2010, 114, 11842-11843; d) H. Langhals, A. J. Esterbauer, A. Walter,

E. Riedle, I. Pugliesi, J. Am. Chem. Soc. 2010, 132, 16777-16782; e) D. Beljonne, C. Curutchet, G. D. Scholes, R. J. Silbey, J. Phys. Chem. B 2009, 113, 6583-6599; f) K. F. Wong, B. Bagchi, P. J. Rossky, J. Phys. Chem. A 2004, 108, 5752-5763.

[6] B. Valeur, M. Berberan-Santos, Molecular Fluorescence: Principles and Applications, Wiley-VCH, Weinheim, 2012.

[7] B. W. v. d. Meer, G. Coker, S. Y. S. Chen, Resonance energy transfer : theory and data, VCH, New York, 1994. 


\section{Appendix $\mid$ REFERENCES}

[8] B. Albinsson, J. Martensson, Phys. Chem. Chem. Phys. 2010, 12, 73387351.

[9] K. Rohatgi-Mukherjee, Fundamentals of Photochemistry, New Age International, 1978.

[10] C.-P. Hsu, Acc. Chem. Res. 2009, 42, 509-518.

[11] D. Gosztola, B. Wang, M. R. Wasielewski, J. Photochem. Photobiol. 1996, 102, 71-80.

[12] C. Curutchet, F. A. Feist, B. V. Averbeke, B. Mennucci, J. Jacob, K. Mullen, T. Basche, D. Beljonne, Phys. Chem. Chem. Phys. 2010, 12, 7378-7385.

[13] N. G. Hendricks, N. M. Lareau, S. M. Stow, J. A. McLean, R. R. Julian, J. Am. Chem. Soc. 2014, 136, 13363-13370.

[14] T. Förster, Naturwissenschaften 1946, 33, 166-175.

[15] T. Förster, Ann. Phys. 1948, 437, 55-75.

[16] T. Förster, Z. Naturforschg. 1949, 4a, 321.

[17] T. Förster, Discuss. Faraday Soc. 1959, 27, 7-17.

[18] D. L. Dexter, J. Chem. Phys. 1953, 21, 836-850.

[19] G. D. Scholes, Annu. Rev. Phys. Chem. 2003, 54, 57-87.

[20] K. Pettersson, A. Kyrychenko, E. Rönnow, T. Ljungdahl, J. Mårtensson, B. Albinsson, J. Phys. Chem. A 2006, 110, 310-318.

[21] W. Lin, L. Yuan, Z. Cao, Y. Feng, J. Song, Angew. Chem. Int. Ed. Engl. 2010, 49, 375-379.

[22] J. Kroon, A. M. Oliver, M. N. Paddon-Row, J. W. Verhoeven, J. Am. Chem. Soc. 1990, 112, 4868-4873.

[23] G.-S. Jiao, L. H. Thoresen, K. Burgess, J. Am. Chem. Soc. 2003, 125, 1466814669.

[24] C.-H. Tung, L.-P. Zhang, Y. Li, H. Cao, Y. Tanimoto, J. Phys. Chem. 1996, $100,4480-4484$.

[25] M. Vestergaard, K. Kerman, I. Hsing, E. Tamiya, Nanobiosensors and Nanobioanalyses, Springer, Tokyo, 2015.

[26] L. Novotny, B. Hecht, Principles of Nano-Optics, Cambridge University Press, 2012. 
[27] R. S. Knox, H. van Amerongen, J. Phys. Chem. B 2002, 106, 5289-5293.

[28] H. Oevering, J. W. Verhoeven, M. N. Paddon-Row, E. Cotsaris, N. S. Hush, Chem. Phys. Lett. 1988, 143, 488-495.

[29] H. M. McConnell, J. Chem. Phys. 1961, 35, 508-515.

[30] B. Di Bartolo, O. Forte, Frontiers of Optical Spectroscopy: Investigating Extreme Physical Conditions with Advanced Optical Techniques, Springer Netherlands, 2005.

[31] M. Gilbert, B. Albinsson, Chem. Soc. Rev. 2015, 44, 845-862.

[32] B. Albinsson, M. P. Eng, K. Pettersson, M. U. Winters, Phys. Chem. Chem. Phys. 2007, 9, 5847-5864.

[33] A. C. Benniston, A. Harriman, Chem. Soc. Rev. 2006, 35, 169-179.

[34] L. Berstis, K. K. Baldridge, Phys. Chem. Chem. Phys. 2015, 17, 3084230853.

[35] M. A. H. Alamiry, J. P. Hagon, A. Harriman, T. Bura, R. Ziessel, Chem. Sci. 2012, 3, 1041-1048.

[36] V. Russo, C. Curutchet, B. Mennucci, J. Phys. Chem. B 2007, 111, 853-863.

[37] S. Speiser, Chem. Rev. 1996, 96, 1953-1976.

[38] O. Schnepp, M. Levy, J. Am. Chem. Soc. 1962, 84, 172-177.

[39] K. Hisada, A. Tsuchida, S. Ito, M. Yamamoto, J. Phys. Chem. B 1998, 102, 2640-2645.

[40] J. W. Park, B. A. Lee, S. Y. Lee, J. Phys. Chem. B 1998, 102, 8209-8215.

[41] B. Chen, X. Sun, R. E. Evans, R. Zhou, J. N. Demas, C. O. Trindle, G. Zhang, J. Phys. Chem. A 2015, 119, 8854-8859.

[42] J. Kwon, J. Hwang, J. Park, G. R. Han, K. Y. Han, S. K. Kim, Sci. Rep. 2015, 5,17804 .

[43] A. V. Saura, M. J. Marin, M. I. Burguete, D. A. Russell, F. Galindo, S. V. Luis, Org. Biomol. Chem. 2015, 13, 7736-7749.

[44] C. C. Hofmann, S. M. Lindner, M. Ruppert, A. Hirsch, S. A. Haque, M. Thelakkat, J. Kohler, Phys. Chem. Chem. Phys. 2010, 12, 14485-14491.

[45] C. C. Hofmann, P. Bauer, S. A. Haque, M. Thelakkat, J. Köhler, J. Chem. Phys. 2009, 131, 144512. 


\section{Appendix $\mid$ REFERENCES}

[46] C. Scharf, K. Peter, P. Bauer, C. Jung, M. Thelakkat, J. Köhler, Chem Phys. 2006, 328, 403-409.

[47] K. J. Lee et al., Phys .Chem. Chem. Phys. 2016, 18, 7875-7887.

[48] M. Tsushima, N. Ohta, J. Chem. Phys. 2004, 120, 6238-6245.

[49] M. Hasegawa, S. Enomoto, T. Hoshi, K. Igarashi, T. Yamazaki, Y. Nishimura, S. Speiser, I. Yamazaki, J. Phys. Chem. B 2002, 106, 4925-4932.

[50] D. M. Guldi, S. González, N. Martín, A. Antón, J. Garín, J. Orduna, J. Org. Chem. 2000, 65, 1978-1983.

[51] M. Asano-Someda, A. van der Est, U. Krüger, D. Stehlik, Y. Kaizu, H. Levanon, J. Phys. Chem. A 1999, 103, 6704-6714.

[52] P. Klán, P. J. Wagner, J. Am. Chem. Soc. 1998, 120, 2198-2199.

[53] D. M. Guldi, M. Maggini, G. Scorrano, M. Prato, J. Am. Chem. Soc. 1997, 119, 974-980.

[54] N. Amdursky, ChemPlusChem 2015, 80, 1075-1095.

[55] M. Wolffs, N. Delsuc, D. Veldman, N. V. Anh, R. M. Williams, S. C. J. Meskers, R. A. J. Janssen, I. Huc, A. P. H. J. Schenning, J. Am. Chem. Soc. 2009, 131, 4819-4829.

[56] A. Shah, B. Adhikari, S. Martic, A. Munir, S. Shahzad, K. Ahmad, H.-B. Kraatz, Chem. Soc. Rev. 2015, 44, 1015-1027.

[57] Y.-T. Long, E. Abu-Irhayem, H.-B. Kraatz, Chem. Eur. J. 2005, 11, 51865194.

[58] J. Juhaniewicz, J. Pawlowski, S. Sek, Isr. J. Chem. 2015, 55, 645-660.

[59] B. Schuler, E. A. Lipman, P. J. Steinbach, M. Kumke, W. A. Eaton, Proc. Natl. Acad. Sci. USA 2005, 102, 2754-2759.

[60] R. B. Best, K. A. Merchant, I. V. Gopich, B. Schuler, A. Bax, W. A. Eaton, Proc. Natl. Acad. Sci. USA 2007, 104, 18964-18969.

[61] M. Hoefling, N. Lima, D. Haenni, C. A. M. Seidel, B. Schuler, H. Grubmüller, PLoS ONE 2011, 6, e19791.

[62] H. E. Zimmerman, T. D. Goldman, T. K. Hirzel, S. P. Schmidt, J. Org. Chem. 1980, 45, 3933-3951. 
[63] H. Langhals, S. Poxleitner, O. Krotz, T. Pust, A. Walter, Eur. J. Org. Chem. 2008, 2008, 4559-4562.

[64] H. Langhals, B. Böck, T. Schmid, A. Marchuk, Chem. Eur. J. 2012, 18, 13188-13194.

[65] P. Kolle, I. Pugliesi, H. Langhals, R. Wilcken, A. J. Esterbauer, R. de VivieRiedle, E. Riedle, Phys. Chem. Chem. Phys. 2015, 17, 25061-25072.

[66] P. Wessig, N. Behrends, M. U. Kumke, U. Eisold, Eur. J. Org. Chem. 2016.

[67] A. M. Oliver, M. N. Paddon-Row, J. Chem. Soc. Perkin Trans. 1 1990, 11451150 .

[68] D. Opris, I. Grosu, L. Toupet, G. Ple, A. Terec, S. Mager, L. Muntean, J. Chem. Soc. Perkin Trans. 12001, 2413-2420.

[69] G. L. Closs, P. Piotrowiak, J. M. Maclnnis, G. R. Fleming, J. Am. Chem. Soc. 1988, 110, 2652-2653.

[70] G. L. Closs, M. D. Johnson, J. R. Miller, P. Piotrowiak, J. Am. Chem. Soc. 1989, 111, 3751-3753.

[71] Z. Z. Wu, H. Morrison, J. Am. Chem. Soc. 1992, 114, 4119-4128.

[72] Y. Zhu, G. B. Schuster, J. Am. Chem. Soc. 1993, 115, 2190-2199.

[73] S. A. Jiang, C. Xiao, H. Morrison, J. Org. Chem. 1996, 61, 7045-7055.

[74] M. S. Vollmer, F. Effenberger, T. Stümpfig, A. Hartschuh, H. Port, H. C. Wolf, J. Org. Chem. 1998, 63, 5080-5087.

[75] J. K. Agyin, L. D. Timberlake, H. Morrison, J. Am. Chem. Soc. 1997, 119, 7945-7953.

[76] L.-P. Zhang, B. Chen, L.-Z. Wu, C.-H. Tung, H. Cao, Y. Tanimoto, J. Phys. Chem. A 2003, 107, 3438-3442.

[77] L.-P. Zhang, B. Chen, L.-Z. Wu, C.-H. Tung, H. Cao, Y. Tanimoto, Chem. Eur. J. 2003, 9, 2763-2769.

[78] B. P. Paulson, J. R. Miller, W.-X. Gan, G. Closs, J. Am. Chem. Soc. 2005, $127,4860-4868$.

[79] K. D. Jordan, M. N. Paddon-Row, Chem. Rev. 1992, 92, 395-410.

[80] G. D. Scholes, K. P. Ghiggino, A. M. Oliver, M. N. Paddon-Row, J. Am. Chem. Soc. 1993, 115, 4345-4349. 


\section{Appendix $\mid$ REFERENCES}

[81] G. D. Scholes, K. P. Ghiggino, A. M. Oliver, M. N. Paddon-Row, J. Phys. Chem. 1993, 97, 11871-11876.

[82] M. N. Paddon-Row, Acc. Chem. Res. 1994, 27, 18-25.

[83] R. M. Williams, M. Koeberg, J. M. Lawson, Y.-Z. An, Y. Rubin, M. N. Paddon-Row, J. W. Verhoeven, J. Org. Chem. 1996, 61, 5055-5062.

[84] N. Lokan, M. N. Paddon-Row, T. A. Smith, M. La Rosa, K. P. Ghiggino, S. Speiser, J. Am. Chem. Soc. 1999, 121, 2917-2918.

[85] G. L. Closs, L. T. Calcaterra, N. J. Green, K. W. Penfield, J. R. Miller, J. Phys. Chem. 1986, 90, 3673-3683.

[86] R. Ziessel, M. A. H. Alamiry, K. J. Elliott, A. Harriman, Angew. Chem. 2009, $121,2810-2814$.

[87] B. P. Krueger, G. D. Scholes, G. R. Fleming, J. Phys. Chem. B 1998, 102, 5378-5386.

[88] B. P. Krueger, G. D. Scholes, I. R. Gould, G. R. Fleming, PhysChemComm 1999, 2, 34-40.

[89] N. Vân Anh, F. Schlosser, M. M. Groeneveld, I. H. M. van Stokkum, F. Würthner, R. M. Williams, J. Phys. Chem. C 2009, 113, 18358-18368.

[90] R. M. Williams, Thesis, 1996.

[91] J. R. Platt, J. Chem. Phys. 1949, 17, 484-495.

[92] P. Belser, S. Bernhard, C. Blum, A. Beyeler, L. De Cola, V. Balzani, Coord. Chem. Rev. 1999, 190-192, 155-169.

[93] J. Ju, C. Ruan, C. W. Fuller, A. N. Glazer, R. A. Mathies, Proc. Natl. Acad. Sci. USA 1995, 92, 4347-4351.

[94] A. Harriman, L. Mallon, R. Ziessel, Chem. - Eur. J. 2008, 14, 11461-11473.

[95] L. E. Hood, M. W. Hunkapiller, L. M. Smith, Genomics 1987, 1, $201-212$.

[96] L. M. Smith, J. Z. Sanders, R. J. Kaiser, P. Hughes, C. Dodd, C. R. Connell, C. Heiner, S. B. H. Kent, L. E. Hood, Nature 1986, 321, 674-679.

[97] C.-P. Hsu, G. R. Fleming, M. Head-Gordon, T. Head-Gordon, J. Chem. Phys. 2001, 114, 3065-3072.

[98] D. Schwarzer, C. Hanisch, P. Kutne, J. Troe, J. Phys. Chem. A 2002, 106, 8019-8028. 
[99] G. J. Hedley, A. Ruseckas, A. C. Benniston, A. Harriman, I. D. W. Samuel, J. Phys. Chem. A 2015, 119, 12665-12671.

[100] J. B. Issa, A. S. Salameh, E. W. Castner, J. F. Wishart, S. S. Isied, J. Phys. Chem. B 2007, 111, 6878-6886.

[101] T. Ikeda, B. Lee, S. Tazuke, A. Takenaka, J. Am. Chem. Soc. 1990, 112, 46504656.

[102] T. Ikeda, B. Lee, S. Kurihara, S. Tazuke, S. Ito, M. Yamamoto, J. Am. Chem. Soc. 1988, 110, 8299-8304.

[103] R. S. Moog, A. Kuki, M. D. Fayer, S. G. Boxer, Biochemistry 1984, 23, 15641571.

[104] J. Hofkens et al., Proc. Natl. Acad. Sci. USA 2003, 100, 13146-13151.

[105] C. G. Hübner, G. Zumofen, A. Renn, A. Herrmann, K. Müllen, T. Basché, Phys. Rev. Lett. 2003, 91, 093903.

[106] A. S. Trevor, P. G. Kenneth, Methods Appl. Fluoresc. 2015, 3, 022001.

[107] P. Kapusta, R. Erdmann, U. Ortmann, M. Wahl, J. Fluoresc. 2003, 13, 179183.

[108] J. R. Lakowicz, Principles of fluorescence spectroscopy, 2nd ed., Kluwer Academic/Plenum, New York, 1999, pp. xxiii, 698.

[109] D. M. Jameson, J. A. Ross, Chem. Rev. 2010, 110, 2685-2708.

[110] K. S. Grußmayer, D.-P. Herten in Advanced Photon Counting: Applications, Methods, Instrumentation, (Eds.: P. Kapusta, M. Wahl, R. Erdmann), Springer International Publishing, Cham, 2015, pp. 159-190.

[111] H. Ta, A. Kiel, M. Wahl, D.-P. Herten, Phys. Chem. Chem. Phys. 2010, 12, 10295-10300.

[112] H. Paul, Rev. Mod. Phys. 1982, 54, 1061-1102.

[113] M. Fox, Quantum Optics: An Introduction, OUP Oxford, 2006.

[114] S. Lukishova, SPIE Newsroom 2014.

[115] J. Sykora, K. Kaiser, I. Gregor, W. Bönigk, G. Schmalzing, J. Enderlein, Anal. Chem. 2007, 79, 4040-4049.

[116] C. Gell, D. Brockwell, A. Smith, Handbook of Single Molecule Fluorescence Spectroscopy, OUP Oxford, 2006. 


\section{Appendix $\mid$ REFERENCES}

[117] P. Tinnefeld, K. D. Weston, T. Vosch, M. Cotlet, T. Weil, J. Hofkens, K. Müllen, F. C. De Schryver, M. Sauer, J. Am. Chem. Soc. 2002, 124, 1431014311.

[118] M. Schwoerer, H. Wolf, Organic Molecular Solids, Wiley, Weinheim, 2008.

[119] P. Nalbach, I. Pugliesi, H. Langhals, M. Thorwart, Phys. Rev. Lett. 2012, 108, 218302.

[120] R. C. Fort, P. v. R. Schleyer, Chem. Rev. 1964, 64, 277-300.

[121] M. R. Wasielewski, Chem. Rev. 1992, 92, 435-461.

[122] C. L. Schenck, J. M. Nadeau, Tetrahedron 2010, 66, 462-466.

[123] W. Y. Huang, S. Y. Huang, Macromolecules 2010, 43, 10355-10365.

[124] S. Dufresne, I. U. Roche, T. Skalski, W. G. Skene, J. Phys. Chem. C 2010, 114, 13106-13112.

[125] A. Amer, A. Burkhardt, A. Nkansah, R. Shabana, A. Galal, H. B. Mark, H. Zimmer, Phosphorus Sulfur Silicon Relat. Elem. 1989, 42, 63-71.

[126] J. Kauffman, P. Litak, J. Novinski, C. Kelley, A. Ghiorghis, Y. Qin, J. Fluoresc. 1995, 5, 295-305.

[127] C. J. Kelley, K. Ansu, W. Budisusetyo, A. Ghiorghis, Y. Qin, J. M. Kauffman, J. Heterocyclic Chem. 2001, 38, 11-23.

[128] E. Genin, V. Hugues, G. Clermont, C. Herbivo, M. C. R. Castro, A. Comel, M. M. M. Raposo, M. Blanchard-Desce, Photochem. Photobiol. Sci. 2012, 11, 1756-1766.

[129] M. Pasini, S. Destri, C. Botta, W. Porzio, Tetrahedron 1999, 55, 1498514994.

[130] M. Pasini, S. Destri, C. Botta, W. Porzio, Synt. Met. 2000, 113, 129-133.

[131] S. A. Lee, S. Hotta, F. Nakanishi, J. Phys. Chem. A 2000, 104, 1827-1833.

[132] H. Pal, S. Nad, M. Kumbhakar, J. Chem. Phys. 2003, 119, 443-452.

[133] G. Jones li, W. R. Jackson, A. M. Halpern, Chem. Phys. Letters 1980, 72, 391-395.

[134] D. R. Lide, R. David, CRC Handbook of Chemistry and Physics, Internet version, CRC Press, Boca Raton, FL, 2005. 
[135] Y. Ooyama, S. Inoue, T. Nagano, K. Kushimoto, J. Ohshita, I. Imae, K. Komaguchi, Y. Harima, Angew. Chem. Int. Ed. 2011, 50, 7429-7433.

[136] E. Buncel, A. R. Norris, K. E. Russell, Q. Rev. Chem. Soc. 1968, 22, 123-146.

[137] K. Rurack, M. Spieles, Anal. Chem. 2011, 83, 1232-1242.

[138] S. Van Mierloo, P. J. Adriaensens, W. Maes, L. Lutsen, T. J. Cleij, E. Botek, B. Champagne, D. J. Vanderzande, J. Org. Chem. 2010, 75, 7202-7209.

[139] S. Gronowitz, Y. Zhang, A.-B. Hörnfeldt, E. Högfeldt, P. Spielbüchler, J. B. Pedersen, P. Krogsgaard-Larsen, Acta Chem. Scand. 1992, 46, 654-660.

[140] U. Harm, R. Bürgler, W. Fürbeth, K. M. Mangold, K. Jüttner, Macromol. Symp. 2002, 187, 65-76.

[141] Z. Wu, Z. An, X. Chen, P. Chen, Org. Lett. 2013, 15, 1456-1459.

[142] C. Herbivo, A. Comel, G. Kirsch, M. M. M. Raposo, Tetrahedron 2009, 65, 2079-2086.

[143] Z. Luo, Y. Huang, G. Wei, X. Cheng, M. Prehm, C. Tschierske, Liquid Crystals 2008, 35, 1237-1249.

[144] T. Cardolaccia, A. M. Funston, M. E. Kose, J. M. Keller, J. R. Miller, K. S. Schanze, J. Phys. Chem. B 2007, 111, 10871-10880.

[145] T. Karstens, K. Kobs, J. Phys. Chem. 1980, 84, 1871-1872.

[146] S. Yu, H. Lin, Z. Zhao, Z. Wang, P. Lu, Tetrahedron Lett. 2007, 48, 91129115.

[147] Z. Xia, R. G. Correa, J. K. Das, L. Farhana, D. J. Castro, J. Yu, R. G. Oshima, J. A. Fontana, J. C. Reed, M. I. Dawson, J. Med. Chem. 2012, 55, 233-249.

[148] H.-M. Kang, Y.-K. Lim, I.-J. Shin, H.-Y. Kim, C.-G. Cho, Org. Lett. 2006, 8, 2047-2050.

[149] O. Loog, U. Mäeorg, U. Ragnarsson, Synthesis 2000, 2000, 1591-1597.

[150] C. Thiebes, C. Thiebes, G. K. S. Prakash, N. A. Petasis, G. A. Olah, Synlett 1998, 1998, 141-142.

[151] M. Kimura, S. Kuwano, Y. Sawaki, H. Fujikawa, K. Noda, Y. Taga, K. Takagi, J. Mater. Chem. 2005, 15, 2393.

[152] K. Shen, Y. Fu, J.-N. Li, L. Liu, Q.-X. Guo, Tetrahedron 2007, 63, 1568-1576. 


\section{Appendix $\mid$ REFERENCES}

[153] Z. Gu et al., J. Polym. Sci. Part A: Polym. Chem. 2012, 50, 3848-3858.

[154] K. Toyota, H. Katsuta, T. Iwamoto, N. Morita, Heteroat. Chem. 2011, 22, 531-537.

[155] D. Magde, R. Wong, P. G. Seybold, Photochem. Photobiol. 2002, 75, 327334.

[156] S. E. E. Jr., W. A. Guice, Patent, US3285926A, 1966.

[157] S. E. Ellzey, W. A. Guice, J. Org. Chem. 1966, 31, 1300-1303.

[158] S. Nizamov, K. I. Willig, M. V. Sednev, V. N. Belov, S. W. Hell, Chem. Eur. J. 2012, 18, 16339-48.

[159] T. S. Zhuk, T. Koso, A. E. Pashenko, N. T. Hoc, V. N. Rodionov, M. Serafin, P. R. Schreiner, A. A. Fokin, J. Am. Chem. Soc. 2015, 137, 6577-6586.

[160] D. N. H. Meineke, M. L. Bossi, H. Ta, V. N. Belov, S. W. Hell, Chem. Eur. J. 2017, 23, 2469-2475.

[161] S. K. Mylona, M. J. Assael, K. D. Antoniadis, S. K. Polymatidou, L. Karagiannidis, J. Chem. Eng. Data 2013, 58, 2805-2808.

[162] W. Becker, Advanced Time-Correlated Single Photon Counting Applications, Springer International Publishing, 2015.

[163] H. Ta, J. Keller, M. Haltmeier, S. K. Saka, J. Schmied, F. Opazo, P. Tinnefeld, A. Munk, S. W. Hell, Nat. Commun. 2015, 6.

[164] B. Fückel, G. Hinze, F. Nolde, K. Müllen, T. Basché, Phys. Rev. Lett. 2009, 103, 103003.

[165] J. Tirado-Rives, W. L. Jorgensen, J. Chem. Theory Comput. 2008, 4, 297306.

[166] M. J. Frisch et al., Gaussian 09, Computer Program, 2009.

[167] C. Adamo, D. Jacquemin, Chem. Soc. Rev. 2013, 42, 845-856.

[168] C. Poriel, Y. Ferrand, S. Juillard, P. Le Maux, G. Simonneaux, Tetrahedron 2004, 60, 145-158.

[169] A. Takadate, T. Masuda, C. Murata, T. Tanaka, M. Irikura, S. Goya, Anal. Sci. 1995, 11, 97-101.

[170] S. Y. Cho, A. C. Grimsdale, D. J. Jones, S. E. Watkins, A. B. Holmes, J. Am. Chem. Soc. 2007, 129, 11910-11911. 
[171] F. Dierschke, A. C. Grimsdale, K. Müllen, Synthesis 2003, 2003, 2470-2472.

[172] S. Yamamura, M. Toda, Y. Hirata, Org. Synth. 1973, 53, 86.

[173] T. A. Kelly, D. W. McNeil, Tetrahedron Lett. 1994, 35, 9003-9006.

[174] J. P. Wolfe, S. L. Buchwald, J. Org. Chem. 2000, 65, 1144-1157.

[175] W. A. Marinaro, L. J. Schieber, E. J. Munson, V. W. Day, V. J. Stella, J. Pharm. Sci. 2012, 101, 3190-3198.

[176] R. M. Kellogg, A. P. Schaap, E. T. Harper, H. Wynbert, J. Org. Chem. 1968, 33, 2902-2909.

[177] F. Liu, J. Wang, H. Wu, C. Wang, Patent, CN102285960, 2011.

[178] J. I. G. Cadogan, D. A. Roy, D. M. Smith, J. Chem. Soc. C 1966, 1249-1250.

[179] M. I. Dawson et al., J. Med. Chem. 2008, 51, 5650-5662.

[180] S. Suzuki, R. Sugimura, M. Kozaki, K. Keyaki, K. Nozaki, N. Ikeda, K. Akiyama, K. Okada, J. Am. Chem. Soc. 2009, 131, 10374-10375.

[181] S. Senthilkumar, S. Nath, H. Pal, Photochem. Photobiol. 2004, 80, 104-111. 



\section{Acknowledgments}

I would like to thank all the people who supported me during my doctoral thesis. Without their commitment and support, this project would not have been possible.

First of all, I would like to thank the members of my thesis committee. I thank Prof. Dr. Stefan Hell for the challenging topic and for giving me the opportunity to work in this fantastic environment. I would like to thank Prof. Dr. Ulf Diederichsen for valuable feedback at my yearly progress reports and reviewing my thesis. I am especially grateful to my supervisor Dr. Vladimir Belov for his guidance and support throughout my thesis and for an open door to discuss problems at any time. I could not have imagined having a better adviser and mentor.

Besides my thesis committee, I would like to thank the rest of the examination board, namely Prof. Dr. Marina Bennati, Prof. Dr. Karl Helmut Grubmüller, Prof. Dr. Claudia Höbartner and Prof. Dr. Konrad Koszinowski.

I am very thankful to Mariano Bossi and Haisen Ta for their contributions to the bichromophore characterization. I thank Mariano especially for the fluorescence anisotropy measurements and interpretation, but also for measuring fluorescence lifetimes. And I want to thank Haisen for carrying out and helping me with the interpretation of the fluorescence antibunching experiments.

I would like to thank the members of the Organic Synthesis Project Group, especially Alexey Butkevich, Kirill Kolmakov, Gyuzel Mitronova, Shamil Nizamov, Benoît Roubinet, Maksim Sednev and Heydar Shojaei, for invaluable discussions and sharing their knowledge. At the Facility for Synthetic Chemistry, where I was kindly allowed to work, I like to thank Wilfried Bosch, Mario Fischer, Jens Schimpfhauser and Jan Seikowski for the nice working atmosphere and for passing on their extensive experience in organic synthesis. A special thanks goes to Jürgen Bienert for numerous MS-ESI, GC-MS and HPLC analysis, but also preparative HPLC purifications.

I am also very thankful to all colleagues at the NanoBiophotonics Department, many of whom have become close friends. I want to especially thank my office mate Sebastian Schnorrenberg for many funny or profound conversations, and 


\section{Appendix $\mid$ ACKNOWLEDGMENTS}

also Philipp Alt, Hannah Barthel, Christian Brüser, Lars Frahm, Klaus Gwosch, Elke Hebisch, Isabelle Jansen, Martina Johannsen, Maria Kamper, Timo Konen, Jennifer Masch, and Jasmin Pape for enjoyable lunch and coffee breaks, as well as free time activities and cool parties. I thank Grazvydas Lukinavicius for the nice time in our shared office and his valuable comment at the last second. From the Nucleic Acid Chemistry Group, I want to thank Anne Bartels for valuable advice to solve chemical problems and nice chats. You all together turned everyday life at the MPI-BPC into a great experience.

I would also like to thank Jaydev Jethwa and Marco Roose for technical support as well as Stefanie Kruse and Sarah Leondarakis for help with organizational issues.

At the Faculty of Chemistry at the University Göttingen, I like to thank Dr. Holm Frauendorf and the whole mass spectrometry department for the fast acquisition of HR-MS spectra.

I thank my family and friends for their support during my doctoral research. But my biggest thanks go to you, Maren. I thank you for always being there for me and for your understanding when there was only little time, but most of all for the wonderful moments together. 


\section{Curriculum vitae}

Name Dirk Norbert Hendrik Meineke

Date of birth $\quad 26.03 .1987$

Place of birth Hamburg

Nationality German

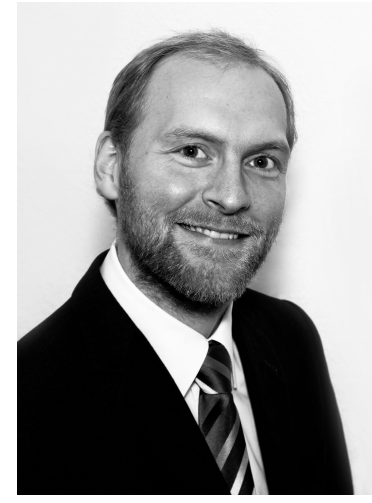

\section{Education}

2013 - present

PhD in Organic Chemistry

University of Göttingen and Max Planck Institute for Biophysical Chemistry, Germany

Thesis: Fluorescent Dyes and Quenchers with rigid Linkers

Supervisors: Prof. Dr. Stefan W. Hell, Prof. Dr. Ulf Diederichsen, Dr. Vladimir N. Belov

Master of Science in Chemistry

University of Göttingen and Max Planck Institute for Biophysical Chemistry, Germany

Master Thesis: Fluorescent Dyes with Multiple Negative Charges and an Amino Group for Reductive Amination of Glycans

Supervisors: Prof. Dr. Stefan W. Hell, Prof. Dr. UIf Diederichsen, Dr. Vladimir N. Belov

$2009-2010 \quad$ Exchange studies in Sweden

Royal Institute of Technology (KTH), Stockholm

Research project: Brønsted Acid Catalyzed Intramolecular

Friedel-Crafts Alkylation: Formation of Spiro Ethers

Supervisor: Dr. Johan Franzén 


\title{
Appendix $\mid$ CURRICULUM VITAE
}

$2006-2009$

\author{
Bachelor of Science in Chemistry \\ University of Göttingen, Germany \\ Bachelor Thesis: Synthese von $\alpha$-hydroxylierten Fettsäuren \\ zum Aufbau von Glycosphingolipiden \\ Supervisors: Prof. Dr. Daniel B. Werz, Dr. Annika Ries
}

\section{Publications}

4. D. N. H. Meineke, M. L. Bossi, H. Ta, V. N. Belov, S. W. Hell, Chem. Eur. J. 2017, 23, 2469-2475.

3. A. N. Butkevich, G. Y. Mitronova, S. C. Sidenstein, J. L. Klocke, D. Kamin, D. N. H. Meineke, E. D’Este, P.-T. Kraemer, J. G. Danzl, V. N. Belov, S. W. Hell, Angew. Chem. Int. Ed. 2016, 55, 3290-3294.

2. G. Y. Mitronova, S. Polyakova, C. A. Wurm, K. Kolmakov, T. Wolfram, D. N. H. Meineke, V. N. Belov, M. John, S. W. Hell, Eur. J. Org. Chem. 2015, 2015, 337-349.

1. K. Kolmakov, C. A. Wurm, D. N. H. Meineke, F. Göttfert, V. P. Boyarskiy, V. N. Belov, S. W. Hell, Chem. Eur. J. 2014, 20, 146-157. 